\title{
Vitamin D and Human Health
}

\author{
Edited by \\ Simon Spedding
}

Printed Edition of the Special Issue Published in Nutrients

MDPI www.mdpi.com/journal/nutrients 
Simon Spedding (Ed.)

\section{Vitamin D and Human Health}

MDPI 
This book is a reprint of papers that appeared in the online open access journal Nutrients (ISSN 2072-6643)

(available at: http://www.mdpi.com/journal/nutrients).

Guest Editor

Simon Spedding

Nutritional Physiology Research Centre

Sansom Institute for Health Research

University of South Australia

GPO Box 2471, Adelaide

South Australia

Australia

Editorial Office

MDPI AG

Klybeckstrasse 64

Basel, Switzerland

\section{Publisher}

Shu-Kun Lin

Assistant Editor

Xiaocen Zhang

\section{Edition 2015}

MDPI • Basel • Beijing • Wuhan

ISBN 978-3-03842-057-6

(C) 2015 by the authors; licensee MDPI, Basel, Switzerland. All articles in this volume are Open Access distributed under the Creative Commons Attribution 3.0 license (http://creativecommons.org/licenses/by/3.0/), which allows users to download, copy and build upon published articles even for commercial purposes, as long as the author and publisher are properly credited, which ensures maximum dissemination and a wider impact of our publications. However, the dissemination and distribution of copies of this book as a whole is restricted to MDPI, Basel, Switzerland. 


\section{Table of Contents}

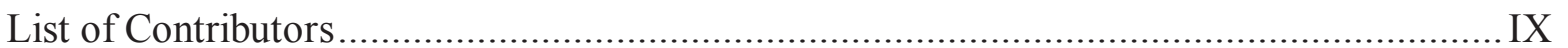

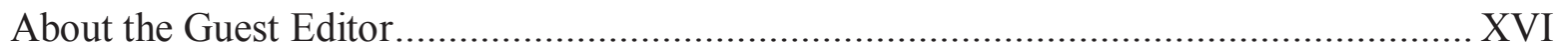

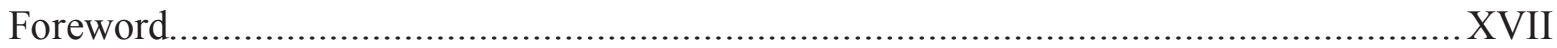

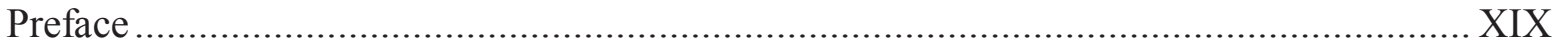

\section{Chapter 1: Overview}

Matthias Wacker and Michael F. Holick

Vitamin D - Effects on Skeletal and Extra-skeletal Health and the Need for Supplementation

Reprinted from: Nutrients 2013, 5, 111-148

http://www.mdpi.com/2072-6643/5/1/111 3

\section{Chapter 2: Vitamin D Intakes and Serum Levels}

\section{Paul J. Veugelers and John Paul Ekwaru}

A Statistical Error in the Estimation of the Recommended Dietary Allowance for Vitamin D Reprinted from: Nutrients 2014, 6, 4472-4475

http://www.mdpi.com/2072-6643/6/10/4472 45

Yi-Sheng Chao, Ludovic Brunel, Peter Faris and Paul J. Veugelers

The Importance of Dose, Frequency and Duration of Vitamin D Supplementation for Plasma 25-Hydroxyvitamin D

Reprinted from: Nutrients 2013, 5(10), 4067-4078

http://www.mdpi.com/2072-6643/5/10/4067. 


\section{Fahad Alshahrani and Naji Aljohani}

Vitamin D: Deficiency, Sufficiency and Toxicity

Reprinted from: Nutrients 2013, 5(9), 3605-3616

http://www.mdpi.com/2072-6643/5/9/3605

\section{Daniel E. Roth, Abdullah Al Mahmud, Rubhana Raqib, Evana Akhtar, Robert E.}

\section{Black and Abdullah H. Baqui}

Pharmacokinetics of High-Dose Weekly Oral Vitamin D3 Supplementation during the Third

Trimester of Pregnancy in Dhaka, Bangladesh

Reprinted from: Nutrients 2013, 5(3), 788-810

http://www.mdpi.com/2072-6643/5/3/788

\section{Chapter 3: Health Impact}

\section{Simon Spedding, Simon Vanlint, Howard Morris and Robert Scragg}

Does Vitamin D Sufficiency Equate to a Single Serum 25-Hydroxyvitamin D Level or Are Different Levels Required for Non-Skeletal Diseases?

Reprinted from: Nutrients 2013, 5(12), 5127-5139

http://www.mdpi.com/2072-6643/5/12/5127 99

\section{Chapter 4: Neoplastic Disorders}

\section{Meis Moukayed and William B Grant}

Molecular Link between Vitamin D and Cancer Prevention

Reprinted from: Nutrients 2013, 5(10), 3993-4021

http://www.mdpi.com/2072-6643/5/10/3993

\section{Aric C. Hall and Mark B. Juckett}

The Role of Vitamin D in Hematologic Disease and Stem Cell Transplantation

Reprinted from: Nutrients 2013, 5(6), 2206-2221

http://www.mdpi.com/2072-6643/5/6/2206 
Lynda Thyer, Emma Ward, Rodney Smith, Maria Giulia Fiore, Stefano Magherini, Jacopo J. V. Branca, Gabriele Morucci, Massimo Gulisano, Marco Ruggiero and Stefania Pacini

A Novel Role for a Major Component of the Vitamin D Axis: Vitamin D Binding Protein-Derived Macrophage Activating Factor Induces Human Breast Cancer Cell Apoptosis through Stimulation of Macrophages

Reprinted from: Nutrients 2013, 5(7), 2577-2589

http://www.mdpi.com/2072-6643/5/7/2577 161

\section{Chapter 5: Cardiovascular Disorders}

Patricia G. Weyland, William B. Grant and Jill Howie-Esquivel

Does Sufficient Evidence Exist to Support a Causal Association between Vitamin D Status and Cardiovascular Disease Risk? An Assessment Using Hill's Criteria for Causality Reprinted from: Nutrients 2014, 6, 3403-3430

http://www.mdpi.com/2072-6643/6/9/3403 177

Michelle C. Mann, Derek V. Exner, Brenda R. Hemmelgarn, Darlene Y. Sola, Tanvir C. Turin, Linda Ellis and Sofia B. Ahmed

Vitamin D Levels Are Associated with Cardiac Autonomic Activity in Healthy Humans Reprinted from: Nutrients 2013, 5(6), 2114-2127

http://www.mdpi.com/2072-6643/5/6/2114 205

Stefania Pacini, Gabriele Morucci, Jacopo J. V. Branca, Stefano Aterini, Marcello Amato, Massimo Gulisano and Marco Ruggiero

Effects of Vitamin D3 and Paricalcitol on Immature Cardiomyocytes: A Novel Role for Vitamin D Analogs in the Prevention of Cardiovascular Diseases

Reprinted from: Nutrients 2013, 5(6), 2076-2092

http://www.mdpi.com/2072-6643/5/6/2076 219

\section{Zhao-Min Liu, Jean Woo, Sheng-Hui Wu and Suzanne C. Ho}

The Role of Vitamin D in Blood Pressure, Endothelial and Renal Function in Postmenopausal Women

Reprinted from: Nutrients 2013, 5(7), 2590-2610

http://www.mdpi.com/2072-6643/5/7/2590 235 


\section{Chapter 6: Metabolic Disorders}

Jia-Yi Dong, Weiguo Zhang, Jiong Jack Chen, Zeng-Li Zhang, Shu-Fen Han and Li-Qiang Qin

Vitamin D Intake and Risk of Type 1 Diabetes: A Meta-Analysis of Observational Studies

Reprinted from: Nutrients 2013, 5(9), 3551-3562

http://www.mdpi.com/2072-6643/5/9/3551 259

\section{Simon Vanlint}

Vitamin D and Obesity

Reprinted from: Nutrients 2013, 5(3), 949-956

http://www.mdpi.com/2072-6643/5/3/949

Brian D. Piccolo, Gregory Dolnikowski, Elias Seyoum, Anthony P. Thomas,

Erik R. Gertz, Elaine C. Souza, Leslie R. Woodhouse, John W. Newman, Nancy L. Keim, Sean H. Adams and Marta D. Van Loan

Association between Subcutaneous White Adipose Tissue and Serum 25-Hydroxyvitamin D in Overweight and Obese Adults

Reprinted from: Nutrients 2013, 5, 3352-3366

http://www.mdpi.com/2072-6643/5/9/3352 278

\section{Chapter 7: Mental Disorders}

\section{Simon Spedding}

Vitamin D and Depression: A Systematic Review and Meta-Analysis Comparing Studies with and without Biological Flaws

Reprinted from: Nutrients 2014, 6(4), 1501-1518

http://www.mdpi.com/2072-6643/6/4/1501 295

Maria A. Polak, Lisa A. Houghton, Anthony I. Reeder, Michelle J. Harper and

Tamlin S. Conner

Serum 25-Hydroxyvitamin D Concentrations and Depressive Symptoms among Young Adult Men and Women

Reprinted from: Nutrients 2014, 6, 4720-4730

http://www.mdpi.com/2072-6643/6/11/4720 312 


\section{Chapter 8: Sports Medicine}

\section{Dana Ogan and Kelly Pritchett}

Vitamin D and the Athlete: Risks, Recommendations, and Benefits

Reprinted from: Nutrients 2013, 5(6), 1856-1868

http://www.mdpi.com/2072-6643/5/6/1856

Tyler Barker, Vanessa T. Henriksen, Thomas B. Martins, Harry R. Hill,

Carl R. Kjeldsberg, Erik D. Schneider, Brian M. Dixon and Lindell K. Weaver

Higher Serum 25-Hydroxyvitamin D Concentrations Associate with a Faster Recovery of Skeletal Muscle Strength after Muscular Injury

Reprinted from: Nutrients 2013, 5(4), 1253-1275

http://www.mdpi.com/2072-6643/5/4/1253 337

\section{Chapter 9: Risk Factors}

\section{Hassanali Vatanparast, Christine Nisbet and Brian Gushulak}

Vitamin D Insufficiency and Bone Mineral Status in a Population of Newcomer Children in Canada

Reprinted from: Nutrients 2013, 5(5), 1561-1572

http://www.mdpi.com/2072-6643/5/5/1561 363

\section{Billie Bonevski, Jamie Bryant, Sylvie Lambert, Irena Brozek and Vanessa Rock}

The ABC of Vitamin D: A Qualitative Study of the Knowledge and Attitudes Regarding Vitamin D Deficiency amongst Selected Population Groups

Reprinted from: Nutrients 2013, 5(3), 915-927

http://www.mdpi.com/2072-6643/5/3/915 375

\section{Ursula Thiem, Bartosz Olbramski and Kyra Borchhardt}

Calcidiol Deficiency in End-Stage Organ Failure and after Solid Organ Transplantation: Status quo

Reprinted from: Nutrients 2013, 5(7), 2352-2371

http://www.mdpi.com/2072-6643/5/7/2352 388

\section{Zohreh Sabbagh, Janet Markland and Hassanali Vatanparast}

Vitamin D Status Is Associated with Disease Activity among Rheumatology Outpatients Reprinted from: Nutrients 2013, 5(7), 2268-2275 
VIII

Anna J. Jovanovich, Adit A. Ginde, John Holmen, Kristen Jablonski,

Rebecca L. Allyn, Jessica Kendrick and Michel Chonchol

Vitamin D Level and Risk of Community-Acquired Pneumonia and Sepsis

Reprinted from: Nutrients 2014, 6(6), 2196-2205

http://www.mdpi.com/2072-6643/6/6/2196

Ulla K. Møller, Susanna við Streym, Lars T. Jensen, Leif Mosekilde, Inez Schoenmakers, Shailja Nigdikar and Lars Rejnmark

Increased Plasma Concentrations of Vitamin D Metabolites and Vitamin D Binding Protein in Women Using Hormonal Contraceptives: A Cross-Sectional Study

Reprinted from: Nutrients 2013, 5(9), 3470-3480

http://www.mdpi.com/2072-6643/5/9/3470

424

Laila Abdel-Wareth, Afrozul Haq, Andrew Turner, Shoukat Khan, Arwa Salem,

Faten Mustafa, Nafiz Hussein, Fasila Pallinalakam, Louisa Grundy,

Gemma Patras and Jaishen Rajah

Total Vitamin D Assay Comparison of the Roche Diagnostics "Vitamin D Total" Electrochemiluminescence Protein Binding Assay with the Chromsystems HPLC Method in a Population with both D2 and D3 forms of Vitamin D

Reprinted from: Nutrients 2013, 5(3), 971-980

http://www.mdpi.com/2072-6643/5/3/971 435 


\section{List of Contributors}

Laila Abdel-Wareth: Pathology \& Laboratory Medicine Institute, Sheikh Khalifa Medical City, Abu Dhabi 51900, UAE

Sean H. Adams: Department of Nutrition \& Graduate Group in Nutritional Biology, University of California, One Shields Avenue, Davis, CA 95616, USA; Obesity \& Metabolism Research Unit, USDA-ARS, Western Human Nutrition Research Center West Health Science Drive, Davis, CA 95616, USA

Sofia B. Ahmed: University of Calgary, 1403 29th St. NW, Calgary, AB T2N 2T9, Canada

Evana Akhtar: International Center for Diarrhoeal Disease Research, Bangladesh (ICDDR,B), GPO Box 128, Dhaka 1000, Bangladesh

Naji Aljohanj: Specialized Diabetes and Endocrine Center, King Fahad Medical City, Riyadh 59046, Saudi Arabia; Faculty of Medicine, King Saud bin Abdulaziz University for Health Sciences, Riyadh 22490, Saudi Arabia; Prince Mutaib Chair for Biomarkers of Osteoporosis, College of Science, King Saud University, Riyadh 11451, Saudi Arabia

Rebecca L. Allyn: Denver Health Medical Center, Denver, CO 80204, USA

Fahad Alshahrani: Department of Medicine, King Abdulaziz Medical City, Riyadh 14611, Saudi Arabia

Marcello Amato: Division of Nephrology and Hemodialysis, Prato Hospital, 59100 Prato, Italy

Stefano Aterini: Division of Nephrology and Hemodialysis, Prato Hospital, 59100 Prato, Italy

Abdullah H. Baqui: Department of International Health, The Johns Hopkins Bloomberg School of Public Health, 615 North Wolfe Street, Baltimore, MD 21205, USA; International Center for Diarrhoeal Disease Research, Bangladesh (ICDDR,B), GPO Box 128, Dhaka 1000, Bangladesh

Tyler Barker: The Orthopedic Specialty Hospital, Murray, UT 84107, USA

Robert E. Black: Department of International Health, The Johns Hopkins Bloomberg School of Public Health, 615 North Wolfe Street, Baltimore,

MD 21205, USA

Billie Bonevski: Priority Research Centre for Translational Neuroscience and Mental Health, Level 5 McAuley Building, Mater Hospital, Cnr Edith \& Platt Streets, Waratah, NSW, 2298, Australia

Kyra Borchhardt: Division of Nephrology and Dialysis, Department of Internal Medicine III, Medical University of Vienna, Spitalgasse 23, Vienna 1090, Austria

Jacopo J. V. Branca: Department of Experimental and Clinical Medicine, University of Firenze, 50134 Firenze, Italy 
Irena Brozek: Translational Cancer Research Unit, Ingham Institute for Applied Medical Research, South Western Sydney Clinical School, UNSW Medicine, The University of New South Wales, Sydney, 2170, Australia

Ludovic Brunel: Santessence, 905 1st Ave NE Calgary, AB T2E 0C5, Canada

Jamie Bryant: Priority Research Centre in Health Behaviour, School of Medicine and Public Health, University of Newcastle, Hunter Medical Research Institute, Newcastle, 2305, Australia

Yi-Sheng Chao: School of Public Health, University of Alberta, 3-50 University Terrace, 8303-112 St, Edmonton, AB T6G 2T4, Canada

Jiong Jack Chen: DSM Nutritional Products, Research Center, 476 Libing Road, Shanghai 201203, China

Michel Chonchol: Division of Renal Diseases and Hypertension, University of Colorado Denver, Denver, CO 80045 USA

Tamlin S. Conner: Department of Psychology, University of Otago, Dunedin 9054, New Zealand

Brian M. Dixon: USANA Health Sciences, Inc., Salt Lake City, UT 84120, USA

Gregory Dolnikowski: Jean Mayer USDA-ARS, Human Nutrition Research Center on Aging at Tufts University, 711 Washington Street, Boston, MA 02111, USA

Jia-Yi Dong: Department of Nutrition and Food Hygiene, School of Public Health, Soochow University, 199 Renai Road, Suzhou 215123, China

Paul Ekwaru: School of Public Health, University of Alberta, 350 University Terrace, Edmonton, AB T6G 2T4, Canada.

Linda Ellis: Libin Cardiovascular Institute of Alberta, 3330 Hospital Dr. NW, Calgary, AB T2N 2T9, Canada

Derek V. Exner: Libin Cardiovascular Institute of Alberta, 3330 Hospital Dr. NW, Calgary, AB T2N 2T9, Canada

Peter Faris: Alberta Bone and Joint Health Institute, Alberta Health Services, 3280 Hospital Dr NW \#400 Calgary, AB T2N 4Z6, Canada

Maria Giulia Fiore: Department of Experimental and Clinical Biomedical Sciences, University of Firenze, 50134 Firenze, Italy

Erik R. Gertz: Obesity \& Metabolism Research Unit, USDA-ARS, Western Human Nutrition Research Center West Health Science Drive, Davis, CA 95616, USA

Adit A. Ginde: Department of Emergency Medicine, University of Colorado Denver, Denver, CO 80045 USA

William B. Grant: Sunlight, Nutrition, and Health Research Center, P.O. Box 641603, San Francisco, CA 94164-1603, USA

Louisa Grundy: Pathology \& Laboratory Medicine Institute, Sheikh Khalifa Medical City, Abu Dhabi 51900, UAE

Massimo Gulisano: Department of Experimental and Clinical Medicine, University of Firenze, 50134 Firenze, Italy 
Brian Gushulak: Migration Health Consultants, Qualicum Beach, British Columbia, V9K 1S9, Canada

Aric C. Hall: Division of Hematology and Medical Oncology, School of Medicine and Public Health, University of Wisconsin, Madison, WI 53705, USA

Shu-Fen Han: Department of Nutrition and Food Hygiene, School of Public Health, Soochow University, 199 Renai Road, Suzhou 215123, China

Michelle J. Harper: Department of Human Nutrition, University of Otago, Dunedin 9054, New Zealand

Afrozul Haq: Pathology \& Laboratory Medicine Institute, Sheikh Khalifa Medical City, Abu Dhabi 51900, UAE

Brenda R. Hemmelgarn: University of Calgary, 1403 29th St. NW, Calgary, AB T2N 2T9, Canada

Vanessa T. Henriksen: The Orthopedic Specialty Hospital, Murray, UT 84107, USA

Harry R. Hill: ARUP Laboratories, Institute for Clinical and Experimental Pathology, Salt Lake City, UT 84108, USA; Department of Pathology, Pediatrics and Medicine, University of Utah, Salt Lake City, UT 84132, USA

Suzanne C. Ho: Division of Epidemiology, The Jockey Club School of Public Health and Primary Care, the Chinese University of Hong Kong, Hong Kong SAR, 999077, China

Michael F. Holick: Vitamin D, Skin and Bone Research Laboratory, Section of Endocrinology, Nutrition, and Diabetes, Department of Medicine, Boston University Medical Center, 85 East Newton Street, M-1013, Boston, MA 02118, USA

John Holmen: Intermountain Healthcare, Salt Lake City, UT 84157, USA

Lisa A. Houghton: Department of Human Nutrition, University of Otago, Dunedin 9054, New Zealand

Jill Howie-Esquivel: Department of Physiological Nursing, School of Nursing, University of California, San Francisco (UCSF), \#2 Koret Way Box 0610, San Francisco, CA 94143, USA

Nafiz Hussein: Pathology \& Laboratory Medicine Institute, Sheikh Khalifa Medical City, Abu Dhabi 51900, UAE

Kristen Jablonski: Division of Renal Diseases and Hypertension, University of Colorado Denver, Denver, CO 80045 USALars T. Jensen: Department of Clinical Physiology, Glostrup University Hospital, Copenhagen DK-2900, Denmark

Anna J. Jovanovich: Division of Renal Diseases and Hypertension, University of Colorado Denver, Denver, CO 80045 USA

Mark B. Juckett: Division of Hematology and Medical Oncology, School of Medicine and Public Health, University of Wisconsin, Madison, WI 53705, USA

Nancy L. Keim: Department of Nutrition \& Graduate Group in Nutritional Biology, University of California, One Shields Avenue, Davis, CA 95616, USA; Obesity \& Metabolism Research Unit, USDA-ARS, Western Human Nutrition Research Center West Health Science Drive, Davis, CA 95616, USA

Jessica Kendrick: Division of Renal Diseases and Hypertension, University of Colorado Denver, Denver, CO 80045 USA; Denver Health Medical Center, Denver, CO 80204, USA 
Shoukat Khan: Pathology \& Laboratory Medicine Department, Military Hospital, Riyadh 11159, Kingdom of Saudi Arabia

Carl R. Kjeldsberg: ARUP Laboratories, Institute for Clinical and Experimental Pathology, Salt Lake City, UT 84108, USA; Department of Pathology, Pediatrics and Medicine, University of Utah, Salt Lake City, UT 84132, USA

Sylvie Lambert: Priority Research Centre in Health Behaviour, School of Medicine and Public Health, University of Newcastle, Hunter Medical Research Institute, Newcastle, 2305, Australia

Zhao-Min Liu: Department of Medicine \& Therapeutics, the Chinese University of Hong Kong, Hong Kong SAR, 999077, China

Stefano Magherini: Department of Experimental and Clinical Medicine, University of Firenze, 50134 Firenze, Italy

Abdullah Al Mahmud: International Center for Diarrhoeal Disease Research, Bangladesh (ICDDR,B), GPO Box 128, Dhaka 1000, Bangladesh

Michelle C. Mann: University of Calgary, 1403 29th St. NW, Calgary, AB T2N 2T9, Canada

Janet Markland: Department of Internal Medicine, College of Medicine, University of Saskatchewan, Saskatoon, SK S7J 5B6, Canada

Thomas B. Martins: ARUP Laboratories, Institute for Clinical and Experimental Pathology, Salt Lake City, UT 84108, USA

Ulla K. Møller: Department of Endocrinology and Internal Medicine, THG, Aarhus University Hospital, Tage Hansens Gade 2, DK, Aarhus 8000, Denmark

Howard Morris: Division of Health Sciences, University of South Australia, Adelaide, SA 5000, Australia; SA Pathology, PO Box 14, Rundle Mall, Adelaide, SA 5000, Australia

Gabriele Morucci: Department of Experimental and Clinical Medicine, University of Firenze, 50134 Firenze, Italy

Leif Mosekilde: Department of Endocrinology and Internal Medicine, THG, Aarhus University Hospital, Tage Hansens Gade 2, DK, Aarhus 8000, Denmark

Meis Moukayed: School of Arts and Sciences, American University in Dubai, P. O. Box 28282, Dubai, UAE

Faten Mustafa: Pathology \& Laboratory Medicine Institute, Sheikh Khalifa Medical City, Abu Dhabi 51900, UAE

John W. Newman: Department of Nutrition \& Graduate Group in Nutritional Biology, University of California, One Shields Avenue, Davis, CA 95616, USA; Obesity \& Metabolism Research Unit, USDA-ARS, Western Human Nutrition Research Center West Health Science Drive, Davis, CA 95616, USA

Shailja Nigdikar: MRC Human Nutrition Research, Cambridge CB1 9NL, UK

Christine Nisbet: Division of Nutrition and Dietetics, College of Pharmacy and Nutrition, University of Saskatchewan, Saskatoon, SK S7N 5A2, Canada

Dana Ogan: Department of Nutrition, Exercise and Health Science, Central Washington University, 400 E. University Way, Ellensburg, WA 98926, USA 
Bartosz Olbramski: Division of Nephrology and Dialysis, Department of Internal Medicine III, Medical University of Vienna, Spitalgasse 23, Vienna 1090, Austria

Stefania Pacini: Department of Experimental and Clinical Medicine, University of Firenze, 50134 Firenze, Italy

Fasila Pallinalakam: Pathology \& Laboratory Medicine Institute, Sheikh Khalifa Medical City, Abu Dhabi 51900, UAE

Gemma Patras: Pathology \& Laboratory Medicine Institute, Sheikh Khalifa Medical City, Abu Dhabi 51900, UAE

Brian D. Piccolo: Department of Nutrition \& Graduate Group in Nutritional Biology, University of California, One Shields Avenue, Davis, CA 95616, USA

Maria A. Polak: Department of Psychology, University of Otago, Dunedin 9054, New Zealand

Kelly Pritchett: Department of Nutrition, Exercise and Health Science, Central Washington University, 400 E. University Way, Ellensburg, WA 98926, USA

Li-Qiang Qin: Department of Nutrition and Food Hygiene, School of Public Health, Soochow University, 199 Renai Road, Suzhou 215123, China

Jaishen Rajah: Pediatric Institute, Sheikh Khalifa Medical City, Abu Dhabi 51900, UAE

Rubhana Raqib: International Center for Diarrhoeal Disease Research, Bangladesh (ICDDR,B), GPO Box 128, Dhaka 1000, Bangladesh

Anthony I. Reeder: Cancer Society Social and Behavioural Research Unit, Department of Preventive and Social Medicine, University of Otago, Dunedin 9054, New Zealand

Lars Rejnmark: Department of Endocrinology and Internal Medicine, THG, Aarhus University Hospital, Tage Hansens Gade 2, DK, Aarhus 8000, Denmark

Vanessa Rock: Cancer Council NSW, PO Box 572 Kings Cross, NSW, 1340, Australia

Daniel E. Roth: Department of International Health, The Johns Hopkins Bloomberg School of Public Health, 615 North Wolfe Street, Baltimore, MD 21205, USA

Marco Ruggiero: Department of Biomedical, Experimental and Clinical Sciences, University of Firenz e, 50134 Firenze, Italy

Arwa Salem: Pathology \& Laboratory Medicine Institute, Sheikh Khalifa Medical City, Abu Dhabi 51900, UAE

Susanna við Streym: Department of Endocrinology and Internal Medicine, THG, Aarhus University Hospital, Tage Hansens Gade 2, DK, Aarhus 8000, Denmark

Zohreh Sabbagh: Department of Internal Medicine, College of Medicine, University of Saskatchewan, Saskatoon, SK S7J 5B6, Canada

Inez Schoenmakers: MRC Human Nutrition Research, Cambridge CB1 9NL, UK

Erik D. Schneider: USANA Health Sciences, Inc., Salt Lake City, UT 84120, USA

Robert Scragg: School of Population Health, Faculty of Medical and Health Sciences, The University of Auckland, Private Bag 92019, Auckland 1142,

New Zealand

Elias Seyoum: Jean Mayer USDA-ARS, Human Nutrition Research Center on Aging at Tufts University, 711 Washington Street, Boston, MA 02111, USA 
Rodney Smith: Macro Innovations Ltd., CB4 0DS Cambridge, UK

Darlene Y. Sola: Libin Cardiovascular Institute of Alberta, 3330 Hospital Dr. NW, Calgary, AB T2N 2T9, Canada

Elaine C. Souza : Department of Nutrition \& Graduate Group in Nutritional Biology, University of California, One Shields Avenue, Davis, CA 95616, USA

Simon Spedding: Nutritional Physiology Research Centre, University of South Australia, City East Campus, North Tce, Adelaide, SA 5000, Australia

Ursula Thiem: Division of Nephrology and Dialysis, Department of Internal Medicine III, Medical University of Vienna, Spitalgasse 23, Vienna 1090, Austria

Anthony P. Thomas: Department of Nutrition \& Graduate Group in Nutritional Biology, University of California, One Shields Avenue, Davis, CA 95616, USA

Lynda Thyer: Macro Innovations Ltd., CB4 0DS Cambridge, UK

Tanvir C. Turin: Libin Cardiovascular Institute of Alberta, 3330 Hospital Dr. NW, Calgary, AB T2N 2T9, Canada

Andrew Turner: Pathology \& Laboratory Medicine Institute, Sheikh Khalifa Medical City, Abu Dhabi 51900, UAE

Marta D. Van Loan: Department of Nutrition \& Graduate Group in Nutritional Biology, University of California, One Shields Avenue, Davis, CA 95616, USA; Obesity \& Metabolism Research Unit, USDA-ARS, Western Human Nutrition Research Center West Health Science Drive, Davis, CA 95616, USA

Simon Vanlint: Discipline of General Practice, School of Population Health, University of Adelaide, Adelaide, South Australia, SA 5005, Australia

Hassanali Vatanparast: Division of Nutrition and Dietetics, College of Pharmacy and Nutrition, School of Public Health, University of Saskatchewan, Saskatoon SK S7N 5C9, Canada

Paul J. Veugelers: School of Public Health, University of Alberta, 350 University Terrace, Edmonton, AB T6G 2T4, Canada.

Matthias Wacker: Vitamin D, Skin and Bone Research Laboratory, Section of Endocrinology, Nutrition, and Diabetes, Department of Medicine, Boston University Medical Center, 85 East Newton Street, M-1013, Boston, MA 02118, USA.

Emma Ward: Macro Innovations Ltd., CB4 0DS Cambridge, UK

Lindell K. Weaver: Hyperbaric Medicine, Intermountain Medical Center, Murray, UT 84107, USA; LDS Hospital, Salt Lake City, UT 84143, USA; School of Medicine, University of Utah, Salt Lake City, UT 84132, USA

Patricia G. Weyland: Department of Physiological Nursing, School of Nursing, University of California, San Francisco (UCSF), \#2 Koret Way Box 0610, San Francisco, CA 94143, USA

Jean Woo: Department of Medicine \& Therapeutics, the Chinese University of Hong Kong, Hong Kong SAR, 999077, China 
Leslie R. Woodhouse : Obesity \& Metabolism Research Unit, USDA-ARS, Western Human Nutrition Research Center West Health Science Drive, Davis,

CA 95616, USA

Sheng-Hui Wu: Division of Epidemiology, Department of Medicine, Vanderbilt University Medical Center, Nashville, TN 37203-1738, USA

Weiguo Zhang: DSM Nutritional Products, Human Nutrition and Health, No.1-3 Xinyuannanlu, Beijing 100027, China

Zeng-Li Zhang: Department of Labor Hygiene and Environmental Health, School of Public Health of Soochow University, 199 Renai Road, Suzhou 215123, China 


\section{About the Guest Editor}

Simon Spedding is a physician, academic and government advisor in nutrition, applied epidemiology and general medicine. He trained in exercise and sports medicine, psychiatry and applied epidemiology in clinical and academic settings including the Australian National University and the Australasian Cochrane Centre.

His research is based at the University of South Australia where Simon is a visiting physician and senior research fellow at the Alliance for Research in Exercise, Nutrition and Activity. He focuses on Vitamin D, nutrients and other integrative medicines in human health. He has been exploring the line of thinking that it is the different understandings of vitamin D physiology, which give rise to contrasting study designs in randomised trials, conflicting results in systematic reviews and ultimately contrary clinical guidelines and policy advice. This situation may apply across the spectrum of nutrients, and he is working towards an era of nutrient research without these limitations that may usher in a new understanding of nutrients. Simon is a member of an advisory committee of the Australian Therapeutic Goods Administration on complementary medicine and was a member of five national medical college committees. He is currently an Editor for the journal Nutrients.

Simon is a passionate author and speaker on a range of lifestyle topics, contributing to medical and nutrition journals on his favourite topics related to nutrition, especially Vitamin D. 


\section{Foreword}

Vitamin D research has expanded greatly over the last 10 years, with a more than two-fold increase in annual publications listed in Pubmed with the key word 'vitamin D' from 1675 in 2005 to 3953 in 2014.

Part of this increase is due to research showing that vitamin D deficiency is associated with a wide range of diseases and health outcomes. Until the 1980s, the primary focus of vitamin D research (in combination with calcium supplementation) was on bone diseases. Since then, observational studies have linked vitamin D deficiency with increased risk of many diseases: both acute and chronic. This book contains publications on several of these disease groups linked to vitamin D deficiency.

The other driver of the increased funding of vitamin D research is the realisation among public health agencies of the potential for disease prevention from vitamin $\mathrm{D}$, either through supplementation or food-fortification. Vitamin D is very attractive for population-based prevention strategies because it is not under patent and, hence, relatively cheap. For example, monthly bolus vitamin D supplementation costs about \$1-2 per year. The very low costs of vitamin $\mathrm{D}$, combined with findings from meta-analyses of previous randomised controlled trials showing reduced all-cause mortality in people allocated to vitamin $\mathrm{D}_{3}$ supplementation [1], have created interest in investigating vitamin D further.

Several large scale randomised controlled trials of vitamin D supplementation are currently underway around the world [2]. They have two distinguishing features from earlier supplementation trials. First, they are using much higher vitamin D doses (by two- to four-fold) than previous trials. Second, they are using vitamin D by itself, without calcium supplementation. The latter is important from a public health perspective, as calcium is water-soluble and needs to be taken daily, making it less attractive than fat-soluble vitamin D which has the potential for intermittent (weekly or monthly) dosing.

The good news is that these mega-trials of vitamin D supplementation are being done. The bad news, for anyone wanting a quick answer, is that they will take several years to finish and report their main findings.

In the meantime, this Open Book makes an important contribution by informing clinicians and researchers interested in vitamin D and its health effects, particularly those wanting an overview of recent developments in this area. 
XVIII

\section{References}

1. Bjelakovic G, Gluud LL, Nikolova D, et al. Vitamin D supplementation for prevention of mortality in adults. Cochrane Database Syst Rev 2014;1:CD007470 doi: 10.1002/14651858.CD007470.pub3[published Online First: Epub Date].

2. Meyer HE, Holvik K, Lips P. Should vitamin D supplements be recommended to prevent chronic diseases? BMJ 2015;350:h321 doi: 10.1136/bmj.h321[published Online First: Epub Date].

Robert Scragg, MBBS, PhD,

School of Population Health,

University of Auckland, New Zealand. 


\section{Preface}

Nutrients Open Book: Vitamin D and Human Health celebrates diversity in vitamin D with articles from bench-to-bedside, examining the mechanisms, epidemiology, and clinical issues in the management of non-skeletal disease. Michael Holick inspired this project, with his review of the evidence that vitamin D supports both skeletal and non-skeletal health [1]. Developing this theme the book identifies progress in vitamin D research published in a special edition Vitamin D and Human Health and other issues of Nutrients ${ }^{1}$.

This book explores evidence for the role of Vitamin D in non-skeletal disease. The chapters reflect how far we have progressed since Casimir Funk's invented the concept of 'vital amines' a hundred years ago. Whilst he portrayed vitamins as amines found in food with a single mode of action and essential for preventing a specific disorder [2], many vitamins do not follow this model. Some authorities such as the Institute of Medicine (IOM) still portray vitamin D as sourced from food with a recommended dietary allowance of less than 1000IU per day, and being synonymous with calcium metabolism and bone disorders, the IOM found little evidence to support the role of vitamin D in non-skeletal disease [3]. Now we have a different understanding of the epidemiology and physiology of vitamin D and the efficacy of vitamin D supplementation in many diseases.

Today we recognise vitamin $\mathrm{D}$ is a secosteroid hormone derived from sunshine with a plethora of physiological functions; autocrine, paracrine, endocrine [4], and epigenetic [5]. Thus many diseases are associated with vitamin D deficiency [1] and high levels of evidence are available for the efficacy of supplementation with vitamin D. However there is still controversy about the causative nature and efficacy of vitamin D in many diseases, as exemplified by conflicting evidence from meta-analyses about the efficacy of vitamin D supplementation originating from limitations in the design of the primary randomised controlled trials (RCTs) [6]. Furthermore, other factors have added to the controversy including confirmation bias, biological flaws, denial of two-way causation and the view that deficiency is represented by a single $25(\mathrm{OH}) \mathrm{D}$ level. This book illustrates how limited acceptance of the complex physiology of vitamin D may have led to flawed trial methodology, produced conflicting results and differences of opinion evident across the literature.

\footnotetext{
1 Vitamin D intake is measured in micrograms ( $\mu \mathrm{g}$ ) or international units (IU), where $100 \mu \mathrm{g}=4000 \mathrm{IU}$. Vitamin D status assessed as serum levels of 25 hydroxy-vitamin $\mathrm{D}(25(\mathrm{OH}) \mathrm{D})$ is measured in millimols per litre $(\mathrm{mmol} / \mathrm{L})$ or nanograms per millilitre $(\mathrm{ng} / \mathrm{ml})$, where $100 \mathrm{nmol} / \mathrm{L}=40 \mathrm{ng} / \mathrm{ml}$. The level taken to signify deficiency of $50 \mathrm{nmol} / \mathrm{L}$ is controversial.
} 


\section{Chapter 1 - Overview}

Foremost vitamin D researcher Michael Holick, and Mathias Wacker, describe the role of vitamin D in both skeletal and non-skeletal health [1] This includes the history of a century of discovery since Finsen's Nobel Prize in 1903 for the treatment of tuberculosis, the physiology revealing the ubiquitous nature of vitamin D metabolism, including the regulation of 2000 genes, the commonly used cures, such as light and cod liver oil, and the public health interventions, such as sunbaths and food fortification. The authors conclude 'large randomized controlled trials (RCTs) using sufficiently high doses of vitamin D are required to resolve the controversy regarding the dose of vitamin $\mathrm{D}$ and the level of $25(\mathrm{OH}) \mathrm{D}$ that should be attained to reduce the risk of diseases associated with deficiency'.

\section{Chapter 2 - Vitamin D Intake and 25(OH)D Levels}

As vitamin D deficiency is pandemic [27], public concern has driven research to investigate what intake and levels of vitamin D are sufficient. In North America, the Institute of Medicine (IOM) recommends a daily intake of $800 \mathrm{IU}$ of vitamin D [3], however Veugelers and colleague [28] demonstrate the IOM made a statistical error in calculating the recommended dietary allowance (RDA) for vitamin $\mathrm{D}$, and demonstrate that a far higher RDA is required.

In Canada where intakes of vitamin D and levels of 25(OH)D are low [7], Chao and colleagues [9] found the level of dose advised by the IOM did not change 25(OH)D levels in vitamin $\mathrm{D}$ deficient participants and higher doses were required.

In Saudi Arabia, where vitamin D deficiency is common, Alshahrani and colleague [30] found that doses higher than previously recognised of 50,000 IU per week were required for initial treatment for deficient patients. They also identified the pleomorphic effects of vitamin D outside its conventional role in calcium and bone homeostasis, highlighting the different levels of vitamin D and its clinical implications.

Across Asia the risk of vitamin D deficiency in pregnancy is high. Roth and colleagues [10] examined the dose response to vitamin D supplementation in pregnant women in Bangladesh demonstrating that it requires 5,000IU per day (equivalent) for most pregnant women safely attain sufficiency $(>80 \mathrm{nmol} / \mathrm{L})$ and that $2,000 \mathrm{IU}$ per day is ineffective.

These authors believe significantly higher doses are required and recommend the RDA for vitamin $\mathrm{D}$ be reconsidered to allow for appropriate public health and clinical decision-making.

\section{Chapter 3 - Health Impact}

The broad health impact of vitamin D was reviewed by Spedding and colleagues [15] examining evidence about the efficacy of vitamin D and the levels of 25(OH)D required in different disease. High levels of evidence for many diseases were found supporting the efficacy of vitamin D supplements, in contrast to previous reviews [3]. These include Level 1 evidence 
from meta-analyses for premature mortality and falls prevention, and Level 2 evidence from randomised controlled trials for cancer, diabetes, pain, depression and respiratory infections.

Whilst there is disagreement about the levels of $25(\mathrm{OH}) \mathrm{D}$ required to manage bone disorders, there is less agreement for non-skeletal diseases. The hypothesis that there is a specific level of $25(\mathrm{OH}) \mathrm{D}$ at which the therapeutic effect is seen in different diseases is supported by evidence from these trials. Specifically higher levels are required for the management of non-skeletal diseases than skeletal disease. This challenges the concept that there is a single level of $25(\mathrm{OH}) \mathrm{D}$ signifying vitamin $\mathrm{D}$ deficiency.

\section{Chapter 4 - Neoplastic Disorders}

The evidence that vitamin D reduces the risk of cancer is presented by the luminary vitamin D researcher, William B Grant, and Meis Moukayed [16]. They focus on the persistent genomic as well as immediate non-genomic effects of vitamin $\mathrm{D}$ that regulate cell proliferation, differentiation, and survival. This is supported by ecological and epidemiological studies and two RCTs. This is further reinforced by a discussion of the role of vitamin D in the normal process of differentiation and activation of cells by Hall and colleagues [19] who also examine the immune modulation effects of vitamin $\mathrm{D}$ and its role in leukaemia and stem cell transplants. Clinicians and policy need to provide advice and treat patients based on the best evidence available at the time, so researchers need to assist this process through better communication of what is currently know. Aware of this predicament, William B Grant and colleague [16] follow their review with recommendations that countries adopt awareness, education, and implementation strategies to increase supplementation with vitamin $\mathrm{D}$ as a preventive measure to reduce colon and breast cancer.

\section{Chapter 5 - Cardiovascular Disorders}

The evidence that vitamin D deficiency is a cardiovascular (CVD) risk factors is presented by Weyland and colleagues including William B Grant, showing that all the relevant Bradford Hills criteria for a causal association between low 25(OH)D levels and CVD risk are satisfied [29].

Furthermore, vitamin D deficiency may increase cardiovascular risk through altering the sympathovagal balance (SVB) and thus blood pressure. Mann and colleagues [20] demonstrated SVB suppression in vitamin D deficient participants and the cardioprotective effect of Vitamin D supplementation was demonstrated through suppression of SVB in cardiocytes by Pacini and colleagues [21]. Moreover blood pressure was found to be dependent on the dose of vitamin D by Liu and colleagues [17]. 


\section{Chapter 6 - Metabolic Disorders}

Evidence for the preventive nature of vitamin D also was found for metabolic disorders such as diabetes and obesity. A systematic review and meta-analysis of vitamin D supplementation in type 1 Diabetes Mellitus by Dong and colleagues [13] showed a 30\% reduction in the risk of developing Type 1 diabetes when vitamin D supplements were taken in infancy. This provides the highest level of evidence of the efficacy of vitamin D for the prevention of diabetes.

Obesity has become a major global health challenge, which is not only increasing but no national success stories have been reported in the past 33 years. The review by Simon Vanlint [18] concluded the conflicting outcomes of obesity studies may reflect the two-way causation of obesity and vitamin D deficiency and reflect the flaws in the studies rather than the effect of vitamin D itself. The first report of the analyses of adipose $25(\mathrm{OH}) \mathrm{D}$ in healthy human subjects from Piccolo and colleagues [32] found that although there was concordance between levels of 25-hydroxyvitamin $\mathrm{D}$ in serum and subcutaneous white adipose tissue, these levels were unaffected by changes in body weight in overweight and obese individuals.

\section{Chapter 7 - Mental Disorders}

Depression can be difficult to treat and uncertainty about the efficacy of antidepressants medication has led to trials of non-pharmaceutical medicines. A systematic review and meta-analysis of 15 RCTs of the efficacy of vitamin D supplementation in depression was conducted by Spedding [24]. Whilst history, physiology, and epidemiological research support the role of vitamin D in the management of depression, previous systematic reviews of RCTs have found insufficient evidence to support the concept. However these reviews had overlooked most of the 15 RCTs of vitamin D supplementation in the management of depression published in peer reviewed journals. This may explain the conclusions of previous reviewers that more research was needed. This new systematic review and meta-analysis compared studies according to the presence or absence of biological flaws [24] and found the studies without flaws demonstrated vitamin D supplementation significantly reduced depressive symptoms, whereas this effect was not shown for trials with biological flaws. Biological flaws in trials make supplementation ineffective such as when the dose of vitamin D is too low or participants are not deficient in vitamin D.

Many of these primary studies were in elderly patients, however the association between depressive symptoms and vitamin D status also occurs in young healthy adults. In New Zealand a third of healthy students were vitamin D deficient $(<50 \mathrm{nmol} / \mathrm{L})$ and deficiency was found to be associated with depression symptoms after adjustment for confounders. Polak and associates [26] further noted a significantly lower $25(\mathrm{OH}) \mathrm{D}$ in the group with a high depression score; the effect was clinically significant being a 4.5 point increase on the depression scale for each 27 $\mathrm{nmol} / \mathrm{L}$ decrease in serum 25(OH)D. 
Supplements and complementary medicines are commonly used for performance enhancement although the evidence is unclear. Whilst vitamin D deficiency is common in athletes; especially indoor athletes, Ogan and colleagues [23] found conflicting evidence about the efficacy of vitamin D on muscle strength or performance. They concluded performance improved only at high 25(OH)D levels above $100 \mathrm{nmol} / \mathrm{L}$ but not at lower levels. Barker and colleagues [22] found post-exercise muscle weakness correlated with having low levels of $25(\mathrm{OH}) \mathrm{D}$. Considering the importance of optimal vitamin D status to prevent stress fractures, and muscle injury and recovery, authors advise that a goal for all athletes is obtaining optimal $25(\mathrm{OH}) \mathrm{D}$ levels, regardless of the limited literature available in support of a positive effect from vitamin $\mathrm{D}$ on performance.

\section{Chapter 9 - Risk Factors}

The factors affecting vitamin D deficiency are diverse including social, behavioural and as well as health such as chronic disease.

In Canada, Vatanparast and colleagues [8] suggest that migration is a significant risk factor for deficiency intakes of vitamin $\mathrm{D}$ as levels of $25(\mathrm{OH}) \mathrm{D}$ are low in migrant and refugee children, and refugee children have half the level of other Canadian children. In Australia, Bonevski and colleagues [12] found beliefs about skin cancer lead to sun avoidance and vitamin D deficiency. For patients with organ failure, Theim and colleagues [33] found although most patients (80-95\%) show vitamin D insufficiency or deficiency, this also occurs in healthier patients before and after transplantation, However, there are few guidelines about vitamin D covering this situation. Similarly, many rheumatology patients $(56 \%)$ have low serum $25(\mathrm{OH}) \mathrm{D}$ levels, and disease activity was found by Sabbagh and colleagues [34] to be five times higher risk of in cases with low plasma $25(\mathrm{OH}) \mathrm{D}$ than in controls. They consider proper evaluation of vitamin D status is needed in this clinical population as there is evidence of a two-way causation between rheumatoid arthritis and vitamin D deficiency. Infectious disease, such as community acquired pneumonia (CAP) and sepsis in a community-living adult population were also inversely associated with Vitamin D status. The strength of this prospective study by Jovanovich and colleagues [25] was that serum 25(OH)D levels were measuring at least three months prior to illness or hospitalization, thus reinforcing the case for low vitamin D status being considered a causative factor, rather than solely a response to illness. The oral contraceptive pill also affects 25(OH)D levels, as Moller and colleagues [11] found, patients on the pill had up to $25 \%$ higher concentrations of vitamin D than patients not on the pill.

Concerns about the diagnostic accuracy for vitamin D testing [1] led Abdel-Wareth and colleagues [14] to compare methods for measuring $25(\mathrm{OH}) \mathrm{D}$ levels. They found good concordance for the Roche system to HPLC for samples containing high levels of vitamin D3 and slightly lower concordance for those with higher vitamin D2 levels, but not of a degree that would affect clinical decision making. 


\section{Conclusion}

This Nutrients Open Book: Vitamin D and Human Health expands the literature on vitamin $\mathrm{D}$ beyond the views of Casimir Funk, and of the Institute of Medicine about the source, intake and serum levels of vitamin $\mathrm{D}$, and the opinion that there is insufficient evidence to support the use of vitamin $\mathrm{D}$ in non-skeletal disease.

Vitamin D deficiency is pandemic occurring frequently in countries with both high and low levels of sunlight. Current IOM recommendations on dietary intake are unlikely to address vitamin D deficiency across the community. Therefore there is an urgent need to resolve questions of whether vitamin D deficiency is important for disorders other than bone disease, and then address the efficacy of vitamin D supplements and the level of $25(\mathrm{OH}) \mathrm{D}$ required to manage these diseases. Clinicians and policy makers treat patients and guide public health policy based on the best evidence available at the time, so researchers need to assist this process through better communication of what is currently know and how sure we can be of these opinions, rather than focusing on the gaps in knowledge.

The current evidence shows Level 1 evidence from meta-analysis for the efficacy of vitamin D from meta-analyses for premature mortality, diabetes and falls prevention, and the treatment of depression There is Level 2 evidence from randomised controlled trials for cancer, chronic pain, and respiratory infections, and lower levels of evidence for reducing cardiovascular risk, sporting injuries and for patients with chronic debilitating disease. Thus these authors consistently advise the goal is to obtain optimal 25(OH)D levels to manage non-skeletal health. However there is still controversy about these findings, as some researchers are sceptical about the complexity of vitamin D physiology. As opposed to the scientific evidence about diverse mechanism of vitamin D presented here, one such physiology sceptic in a British Medical Journal editorial states 'the putative mechanistic actions of vitamin D's are so numerous that they have been invoked as an explanation for why good triumphs over evil in JRR Tolkien's The Hobbit' [31].

Reinforcing this cynicism are methodological issues highlighted in this Book. Two of these issues affecting trial methodology are biological flaws and conceptualising deficiency as a single level of $25(\mathrm{OH}) \mathrm{D}$, whilst two others issues affecting review methodology are denial of two-way causation and confirmation bias.

- Scepticism may be reinforced by evidence from trials with biological flaws, such as using low ineffective doses of Vitamin D or treating participants who were not deficient at baseline. Mirroring pharmaceutical trials, the trial methodology overlooks the sources and physiology of vitamin, making a null result more likely.

- Another issue concerning researchers is the concept that vitamin D deficiency is indicated by a single serum $25(\mathrm{OH}) \mathrm{D}$ level. However as vitamin D metabolism is tissue dependent, deficiency is disease dependent and a single number may not truly represent deficiency [15]. The evidence indicates that there are different levels of $25(\mathrm{OH}) \mathrm{D}$ representing deficiency in different tissues and thus for different diseases. 
- The two-way causation of disease and vitamin D deficiency was demonstrated for infectious disease and may be involved in rheumatoid arthritis and obesity. Although many chronic diseases lead to vitamin D deficiency, this does not exclude the possibility that deficiency increases the risk of disease. When an association between deficiency and a disease is discovered, it is prone to be interpreted as disease causing deficiency without evidence to exclude the competing explanation that deficiency caused disease. Whereas the causal nature of deficiency in many disorders is supported by RCTs and the Bradford Hill criteria, as well as prospective studies that assessed vitamin D levels before the disorder arose.

- The accepted wisdom was that the role of vitamin D was limited to bone disease and further research is needed to establish its role in non-skeletal disease. This presumes that the evidence is not already available. However previous reviews have demonstrably failed to identify many published trials, leading to a suspicion that some opinion pieces and reviews may suffer from confirmation bias finding only evidence that supports the status quo.

Denying the complex nature of vitamin physiology has led to trial methodology with biological flaws that did not consider supplement doses or levels of deficiency. This led to conflicting results in RCTs and meta-analyses about the efficacy of vitamin D supplementation. Unless these flaws are minimised in future trials, knowledge about vitamin D will not increase and may even promulgate disinformation. Confirmation bias and the resistance to considering two-way causation in reviews and opinion pieces may spuriously have reinforced the accepted wisdom about a lack of evidence for vitamin D in non-skeletal disease. These issues may have contributed to the difference between the evidence presented in this book and the conclusions of the IOM and physiology sceptics.

Scepticism, confirmation bias, deficiency levels, biological flaws, and two-way causation rarely occur in drug trials however are characteristic of vitamin D research and may occur across the spectrum of nutrients. The future of research without these limitations may usher in a new age in the understanding of vitamin D and other nutrients.

\section{References}

1. Wacker, M.; Holick, M.F. Vitamin D-Effects on skeletal and extra skeletal health and the need for supplementation. Nutrients 2013, 5, 111-148.

2. Spedding, S. Vitamins are more Funky than Casimir thought. AMJ 2013, 6, 104-106.

3. National Research Council. Dietary Reference Intakes for Calcium and Vitamin D; The National Academies Press: Washington, DC, USA, 2011.

4. Morris, H.; Anderson, P. Autocrine and paracrine actions of vitamin D. Clin. Biochem. Rev. 2010, 31, 129-138.

5. Hossein-Nezhad, A.; Spira, A.; Holick, M.F. Influence of vitamin D status and vitamin D3 supplementation on genome wide expression of white blood cells: A randomized double-blind clinical trial. PLoS One 2013, 8, e58725IOF and DSM. 
6. Scragg, R. Do we need to take calcium with vitamin D supplements to prevent falls, fractures, and death? Curr Opin Clin Nutr Metab Care. 2012;15(6):614-24.

7. Vitamin D and Calcium: Updated Dietary Reference Intakes. Health Canada Sante Canada. Available online: http://www.hc-sc.gc.ca/fn-an/nutrition/vitamin/vita-d-eng.php (accessed on 22 March 2012).

8. Vatanparast, H.; Nisbet, C.; Gushulak, B. Vitamin D insufficiency and bone mineral status in a population of newcomer children in Canada. Nutrients 2013, 5, 1561-1572.

9. Chao, Y.S.; Brunel, L.; Faris, P.; Veugelers, P.J. The importance of dose, frequency and duration of vitamin D supplementation for plasma 25-hydroxyvitamin D. Nutrients 2013, 5, 4067-4078.

10. Roth, D.E.; Mahmud, A.A.; Raqib, R.; Akhtar, E.; Black, R.E.; Baqui, A.H. Pharmacokinetics of high-dose weekly oral vitamin D3 supplementation during the third trimester of pregnancy in Dhaka, Bangladesh. Nutrients 2013, 5, 788-810.

11. Møller, U.K.; Streym, S.V.; Jensen, L.T.; Mosekilde, L.; Schoenmakers, I.; Nigdikar, S.; Rejnmark, L. Increased plasma concentrations of vitamin $d$ metabolites and vitamin $\mathrm{d}$ binding protein in women using hormonal contraceptives: A cross-sectional study. Nutrients 2013, 5, 3470-3480.

12. Bonevski, B.; Bryant, J.; Lambert, S.; Brozek, I.; Rock, V. The ABC of Vitamin D: A qualitative study of the knowledge and attitudes regarding vitamin d deficiency amongst selected population groups. Nutrients 2013, 5, 915-927.

13. Dong, J.-Y.; Zhang, W.-G.; Chen, J.J.; Zhang, Z.-L.; Han, S.-F.; Qin, L.-Q. Vitamin D intake and risk of type 1 diabetes: A meta-analysis of observational studies. Nutrients 2013, 5, 3551-3562. Nutrients 2014, 614.

14. Abdel-Wareth, L.; Haq, A.; Turner, A.; Khan, S.; Salem, A.; Mustafa, F.; Hussein, N.; Pallinalakam, F.; Grundy, L.; Patras, G.; et al. Total vitamin d assay comparison of the roche diagnostics "Vitamin D Total" electrochemiluminescence protein binding assay with the chromsystems HPLC method in a population with both D2 and D3 forms of vitamin D. Nutrients 2013, 5, 971-980.

15. Spedding, S.; Vanlint, S.; Morris, H.; Scragg, R. Does vitamin D sufficiency equate to a single serum 25-hydroxyvitamin D level or are different levels required for non-skeletal diseases. Nutrients 2013, 5, 5127-5139.

16. Moukayed, M.; Grant, W.B. Molecular link between vitamin D and cancer prevention. Nutrients 2013, 5, 3993-4021.

17. Liu, Z.-M.; Woo, J.; Wu, S.-H.; Ho, S.C. The role of vitamin D in blood pressure, endothelial and renal function in postmenopausal women. Nutrients 2013, 5, 2590-2610.

18. Vanlint, S. Vitamin D and obesity. Nutrients 2013, 5, 949-956.

19. Hall, A.C.; Juckett, M.B. The role of vitamin D in hematologic disease and stem cell transplantation. Nutrients 2013, 5, 2206-2221.

20. Mann, M.C.; Exner, D.V.; Hemmelgarn, B.R.; Sola, D.Y.; Turin, T.C.; Ellis, L.; Ahmed, S.B. Vitamin D levels are associated with cardiac autonomic activity in healthy humans. Nutrients 2013, 5, 2114-2127. 
21. Pacini, S.; Morucci, G.; Branca, J.J.V.; Aterini, S.; Amato, M.; Gulisano, M.; Ruggiero, M. Effects of vitamin D3 and paricalcitol on immature cardiomyocytes: A novel role for vitamin D analogs in the prevention of cardiovascular diseases. Nutrients 2013, 5, 2076-2092.

22. Barker, T.; Henriksen, V.T.; Martins, T.B.; Hill, H.R.; Kjeldsberg, C.R.; Schneider, E.D.; Dixon, B.M.; Weaver, L.K. Higher serum 25-hydroxyvitamin D concentrations associate with a faster recovery of skeletal muscle strength after muscular injury. Nutrients 2013, 5, $1253-1275$.

23. Ogan, D.; Pritchett, K. Vitamin D and the Athlete: Risks, recommendations, and benefits. Nutrients 2013, 5, 1856-1868.

24. Spedding, S.; Vitamin D and Depression: A Systematic Review and Meta-Analysis Comparing Studies with and without Biological Flaws. Nutrients 2014, 6(4), 1501-1518.

25. Jovanovich, A.J.; Ginde, A.A.; Holmen, J.; Jablonski, K.; Allyn, R.L.; Kendrick, J.; Chonchol, M.; Vitamin D Level and Risk of Community-Acquired Pneumonia and Sepsis. Nutrients 2014, 6(6), 2196-2205.

26. Polak, M.A.; Houghton, L.A.; Reeder, A.I.; Harper, M.J.; Conner, T.S. Serum 25-Hydroxyvitamin D Concentrations and Depressive Symptoms among Young Adult Men and Women. Nutrients 2014, 6, 4720-4730.

27. IOF Vitamin D Map. Available online:

http://www.iofbonehealth.org/facts-and-statistics/vitamin-d-studies-map (accessed on 8 July 2013).

28. Veugelers, P.J.; Ekwaru, J.P. A Statistical Error in the Estimation of the Recommended Dietary Allowance for Vitamin D. Nutrients 2014, 6, 4472-4475.

29. Weyland, P.G.; Grant, W.B.; Howie-Esquivel, J. Does Sufficient Evidence Exist to Support a Causal Association between Vitamin D Status and Cardiovascular Disease Risk? An Assessment Using Hill's Criteria for Causality. Nutrients 2014, 6, 3403-3430.

30. Alshahrani, F.; Aljohani, N. Vitamin D: Deficiency, Sufficiency and Toxicity. Nutrients 2013, 5, 3605-3616.

31. Sattar,N.,and Welsh,P., Editorial : Vitamin D and chronic disease prevention; Multiple meta-analysis but still no magic bullet. BMJ 2014; 348:g2280.

32. Piccolo, B.D.; Dolnikowski, G.; Seyoum, E.; Thomas, A.P.; Gertz, E.R.; Souza, E.C.; Woodhouse, L.R.; Newman, J.W.; Keim, N.L.; Adams, S.H.; Van Loan, M.D. Association between Subcutaneous White Adipose Tissue and Serum 25-Hydroxyvitamin D in Overweight and Obese Adults. Nutrients 2013, 5, 3352-3366.

33. Thiem, U.; Olbramski, B.; Borchhardt, K. Calcidiol Deficiency in End-Stage Organ Failure and after Solid Organ Transplantation: Status quo. Nutrients 2013, 5, 2352-2371.

34 Sabbagh, Z.; Markland, J.; Vatanparast, H. Vitamin D Status Is Associated with Disease Activity among Rheumatology Outpatients. Nutrients 2013, 5, 2268-2275.

(C) 2014 by the authors; licensee MDPI, Basel, Switzerland. This article is an open access article distributed under the terms and conditions of the Creative Commons Attribution license (http://creativecommons.org/licenses/by/3.0/). 

Chapter 1

Overview 



\title{
Vitamin D-Effects on Skeletal and Extraskeletal Health and the Need for Supplementation
}

\author{
Matthias Wacker and Michael F. Holick
}

\begin{abstract}
Vitamin D, the sunshine vitamin, has received a lot of attention recently as a result of a meteoric rise in the number of publications showing that vitamin D plays a crucial role in a plethora of physiological functions and associating vitamin $\mathrm{D}$ deficiency with many acute and chronic illnesses including disorders of calcium metabolism, autoimmune diseases, some cancers, type 2 diabetes mellitus, cardiovascular disease and infectious diseases. Vitamin D deficiency is now recognized as a global pandemic. The major cause for vitamin D deficiency is the lack of appreciation that sun exposure has been and continues to be the major source of vitamin D for children and adults of all ages. Vitamin D plays a crucial role in the development and maintenance of a healthy skeleton throughout life. There remains some controversy regarding what blood level of 25-hydroxyvitamin $\mathrm{D}$ should be attained for both bone health and reducing risk for vitamin D deficiency associated acute and chronic diseases and how much vitamin D should be supplemented.
\end{abstract}

Reprinted from Nutrients. Cite as: Wacker, M.; Holick, M.F. Vitamin D_Effects on Skeletal and Extraskeletal Health and the Need for Supplementation. Nutrients 2013, 5, 111-148.

\section{Introduction}

Vitamin D has been produced by phytoplankton for more than 500 million years [1] and is thought to be the oldest of all hormones whose function initially could have been the protection of ultraviolet-sensitive macromolecules including proteins, DNA and RNA, when these early forms of life were exposed to sunlight for photosynthesis. Later, after the evolution of ocean dwelling animals with vertebral skeletons ventured onto land, the maintenance of calcium homeostasis was a major physiological problem (as opposed to living in the calcium-rich ocean). It was vitamin D that ensured the efficient intestinal calcium absorption from dietary sources and ultimately was essential for the development and maintenance of a calcified mammalian skeleton [2]. Obtaining vitamin D from either sunlight or diet is still critical for most vertebrates for their skeletal health [1,3-5]. Over time, vitamin $\mathrm{D}$ has evolved into a hormone having numerous extraskeletal effects by regulating up to estimated 2000 genes [6,7].

Ethnical and gender differences in skin pigmentation indicate the evolutionary importance of a sufficient vitamin D supply. The varying degrees of depigmentation that evolved in order to permit UVB-induced synthesis of previtamin $\mathrm{D}_{3}$ when hominids migrated outside the tropics can be considered as a compromise solution to the conflicting physiological requirements of vitamin D synthesis and photoprotection that differ depending on latitude and thus warrant different degrees of skin pigmentation. An evolutionary selection pressure towards a lighter skin coloration going along with a higher ability to produce vitamin D seems not only to be exerted by living in geographic regions with a lower UV intensity but also by being female. Gender differences in skin pigmentation with females being lighter skinned than males in all populations for which data about the skin 
reflectance was available could be explained by the higher needs of vitamin D during pregnancy and lactation [8].

\section{Vitamin D-Sources}

The main sources of vitamin D are sunlight, supplements and diet [7] (Table 1).

Table 1. Sources of vitamin $\mathrm{D}_{2}$ and vitamin $\mathrm{D}_{3}$ [7]. Note: This table is modified and reproduced with permission from [7], Copyright (C) 2007 Massachusetts Medical Society.

\begin{tabular}{|c|c|}
\hline Source & $\begin{array}{c}\text { Vitamin D Content } \\
I \mathrm{IU}=\mathbf{2 5} \mathrm{ng}\end{array}$ \\
\hline & Chemical structures of vitamin $\mathrm{D}_{2}[9]$ and vitamin $\mathrm{D}_{3}[10]$. \\
\hline & Vitamin $\mathrm{D}_{2}$ (Ergocalciferol) $\quad$ Vitamin $\mathrm{D}_{3}$ (Cholecalciferol) \\
\hline \multicolumn{2}{|l|}{ Natural sources } \\
\hline Cod liver oil & $\sim 400-1000 \mathrm{IU} / \mathrm{tsp}$ vitamin $\mathrm{D}_{3}$ \\
\hline Egg yolk & $\sim 20 \mathrm{IU} /$ yolk vitamin $\mathrm{D}_{3}$ or $\mathrm{D}_{2}$ \\
\hline Mackerel, canned & $\sim 250 \mathrm{IU} / 3.5 \mathrm{oz}$ vitamin $\mathrm{D}_{3}$ \\
\hline Salmon, canned & $\sim 300-600 \mathrm{IU} / 3.5 \mathrm{oz}$ vitamin $\mathrm{D}_{3}$ \\
\hline Salmon, fresh farmed & $\sim 100-250 \mathrm{IU} / 3.5 \mathrm{oz}$ vitamin $\mathrm{D}_{3}$, vitamin $\mathrm{D}_{2}$ \\
\hline Sardines, canned & $\sim 300 \mathrm{IU} / 3.5 \mathrm{oz}$ vitamin $\mathrm{D}_{3}$ \\
\hline Shiitake mushrooms, fresh & $\sim 100 \mathrm{IU} / 3.5 \mathrm{oz}$ vitamin $\mathrm{D}_{2}$ \\
\hline \multicolumn{2}{|c|}{ Shiitake mushrooms, sun dried $\sim 1600 \mathrm{IU} / 3.5 \mathrm{oz}$ vitamin $\mathrm{D}_{2}$} \\
\hline \multirow[t]{2}{*}{ Sunlight/UVB radiation } & $\sim 20,000$ IU equivalent to exposure to 1 minimal erythemal dose (MED) \\
\hline & $\begin{array}{l}\text { in a bathing suit. Thus, exposure of arms and legs to } 0.5 \mathrm{MED} \text { is } \\
\text { equivalent to ingesting } \sim 3000 \mathrm{IU} \text { vitamin } \mathrm{D}_{3}\end{array}$ \\
\hline Tuna, canned & $236 \mathrm{IU} / 3.5 \mathrm{oz}$ vitamin $\mathrm{D}_{3}$ \\
\hline
\end{tabular}


Table 1. Cont.

\begin{tabular}{ll}
\hline Fortified foods & \\
Fortified breakfast cereals & $\sim 100 \mathrm{IU} /$ serving usually vitamin $\mathrm{D}_{3}$ \\
Fortified butter & $56 \mathrm{IU} / 3.5$ oz usually vitamin $\mathrm{D}_{3}$ \\
Fortified cheeses & $100 \mathrm{IU} / 3$ oz usually vitamin $\mathrm{D}_{3}$ \\
Fortified margarine & $429 / 3.5 \mathrm{oz}$ usually vitamin $\mathrm{D}_{3}$ \\
Fortified milk & $100 \mathrm{IU} / 8$ oz usually vitamin $\mathrm{D}_{3}$ \\
Fortified orange juice & $100 \mathrm{IU} / 8$ oz vitamin $\mathrm{D}_{3}$ \\
Fortified yogurts & $100 \mathrm{IU} / 8$ oz usually vitamin $\mathrm{D}_{3}$ \\
Infant formulas & $100 \mathrm{IU} / 8$ oz vitamin $\mathrm{D}_{3}$ \\
\hline
\end{tabular}

Pharmaceutical Sources in the

United States

Drisdol (vitamin $\mathrm{D}_{2}$ ) liquid $8000 \mathrm{IU} / \mathrm{mL}$

Vitamin $\mathrm{D}_{2}$ (Ergocalciferol) $\quad 50,000 \mathrm{IU} /$ capsule

Supplemental Sources

Multivitamin 400,500 , and $1000 \mathrm{IU}$ vitamin $\mathrm{D}_{3}$ or vitamin $\mathrm{D}_{2}$

Vitamin $\mathrm{D}_{3} \quad 400,800,1000,2000,5000,10,000,14,000$, and 50,000 IU

Exposure of human skin to solar UVB radiation (wavelengths: 290-315 nm) leads to the conversion of 7-dehydrocholesterol to previtamin $\mathrm{D}_{3}$ in the skin. Previtamin $\mathrm{D}_{3}$ is then rapidly converted to vitamin $\mathrm{D}_{3}$ (cholecalciferol) by temperature- and membrane-dependent processes $[7,11,12]$ (Figure 1).

Figure 1. Schematic representation of the synthesis and metabolism of vitamin D for regulating calcium, phosphorus and bone metabolism [7]. During exposure to sunlight, 7-dehydrocholesterol in the skin is converted to previtamin $\mathrm{D}_{3}$. Previtamin $\mathrm{D}_{3}$ immediately converts by a heat dependent process to vitamin $D_{3}[7,11,12]$. Excessive exposure to sunlight degrades previtamin $\mathrm{D}_{3}$ and vitamin $\mathrm{D}_{3}$ into inactive photoproducts [13]. Vitamin $\mathrm{D}_{2}$ and vitamin $\mathrm{D}_{3}$ from dietary sources is incorporated into chylomicrons, transported by the lymphatic system into the venous circulation [14]. Vitamin D (D represents $D_{2}$ or $D_{3}$ ) made in the skin or ingested in the diet can be stored in and then released from fat cells. Vitamin D in the circulation is bound to the vitamin D binding protein which transports it to the liver where vitamin $\mathrm{D}$ is converted by the vitamin D-25-hydroxylase to 25-hydroxyvitamin D [25(OH)D]. This is the major circulating form of vitamin $D$ that is used by clinicians to measure vitamin D status $[7,15]$ (although most reference laboratories report the normal range to be $20-100 \mathrm{ng} / \mathrm{mL}$, the preferred healthful range is 30-60 $\mathrm{ng} / \mathrm{mL}$ ) [7]. It is biologically inactive and must be converted in the kidneys by the 25-hydroxyvitamin D-1 $\alpha$-hydroxylase (1-OHase) to its biologically active form 1,25-dihydroxyvitamin D [1,25(OH) $\left.)_{2} \mathrm{D}\right]$ [7,15-17]. Serum phosphorus, calcium, fibroblast growth factors (FGF-23) and other factors can either increase $(+)$ or decrease (-) the renal production of $1,25(\mathrm{OH})_{2} \mathrm{D}[7] .1,25(\mathrm{OH})_{2} \mathrm{D}$ feedback regulates its own synthesis and decreases the synthesis and secretion of parathyroid hormone (PTH) in the parathyroid glands [6,7]. $1,25(\mathrm{OH})_{2} \mathrm{D}$ increases the expression of the 25-hydroxyvitamin D-24-hydroxylase (24-OHase) to catabolize $1,25(\mathrm{OH})_{2} \mathrm{D}$ to the water 
soluble biologically inactive calcitroic acid which is excreted in the bile $[7,18]$. $1,25(\mathrm{OH})_{2} \mathrm{D}$ enhances intestinal calcium absorption in the small intestine by stimulating the expression of the epithelial calcium channel $(\mathrm{ECaC})$ and the calbindin $9 \mathrm{~K}$ (calcium binding protein; $\mathrm{CaBP})[7,19,20] .1,25(\mathrm{OH})_{2} \mathrm{D}$ is recognized by its receptor in osteoblasts causing an increase in the expression of receptor activator of NFKB ligand (RANKL). Its receptor RANK on the preosteoclast binds RANKL which induces the preosteoclast to become a mature osteoclast. The mature osteoclast removes calcium and phosphorus from the bone to maintain blood calcium and phosphorus levels [7,17]. Adequate calcium and phosphorus levels promote the mineralization of the skeleton [7]. Note: This figure is reproduced with permission from [21], Copyright (C) 2007 Michael F. Holick.

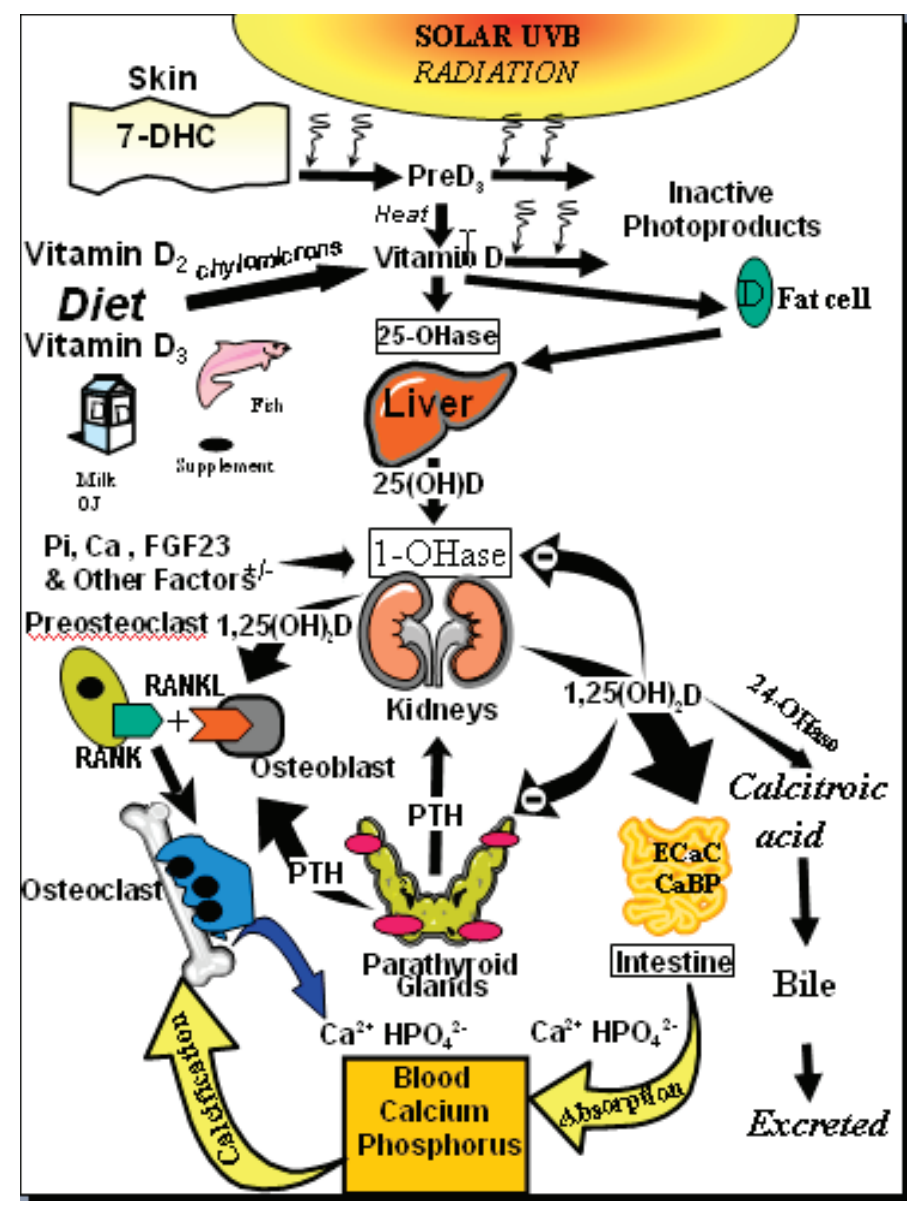

The amount of vitamin D production in the skin depends on the incident angle of the sun and thus on latitude, season and time of the day. It is highest when the sun is in the zenith and a flattening of the incident angle leads to a reduced vitamin D production [17]. Whole body exposure to sunlight with one minimal erythema dose (MED), i.e., the minimal dose leading to pink coloration of the skin $24 \mathrm{~h}$ after exposure, leads to vitamin D levels comparable to oral intake of 10,000 to up to 25,000 IU vitamin $\mathrm{D}_{2}$ [16,22]. However, sun exposure during most of the winter at latitudes above and below $\sim 33$ degrees North and South, respectively, doesn't lead to any production of vitamin $\mathrm{D}_{3}$ in the skin [16,23] (Figure 2). Other factors influencing the cutaneous vitamin D production 
adversely are an increase in skin pigmentation, aging, especially age $>65$ years and the topical application of a sunscreen [17].

Figure 2. Influence of season, time of day, and latitude on the synthesis of previtamin $\mathrm{D}_{3}$ in Northern (A and $\mathbf{C}$ ) and Southern hemispheres (B and $\left.\mathbf{D}\right)$. The hour indicated in $\mathbf{C}$ and $\mathbf{D}$ is the end of the 1-h exposure time. Note: This figure is reproduced with permission from [13], Copyright (C) 2010 Humana Press.
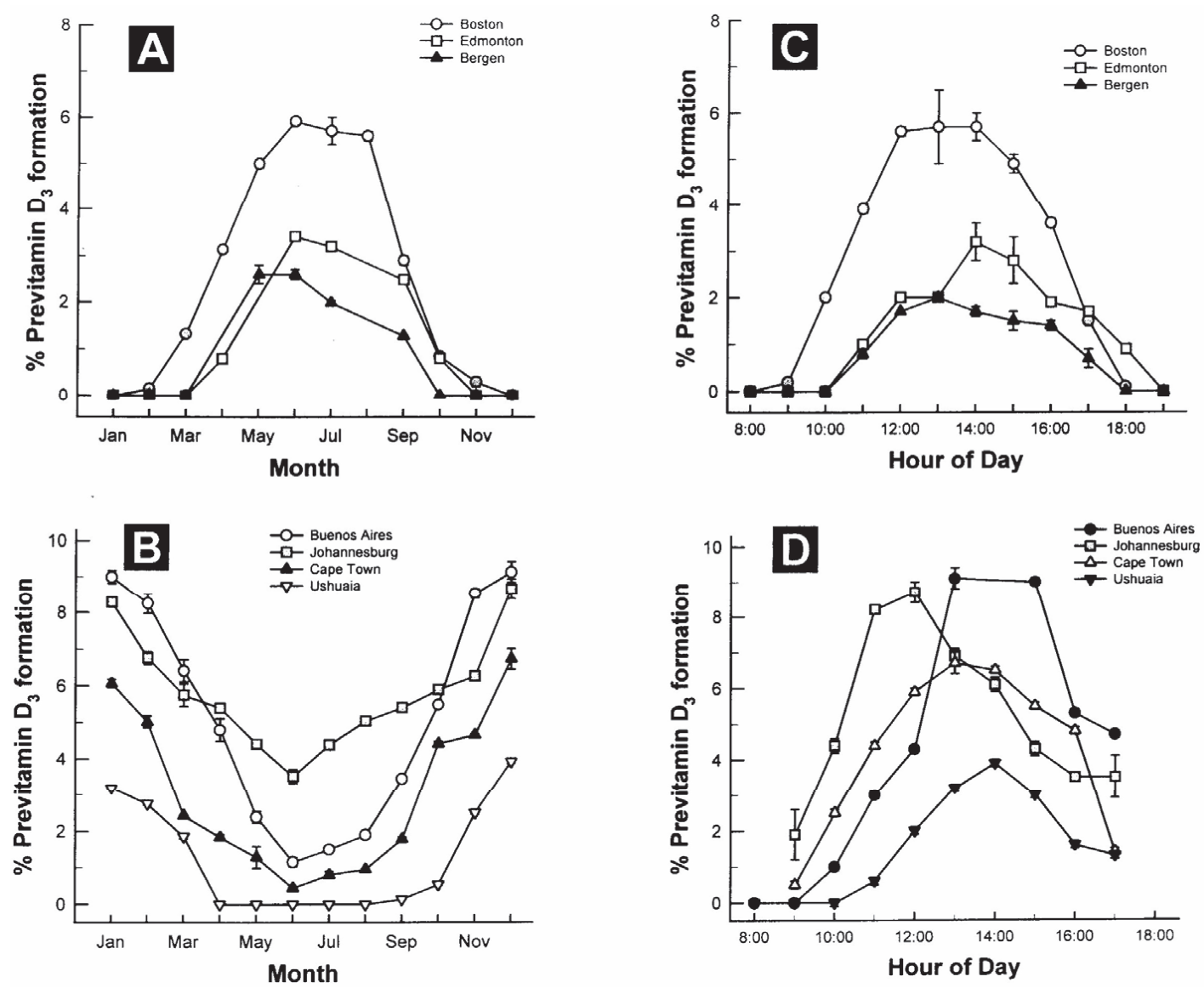

The number of foods naturally containing vitamin D in significant amounts is very limited. Among these are oily fish such as salmon, sardines and tuna, and oils of the liver of some fish such as cod as well as sun-exposed mushrooms [7] (Table 1). To increase the content of vitamin $\mathrm{D}_{2}$ in mushrooms producers are irradiating them with UV radiation [24,25].

In the 1930s, the fortification of milk, sodas, bread and even beer became popular [26]; however, after several cases of presumed vitamin D intoxication in infants in the 1950s in Great Britain [27] strict regulations limiting vitamin D fortification to only margarine were introduced in Europe $[14,28]$. Due to a relatively high prevalence of lactose intolerance leading to an avoidance of milk by many adults, the fortification of orange juice in the US was introduced as a novel approach of enhancing the vitamin D status of the public in the 2003 and proved to be as effective as oral supplementation [26,29]. Other fortified foods include margarine, yogurt, infant formula, butter, cheese and breakfast cereals [7] (Table 1). 
Vitamin $\mathrm{D}_{2}$ and vitamin $\mathrm{D}_{3}$ are available as oral over-the-counter supplements. In the US, only vitamin $\mathrm{D}_{2}$ is available as prescription drug [7,17]. Although there has been debate as to whether vitamin $\mathrm{D}_{2}$ is as effective as vitamin $\mathrm{D}_{3}$ in maintaining vitamin $\mathrm{D}$ status [30-36], other studies in children and adults have demonstrated that they are equally effective [29,37-40].

\section{Vitamin D-Metabolism}

Vitamin D from cutaneous synthesis or dietary/supplemental intake, is transported to the fat where it can be stored or to the liver for the first step of activation, the hydroxylation to 25-hydroxyvitamin $\mathrm{D}[25(\mathrm{OH}) \mathrm{D}]$, which is the major circulating form of vitamin $\mathrm{D}[7,15]$ and measured to assess a patient's vitamin D status [7,16,41,42] (Figure 1).

$25(\mathrm{OH}) \mathrm{D}$ is metabolized in the kidneys by the mitochondrial enzyme 25-hydroxyvitamin D-1 $\alpha$-hydroxylase (CYP27B1) to generate the systemically circulating active form, 1,25dihydroxyvitamin $\mathrm{D}\left[1,25(\mathrm{OH})_{2} \mathrm{D}\right][7,15-17]$. The renal synthesis of $1,25(\mathrm{OH})_{2} \mathrm{D}$ is regulated by several factors including serum phosphorus, calcium, fibroblast growth factor 23 (FGF-23), parathormone (PTH) and itself [7]. CYP27B1 is also expressed extrarenally in a multitude of tissues [17,43], including bone, placenta, prostate, keratinocytes, macrophages, T-lymphocytes, dendritic cells, several cancer cells [44], and the parathyroid gland [45] and enables the production of $1,25(\mathrm{OH})_{2} \mathrm{D}$. This active form of vitamin $\mathrm{D}$ is locally active and exerts auto- or paracrine effects $[15,17]$.

$1,25(\mathrm{OH})_{2} \mathrm{D}$ induces its own destruction by rapidly inducing the 25-hydroxyvitamin D-24-hydroxylase (CYP24A1), which leads to the multistep catabolism of both 25(OH)D and $1,25(\mathrm{OH})_{2} \mathrm{D}$ into biologically inactive, water-soluble metabolites including calcitroic acid [7,18] (Figure 1).

\section{Vitamin D Receptor (VDR)—Distribution and Function}

$1,25(\mathrm{OH})_{2} \mathrm{D}$, either produced in the kidneys [7] or extrarenally in the target tissues $[15,17]$, is the ligand of the vitamin D receptor (VDR) whose widespread distribution across many tissues explains the myriad of physiological actions of vitamin D. By interacting with the VDR, a transcription factor $[17,46], 1,25(\mathrm{OH})_{2} \mathrm{D}$ regulates directly and indirectly the expression of up to 2000 genes [6,7], many of whose promoters contain specific vitamin D response elements (VDRE). The VDR partners with other transcription factors, most importantly the retinoid X receptor (RXR) [47], and coactivators and corepressors provide target gene specificity [48-50]. A membrane-bound VDR may also exist and mediate more immediate, non-genomic actions of $1,25(\mathrm{OH})_{2} \mathrm{D}[44,51,52]$.

\section{Prevalence of Vitamin D Deficiency and Insufficiency}

$25(\mathrm{OH}) \mathrm{D}$ is the vitamin D metabolite that is measured to assess a patient's vitamin D status $[7,17]$. Vitamin D deficiency is diagnosed when $25(\mathrm{OH}) \mathrm{D}<20 \mathrm{ng} / \mathrm{mL}[16,53]$, vitamin D insufficiency is defined as $25(\mathrm{OH}) \mathrm{D}$ of $21-29 \mathrm{ng} / \mathrm{mL}$, and $25(\mathrm{OH}) \mathrm{D}>30 \mathrm{ng} / \mathrm{mL}$ is considered sufficient, with 40-60 ng/mL being the preferred range [16]. Vitamin D intoxication usually doesn't occur until $25(\mathrm{OH}) \mathrm{D}>150 \mathrm{ng} / \mathrm{mL}[7,16,23]$. 
These reference values are in part based on the finding, that the decline of parathyroid hormone (PTH) concentrations with increasing $25(\mathrm{OH}) \mathrm{D}$ levels in adults reached its nadir asymptotically at a $25(\mathrm{OH}) \mathrm{D}$ of $\sim 30-40 \mathrm{ng} / \mathrm{mL}$ in several studies [7,16,23,54-56]. However, a recent cross-sectional analysis of more than 300,000 paired serum PTH and 25(OH)D levels revealed no threshold, even at $25(\mathrm{OH}) \mathrm{D}$ levels $>60 \mathrm{ng} / \mathrm{mL}$, above which a further increase of the $25(\mathrm{OH}) \mathrm{D}$ level failed to further suppress PTH levels. The analysis also showed a strong age-dependency of the PTH-25(OH)D relationship [57].

According to studies in Canada, 30\%-50\% of children and adults are vitamin D deficient [5860]. The National Health and Nutrition Examination Surveys 2001-2006 showed a prevalence of vitamin D deficiency of 33\% [60,61]. Studies in Indian school children revealed a prevalence of severe vitamin D deficiency $(<9 \mathrm{ng} / \mathrm{mL})$ in more than $35 \%$ [62] and over $80 \%$ of pregnant women in India had 25(OH)D levels $<22.5 \mathrm{ng} / \mathrm{mL}$ [63]. Also reports from Africa [64], Australia [65], Brazil [66], Middle East [67,68], Mongolia [69], and New Zealand [70] documented a high risk for vitamin $\mathrm{D}$ deficiency in both adults and children [60,71].

Based on these findings, it has been estimated that 1 billion people worldwide are vitamin $\mathrm{D}$ deficient or insufficient [7,60] (Figure 3A-C).

Figure 3. (A) Prevalence at risk of vitamin D deficiency defined as a 25 -hydroxyvitamin $\mathrm{D}<12-20 \mathrm{ng} / \mathrm{mL}$ by age and sex: United States, 2001-2006. (B) Mean intake of vitamin D (IU) from food and food plus dietary supplements from Continuing Survey of Food Intakes by Individuals (CSFII) 1994-1996, 1998 and the Third National Health and Nutrition Examination Survey (NHANES III) 1988-1994. (C) Reported incidence of vitamin $\mathrm{D}$ deficiency defined as a 25 -hydroxyvitamin $\mathrm{D}<20 \mathrm{ng} / \mathrm{mL}$ around the globe including Australia (AU), Canada (CA), China (CH), India (IN), Korea (KR), Malaysia (MA), Middle East (ME), Mongolia (MO), New Zealand (NZ), North Africa (NA), Northern Europe (NE), United States (USA) [60]. Note: This figure is reproduced with permission from [60], Copyright (C) 2012 The Endocrine Society.

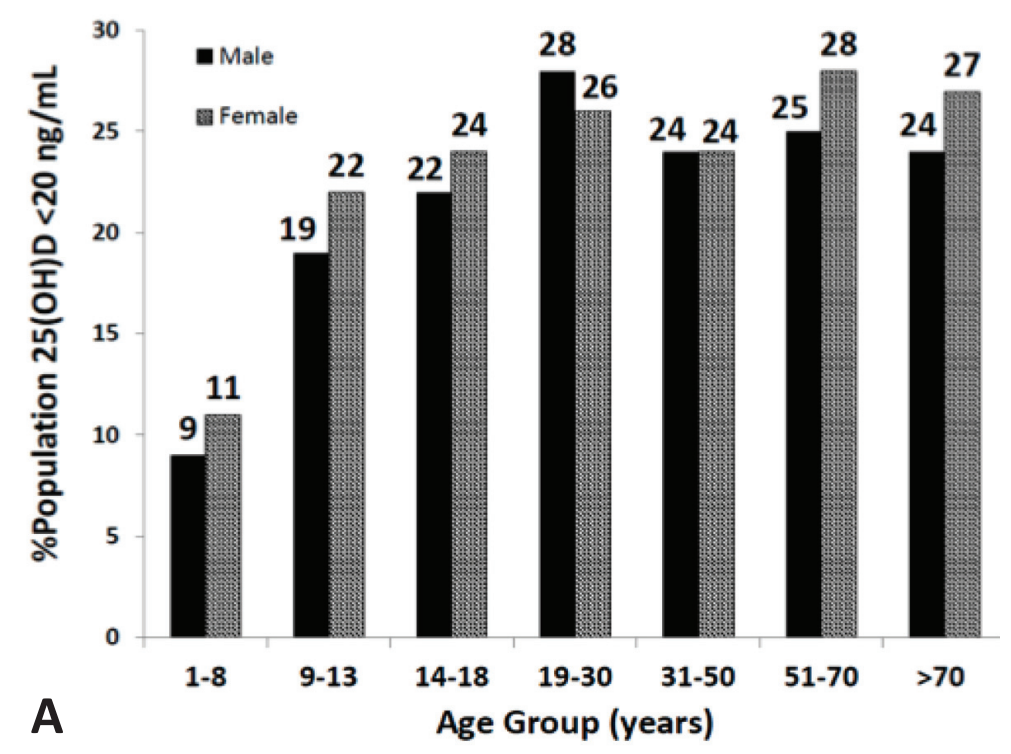


Figure 3. Cont.
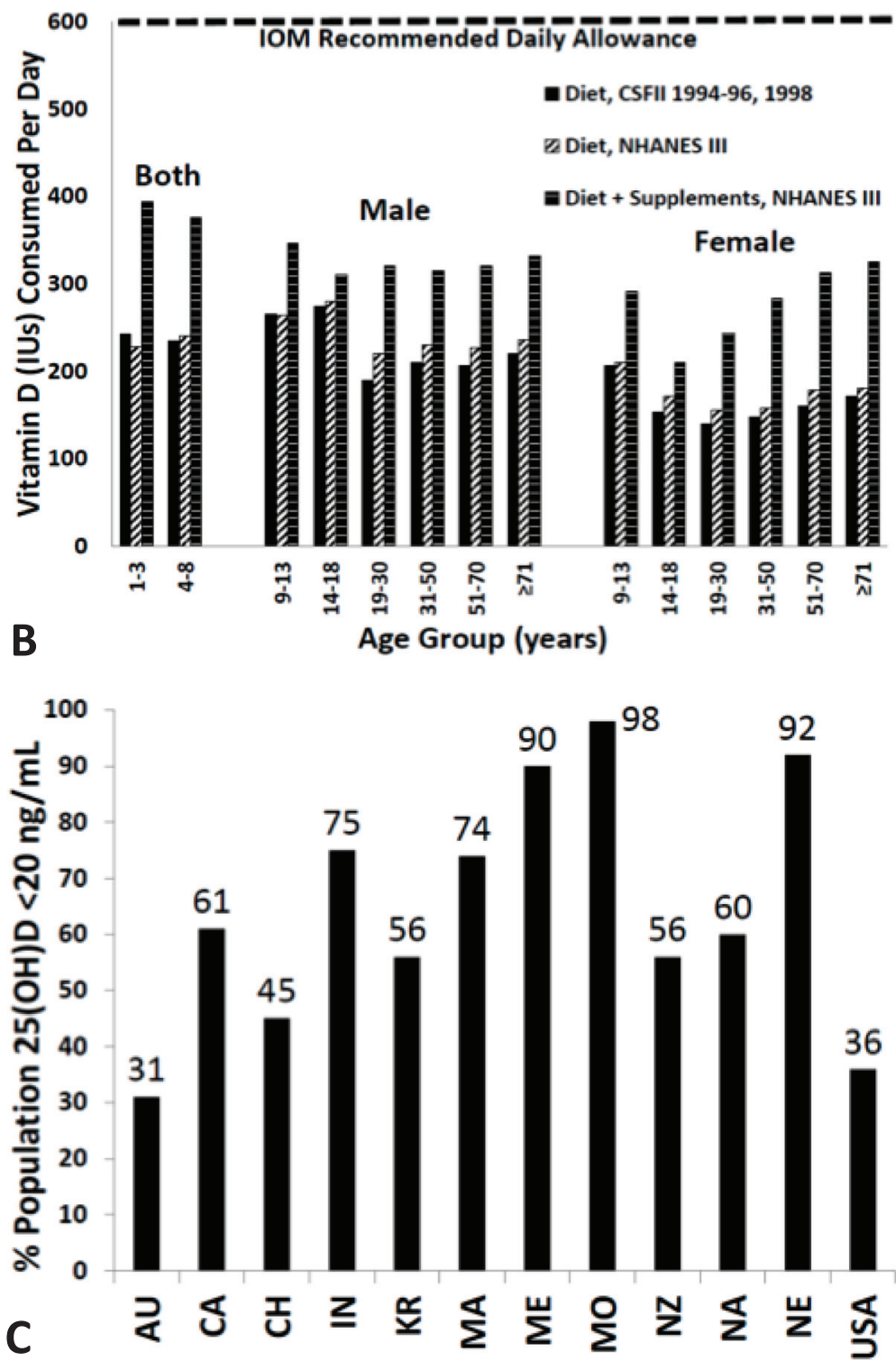

\section{Vitamin D and Calcium and Phosphorus Metabolism}

Vitamin D plays an important role in the calcium and phosphorus metabolism and helps ensure adequate levels of these minerals for metabolic functions and bone mineralization [7]. $1,25(\mathrm{OH})_{2} \mathrm{D}$ increases the efficiency of intestinal calcium absorption from $10 \%-15 \%$ to $30 \%-40 \%$ by interacting with the VDR-RXR and thereby promoting the expression of an epithelial calcium channel and a calcium-binding protein $[7,19,20]$. Based on several experiments conducted in rodents $[72,73]$ it has been estimated that $1,25(\mathrm{OH})_{2} \mathrm{D}$ also increases the intestinal phosphorus absorption from $50 \%-$ $60 \%$ to approximately $80 \%[7,14]$.

Vitamin D also mediates indirect effects on calcium and phosphorus by regulating the PTH levels. The parathyroid glands have CYP27B1 activity and the local production of $1,25(\mathrm{OH})_{2} \mathrm{D}$ using $25(\mathrm{OH}) \mathrm{D}$ as substrate could inhibit the synthesis of PTH [74]. However, 25(OH)D could also directly suppress PTH synthesis by directly activating the VDR [75]. Vitamin D deficiency is associated with 
lower levels of serum-ionized calcium, a stimulus leading to increased PTH levels. Conversely, higher calcium levels that are associated with higher $25(\mathrm{OH}) \mathrm{D}$ levels, suppress the PTH secretion. PTH increases tubular calcium and decreases renal phosphorus reabsorption [14] (Figure 1). PTH also stimulates the production of $1,25(\mathrm{OH})_{2} \mathrm{D}$ with the above mentioned effects on calcium and phosphorus homeostasis [7,14]. Moreover, both PTH and $1,25(\mathrm{OH})_{2} \mathrm{D}$ stimulate osteoblasts to mobilize skeletal calcium stores [7,17] (Figure 1). Vitamin D deficiency leads to secondary hyperparathyroidism with $\mathrm{PTH}$-enhanced $1,25(\mathrm{OH})_{2} \mathrm{D}$ production and is often associated with normal to high $1,25(\mathrm{OH})_{2} \mathrm{D}$ levels [7].

\section{Bone Health}

In the mid-1600s most children living in the crowded and polluted industrialized cities in Northern Europe developed a severe bone-deforming disease, rickets, that was characterized by growth retardation, enlargement of the epiphyses of the long bones, deformities of the legs, bending of the spine, knobby projections of the ribcage, and weak and toneless muscles [14,76] (Figure 4). Autopsy studies in children in the Netherlands and Boston in the early 1900s showed a rickets prevalence of $80 \%-90 \%$ [14]. In the 19th and 20th century, the major discoveries regarding the pathogenesis and prevention of rickets were made. In 1822, the importance of sun exposure for the prevention and cure of rickets was recognized by Sniadecki [77]. In 1890, these observations were extended and the recommendation of sun baths to prevent rickets was promoted by Palm [78]. In 1919, Huldschinski $[79,80]$ found that exposing children to UV radiation from a sun quartz lamp (mercury arc lamp) or carbon arc lamp was effective in treating rickets. In 1918, Mellanby et al. [81] prevented rickets in puppies with cod liver oil. McCollum et al. [82] called this new nutritional factor vitamin D. Hess and Weinstock [83] and Steenbock and Black [84] observed that UV irradiation of various foods and oils imparted antirachitic activity [14].

Vitamin D sufficiency is pivotal for normal skeletal development both in utero $[7,85]$ and in childhood [14], and for achieving and maintaining bone health in adults [23]. This is due to the fact that vitamin D sufficiency leads to an adequate calcium-phosphorus product $\left(\mathrm{Ca}^{2+} \times \mathrm{HPO}^{2-}\right)$ resulting in an effective bone mineralization [14]. Maternal vitamin D insufficiency during pregnancy was associated with a significant reduction in bone mineral acquisition in infants [85] that still persisted 9 years after birth [86]. In children whose epiphyseal plates haven't closed, vitamin D deficiency with $25(\mathrm{OH}) \mathrm{D}$ levels $<15 \mathrm{ng} / \mathrm{mL}$ causes chondrocyte disorganization and hypertrophy at the mineralization front as well as skeletal mineralization defects. This results in bone deformities and short stature, the typical signs of vitamin D deficiency rickets [14,87].

In adults low 25(OH)D and high PTH also lead to a low serum calcium $\times$ phosphorus product, resulting in osteomalacia, i.e., a defective mineralization of the collagen matrix causing a reduction of structural support and being associated with an increased risk of fracture [17,28]. Results from the National Health and Nutrition Examination Survey III (NHANES III) showed that bone density in the hip was directly related to the serum $25(\mathrm{OH}) \mathrm{D}$ level in both genders of all ethnicities $[88,89]$. A German study examined 25(OH)D serum levels and transiliac crest bone specimens of 675 individuals mainly in the 6th and 7th decade of life (401 males, mean age $58.7 \pm 17$ years, and 274 females, mean age: $68.3 \pm 17.3$ years) dying of unnatural death, such as a motor vehicle accident. 
The bone biopsies were taken within $48 \mathrm{~h}$ after death as well as the blood samples. Various previous experiments had shown that the $25(\mathrm{OH}) \mathrm{D}$ serum levels were stable for at least 10 days postmortem. While there's no uniformly accepted osteoid volume cut-off for the histologic diagnosis of osteomalacia, the study showed a prevalence of osteomalacia of over $25 \%$ when using a threshold of $>2 \%$ osteoid volume/bone volume (OV/BV) for the diagnosis of osteomalacia and a prevalence of $>43 \%$ when using a threshold of $1.2 \% \mathrm{OV} / \mathrm{BV}$ as described by Delling in 1975 [90]. Osteomalacia was absent in all individuals with $25(\mathrm{OH}) \mathrm{D}>30 \mathrm{ng} / \mathrm{mL}$, suggesting this as minimum serum level for maintenance of bone health. However, no minimum 25(OH)D level could be determined that was inevitably associated with mineralization defects [91].

One possible explanation is that obtaining a single blood level of $25(\mathrm{OH}) \mathrm{D}$ doesn't provide information about the long-term vitamin $\mathrm{D}$ status of the individual. It is possible that for example that the subject became ill during the winter and stopped ingesting foods containing vitamin D or decreased sun exposure during the summer that would acutely lower blood levels of $25(\mathrm{OH}) \mathrm{D}$ without causing osteomalacia.

Figure 4. Sister (right) and brother (left) ages 4 years and 6.5 years, respectively, demonstrating classic knock-knees and bow legs, growth retardation, and other skeletal deformities [14]. Note: This figure is reproduced with permission from [14], Copyright (C) 2006 American Society for Clinical Investigation.

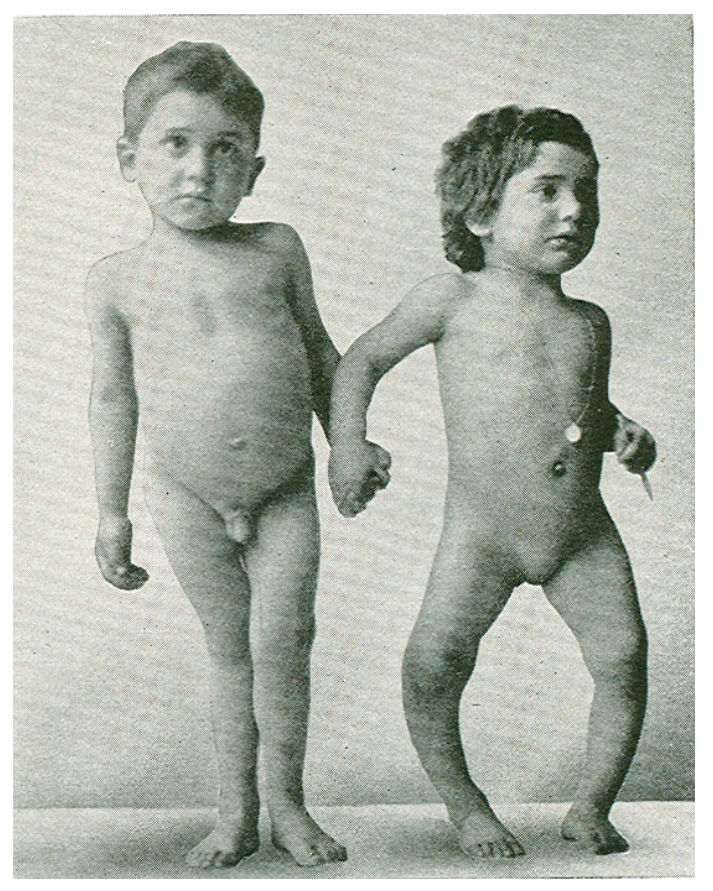

\section{Osteoporosis and Fractures}

As a decrease in $25(\mathrm{OH}) \mathrm{D}$ leads to secondary hyperparathyroidism associated with osteoclastogenesis and an increase in bone resorption exceeding osteoblast-mediated bone formation [88], this can precipitate and exacerbate osteopenia and osteoporosis in adults [17,92,93]. 
Osteoporosis has a prevalence of $\sim 1 / 3$ in women $60-70$ years of age and of $\sim 2 / 3$ in women 80 years of age or older [7]. It's estimated that currently 10 million Americans have osteoporosis with 1.5 to 2 million osteoporosis-related fractures annually [94]. An osteoporosis-related fracture will be experienced by one in eight men over age 50 years in their lifetime [95].

Vitamin D promotes bone health by maintaining the PTH levels in a physiologically healthy level, stimulating osteoblastic activity, and promoting bone mineralization as well as reducing risk of falls thereby reducing risk of fracture $[93,96]$.

According to data from the Women's Health Initiative [97], the odds ratio of risk for hip fracture was inversely related to the serum $25(\mathrm{OH}) \mathrm{D}$ level [88]. There's evidence that patients with $25(\mathrm{OH}) \mathrm{D}$ levels $>30 \mathrm{ng} / \mathrm{mL}$ have a lower risk of fracture. Several studies have been conducted to evaluate the effect of vitamin D supplementation on the fracture risk, with some studies showing a significant reduction of the risk of fractures while others didn't [98]. One of these showed that the supplementation with calcium (1200 mg) and vitamin $\mathrm{D}_{3}$ (800 IU/day) decreased the number of hip fractures by $43 \%(p=0.043)$ and the total number of nonvertebral fractures by $32 \%$ [99]. The RECORD study however, did not show a reduction in fracture risk with supplementation with vitamin D (800 IU/day), or calcium (1000 mg/day), or both [100], but often compliance was poor and serum $25(\mathrm{OH}) \mathrm{D}$ levels were not measured at the end of the study in most participants $[7,98,100]$. A meta-analysis of more than 30,000 participants did show that supplementation with vitamin D ( $\geq 792 \mathrm{IU} /$ day) led to a significant reduction in the risk of fracture; the risk of hip fracture was reduced by $30 \%$, the risk of any non-vertebral fracture by $14 \%$ [98-106].

\section{Muscular Health and Falls}

Vitamin D exerts multiple effects on muscle health [107]. Its active form $1,25(\mathrm{OH})_{2} \mathrm{D}$ could be produced locally in muscle cells as suggested by the recent identification of CYP27B1 bioactivity in regenerating mouse muscle and skeletal muscle cells [108], however other studies have failed to detect this enzyme in muscle cells [109]. $1,25(\mathrm{OH})_{2} \mathrm{D}$ is thought to modulate muscle function via the VDR, which seems to be expressed in skeletal muscles [109-113], by regulating gene transcription and promoting de-novo protein synthesis [107]. Also, rapid non-genomic pathways involving a membrane-bound vitamin $\mathrm{D}$ receptor could exist and affect the calcium handling involving the sarcoplasmic reticulum and the calcium signaling in muscle cells [109]. Several studies indicate that the muscle function depends on the VDR genotype in the muscle cell $[114,115]$. The possibility of a direct interaction between 25(OH)D and the VDR has been proposed in CYP27B1 ${ }^{-/}$cells $[109,116]$. However, the existence of a VDR in muscle cells is discussed highly controversially, as a more recent study failed to detect the VDR in muscle cells and as the antibodies used for immunocytochemical staining to detect the VDR in previous studies have been shown to be not exclusively specific for the VDR and could explain potentially false-positive results in these previous studies [117].

Vitamin D deficiency is associated with diffuse muscle pain, muscle weakness $[7,118]$, predominantly in the proximal muscle groups [115], and a reduction in performance speed [107,119]. This is caused by muscle atrophy of mainly type II muscle fibers [115]. Proximal muscle weakness in severe vitamin D deficiency could also be caused by secondary hyperparathyroidism and resultant hypophosphatemia $[60,106,120]$. 
There is a positive association between $25(\mathrm{OH}) \mathrm{D}$, lower extremity function, proximal muscle strength and physical performance [107,121,122]. Muscle strength [123] and postural and dynamic balance [124] were increased by vitamin D supplementation [107]. The effect of vitamin D supplementation on the risk of falls was examined in a randomized, controlled multi-dose study, showing that the supplementation of $800 \mathrm{IU} /$ day lowered the adjusted-incidence rate ratio of falls by $72 \%$ compared to those taking placebo over 5 months [125]. A meta-analysis of 8 randomized controlled trials $(n=2426)$ showed that supplemental vitamin D of $700-1000 \mathrm{IU} /$ day or a serum $25(\mathrm{OH}) \mathrm{D}$ of $\geq 24 \mathrm{ng} / \mathrm{mL}$ reduced the risk of falls by $19 \%$ and $23 \%$ respectively. No benefit was observed with lower supplemental doses or lower serum $25(\mathrm{OH}) \mathrm{D}$ concentrations [126].

\section{Cancer}

Living at higher latitudes with lower UV exposure and thus lower vitamin D production is associated with an increased risk for the occurrence of a variety of cancers and with an increased likelihood of dying from them, as compared to living at lower latitudes $[7,17,127,128]$. A recent review of ecological studies associating solar UVB exposure-vitamin D and cancers found strong inverse correlations with solar UVB irradiance for 15 types of cancer: bladder, breast, cervical, colon, endometrial, esophageal, gastric, lung, ovarian, pancreatic, rectal, renal, and vulvar cancer; and Hodgkin's and non-Hodgkin's lymphoma [129].

An inverse association between $25(\mathrm{OH}) \mathrm{D}$ and the incidence of several cancers and mortality from these cancers has been shown in case-control studies, prospective and retrospective studies [130-140], especially for cancers of the colon, breast and prostate [7]. Regarding colon cancer, the Nurses' Health cohort study $(n=32,826)$ showed an inverse association of the odds ratios for colorectal cancer with the median 25(OH)D serum levels. At $16.2 \mathrm{ng} / \mathrm{mL}$ the odds ratio was 1 and 0.53 at $39.9 \mathrm{ng} / \mathrm{mL}(p \leq 0.01)[7,140]$.

These associational studies have certain limitations regarding the establishment of a causality between vitamin D status and a reduced risk of cancer, e.g., as low serum 25(OH)D levels are also linked with confounding factors related to higher cancer risk, including obesity (vitamin D is sequestered in adipose tissue), and lack of physical activity (correlated with less time outdoors and less solar exposure) [138]. However, a population-based, double-blind, randomized placebo-controlled trial of 4 years duration with more than thousand postmenopausal women, whose principal secondary outcome was cancer incidence, showed that the supplementation with calcium (1400-1500 mg/day) and vitamin $\mathrm{D}_{3}$ (1100 IU/day) reduced the relative risk (RR) of cancer by $\sim 60 \%(p<0.01)$. The repetition of a cancer free survival analysis after the first 12 months revealed, that the relative risk for the calcium + vitamin D group was reduced by $\sim 77 \%$ (confidence interval $[\mathrm{CI}]: 0.09-0.60$; $p<0.005)$. Multiple regression models also showed that both treatment and serum $25(\mathrm{OH}) \mathrm{D}$ concentrations were significant, independent predictors of cancer risk [137].

Mounting evidence suggests a biological plausibility for anti-carcinogenic effects of vitamin D, which could explain these results. $1,25(\mathrm{OH})_{2} \mathrm{D}$, which has been shown to be produced locally by various cancer cells metabolizing the substrate 25(OH)D [38], inhibits carcinogenesis by several mechanisms [141]. 1,25(OH $)_{2} \mathrm{D}$ exerts anti-proliferative effects on cancer cells by promoting cyclin-dependent kinase (CDK) inhibitor synthesis, and by influencing several growth factors and 
their signaling pathways including insulin-like growth factor 1 (IGF-1), transforming growth factor $\beta$ (TGF $\beta)$, Wnt/ $\beta$-catenin, MAP kinase 5 (MAPK5) and nuclear factor $\kappa \mathrm{B}(\mathrm{NF}-\mathrm{kB})$ [142] (Figure 5).

Figure 5. Metabolism of 25-hydroxyvitamin D [25(OH)D] to 1,25 dihydroxyvitamin D $1,25(\mathrm{OH})_{2} \mathrm{D}$ for non-skeletal functions. When a monocyte/macrophage is stimulated through its toll-like receptor 2/1 (TLR2/1) by an infective agent such as Mycobacterium tuberculosis (TB), or its lipopolysaccharide (LPS) the signal upregulates the expression of vitamin D receptor (VDR) and the 25-hydroxyvitamin D-1-hydroxylase (1-OHase). $25(\mathrm{OH}) \mathrm{D}$ levels $>30 \mathrm{ng} / \mathrm{mL}$ provides adequate substrate for the 1-OHase to convert it to $1,25(\mathrm{OH})_{2} \mathrm{D}$. $1,25(\mathrm{OH})_{2} \mathrm{D}$ returns to the nucleus where it increases the expression of cathelicidin which is a peptide capable of promoting innate immunity and inducing the destruction of infective agents such as TB. It is also likely that the $1,25(\mathrm{OH})_{2} \mathrm{D}$ produced in the monocytes/macrophage is released to act locally on activated $\mathrm{T}$ (AT) and activated $\mathrm{B}(\mathrm{AB})$ lymphocytes which regulate cytokine and immunoglobulin synthesis respectively [143-147]. When $25(\mathrm{OH}) \mathrm{D}$ levels are $\sim 30 \mathrm{ng} / \mathrm{mL}$, it reduces risk of many common cancers [130-140]. It is believed that the local production of $1,25(\mathrm{OH})_{2} \mathrm{D}$ in the breast, colon, prostate, and other cells regulates a variety of genes that control proliferation. Once $1,25(\mathrm{OH})_{2} \mathrm{D}$ completes the task of maintaining normal cellular proliferation and differentiation, it induces the 25-hydroxyvitamin D-24-hydroxylase (24-OHase). The 24-OHase enhances the metabolism of $1,25(\mathrm{OH})_{2} \mathrm{D}$ to calcitroic acid which is biologically inert $[7,18]$. Thus, the local production of $1,25(\mathrm{OH})_{2} \mathrm{D}$ does not enter the circulation and has no influence on calcium metabolism. The parathyroid glands have 1-OHase activity [45] and the local production of $1,25(\mathrm{OH})_{2} \mathrm{D}$ inhibits the expression and synthesis of PTH [74]. The production of $1,25(\mathrm{OH})_{2} \mathrm{D}$ in the kidney enters the circulation and is able to downregulate renin production in the kidney $[148,149]$ and to stimulate insulin secretion in the $\beta$-islet cells of the pancreas $[148,150]$. Note: This figure is reproduced with permission from [21], Copyright (C) 2007 Michael F. Holick.

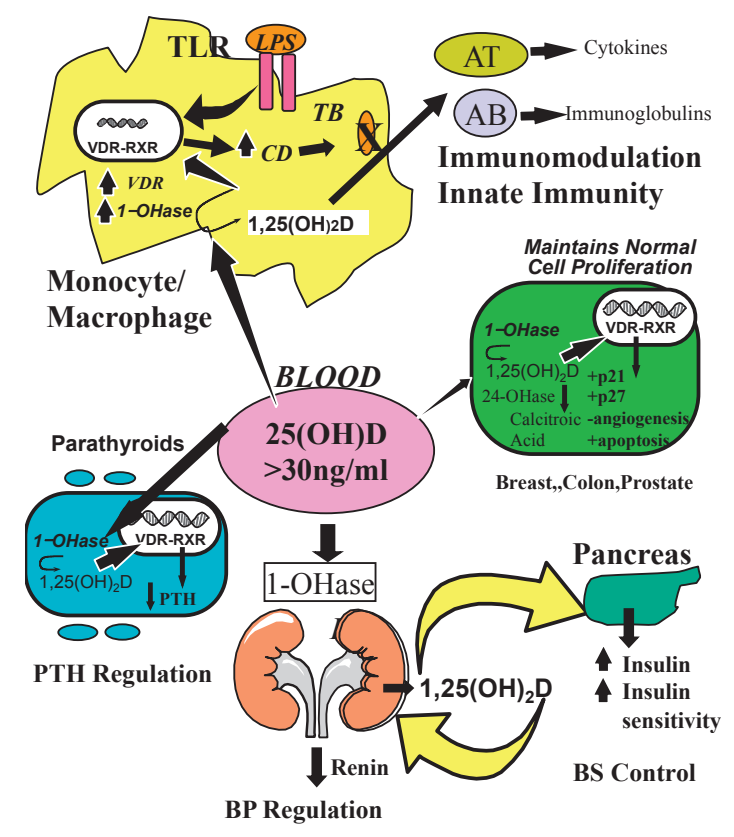


Apoptosis is characterized as programmed cell death permitting the removal of damaged cells including cancer cells in multicellular organisms without impairing the cellular microenvironment. Defective apoptosis plays a major role in the development and progression of cancer [151]. It has been shown, that both immunobiological mechanisms of cancer immunosurveillance and cancer immunoediting [152], as well as chemotherapeutic agents and radiation, utilize the apoptotic pathway to induce cancer cell death $[151,153]$. $1,25(\mathrm{OH})_{2} \mathrm{D}_{3}$ might exert anti-carcinogenic effects by promoting various pro-apoptotic mechanisms including the downregulation of the anti-apoptotic gene Bcl-2 [154] and by upregulating of the pro-apoptotic gene Bax [155], 1,25(OH) ${ }_{2} \mathrm{D}_{3}$ induces differentiation, partly by reducing the expression of the $c$-myc oncogene $[141,156]$. It regulates the prostaglandin (PG) metabolism and signaling, thus decreasing PG-mediated promotion of carcinogenesis $[141,157]$. It suppresses tumor angiogenesis, e.g., mediated by $1,25(\mathrm{OH})_{2} \mathrm{D}$ 's effects on the PG synthesis and by regulating the expression of crucial factors controlling the angiogenesis. $1,25(\mathrm{OH})_{2} \mathrm{D}_{3}$ suppresses tumor invasion and metastasis by various mechanisms [141], e.g., by decreasing the expression and activity of cell invasion-associated serine proteases and metalloproteinases and inducing their inhibitors [158], and by inducing $E$-cadherin expression, contributing to adhesive properties of cells [141,159]. Other effects mediated by $1,25(\mathrm{OH})_{2} \mathrm{D}$ are thought to be the induction of autophagy as process to trigger the death of cancer cells and to block tumor growth and by inducing enzymes involved in antioxidant defense mechanisms and DNA-repair [142]. 1,25(OH) $2 \mathrm{D}$ also regulates androgen and estrogen receptor signaling, thereby inhibiting tumor growth of some sex hormone-dependent tumors such as prostate and breast cancer. It has also been shown to reduce the expression of aromatase, thereby inhibiting breast cancer growth [141].

\section{Vitamin D and Cardiovascular Risk}

Most epidemiological and prospective studies as well as meta-analyses [148,160-163] suggest a significant inverse association between $25(\mathrm{OH}) \mathrm{D}$ serum levels and cardiovascular risk. The prospective Intermountain Heart Collaborative Study with more than 40,000 participants revealed that 25(OH)D $<15 \mathrm{ng} / \mathrm{mL}$ compared to $25(\mathrm{OH}) \mathrm{D}>30 \mathrm{ng} / \mathrm{mL}$ was associated with highly significant increases in the prevalence of type 2 diabetes mellitus, hypertension, hyperlipidemia, and peripheral vascular disease, coronary artery disease, myocardial infarction, heart failure, and stroke $(p<0.0001)$, as well as with incident death (all-cause mortality was used as primary survival measure), heart failure, coronary artery disease/myocardial infarction $(p<0.0001)$, stroke $(p=0.003)$, and their composite $(p<0.0001)$ [164].

A meta-analysis examining the association between vitamin $D$ status and the risk of cerebrovascular events including $>1200$ stroke cases found that the pooled relative risk for stroke was $52 \%$ higher when comparing $25(\mathrm{OH}) \mathrm{D}$ levels $\leq 12.4 \mathrm{ng} / \mathrm{mL}$ with $25(\mathrm{OH}) \mathrm{D}$ levels $>18.8 \mathrm{ng} / \mathrm{mL}$ [165].

Many of these associations are well established, causation however is yet to be proven [166]. Individuals spending less time exercising outdoors in the sun, e.g., have a higher risk of developing cardiovascular diseases, and those individuals also will likely have lower $25(\mathrm{OH}) \mathrm{D}$ levels coincidentally [166,167]. Also, obesity, a condition associated with cardiovascular disease [168], is associated with a lower vitamin D status due to a sequestration and volumetric dilution of the lipophilic vitamin $\mathrm{D}$ in the fat tissue $[23,166,169,170]$, potentially explaining the described 
correlations [166]. Despite these limitations many studies suggest a biological plausibility for the beneficial effects of vitamin D on cardiovascular risk factors and cardiovascular health.

The vitamin D receptor is present in endothelium, vascular smooth muscle, and cardiomyocytes [162,166] and may protect against atherosclerosis through the inhibition of macrophage cholesterol uptake and foam cell formation, reduced vascular smooth muscle cell proliferation, and reduced expression of adhesion molecules in endothelial cells [166] and through inhibition of cytokine release from lymphocytes [162]. Several meta-analyses indicate an inverse association between vitamin D status and hypertension [171]. Studies showed, that antihypertensive effects were associated with raising $25(\mathrm{OH}) \mathrm{D}$ levels with vitamin D supplementation [172-174] or UVB exposure [175].

Mechanistically, this effect could be partly mediated by vitamin D's capability to suppress the levels of PTH, which can cause arrhythmias and lead to myocardial hypertrophy and increased blood pressure $[148,176] .1,25(\mathrm{OH})_{2} \mathrm{D}_{3}$ has also been shown to suppress the levels of renin and could contribute to vitamin D's potential antihypertensive properties $[148,149]$.

A meta-analysis examining the association between vitamin $\mathrm{D}$ status or vitamin $\mathrm{D}$ supplementation, and incident type 2 diabetes showed that individuals with $25(\mathrm{OH}) \mathrm{D}$ levels $>25 \mathrm{ng} / \mathrm{mL}$ compared to those with $25(\mathrm{OH}) \mathrm{D}<14 \mathrm{ng} / \mathrm{mL}$ had a $43 \%$ lower risk of developing type 2 diabetes and that a vitamin D supplementation with $>500 \mathrm{IU} /$ day compared to $<200 \mathrm{IU} /$ day reduced the risk by $13 \%$ [177]. In the Nurses' Health Study $>83,000$ women were followed-up prospectively and it was shown, that a combined daily intake of $>1200 \mathrm{mg}$ calcium and $>800$ IU vitamin D was associated with a 33\% lower risk of type 2 diabetes with RR of 0.67 (CI: 0.49-0.90) compared with an intake of $<600 \mathrm{mg}$ calcium and $400 \mathrm{IU}$ vitamin D [178]. A prospective study following-up more than 2000 participants showed, that the risk of progression from prediabetes to diabetes was reduced by $62 \%$ when comparing the highest quartile of $25(\mathrm{OH}) \mathrm{D}$ levels with the lowest quartile $[179,180]$.

This could be explained by experimental findings indicating that vitamin $\mathrm{D}$ exerts various antidiabetic effects. The VDR is expressed in pancreatic beta cells and $1,25(\mathrm{OH})_{2} \mathrm{D}$ stimulates insulin secretion [148,150]. Improvement in vitamin D status also leads to a improvement of insulin sensitivity, mediated for example by upregulation of insulin receptors [148], and modulates inflammation, which is also thought to play a role in type 2 diabetes [150,179] (Figure 5).

\section{Vitamin D's Role in Autoimmune Disease}

Ecological studies have shown that the prevalence of certain autoimmune diseases was associated with latitude, suggesting a potential role of sunlight exposure, and thus vitamin D production, on the pathogenesis of type 1 diabetes mellitus, multiple sclerosis and Crohn's disease [181]. The increased prevalence at higher latitudes has been shown for multiple sclerosis (MS) [181,182], inflammatory bowel disease [183], rheumatoid arthritis [184] and type 1 diabetes [181,182,185].

A few case-control studies relate the vitamin D status to the risk of developing these autoimmune diseases [181]. One of them, a prospective, nested case-control study analyzed serum samples and the data of disability databases of more than seven million US military personnel, and showed, that among whites (148 cases, 296 controls), the risk of multiple sclerosis significantly decreased with increasing levels of $25(\mathrm{OH}) \mathrm{D}$ (odds ratio for a $20 \mathrm{ng} / \mathrm{mL}$ increase in $25(\mathrm{OH}) \mathrm{D}$ was $0.59(95 \% \mathrm{CI}$ : 
$0.36-0.97)$. When comparing the highest quintile of $25(\mathrm{OH}) \mathrm{D}$ with the lowest, the odds ratio for developing MS was 0.38 (95\% CI: $0.19-0.75 ; p=0.006)$, with an particularly strong inverse association for $25(\mathrm{OH}) \mathrm{D}$ levels measured before age 20 years [186].

A study addressing vitamin D's effect on multiple sclerosis showed the safety of high-dose vitamin D ( 14,000 IU/day). It appeared to have immunomodulatory effects including a persistent reduction in T-cell proliferation and resulted in a trend for fewer relapse events [187]. When examining the association between 25(OH)D serum levels and the relapse rate in MS patients before and after supplementation with $\sim 3000$ IU vitamin D per day, a significant strong inverse relationship between the relapse incidence rate and the 25(OH)D level $(p<0.0001)$ was found [188].

An inverse association between maternal 25(OH)D levels and the risk for type 1 diabetes in the offspring has been shown in a population-based, nested cohort study of $\sim 30,000$ pregnant women. Compared to the upper quartile of $25(\mathrm{OH}) \mathrm{D}$ levels, the odds of type 1 diabetes in the women with the lowest quartile was more than twofold higher [189]. A birth-cohort study with $>10,000$ children showed, that regular supplementation with 2000 IU vitamin D per day in the first year of life was associated with a $88 \%$ reduction of the risk for type 1 diabetes later in life when compared to those without supplementation [190]. However, another study did not show a statistically significant association between taking cod liver oil or other vitamin D supplements in the first year of life and the risk of type 1 diabetes mellitus [191].

Merlino et al. [192] showed in a prospective cohort study of 29,368 women of ages 55-69 years without a history of rheumatoid arthritis at study baseline, that greater intake (highest versus lowest tertile) of vitamin D was inversely associated with risk of rheumatoid arthritis (RR $0.67 ; 95 \% \mathrm{CI}$ : $0.44-1.00 ; p$ for trend $=0.05$ ).

These associations indicate a contributory role of vitamin D in the pathophysiology of autoimmune diseases. This is further supported by various experimental findings showing vitamin D's capability to regulate chemokine production, counteracting autoimmune inflammation and to induce differentiation of immune cells in a way that promotes self-tolerance. This involves the enhancement of the innate and the inhibition of the adaptive immune system by regulating the interactions between lymphocytes and antigen presenting cells. By increasing the quantity of Th2 lymphocytes and by inducing proliferation of dendritic cells with tolerance properties, vitamin D exerts anti-inflammatory and immunoregulatory effects [181].

Immune cells possess both the enzymatic machinery to produce $1,25(\mathrm{OH})_{2} \mathrm{D}$ and a VDR. This could explain, why certain polymorphisms in the VDR gene seem to affect the risk for multiple autoimmune diseases, the time of onset of disease and disease activity [181,193-197].

\section{Vitamin D and Infectious Diseases}

The plethora of effects of vitamin D on regulating the immune system plays a role in fighting infectious diseases [198]. Vitamin D enhances the innate immunity against various infections [143], especially tuberculosis, influenza and viral upper respiratory tract infections [198].

Historically, cod liver oil (one of only a few natural sources of vitamin D) was given to tuberculosis patients in 19th and 20th century [199-201]. Later in the nineteenth century, tuberculosis patients were treated in sanatoriums with heliotherapy, i.e., sun exposure. In 1903, Niels 
Ryberg Finsen was awarded the Nobel prize for medicine "in recognition of his contribution to the treatment of diseases, especially lupus vulgaris (tuberculosis of the skin), with concentrated light radiation, whereby he has opened a new avenue for medical science" [199,202]. After vitamin D had been identified as the active ingredient in cod-liver oil $[199,203]$, vitamin $\mathrm{D}_{2}$ was used successfully in the treatment of lupus vulgaris in several studies. In 1946 a report in Proc. R. Soc. Med. [204] stated that there was no room for doubt that calciferol (vitamin D) in adequate dosage will cure a substantial proportion of cases of lupus vulgaris [199,204]. In 1947 the first reference to successful treatment of pulmonary tuberculosis with vitamin D was published $[199,205]$. In the wake of the antibiotic era both heliotherapy and vitamin D therapy for treating tuberculosis patients were quickly forgotten $[199,206]$. However recent studies have suggested that vitamin D may have an important role to play in reducing risk for acquiring one of the most common and deadly infectious diseases that plague third world countries [206].

One case-control study examining the association between vitamin D status and tuberculosis showed, that the mean 25(OH)D levels were statistically significant different $(p<0.005)$ between patients with pulmonary and extrapulmonary tuberculosis $(10.7 \mathrm{ng} / \mathrm{mL})$ and controls $(19.5 \mathrm{ng} / \mathrm{mL})$ [207]. In another study, 25(OH)D levels $<10 \mathrm{ng} / \mathrm{mL}$ were significantly associated with active tuberculosis (OR 2.9; 95\% Cl: 1.3-6.5; $p=0.008$ ) [208]. A meta-analysis showed, that low serum 25(OH)D levels were associated with higher risk of active tuberculosis, and that the pooled effect size in random effects meta-analysis was 0.68 (95\% CI: 0.43-0.93), representing a medium to large effect [209]. A double-blind, placebo-controlled study in Mongolian school children $(n=120)$ examining the effect of vitamin D supplementation (800 IU/day) on tuberculin skin test conversion to positive showed a trend towards fewer conversions in the vitamin D group $(p=0.06)$, suggesting a potential role of vitamin D in reducing the rate of acquisition of latent tuberculosis infection [210].

Several interventional studies examining the effect of vitamin D supplementation in patients with active tuberculosis have been conducted. Some of them showed an improved immunity against mycobacteria [211], a significantly improved sputum conversion rate and a higher rate of radiological improvement [212], and a significantly hastened sputum culture conversion in participants with the tt genotype of the TaqI vitamin D receptor polymorphism [213]. There was also a higher rate of tuberculosis symptom improvement and a significantly higher weight gain $(p<0.005)$ in children [214]. A prospective, randomized placebo-controlled trial examining the effect of adjunctive vitamin $\mathrm{D}$ supplementation in patients receiving antimicrobial therapy showed that vitamin D supplementation led to an accelerated sputum smear conversion and an accelerated resolution of inflammation [215]. Another study however in which three doses of 100,000 IU vitamin D3 each were given during 8 months did not lead to a reduction in the clinical severity score or mortality [216].

Some studies examined the effect of vitamin D supplementation on the risk of influenza [217,218].

In 1981, R. Edgar Hope-Simpson proposed that a "seasonal stimulus" was intimately associated with solar radiation and explained the remarkable seasonality of epidemic influenza [219,220]. As the vitamin D status changes during the seasons, it has been suggested, that vitamin D could be this "seasonal stimulus" [219]. A randomized trial of vitamin D3 supplementation (1200 IU/day) in school children $(n=334)$ showed a significantly reduced risk for influence A as determined by 
both antibody and sputum testing compared to the placebo group (RR 0.58 ; 95\% CI: $0.34-0.99$; $p=0.04)$ [218].

One study using questionnaires to retrospectively determine the occurrence of influenza-like disease in participants of 10 different clinical trials $(n=569)$, receiving 1111-6800 IU/day, however did not show a significant difference in the incidence and severity of influenza-like disease [217].

The NHANES III study $(n>18)$ revealed an inverse association between serum 25(OH)D levels and recent upper respiratory tract infections (URTI). Lower 25(OH)D levels were independently associated with recent URTI compared with $25(\mathrm{OH}) \mathrm{D}$ levels of $\geq 30 \mathrm{ng} / \mathrm{mL}$ (OR 1.36; 95\% CI: $1.01-1.84$ for $<10 \mathrm{ng} / \mathrm{mL}$ and OR $1.24 ; 95 \% \mathrm{CI}: 1.07-1.43$ for 10 to $<30 \mathrm{ng} / \mathrm{mL}$ ). In individuals with asthma or chronic obstructive airway disease this association was stronger (OR of 5.67 in asthma respectively OR of 2.26 in chronic obstructive airway disease) [221]. A study in Finish men $(n=800)$ found a significant association between $25(\mathrm{OH}) \mathrm{D}$ serum levels $<16 \mathrm{ng} / \mathrm{mL}$ and significantly more days of absence from duty due to respiratory infections $(p=0.004)$ [222]. In Indian children $(n=150)$ vitamin $\mathrm{D}$ deficiency has been associated with a significantly higher risk of acute lower respiratory infections [223].

A study with $>200$ participants whose primary endpoint was the effect of vitamin D supplementation on bone loss also revealed, that the vitamin $\mathrm{D}_{3}$ supplementation for 2 years with $800 \mathrm{IU} /$ day and for 1 year with $2000 \mathrm{IU} /$ day was associated with a significantly reduced risk of cold and influenza symptoms, an effect that was magnified with the supplementation of 2000 IU/day [198,224]. Other studies however did not show a statistically significant difference, possibly due to poor compliance $[225,226]$. Certain VDR polymorphisms were also associated with a significantly increased risk of acute lower respiratory tract infections [227].

Several mechanisms could explain vitamin D's potentially beneficial effects on infectious diseases. Monocytes and macrophages can sense pathogen-associated molecular patterns (PAMPs) of, e.g., tuberculosis by utilizing their toll-like receptors (TLRs). This induces both VDR and CYP27B1, which increases the local production of $1,25(\mathrm{OH})_{2} \mathrm{D}$ that is dependent on the serum $25(\mathrm{OH}) \mathrm{D}$ concentration $[145,228] .1,25(\mathrm{OH})_{2} \mathrm{D}$ enhances the innate immune system by inducing the production of antimicrobial peptides like cathelicidin, reactive oxygen species by the (reduced) nicotinamide adenine dinucleotide phosphate (NADPH) oxidase and potentially reactive nitrogen species by inducible nitric oxide synthase (iNOS), and by inducing autophagy [143-147] (Figure 4).

\section{Vitamin D and Respiratory Diseases}

Although some studies did not find a consistent association between 25(OH)D levels in cord blood, maternal vitamin D intake or status during pregnancy and the risk for asthma in childhood [229-236], in children with asthma, 25(OH)D levels seem to correlate positively with asthma control [237] and lung function [238], and inversely with corticosteroid use [239]. A few interventional studies examining vitamin D's effect on asthma exist [229]. One of them showed as secondary outcome that vitamin $\mathrm{D}_{3}$ supplementation (1200 IU/day) in school children was associated with a significant $83 \%$ reduced risk for asthma exacerbations [218]. Presumably vitamin D's immunmodulatory and pulmonary effects could play a role [229]. 


\section{Prevention and Treatment of Vitamin D Deficiency}

According to the Endocrine Society Practice Guidelines a screening for vitamin D deficiency by measuring the $25(\mathrm{OH}) \mathrm{D}$ serum level is only recommended for individuals at risk (the most important risk factors are listed in Figure 6), and not for the general population [16]. To prevent vitamin D deficiency, the Institute of Medicine (IOM) recommends, that infants should immediately receive a daily supplementation of vitamin D of 400 IUs during the first year of life. Individuals between 1 and 70 years should receive 600 IU of vitamin D daily and adults $>70$ years should receive a daily dose of 800 IU vitamin D [53] (Table 2). The serum 25(OH)D level increases for every $100 \mathrm{IU} /$ day by $\sim 0.6-1.0 \mathrm{ng} / \mathrm{mL}[29,37,240,241]$. The doses recommended by IOM will likely increase the $25(\mathrm{OH}) \mathrm{D}$ level to $20 \mathrm{ng} / \mathrm{mL}$, which they considered to be adequate for bone health, but not to levels $>30 \mathrm{ng} / \mathrm{mL}$, as recommended by the Endocrine Society.

That's why the Endocrine Society recommended in its Practice Guidelines that infants during their first year of life receive a daily supplementation of 400-1000 IU (up to 2000 IU is safe), children and adolescents between 1 and 18 years a daily supplementation of 600-1000 IU (up to 4000 IU is safe), and adults $>18$ years a daily supplementation of 1500-2000 IU (up to 10,000 IU is safe) for the prevention of vitamin D deficiency [16,53] (Table 2).

Figure 6. A Schematic representation of the major causes for vitamin D deficiency and potential health consequences. Note: This figure is reproduced with permission from [21], Copyright (C) 2007 Michael F. Holick.

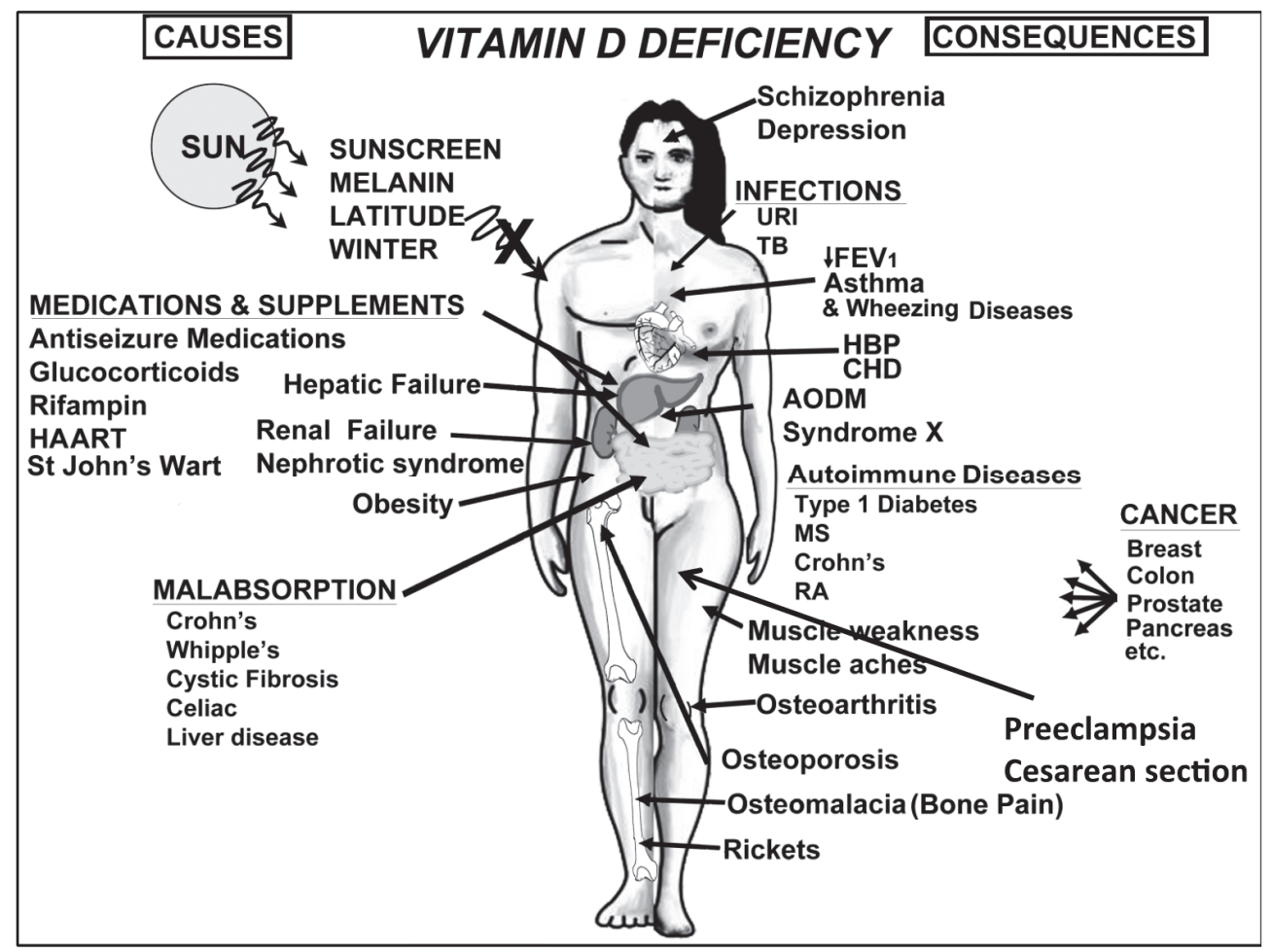


Table 2. Recommendations of the Institute of Medicine and the Endocrine Society Practice Guidelines for daily vitamin D supplementation to prevent vitamin D deficiency. This table is reproduced with permission from [16], Copyright (C) 2011 The Endocrine Society.

\begin{tabular}{|c|c|c|c|c|c|c|}
\hline \multirow[b]{2}{*}{$\begin{array}{l}\text { Life Stage } \\
\text { Group }\end{array}$} & \multicolumn{4}{|c|}{ IOM Recommendations } & \multicolumn{2}{|c|}{$\begin{array}{l}\text { Endocrine Society's } \\
\text { Recommendations }\end{array}$} \\
\hline & AI & EAR & RDA & $\mathbf{U L}$ & $\begin{array}{l}\text { Daily Allowance } \\
\text { (IU/day) }\end{array}$ & UL (IU) \\
\hline \multicolumn{7}{|l|}{ Infants } \\
\hline 0 to 6 months & $400 \mathrm{IU}(10 \mu \mathrm{g})$ & & & $1000 \mathrm{IU}(25 \mu \mathrm{g})$ & $400-1000$ & 2000 \\
\hline 6 to 12 months & $400 \mathrm{IU}(10 \mu \mathrm{g})$ & & & $1500 \mathrm{IU}(38 \mu \mathrm{g})$ & $400-1000$ & 2000 \\
\hline \multicolumn{7}{|l|}{ Children } \\
\hline $1-3$ years & & $400 \mathrm{IU}(10 \mu \mathrm{g})$ & $600 \mathrm{IU}(15 \mu \mathrm{g})$ & $2500 \mathrm{IU}(63 \mu \mathrm{g})$ & $600-1000$ & 4000 \\
\hline $4-8$ years & & $400 \mathrm{IU}(10 \mu \mathrm{g})$ & $600 \mathrm{IU}(15 \mu \mathrm{g})$ & $3000 \mathrm{IU}(75 \mu \mathrm{g})$ & $600-1000$ & 4000 \\
\hline \multicolumn{7}{|l|}{ ales } \\
\hline $9-13$ years & & $400 \mathrm{IU}(10 \mu \mathrm{g})$ & $600 \mathrm{IU}(15 \mu \mathrm{g})$ & $4000 \mathrm{IU}(100 \mu \mathrm{g})$ & $600-1000$ & 4000 \\
\hline $14-18$ years & & $400 \mathrm{IU}(10 \mu \mathrm{g})$ & $600 \mathrm{IU}(15 \mu \mathrm{g})$ & $4000 \mathrm{IU}(100 \mu \mathrm{g})$ & $600-1000$ & 4000 \\
\hline 19-30 years & & $400 \mathrm{IU}(10 \mu \mathrm{g})$ & $600 \mathrm{IU}(15 \mu \mathrm{g})$ & $4000 \mathrm{IU}(100 \mu \mathrm{g})$ & $1500-2000$ & 10,000 \\
\hline $31-50$ years & & $400 \mathrm{IU}(10 \mu \mathrm{g})$ & $600 \mathrm{IU}(15 \mu \mathrm{g})$ & $4000 \mathrm{IU}(100 \mu \mathrm{g})$ & $1500-2000$ & 10,000 \\
\hline $51-70$ years & & $400 \mathrm{IU}(10 \mu \mathrm{g})$ & $600 \mathrm{IU}(15 \mu \mathrm{g})$ & $4000 \mathrm{IU}(100 \mu \mathrm{g})$ & $1500-2000$ & 10,000 \\
\hline$>70$ years & & $400 \mathrm{IU}(10 \mu \mathrm{g})$ & $800 \mathrm{IU}(20 \mu \mathrm{g})$ & $4000 \mathrm{IU}(100 \mu \mathrm{g})$ & $1500-2000$ & 10,000 \\
\hline \multicolumn{7}{|l|}{ Females } \\
\hline $9-13$ years & & $400 \mathrm{IU}(10 \mu \mathrm{g})$ & $600 \mathrm{IU}(15 \mu \mathrm{g})$ & $4000 \mathrm{IU}(100 \mu \mathrm{g})$ & $600-1000$ & 4000 \\
\hline $14-18$ years & & $400 \mathrm{IU}(10 \mu \mathrm{g})$ & $600 \mathrm{IU}(15 \mu \mathrm{g})$ & $4000 \mathrm{IU}(100 \mu \mathrm{g})$ & $600-1000$ & 4000 \\
\hline 19-30 years & & $400 \mathrm{IU}(10 \mu \mathrm{g})$ & $600 \mathrm{IU}(15 \mu \mathrm{g})$ & $4000 \mathrm{IU}(100 \mu \mathrm{g})$ & $1500-2000$ & 10,000 \\
\hline $31-50$ years & & $400 \mathrm{IU}(10 \mu \mathrm{g})$ & $600 \mathrm{IU}(15 \mu \mathrm{g})$ & $4000 \mathrm{IU}(100 \mu \mathrm{g})$ & $1500-2000$ & 10,000 \\
\hline $51-70$ years & & $400 \mathrm{IU}(10 \mu \mathrm{g})$ & $600 \mathrm{IU}(15 \mu \mathrm{g})$ & $4000 \mathrm{IU}(100 \mu \mathrm{g})$ & $1500-2000$ & 10,000 \\
\hline$>70$ years & & $400 \mathrm{IU}(10 \mu \mathrm{g})$ & $800 \mathrm{IU}(20 \mu \mathrm{g})$ & $4000 \mathrm{IU}(100 \mu \mathrm{g})$ & $1500-2000$ & 10,000 \\
\hline \multicolumn{7}{|l|}{ Pregnancy } \\
\hline 14-18 years & & $400 \mathrm{IU}(10 \mu \mathrm{g})$ & $600 \mathrm{IU}(15 \mu \mathrm{g})$ & $4000 \mathrm{IU}(100 \mu \mathrm{g})$ & $600-1000$ & 4000 \\
\hline 19-30 years & & $400 \mathrm{IU}(10 \mu \mathrm{g})$ & $600 \mathrm{IU}(15 \mu \mathrm{g})$ & $4000 \mathrm{IU}(100 \mu \mathrm{g})$ & $1500-2000$ & 10,000 \\
\hline $31-50$ years & & $400 \mathrm{IU}(10 \mu \mathrm{g})$ & $600 \mathrm{IU}(15 \mu \mathrm{g})$ & $4000 \mathrm{IU}(100 \mu \mathrm{g})$ & $1500-2000$ & 10,000 \\
\hline \multicolumn{7}{|l|}{ Lactation * } \\
\hline $14-18$ years & & $400 \mathrm{IU}(10 \mu \mathrm{g})$ & $600 \mathrm{IU}(15 \mu \mathrm{g})$ & $4000 \mathrm{IU}(100 \mu \mathrm{g})$ & $600-1000$ & 4000 \\
\hline 19-30 years & & $400 \mathrm{IU}(10 \mu \mathrm{g})$ & $600 \mathrm{IU}(15 \mu \mathrm{g})$ & $4000 \mathrm{IU}(100 \mu \mathrm{g})$ & $1500-2000$ & 10,000 \\
\hline $31-50$ years & & $400 \mathrm{IU}(10 \mu \mathrm{g})$ & $600 \mathrm{IU}(15 \mu \mathrm{g})$ & $4000 \mathrm{IU}(100 \mu \mathrm{g})$ & $1500-2000$ & 10,000 \\
\hline
\end{tabular}

* Mother's requirement 4000-6000 (mother's intake for infant's requirement if infant is not receiving 400 IU/day); $\mathrm{AI}=$ Adequate Intake; EAR = Estimated Average Requirement; IU = International Units; RDA = Recommended Dietary Allowance; UL = Tolerable Upper Intake Level.

However, obese individuals, patients with malabsorption syndromes, and patients on glucocorticoids, anti-seizure and AIDS medications may require higher doses of vitamin D than individuals without these conditions [16]. The Endocrine Society's Clinical Practice Guidelines also recommended sensible sun exposure, which for most individuals is the main physiological source of vitamin $\mathrm{D}$, and provided a list of the foods rich in vitamin $\mathrm{D}$, and encouraged taking a daily vitamin $\mathrm{D}$ supplement to ensure adequate $25(\mathrm{OH}) \mathrm{D}$ levels. 
The Endocrine Society's Practice Guidelines also recommended treatment strategies for patients with vitamin $\mathrm{D}$ deficiency depending on age and underlying medical conditions. For vitamin D deficient infants $0-1$ years old, a treatment with $2000 \mathrm{IU} /$ day of vitamin $\mathrm{D}_{2}$ or vitamin $\mathrm{D}_{3}$ or with 50,000 IU of vitamin $\mathrm{D}_{2}$ or vitamin $\mathrm{D}_{3}$ once weekly for 6 weeks was suggested, followed by maintenance therapy of 400-1000 IU/day. For vitamin D deficient children aged 1-18 years who are vitamin D deficient, treatment with $2000 \mathrm{IU} /$ day of vitamin $\mathrm{D}_{2}$ or vitamin $\mathrm{D}_{3}$ or with $50,000 \mathrm{IU}$ of vitamin $\mathrm{D}_{2}$ once a week, both for at least 6 weeks, was suggested, followed by maintenance therapy of 600-1000 IU/day. Vitamin D deficient adults should be treated with 50,000 IU of vitamin $\mathrm{D}_{2}$ or vitamin $\mathrm{D}_{3}$ once a week for 8 weeks or with $\sim 6000 \mathrm{IU} /$ day of vitamin $\mathrm{D}_{2}$ or vitamin $\mathrm{D}_{3}$, followed by maintenance therapy of 1500-2000 IU/day. In obese patients, patients with malabsorption syndromes, and patients on medications affecting vitamin D metabolism, two to three times higher doses are (at least 6000-10,000 IU/day) of vitamin D to treat vitamin D deficiency are recommended, followed by maintenance therapy of at least 3000-6000 IU/day [16]. This strategy of giving 50,000 IU of vitamin D twice monthly to treat or prevent recurrence of vitamin D deficiency or insufficiency was without any toxicity for up to six years [242] (Figure 7).

Figure 7. (A) Mean serum 25-hydroxyvitamin D [25(OH)D] levels in all patients: includes patients treated with 50,000 IU vitamin $\mathrm{D}_{2}$ every 2 weeks (maintenance therapy, $n=81$ ), including those patients with vitamin D insufficiency who were initially treated with 8 weeks of 50,000 IU vitamin $\mathrm{D}_{2}$ weekly prior to maintenance therapy $(n=39)$. Error bars represent standard error of the mean, mean result over 5 years shown. Time 0 is initiation of treatment, results shown as mean values averaged for 6 month intervals. When mean 25(OH)D in each 6 month group was compared to mean initial $25(\mathrm{OH}) \mathrm{D}$, a significant difference was shown with $p<0.001$ up until month 43 and $p<0.001$ when all remaining values after month 43 were compared to mean initial 25(OH)D. (B) Mean serum 25(OH)D levels in patients receiving maintenance therapy only: Levels for 37 patients who were vitamin D insufficient $(25(\mathrm{OH}) \mathrm{D}$ levels $<30 \mathrm{ng} / \mathrm{mL})$ and 5 patients who were vitamin D sufficient $(25(\mathrm{OH}) \mathrm{D}$ levels $\geq 30 \mathrm{ng} / \mathrm{mL})$ who were treated with maintenance therapy of 50,000 IU vitamin $\mathrm{D}_{2}$ every two weeks. Error bars represent standard error of the mean, mean result over 5 years shown. Time 0 is initiation of treatment, results shown as mean values averaged for 6 month intervals. When mean $25(\mathrm{OH}) \mathrm{D}$ in each 6 month group were compared to mean initial 25(OH)D, a significant difference was shown with $p<0.001$ up until month 37 and $p<0.001$ when all remaining values after month 43 were compared to mean initial 25(OH)D. (C) Serum calcium levels: Results for all 81 patients who were treated with 50,000 IU of vitamin $\mathrm{D}_{2}$. Error bars represent standard error of the mean. Time 0 is initiation of treatment, results shown as mean values averaged for 6 month intervals. Normal serum calcium: $8.5-10.2 \mathrm{mg} / \mathrm{dL}$. Note: This figure is reproduced with permission from [242], Copyright (C) 2009 American Medical Association. 

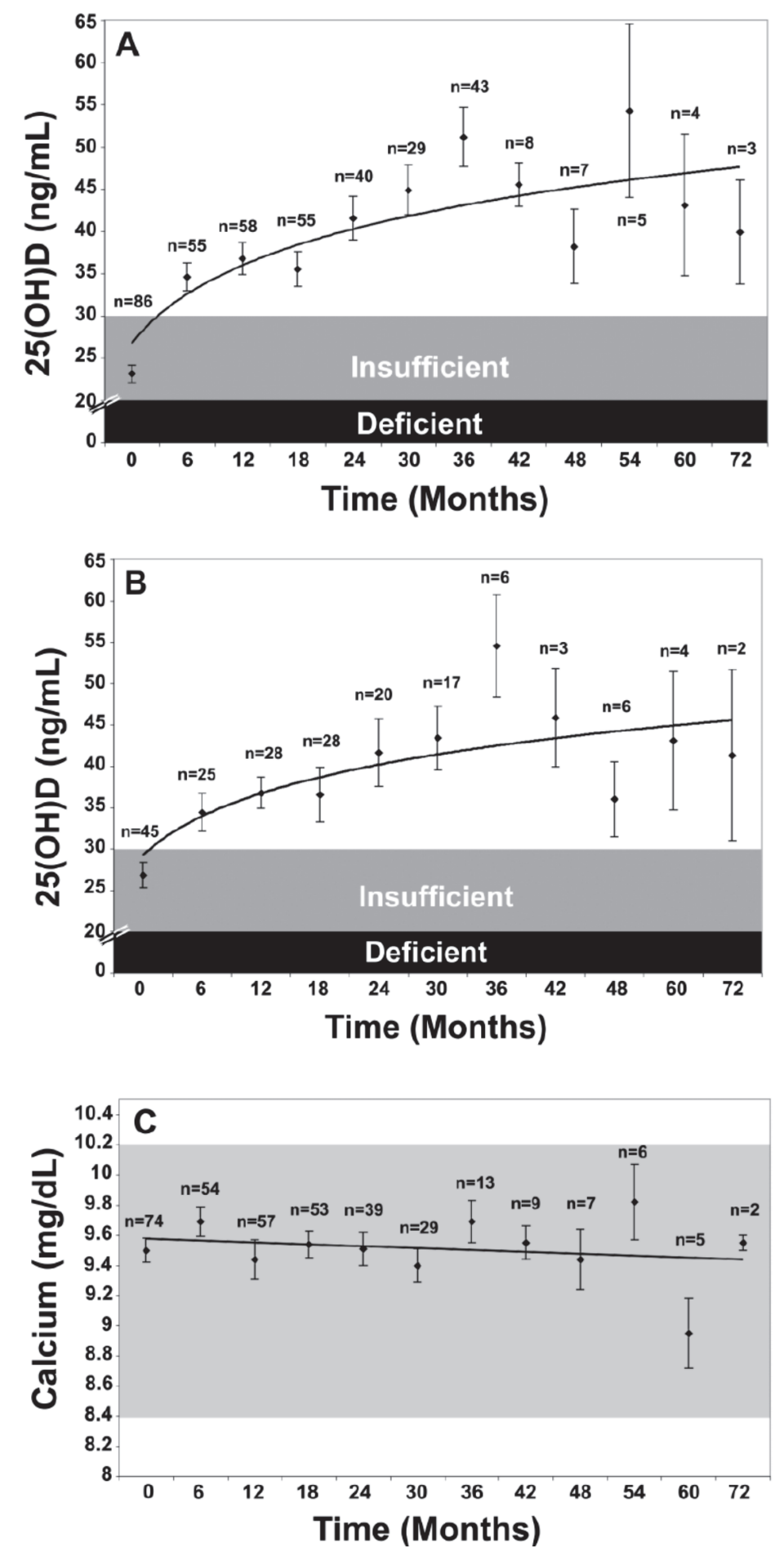

However, certain conditions like granulomatous conditions [243], genetic disorders [244] or rare polymorphisms of enzymes involved in vitamin D metabolism [245] are associated with an increased risk for vitamin $\mathrm{D}$ toxicity.

\section{Conclusions}

What continues to be needed are randomized controlled interventional studies with high power and using sufficiently high doses of vitamin D examining vitamin D's effects on various health outcomes. 
However, the present body of evidence of experimental findings, ecological, case-control, retroand prospective observational and interventional studies is substantial and suggests a pivotal role of vitamin D for a plethora of physiological functions and health outcomes including neuropsychiatric disorders [246], justifying the recommendation to enhance children's and adults' vitamin D status by following recommendations for sensible sun exposure, ingesting foods that contain vitamin $\mathrm{D}$ and vitamin D supplementation. Increasing the vitamin D status worldwide in the general adult and children population without rare conditions associated with an increased risk for vitamin D toxicity will help improve their overall health and well-being (Figure 6).

\section{Acknowledgments}

This work was supported in part by the UV Foundation (2880 Zanker Road, Suite 203, San Jose, CA 95134, USA) and the Mushroom Council (6620 Fletcher Lane, McLean, VA, USA).

\section{Conflicts of Interest}

The authors declare no conflict of interest.

\section{References}

1. Holick, M. Phylogenetic and Evolutionary Aspects of Vitamin D from Phytoplankton to Humans. In Verebrate Endocrinology: Fundamentals and Biomedical Implications; Pang, P.K.T., Schreibman, M.P., Eds.; Academic Press, Inc.: Orlando, FL, USA, 1989.

2. Holick, M.F. Vitamin D: Evolutionary, physiological and health perspectives. Curr. Drug Targets 2011, 12, 4-18.

3. Yoshida, T.; Stern, P.H. How vitamin D works on bone. Endocrinol. Metab. Clin. North Am. 2012, 41, 557-569.

4. Sai, A.J.; Walters, R.W.; Fang, X.; Gallagher, J.C. Relationship between vitamin D, parathyroid hormone, and bone health. J. Clin. Endocrinol. Metab. 2011, 96, E436-E446.

5. Lips, P.; van Schoor, N.M. The effect of vitamin D on bone and osteoporosis. Best Pract. Res. Clin. Endocrinol. Metab. 2011, 25, 585-591.

6. Nagpal, S.; Na, S.; Rathnachalam, R. Noncalcemic actions of vitamin D receptor ligands. Endocr. Rev. 2005, 26, 662-687.

7. Holick, M.F. Vitamin D deficiency. N. Engl. J. Med. 2007, 357, 266-281.

8. Jablonski, N.G.; Chaplin, G. The evolution of human skin coloration. J. Hum. Evol. 2000, 39, $57-106$.

9. Ergocalciferol. Available online: http://www.chemspider.com/Chemical-Structure.4444351. html (accessed on 21 December 2012).

10. Cholecalciferol. Available online: http://www.chemspider.com/Chemical-Structure.9058792. html (accessed on 21 December 2012).

11. Holick, M.F.; Tian, X.Q.; Allen, M. Evolutionary importance for the membrane enhancement of the production of vitamin $\mathrm{D}_{3}$ in the skin of poikilothermic animals. Proc. Natl. Acad. Sci. USA 1995, 92, 3124-3126. 
12. Tian, X.Q.; Chen, T.C.; Matsuoka, L.Y.; Wortsman, J.; Holick, M.F. Kinetic and thermodynamic studies of the conversion of previtamin $\mathrm{D}_{3}$ to vitamin $\mathrm{D}_{3}$ in human skin. J. Biol. Chem. 1993, 268, 14888-14892.

13. Chen, T.; Lu, Z.; Holick, M. Photobiology of Vitamin D. In Vitamin D: Physiology, Molecular Biology, and Clinical Applications, 2nd ed.; Holick, M.F., Ed.; Humana Press: New York, NY, USA, 2010; pp. 35-60.

14. Holick, M.F. Resurrection of vitamin D deficiency and rickets. J. Clin. Invest. 2006, 116, 2062-2072.

15. Jones, G. Phosphorus metabolism and management in chronic kidney disease: Expanding role for vitamin D in chronic kidney disease: Importance of blood 25-OH-D levels and extra-renal $1 \alpha$-hydroxylase in the classical and nonclassical actions of $1 \alpha, 25$-dihydroxyvitamin $\mathrm{D}_{3}$. Semin. Dial. 2007, 20, 316-324.

16. Holick, M.F.; Binkley, N.C.; Bischoff-Ferrari, H.A.; Gordon, C.M.; Hanley, D.A.; Heaney, R.P.; Murad, M.H.; Weaver, C.M. Evaluation, treatment, and prevention of vitamin D deficiency: An endocrine society clinical practice guideline. J. Clin. Endocrinol. Metab. 2011, 96, 1911-1930.

17. Holick, M.F. Vitamin D and health: Evolution, biologic functions, and recommended dietary intakes for vitamin D. Clin. Rev. Bone Miner. Metab. 2009, 7, 2-19.

18. Bosworth, C.R.; Levin, G.; Robinson-Cohen, C.; Hoofnagle, A.N.; Ruzinski, J.; Young, B.; Schwartz, S.M.; Himmelfarb, J.; Kestenbaum, B.; de Boer, I.H. The serum 24,25-dihydroxyvitamin D concentration, a marker of vitamin D catabolism, is reduced in chronic kidney disease. Kidney Int. 2012, 92, 693-700.

19. Christakos, S.; Dhawan, P.; Porta, A.; Mady, L.J.; Seth, T. Vitamin D and intestinal calcium absorption. Mol. Cell. Endocrinol. 2011, 347, 25-29.

20. Christakos, $\mathrm{S}$. Recent advances in our understanding of 1,25-dihydroxyvitamin $\mathrm{D}_{3}$ regulation of intestinal calcium absorption. Arch. Biochem. Biophys. 2012, 523, 73-76.

21. Holick, M.F. The vitamin D deficiency pandemic: A forgotten hormone important for health. Public Health Rev. 2010, 32, 267-283.

22. Holick, M.F. Environmental factors that influence the cutaneous production of vitamin D. Am. J. Clin. Nutr. 1995, 61, 638S-645S.

23. Holick, M.F.; Chen, T.C. Vitamin D deficiency: A worldwide problem with health consequences. Am. J. Clin. Nutr. 2008, 87, 1080S-1086S.

24. Urbain, P.; Singler, F.; Ihorst, G.; Biesalski, H.K.; Bertz, H. Bioavailability of vitamin $\mathrm{D}_{2}$ from UV-B-irradiated button mushrooms in healthy adults deficient in serum 25-hydroxyvitamin D: A randomized controlled trial. Eur. J. Clin. Nutr. 2011, 65, 965-971.

25. Mau, J.-L.; Chen, P.-R.; Yang, J.-H. Ultraviolet irradiation increased vitamin $\mathrm{D}_{2}$ content in edible mushrooms. J. Agric. Food Chem. 1998, 46, 5269-5272.

26. Tangpricha, V.; Koutkia, P.; Rieke, S.M.; Chen, T.C.; Perez, A.A.; Holick, M.F. Fortification of orange juice with vitamin D: A novel approach for enhancing vitamin D nutritional health. Am. J. Clin. Nutr. 2003, 77, 1478-1483.

27. Hypercalcaemia in infants and vitamin D. Br. Med. J. 1956, 2, 149. 
28. Holick, M.F. Sunlight and vitamin D for bone health and prevention of autoimmune diseases, cancers, and cardiovascular disease. Am. J. Clin. Nutr. 2004, 80, 1678S-1688S.

29. Biancuzzo, R.M.; Young, A.; Bibuld, D.; Cai, M.H.; Winter, M.R.; Klein, E.K.; Ameri, A.; Reitz, R.; Salameh, W.; Chen, T.C.; Holick, M.F. Fortification of orange juice with vitamin $\mathrm{D}_{2}$ or vitamin $\mathrm{D}_{3}$ is as effective as an oral supplement in maintaining vitamin $\mathrm{D}$ status in adults. Am. J. Clin. Nutr. 2010, 91, 1621-1626.

30. Armas, L.A.G.; Hollis, B.W.; Heaney, R.P. Vitamin $\mathrm{D}_{2}$ is much less effective than vitamin $\mathrm{D}_{3}$ in humans. J. Clin. Endocrinol. Metab. 2004, 89, 5387-5391.

31. Trang, H.M.; Cole, D.E.; Rubin, L.A.; Pierratos, A.; Siu, S.; Vieth, R. Evidence that vitamin $\mathrm{D}_{3}$ increases serum 25-hydroxyvitamin $\mathrm{D}$ more efficiently than does vitamin $\mathrm{D}_{2}$. Am. J. Clin. Nutr. 1998, 68, 854-858.

32. Houghton, L.A.; Vieth, R. The case against ergocalciferol (vitamin $\mathrm{D}_{2}$ ) as a vitamin supplement. Am. J. Clin. Nutr. 2006, 84, 694-697.

33. Romagnoli, E.; Mascia, M.L.; Cipriani, C.; Fassino, V.; Mazzei, F.; D’Erasmo, E.; Carnevale, V.; Scillitani, A.; Minisola, S. Short and long-term variations in serum calciotropic hormones after a single very large dose of ergocalciferol (vitamin $\mathrm{D}_{2}$ ) or cholecalciferol (vitamin $\mathrm{D}_{3}$ ) in the elderly. J. Clin. Endocrinol. Metab. 2008, 93, 3015-3020.

34. Heaney, R.P.; Recker, R.R.; Grote, J.; Horst, R.L.; Armas, L.A.G. Vitamin $\mathrm{D}_{3}$ is more potent than vitamin $\mathrm{D}_{2}$ in humans. J. Clin. Endocrinol. Metab. 2011, 96, E447-E452.

35. Leventis, P.; Kiely, P.D. The tolerability and biochemical effects of high-dose bolus vitamin $\mathrm{D}_{2}$ and $\mathrm{D}_{3}$ supplementation in patients with vitamin D insufficiency. Scand. J. Rheumatol. 2009, 38, 149-153.

36. Tripkovic, L.; Lambert, H.; Hart, K.; Smith, C.P.; Bucca, G.; Penson, S.; Chope, G.; Hyppönen, E.; Berry, J.; Vieth, R.; Lanham-New, S. Comparison of vitamin $\mathrm{D}_{2}$ and vitamin $\mathrm{D}_{3}$ supplementation in raising serum 25-hydroxyvitamin D status: A systematic review and meta-analysis. Am. J. Clin. Nutr. 2012, 95, 1357-1364.

37. Holick, M.F.; Biancuzzo, R.M.; Chen, T.C.; Klein, E.K.; Young, A.; Bibuld, D.; Reitz, R.; Salameh, W.; Ameri, A.; Tannenbaum, A.D. Vitamin $\mathrm{D}_{2}$ is as effective as vitamin $\mathrm{D}_{3}$ in maintaining circulating concentrations of 25-hydroxyvitamin D. J. Clin. Endocrinol. Metab. 2008, 93, 677-681.

38. Thacher, T.D.; Obadofin, M.O.; O'Brien, K.O.; Abrams, S.A. The Effect of vitamin $\mathrm{D}_{2}$ and vitamin $\mathrm{D}_{3}$ on intestinal calcium absorption in Nigerian children with rickets. J. Clin. Endocrinol. Metab. 2009, 94, 3314-3321.

39. Gordon, C.M.; Williams, A.L.; Feldman, H.A.; May, J.; Sinclair, L.; Vasquez, A.; Cox, J.E. Treatment of hypovitaminosis D in infants and toddlers. J. Clin. Endocrinol. Metab. 2008, 93, 2716-2721.

40. Rapuri, P.B.; Gallagher, J.C.; Haynatzki, G. Effect of vitamins $\mathrm{D}_{2}$ and $\mathrm{D}_{3}$ supplement use on serum 25OHD concentration in elderly women in summer and winter. Calcif. Tissue Int. 2004, $74,150-156$.

41. Hollis, B. Assessment of vitamin D nutritional and hormonal status: What to measure and how to do it. Calcif. Tissue Int.1996, 58, 4-5. 
42. DeLuca, H.F. Overview of general physiologic features and functions of vitamin D. Am. J. Clin. Nutr. 2004, 80, 1689S-1696S.

43. Zehnder, D.; Bland, R.; Williams, M.C.; McNinch, R.W.; Howie, A.J.; Stewart, P.M.; Hewison, M. Extrarenal expression of 25-hydroxyvitamin $\mathrm{D}(3) 1$ alpha-hydroxylase. $J$. Clin. Endocrinol. Metab. 2001, 86, 888-894.

44. Lehmann, B.; Meurer, M. Vitamin D metabolism. Dermatol. Ther. 2010, 23, 2-12.

45. Ritter, C.S.; Haughey, B.H.; Armbrecht, H.J.; Brown, A.J. Distribution and regulation of the 25-hydroxyvitamin D3 1 $\alpha$-hydroxylase in human parathyroid glands. J. Steroid Biochem. Mol. Biol. 2012, 130, 73-80.

46. Rosen, C.J.; Adams, J.S.; Bikle, D.D.; Black, D.M.; Demay, M.B.; Manson, J.E.; Murad, M.H.; Kovacs, C.S. The nonskeletal effects of vitamin D: An endocrine society scientific statement. Endocr. Rev. 2012, 33, 456-492.

47. Carlberg, C.; Bendik, I.; Wyss, A.; Meier, E.; Sturzenbecker, L.J.; Grippo, J.F.; Hunziker, W. Two nuclear signalling pathways for vitamin D. Nature 1993, 361, 657-660.

48. McKenna, N.J.; Lanz, R.B.; O’Malley, B.W. Nuclear receptor coregulators: Cellular and molecular biology. Endocr. Rev. 1999, 20, 321-344.

49. Smith, C.L.; O’Malley, B.W. Coregulator function: A key to understanding tissue specificity of selective receptor modulators. Endocr. Rev. 2004, 25, 45-71.

50. Dunlop, T.W.; Vaisanen, S.; Frank, C.; Carlberg, C. The genes of the coactivator TIF2 and the corepressor SMRT are primary 1alpha,25(OH)2D3 targets. J. Steroid Biochem. Mol. Biol. 2004, 89-90, 257-260.

51. Fleet, J.C. Vitamin D receptors: Not just in the nucleus anymore. Nutr. Rev. 1999, 57, 60-62.

52. Norman, A.W. Minireview: Vitamin D receptor: New assignments for an already busy receptor. Endocrinology 2006, 147, 5542-5548.

53. Institute of Medicine of the National Academies. Dietary Reference Intakes for Calcium and Vitamin D; Catharine Ross, A., Taylor, C.L., Yaktine, A.L., Eds.; The National Academy of Sciences: Washington, DC, USA, 2011.

54. Chapuy, M.C.; Schott, A.M.; Garnero, P.; Hans, D.; Delmas, P.D.; Meunier, P.J. Healthy elderly French women living at home have secondary hyperparathyroidism and high bone turnover in winter. EPIDOS Study Group. J. Clin. Endocrinol. Metab. 1996, 81, 1129-1133.

55. Holick, M.F.; Siris, E.S.; Binkley, N.; Beard, M.K.; Khan, A.; Katzer, J.T.; Petruschke, R.A.; Chen, E.; de Papp, A.E. Prevalence of vitamin D inadequacy among postmenopausal north American women receiving osteoporosis therapy. J. Clin. Endocrinol. Metab. 2005, 90, 3215-3224.

56. Thomas, M.K.; Lloyd-Jones, D.M.; Thadhani, R.I.; Shaw, A.C.; Deraska, D.J.; Kitch, B.T.; Vamvakas, E.C.; Dick, I.M.; Prince, R.L.; Finkelstein, J.S. Hypovitaminosis D in medical inpatients. N. Engl. J. Med. 1998, 338, 777-783.

57. Valcour, A.; Blocki, F.; Hawkins, D.M.; Rao, S.D. Effects of age and serum 25-OH-vitamin D on serum parathyroid hormone levels. J. Clin. Endocrinol. Metab. 2012, 97, 3989-3995. 
58. Whiting, S.J.; Langlois, K.A.; Vatanparast, H.; Greene-Finestone, L.S. The vitamin D status of Canadians relative to the 2011 Dietary Reference Intakes: An examination in children and adults with and without supplement use. Am. J. Clin. Nutr. 2011, 94, 128-135.

59. Hanley, D.A.; Cranney, A.; Jones, G.; Whiting, S.J.; Leslie, W.D.; Cole, D.E.; Atkinson, S.A.; Josse, R.G.; Feldman, S.; Kline, G.A.; Rosen, C. Vitamin D in adult health and disease: A review and guideline statement from Osteoporosis Canada. CMAJ 2010, 182, E610-E618.

60. Holick, M.F.; Binkley, N.C.; Bischoff-Ferrari, H.A.; Gordon, C.M.; Hanley, D.A.; Heaney, R.P.; Murad, M.H.; Weaver, C.M. Guidelines for preventing and treating vitamin D deficiency and insufficiency revisited. J. Clin. Endocrinol. Metab. 2012, 97, 1153-1158.

61. Looker, A.C.; Johnson, C.L.; Lacher, D.A.; Pfeiffer, C.M.; Schleicher, R.L.; Sempos, C.T. Vitamin D status: United States, 2001-2006. NCHS Data Brief 2011, 59, 1-8.

62. Marwaha, R.K.; Tandon, N.; Reddy, D.R.H.; Aggarwal, R.; Singh, R.; Sawhney, R.C.; Saluja, B.; Ganie, M.A.; Singh, S. Vitamin D and bone mineral density status of healthy schoolchildren in northern India. Am. J. Clin. Nutr. 2005, 82, 477-482.

63. Sachan, A.; Gupta, R.; Das, V.; Agarwal, A.; Awasthi, P.K.; Bhatia, V. High prevalence of vitamin $\mathrm{D}$ deficiency among pregnant women and their newborns in northern India. Am. $J$. Clin. Nutr. 2005, 81, 1060-1064.

64. Prentice, A.; Schoenmakers, I.; Jones, K.; Jarjou, L.; Goldberg, G. Vitamin D deficiency and its health consequences in Africa. Clin. Rev. Bone Miner. Metab. 2009, 7, 94-106.

65. Van der Mei, I.A.; Ponsonby, A.L.; Engelsen, O.; Pasco, J.A.; McGrath, J.J.; Eyles, D.W.; Blizzard, L.; Dwyer, T.; Lucas, R.; Jones, G. The high prevalence of vitamin D insufficiency across Australian populations is only partly explained by season and latitude. Environ. Health Perspect. 2007, 115, 1132-1139.

66. Maeda, S.S.; Kunii, I.S.; Hayashi, L.; Lazaretti-Castro, M. The effect of sun exposure on 25-hydroxyvitamin D concentrations in young healthy subjects living in the city of Sao Paulo, Brazil. Braz. J. Med. Biol. Res. 2007, 40, 1653-1659.

67. Sedrani, S.H. Low 25-hydroxyvitamin D and normal serum calcium concentrations in Saudi Arabia: Riyadh region. Ann. Nutr. Metab. 1984, 28, 181-185.

68. El-Hajj Fuleihan, G. Vitamin D Deficiency in the Middle East and Its Health Consequences. In Vitamin D: Physiology, Molecular Biology, and Clinical Applications; Holick, M.F., Ed.; Humana Press: New York, NY, USA, 2010; pp. 469-494.

69. Rich-Edwards, J.W.; Ganmaa, D.; Kleinman, K.; Sumberzul, N.; Holick, M.F.; Lkhagvasuren, T.; Dulguun, B.; Burke, A.; Frazier, A.L. Randomized trial of fortified milk and supplements to raise 25-hydroxyvitamin D concentrations in schoolchildren in Mongolia. Am. J. Clin. Nutr. 2011, 94, 578-584.

70. Rockell, J.; Skeaff, C.; Williams, S.; Green, T. Serum 25-hydroxyvitamin D concentrations of New Zealanders aged 15 years and older. Osteoporos. Int. 2006, 17, 1382-1389.

71. Prentice, A. Vitamin D deficiency: A global perspective. Nutr. Rev. 2008, 66, S153-S164.

72. Marks, J.; Srai, S.K.; Biber, J.; Murer, H.; Unwin, R.J.; Debnam, E.S. Intestinal phosphate absorption and the effect of vitamin D: A comparison of rats with mice. Exp. Physiol. 2006, 91, 531-537. 
73. Chen, T.C.; Castillo, L.; Korycka-Dahl, M.; DeLuca, H.F. Role of vitamin D metabolites in phosphate transport of rat intestine. J. Nutr. 1974, 104, 1056-1060.

74. Segersten, U.; Correa, P.; Hewison, M.; Hellman, P.; Dralle, H.; Carling, T.; Åkerström, G.; Westin, G. 25-Hydroxyvitamin $\mathrm{D}(3)$-1 $\alpha$-hydroxylase expression in normal and pathological parathyroid glands. J. Clin. Endocrinol. Metab. 2002, 87, 2967-2972.

75. Ritter, C.S.; Armbrecht, H.J.; Slatopolsky, E.; Brown, A.J. 25-Hydroxyvitamin D3 suppresses PTH synthesis and secretion by bovine parathyroid cells. Kidney Int. 2006, 70, 654-659.

76. Rajakumar, K. Vitamin D, cod-liver oil, sunlight, and rickets: A historical perspective. Pediatrics 2003, 112, e132-e135.

77. Mozołowski, W. Jędrzej Sniadecki (1768-1838) on the cure of rickets. Nature 1939, 143, 121.

78. Palm, T.A. The geographical distribution and etiology of rickets. Practitioner 1890, 45, 270-342.

79. Huldschinsky, K. Heilung von Rachitis durch künstliche Höhensonne. Dtsch. Med. Wochenschr. 1919, 45, 712-713.

80. Huldschinsky, K. The Ultra-Violet Light Treatment of Rickets; Alpine Press: Newark, NJ, USA, 1928; pp. 3-19.

81. Mellanby, T. The part played by an "accessory factor" in the production of experimental rickets. J. Physiol. 1918, 52, 11-14.

82. McCollum, E.F.; Simmonds, N.; Becker, J.E.; Shipley, P.G. Studies on experimental rickets; and experimental demonstration of the existence of a vitamin which promotes calcium deposition. J. Biol. Chem. 1922, 53, 293-312.

83. Hess, A.F.; Weinstock, M. Antirachitic properties imparted to inert fluids and to green vegetables by ultraviolet irradiation. J. Biol. Chem. 1924, 62, 301-313.

84. Steenbock, H.; Black, A. The reduction of growth-promoting and calcifying properties in a ration by exposure to ultraviolet light. J. Biol. Chem. 1924, 61, 408-422.

85. Cooper, C.; Javaid, K.; Westlake, S.; Harvey, N.; Dennison, E. Developmental origins of osteoporotic fracture: The role of maternal vitamin D insufficiency. J. Nutr. 2005, 135, 2728S-2734S.

86. Javaid, M.K.; Crozier, S.R.; Harvey, N.C.; Gale, C.R.; Dennison, E.M.; Boucher, B.J.; Arden, N.K.; Godfrey, K.M.; Cooper, C. Maternal vitamin D status during pregnancy and childhood bone mass at age 9 years: A longitudinal study. Lancet 2006, 367, 36-43.

87. Holick, M.F. Vitamin D and bone health. J. Nutr. 1996, 126, 1159S-1164S.

88. Adams, J.S.; Hewison, M. Update in vitamin D. J. Clin. Endocrinol. Metab. 2010, 95, 471-478.

89. Bischoff-Ferrari, H.A.; Kiel, D.P.; Dawson-Hughes, B.; Orav, J.E.; Li, R.; Spiegelman, D.; Dietrich, T.; Willett, W.C. Dietary calcium and serum 25-hydroxyvitamin D status in relation to BMD among U.S. adults. J. Bone Miner. Res. 2009, 24, 935-942.

90. Delling, G. Endokrine Osteopathien; Morphologie, Histomorphometrie und Differentialdiagnose. Endocrine Bone Diseases; Morphology, Histomorphometry and Differential Diagnosis; Fischer: Stuttgart, Germany, 1975. 
91. Priemel, M.; von Domarus, C.; Klatte, T.O.; Kessler, S.; Schlie, J.; Meier, S.; Proksch, N.; Pastor, F.; Netter, C.; Streichert, T.; et al. Bone mineralization defects and vitamin D deficiency: Histomorphometric analysis of iliac crest bone biopsies and circulating 25-hydroxyvitamin D in 675 patients. J. Bone Miner. Res. 2010, 25, 305-312.

92. Holick, M.F. Vitamin D: Importance in the prevention of cancers, type 1 diabetes, heart disease, and osteoporosis. Am. J. Clin. Nutr. 2004, 79, 362-371.

93. Holick, M.F. Optimal vitamin D status for the prevention and treatment of osteoporosis. Drugs Aging 2007, 24, 1017-1029.

94. Becker, D.; Kilgore, M.; Morrisey, M. The societal burden of osteoporosis. Curr. Rheumatol. Rep. 2010, 12, 186-191.

95. Khosla, S.; Amin, S.; Orwoll, E. Osteoporosis in Men. Endocr. Rev. 2008, 29, 441-464.

96. Dawson-Hughes, B.; Heaney, R.P.; Holick, M.F.; Lips, P.; Meunier, P.J.; Vieth, R. Estimates of optimal vitamin D status. Osteoporos. Int. 2005, 16, 713-716.

97. Cauley, J.A.; LaCroix, A.Z.; Wu, L.; Horwitz, M.; Danielson, M.E.; Bauer, D.C.; Lee, J.S.; Jackson, R.D.; Robbins, J.A.; Wu, C.; et al. Serum 25-hydroxyvitamin D concentrations and risk for hip fractures. Ann. Int. Med. 2008, 149, 242-250.

98. Bischoff-Ferrari, H.A.; Willett, W.C.; Orav, E.J.; Lips, P.; Meunier, P.J.; Lyons, R.A.; Flicker, L.; Wark, J.; Jackson, R.D.; Cauley, J.A.; et al. A pooled analysis of vitamin D dose requirements for fracture prevention. N. Engl. J. Med. 2012, 367, 40-49.

99. Chapuy, M.C.; Arlot, M.E.; Duboeuf, F.; Brun, J.; Crouzet, B.; Arnaud, S.; Delmas, P.D.; Meunier, P.J. Vitamin D 3 and calcium to prevent hip fractures in elderly women. N. Engl. J. Med. 1992, 327, 1637-1642.

100. Grant, A.M.; Avenell, A.; Campbell, M.K.; McDonald, A.M.; MacLennan, G.S.; McPherson, G.C.; Anderson, F.H.; Cooper, C.; Francis, R.M.; Donaldson, C.; et al. Null Oral vitamin $\mathrm{D}_{3}$ and calcium for secondary prevention of low-trauma fractures in elderly people (Randomised Evaluation of Calcium Or vitamin D, RECORD): A randomised placebo-controlled trial. Lancet 2005, 365, 1621-1628.

101. Dawson-Hughes, B.; Harris, S.S.; Krall, E.A.; Dallal, G.E. Effect of calcium and vitamin D supplementation on bone density in men and women 65 years of age or older. N. Engl. J. Med. 1997, 337, 670-676.

102. Lips, P.; Graafmans, W.C.; Ooms, M.E.; Bezemer, P.D.; Bouter, L.M. Vitamin D supplementation and fracture incidence in elderly persons. A randomized, placebo-controlled clinical trial. Ann. Int. Med. 1996, 124, 400-406.

103. Meyer, H.E.; Smedshaug, G.B.; Kvaavik, E.; Falch, J.A.; Tverdal, A.; Pedersen, J.I. Can vitamin D supplementation reduce the risk of fracture in the elderly? A randomized controlled trial. J. Bone Miner. Res. 2002, 17, 709-715.

104. Jackson, R.D.; LaCroix, A.Z.; Gass, M.; Wallace, R.B.; Robbins, J.; Lewis, C.E.; Bassford, T.; Beresford, S.A.A.; Black, H.R.; Blanchette, P.; et al. Calcium plus vitamin D supplementation and the risk of fractures. N. Engl. J. Med. 2006, 354, 669-683. 
105. Pfeifer, M.; Begerow, B.; Minne, H.W.; Abrams, C.; Nachtigall, D.; Hansen, C. Effects of a short-term vitamin $\mathrm{D}$ and calcium supplementation on body sway and secondary hyperparathyroidism in elderly women. J. Bone Miner. Res. 2000, 15, 1113-1118.

106. Pfeifer, M.; Begerow, B.; Minne, H.W.; Suppan, K.; Fahrleitner-Pammer, A.; Dobnig, H. Effects of a long-term vitamin D and calcium supplementation on falls and parameters of muscle function in community-dwelling older individuals. Osteoporos. Int. 2009, 20, 315-322.

107. Bischoff-Ferrari, H. Relevance of vitamin D in muscle health. Rev. Endocr. Metab. Disord. 2012, 13, 71-77.

108. Srikuea, R.; Zhang, X.; Park-Sarge, O.-K.; Esser, K.A. VDR and CYP27B1 are expressed in $\mathrm{C} 2 \mathrm{C} 12$ cells and regenerating skeletal muscle: Potential role in suppression of myoblast proliferation. Am. J. Physiol. Cell Physiol. 2012, 303, C396-C405.

109. Ceglia, L.; Harris, S.S. Vitamin D and its role in skeletal muscle. Calcif. Tissue Int. 2012, doi:10.1007/s00223-012-9645-y.

110. Simpson, R.U.; Thomas, G.A.; Arnold, A.J. Identification of 1,25-dihydroxyvitamin D3 receptors and activities in muscle. J. Biol. Chem. 1985, 260, 8882-8891.

111. Bischoff, H.A.; Borchers, M.; Gudat, F.; Duermueller, U.; Theiler, R.; Stähelin, H.B.; Dick, W. In situ detection of 1,25-dihydroxyvitamin D3 receptor in human skeletal muscle tissue. Histochem. J. 2001, 33, 19-24.

112. Costa, E.M.; Blau, H.M.; Feldman, D. 1,25-Dihydroxyvitamin D3 receptors and hormonal responses in cloned human skeletal muscle cells. Endocrinology 1986, 119, 2214-2220.

113. Boland, R.; Norman, A.; Ritz, E.; Hasselbach, W. Presence of a 1,25-dihydroxy-vitamin D3 receptor in chick skeletal muscle myoblasts. Biochem. Biophys. Res. Commun. 1985, 128, 305-311.

114. Pfeifer, M.; Begerow, B.; Minne, H.W. Vitamin D and muscle function. Osteoporos. Int. 2002, 13, 187-194.

115. Janssen, H.C.; Samson, M.M.; Verhaar, H.J. Vitamin D deficiency, muscle function, and falls in elderly people. Am. J. Clin. Nutr. 2002, 75, 611-615.

116. Lou, Y.-R.; Molnár, F.; Peräkylä, M.; Qiao, S.; Kalueff, A.V.; St-Arnaud, R.; Carlberg, C.; Tuohimaa, P. 25-Hydroxyvitamin D(3) is an agonistic vitamin D receptor ligand. J. Steroid Biochem. Mol. Biol. 2010, 118, 162-170.

117. Wang, Y.; DeLuca, H.F. Is the vitamin D receptor found in muscle? Endocrinology 2011, 152, 354-363.

118. Schott, G.D.; Wills, M.R. Muscle weakness in osteomalacia. Lancet 1976, 307, 626-629.

119. Bischoff-Ferrari, H.A.; Giovannucci, E.; Willett, W.C.; Dietrich, T.; Dawson-Hughes, B. Estimation of optimal serum concentrations of 25-hydroxyvitamin D for multiple health outcomes. Am. J. Clin. Nutr. 2006, 84, 18-28.

120. Glerup, H.; Mikkelsen, K.; Poulsen, L.; Hass, E.; Overbeck, S.; Andersen, H.; Charles, P.; Eriksen, E.F. Hypovitaminosis D myopathy without biochemical signs of osteomalacic bone involvement. Calcif. Tissue Int. 2000, 66, 419-424. 
121. Wicherts, I.S.; van Schoor, N.M.; Boeke, A.J.P.; Visser, M.; Deeg, D.J.H.; Smit, J.; Knol, D.L.; Lips, P. Vitamin D status predicts physical performance and its decline in older persons. $J$. Clin. Endocrinol. Metab. 2007, 92, 2058-2065.

122. Bischoff-Ferrari, H.A.; Dietrich, T.; Orav, E.J.; Hu, F.B.; Zhang, Y.; Karlson, E.W.; Dawson-Hughes, B. Higher 25-hydroxyvitamin D concentrations are associated with better lower-extremity function in both active and inactive persons aged $\geq 60$ y. Am. J. Clin. Nutr. 2004, 80, 752-758.

123. Bischoff, H.A.; Stähelin, H.B.; Dick, W.; Akos, R.; Knecht, M.; Salis, C.; Nebiker, M.; Theiler, R.; Pfeifer, M.; Begerow, B.; et al. Effects of vitamin D and calcium supplementation on falls: A randomized controlled trial. J. Bone Miner. Res. 2003, 18, 343-351.

124. Bischoff-Ferrari, H.; Conzelmann, M.; Stähelin, H.; Dick, W.; Carpenter, M.; Adkin, A.; Theiler, R.; Pfeifer, M.; Allum, J. Is fall prevention by vitamin D mediated by a change in postural or dynamic balance? Osteoporos. Int. 2006, 17, 656-663.

125. Broe, K.E.; Chen, T.C.; Weinberg, J.; Bischoff-Ferrari, H.A.; Holick, M.F.; Kiel, D.P. A higher dose of vitamin D reduces the risk of falls in nursing home residents: A randomized, multiple-dose study. J. Am. Geriatr. Soc. 2007, 55, 234-239.

126. Bischoff-Ferrari, H.A.; Dawson-Hughes, B.; Staehelin, H.B.; Orav, J.E.; Stuck, A.E.; Theiler, R.; Wong, J.B.; Egli, A.; Kiel, D.P.; Henschkowski, J. Fall prevention with supplemental and active forms of vitamin D: A meta-analysis of randomised controlled trials. BMJ 2009, 339, doi:10.1136/bmj.b3692.

127. Apperly, F.L. The relation of solar radiation to cancer mortality in north America. Cancer Res. 1941, 1, 191-195.

128. Garland, C.F.; Garland, F.F. Do sunlight and vitamin D reduce the likelihood of colon cancer? Int. J. Epidemiol. 1980, 9, 227-231.

129. Grant, W.B. Ecological studies of the UVB-vitamin D-cancer hypothesis. Anticancer Res. 2012, 32, 223-236.

130. Drake, M.T.; Maurer, M.J.; Link, B.K.; Habermann, T.M.; Ansell, S.M.; Micallef, I.N.; Kelly, J.L.; Macon, W.R.; Nowakowski, G.S.; Inwards, D.J.; et al. Vitamin D insufficiency and prognosis in non-Hodgkin's lymphoma. J. Clin. Oncol. 2010, 28, 4191-4198.

131. Freedman, D.M.; Looker, A.C.; Chang, S.-C.; Graubard, B.I. Prospective study of serum vitamin D and cancer mortality in the United States. J. Natl. Cancer Inst. 2007, 99, 1594-1602.

132. Garland, C.F.; Garland, F.C.; Shaw, E.K.; Comstock, G.W.; Helsing, K.J.; Gorham, E.D. Serum 25-hydroxyvitamin D and colon cancer: Eight-year prospective study. Lancet 1989, 334, 1176-1178.

133. Garland, C.; Shekelle, R.B.; Barrett-Connor, E.; Criqui, M.H.; Rossof, A.H.; Paul, O. Dietary vitamin D and calcium and risk of colorectal cancer: A 19-year prospective study in men. Lancet 1985, 1, 307-309.

134. Garland, C.F.; Garland, F.C.; Gorham, E.D.; Lipkin, M.; Newmark, H.; Mohr, S.B.; Holick, M.F. The role of itamin D in cancer prevention. Am. J. Public Health 2006, 96, 252-261. 
135. Giovannucci, E.; Liu, Y.; Rimm, E.B.; Hollis, B.W.; Fuchs, C.S.; Stampfer, M.J.; Willett, W.C. Prospective study of predictors of vitamin D status and cancer incidence and mortality in men. J. Natl. Cancer Inst. 2006, 98, 451-459.

136. John, E.M.; Schwartz, G.G.; Dreon, D.M.; Koo, J. Vitamin D and breast cancer risk: The NHANES I Epidemiologic Follow-up Study, 1971-1975 to 1992. National Health and Nutrition Examination Survey. Cancer Epidemiol. Biomarkers Prev. 1999, 8, 399-406.

137. Lappe, J.M.; Travers-Gustafson, D.; Davies, K.M.; Recker, R.R.; Heaney, R.P. Vitamin D and calcium supplementation reduces cancer risk: Results of a randomized trial. Am. J. Clin. Nutr. 2007, 85, 1586-1591.

138. Manson, J.E.; Mayne, S.T.; Clinton, S.K. Vitamin D and prevention of cancer-Ready for prime time? N. Engl. J. Med. 2011, 364, 1385-1387.

139. Shin, M.-H.; Holmes, M.D.; Hankinson, S.E.; Wu, K.; Colditz, G.A.; Willett, W.C. Intake of dairy products, calcium, and vitamin D and risk of breast cancer. J. Natl. Cancer Inst. 2002, 94, 1301-1310.

140. Feskanich, D.; Ma, J.; Fuchs, C.S.; Kirkner, G.J.; Hankinson, S.E.; Hollis, B.W.; Giovannucci, E.L. Plasma vitamin D metabolites and risk of colorectal cancer in women. Cancer Epidemiol. Biomarkers Prev. 2004, 13, 1502-1508.

141. Krishnan, A.V.; Feldman, D. Mechanisms of the anti-cancer and anti-inflammatory actions of vitamin D. Annu. Rev. Pharmacol. Toxicol. 2011, 51, 311-336.

142. Fleet, J.C.; Desmet, M.; Johnson, R.; Li, Y. Vitamin D and cancer: A review of molecular mechanisms. Biochem. J. 2012, 441, 61-76.

143. Vazirnia, A.; Liu, P.T. Vitamin D and the Innate Immune Response. In Vitamin D and the Lung; Litonjua, A.A., Ed.; Humana Press: New York, NY, USA, 2012; pp. 59-84.

144. Campbell, G.R.; Spector, S.A. Autophagy induction by vitamin D inhibits both Mycobacterium tuberculosis and human immunodeficiency virus type 1. Autophagy 2012, 8, 1523-1525.

145. Liu, P.T.; Stenger, S.; Li, H.; Wenzel, L.; Tan, B.H.; Krutzik, S.R.; Ochoa, M.T.; Schauber, J.; $\mathrm{Wu}, \mathrm{K}$; Meinken, C.; et al. Toll-like receptor triggering of a vitamin D-mediated human antimicrobial response. Science 2006, 311, 1770-1773.

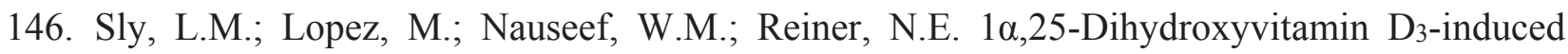
monocyte antimycobacterial activity is regulated by phosphatidylinositol 3-kinase and mediated by the NADPH-dependent phagocyte oxidase. J. Biol. Chem. 2001, 276, 35482-35493.

147. Baeke, F.; Takiishi, T.; Korf, H.; Gysemans, C.; Mathieu, C. Vitamin D: Modulator of the immune system. Curr. Opin. Pharmacol. 2010, 10, 482-496.

148. Pilz, S.; Tomaschitz, A.; März, W.; Drechsler, C.; Ritz, E.; Zittermann, A.; Cavalier, E.; Pieber, T.R.; Lappe, J.M.; Grant, W.B.; et al. Vitamin D, cardiovascular disease and mortality. Clin. Endocrinol. 2011, 75, 575-584.

149. Li, Y.C.; Qiao, G.; Uskokovic, M.; Xiang, W.; Zheng, W.; Kong, J. Vitamin D: A negative endocrine regulator of the renin-angiotensin system and blood pressure. J. Steroid Biochem. Mol. Biol. 2004, 89-90, 387-392. 
150. Wolden-Kirk, H.; Overbergh, L.; Christesen, H.T.; Brusgaard, K.; Mathieu, C. Vitamin D and diabetes: Its importance for beta cell and immune function. Mol. Cell. Endocrinol. 2011, 347, $106-120$.

151. Russo, A.; Terrasi, M.; Agnese, V.; Santini, D.; Bazan, V. Apoptosis: A relevant tool for anticancer therapy. Ann. Oncol. 2006, 17, vii115-vii123.

152. Dunn, G.P.; Old, L.J.; Schreiber, R.D. The Immunobiology of cancer immunosurveillance and immunoediting. Immunity 2004, 21, 137-148.

153. Kerr, J.F.R.; Winterford, C.M.; Harmon, B.V. Apoptosis. Its significance in cancer and cancer therapy. Cancer 1994, 73, 2013-2026.

154. Blutt, S.E.; McDonnell, T.J.; Polek, T.C.; Weigel, N.L. Calcitriol-induced apoptosis in LNCaP cells is blocked by overexpression of Bcl-2. Endocrinology 2000, 141, 10-17.

155. Deeb, K.K.; Trump, D.L.; Johnson, C.S. Vitamin D signalling pathways in cancer: Potential for anticancer therapeutics. Nat. Rev. Cancer 2007, 7, 684-700.

156. Rohan, J.N.P.; Weigel, N.L. 1Alpha,25-dihydroxyvitamin $\mathrm{D}_{3}$ reduces c-Myc expression, inhibiting proliferation and causing G1 accumulation in C4-2 prostate cancer cells. Endocrinology 2009, 150, 2046-2054.

157. Hawk, E.T.; Viner, J.L.; Dannenberg, A.; DuBois, R.N. COX-2 in cancer-A player that's defining the rules. J. Natl. Cancer Inst. 2002, 94, 545-546.

158. Koli, K.; Keski-Oja, J. 1Alpha,25-dihydroxyvitamin $\mathrm{D}_{3}$ and its analogues down-regulate cell invasion-associated proteases in cultured malignant cells. Cell Growth Differ. 2000, 11, 221-229.

159. Peña, C.; García, J.M.; Silva, J.; García, V.; Rodríguez, R.; Alonso, I.; Millán, I.; Salas, C.; de Herreros, A.G.; Muñoz, A.; Bonilla, F. E-cadherin and vitamin D receptor regulation by SNAIL and ZEB1 in colon cancer: Clinicopathological correlations. Hum. Mol. Genet. 2005, 14, 3361-3370.

160. Grandi, N.C.; Breitling, L.P.; Brenner, H. Vitamin D and cardiovascular disease: Systematic review and meta-analysis of prospective studies. Prev. Med. 2010, 51, 228-233.

161. Vacek, J.L.; Vanga, S.R.; Good, M.; Lai, S.M.; Lakkireddy, D.; Howard, P.A. Vitamin D deficiency and supplementation and relation to cardiovascular health. Am. J. Cardiol. 2012, 109, 359-363.

162. Wang, T.J.; Pencina, M.J.; Booth, S.L.; Jacques, P.F.; Ingelsson, E.; Lanier, K.; Benjamin, E.J.; D'Agostino, R.B.; Wolf, M.; Vasan, R.S. Vitamin D deficiency and risk of cardiovascular disease. Circulation 2008, 117, 503-511.

163. Eaton, C.B.; Young, A.; Allison, M.A.; Robinson, J.; Martin, L.W.; Kuller, L.H.; Johnson, K.C.; Curb, J.D.; van Horn, L.; McTiernan, A.; et al. Prospective association of vitamin D concentrations with mortality in postmenopausal women: Results from the Women's Health Initiative (WHI). Am. J. Clin. Nutr. 2011, 94, 1471-1478.

164. Anderson, J.L.; May, H.T.; Horne, B.D.; Bair, T.L.; Hall, N.L.; Carlquist, J.F.; Lappé, D.L.; Muhlestein, J.B. Relation of vitamin D deficiency to cardiovascular risk factors, disease status, and incident events in a general healthcare population. Am. J. Cardiol. 2010, 106, 963-968.

165. Sun, Q.; Pan, A.; Hu, F.B.; Manson, J.E.; Rexrode, K.M. 25-Hydroxyvitamin D levels and the risk of stroke. Stroke 2012, 43, 1470-1477. 
166. Reid, I.R.; Bolland, M.J. Role of vitamin D deficiency in cardiovascular disease. Heart 2012, 98, 609-614.

167. Tall, A.R. Exercise to reduce cardiovascular risk-How much is enough? N. Engl. J. Med. 2002, 347, 1522-1524.

168. Wilson, P.W.; D’Agostino, R.B.; Sullivan, L.; Parise, H.; Kannel, W.B. Overweight and obesity as determinants of cardiovascular risk: The Framingham experience. Arch. Intern. Med. 2002, 162, 1867-1872.

169. Wortsman, J.; Matsuoka, L.Y.; Chen, T.C.; Lu, Z.; Holick, M.F. Decreased bioavailability of vitamin D in obesity. Am. J. Clin. Nutr. 2000, 72, 690-693.

170. Drincic, A.T.; Armas, L.A.G.; van Diest, E.E.; Heaney, R.P. Volumetric dilution, rather than sequestration best explains the low vitamin D status of obesity. Obesity 2012, 20, 1444-1448.

171. Burgaz, A.; Orsini, N.; Larsson, S.C.; Wolk, A. Blood 25-hydroxyvitamin D concentration and hypertension: A meta-analysis. J. Hypertens. 2011, 29, 636-645.

172. Witham, M.D.; Nadir, M.A.; Struthers, A.D. Effect of vitamin D on blood pressure: A systematic review and meta-analysis. J. Hypertens. 2009, 27, 1948-1954.

173. Wu, S.H.; Ho, S.C.; Zhong, L. Effects of vitamin D supplementation on blood pressure. South Med. J. 2010, 103, 729-737.

174. Elamin, M.B.; Abu Elnour, N.O.; Elamin, K.B.; Fatourechi, M.M.; Alkatib, A.A.; Almandoz, J.P.; Liu, H.; Lane, M.A.; Mullan, R.J.; Hazem, A.; et al. Vitamin D and cardiovascular outcomes: A systematic review and meta-analysis. J. Clin. Endocrinol. Metab. 2011, 96, 1931-1942.

175. Krause, R.; Bühring, M.; Hopfenmüller, W.; Holick, M.F.; Sharma, A.M. Ultraviolet B and blood pressure. Lancet 1998, 352, 709-710.

176. Fitzpatrick, L.; Bilezikian, J.; Silverberg, S. Parathyroid hormone and the cardiovascular system. Curr. Osteoporos. Rep. 2008, 6, 77-83.

177. Mitri, J.; Muraru, M.D.; Pittas, A.G. Vitamin D and type 2 diabetes: A systematic review. Eur. J. Clin. Nutr. 2011, 65, 1005-1015.

178. Pittas, A.G.; Dawson-Hughes, B.; Li, T.; van Dam, R.M.; Willett, W.C.; Manson, J.E.; Hu, F.B. Vitamin D and calcium intake in relation to type 2 diabetes in women. Diabetes Care 2006, 29, 650-656.

179. Holick, M.F. Nutrition: D-iabetes and D-eath D-efying vitamin D. Nat. Rev. Endocrinol. 2012, $8,388-390$.

180. Deleskog, A.; Hilding, A.; Brismar, K.; Hamsten, A.; Efendic, S.; Östenson, C.G. Low serum 25-hydroxyvitamin $\mathrm{D}$ level predicts progression to type 2 diabetes in individuals with prediabetes but not with normal glucose tolerance. Diabetologia 2012, 55, 1668-1678.

181. Antico, A.; Tampoia, M.; Tozzoli, R.; Bizzaro, N. Can supplementation with vitamin D reduce the risk or modify the course of autoimmune diseases? A systematic review of the literature. Autoimmun. Rev. 2012, 12, 127-136.

182. Ponsonby, A.-L.; McMichael, A.; van der Mei, I. Ultraviolet radiation and autoimmune disease: Insights from epidemiological research. Toxicology 2002, 181-182, 71-78. 
183. Peyrin-Biroulet, L.; Oussalah, A.; Bigard, M.-A. Crohn's disease: The hot hypothesis. Med. Hypotheses 2009, 73, 94-96.

184. Vieira, V.M.; Hart, J.E.; Webster, T.F.; Weinberg, J.; Puett, R.; Laden, F.; Costenbader, K.H.; Karlson, E.W. Association between residences in U.S. northern latitudes and rheumatoid arthritis: A spatial analysis of the Nurses' Health Study. Environ. Health Perspect. 2010, 118, 957-961.

185. Mohr, S.; Garland, C.; Gorham, E.; Garland, F. The association between ultraviolet B irradiance, vitamin D status and incidence rates of type 1 diabetes in 51 regions worldwide. Diabetologia 2008, 51, 1391-1398.

186. Munger, K.L.; Levin, L.I.; Hollis, B.W.; Howard, N.S.; Ascherio, A. Serum 25-hydroxyvitamin D levels and risk of multiple sclerosis. JAMA 2006, 296, 2832-2838.

187. Burton, J.M.; Kimball, S.; Vieth, R.; Bar-Or, A.; Dosch, H.-M.; Cheung, R.; Gagne, D.; D’Souza, C.; Ursell, M.; O'Connor, P. A phase I/II dose-escalation trial of vitamin D3 and calcium in multiple sclerosis. Neurology 2010, 74, 1852-1859.

188. Pierrot-Deseilligny, C.; Rivaud-Pechoux, S.; Clerson, P.; de Paz, R.; Souberbielle, J.C. Relationship between 25-OH-D serum level and relapse rate in multiple sclerosis patients before and after vitamin D supplementation. Ther. Adv. Neurol. Disord. 2012, 5, 187-198.

189. Sørensen, I.M.; Joner, G.; Jenum, P.A.; Eskild, A.; Torjesen, P.A.; Stene, L.C. Maternal serum levels of 25-hydroxy-vitamin $\mathrm{D}$ during pregnancy and risk of type 1 diabetes in the offspring. Diabetes 2012, 61, 175-178.

190. Hyppönen, E.; Läärä, E.; Reunanen, A.; Järvelin, M.-R.; Virtanen, S.M. Intake of vitamin D and risk of type 1 diabetes: A birth-cohort study. Lancet 2001, 358, 1500-1503.

191. Stene, L.C.; Ulriksen, J.; Magnus, P.; Joner, G. Use of cod liver oil during pregnancy associated with lower risk of Type I diabetes in the offspring. Diabetologia 2000, 43, 1093-1098.

192. Merlino, L.A.; Curtis, J.; Mikuls, T.R.; Cerhan, J.R.; Criswell, L.A.; Saag, K.G. Vitamin D intake is inversely associated with rheumatoid arthritis: Results from the Iowa Women's Health Study. Arthritis Rheum. 2004, 50, 72-77.

193. Vogel, A.; Strassburg, C.P.; Manns, M.P. Genetic association of vitamin D receptor polymorphisms with primary biliary cirrhosis and autoimmune hepatitis. Hepatology 2002, 35, 126-131.

194. Ban, Y.; Taniyama, M.; Ban, Y. Vitamin D receptor gene polymorphism is associated with Graves' disease in the Japanese population. J. Clin. Endocrinol. Metab. 2000, 85, 4639-4643.

195. Škrabić, V.; Zemunik, T.; Šitum, M.; Terzić, J. Vitamin D receptor polymorphism and susceptibility to type 1 diabetes in the Dalmatian population. Diabetes Res. Clin. Pract. 2003, 59, 31-35.

196. Garcia-Lozano, J.R.; Gonzalez-Escribano, M.F.; Valenzuela, A.; Garcia, A.; Núñez-Roldán, A. Association of vitamin D receptor genotypes with early onset rheumatoid arthritis. Eur. $J$. Immunogenet. 2001, 28, 89-93.

197. Gómez-Vaquero, C.; Fiter, J.; Enjuanes, A.; Nogués, X.; Díez-Pérez, A.; Nolla, J.M. Influence of the BsmI polymorphism of the vitamin D receptor gene on rheumatoid arthritis clinical activity. J. Rheumatol. 2007, 34, 1823-1826. 
198. Yamshchikov, A.V.; Desai, N.S.; Blumberg, H.M.; Ziegler, T.R.; Tangpricha, V. Vitamin D for treatment and prevention of infectious diseases: A systematic review of randomized controlled trials. Endocr. Pract. 2009, 15, 438-449.

199. Battersby, A.J.; Kampmann, B.; Burl, S. Vitamin D in early childhood and the effect on immunity to mycobacterium tuberculosis. Clin. Dev. Immunol. 2012, 2012, 430972.

200. Hart, P.D. Chemotherapy of tuberculosis; research during the past 100 years. Br. Med. J. 1946, $2,805-849$.

201. Everett, D. On the use of cod-liver oil in tubercular disease. Prov. Med. Surg. J. 1846, 10, 538-539.

202. The Nobel Prize in Physiology or Medicine 1903. Niels Ryberg Finsen. Available online: http://www.nobelprize.org/nobel_prizes/medicine/laureates/1903/ (accessed on 8 September 2012).

203. Rider, A.A. Elmer Verner McCollum-A biographical sketch (1879-1967). J. Nutr. 1970, 100, $1-10$.

204. Dowling, G.B.; Thomas, E.W.; Wallace, H.J. Lupus Vulgaris treated with Calciferol.Proc. R. Soc. Med. 1946, 39, 225-227.

205. Phelan, J.J. Calciferol in pulmonary tuberculosis. Lancet 1947, 1, 764.

206. Martineau, A.R. Old wine in new bottles: Vitamin D in the treatment and prevention of tuberculosis. Proc. Nutr. Soc. 2012, 71, 84-89.

207. Sasidharan, P.K.; Rajeev, E.; Vijayakumari, V. Tuberculosis and vitamin D deficiency. J. Assoc. Physicians India 2002, 50, 554-558.

208. Wilkinson, R.J.; Llewelyn, M.; Toossi, Z.; Patel, P.; Pasvol, G.; Lalvani, A.; Wright, D.; Latif, M.; Davidson, R.N. Influence of vitamin D deficiency and vitamin D receptor polymorphisms on tuberculosis among Gujarati Asians in west London: A case-control study. Lancet 2000, 355, 618-621.

209. Nnoaham, K.E.; Clarke, A. Low serum vitamin D levels and tuberculosis: A systematic review and meta-analysis. Int. J. Epidemiol. 2008, 37, 113-119.

210. Ganmaa, D.; Giovannucci, E.; Bloom, B.R.; Fawzi, W.; Burr, W.; Batbaatar, D.; Sumberzul, N.; Holick, M.F.; Willett, W.C. Vitamin D, tuberculin skin test conversion, and latent tuberculosis in Mongolian school-age children: A randomized, double-blind, placebo-controlled feasibility trial. Am. J. Clin. Nutr. 2012, 96, 391-396.

211. Martineau, A.R.; Wilkinson, R.J.; Wilkinson, K.A.; Newton, S.M.; Kampmann, B.; Hall, B.M.; Packe, G.E.; Davidson, R.N.; Eldridge, S.M.; Maunsell, Z.J.; et al. A single dose of vitamin D enhances immunity to mycobacteria. Am. J. Respir. Crit. Care Med. 2007, 176, 208-213.

212. Nursyam, E.W.; Amin, Z.; Rumende, C.M. The effect of vitamin D as supplementary treatment in patients with moderately advanced pulmonary tuberculous lesion. Acta Med. Indones. 2006, $38,3-5$.

213. Martineau, A.R.; Timms, P.M.; Bothamley, G.H.; Hanifa, Y.; Islam, K.; Claxton, A.P.; Packe, G.E.; Moore-Gillon, J.C.; Darmalingam, M.; Davidson, R.N.; et al. High-dose vitamin $\mathrm{D}_{3}$ during intensive-phase antimicrobial treatment of pulmonary tuberculosis: A double-blind randomised controlled trial. Lancet 2011, 377, 242-250. 
214. Morcos, M.M.; Gabr, A.A.; Samuel, S.; Kamel, M.; el Baz, M.; el Beshry, M.; Michail, R.R. Vitamin D administration to tuberculous children and its value. Boll. Chim. Farm. 1998, 137, $157-164$.

215. Coussens, A.K.; Wilkinson, R.J.; Hanifa, Y.; Nikolayevskyy, V.; Elkington, P.T.; Islam, K.; Timms, P.M.; Venton, T.R.; Bothamley, G.H.; Packe, G.E.; et al. Vitamin D accelerates resolution of inflammatory responses during tuberculosis treatment. Proc. Natl. Acad. Sci. USA 2012, 109, doi:10.1073/pnas.1216789109.

216. Wejse, C.; Gomes, V.F.; Rabna, P.; Gustafson, P.; Aaby, P.; Lisse, I.M.; Andersen, P.L.; Glerup, H.; Sodemann, M. Vitamin D as supplementary treatment for tuberculosis. Am. J. Respir. Crit. Care Med. 2009, 179, 843-850.

217. Jorde, R.; Witham, M.; Janssens, W.; Rolighed, L.; Borchhardt, K.; de Boer, I.H.; Grimnes, G.; Hutchinson, M.S. Vitamin D supplementation did not prevent influenza-like illness as diagnosed retrospectively by questionnaires in subjects participating in randomized clinical trials. Scand. J. Infect. Dis. 2012, 44, 126-132.

218. Urashima, M.; Segawa, T.; Okazaki, M.; Kurihara, M.; Wada, Y.; Ida, H. Randomized trial of vitamin D supplementation to prevent seasonal influenza A in schoolchildren. Am. J. Clin. Nutr. 2010, 91, 1255-1260.

219. Cannell, J.J.; Vieth, R.; Umhau, J.C.; Holick, M.F.; Grant, W.B.; Madronich, S.; Garland, C.F.; Giovannucci, E. Epidemic influenza and vitamin D. Epidemiol. Infect. 2006, 134, 1129-1140.

220. Hope-Simpson, R.E. The role of season in the epidemiology of influenza. J. Hyg. (Lond.) 1981, $86,35-47$.

221. Ginde, A.; Mansbach, J.M.; Camargo, C.A. Association between serum 25-hydroxyvitamin D level and upper respiratory tract infection in the third national health and nutrition examination survey. Arch. Intern. Med. 2009, 169, 384-390.

222. Laaksi, I.; Ruohola, J.-P.; Tuohimaa, P.; Auvinen, A.; Haataja, R.; Pihlajamäki, H.; Ylikomi, T. An association of serum vitamin $\mathrm{D}$ concentrations $<40 \mathrm{nmol} / \mathrm{L}$ with acute respiratory tract infection in young Finnish men. Am. J. Clin. Nutr. 2007, 86, 714-717.

223. Wayse, V.; Yousafzai, A.; Mogale, K.; Filteau, S. Association of subclinical vitamin D deficiency with severe acute lower respiratory infection in Indian children under 5 y. Eur. $J$. Clin. Nutr. 2004, 58, 563-567.

224. Aloia, J.F.; Li-Ng, M. Re: Epidemic influenza and vitamin D. Epidemiol. Infect. 2007, 135, 1095-1096.

225. Avenell, A.; Cook, J.A.; MacLennan, G.S.; MacPherson, G.C. Vitamin D supplementation to prevent infections: A sub-study of a randomised placebo-controlled trial in older people (RECORD trial, ISRCTN 51647438). Age Ageing 2007, 36, 574-577.

226. Li-Ng, M.; Aloia, J.F.; Pollack, S.; Cunha, B.A.; Mikhail, M.; Yeh, J.; Berbari, N. A randomized controlled trial of vitamin D3 supplementation for the prevention of symptomatic upper respiratory tract infections. Epidemiol. Infect. 2009, 137, 1396-1404.

227. Roth, D.E.; Jones, A.B.; Prosser, C.; Robinson, J.L.; Vohra, S. Vitamin D receptor polymorphisms and the risk of acute lower respiratory tract infection in early childhood. J. Infect. Dis. 2008, 197, 676-680. 
228. Hewison, M. An update on vitamin D and human immunity. Clin. Endocrinol. 2012, 76, $315-325$.

229. Brown, S.D.; Calvert, H.H.; Fitzpatrick, A.M. Vitamin D and asthma. Dermato-Endocrinol. 2012, 4, 137-145.

230. Camargo, C.A., Jr.; Rifas-Shiman, S.L.; Litonjua, A.A.; Rich-Edwards, J.W.; Weiss, S.T.; Gold, D.R.; Kleinman, K.; Gillman, M.W. Maternal intake of vitamin D during pregnancy and risk of recurrent wheeze in children at 3 y of age. Am. J. Clin. Nutr. 2007, 85, 788-795.

231. Camargo, C.A., Jr.; Ingham, T.; Wickens, K.; Thadhani, R.; Silvers, K.M.; Epton, M.J.; Town, G.I.; Pattemore, P.K.; Espinola, J.A.; Crane, J. Cord-blood 25-hydroxyvitamin D levels and risk of respiratory infection, wheezing, and asthma. Pediatrics 2011, 127, e180-e187.

232. Carroll, K.N.; Gebretsadik, T.; Larkin, E.K.; Dupont, W.D.; Liu, Z.; van Driest, S.; Hartert, T.V. Relationship of maternal vitamin D level with maternal and infant respiratory disease. Am. J. Obstet. Gynecol. 2011, 205, e211-e217.

233. Devereux, G.; Litonjua, A.A.; Turner, S.W.; Craig, L.C.; McNeill, G.; Martindale, S.; Helms, P.J.; Seaton, A.; Weiss, S.T. Maternal vitamin D intake during pregnancy and early childhood wheezing. Am. J. Clin. Nutr. 2007, 85, 853-859.

234. Erkkola, M.; Kaila, M.; Nwaru, B.I.; Kronberg-Kippila, C.; Ahonen, S.; Nevalainen, J.; Veijola, R.; Pekkanen, J.; Ilonen, J.; Simell, O.; et al. Maternal vitamin D intake during pregnancy is inversely associated with asthma and allergic rhinitis in 5-year-old children. Clin. Exp. Allergy 2009, 39, 875-882.

235. Gale, C.R.; Robinson, S.M.; Harvey, N.C.; Javaid, M.K.; Jiang, B.; Martyn, C.N.; Godfrey, K.M.; Cooper, C. Maternal vitamin D status during pregnancy and child outcomes. Eur. J. Clin. Nutr. 2008, 62, 68-77.

236. Rothers, J.; Wright, A.L.; Stern, D.A.; Halonen, M.; Camargo, C.A., Jr. Cord blood 25-hydroxyvitamin D levels are associated with aeroallergen sensitization in children from Tucson, Arizona. J. Allergy Clin. Immunol. 2011, 128, 1093-1099.

237. Chinellato, I.; Piazza, M.; Sandri, M.; Peroni, D.; Piacentini, G.; Boner, A.L. Vitamin D serum levels and markers of asthma control in Italian children. J. Pediatr. 2011, 158, 437-441.

238. Chinellato, I.; Piazza, M.; Sandri, M.; Peroni, D.G.; Cardinale, F.; Piacentini, G.L.; Boner, A.L. Serum vitamin D levels and exercise-induced bronchoconstriction in children with asthma. Eur. Respir. J. 2011, 37, 1366-1370.

239. Searing, D.A.; Zhang, Y.; Murphy, J.R.; Hauk, P.J.; Goleva, E.; Leung, D.Y. Decreased serum vitamin $\mathrm{D}$ levels in children with asthma are associated with increased corticosteroid use. J. Allergy Clin. Immunol. 2010, 125, 995-1000.

240. Heaney, R.P.; Davies, K.M.; Chen, T.C.; Holick, M.F.; Barger-Lux, M.J. Human serum 25-hydroxycholecalciferol response to extended oral dosing with cholecalciferol. Am. J. Clin. Nutr. 2003, 77, 204-210.

241. Godar, D.E.; Pope, S.J.; Grant, W.B.; Holick, M.F. Solar UV doses of adult Americans and vitamin D(3) production. Dermato-Endocrinol. 2011, 3, 243-250.

242. Pietras, S.M.; Obayan, B.K.; Cai, M.H.; Holick, M.F. Vitamin D2 treatment for vitamin D deficiency and insufficiency for up to 6 years. Arch. Intern. Med. 2009, 169, 1806-1808. 
243. Jones, G. Pharmacokinetics of vitamin D toxicity. Am. J. Clin. Nutr. 2008, 88, 582S-586S.

244. Taylor, A.B.; Stern, P.H.; Bell, N.H. Abnormal regulation of circulating 25-hydroxyvitamin D in the Williams syndrome. N. Engl. J. Med. 1982, 306, 972-975.

245. Schlingmann, K.P.; Kaufmann, M.; Weber, S.; Irwin, A.; Goos, C.; John, U.; Misselwitz, J.; Klaus, G.; Kuwertz-Bröking, E.; Fehrenbach, H.; et al. Mutations in CYP24A1 and Idiopathic Infantile Hypercalcemia. N. Engl. J. Med. 2011, 365, 410-421.

246. Eyles, D.W.; Burne, T.H.J.; McGrath, J.J. Vitamin D, effects on brain development, adult brain function and the links between low levels of vitamin D and neuropsychiatric disease. Front. Neuroendocrinol. 2012, in press. 



\section{Chapter 2}

\section{Vitamin D Intakes and Serum Levels}





\title{
A Statistical Error in the Estimation of the Recommended Dietary Allowance for Vitamin D
}

\author{
Paul J. Veugelers and John Paul Ekwaru
}

Reprinted from Nutrients. Cite as: Veugelers, P.J.; Ekwaru, J.P. A Statistical Error in the Estimation of the Recommended Dietary Allowance for Vitamin D. Nutrients 2014, 6, 4472-4475.

The Institute of Medicine (IOM) issues dietary recommendations on the request of the U.S. and Canadian governments. One of these recommendations is the Recommended Dietary Allowance (RDA). The RDA is the nutrient intake considered to be sufficient to meet the requirements of $97.5 \%$ of healthy individuals [1]. The RDA for vitamin D is $600 \mathrm{IU}$ per day for individuals 1 to 70 years of age and is assumed to achieve serum 25-hydroxyvitamin $\mathrm{D}(25(\mathrm{OH}) \mathrm{D})$ levels of $50 \mathrm{nmol} / \mathrm{L}$ or more in $97.5 \%$ of healthy individuals [1]. Serum $25(\mathrm{OH}) \mathrm{D}$ is the established proxy for vitamin D status and levels of $50 \mathrm{nmol} / \mathrm{L}$ or more have been shown to benefit bone health and to prevent disease and injury [1].

The IOM based their RDA for vitamin D on an aggregation of 10 supplementation studies that were carried out during winter months and at locations with latitudes above the 50th parallel north to minimize the influence of cutaneous vitamin D synthesis [2-11]. As several of these 10 studies examined more than one supplementation dose, collectively they provided 32 study averages of serum 25(OH)D levels. These are replicated as the green diamonds in Figure 1. The IOM regressed the 32 study averages against vitamin D intake to yield the dose response relationship of vitamin D intake and serum 25(OH)D (green solid line in Figure 1). The IOM further calculated the lower and upper $95 \%$ confidence prediction interval based on the 32 study averages and the standard deviation of these 32 study averages (green dashed lines in Figure 1). On the basis of this, the IOM estimated that $600 \mathrm{IU}$ of vitamin D would achieve an average 25(OH)D level of $63 \mathrm{nmol} / \mathrm{L}$ and a lower $95 \%$ confidence prediction limit ( 2.5 percentile) of $56 \mathrm{nmol} / \mathrm{L}$. The latter value was rounded downwards to $50 \mathrm{nmol} / \mathrm{L}$ to accommodate uncertainty in the estimation [1]. This data point (600 IU vitamin D, $50 \mathrm{nmol} / \mathrm{L}$ ) is the basis for the current RDA and for the IOM's conclusion that an intake of $600 \mathrm{IU}$ of vitamin D per day will achieve serum $25(\mathrm{OH}) \mathrm{D}$ levels of $50 \mathrm{nmol} / \mathrm{L}$ or more in $97.5 \%$ of individuals. This conclusion, however, is incorrect. 
Figure 1. Dose response relationship of vitamin D intake and serum 25 hydroxyvitamin D.

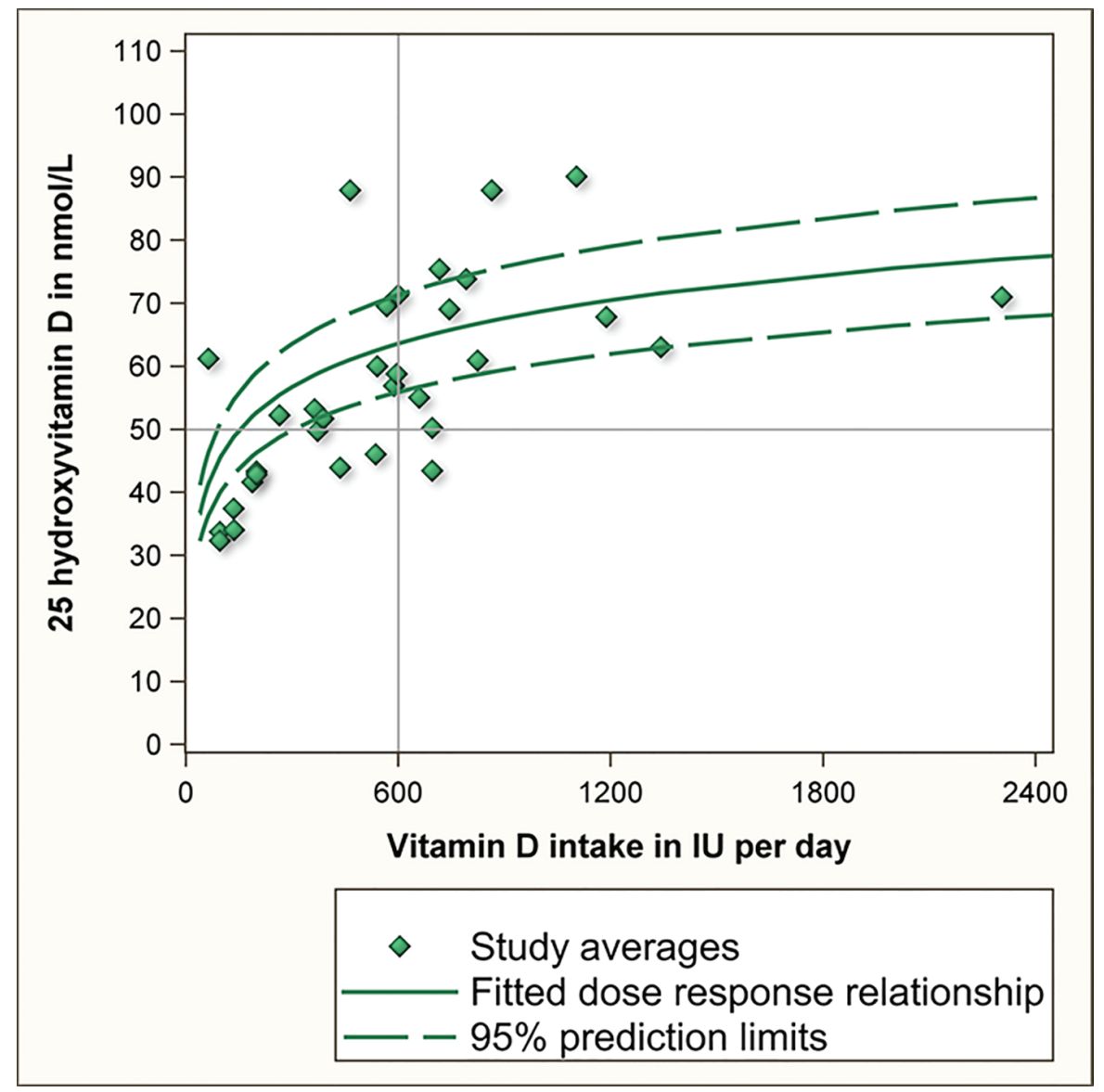

The correct interpretation of the lower prediction limit is that $97.5 \%$ of study averages are predicted to have values exceeding this limit. This is essentially different from the IOM's conclusion that $97.5 \%$ of individuals will have values exceeding the lower prediction limit. To illustrate the difference between the former and latter interpretation, we estimated how much vitamin $\mathrm{D}$ is needed to achieve that $97.5 \%$ of individuals achieve serum $25(\mathrm{OH}) \mathrm{D}$ values of $50 \mathrm{nmol} / \mathrm{L}$ or more. For this purpose we reviewed each of the 10 studies used by the IOM. Eight studies reported both the average and standard deviation [2,5-11]. These eight studies had examined a total of 23 supplementation doses [2,5-11]. For each of these 23 study averages we calculated the 2.5 th percentile by subtracting 2 standard deviations from the average (depicted by yellow dots in Figure 2). Next, we regressed these 23 values against vitamin D intake to yield the lower prediction limit (red line in Figure 2). This regression line revealed that $600 \mathrm{IU}$ of vitamin D per day achieves that $97.5 \%$ of individuals will have serum $25(\mathrm{OH}) \mathrm{D}$ values above $26.8 \mathrm{nmol} / \mathrm{L}$ rather than above $50 \mathrm{nmol} / \mathrm{L}$ which is currently assumed. It also estimated that 8895 IU of vitamin D per day may be needed to accomplish that $97.5 \%$ of individuals achieve serum $25(\mathrm{OH}) \mathrm{D}$ values of $50 \mathrm{nmol} / \mathrm{L}$ or more. As this dose is far beyond the range of studied doses, caution is warranted when interpreting this estimate. Regardless, the very high estimate illustrates that the dose is well in excess of the current RDA of 600 IU per day and the tolerable upper intake of 4000 IU per day [1]. 
Figure 2. Dose response relationship of vitamin D intake and serum 25 hydroxyvitamin D.

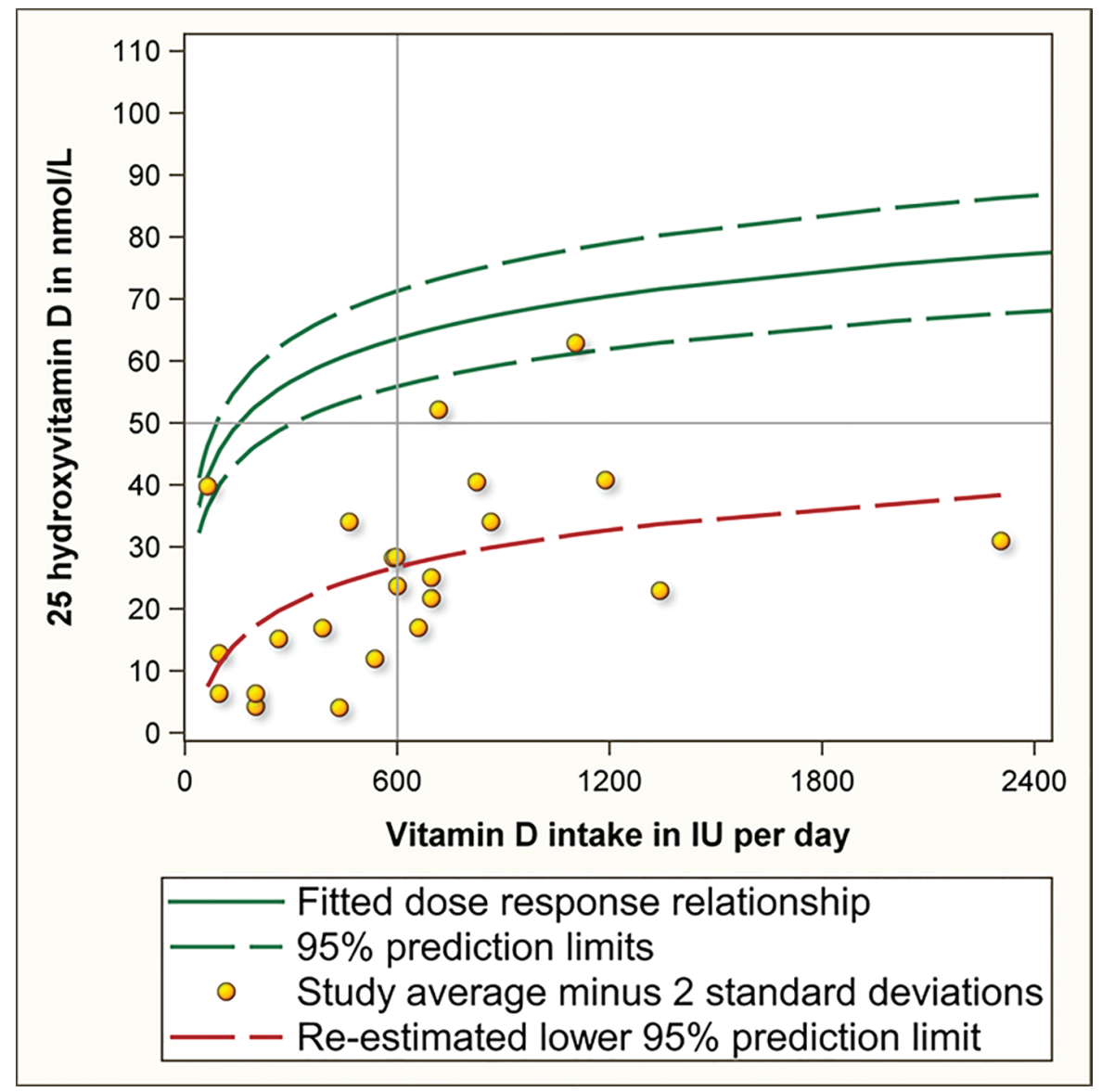

The public health and clinical implications of the miscalculated RDA for vitamin D are serious. With the current recommendation of $600 \mathrm{IU}$, bone health objectives and disease and injury prevention targets will not be met. This became apparent in two studies conducted in Canada where, because of the Northern latitude, cutaneous vitamin D synthesis is limited and where diets contribute an estimated 232 IU of vitamin D per day [12]. One study estimated that despite Vitamin D supplementation with $400 \mathrm{IU}$ or more (including dietary intake that is a total intake of $632 \mathrm{IU}$ or more) $10 \%$ of participants had values of less than $50 \mathrm{nmol} / \mathrm{L}$ [13]. The second study reported serum 25(OH)D levels of less than $50 \mathrm{nmol} / \mathrm{L}$ for $15 \%$ of participants who reported supplementation with vitamin D [14]. If the RDA had been adequate, these percentages should not have exceeded $2.5 \%$. Herewith these studies show that the current public health target is not being met.

We recommend that the RDA for vitamin D be reconsidered to allow for appropriate public health and clinical decision-making.

\section{References}

1. Food and Nutrition Board, Institute of Medicine. Dietary Reference Intakes for Calcium and Vitamin D; The National Academic Press: Washington, DC, USA, 2011.

2. Ala-Houhala, M.; Koskinen, T.; Koskinen, M.; Visakorpi, J.K. Double blind study on the need for vitamin D supplementation in prepubertal children. Acta Paediatr. Scand. 1988, 77, 89-93. 
3. Cashman, K.D.; Hill, T.R.; Lucey, A.J.; Taylor, N.; Seamans, K.M.; Muldowney, S.; FitzGerald, A.P.; Flynn, A.; Barnes, M.S.; Horigan, G.; et al. Estimation of the dietary requirement for vitamin D in healthy adults. Am. J. Clin. Nutr. 2008, 88, 1535-1542.

4. Cashman, K.D.; Wallace, J.M.; Horigan, G.; Hill, T.R.; Barnes, M.S.; Lucey, A.J.; Bonham, M.P.; Taylor, N.; Duffy, E.M.; Seamans, K.; et al. Estimation of the dietary requirement for vitamin $\mathrm{D}$ in free-living adults $>=64$ y of age. Am. J. Clin. Nutr. 2009, 89, 1366-1374.

5. Larsen, E.R.; Mosekilde, L.; Foldspang, A. Vitamin D and calcium supplementation prevents osteoporotic fractures in elderly community dwelling residents: A pragmatic population-based 3-year intervention study. J. Bone Miner. Res. 2004, 19, 370-378.

6. Schou, A.J.; Heuck, C.; Wolthers, O.D. A randomized, controlled lower leg growth study of vitamin D supplementation to healthy children during the winter season. Ann. Hum. Biol. 2003, 30, 214-219.

7. Smith, S.M.; Gardner, K.K.; Locke, J.; Zwart, S.R. Vitamin D supplementation during Antarctic winter. Am. J. Clin. Nutr. 2009, 89, 1092-1098.

8. Van der Klis, F.R.; Jonxis, J.H.; van Doormaal, J.J.; Sikkens, P.; Saleh, A.E.; Muskiet, F.A. Changes in vitamin-D metabolites and parathyroid hormone in plasma following cholecalciferol administration to pre- and postmenopausal women in the Netherlands in early spring and to postmenopausal women in Curacao. Br. J. Nutr. 1996, 75, 637-646.

9. Viljakainen, H.T.; Natri, A.M.; Karkkainen, M.; Huttunen, M.M.; Palssa, A.; Jakobsen, J.; Cashman, K.D.; Mølgaard, C.; Lamberg-Allardt, C. A positive dose-response effect of vitamin D supplementation on site-specific bone mineral augmentation in adolescent girls: A double-blinded randomized placebo-controlled 1-year intervention. J. Bone Miner. Res. 2006, 21, 836-844.

10. Viljakainen, H.T.; Palssa, A.; Karkkainen, M.; Jakobsen, J.; Lamberg-Allardt, C. How much vitamin D3 do the elderly need? J. Am. Coll. Nutr. 2006, 25, 429-435.

11. Viljakainen, H.T.; Vaisanen, M.; Kemi, V.; Rikkonen, T.; Kröger, H.; Laitinen, E.K.; Rita, H.; Lamberg-Allardt, C. Wintertime vitamin D supplementation inhibits seasonal variation of calcitropic hormones and maintains bone turnover in healthy men. J. Bone Miner. Res. 2009, 24, 346-352.

12. Vatanparast, H.; Calvo, M.S.; Green, T.J.; Whiting, S.J. Despite mandatory fortification of staple foods, vitamin D intakes of Canadian children and adults are inadequate. J. Steroid Biochem. Mol. Biol. 2010, 121, 301-303.

13. Greene-Finestone, L.S.; Berger, C.; de Groh, M.; Hanley, D.A.; Hidiroglou, N.; Sarafin, K.; Poliquin, S.; Krieger, J.; Richards, J.B.; Goltzman, D.; et al. 25-Hydroxyvitamin D in Canadian adults: Biological, environmental, and behavioral correlates. Osteoporos. Int. 2011, 22, 1389-1399.

14. Janz, T.; Pearson, C. Vitamin D blood levels of Canadians. In Health at a Glance; (Catalogue no 82-624-X); Statistics Canada: Ottawa, ON, Canada, 2013. 


\title{
The Importance of Dose, Frequency and Duration of Vitamin D Supplementation for Plasma 25-Hydroxyvitamin D
}

\author{
Yi-Sheng Chao, Ludovic Brunel, Peter Faris and Paul J. Veugelers
}

\begin{abstract}
The importance of dose, frequency and duration of vitamin D supplementation for plasma $25(\mathrm{OH}) \mathrm{D}$ levels is not well described and rarely reported for supplementation that exceeds $2000 \mathrm{IU}$ per day. The objective is to examine dose, frequency and duration of supplementation in relation to plasma $25(\mathrm{OH}) \mathrm{D}$ in a large population-based sample. We accessed data on 2714 volunteers that contributed to 4224 visits and applied multilevel regression. Compared to not using supplements, a minimum regimen of 1000-2000 IU once or twice per week for one month was not effective in raising 25(OH)D. Compared to this minimum regimen, higher doses of 2000-3000, 3000-4000, and $5000 \mathrm{IU}$ or more were associated with a 7.49, 13.19 and $30.22 \mathrm{nmol} / \mathrm{L} 25(\mathrm{OH}) \mathrm{D}$ increase, respectively; frequencies of three to four, five to six and seven times/week were associated with a 5.44, 16.52 and $30.69 \mathrm{nmol} / \mathrm{L}$ increase, respectively; and supplementation of five months or longer was associated with an increase of $6.68 \mathrm{nmol} / \mathrm{L}$ ( $p<0.01$ for all). Age, body weight, physical activity, smoking, and self-rated health were significantly associated with $25(\mathrm{OH}) \mathrm{D}$. Whereas dose, frequency and duration of supplementation are important to healthy subjects committed to optimizing their nutritional status, to the design of clinical trials, individual characteristics and lifestyle contribute substantially to $25(\mathrm{OH}) \mathrm{D}$.
\end{abstract}

Reprinted from Nutrients. Cite as: Chao, Y.-S.; Brunel, L.; Faris, P.; Veugelers, P.J. The Importance of Dose, Frequency and Duration of Vitamin D Supplementation for Plasma 25-Hydroxyvitamin D. Nutrients 2013, 5, 4067-4078.

\section{Introduction}

Vitamin D plays an important role in maintaining bone health and has benefits for extra-skeletal health [1]. Daily intake of 600 IU of vitamin D [2] and other dosages have been recommended [3]. However, vitamin D intake is not an effective measure of vitamin D status in human bodies. Plasma 25-hydroxy-vitamin D [25(OH)D] levels are the established proxy to assess health benefits $[4,5]$.

The dose response relationship between supplementation and plasma $25(\mathrm{OH}) \mathrm{D}$ is the key to understanding the intervention effect. Researchers studied the relationship between vitamin D daily doses and plasma $25(\mathrm{OH}) \mathrm{D}$ levels in the elderly or postmenopausal women with a maximum dose of 1000 or $1400 \mathrm{IU} /$ day [6,7]. Heaney suggested that plasma $25(\mathrm{OH}) \mathrm{D}$ levels rise by $1 \mathrm{ng} / \mathrm{mL}$ $(2.5 \mathrm{nmol} / \mathrm{L})$ for every $100 \mathrm{IU}$ (2500 ng) of daily vitamin D intake [8]. Where he and other researchers confirmed the linearity in the relationship between vitamin D supplementation and 25(OH)D in other populations [3,9], others reported a non-linear relationship for doses exceeding 1600-4000 IU per day [10-12]. In addition to daily supplementation, other supplementation frequencies (i.e., variable times per week or weekly, monthly or annually) were tested in various trials of varying periods of time $[3,13,14]$. Recent studies focused on the long half-life of vitamin D and investigated the long-term effects of supplementation [7,15]. However, these studies have not investigated the importance of dose, frequency and duration of supplementation for plasma $25(\mathrm{OH}) \mathrm{D}$. 
The objective of the present study is to examine the independent associations of dose, frequency and duration of vitamin D supplementation to plasma $25(\mathrm{OH}) \mathrm{D}$ in a large population-based sample of healthy participants.

\section{Experimental Section}

The Pure North S'Energy Foundation, a not-for-profit charitable organization, has provided free health and wellness services for volunteers since October 2007 [16]. Details of the program and data collection protocol are described elsewhere [16]. In brief, volunteer participants, mostly residing in the Canadian province of Alberta, were offered health promotion counseling and nutritional supplementation, with a particular focus on vitamin D given the Northern latitude of the locations where the program was offered. Participants were invited to complete a lifestyle questionnaire, have their heights and weights measured, and have blood drawn for the assessment of plasma 25(OH)D [17], among others [16].

Questionnaires included questions on dose, frequency, and duration of vitamin D supplementation. The dose was recorded as 1000-2000 IU, 2000-3000 IU, 3000-4000 IU and more than 5000 IU. Frequency of supplementation was recorded as never, one or two times, three to four times, five to six times and seven times per week, and duration of supplementation as one, two, three, four or five (or more) months. In the present study, we used 1000-2000 IU, once or twice per week, for one month as the minimum regimen for comparisons with higher doses, frequencies and durations, as well as for comparisons with those not taking vitamin D supplements.

The questionnaire included questions on physical activity, diet, smoking, season and place of residence. Physical activity was categorized as low, moderate and high [18]. Participants were asked to rate their general health as excellent, very good, good, fair, or needs improvement. As self-selection in the participation in the health and wellness program could be motivated by participants' health status [19], and the program could be particularly attractive to those with relatively poor health, we also considered individuals' health status at first visits in our analyses.

The Foundation anonymised their data prior to forwarding it to the University of Alberta for analyses. The present study made use of 4224 records of 2714 participants that completed the questionnaire and had plasma 25(OH)D measured between October 2007 and April 2012. One participant was excluded due to advanced kidney disease (estimated glomerular filtration rate $<15 \mathrm{~mL} / \mathrm{min} / 1.73 \mathrm{~m}^{2}$ ), which affects the metabolism of vitamin $\mathrm{D}$ and the production of its active metabolites [20].

Plasma 25(OH)D values constituted the outcome in multilevel regressions models that tested the importance of dose, frequency and duration of vitamin D supplementation. Dose, frequency and duration were first analyzed univariably and subsequently multivariably to adjust for the potential confounders listed above. We applied multilevel regression to accommodate the hierarchical structure of the data, as records from one, two or more study visits were nested within observations of participants. Participants' characteristics were considered at level 1 and visit specific characteristics at level 2. The regression analyses of the contribution of dose, frequency and duration to raising $25(\mathrm{OH}) \mathrm{D}$ were first analyzed without consideration of potential confounders (univariable) and subsequently with consideration (multivariable) of potential confounding of age, gender, body 
weight status, general health, season, physical activity, smoking status, and the consumption of milk (daily servings), fish (weekly servings) and margarine (frequency). To allow for our regression analyses to simultaneously analyze the associations of (1) no supplementation and (2) varying levels of supplementation in relation to $25(\mathrm{OH}) \mathrm{D}$, we introduced interaction terms of (1) supplementation (Yes/No) and (2) dose, frequency and duration of supplementation. The resulting regression coefficients were to be interpreted as the changes in plasma $25(\mathrm{OH}) \mathrm{D}$ associated with (1) a minimum regimen relative to no supplementation and (2) a one-unit change in dose, frequency and duration of vitamin D supplementation relative to the minimum regimen. All analyses were conducted using STATA version 12 (College Station, TX, USA). Statistical significance was defined as p values less than 0.05 (two tails).

The Human Research Ethics Board of the University of Alberta had approved access to and analysis of the Foundation's data for the purpose of the present analyses.

\section{Results}

From 2007 to 2012, there were 4224 visits made by 2714 participants. The mean age at visits was $42.48 \pm 11.00($ mean $\pm S D$, range $=9-85)$ years and the latitudes of their residence were $53.36 \pm 2.49$ (range $=42.93-68.30)$. The mean plasma 25(OH)D level was $96.41 \pm 47.17($ range $=10-522)$ and 2068 visits (48.96\%) were made between April and October. At visits with reported use of vitamin D supplementation, plasma 25(OH)D levels were, on average, $103.31 \pm 47.58 \mathrm{nmol} / \mathrm{L}$ (range $=10-522)$, which is statistically significant more than $64.92 \pm 28.93 \mathrm{nmol} / \mathrm{L}$ (range $=13.1-211$ ) at visits without reported vitamin D supplementation (Table 1). Relative to visits without reporting supplementation use, visits with reported supplementations were more likely to be made by older participants, by those residing at lower latitudes, by overweight and obese participants, and by those reporting better health $(p<0.05$ for all) (Table 1).

Table 1. Characteristics of 4224 visits by 2714 participants according to vitamin D supplementation use.

\begin{tabular}{|c|c|c|c|c|c|}
\hline & \multicolumn{2}{|c|}{$\begin{array}{l}\text { Not taking vitamin D } \\
\text { supplements }(N=759)\end{array}$} & \multicolumn{2}{|c|}{$\begin{array}{c}\text { Taking any vitamin } D \\
\text { supplements }(N=3465)\end{array}$} & \multirow[t]{2}{*}{$P$} \\
\hline & Mean & SD & Mean & SD & \\
\hline 25-hydroxyvitamin D (nmol/L) & 64.92 & 28.93 & 103.31 & 47.58 & $<0.001$ \\
\hline Age (years) & 35.78 & 11.03 & 42.48 & 11 & $<0.001$ \\
\hline Male (\%) & $68.25 \%$ & & $69.99 \%$ & & 0.35 \\
\hline Latitude (degrees) & 53.8 & 2.91 & 53.36 & 2.49 & $<0.001$ \\
\hline Body weight categories (\%) & & & & & $<0.01$ \\
\hline Underweight & $1.19 \%$ & & $0.52 \%$ & & \\
\hline Normal weight & $28.59 \%$ & & $24.01 \%$ & & \\
\hline Overweight & $36.50 \%$ & & $37.09 \%$ & & \\
\hline Obesity & $33.73 \%$ & & $38.38 \%$ & & \\
\hline General Health (first visits) (\%) & & & & & $<0.01$ \\
\hline Excellent & $4.87 \%$ & & $6.58 \%$ & & \\
\hline Very good & $23.98 \%$ & & 0.2771 & & \\
\hline Good & $41.90 \%$ & & $42.60 \%$ & & \\
\hline
\end{tabular}


Table 1. Cont.

\begin{tabular}{|c|c|c|c|}
\hline & $\begin{array}{l}\text { Not taking vitamin } D \\
\text { supplements }(N=759)\end{array}$ & $\begin{array}{c}\text { Taking any vitamin D } \\
\text { supplements }(N=3465)\end{array}$ & $\boldsymbol{P}$ \\
\hline General Health (first visits) (\%) & & & $<0.01$ \\
\hline Fair & $17.13 \%$ & $14.31 \%$ & \\
\hline Needs improvement & $12.12 \%$ & $8.80 \%$ & \\
\hline April to October (\%) & $49.28 \%$ & $48.89 \%$ & 0.85 \\
\hline \multicolumn{4}{|l|}{ Health behaviors } \\
\hline Physical activity level (\%) & & & $<0.01$ \\
\hline Low & $37.88 \%$ & $33.58 \%$ & \\
\hline Moderate & $43.29 \%$ & $51.87 \%$ & \\
\hline High & $18.82 \%$ & $14.56 \%$ & \\
\hline Smoking (\%) & $16.16 \%$ & $15.48 \%$ & 0.64 \\
\hline \multicolumn{4}{|l|}{ Food servings } \\
\hline Milk (per day) (\%) & & & 0.49 \\
\hline $\mathbf{0}$ & $6.18 \%$ & $6.82 \%$ & \\
\hline 1 to 2 & $64.61 \%$ & $66.69 \%$ & \\
\hline 3 or more & $29.22 \%$ & $26.49 \%$ & \\
\hline Fish (per week) (\%) & & & $<0.001$ \\
\hline $\mathbf{0}$ & $28.25 \%$ & $19.17 \%$ & \\
\hline 1 to 2 & $50.28 \%$ & $59.11 \%$ & \\
\hline 3 or more & $21.47 \%$ & $21.72 \%$ & \\
\hline Margarine consumption frequency $(\%)$ & & & 0.11 \\
\hline Never & $24.04 \%$ & $27.99 \%$ & \\
\hline Rarely & $34.13 \%$ & $32.16 \%$ & \\
\hline Often & $34.38 \%$ & $30.28 \%$ & \\
\hline Always & $7.45 \%$ & $9.57 \%$ & \\
\hline
\end{tabular}

Note: 25(OH)D: plasma 25-hydroxyvitamin D levels. Categorical and continuous variables were tested with chi-square and two-sample $t$ tests, respectively.

At those visits that participants reported to be taking vitamin D supplements, Table 2 shows the average plasma 25(OH)D levels by dose, frequency and duration of supplementation. The incremental increases of plasma 25(OH)D levels in each of these three dimensions were statistically significant ( $p<0.001$ for each of dose, frequency and duration). Compared to those using supplements one or two times per week $(75.15 \pm 31.86 \mathrm{nmol} / \mathrm{L})$, those taking vitamin $\mathrm{D}$ supplements everyday had significantly higher 25(OH)D $(129.37 \pm 53.20 \mathrm{nmol} / \mathrm{L}, p<0.001)$. Similarly, participants taking dosages of 5000 or more IU at a time $(129.63 \pm 55.88 \mathrm{nmol} / \mathrm{L})$ had significantly higher $25(\mathrm{OH}) \mathrm{D}$ than those taking $1000-2000 \mathrm{IU}$ each time $(82.99 \pm 34.77 \mathrm{nmol} / \mathrm{L}, p<0.001)$. The average $25(\mathrm{OH}) \mathrm{D}$ for those taking vitamin $\mathrm{D}$ for five or more months $(113.96 \pm 48.89 \mathrm{nmol} / \mathrm{L})$ was also significantly higher than for those only using vitamin D supplements for one month $(83.35 \pm 41.08 \mathrm{nmol} / \mathrm{L}, p<0.001)$. The mean 25(OH)D levels associated with the highest doses, most frequent use and longest time of supplementation were significantly higher than those associated with the least intense supplementation regimens. 
Table 2. Plasma 25-hydroxyvitamin D [25(OH)D] levels by dose, frequency and duration of vitamin D supplementation of 4224 study visits by 2714 participants.

\begin{tabular}{|c|c|c|c|c|c|c|c|}
\hline & \multicolumn{3}{|c|}{ Not taking vitamin D supplementation } & \multicolumn{3}{|c|}{ Taking any supplements } & \multirow[t]{2}{*}{$\boldsymbol{P}$} \\
\hline & No. of obs. & Mean & SD & No. of obs. & Mean & SD & \\
\hline & 759 & 64.76 & 28.86 & 3465 & 103.31 & 47.58 & $<0.001 *$ \\
\hline \multicolumn{8}{|l|}{ Dose (IU) } \\
\hline 1000 to 2000 & & & & 1433 & 82.99 & 34.77 & $<0.001 * *$ \\
\hline 2000 to 3000 & & & & 524 & 98.89 & 36.53 & \\
\hline 3000 to 4000 & & & & 432 & 110.56 & 39.93 & \\
\hline 5000 or more & & & & 1076 & 129.63 & 55.88 & \\
\hline \multicolumn{8}{|c|}{ Frequency, times/week } \\
\hline 1-2/week & & & & 738 & 75.15 & 31.86 & $<0.001 * *$ \\
\hline 3-4/week & & & & 762 & 89.28 & 37.94 & \\
\hline 5-6/week & & & & 935 & 108.27 & 41.76 & \\
\hline 7/week & & & & 1030 & 129.37 & 53.2 & \\
\hline \multicolumn{8}{|l|}{ Duration (months) } \\
\hline 1 & & & & 629 & 83.35 & 41.08 & $<0.001 * *$ \\
\hline 2 & & & & 258 & 85.72 & 36.99 & \\
\hline 3 & & & & 281 & 90.84 & 40.63 & \\
\hline 4 & & & & 225 & 96.8 & 42.23 & \\
\hline 5 or more & & & & 2072 & 113.96 & 48.89 & \\
\hline
\end{tabular}

Note: IU: international units; * Two-sample $t$ test, comparing the mean values between those taking supplements or not; ** One-way ANOVA (analysis of variance) for different doses or frequencies or durations among those taking any supplements.

Table 3 shows univariable and multivariable analysis of the association of dose, frequency and duration and other factors with plasma $25(\mathrm{OH}) \mathrm{D}$ levels. The multivariable analysis revealed that plasma 25(OH)D levels associated with minimum supplementation regimen (1000-2000 IU once or twice per week for a duration of one month) was not statistically significant higher than that when not taking supplements $(\beta=3.08 ; 95 \% \mathrm{CI}:-1.10-7.26)$. Dose, frequency and duration of supplementation were each significantly associated with 25(OH)D levels (Table 3). Relative to the minimum regimen dose, those taking 2000-3000 IU was associated with $7.49 \mathrm{nmol} / \mathrm{L}$ (95\% CI: 3.73-11.24) higher plasma 25(OH)D levels and higher doses were associated with higher plasma levels (Table 3). With respect to frequency, taking supplements 3-4 times per week was associated with $5.44 \mathrm{nmol} / \mathrm{L}(95 \% \mathrm{CI}: 1.52-9.35)$ higher plasma levels compared to the minimum regimen. Any further increases in frequency, 5-6 times or 7 times per week, were associated with higher increase in plasma $25(\mathrm{OH}) \mathrm{D}$ levels. For those who had been supplementing vitamin D for five months or more, there were statistically significant increases of $6.68 \mathrm{nmol} / \mathrm{L}$ (95\% CI: 3.05-10.31) in plasma $25(\mathrm{OH}) \mathrm{D}$ levels relative to those taking the minimum regimen. Figure 1 visualizes the dose response relationship of vitamin D supplementation and plasma $25(\mathrm{OH}) \mathrm{D}$ based on the figures presented in Table 3. The first bar drawn on the left shows the plasma 25(OH)D for those not taking supplements, compared to the plasma 25(OH)D level for a minimum regimen (1000-2000 IU once or twice per 
week for one month). The other bars visualize the estimated increase in plasma 25(OH)D resulting from doses, frequencies and durations exceeding those of the minimum regimen.

Table 3. The effects of dose, frequency and duration of vitamin D supplementation and individual characteristics on plasma $25(\mathrm{OH}) \mathrm{D}$ levels $(\mathrm{nmol} / \mathrm{L})$ among participants of a health and wellness program.

\begin{tabular}{|c|c|c|c|c|c|c|}
\hline \multirow[t]{2}{*}{ Models } & \multicolumn{2}{|c|}{ Univariable analysis } & \multicolumn{4}{|c|}{ Multivariable analysis } \\
\hline & $\beta$ & $(95 \% \mathrm{CI})$ & & $\beta$ & $(95 \%$ CI) & \\
\hline \multicolumn{7}{|l|}{ Vitamin D supplementation } \\
\hline $\begin{array}{lr}\text { Taking } & \text { minimum } \\
\text { supplementation } & \text { (compared } \\
\text { to not taking any } \\
\text { supplements) }\end{array}$ & $36.84 * * *$ & (33.39 to & 40.29) & 3.08 & $(-1.10$ to & $7.26)$ \\
\hline \multicolumn{7}{|l|}{ Dose (IU) } \\
\hline 1000 to 2000 & \multicolumn{3}{|c|}{ (minimum regimen as reference) } & \multicolumn{3}{|c|}{ (minimum regimen as reference) } \\
\hline 2000 to 3000 & $14.27 * * *$ & (10.47 to & 18.08) & $7.49 * * *$ & (3.73 to & 11.24) \\
\hline 3000 to 4000 & $26.56 * * *$ & (22.44 to & $30.68)$ & $13.19 * * *$ & (9.02 to & 17.36) \\
\hline 5000 or more & $47.33 * * *$ & (44.25 to & $50.41)$ & $30.22 * * *$ & (26.86 to & $33.59)$ \\
\hline \multicolumn{7}{|l|}{ Dose frequency } \\
\hline 1-2/week & \multicolumn{3}{|c|}{ (minimum regimen as reference) } & \multicolumn{3}{|c|}{ (minimum regimen as reference) } \\
\hline $3-4 /$ week & $14.09 * * *$ & (10.07 to & 18.10) & $5.44 * *$ & (1.52 to & $9.35)$ \\
\hline 5-6/week & $33.05 * * *$ & (29.13 to & $36.97)$ & $16.52 * * *$ & (12.41 to & 20.64) \\
\hline 7/week & $53.02 * * *$ & (49.09 to & $56.95)$ & $30.69 * * *$ & (26.35 to & $35.03)$ \\
\hline \multicolumn{7}{|l|}{ Duration (months) } \\
\hline 1 & \multicolumn{3}{|c|}{ (minimum regimen as reference) } & \multicolumn{3}{|c|}{ (minimum regimen as reference) } \\
\hline 2 & 2.87 & $(-3.11$ to & $8.85)$ & 0.18 & $(-5.07$ to & $5.44)$ \\
\hline 3 & $6.61 *$ & ( 0.82 to & 12.39) & 3.22 & $(-1.94$ to & $8.38)$ \\
\hline 4 & $11.10 * * *$ & (4.86 to & 17.35) & 3.31 & $(-2.34$ to & $8.97)$ \\
\hline 5 or more & $28.56 * * *$ & (24.82 to & $32.30)$ & $6.68 * * *$ & (3.05 to & 10.31) \\
\hline Age (per 10 years) & $7.65 * * *$ & (6.35 to & $8.96)$ & $1.46^{*}$ & $(0.28$ to & 2.63) \\
\hline Male (female as reference) & $-6.79 * * *$ & $(-10.19$ to & $-3.38)$ & -1.23 & $(-4.10$ to & 1.64) \\
\hline \multicolumn{7}{|l|}{ Body weight status } \\
\hline Underweight & -10.27 & $(-28.06$ to & $7.52)$ & -3.45 & $(-17.87$ to & 10.96) \\
\hline Normal & \multicolumn{3}{|c|}{ (reference) } & \multicolumn{3}{|c|}{ (reference) } \\
\hline Overweight & $-6.56 * *$ & $(-10.36$ to & $-2.76)$ & $-7.13 * * *$ & $(-10.33$ to & $-3.93)$ \\
\hline Obesity & $-14.85 * * *$ & $(-18.71$ to & $-11.00)$ & $-14.59 * * *$ & $(-17.97$ to & $-11.21)$ \\
\hline \multicolumn{7}{|l|}{ General health (first visit) } \\
\hline Excellent & \multicolumn{3}{|c|}{ (reference) } & \multicolumn{3}{|c|}{ (reference) } \\
\hline Very good & $-11.01 * *$ & $(-17.89$ to & $-4.14)$ & -4.29 & $(-9.94$ to & 1.36) \\
\hline Good & $-19.08 * * *$ & $(-25.70$ to & $-12.46)$ & $-7.98 * *$ & $(-13.52$ to & $-2.44)$ \\
\hline Fair & $-27.34 * * *$ & $(-34.73$ to & $-19.96)$ & $-12.92 * * *$ & $(-19.15$ to & $-6.69)$ \\
\hline Needs improvement & $-30.20 * * *$ & $(-38.11$ to & $-22.29)$ & $-11.24 * *$ & $(-17.97$ to & $-4.51)$ \\
\hline Latitude (per $10^{\circ}$ ) & $-17.50 * * *$ & $(-23.37$ to & $-11.63)$ & $-5.39 *$ & $(-10.17$ to & $-0.61)$ \\
\hline $\begin{array}{l}\text { Summer months (April to } \\
\text { October) }\end{array}$ & 1.18 & $(-1.50$ to & $3.86)$ & $2.91 * *$ & (0.74 to & $5.09)$ \\
\hline
\end{tabular}


Table 3. Cont.

\begin{tabular}{|c|c|c|c|c|c|c|}
\hline \multirow[t]{2}{*}{ Models } & \multicolumn{2}{|c|}{ Univariable analysis } & \multicolumn{4}{|c|}{ Multivariable analysis } \\
\hline & $\beta$ & $(95 \% \mathrm{CI})$ & & $\beta$ & $(95 \% \mathrm{CI})$ & \\
\hline \multicolumn{7}{|c|}{ Physical activity levels } \\
\hline Low & \multicolumn{3}{|c|}{ (reference) } & \multicolumn{3}{|c|}{ (reference) } \\
\hline Moderate & $6.05 * *$ & (2.58 to & $9.52)$ & $1.96 *$ & $(-1.01$ to & 4.92) \\
\hline High & $9.99 * * *$ & (5.06 to & 14.93) & 4.36 & (0.04 to & $8.68)$ \\
\hline \multicolumn{7}{|l|}{ Smoking } \\
\hline Non-smoker & \multicolumn{3}{|c|}{ (reference) } & \multicolumn{3}{|c|}{ (reference) } \\
\hline Smoker & -0.91 & $(-4.79$ to & $2.97)$ & $-5.52 * *$ & $(-8.88$ to & $-2.17)$ \\
\hline \multicolumn{7}{|l|}{ Milk servings/day } \\
\hline $\mathbf{0}$ & \multicolumn{2}{|l|}{ (reference) } & \multicolumn{4}{|c|}{ (reference) } \\
\hline 1 to 2 & -5.14 & $(-11.69$ to & 1.41) & -3.92 & $(-9.46$ to & 1.62) \\
\hline 3 or more & -2.56 & $(-9.58$ to & 4.46) & -1.76 & $(-7.72$ to & 4.19) \\
\hline \multicolumn{7}{|l|}{ Fish servings/week } \\
\hline $\mathbf{0}$ & \multicolumn{2}{|l|}{ (reference) } & \multicolumn{4}{|c|}{ (reference) } \\
\hline 1 to 2 & $0.33 *$ & (9.45 to & 2.10) & -0.51 & $(-4.31$ to & $3.30)$ \\
\hline 3 or more & $2.52 * *$ & (13.51 to & 2.86) & 2.59 & $(-2.00$ to & 7.18) \\
\hline \multicolumn{7}{|l|}{$\begin{array}{l}\text { Margarine } \\
\text { frequency }\end{array}$} \\
\hline Never & \multicolumn{2}{|l|}{ (reference) } & \multicolumn{4}{|c|}{ (reference) } \\
\hline Rarely & $-6.12 * *$ & $(-10.33$ to & $-1.91)$ & -2.22 & $(-5.78$ to & 1.34) \\
\hline Often & $-12.89 * * *$ & $(-17.19$ to & $-8.59)$ & $-4.75 *$ & $(-8.43$ to & $-1.08)$ \\
\hline Always & $-7.60 *$ & $(-13.74$ to & $-1.47)$ & -2.74 & $(-7.96$ to & 2.47) \\
\hline Constant & (not shown) & & & $114.46 * * *$ & (87.25 to & 141.68) \\
\hline No. of visits & 4224 & & & 4224 & & \\
\hline No. of individuals & 2714 & & & 2714 & & \\
\hline \multicolumn{7}{|c|}{$\begin{array}{l}\text { Note: } \beta=\text { regression coefficients representing changes in plasma } 25 \text {-hydroxyvitamin } \mathrm{D}(25(\mathrm{OH}) \mathrm{D})(\mathrm{nmol} / \mathrm{L}) \text {. } \\
\text { Minimum regimen: } 1000-2000 \mathrm{IU} \text { once or twice per week for one month. } * p<0.05 ; * * p<0.01 ; * * *<0.001 \text {. The } \\
\text { univariable analyses considered each covariate in separate analyses with the exception of dose, frequency and duration } \\
\text { that were considered simultaneous. The constants of these univariable models were not shown. The multivariable } \\
\text { analysis considered all variables included in the table as these have been reported to affect plasma } 25 \text {-hydroxyvitamin } \\
\text { D levels in the literature. }\end{array}$} \\
\hline
\end{tabular}

Age, summer season (April to October), and high levels of physical activity were associated in a statistically significant manner with higher plasma 25(OH)D levels ( $p<0.05$ for all). Males, northern latitudes, overweight or obesity, poor self-reported health and margarine intake were associated with lower 25(OH)D levels $(p<0.05$ for all). 
Figure 1. The dose responses between 25-hydroxyvitamin D levels and vitamin D supplementation based on the estimates from the multivariable regression model.

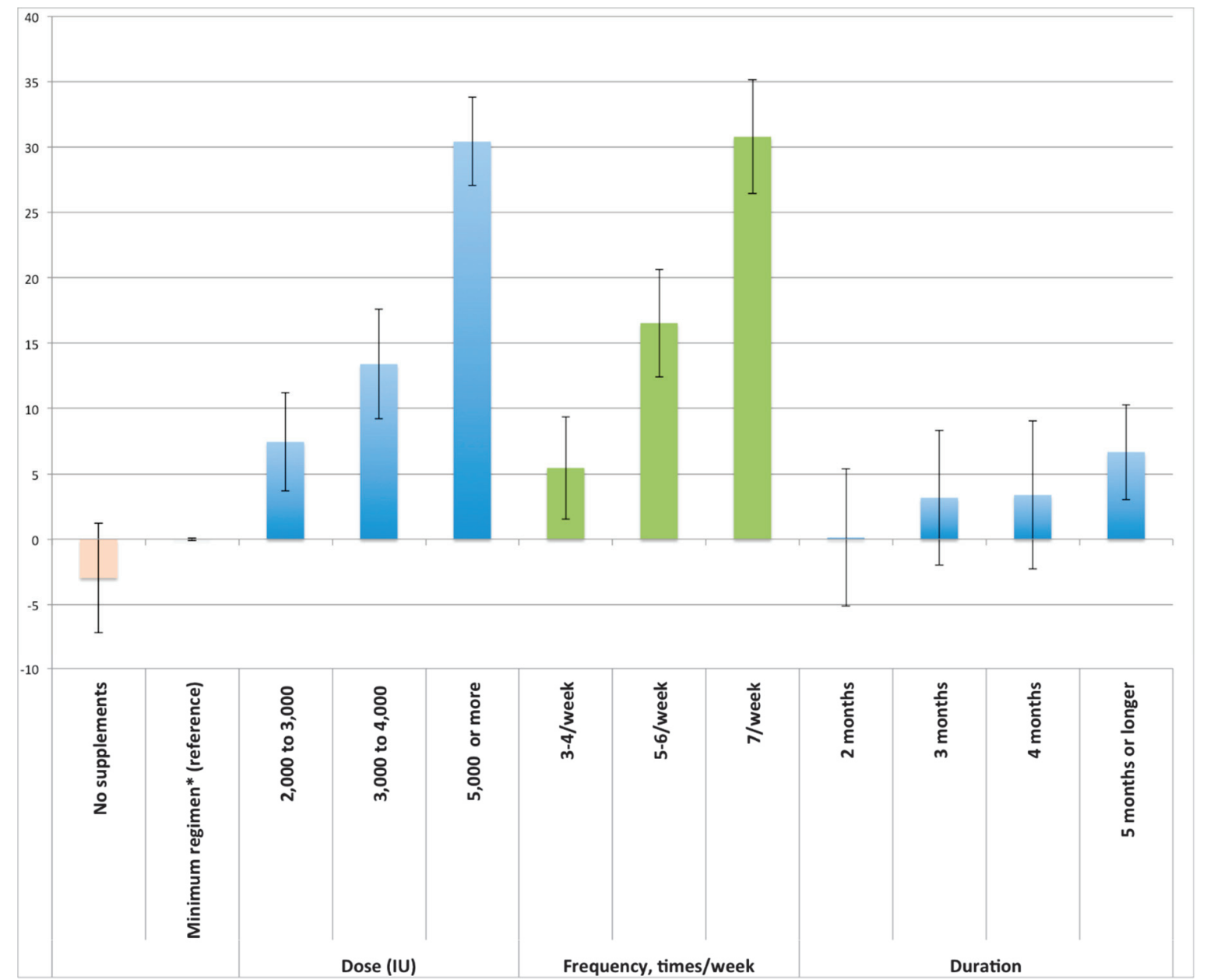

Note: All estimated changes in plasma 25-hydroxyvitamin D were compared to the minimum regimen* (1000-2000 IU once per week for one month). The error bars indicate the range of $95 \%$ confidence intervals. All estimates were adjusted for gender, age, body weight status, general health at first visits, latitudes, summer months (April to October), physical activity levels, smoking, and diet (milk and fish servings and frequency of margarine use).

\section{Discussion}

We showed that participants with a minimum regimen (1000-2000 IU of vitamin D once or twice per week for one month) had plasma $25(\mathrm{OH}) \mathrm{D}$ levels that were not statistically higher than those of participants not taking supplements. This minimum regimen (1000-2000 IU, one or two times a week) is quantitatively similar to a daily intake of 143-571 IU. Clinical trials have tested compatible doses of $400 \mathrm{IU}$ daily and showed benefits to bone density, but equivalent doses are not sufficient to prevent hip fractures or colorectal cancer [21,22]. To achieve the latter, individuals need higher plasma 25(OH)D levels, usually more than $75 \mathrm{nmol} / \mathrm{L}[23,24]$. The effectiveness of the minimum regimen on improving plasma $25(\mathrm{OH}) \mathrm{D}$ levels is consistent with biological evidence. Lips suggested that catabolism rates of vitamin $\mathrm{D}$ are lower when plasma 25(OH)D levels are low [25]. This increases the propensity of accumulation at lower dosages. 
We quantified the associations of dose, frequency and duration with plasma $25(\mathrm{OH}) \mathrm{D}$ and showed that the effects of dose and frequency were more pronounced than the effect of duration. We showed that only extending the supplementation duration to three or five months (or more) was correlated with substantial increases in plasma 25(OH)D. Whole-body distribution [26], half-life time [4], and plateau effects $[12,23]$ may be the biological mechanisms underlying the modest contribution of regimens with duration of less than five months.

The dosing regimen promoted by the IOM is $600 \mathrm{IU}$ for males and females between the ages of 1-70 years [2]. Others have advocated higher vitamin D intake [4,27]. The IOM recommendations are uniform and not distinct for individuals of varying body weight, sun exposure, health status and life style (physical activity, smoking status and diet). The present study revealed substantial contributions of these factors such that we believe they should be taken into consideration when choosing supplementation dosages to reach a target plasma 25(OH)D level.

The effects of body weight, sun exposure, and lifestyle on $25(\mathrm{OH}) \mathrm{D}[4]$ are also important to the design of clinical trials. Clinical trials testing varying vitamin D regimens may successfully control for heterogeneity between groups by random assignment, but may fail to adjust for the heterogeneity within groups [3]. To improve clinical trials of vitamin D regimens, we recommend that consideration be given not only to supplementation dose and frequency, but also to the individual characteristics. Examples include sun exposure advisories and season specific sun exposure advisories to trial participants, quantification of dietary intake, and an analytic approach that addresses body weight status.

We had observed that a minimum regimen (1000-2000 IU, one or two times a week, quantitatively similar to a daily intake of 143-571 IU) did not result in a substantial or statistically significant increase relative to not taking supplements (see Supplemental Table 1 to compare the results from previous studies). The Women's Health Initiative used a regimen of 400 IU daily (which is in the range of our minimum regimen) and did not observe a reduction in all cardiometabolic outcomes examined [28,29]. Pittas et al. and Scragg [28,29] reported that 400 IU daily may have been too low a dose to achieve these benefits, which seem consistent with our observation of an insignificant effect of the minimum regimen.

The population-based approach with the consideration of various confounding factors and a large sample size should be considered as strengths of the present study [8,30]. As this study was conducted among healthy individuals, the results are generalizable to healthy individuals who seek to improve their vitamin D status. Other studies and trials had targeted populations with very specific characteristics, such as postmenopausal women [31] or the elderly only [32], or a small number of healthy men [9]. The adjustment of a number of confounding factors, such as obesity and physical activity, also makes the findings relevant to a broader population. However, we acknowledge that there are other existing confounding factors not taken into consideration. This study evaluated participants of a health and wellness program that is mostly offered to active workers. For this reason, socioeconomic status was considered to be homogenous. No questions related to income, education, job status, race and ethnicity, or family composition was included in the program's questionnaires, which had been associated with 25(OH)D levels [33-35]. Limitations include the self-reported nature and reporting imprecision of some of the factors studied including dose, frequency and duration. 
Lastly, in the absence of better information on sun exposure, we used latitude and season as proxies for sunlight exposure, which may have introduced error and bias.

\section{Conclusions}

Each of the three dimensions of vitamin D supplementation - dose, frequency and duration - are independently associated with plasma $25(\mathrm{OH}) \mathrm{D}$ levels. The multivariable analysis reveals that plasma $25(\mathrm{OH}) \mathrm{D}$ levels associated with a minimum supplementation regimen (similar to a daily intake of 143-571 IU are not significantly higher than not taking supplements. These characteristics of the dose response relationship are not only useful for individuals to optimize plasma $25(\mathrm{OH}) \mathrm{D}$, but also important for researchers to consider that, besides the assigned doses, there are other factors that influence plasma 25(OH)D levels and these should be adjusted for.

\section{Acknowledgments}

The authors wish to thank the Pure North S'Energy Foundation for allowing their data to be analyzed for the purpose of this contribution. They also wish to thank Peter Tran and Jason Liang for data management and validation.

\section{Conflicts of Interest}

The authors did not declare any conflict of interest.

\section{References}

1. Ceglia, L.; Simpson, R.U. Vitamin D and Skeletal Muscle Function. In Vitamin D; Feldman, D., Pike, J.W., Adams, J.S., Eds.; Elsevier Science: Amsterdam, The Netherlands, 2011.

2. Institute of Medicine Committee to Review Dietary Reference Intakes for Vitamin D and Calcium Institute of Medicine. Dietary Reference Intakes for Calcium and Vitamin D; The National Academies Press: Washington, DC, USA, 2011.

3. Chung, M.; Balk, E.; Brendel, M.; Ip, S.; Lau, J.; Lee, J.; Lichtenstein, A.; Patel, K.; Raman, G.; Tatsioni, A.; et al. Vitamin D and Calcium: Systematic Review of Health Outcomes; Evidence Report/Technology Assessment No. 183. (Prepared by Tufts Evidence-Based Practice Center under Contract No. 290-2007-10055-i); Agency for Healthcare Research and Quality: Rockville, MD, USA, 2009.

4. Holick, M.F. Vitamin D: Physiology, Molecular Biology, and Clinical Applications; Humana Press: New York, NY, USA, 2010.

5. Heaney, R.P. Serum 25-hydroxyvitamin d is a reliable indicator of vitamin D status. Am. J. Clin. Nutr. 2011, 94, 619-620.

6. Lappe, J.M.; Davies, K.M.; Travers-Gustafson, D.; Heaney, R.P. Vitamin D status in a rural postmenopausal female population. J. Am. Coll. Nutr. 2006, 25, 395-402.

7. Viljakainen, H.T.; Palssa, A.; Karkkainen, M.; Jakobsen, J.; Lamberg-Allardt, C. How much vitamin D3 do the elderly need? J. Am. Coll. Nutr. 2006, 25, 429-435. 
8. Heaney, R.P. Vitamin d in health and disease. Clin. J. Am. Soc. Nephrol. 2008, 3, 1535-1541.

9. Heaney, R.P. The vitamin D requirement in health and disease. J. Steroid Biochem. Mol. Biol. 2005, 97, 13-19.

10. Vieth, R. Vitamin D supplementation, 25-hydroxyvitamin d concentrations, and safety. Am. J. Clin. Nutr. 1999, 69, 842-856.

11. Vieth, R. The Pharmacology of Vitamin D, Including Fortification Strategies. In Vitamin D; Feldman, D., Glorieux, F., Pike, J., Eds.; Elsevier: New York, NY, USA, 2005; pp. 995-1015.

12. Holick, M.F. Vitamin D. In Modern Nutrition in Health and Disease; Shils, M.E., Shike, M.E.S.M., Eds.; Lippincott Williams \& Wilkins: Philadelphia, PA, USA, 2006; Volume 2, 376-393.

13. Hathcock, J.N.; Shao, A.; Vieth, R.; Heaney, R. Risk assessment for vitamin D. Am. J. Clin. Nutr. 2007, 85, 6-18.

14. Plum, L.A.; DeLuca, H.F. Vitamin d, disease and therapeutic opportunities. Nat. Rev. Drug Discov. 2010, 9, 941-955.

15. Saliba, W.; Barnett, O.; Stein, N.; Kershenbaum, A.; Rennert, G. The longitudinal variability of serum 25(OH)D levels. Eur. J. Intern. Med. 2012, 23, e106-e111.

16. Heaney, R.P.; French, C.B.; Nguyen, S.; Baggerly, L.L.; Brunel, L.; Veugelers, P.J. A novel approach localizes the association of vitamin $\mathrm{D}$ status with insulin resistance to one region of the 25-hydroxyvitamin d continuum. Adv. Nutr. 2013, 4, 303-310.

17. Calgary Laboratry Services. Vitamin D, 25 Hydroxy. Available online: http://www. calgarylabservices.com/lab-services-guide/lab-tests/AlphabeticalListing/V/Vitamin-D-25-Hydroxy. $\mathrm{htm}$ (accessed on 9 December 2012).

18. Craig, C.L.; Marshall, A.L.; Sjostrom, M.; Bauman, A.E.; Booth, M.L.; Ainsworth, B.E.; Pratt, M.; Ekelund, U.; Yngve, A.; Sallis, J.F.; et al. International physical activity questionnaire: 12-Country reliability and validity. Med. Sci. Sports Exerc. 2003, 35, 1381-1395.

19. Mello, M.M.; Stearns, S.C.; Norton, E.C.; Ricketts, T.C., III. Understanding biased selection in Medicare HMOs. Health Serv. Res. 2003, 38, 961-992.

20. Holick, M.F. Vitamin D deficiency. N. Engl. J. Med. 2007, 357, 266-281.

21. Jackson, R.D.; LaCroix, A.Z.; Gass, M.; Wallace, R.B.; Robbins, J.; Lewis, C.E.; Bassford, T.; Beresford, S.A.A.; Black, H.R.; Blanchette, P.; et al. Calcium plus vitamin D supplementation and the risk of fractures. N. Engl. J. Med. 2006, 354, 669-683.

22. Wactawski-Wende, J.; Kotchen, J.M.; Anderson, G.L.; Assaf, A.R.; Brunner, R.L.; O’Sullivan, M.J.; Margolis, K.L.; Ockene, J.K.; Phillips, L.; Pottern, L.; et al. Calcium plus vitamin D supplementation and the risk of colorectal cancer. N. Engl. J. Med. 2006, 354, 684-696.

23. Stroud, M.L.; Stilgoe, S.; Stott, V.E.; Alhabian, O.; Salman, K. Vitamin D-A review. Aust. Fam. Phys. 2008, 37, 1002-1005.

24. Holick, M.F. Vitamin D deficiency: What a pain it is. Mayo Clin. Proc. 2003, 78, 1457-1459.

25. Lips, P. Vitamin D deficiency and secondary hyperparathyroidism in the elderly: Consequences for bone loss and fractures and therapeutic implications. Endocr. Rev. 2001, 22, 477-501. 
26. Drincic, A.T.; Armas, L.A.; van Diest, E.E.; Heaney, R.P. Volumetric dilution, rather than sequestration best explains the low vitamin D status of obesity. Obesity (Silver Spring) 2012, 20, 1444-1448.

27. Heaney, R.P.; Holick, M.F. Why the iom recommendations for vitamin D are deficient. J. Bone Miner. Res. 2011, 26, 455-457.

28. Pittas, A.G.; Chung, M.; Trikalinos, T.; Mitri, J.; Brendel, M.; Patel, K.; Lichtenstein, A.H.; Lau, J.; Balk, E.M. Systematic review: Vitamin D and cardiometabolic outcomes. Ann. Intern. Med. 2010, 152, 307-314.

29. Scragg, R. Vitamin D and Cardiovascular Disease: A Review of the Epidemiological and Clinical Evidence. In Vitamins in the Prevention of Human Diseases; Herrmann, W., Obeid, R., Eds.; Walter de Gruyter GmbH \& Company KG: Berlin, Germany, 2011.

30. Heaney, R.P. Vitamin D-Baseline status and effective dose. N. Engl. J. Med. 2012, 367, 77-78.

31. Gallagher, J.C.; Sai, A.; Templin, T., II; Smith, L. Dose response to vitamin D supplementation in postmenopausal women: A randomized trial. Ann. Intern. Med. 2012, 156, 425-437.

32. Lips, P.; Wiersinga, A.; van Ginkel, F.C.; Jongen, M.J.; Netelenbos, J.C.; Hackeng, W.H.; Delmas, P.D.; van der Vijgh, W.J. The effect of vitamin D supplementation on vitamin d status and parathyroid function in elderly subjects. J. Clin. Endocrinol. Metab. 1988, 67, 644-650.

33. Ginde, A.A.; Sullivan, A.F.; Mansbach, J.M.; Camargo, C.A., Jr. Vitamin D insufficiency in pregnant and nonpregnant women of childbearing age in the united states. Am. J. Obstet. Gynecol. 2010, 202, 431-438.

34. Ginde, A.A.; Liu, M.C.; Camargo, C.A., Jr. Demographic differences and trends of vitamin D insufficiency in the US population, 1988-2004. Arch. Intern. Med. 2009, 169, 626-632.

35. Langlois, K.; Greene-Finestone, L.; Little, J.; Hidiroglou, N.; Whiting, S. Vitamin D status of canadians as measured in the 2007 to 2009 Canadian Health Measures Survey. Health Reports 2010, 21, 47-55. 


\title{
Vitamin D: Deficiency, Sufficiency and Toxicity
}

\section{Fahad Alshahrani and Naji Aljohani}

\begin{abstract}
The plethora of vitamin D studies over the recent years highlight the pleomorphic effects of vitamin D outside its conventional role in calcium and bone homeostasis. Vitamin D deficiency, though common and known, still faces several challenges among the medical community in terms of proper diagnosis and correction. In this review, the different levels of vitamin D and its clinical implications are highlighted. Recommendations and consensuses for the appropriate dose and duration for each vitamin D status are also emphasized.
\end{abstract}

Reprinted from Nutrients. Cite as: Alshahrani, F.; Aljohani, N. Vitamin D: Deficiency, Sufficiency and Toxicity. Nutrients 2013, 5, 3605-3616.

\section{Introduction}

Vitamin D plays an essential role in the regulation of metabolism, calcium and phosphorus absorption of bone health. However, the effects of vitamin D are not limited to mineral homeostasis and skeletal health maintenance. The presence of vitamin D receptors (VDR) in other tissue and organs suggest that vitamin D physiology extends well above and beyond bone homeostasis [1]. Additionally, the enzyme responsible for the conversion of $25[\mathrm{OH}] \mathrm{D}$ to its biologically active form [Vitamin D $\left.\left(1,25[\mathrm{OH}]_{2} \mathrm{D}\right)\right]$ has been identified in other tissues aside from kidneys [2,3], and that extra renal synthesis of $1,23[\mathrm{OH}]_{2} \mathrm{D}$ may be equally important in regulating cell growth and differentiation via paracrine or autocrine regulatory mechanisms [4].

The mechanism of action of vitamin $\mathrm{D}_{3}$ through its hormonal form, dihydroxyvitamin $\mathrm{D}_{3}$, involves a nuclear VDR that regulates the transcription of several target genes in a variety of vitamin D target cells that are primarily involved in the calcium homeostasis of cell differentiation [5]. Hypervitaminosis D occurs when pharmacologic doses of vitamin D are consumed for prolonged periods of time or from a single megadose translating to a large increase in circulating $25[\mathrm{OH}] \mathrm{D}$ concentrations [6].

\section{Vitamin D Metabolism}

Vitamin $\mathrm{D}$ has two distinct forms: vitamins $\mathrm{D}_{2}$ and $\mathrm{D}_{3}$. Vitamin $\mathrm{D}_{2}$ is a 28-carbon molecule derived from ergosterol (a component of fungal cell membranes), while vitamin $\mathrm{D}_{3}$ is a 27-carbon derived from cholesterol [7]. UV-B irradiation of skin triggers photolysis of 7-dehydrocholesterol (pro-vitamin $\mathrm{D}_{3}$ ) to pre-vitamin $\mathrm{D}_{3}$, which is rapidly converted to vitamin $\mathrm{D}_{3}$ by the skin's temperature. Vitamin $\mathrm{D}\left(\mathrm{D}_{2}\right.$ and $\left.\mathrm{D}_{3}\right)$ from the skin and diet undergo two sequential hydroxylations: first in the liver $(25[\mathrm{OH}] \mathrm{D})$ and then in the kidney, leading to its biologically active form 1,25-dihydroxyvitamin $\mathrm{D}\left(1,25[\mathrm{OH}]_{2} \mathrm{D}\right)$ [8]. Table 1 shows the nomenclature for vitamin $\mathrm{D}$ precursors and metabolites. 
Table 1. Nomenclature of vitamin D precursors and metabolites.

\begin{tabular}{|c|c|c|c|}
\hline Common Name & Clinical Name & Abbreviation & Comments \\
\hline 7-Dehydrocholesterol & Pro-vitamin $\mathrm{D}_{3}$ & 7DHC & Lipid in cell membranes \\
\hline Cholecalciferol & Pre-vitamin $\mathrm{D}_{3}$ & & Photosynthesized in skin or diet \\
\hline Ergocalciferol & Pre-vitamin $\mathrm{D}_{2}$ & & $\begin{array}{l}\text { Obtained from diet. Equivalent to } \\
\text { vitamin } \mathrm{D}_{3} \text { as precursor for active } \\
\text { vitamin } \mathrm{D}\end{array}$ \\
\hline Calcidiol & 25-Hydroxyvitamin D & $25[\mathrm{OH}] \mathrm{D}$ & Best reflects vitamin D status \\
\hline Calcitriol & 1,25-Dihydroxvitamin D & $1,25[\mathrm{OH}] \mathrm{D}_{2}$ & $\begin{array}{c}\text { Active form of vitamin D, tightly } \\
\text { regulated }\end{array}$ \\
\hline
\end{tabular}

The $1,25[\mathrm{OH}]_{2} \mathrm{D}$ ligand binds with high affinity to vitamin $\mathrm{D}$ receptors (VDRs), which then increases intestinal absorption of both calcium and phosphorus. In addition, vitamin D is actively involved in bone formation, resorption, mineralization, and in maintenance of neuromuscular function. Circulating $1,25[\mathrm{OH}]_{2} \mathrm{D}$ inhibits serum parathyroid hormone (PTH) levels by negative feedback mechanism and by increased serum calcium levels. It also regulates bone metabolism through activation of the VDRs found in osteoblasts, releasing biochemical signals and leading to the formation of mature osteoclasts [9].

In a low vitamin $\mathrm{D}$ state, the small intestine can absorb approximately $10 \%-15 \%$ of dietary calcium. When adequate however, intestinal absorption of dietary calcium rises to approximately $30 \%-40 \%[9,10]$. Hence, low vitamin D levels $(25[\mathrm{OH}] \mathrm{D})$ may lead to insufficient calcium absorption, and this has clinical implications not only for bone health but also for most metabolic functions. The increase in PTH restores calcium homeostasis by increasing tubular reabsorption of calcium in the kidney, increasing bone calcium mobilization and enhancing $1,25[\mathrm{OH}]_{2} \mathrm{D}$ production [10].

\section{Optimum 25[OH]D Levels}

The vitamin D level needed to optimize intestinal calcium absorption $(34 \mathrm{ng} / \mathrm{mL})$ is lower than the level needed for neuromuscular performance $(38 \mathrm{ng} / \mathrm{mL})[11,12]$. Experts however believe that the lower limit of adequate 25[OH]D levels should be $30 \mathrm{ng} / \mathrm{mL}$ [13]. Still others recommend a lower limit of $40 \mathrm{ng} / \mathrm{mL}$, since impaired calcium metabolism due to low serum 25[OH]D levels may trigger secondary hyperparathyroidism, increased bone turnover and progressive bone loss [14,15].

The proposed $25[\mathrm{OH}] \mathrm{D}$ cut-off for optimum skeletal health is the level that reduces PTH to a minimum and increases calcium absorption to its maximum $[11,16]$. Several studies have shown that PTH levels plateau at a minimum steady-state level as serum 25[OH]D levels approach and rise above approximately $30 \mathrm{ng} / \mathrm{mL}(75 \mathrm{nmol} / \mathrm{L})$ [16-18]. The established consensus of several vitamin D cut-offs is presented in Table 2 [18-20]. It is noteworthy, however, that there is a continued debate and exchange of knowledge with respect to the optimum cut-off for 25(OH)D. 
Table 2. Diagnostic Cut-Offs of levels of serum 25[OH]D.

\begin{tabular}{ccc}
\hline $\mathbf{2 5}[\mathbf{O H}]$ Level $(\mathbf{n g} / \mathbf{m L})$ & $\mathbf{2 5}[\mathbf{O H}] \mathbf{D}$ Level $(\mathbf{n m o L} / \mathbf{L})$ & Laboratory Diagnosis \\
\hline$<20$ & $<50$ & Deficiency \\
$20-32$ & $50-80$ & Insufficiency \\
$54-90$ & $135-225$ & Normal in sunny countries \\
$>100$ & $>250$ & Excess \\
$>150$ & $>325$ & Intoxication \\
\hline
\end{tabular}

\section{Measurements of $25[\mathrm{OH}] \mathrm{D}$ versus $1,25[\mathrm{OH}]_{2} \mathrm{D}_{3}$}

The clinical advantages of choosing 25[OH]D instead of calcitriol as a marker for vitamin D status has been listed by Rajasree et al. [21]. First, 25[OH]D has the highest concentration of all vitamin D metabolites. Second, its levels remain stable for almost two weeks. Lastly, vitamin D toxicity is thought to be a function of 25[OH]D instead of calcitriol. It has been observed that serum 25[OH]D is the best indicator of vitamin D status among individuals without kidney disease [22]. Furthermore, 25[OH]D in large amounts can replace calcitriol to stimulate bone calcium metabolism [23]. Although nephrectomy abolishes a response to physiological dose of $25[\mathrm{OH}] \mathrm{D}$, a large dose $(1000$ fold) of $25[\mathrm{OH}] \mathrm{D}$ can stimulate intestinal calcium absorption and bone calcium metabolism in nephrectomized rats [24]. Hughes et al., studied vitamin D intoxication in two human patients with normal kidney function and showed that both patients had 16-fold above normal concentrations of plasma 25[OH]D levels $(500-600 \mathrm{ng} / \mathrm{mL})$, while $1,25[\mathrm{OH}] \mathrm{D}_{2} \mathrm{D}_{3}$ plasma concentrations were only modestly elevated $(40-56 \mathrm{pg} / \mathrm{mL})$ [25]. Differences in calcidiol versus calcitriol are presented in Table 3.

Table 3. Calcidiol versus Calcitriol.

\begin{tabular}{|c|c|c|}
\hline Metabolite function & $25[\mathrm{OH}] \mathrm{D}$ & $1,25[\mathrm{OH}]_{2} \mathrm{D}_{3}$ \\
\hline Nutritional Status & Best indicator & $\begin{array}{l}\text { Does not indicate nutritional } \\
\text { status }\end{array}$ \\
\hline Half life & $>15$ days & $<15 \mathrm{~h}$ \\
\hline Stability in serum & Stable & Unstable \\
\hline Hypovitaminosis D & Indicative (low) & $\begin{array}{c}\text { Non-indicative } \\
\text { (normal to elevated) }\end{array}$ \\
\hline Hypervitaminosis D & Indicative (elevated) & $\begin{array}{l}\text { Non-indicative (low to normal or } \\
\text { mild elevated) }\end{array}$ \\
\hline Calcium regulation & $\begin{array}{c}\text { Possible under } \\
\text { non-physiological conditions }\end{array}$ & $\begin{array}{c}\text { Tight under } \\
\text { physiological conditions }\end{array}$ \\
\hline PTH regulation & Depends on vitamin D status & Tight \\
\hline DBP binding & $\begin{array}{l}\text { High affinity (releases the free } \\
\text { metabolite once DBP is saturated }\end{array}$ & $\begin{array}{l}\text { Low affinity to exert the } \\
\text { physiological function }\end{array}$ \\
\hline VDR binding & $\begin{array}{l}\text { Strongest among metabolite other } \\
\text { than calcitriol }\end{array}$ & $\begin{array}{l}\text { High affinity to elicit the } \\
\text { biological function }\end{array}$ \\
\hline
\end{tabular}

Note: VDR: vitamin D receptor; DBP: vitamin D binding protein; PTH: parathyroid hormone. 


\section{Supplementation of Vitamin $D_{2}$ versus Vitamin $D_{3}$}

Multiple preparations of vitamin D and its metabolites are commercially available for supplement use. The two most common supplements are ergocalciferol (vitamin $\mathrm{D}_{2}$ ) and cholecalciferol (vitamin $\mathrm{D}_{3}$ ). Some studies [26,27], but not all [28], suggest that vitamin $\mathrm{D}_{3}$ increases serum 25[OH]D more efficiently than vitamin $\mathrm{D}_{2}$. A large, single dose of vitamin $\mathrm{D}_{2}$ does not last longer than a large dose of $\mathrm{D}_{3}$. In a study conducted by Armas et al., [27], subjects were given one dose of 50,000 IU of either vitamin $\mathrm{D}_{2}$ or vitamin $\mathrm{D}_{3}$. Vitamin $\mathrm{D}_{2}$ was absorbed just as well as vitamin $\mathrm{D}_{3}$, yet blood levels of $25[\mathrm{OH}] \mathrm{D}$ started dropping rapidly after 3 days among subjects given vitamin $\mathrm{D}_{2}$ whereas those on vitamin $\mathrm{D}_{3}$ sustained high levels for two weeks before dropping gradually.

A daily dose of $4000 \mathrm{IU}$ of vitamin $\mathrm{D}_{3}$ for two weeks was observed to be 1.7 times more effective in raising 25[OH]D levels than $4000 \mathrm{IU}$ of vitamin $\mathrm{D}_{2}$ [26]. On the other hand, Holick et al. found that a daily dose of $1000 \mathrm{IU}$ of vitamin $\mathrm{D}_{2}$ over 11 weeks duration increased 25[OH]D levels from 42 to $67 \mathrm{nmoL} / \mathrm{L}$ (16.9 to $26.8 \mathrm{ng} / \mathrm{mL}$ ) [28]. Consequently, vitamin $\mathrm{D}_{3}$ levels also increased from 49 to $72 \mathrm{nmoL} / \mathrm{L}$ (19.6 to $28.9 \mathrm{ng} / \mathrm{mL}$ ). It took 6 weeks for 25[OH]D levels to plateau on that regimen. In another study, Glendenning et al. compared 1000 IU of $\mathrm{D}_{2}$ versus $\mathrm{D}_{3}$ in patients who had vitamin D insufficiency with subsequent hip fractures. After three months, those who were supplemented with $\mathrm{D}_{3}$ had a $31 \%-52 \%$ greater increase in $25[\mathrm{OH}] \mathrm{D}$ levels than those supplemented with $\mathrm{D}_{2}$. However, parathyroid hormone levels did not differ between groups [29].

In children, Gordon et al, assigned 40 infants and toddlers with vitamin D deficiency to one of three regimens (2000 IU oral vitamin $\mathrm{D}_{2}$ daily, 50,000 IU vitamin $\mathrm{D}_{2}$ weekly or $2000 \mathrm{IU}$ vitamin $\mathrm{D}_{3}$ daily) for 6 weeks. At the end of the trial, 25[OH]D levels increased from 42.5 to $90 \mathrm{nmoL} / \mathrm{L}$ and there were no significant differences between treatment groups [30].

In terms of bioavailability, Biancuzzo et al., tested changes in 25[OH]D status from a daily dose of $1000 \mathrm{IU}$ of vitamin $\mathrm{D}_{2}$ or $\mathrm{D}_{3}$ from either calcium-fortified orange juice with vitamin $\mathrm{D}$ or supplement capsules for 11 weeks. The average 25[OH]D levels of all groups $\left(\mathrm{D}_{2}\right.$ from orange juice, $\mathrm{D}_{2}$ from capsules, $\mathrm{D}_{3}$ from orange juice, $\mathrm{D}_{3}$ from capsules) went up to about $25 \mathrm{nmoL} / \mathrm{L}$ with no significant differences between groups [31].

Treatment for most studies found $\mathrm{D}_{2}$ to be less effective than $\mathrm{D}_{3}$, whereas in studies finding them equally effective, the treatment was daily amounts between 400 and 2000 IU [32]. Houghton and Vieth indicated that vitamin $\mathrm{D}_{3}$ is the most potent form of vitamin $\mathrm{D}$ in all primate species, including humans, owing to the diminished binding of vitamin $\mathrm{D}_{2}$ metabolites to DBP in plasma [33]. They also confirmed the finding of Hollick [34], which indicated that the difference in binding capacity is potentially explained by the presence of a methyl group at carbon-24 position on the $\mathrm{D}_{2}$ molecule. The different hydroxylation sites of two forms of vitamin D leads to the production of unique biologically active metabolites. Based on this, the 24-hydroxylation after the 25-hydroxylation results in the formation of $1,24,25[\mathrm{OH}]_{3} \mathrm{D}_{2}$ and the deactivation of vitamin $\mathrm{D}_{2}$ molecule. On the other hand, the vitamin $\mathrm{D}_{3}$ metabolite $1,24,25[\mathrm{OH}]_{3} \mathrm{D}_{3}$ must undergo an additional side chain oxidation to be biologically deactivated [35]. Interestingly, $1,24,25[\mathrm{OH}]_{3} \mathrm{D}_{3}$ has the ability to bind VDR with $\sim 40 \%$ capacity higher than with $1,25[\mathrm{OH}]_{2} \mathrm{D}_{3}[36]$. 


\section{Candidates for Calcidiol (25-OHD) Measurements}

The best indicator of vitamin D status is 25-OHD because it reflects cutaneous and dietary intake, not to mention it is the major circulating form of vitamin D [37]. While there are many established causes of vitamin D deficiency, as listed in Table 4, screening for the general population warrants further investigation. The United States Preventive Services Task Force (USPSTF) did not comment for or against routine screening for vitamin D deficiency. One approach is to consider serum testing in patients at high risk for vitamin D deficiency, and treating without testing those at a lower risk [38]. Just recently, a statement from Osteoporosis Canada suggested that based on clinical suspicion for vitamin D insufficiency and its complications the clinical approach can take into account three settings (Table 5).

Table 4. Major causes of vitamin D deficiency [13].

\begin{tabular}{cc}
\hline Causes & Example \\
\hline Reduced skin synthesis & $\begin{array}{c}\text { Sunscreen, skin pigment, season/time of day, aging } \\
\text { Cystic fibrosis, celiac disease, Crohn's disease, } \\
\text { gecreased absorption }\end{array}$ \\
Increased sequestration & cholesterol absorption \\
Increased catabolism & Obesity (BMI > 30) \\
Breastfeeding & Anti-convulsant, glucocorticoid \\
Decreased synthesis of 25-hydroxyvitamin D & Exclusively without vitamin D supplementation \\
Increased urinary loss of 25-hydroxyvitmain D & Hepatic failure \\
Decreased synthesis of 1,25-dihydroxyvitmain D & Nephrotic proteinuria \\
Inherited disorders & Chronic renal failure \\
\hline
\end{tabular}

Table 5. Approach to vitamin D correction [39].

\begin{tabular}{|c|c|c|}
\hline Risk Category & Action & Level of Evidence \\
\hline Low: & & \\
\hline Adult $<50$ years & 400-1000 IU & Level 3 \\
\hline $\begin{array}{l}\text { Without comorbid conditions } \\
\text { affecting vitamin D absorption } \\
\text { or action }\end{array}$ & $\begin{array}{c}\text { No calcidiol } \\
\text { measurement required }\end{array}$ & Evidence grade D \\
\hline $\begin{array}{c}\text { Moderate: } \\
\text { Adult }>50 \text { years } \\
\text { With or without osteoporosis } \\
\text { but without comorbid conditions } \\
\text { that affect vitamin D absorption } \\
\text { or action }\end{array}$ & $\begin{array}{l}\qquad 800-2000 \mathrm{IU} \\
\text { Calcidiol measurement in initial } \\
\text { assessment but if therapy for } \\
\text { osteoporosis is prescribed, } \\
\text { calcidiol should be measured } \\
\text { after three to four months, of an } \\
\text { adequate dose. }\end{array}$ & $\begin{array}{c}\text { Level } 2 \\
\text { Evidence grade B } \\
\text { Level } 3 \\
\text { Evidence grade D }\end{array}$ \\
\hline $\begin{array}{c}\text { High: } \\
\text { Co-morbid conditions that affect } \\
\text { vitamin D absorption or action } \\
\text { and/or recurrent fractures } \\
\text { or bone loss despite } \\
\text { osteoporosis treatment }\end{array}$ & $\begin{array}{l}\text { Calcidiol should be measured } \\
\text { and supplementation based on } \\
\text { the measured value. }\end{array}$ & $\begin{array}{c}\text { Grade B } \\
\text { Recommendation }\end{array}$ \\
\hline
\end{tabular}




\section{Vitamin D Correction}

In patients with normal absorptive capacity, for every $40 \mathrm{IU} /$ day $(1 \mu \mathrm{g} /$ day $)$ of vitamin $\mathrm{D}_{3}$, serum $25(\mathrm{OH}) \mathrm{D}$ concentrations increase by approximately 0.3 to $0.4 \mathrm{ng} / \mathrm{mL}(0.7$ to $1.0 \mathrm{nmol} / \mathrm{L})$ [40]. Largest increments are seen in patients with the lowest starting $25(\mathrm{OH}) \mathrm{D}$ level, but subsequently declines as 25(OH)D concentration reaches $40 \mathrm{ng} / \mathrm{mL}(100 \mathrm{nmol} / \mathrm{L})$ [41]. Nutritional deficiency $(25 \mathrm{OHD}<50 \mathrm{nmol} / \mathrm{L})$ requires initial treatment with 50,000 units of vitamin $\mathrm{D}_{2}$ or vitamin $\mathrm{D}_{3}$ orally once per week for 6-8 weeks, and then 800 to $1000 \mathrm{IU}$ of vitamin $\mathrm{D}_{3}$ orally thereafter [42]. Intramuscular cholecalciferol (300,000 IU) in one or two doses per year is also an option for increasing serum 25 OHD level [43].

Nutritional insufficiency (25 OHD 50-75 nmol/L) requires treatment with 800 to $1000 \mathrm{IU}$ of vitamin $\mathrm{D}_{3}$ daily. This intake will bring the average adult's vitamin $\mathrm{D}$ status to $7 \mathrm{nmol} / \mathrm{L}$ higher over a three-month period. Still, many individuals might need higher doses. In malabsorptive states, oral dosing and duration of treatment is dependent on the individual patient's on vitamin $\mathrm{D}$ absorptive capacity. High doses of vitamin D (10,000 to 50,000 IU daily) may be necessary for patients who had gastrectomy or malabsorption history. Patients who remain deficient or insufficient on such doses need to be treated with hydroxylated vitamin D metabolites, since they are more readily absorbed than with ordinary sun or sun camp exposure. All patients should maintain a daily calcium intake of at least $1000 \mathrm{mg}$ (for ages 31 to 50 years) to $1200 \mathrm{mg}$ (for ages 51 and older) per day [44].

\section{Vitamin D Toxicity}

Vitamin D as a fat-soluble vitamin raised concerns about toxicity from excessive supplementation. Widespread vitamin D fortification of foods and drinks from the 1930s to 1950s in the United States and Europe led to reported cases of toxicity [45]. Hypercalcemia is responsible for producing most of the symptoms of vitamin D toxicity. Early symptoms of vitamin D toxicity include gastrointestinal disorders like anorexia, diarrhea, constipation, nausea, and vomiting. Bone pain, drowsiness, continuous headaches, irregular heartbeat, loss of appetite, muscle and joint pain are other symptoms that are likely to appear within a few days or weeks; frequent urination, especially at night, excessive thirst, weakness, nervousness and itching; kidney stones [46].

There are three major hypotheses for vitamin D toxicity [47]:

(i) Raised plasma $1,25[\mathrm{OH}] \mathrm{D}$ concentrations lead to increased intracellular $1,24[\mathrm{OH}] \mathrm{D}$ concentrations. This hypothesis is not widely supported as many studies revealed that vitamin D toxicity is associated with normal or marginally elevated 1,25[OH]D [23]. It was only Mawer et al. who reported elevated 1,25[OH]D with vitamin D toxicity [48].

(ii) Vitamin D intake raises plasma $25[\mathrm{OH}] \mathrm{D}$ levels to concentrations that exceed DBP binding capacity, and free 25[OH]D has direct effects on gene expression once it enters target cells. High dietary vitamin D intake alone increases plasma 25[OH]D. The low affinity of $1,25[\mathrm{OH}] \mathrm{D}$ for the transport protein DBP and its high affinity for VDR dominate normal physiology. This makes it the only ligand with access to the transcriptional signal transduction machinery. However, in vitamin $\mathrm{D}$ intoxication, overloading by various vitamin D metabolites significantly compromises the capacity of the DBP by allowing other 
metabolites to enter the cell nucleus. Of all the inactive metabolites, $25[\mathrm{OH}] \mathrm{D}$ has the strongest affinity for the VDR, and thus at sufficiently high concentrations, could stimulate transcription [47].

(iii) Vitamin D intake raises the concentrations of many vitamin D metabolites, including vitamin D itself and 25[OH]D, and these concentrations exceed the DBP binding capacity and release of "free" $1,25[\mathrm{OH}] \mathrm{D}$ which enters target cells [47].

The amount of UVB radiation required for vitamin D sufficiency can be calculated from the amount of vitamin D produced from one minimal erythemal dose (MED), or 10,000-25,000 IU of oral vitamin D [9].The MED can be defined as the amount of time needed to cause skin to turn pink. The length of time varies with geographical location, skin pigmentation, percent of body fat, and age. Excessive exposure to sunlight will not cause vitamin D intoxication because sunlight degrades any excess vitamin D [48].

The highest recorded individual serum 25[OH]D concentration obtained from sunshine was from a farmer in Puerto Rico with a level of $225 \mathrm{nmol} / \mathrm{L}$ [49]. On the other hand, the highest recorded individual 25[OH]D achieved from artificial ultraviolet light treatment sessions was $275 \mathrm{nmol} / \mathrm{L}$ [50]. Vieth reported that vitamin D toxicity probably begins to occur after chronic consumption of approximately 40,000 IU/day (100 of the 400 IU capsules) [6]. Reports in which pharmacologic doses of vitamin $\mathrm{D}$ were given for a prolonged time, the indications why it was given and in which the final serum 25[OH]D concentrations are provided and summarized in Table 6.

\section{Hypersensitivity to Vitamin D}

Vitamin D hypersensitivity syndromes are often mistaken for vitamin D toxicity. The most common is primary hyperparathyroidism. Granulomatous diseases, such as sarcoidosis, granulomatous TB and some cancers also cause vitamin D hypersensitivity, as the granuloma or the tumor may make excessive amounts of calcitriol, thus raising serum calcium levels [6].

Table 6. Studies reporting elevated vitamin D status and associated diseases.

\begin{tabular}{|c|c|c|c|}
\hline $\begin{array}{c}\text { Reference, year, and daily } \\
\text { dosage }(\mu \mathrm{g})\end{array}$ & Duration & $\begin{array}{c}\text { Final } 25[\mathrm{OH}] \mathrm{D} \\
\text { concentration }(\mathrm{nmoL} / \mathrm{L})\end{array}$ & Indication \\
\hline Mason et al., [51], 19801250 & $>52$ weeks & 717 & Hypoparathyroidism \\
\hline $\begin{array}{l}\text { Haddock et al., [49], } \\
\qquad 19821875\end{array}$ & $>100$ weeks & 1707.5 & Hypoparathyroidism \\
\hline $\begin{array}{l}\text { Gertner and Domenech [52], } \\
1977 \text { 500-2000 }\end{array}$ & $12-52$ weeks & $442-1022$ & Various \\
\hline $\begin{array}{c}\text { Counts et al., [53], } \\
19752500\end{array}$ & 12 weeks & 1550 & Anephric \\
\hline $\begin{array}{c}\text { Hughes et al., [25], } 1976 \\
2500-6250 n=3\end{array}$ & $>52$ weeks & $1000-1600$ & Not stated \\
\hline $\begin{array}{l}\text { Streck et al., [54], } \\
19792500\end{array}$ & 3.8 years & 707.5 & Hypoparathyroidism \\
\hline
\end{tabular}


Table 6. Cont.

\begin{tabular}{|c|c|c|c|}
\hline $\begin{array}{c}\text { Reference, year, and daily } \\
\text { dosage }(\mu \mathrm{g})\end{array}$ & Duration & $\begin{array}{c}\text { Final } 25[\mathrm{OH}] \mathrm{D} \\
\text { concentration }(\mathrm{nmoL} / \mathrm{L})\end{array}$ & Indication \\
\hline \multicolumn{4}{|l|}{ Davies and Adams [55], 1978} \\
\hline 3750 & 364 weeks & 1125 & Paget disease \\
\hline 2500 & 520 weeks & 1000 & Thyroidectomy \\
\hline Mawer et al., [48], 1985 & & & Hypoparathyroidism \\
\hline 1875 & 520 weeks & 568 & Hypophosphatemic \\
\hline 5000 & 520 weeks & 1720 & rickets \\
\hline 2500 & 520 weeks & 995 & Carpal tunnel \\
\hline 1250 & 1248 weeks & 632 & syndrome \\
\hline 4285 & 26 weeks & 908 & Celiac disease \\
\hline 2500 & 520 weeks & 856 & Chilblain \\
\hline 2500 & 312 weeks & 778 & Thyroidectomy \\
\hline \multirow[t]{2}{*}{1250} & 1040 weeks & 903 & Arthritis \\
\hline & & & Hypoparathyroidism \\
\hline \multicolumn{4}{|l|}{ Allen and Skah [56], 1992} \\
\hline 1875 & 19 years & 267 & Hypoparathyroidism \\
\hline \multicolumn{4}{|l|}{ Rizzoli et al., [57], 1994} \\
\hline 15,000 & 96 weeks & 221 & \\
\hline 7500 & 3 weeks & 801 & Osteoporosis \\
\hline 7500 & 74 weeks & 1692 & Osteoporosis \\
\hline 1075 & 12 weeks & 374 & Hypoparathyroidism \\
\hline 7500 & 4 weeks & 650 & Osteoporosis \\
\hline 7500 & 4 weeks & 621 & Osteoporosis \\
\hline 250 & 390 weeks & 608 & Osteomalacia \\
\hline \multicolumn{3}{|l|}{ Pettifor et al., [58] 1995} & \multirow{2}{*}{ Not stated } \\
\hline $50,000(n=11)$ & 10 days & $847-1652$ & \\
\hline \multicolumn{3}{|l|}{ Jacobus et al., [59] 1992} & \multirow{2}{*}{ Not stated } \\
\hline $725-4364(n=8)$ & 6 years & "mean" 731 & \\
\hline
\end{tabular}

\section{Conclusions}

The present review discussed current knowledge on vitamin D physiology, its clinical relevance and evidence-based treatment options on vitamin D status correction. Caution should still be practiced by clinicians in providing vitamin D supplementation among vitamin D deficient populations, with proper monitoring using approved and certified methods. Indications for vitamin D supplementation outside the conventional calcium homeostasis should also be considered to maximize extra-skeletal benefits of vitamin D correction.

\section{Conflicts of Interest}

The authors declare no conflict of interest. 


\section{References}

1. DeLuca, H. Overview of General physiological tenures and function of vitamin D. Am. J. Clin. Nutr. 2004, 80, 16895-16965.

2. Mawer, E.B.; Hayes, M.E.; Heys, S.E.; Davies, M.; White, A.; Stewart, M.F.; Smith, G.N. Constitutive synthesis of 1,25 dihydroxy vitamin $\mathrm{D}_{3}$ by a human small cell lung cancer cell line. J. Clin. Endocrinol. Metab. 1994, 79, 554-560.

3. Schwartz, G.G.; Whitlutch, L.W.; Chen, T.C.; Lokeshwar, B.L.; Holick, M.F. Human prostate cells synthesize 1,25 dihydroxyvitamin D3. Cancer Epidemiol. Biomark. Prev. 1998, 7, 391-395.

4. Holick, M.F. Sunlight, vitamin D and health: A D-lightful story. Nor. Acad. Sci. Lett. 2008, 2008, 147-166.

5. Jones, G.; Strugnell, S.; DeLuca, H.F. Current understanding of the molecular action of vitamin D. Physiol. Rev. 1998, 78, 1193-1231.

6. Vieth, R. Vitamin D supplementation, 25 hydroxy-vitamin D concentrations, and safety. Am. J. Clin. Nutr. 1999, 69, 842-856.

7. Ahmed, M.S.; Shoker, A. Vitamin D metabolites; protective versus toxic properties: Molecular and cellular perspectives. Nephrol. Rev. 2010, 2, 19-26.

8. Holick, M.F. High prevalence of vitamin D inadequacy and implications for health. Mayo Clin. Proc. 2006, 81, 353-373.

9. Holick, M.F. Sunlight and vitamin D for bone health and prevention of autoimmune diseases, cancers and cardiovascular disease. Am. J. Clin. Nutr. 2004, 80, 1678S-1688S.

10. Holick, M.F. Vitamin D: the underappreciated D-lightful hormone that is important for skeletal and cellular health. Curr. Opin. Endocrinol. Diabetes 2002, 9, 87-98.

11. Heaney, R.P.; Dowell, M.S.; Hale, C.A.; Bendich, A. Calcium absorption varies within the reference range for serum 25-hydroxyvitamin D. J. Am. Coll. Nutr. 2003, 22, 142-146.

12. Bischoff-Ferrari, H.A.; Dietrich, T.; Orav, E.J.; Hu, F.B.; Zhang, Y.; Karlson, E.W.; Dawson-Hughes, E.B. Higher 25 hydroxy-vitamin D concentration are associated with better lower-extremity function in both active and inactive persons aged $>60 \mathrm{yrs}$. Am. J. Clin. Nutr. 2004, 80, 752-758.

13. Holick, M.F. Vitamin D deficiency. N. Engl. J. Med. 2007, 357, 266-281.

14. Cannell, J.J.; Hollis, B.W.; Zasloff, M.; Heaney, R.P. Diagnosis and treatment of vitamin D deficiency. Expert Opin. Pharmacother. 2008, 9, 107-118.

15. Lips, P. Vitamin D deficiency of secondary hyperparathyroidism in the elderly: Consequences for bone loss and fractures and therapeutic implications. Endocr. Rev. 2001, 22, 477-501.

16. Chapuy, M.C.; Preziosi, P.; Maamer, M.; Arnaud, S.; Galan, P.; Hercberg, S.; Meunier, P.J.; Prevalence of vitamin D insufficiency in an adult normal population. Osteopros. Int. 1997, 7, 439-443.

17. Holick, M.F.; Siris, E.S.; Binkley, N.; Beard, M.K.; Khan, A.; Katzer, J.T.; Petruschke, R.A.; Chen, E.; de Papp, A.E. Prevalence of vitamin D inadequacy among postmenopausal North American women receiving osteoporosis therapy. J. Clin. Endocrinol. Metab. 2005, 90, 3215-3224. 
18. Heaney, P.R. Functional indices of vitamin D status and ramifications of vitamin D deficiency. Am. J. Clin. Nutr. 2004, 80, 1706S-1709S.

19. Hanley, D.A.; Davison, K.S. Vitamin D insufficiency in North America. J. Nutr. 2005, 135, 332-337.

20. Hollis, B.W.; Wagner, C.L. Assessment of dietary vitamin D requirements during pregnancy and lactation. Am. J. Clin. Nutr. 2004, 79, 717-726.

21. Rajasree, S.; Rajpal, K.; Kartha, C.C.; Sarma, P.S.; Kutty, V.R.; Iyer, C.S.; Girija, G. Serum 25-dihyroxyvitamin $\mathrm{D}_{3}$ levels are elevated in South Indian patients with ischemic heart disease. Eur. J. Epidemiol. 2001, 17, 567-571.

22. Wang, T.J.; Pencina, M.J.; Booth, S.L.; Jacques, P.F.; Ingelsson, E.; Lanier, K.; Benjamin, E.J.; DÁgostino, R.B.; Wolf, M.; Vasan, R.S. Vitamin D deficiency and risk of cardiovascular disease. Circulation 2008, 117, 503-511.

23. Shepard, M.R.; Deluca, H.F. Plasma concentrations of vitamin $D_{3}$ and its metabolites in the rat as infused by vitamin D3 intake. Arch. Biochem. Biophys. 1980, 202, 43-53.

24. Paulovitch, H.; Gurabedian, M.; Bulsan, S. Calcium mobilizing effect of large doses of 25-dyroxycalciferol ion anephric rats. J. Clin. Investig. 1973, 52, 2656-2659.

25. Hughes, M.R.; Baylink, D.J.; Jones, P.J.; Haussler, M.R. Radioligand receptor assay for 25-hydroxvitamin $\mathrm{D}_{2} / \mathrm{D}_{3}$ and 1 alpha,25-hydroxyvitamin. J. Clin. Investig. 1976, 58, 61-70.

26. Trang, H.M.; Cole, D.E.; Rubin, L.A.; Pierratos, A.; Siu, S.; Vieth, R. Evidence that vitamin D3 increases serum 25-hydroxyvitamin $\mathrm{D}$ more efficiently than does vitamin $\mathrm{D}_{2}$. Am. J. Clin. Nutr. 1998, 68, 854-858.

27. Armas, L.A.; Hollis, B.W.; Heaney, R.P. Vitamin $\mathrm{D}_{2}$ is much less effective than vitamin $\mathrm{D}_{3}$ in humans. J. Clin. Endocrinol. Metab. 2004, 89, 5387-5391.

28. Holick, M.F.; Biancuzzo, R.M.; Chen, T.C.; Klein, E.K.; Young, A.; Bibuld, D.; Reitz, R.; Salameh, W.; Ameri, A.; Tannenbaum, A.D. Vitamin $\mathrm{D}_{2}$ is as effective as vitamin $\mathrm{D}_{3}$ in maintaining circulating concentrations of 25-hydroxyvitamin D. J. Clin. Endocrinol. Metab. 2008, 93, 677-681.

29. Glendenning, P.; Chew, G.T.; Seymour, M.J.; Goldswain, P.R.; Inderjeeth, C.A.; Vasikaran, S.D.; Toronto, M.; Musk, A.A.; Fraser, W.D. Serum 25 hydroxyvitamin D levels in vitamin D insufficient hip fracture patients after supplementation wit ergocalciferol and cholecalciferol. Bone 2009, 45, 870-875.

30. Gordon, C.M.; Williams, A.L.; Feldman, H.A.; May, J.; Sinclair, L.; Vasquez, A.; Coz, J.E. Treatment of hypovitaminosis D in infants and toddlers. J. Clin. Endocrinol. Metab. 2008, 93, 2716-2721.

31. Biancuzzo, R.M.; Young, A.; Bibuld, D.; Cai, M.H.; Winter, M.R.; Klein, E.K.; Ameri, A.; Reitz, R.; Salameh, W.; Chen, T.C.; et al. Fortification of orange juice with vitamin $\mathrm{D}_{2}$ or vitamin $\mathrm{D}_{3}$ is as effective as an oral supplement in maintaining vitamin $\mathrm{D}$ status in an adult. Am. J. Clin. Nutr. 2010, 91, 162-166.

32. Chan, J. Vitamin D update for nutrition professionals. Veget. Nutr. 2009, 18, 1-2.

33. Houghton, A.L.; Vieth, R. The case against ergocalciferol (vitamin $\mathrm{D}_{2}$ as a vitamin supplement). Am. J. Clin. Nutr. 2006, 84, 694-697. 
34. Hollis, B.W. Comparison of equilibrium and disequilibrium assay conditions of ergocalciferol and cholicalciferol and their metabolites. J. Steroid Biochem. 1989, 21, 81-86.

35. Horst, R.L.; Reinhardt, T.A.; Ramberg, C.F.; Koszewski, N.J.; Napoli, J.L. 24-Hydroxylation of 1,25-dihydroxyergocalciferol: An unambiguous deactivation process. J. Biol. Chem. 1986, 261, 9250-9256.

36. Jones, G. Analog Metabolism in Vitamin D; Feldman, D., Glorieux, F., Pike, J.W., Eds.; Elsevier Academic Press: Melville, NY, USA, 1997; pp. 73-94.

37. Institute of Medicine Standing Committee on the Scientific Evaluation of Dietary Reference Intakes. Dietary Reference Intakes for Calcium, Phosphorus, Magnesium, Vitamin D, and Fluoride; National Academy Press: Washington, DC, USA, 1997.

38. Teresa, K.; Amy, G.; Jackie, R.; Jennie, H.; Sarina, S. Vitamin D: An evidence based review. J. Am. Board. Fam. Med. 2009, 22, 698-706.

39. Hanley, D.A.; Cranney, A.; Jones, G.; Whiting, S.J.; Leslie, W.D. Guidelines Committee of the Scientific Advisory Council of Osteoporosis of Canada. Vitamin D in adult health and disease; a review and guideline statement from Osteoporosis Canada (summary). Can. Med. Assoc. J. 2010, 182, 1315-1319.

40. Dawson-Hughes, B. Treatment of Vitamin D Deficient States, 2010. Wolters Kluwer Health Website. Available online: http://www.update.com/contents/treatment-of-vitamin-d-deficiencyin-adults (accessed on 11 September 2013).

41. Vieth, R. Critique of the consideration for establishing the tolerable upper intake level for vitamin D: Critical need for revision upwards. J. Nutr. 2006, 136, 1117-1122.

42. Dawson-Hughes, B.; Heaney, R.P.; Holick, M.F.; Lips, P.; Meunier, P.J.; Vieth, R. Estimates of optimal vitamin D status. Osteoporos. Int. 2005, 16, 713-716.

43. De Torrente de la Jara, G.; Pecoud, A.; Favrat, B. Female asylum seekers with musculoskeletal pain; the importance of diagnosis and treatment of hypovitaminosis D. BMC Fam. Pract. 2006, 7,4 .

44. Institute of Medicine (US) Standing Committee on the Scientific Evaluation of Dietary Reference Intakes. Dietary Reference Intakes for Calcium, Phosphorus, Magnesium, Vitamin D, and Fluoride; National Academies Press: Washington, DC, USA, 1997.

45. Holick, M.F. Vitamin D deficiency: What a pain it is. Mayo Clin. Proc. 2003, 78, 1457-1459.

46. Schwalfenberg, G. Not enough vitamin D: health consequences for Canadians. Can. Fam. Phys. 2007, 53, 841-854.

47. Jones, G. Pharmacokinetics of vitamin D toxicity. Am. J. Clin. Nutr. 2008, 88, 5825-5865.

48. Mawer, E.B.; Hann, J.T.; Berr, J.L.; Davies, M. Vitamin D metabolism in patients intoxicated with ergocalciferol. Clin. Sci. (Lond.) 1985, 68, 135-141.

49. Haddock, L.; Corcino, J.; Vazquez, M.D. 25 OHD serum level in the normal Puerto Rican population and in subject with tropical sprue and parathyroid disease. Puerto Rico Health Sci. $J$. 1982, 1, 85-91.

50. Krause, R.; Buhring, M.; Hopfenmuller, W.; Holick, M.F.; Sharma, A.M. Ultraviolet B and blood pressure. Lancet 1998, 352, 709-710. 
51. Mason, R.S.; Lissner, D.; Grunstein, H.S.; Posen, S. A simplified assay for dihydroxylated vitamin D metabolites in human serum: Application to hyper- and hypovitaminosis D. Clin. Chem. 1980, 26, 444-450.

52. Gertner, J.M.; Domenech, M. 25-Hydroxyvitamin D levels in patients treated with high-dosage ergo- and cholecalciferol. Clin. Pathol. 1977, 30, 144-150.

53. Counts, S.J.; Baylink, D.J.; Shen, F.H.; Sherrard, D.J.; Hickman, R.O. Vitamin D intoxication in an anephric child. Ann. Intern. Med. 1975, 82, 196-200.

54. Streck, W.F.; Waterhouse, C.; Haddad, J.G. Glucocorticoid effects in vitamin D intoxication. Arch. Intern. Med. 1979, 139, 974-977.

55. Davies, M.; Adams, P.H. The continuing risk of vitamin-D intoxication. Lancet 1978, 2, 621-623.

56. Allen, S.H.; Shah, J.H. Calcinosis and metastatic calcification due to vitamin D intoxication. A case report and review. Horm. Res. 1992, 37, 68-77.

57. Rizzoli, R.; Stoermann, C.; Ammann, P.; Bonjour, J.P. Hypercalcemia and hyperosteolysis in vitamin D intoxication: Effects of clodronate therapy. Bone 1994, 15, 193-198.

58. Pettifor, J.M.; Bikle, D.D.; Cavaleros, M.; Zachen, D.; Kamdar, M.C.; Ross, F.P. Serum levels of free 1,25-dihydroxyvitamin D in vitamin D toxicity. Ann. Intern. Med. 1995, 122, 511-513.

59. Jacobus, C.H.; Holick, M.F.; Shao, Q.; Chen, T.C.; Holm, I.A.; Kolodny, J.M.; Fuleihan, G.E.; Seely, E.W. Hypervitaminosis D associated with drinking milk. N. Engl. J. Med. 1992, $326,1173-1177$. 


\title{
Pharmacokinetics of High-Dose Weekly Oral Vitamin D3 Supplementation during the Third Trimester of Pregnancy in Dhaka, Bangladesh
}

\section{Daniel E. Roth, Abdullah Al Mahmud, Rubhana Raqib, Evana Akhtar, Robert E. Black and}

Abdullah H. Baqui

\begin{abstract}
A pharmacokinetic study was conducted to assess the biochemical dose-response and tolerability of high-dose prenatal vitamin D3 supplementation in Dhaka, Bangladesh $\left(23^{\circ} \mathrm{N}\right)$. Pregnant women at 27-30 weeks gestation $(n=28)$ were randomized to 70,000 IU once $+35,000 \mathrm{IU} /$ week vitamin D3 (group PH: pregnant, higher dose) or 14,000 IU/week vitamin D3 (PL: pregnant, lower dose) until delivery. A group of non-pregnant women $(n=16)$ was similarly administered 70,000 IU once $+35,000$ IU/week for 10 weeks (NH: non-pregnant, higher-dose). Rise $(\Delta)$ in serum 25-hydroxyvitamin D concentration $([25(\mathrm{OH}) \mathrm{D}])$ above baseline was the primary pharmacokinetic outcome. Baseline mean [25(OH)D] were similar in PH and PL (35 nmol/L vs. $31 \mathrm{nmol} / \mathrm{L}, p=0.34)$. A dose-response effect was observed: $\Delta[25(\mathrm{OH}) \mathrm{D}]$ at modeled steady-state was $19 \mathrm{nmol} / \mathrm{L}$ (95\% CI, 1 to 37$)$ higher in PH vs. PL $(p=0.044) . \Delta[25(\mathrm{OH}) \mathrm{D}]$ at modeled steady-state was lower in $\mathrm{PH}$ versus $\mathrm{NH}$ but the difference was not significant $(-15 \mathrm{nmol} / \mathrm{L}, 95 \% \mathrm{CI}-34$ to $5 ; p=0.13)$. In PH, $100 \%$ attained $[25(\mathrm{OH}) \mathrm{D}] \geq 50 \mathrm{nmol} / \mathrm{L}$ and $90 \%$ attained $[25(\mathrm{OH}) \mathrm{D}] \geq 80 \mathrm{nmol} / \mathrm{L}$; in $\mathrm{PL}, 89 \%$ attained $[25(\mathrm{OH}) \mathrm{D}] \geq 50 \mathrm{nmol} / \mathrm{L}$ but $56 \%$ attained $[25(\mathrm{OH}) \mathrm{D}] \geq 80 \mathrm{nmol} / \mathrm{L}$. Cord $[25(\mathrm{OH}) \mathrm{D}](n=23)$ was slightly higher in PH versus $\mathrm{PL}(117 \mathrm{nmol} / \mathrm{L} v s$. $98 \mathrm{nmol} / \mathrm{L} ; p=0.07)$. Vitamin D3 was well tolerated; there were no supplement-related serious adverse clinical events or hypercalcemia. In summary, a regimen of an initial dose of 70,000 IU and 35,000 IU/week vitamin D3 in the third trimester of pregnancy was non-hypercalcemic and attained [25(OH)D] $\geq 80 \mathrm{nmol} / \mathrm{L}$ in virtually all mothers and newborns. Further research is required to establish the safety of high-dose vitamin D3 in pregnancy and to determine if supplement-induced [25(OH)D] elevations lead to maternal-infant health benefits.
\end{abstract}

Reprinted from Nutrients. Cite as: Roth, D.E.; Mahmud, A.A.; Raqib, R.; Akhtar, E.; Black, R.E.; Baqui, A.H. Pharmacokinetics of High-Dose Weekly Oral Vitamin D3 Supplementation during the Third Trimester of Pregnancy in Dhaka, Bangladesh. Nutrients 2013, 5, 788-810.

\section{Introduction}

The maternal-infant health benefits of vitamin $\mathrm{D}$ supplementation during pregnancy remain uncertain $[1,2]$. However, observational studies have suggested associations between vitamin D status during pregnancy and postnatal infant health outcomes [3-5]. Serum 25-hydroxyvitamin D concentration $([25(\mathrm{OH}) \mathrm{D}]) \geq 50 \mathrm{nmol} / \mathrm{L}$ is associated with skeletal health benefits [1], but some data suggest that improving vitamin $\mathrm{D}$ status to attain serum $[25(\mathrm{OH}) \mathrm{D}] \geq 80 \mathrm{nmol} / \mathrm{L}$ may enhance a range of vitamin D-related functions [6-8]. However, there have been relatively few published studies of vitamin D3 pharmacokinetics, safety and clinical effects during pregnancy [9].

The possible association between maternal-fetal vitamin D status and infant health outcomes may be particularly relevant to South Asian countries such as Bangladesh, where adverse perinatal outcomes 
and infant mortality are public health priorities [10], and where vitamin D deficiency has been observed among women of reproductive age [11] and young infants [12]. Therefore, to guide the design of clinical trials of antenatal vitamin D supplementation in Bangladesh, we conducted a randomized open-label pilot trial of two antenatal vitamin D3 supplementation doses that were several fold higher than those in typical prenatal supplements. The primary aims were to establish the biochemical dose-response in terms of the change in serum $[25(\mathrm{OH}) \mathrm{D}]$, and to specifically assess whether the regimens achieved $[25(\mathrm{OH}) \mathrm{D}]$ $\geq 80 \mathrm{nmol} / \mathrm{L}$ in most participants. The response to the higher-dose supplement regimen was also assessed in a cohort of non-pregnant participants that served as a separate comparison group. The present study builds on previously reported observations of single-dose vitamin D3 pharmacokinetics in the same setting [13].

\section{Experimental Section}

\subsection{Participants}

Pregnant women were enrolled at a maternal health clinic in inner-city Dhaka, Bangladesh $\left(23^{\circ} \mathrm{N}\right)$ in February 2010 if they were: Aged 18 to $<35$ years; at 27 to $<31$ completed weeks of gestation based on the reported first day of the last menstrual period; held permanent residence in Dhaka at a fixed address; and, planned to stay in Dhaka for at least four months. Reasons for exclusion were: preexisting medical condition; current vitamin D supplement use; anti-convulsant or anti-mycobacterial medications; severe anemia (hemoglobin concentration $<70 \mathrm{~g} / \mathrm{L}$ ); hypertension at enrollment (systolic blood pressure $\geq 140 \mathrm{mmHg}$ or diastolic blood pressure $\geq 90 \mathrm{mmHg}$ on at least two measurements); major risk factors for preterm delivery or pregnancy complications; or previous delivery of an infant with a congenital anomaly or perinatal death. Healthy non-pregnant women attending the same clinic for health maintenance (e.g., contraception), or because they were accompanying pregnant women, were enrolled in August-September 2009 if they were non-lactating, had not missed a recent menses at the expected date, and had a negative urine pregnancy test (First Response Early Results, Church \& Dwight Company, Inc., Princeton, NJ, USA). Otherwise, inclusion and exclusion criteria were similar to the pregnant participants.

The study was approved by the Institutional Review Board at The Johns Hopkins Bloomberg School of Public Health and the International Center for Diarrheal Disease Research, Bangladesh (ICDDR, B). All participants gave signed informed consent prior to participation. The trial was registered at ClinicalTrials.gov (NCT00938600).

\subsection{Study Design and Interventions}

Pregnant participants were randomized at enrollment to receive a single dose of vitamin D3 70,000 IU (1.75 mg, where $1 \mathrm{mg}=40,000 \mathrm{IU})$ on day 0 followed by vitamin D3 35,000 IU (0.875 mg) per week starting on day 7 and continuing until delivery (referred to as group "PH"; pregnant, higher dose), or to vitamin D3 14,000 IU (0.350 mg) per week starting on day 0 and continuing until delivery ("PL"; pregnant, lower dose). Participants in the non-pregnant cohort ("NH"; non-pregnant, higher dose) received the same higher-dose intervention as $\mathrm{PH}$, i.e., a single dose of vitamin D3 70,000 IU on day 0 followed by vitamin D3 35,000 IU per week starting on day 7 and continuing until the last dose on day 63 (total of 10 doses). Vitamin D3 was administered as Vigantol Oil (Merck KGaA, Germany), 
a liquid supplement (20,000 IU D3/mL) commercially available in Bangladesh (see Ref 13 for details regarding quality assurance). Participants were advised not to take other vitamin D-containing supplements during the study period. Pregnant participants were provided with standard prenatal supplemental iron (60 mg/day) and folic acid (400 mcg/day). NH was studied before enrolment of $\mathrm{PH}$, to establish safety of the high-dose regimen in non-pregnant women prior to its use in pregnant women. As an additional safety measure, the response to a single initial dose vitamin D3 (70,000 IU) was observed in a separate cohort, prior to the initiation of enrollment of cohorts of participants who received weekly doses [13]. A preceding report of single-dose vitamin D3 pharmacokinetics included data from participants in weekly-dose groups $\mathrm{PH}$ and $\mathrm{NH}$, but only from days 0 to 7 (i.e., preceding the administration of a second vitamin D dose) [13]. Women who received only the single 70,000 IU dose are not included in any of the present analyses.

\subsection{Data Collection Procedures}

Pregnant women were assessed weekly until delivery. Non-pregnant participants had weekly followups for 10 weeks (the last visit was on day 70, one week after the final D3 dose). Weekly assessments included a checklist of symptoms and blood pressure measurement. In $\mathrm{NH}$ and $\mathrm{PH}$, participants provided up to six scheduled blood specimens and at least seven urine samples during a 10-week follow-up period beginning on the day of supplement administration (day 0), according to one of two randomly assigned sampling schedules, A or B (Figure 1). Specimens on days 65 and 67 were intended to measure inter-dose fluctuations in $[25(\mathrm{OH}) \mathrm{D}]$ and serum calcium. Urine was collected at visits without scheduled blood collection up to day 70 (Figure 1). Participants in PL were asked to provide three blood specimens and four urine specimens (schedule $\mathrm{C}$ in Figure 1). From day 70, pregnant participants provided urine specimens on a weekly basis until delivery.

Figure 1. Blood and urine specimen collection schedules. Participants in groups $\mathrm{PH}$ and $\mathrm{NH}$ were randomly assigned to either scheduled "A" or "B". Participants in group PL all followed schedule "C".

\begin{tabular}{|c|c|c|c|c|c|c|c|c|c|c|c|c|c|c|c|}
\hline & \multicolumn{15}{|c|}{ Day } \\
\hline & 0 & 2 & 4 & 7 & 14 & 21 & 28 & 35 & 42 & 49 & 56 & 63 & 65 & 67 & 70 \\
\hline$\overline{\mathbf{A}}$ & $0 \square$ & & 0 & 0 & $\bar{\square}$ & $\bar{\pi}$ & $\bar{\square}$ & 0 & $\bar{\square}$ & $\bar{\square}$ & $\bar{\square}$ & 0 & & 0 & $\bar{\square}$ \\
\hline B & OI & & 0 & $\square$ & " & 0 & 口 & $\square$ & $\bar{\square}$ & 0 & $\bar{\square}$ & $\bar{\square}$ & 0 & & 0 \\
\hline $\bar{C}$ & 0 I & & & & & $\bar{\square}$ & 0 & & $\square$ & & & Or & & & \\
\hline 0 & Blood c & lect & & & & & & & & & & & & & \\
\hline घ & Urine c & lect & & & & & & & & & & & & & \\
\hline
\end{tabular}

\subsection{Specimen Collection and Biochemical Analyses}

Maternal and cord serum samples were collected by standard techniques and maintained at $4{ }^{\circ} \mathrm{C}$ prior to same-day transfer to the laboratory. Spot urine specimens were collected in sterile plastic containers and maintained at $4{ }^{\circ} \mathrm{C}$ until same or next-day analysis of the calcium:creatinine ratio (ca:cr). Serum aliquots for the $25(\mathrm{OH}) \mathrm{D}$ assay were frozen at $-20{ }^{\circ} \mathrm{C}$ for up to five months prior to shipment from 
Bangladesh to Toronto. Total serum [25(OH)D] was measured with the Diasorin Liaison Total assay in the laboratory of Reinhold Vieth in Toronto [14], which meets the International Vitamin D External Quality Assessment Scheme (DEQAS) performance targets [15]. Mean within-run coefficient of variation $(\mathrm{CV} \%$ ) was $7.8 \%$ (5.8\% for specimens with values $<150 \mathrm{nmol} / \mathrm{L})$ and mean between-run $\mathrm{CV} \%$ was $10.5 \%(9.0 \%$ for specimens $<150 \mathrm{nmol} / \mathrm{L})$. Serum calcium, serum albumin, and urine calcium:creatinine ratio (ca:cr) were routinely measured using the AU640 Olympus Autoanalyzer (Olympus Corporation, Japan) in the Clinical Biochemistry Laboratory at the International Center for Diarrheal Disease Research, Bangladesh (ICDDR, B) in Dhaka within 24-48 h of collection of serum or urine aliquots. Total serum calcium concentration ([Ca]) was adjusted for the serum albumin concentration by the following conventional formula: $[\mathrm{Ca}]+(0.02 \times(40$-albumin $))$. Intact parathyroid hormone (PTH) was measured using a chemiluminescent assay on the i1000SR Architect Autoanalyzer (Abbott Diagnostics, Lake Forest, IL, USA), with a reference range of 1.59-7.23 pmol/L (Clinical Biochemistry Lab, icddr,b).

\subsection{Safety Monitoring}

The adjusted $[\mathrm{Ca}$ ] reference range was $2.10-2.60 \mathrm{mmol} / \mathrm{L}$. Umbilical cord venous serum $[\mathrm{Ca}]$ was considered elevated if greater than $3.0 \mathrm{mmol} / \mathrm{L}$ [16]. Urine ca:cr were expressed as $\mathrm{mmol} \mathrm{Ca} / \mathrm{mmol} \mathrm{Cr}$, considering 1.0 as the upper limit of the reference range [17]. An albumin-adjusted serum calcium concentration $>2.60 \mathrm{mmol} / \mathrm{L}$ prompted a repeat measurement on a new specimen as soon as possible. Confirmed hypercalcemia was a priori defined as albumin-adjusted serum calcium concentration $>2.60 \mathrm{mmol} / \mathrm{L}$ on both specimens (since hypercalcemia caused by vitamin $\mathrm{D}$ intoxication would not be expected to resolve within a few days without intervention). Episodes of urinary calcium:creatinine ratio (ca:cr) $>1.0 \mathrm{mmol} / \mathrm{mmol}$ prompted a repeat urine ca:cr measurement within one week. A ca:cr $>0.85 \mathrm{mmol} / \mathrm{mmol}$ that was also 2 -fold or greater relative to the lowest previously observed value in the same participant prompted repeat urine assessment. Persistent hypercalciuria was defined as ca:cr $>1.0 \mathrm{mmol} / \mathrm{mmol}$ on two consecutive results, or on two non-consecutive measurements but in the presence of persistent symptoms suggestive of possible hypercalcemia. Persistent hypercalciuria or persistent ca:cr $>0.85 \mathrm{mmol} / \mathrm{mmol}$ that was also 2 -fold or greater relative to the lowest previously observed value were indications for unscheduled measurement of serum calcium. Abnormal urinalyses, hypertension, reported severe symptoms, or persistence of any mild symptomatic complaints prompted referral to the study physician for further evaluation. Participants were referred to an antenatal care physician at the maternity clinic for treatment of urinary tract infections, hypertension, or other medical problems. Participants with obstetric complications were transported to a local tertiary-care hospital with advanced neonatal care facilities. All costs of medical and obstetric care were borne by the study.

\subsection{Statistical Analysis}

Pharmacokinetic outcomes were expressed as the attained maternal/cord [25(OH)D] and the rise in maternal $[25(\mathrm{OH}) \mathrm{D}]$ above baseline $(\Delta[25(\mathrm{OH}) \mathrm{D}])$. Distributions in each group and at specific time points were summarized as geometric mean $[25(\mathrm{OH}) \mathrm{D}]$ and $95 \%$ confidence intervals $(\mathrm{CI})$. Between-group differences were analyzed by linear regression of log-transformed $[25(\mathrm{OH}) \mathrm{D}]$. To facilitate comparisons to other studies, the $\Delta[25(\mathrm{OH}) \mathrm{D}]$ at days 63 and beyond was also expressed as a function of the 
equivalent daily dose administered to each group, in micrograms (i.e., $125 \mathrm{mcg} /$ day in groups $\mathrm{NH}$ and $\mathrm{PH}$, and $50 \mathrm{mcg} /$ day in group PL). To investigate inter-dose fluctuations, the mean [25(OH)D] at days 65, 67, and 70 were compared to day 63 in groups $\mathrm{NH}$ and $\mathrm{PH}$. The proportion of participants and cord blood specimens with $[25(\mathrm{OH}) \mathrm{D}] \geq 50 \mathrm{nmol} / \mathrm{L}$ or $\geq 80 \mathrm{nmol} / \mathrm{L}$ were compared across groups using logbinomial regression. Mean changes in $[25(\mathrm{OH}) \mathrm{D}]$ over time in each group were also modeled as continuous non-linear parametric functions (see Appendix). These analyses used all available individual participant-level data; standard errors were corrected to account for the within-subject correlation of repeated outcomes. Serum [Ca] and urine log-transformed ca:cr were each modeled as functions of time using fixed indicator variables for baseline, weeks 2 to 5 (days 4 to 34), and week 6 and later (day 35 and thereafter). Comparisons of PH to NH or PL were analyzed using group-by-time interaction terms. Serum [Ca] and urine ca:cr were also expressed in terms of the proportions of episodes above the references ranges. In all analyses, $p<0.05$ was considered statistically significant; however, the Holm procedure was used for multiple pair-wise comparisons [18]. Where appropriate, generalized estimating equations (GEE) with robust error estimation were used to account for non-independence of repeated measures. Analyses were conducted using Stata versions 10.1 and 11.1 (Stata Corporation, College Station, TX, USA).

\section{Results}

\subsection{Participant Characteristics and Retention}

Twenty-eight pregnant women were recruited and randomly assigned to one of two groups, PH $(n=14)$ and PL $(n=14)$. Sixteen non-pregnant women were enrolled (Figure 2).

Figure 2. Study flow diagram. Participant screening, enrollment, exclusions, and withdrawal over the course of the study.

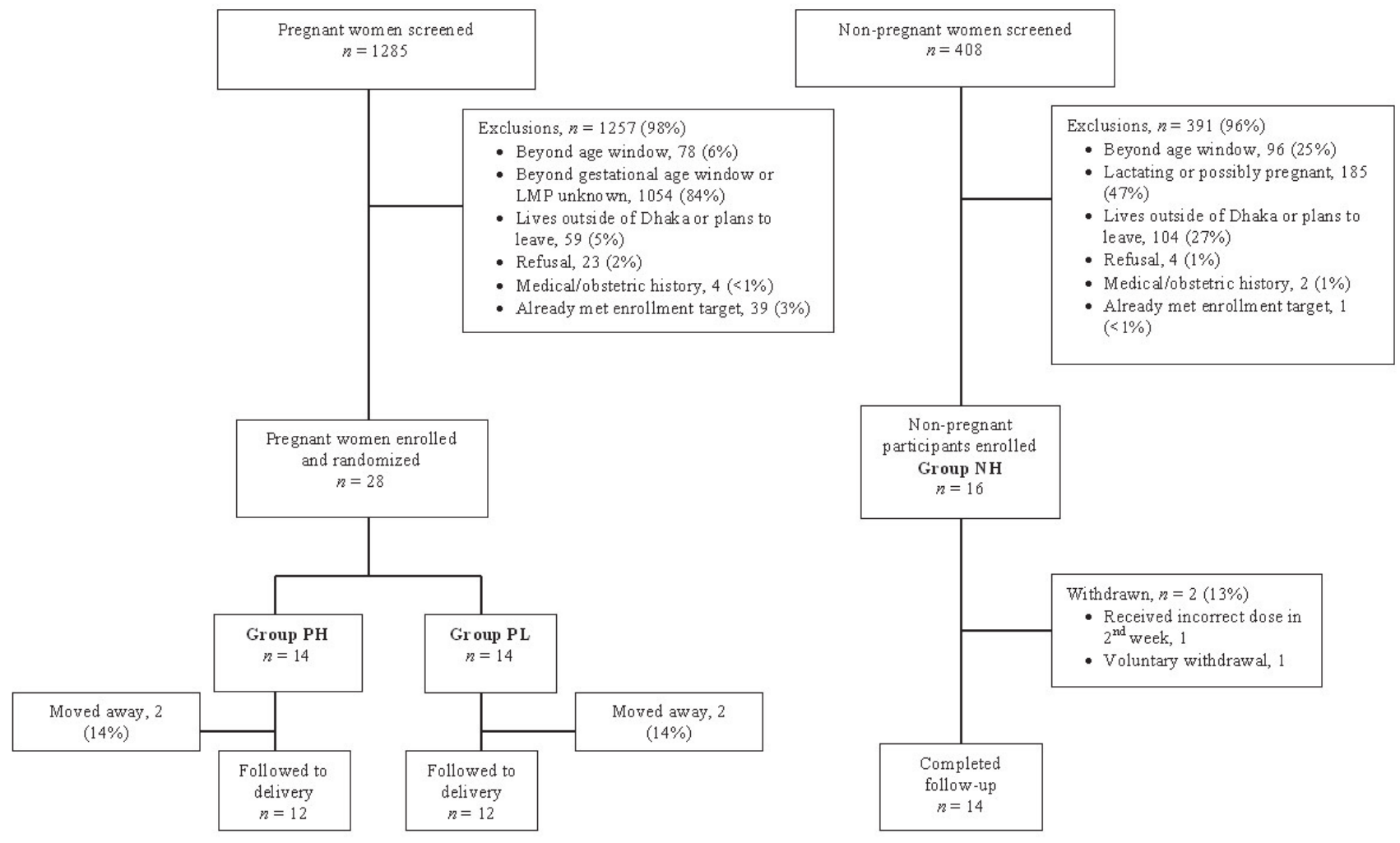


Of 28 randomized pregnant participants, $4(14 \%)$ left the Dhaka area prior to completion of the study protocol (2 in PH and 2 in PL). Specimens in the 10th week were available in 10/14 women in PH and 9/14 in PL. Cord specimens were available in 23 (82\%) of enrolled participants. PH and PL were generally similar with respect to baseline characteristics (Table 1) and [25(OH)D] (Table 2). However, $\mathrm{NH}$ enrollment occurred in the summer rather than mid-winter and $\mathrm{NH}$ participants had higher average baseline $[25(\mathrm{OH}) \mathrm{D}]$ compared to the pregnant participants (Table 1).

Table 1. Participant characteristics at enrollment.

\begin{tabular}{lcccc}
\hline & NH & PH & PL & $p$ \\
\hline \# Enrolled & 16 & 14 & 14 & \\
\hline Age (years), Mean $( \pm$ SD) & $24.6( \pm 4.5)$ & $22.2( \pm 3.1)$ & $22.1( \pm 4.8)$ & 0.190 \\
\hline Gestational age at enrollment (weeks) & & & & \\
$\quad$ Mean ( \pm SD) & - & $28.4( \pm 1.2)$ & $28.5( \pm 1.3)$ & 0.760 \\
$\quad$ Range & - & $26.1-30.6$ & $27-30.7$ & \\
\hline Married & $12(75 \%)$ & $14(100 \%)$ & $13(93 \%)$ & 0.110 \\
\hline Education level attained & & & & \\
$\quad$ None & $2(13 \%)$ & $4(29 \%)$ & $3(21 \%)$ & 0.285 \\
$\quad$ Primary & $10(63 \%)$ & $9(64 \%)$ & $11(79 \%)$ & \\
$\quad$ Secondary or higher & $4(25 \%)$ & $1(7 \%)$ & 0 & \\
\hline Height $(\mathbf{c m})$, mean $( \pm$ SD) & $152.1( \pm 4.7)$ & $150.7( \pm 4.7)$ & $148.9( \pm 4.5)$ & 0.179 \\
\hline
\end{tabular}

Table 2. Serum 25-hydroxyvitamin D concentrations at baseline and through 10 weeks of supplementation in non-pregnant and pregnant participants ${ }^{1}$.

\begin{tabular}{|c|c|c|c|c|c|}
\hline & $\begin{array}{c}\text { Non-pregnant } \\
\text { NH }\end{array}$ & $\begin{array}{c}\text { Pregnant } \\
\text { PH }\end{array}$ & $\begin{array}{c}\text { Pregnant } \\
\text { PL }\end{array}$ & & \\
\hline \# Enrolled & 16 & 14 & 14 & & \\
\hline \multicolumn{6}{|l|}{ Vitamin D3 regimen } \\
\hline Loading dose & $70,000 \mathrm{IU}$ & $70,000 \mathrm{IU}$ & 0 & & \\
\hline Weekly doses & $35,000 \mathrm{IU}$ & $35,000 \mathrm{IU}$ & $14,000 \mathrm{IU}$ & & \\
\hline Duration of supplementation & 10 weeks & $\begin{array}{c}\text { 27-30 weeks } \\
\text { gestation until } \\
\text { delivery }\end{array}$ & $\begin{array}{c}27-30 \text { weeks } \\
\text { gestation until } \\
\text { delivery }\end{array}$ & & \\
\hline Dates of enrollment & $\begin{array}{c}17 \text { Aug-6 Sep } \\
2009\end{array}$ & 3-16 Feb 2010 & 3-16 Feb 2010 & & \\
\hline $\begin{array}{l}\text { Participants with }[25(\mathrm{OH}) \mathrm{D}] \text { measured } \\
\text { during 10th week (days } 63 \text { to } 70), n(\%)\end{array}$ & $14(88 \%)$ & $10(71 \%)$ & $9(64 \%)$ & $p$ va & $u^{2}$ \\
\hline $\begin{array}{l}\text { Number of specimens per participant, } \\
\text { Median }\end{array}$ & 6 & 6 & 3 & $\begin{array}{c}\text { PH vs. } \\
\text { NH }\end{array}$ & $\begin{array}{c}\text { PH vs. } \\
\text { PL }\end{array}$ \\
\hline \multicolumn{6}{|l|}{ Baseline [25(OH)D] } \\
\hline Mean $[95 \% \mathrm{CI}]$ & $57[47,69]$ & $35[30,42]$ & $31[26,38]$ & $<0.001$ & 0.341 \\
\hline Range (min, max) & 27,93 & 21,55 & 20,57 & & \\
\hline \multicolumn{6}{|l|}{ Attained $[25(\mathrm{OH}) \mathrm{D}]$ in 10 th week } \\
\hline Mean $[95 \% \mathrm{CI}]$ & $139[121,160]$ & $98[89,109]$ & $76[61,95]$ & $<0.001$ & 0.038 \\
\hline Range (min, max) & 85,238 & 71,153 & 36,119 & & \\
\hline
\end{tabular}


Table 2. Cont.

\begin{tabular}{|c|c|c|c|c|c|}
\hline \multicolumn{6}{|l|}{$\Delta[25(\mathrm{OH}) \mathrm{D}]$ in 10 th week } \\
\hline Mean $[95 \% \mathrm{CI}]$ & $76[61,96]$ & $57[44,73]$ & $36[22,61]$ & 0.082 & 0.128 \\
\hline Range on days 63 to 70 & 28,160 & 19,130 & 7,75 & & \\
\hline \multicolumn{6}{|l|}{$\begin{array}{l}\Delta[25(\mathrm{OH}) \mathrm{D}] \text { at days } 63 \text { to } 70 \text { per daily } \\
\text { vitamin } \mathrm{D} 3 \mathrm{dose}(\mathrm{nmol} / \mathrm{L} / \mathrm{mcg})\end{array}$} \\
\hline Mean $[95 \% \mathrm{CI}]$ & $0.61[0.48,0.79]$ & $0.46[0.34,0.61]$ & $0.73[0.38,1.38]$ & 0.220 & 0.081 \\
\hline $\begin{array}{l}\text { Area under the } \Delta[25(\mathrm{OH}) \mathrm{D}] \text {-time curve } \\
(\mathrm{nmol} \cdot \mathrm{d} / \mathrm{L}) \text { to day } 63 / 65\left(\mathrm{AUC}_{63}\right)^{3}\end{array}$ & $3500[2886,4245]$ & $2925[2331,3670]$ & $1678[923,3053]$ & 0.383 & 0.020 \\
\hline \multicolumn{6}{|l|}{ Participants with mean $[25(\mathrm{OH}) \mathrm{D}]$} \\
\hline $\begin{array}{l}\text { Participants with mean }[25(\mathrm{OH}) \mathrm{D}] \\
\geq 80 \mathrm{nmol} / \mathrm{L} \text { in } 10 \text { th week, } \# / n(\%)^{4}\end{array}$ & $14 / 14(100 \%)$ & $9 / 10(90 \%)$ & $5 / 9(56 \%)$ & 0.152 & 0.127 \\
\hline \multicolumn{6}{|l|}{ PTH } \\
\hline Baseline $(n=28)$, mean $[95 \% \mathrm{CI}]$ & - & $2.10[1.26,3.52]$ & $1.53[0.94,2.49]$ & & \\
\hline Final $(n=22)$, mean $[95 \% \mathrm{CI}]$ & - & $1.63[1.01,2.66]$ & $2.49[1.61,3.85]$ & - & $0.011^{5}$ \\
\hline \multicolumn{6}{|l|}{ Cord serum $[25(\mathrm{OH}) \mathrm{D}](n=23)$} \\
\hline Mean $[95 \%$ CI] & - & $117[99,137]$ & $98[84,115]$ & - & 0.074 \\
\hline Range (min, max) & - & 74,168 & 53,124 & & \\
\hline Cord $[25(\mathrm{OH}) \mathrm{D}] \geq 50 \mathrm{nmol} / \mathrm{L}, \# / n(\%)$ & - & $12 / 12(100 \%)$ & $11 / 11(100 \%)$ & - & 1.000 \\
\hline Cord $[25(\mathrm{OH}) \mathrm{D}] \geq 80 \mathrm{nmol} / \mathrm{L}, \# / n(\%)$ & - & $11 / 12(92 \%)$ & $10 / 11(91 \%)$ & - & 0.949 \\
\hline
\end{tabular}

${ }^{1}$ Summary measures are geometric means with $95 \%$ confidence intervals, unless otherwise indicated. ${ }^{2}$ Linear regression models (GEE was implemented where there were repeated measures for the same individuals) unless otherwise indicated; all $p$ values $<0.05$ remained significant after correction for multiple pairwise comparisons using the Holm method. ${ }^{3}$ AUC for each group was the geometric mean (and 95\% confidence intervals) of individual participants' AUCs; the analyses included 33 participants who were followed-up to at least week 10 (day 63 or 65, depending on serum sampling schedule): NH, $n=14$ participants; PH, $n=10$; PL, $n=9$. Comparison of the AUC based on only 3 datapoints (baseline, day 21/28/35, and day 63/65) was undertaken as a sensitivity analysis because group PL participants only had [25(OH)D] measured at a maximum of three visits at which blood collection was scheduled; the latter analysis involved the same 33 participants as in the preceding analysis. ${ }^{4}$ Proportion of participants in each group with average $[25(\mathrm{OH}) \mathrm{D}] \geq 50 \mathrm{nmol} / \mathrm{L}$ or $\geq 80 \mathrm{nmol} / \mathrm{L}$ in specimens collected on days 63 to 70 ; comparisons between groups were assessed by binomial regression. None of the pairwise comparisons were statistically significant after correction for multiplicity using the Holm method. ${ }^{5} p$ value for the group-by-time interaction term in a GEE model (exchangeable correlation and robust standard errors), using log-transformed PTH as the outcome, indicating that the change from baseline over time significantly differed between the two groups.

\subsection{Effect of Prenatal Vitamin D3 Supplementation on Vitamin D Status}

Mean [25(OH)D] rose gradually above baseline in all groups during follow-up (Table 2; Figure 3). Final mean $[25(\mathrm{OH}) \mathrm{D}]$ during the 10th week of supplementation was significantly higher in $\mathrm{PH}$ versus PL (98 vs. $76 \mathrm{nmol} / \mathrm{L}$, respectively; $p=0.038)$ and significantly lower versus NH (98 vs. $139 \mathrm{nmol} / \mathrm{L}$; $p<0.001$ ) (Table 2). However, $\Delta[25(\mathrm{OH}) \mathrm{D}]$ in $\mathrm{PH}$ was not significantly lower in the 10 th week compared to $\mathrm{NH}$ (Table 2). The $[25(\mathrm{OH}) \mathrm{D}]$ threshold of $50 \mathrm{nmol} / \mathrm{L}$ was attained by nearly all participants, but only the higher-dose regimen reliably led to $[25(\mathrm{OH}) \mathrm{D}] \geq 80 \mathrm{nmol} / \mathrm{L}$ by the 10 th week in pregnant women. During the 10th week, there were no notable inter-dose fluctuations in $\mathrm{NH}$ and $\mathrm{PH}$ (Figure 4); mean [25(OH)D] at days 65, 67, and 70 differed from day 63 by $<6 \mathrm{nmol} / \mathrm{L}$ (all 
$p$ values $>0.5)$. There was substantial inter-subject variability in the response to vitamin $\mathrm{D}$ supplementation, with one PL participant demonstrating only a $7 \mathrm{nmol} / \mathrm{L}$ final increase in $[25(\mathrm{OH}) \mathrm{D}]$ above her baseline. Among participants who received the higher-dose regimen, there was as much as a 7 -fold difference between the lowest and highest responders based on $\Delta[25(\mathrm{OH}) \mathrm{D}]$ at week 10 (Table 2). Three participants in $\mathrm{NH}$ had $[25(\mathrm{OH}) \mathrm{D}]>200 \mathrm{nmol} / \mathrm{L}$, but the highest $[25(\mathrm{OH}) \mathrm{D}]$ in any pregnant participant was $153 \mathrm{nmol} / \mathrm{L}$. There was no significant association between baseline vitamin $\mathrm{D}$ status and $\Delta[25(\mathrm{OH}) \mathrm{D}]$ (data not shown).

Figure 3. Changes in serum 25-hydroxyvitamin D concentration from baseline resulting from weekly vitamin D3 administration to non-pregnant women who received an initial dose of 70,000 IU and then 35,000 IU/week thereafter (NH), pregnant women who received an initial dose of 70,000 IU and then 35,000 IU/week thereafter (PH), and pregnant women who received 14,000 IU/week (PL). Lines connect the group means at each follow-up visit.

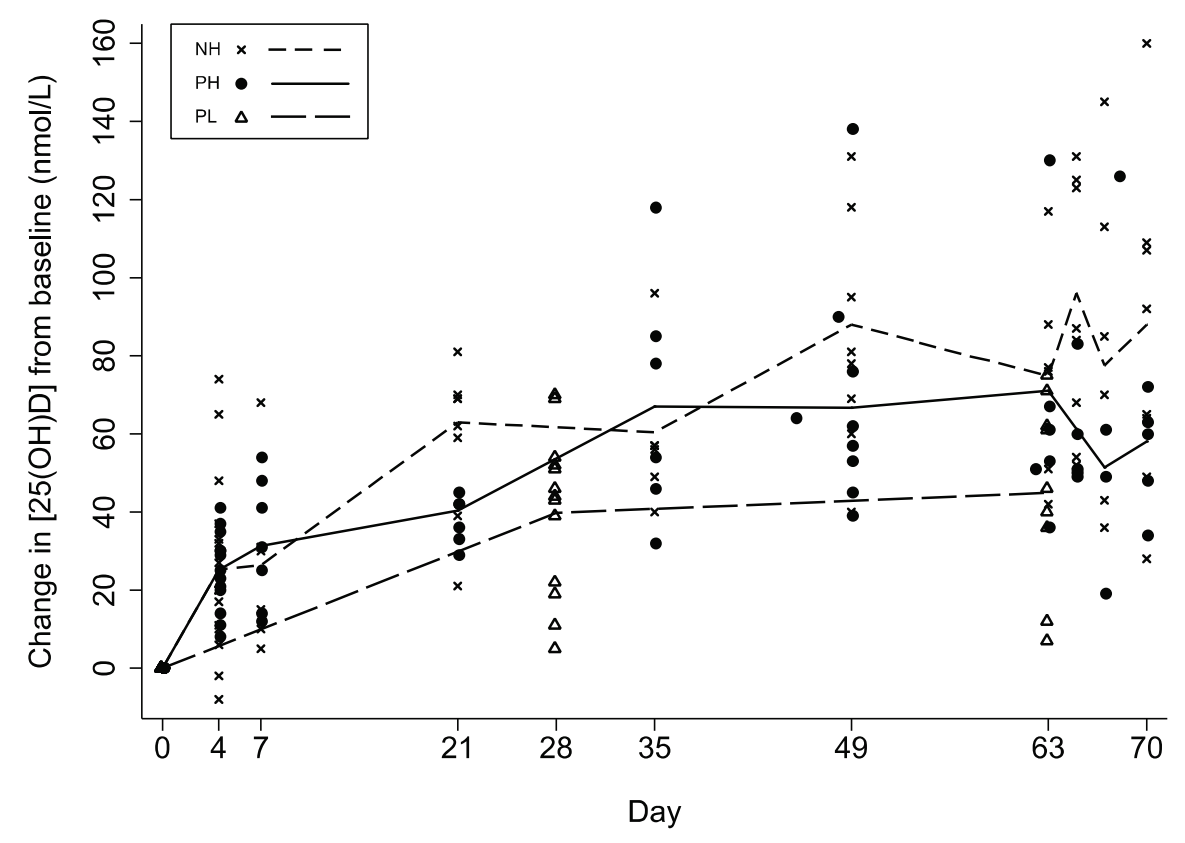


Figure 4. Lack of inter-dose fluctuations in mean serum 25-hydroxyvitamin D concentrations among non-pregnant $(\mathrm{NH})$ and pregnant women $(\mathrm{PH})$ during the 10th week of supplementation with 35,000 IU vitamin D3 per week, with the most recent dose administered on day 63. Lines connect the group means at each day; 95\% confidence intervals are represented by vertical capped bars.

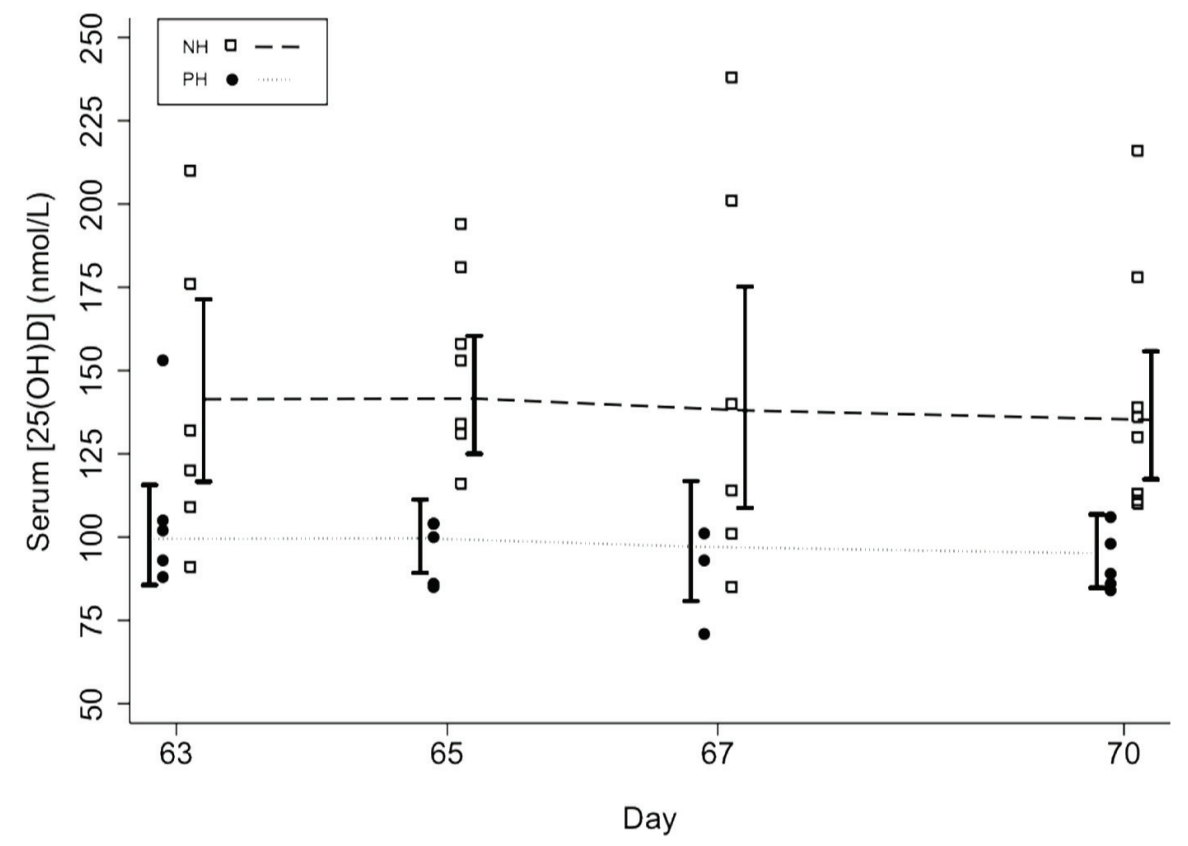

Non-linear parametric models representing the change in $[25(\mathrm{OH}) \mathrm{D}]$ as a continuous function of time yielded inferences regarding baseline and modeled steady-state mean $[25(\mathrm{OH}) \mathrm{D}]$ that were consistent with the empiric cross-sectional estimates (Table 3) and provided appropriate fits to the data (Figure 5). Extended models indicated that the $\Delta[25(\mathrm{OH}) \mathrm{D}]$ at modeled steady-state was $19 \mathrm{nmol} / \mathrm{L}$ greater in $\mathrm{PH}$ compared to PL $(p=0.044)$ (Table 3$)$. Mean modeled steady-state $\Delta[25(\mathrm{OH}) \mathrm{D}]$ was lower in $\mathrm{PH}$ compared to NH but the difference was not statistically significant (Table 3).

Table 3. Estimates of the change in serum 25-hydroxyvitamin D concentration over time in response to weekly vitamin D3 supplementation in non-pregnant women who received an initial dose of 70,000 IU and then 35,000 IU/week (NH), pregnant women who received an initial dose of 70,000 IU and then 35,000 IU/week (PH), and pregnant women who received a weekly dose of 14,000 IU/week (PL). Results are based on negative exponential models, and shown as mean (lower 95\% confidence bound, upper 95\% confidence bound).

\begin{tabular}{lccccc}
\hline & Model 1 & Model 2 & Model 3 & Model 4 & Model 5 \\
\hline & $\begin{array}{c}\text { Non-pregnant } \\
(\mathbf{N H})\end{array}$ & $\begin{array}{c}\text { Pregnant, } \\
\text { higher-dose } \\
(\mathbf{P H})\end{array}$ & $\begin{array}{c}\text { Pregnant, lower- } \\
\text { dose } \\
(\text { PL) }\end{array}$ & $\begin{array}{c}\text { Pregnant } \\
\text { (PL \& PH) }\end{array}$ & $\begin{array}{c}\text { Higher dose } \\
\text { (NH \& PH) }\end{array}$ \\
\hline $\begin{array}{l}\text { Number of } \\
\text { participants }\end{array}$ & 16 & 14 & 14 & 28 & 29 \\
$\begin{array}{l}\text { Number of } \\
\text { specimens }\end{array}$ & 89 & 75 & 36 & 111 & 162 \\
\hline
\end{tabular}


Table 3. Cont.

\begin{tabular}{|c|c|c|c|c|c|c|}
\hline & & Model 1 & Model 2 & Model 3 & Model 4 & Model 5 \\
\hline & & $\begin{array}{c}\text { Non-pregnant } \\
\text { (NH) }\end{array}$ & $\begin{array}{c}\text { Pregnant, } \\
\text { higher-dose } \\
\text { (PH) }\end{array}$ & $\begin{array}{c}\text { Pregnant, lower- } \\
\text { dose } \\
\text { (PL) } \\
\end{array}$ & $\begin{array}{c}\text { Pregnant } \\
\text { (PL \& PH) }\end{array}$ & $\begin{array}{c}\text { Higher dose } \\
\text { (NH \& PH) }\end{array}$ \\
\hline $\begin{array}{l}\text { Baseline } \\
{[25(\mathrm{OH}) \mathrm{D}]}\end{array}$ & $\mathrm{nmol} / \mathrm{L}$ & $58[48,69]$ & $36[29,42]$ & $31[25,38]$ & $31[25,37]$ & $57[47,67]$ \\
\hline $\begin{array}{l}\Delta[25(\mathrm{OH}) \mathrm{D}] \text { at } \\
\text { steady-state }(a)\end{array}$ & $\mathrm{nmol} / \mathrm{L}$ & $79[60,97]$ & $62[48,75]$ & $45[23,67]$ & $43[29,57]$ & $77[62,93]$ \\
\hline $\begin{array}{l}\Delta[25(\mathrm{OH}) \mathrm{D}] \text { at } \\
\text { steady-state per } \\
\text { daily dose } \\
\text { equivalent }\end{array}$ & $\begin{array}{c}\mathrm{nmol} / \mathrm{L} / \mathrm{mcg} \\
\mathrm{D} 3 \text { per day }\end{array}$ & $\begin{array}{c}0.63[0.48 \\
0.78]\end{array}$ & $0.49[0.38,0.60]$ & $0.90[0.47,1.34]$ & - & - \\
\hline $\begin{array}{l}\text { Steady-state } \\
{[25(\mathrm{OH}) \mathrm{D}]} \\
\left([25(\mathrm{OH}) \mathrm{D}]_{t 0}+a\right)\end{array}$ & $\mathrm{nmol} / \mathrm{L}$ & $137[116,157]$ & $97[87,108]$ & $76[54,98]$ & $74[61,87]$ & $134[117,151]$ \\
\hline Decay rate $(k)$ & days $^{-1}$ & $0.08[0.03,0.12]$ & $0.11[0.07,0.15]$ & $0.07[-0.01,0.16]$ & $0.11[0.07,0.15]$ & $0.09[0.06,0.12]$ \\
\hline Group (g) & $\begin{array}{c}0 \text { (Ref) } \\
1 \\
\end{array}$ & $\begin{array}{l}- \\
-\end{array}$ & - & - & $\begin{array}{l}\text { PW-C } \\
\text { PW-H }\end{array}$ & $\begin{array}{l}\text { NP-H } \\
\text { PW-H }\end{array}$ \\
\hline $\begin{array}{l}\text { Difference in } \\
{[25(\mathrm{OH}) \mathrm{D}]} \\
\text { between groups } \\
\text { at baseline }(\beta)\end{array}$ & $\mathrm{nmol} / \mathrm{L}$ & - & - & - & $4[-4,13]$ & $-21[-33,-9]$ \\
\hline $\begin{array}{l}\text { Difference in } \\
\Delta[25(\mathrm{OH}) \mathrm{D}] \\
\text { between groups } \\
\text { at steady-state } \\
\text { (d) }\end{array}$ & $\mathrm{nmol} / \mathrm{L}$ & - & - & - & $\begin{array}{l}19[1,37] \\
p=0.044\end{array}$ & $\begin{array}{c}-15[-34,5] \\
p=0.131\end{array}$ \\
\hline Adjusted R $^{2}$ & & 0.55 & 0.71 & 0.63 & 0.72 & 0.69 \\
\hline
\end{tabular}


Figure 5. Negative exponential models predicting serum 25-hydroxyvitamin D concentrations in response to weekly vitamin D3 supplementation in non-pregnant women who received an initial dose of 70,000 IU and then 35,000 IU/week (NH), pregnant women who received an initial dose of 70,000 IU and then 35,000 IU/week (PH), and pregnant women who received a weekly dose of 14,000 IU/week (PL). Vertical bars represent the $95 \%$ confidence intervals of the empiric geometric means at each scheduled follow-up time.

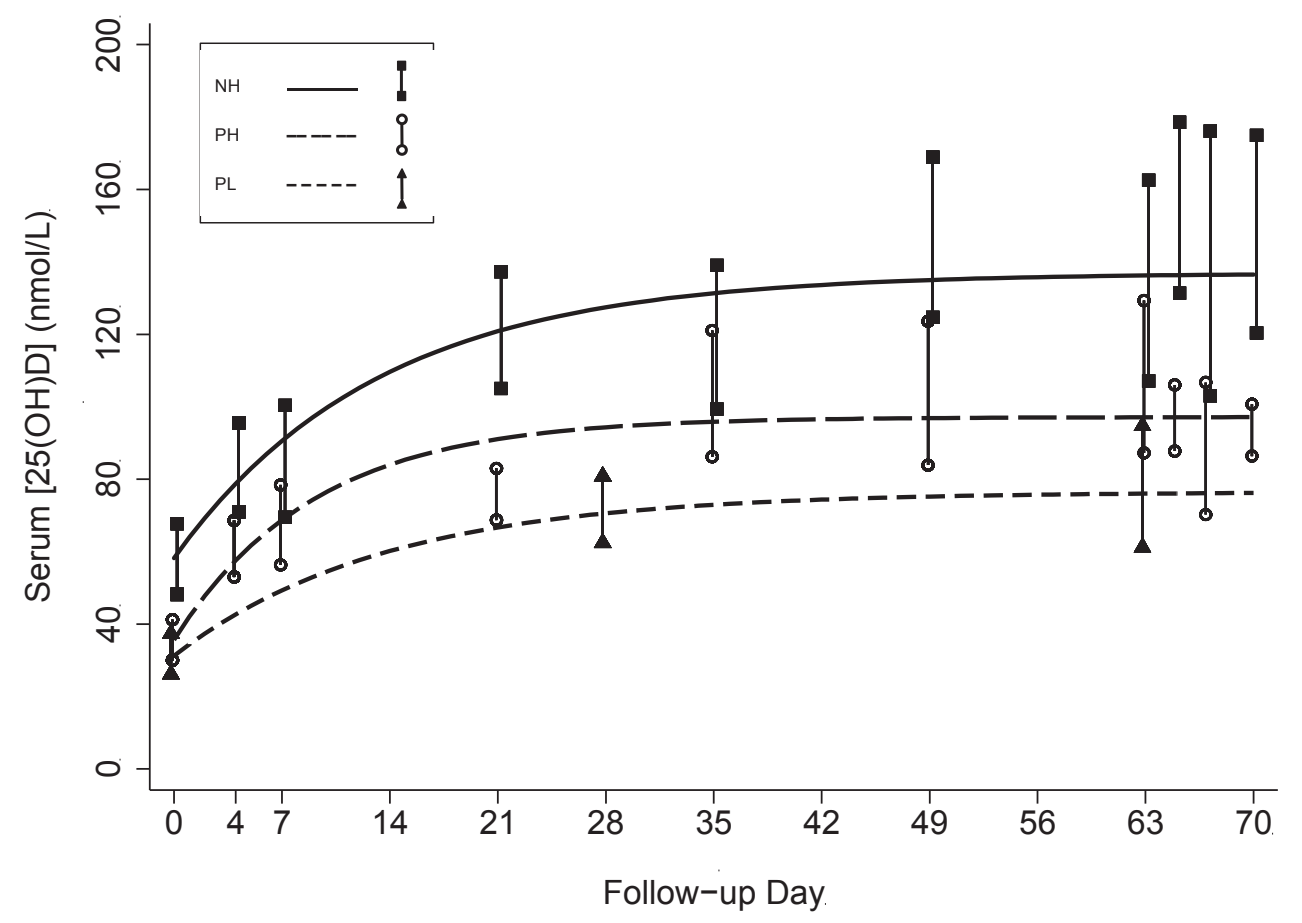

Mean cord serum $[25(\mathrm{OH}) \mathrm{D}]$ was higher in PH $(117 \mathrm{nmol} / \mathrm{L})$ versus $\mathrm{PL}(98 \mathrm{nmol} / \mathrm{L})$ but the difference was not significant (Table 2). The proportions of newborns with $[25(\mathrm{OH}) \mathrm{D}] \geq 80 \mathrm{nmol} / \mathrm{L}(\mathrm{PH}$ : 92\%; PL: 91\%) and $\geq 50 \mathrm{nmol} / \mathrm{L}$ (PH: 100\%; PL: 100\%) were similar in the two groups. There was a moderate association between cord and maternal $[25(\mathrm{OH}) \mathrm{D}](\rho=0.67, p<0.001)$.

\subsection{Ancillary Biochemical Parameters}

Mean albumin-adjusted serum [Ca] increased significantly within the reference range during followup in PH but it did not change significantly in the comparison groups (Table 4; Figure 6). The increase in PH was significantly greater than in PL or NH (Table 4). There was a single episode of albuminadjusted [Ca] $>2.60 \mathrm{mmol} / \mathrm{L}$ in a $\mathrm{PH}$ participant during an episode of acute gastroenteritis that occurred after two weeks of supplementation. Her albumin-adjusted [Ca] of $2.61 \mathrm{mmol} / \mathrm{L}$ declined to $2.39 \mathrm{mmol} / \mathrm{L}$ in a repeat specimen on the same day, the illness was self-limited, and there was no other biochemical or clinical evidence of vitamin D toxicity; furthermore, the participant continued to receive the supplement and had increasing [25(OH)D] (range, 52 to $98 \mathrm{nmol} / \mathrm{L}$ during follow-up) but did not develop any further episodes of hypercalcemia or elevations in urine ca:cr. There were no episodes of confirmed hypercalcemia according to a priori study definitions. 


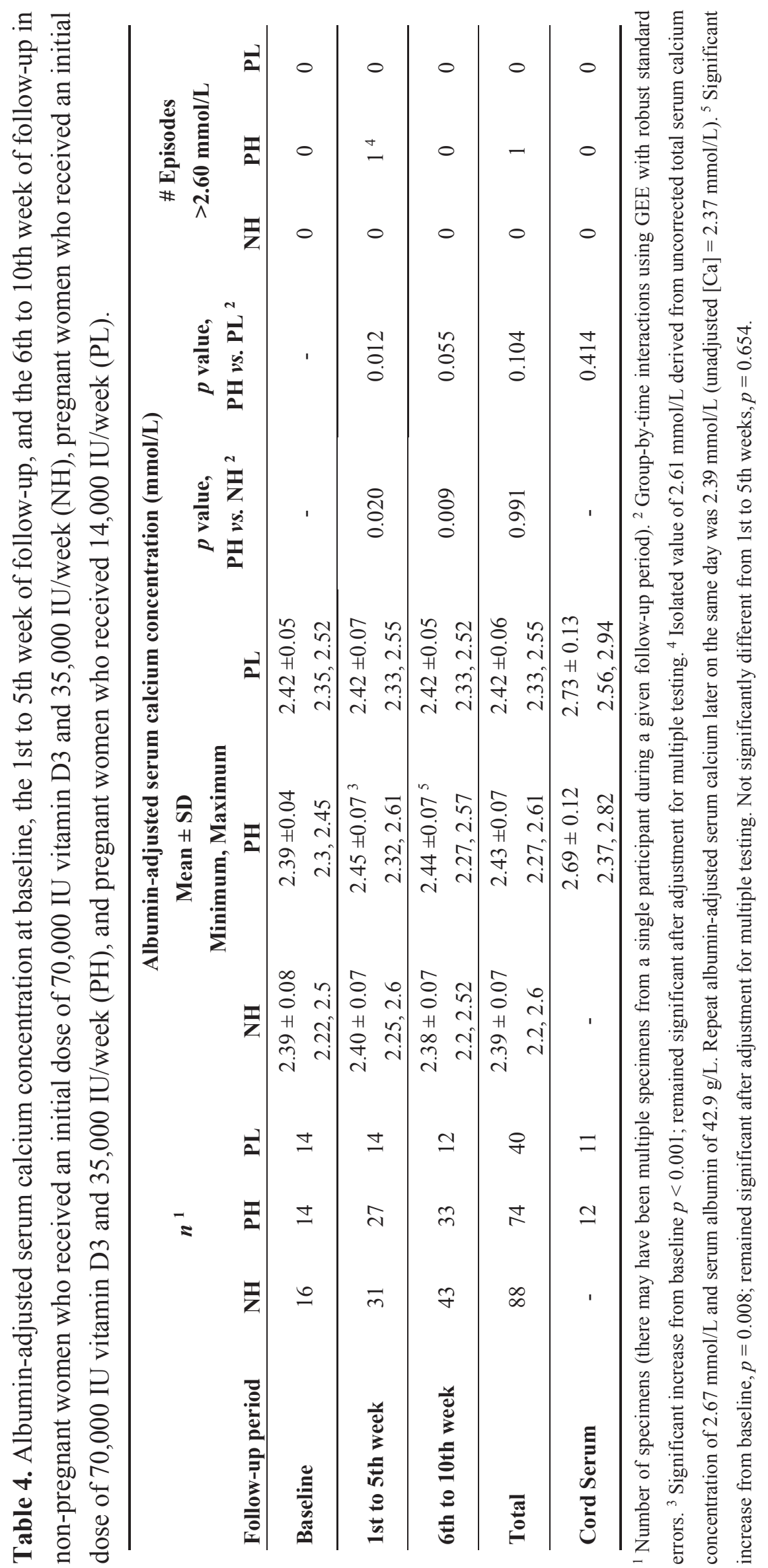


Figure 6. Mean albumin-adjusted serum calcium concentrations in the three participant groups. (A) Mean albumin-adjusted serum calcium concentration in pregnant participants who received an initial dose of 70,000 IU and then 35,000 IU/week (PH) and pregnant participants who received a weekly dose of 14,000 IU/week (PL); (B) Mean albumin-adjusted serum calcium concentration in non-pregnant participants who received an initial dose of 70,000 IU and then 35,000 IU/week (NH). Vertical bars represent the $95 \%$ confidence intervals of the means at each scheduled follow-up time. Horizontal line indicates the upper limit of the reference range $(2.60 \mathrm{mmol} / \mathrm{L})$.

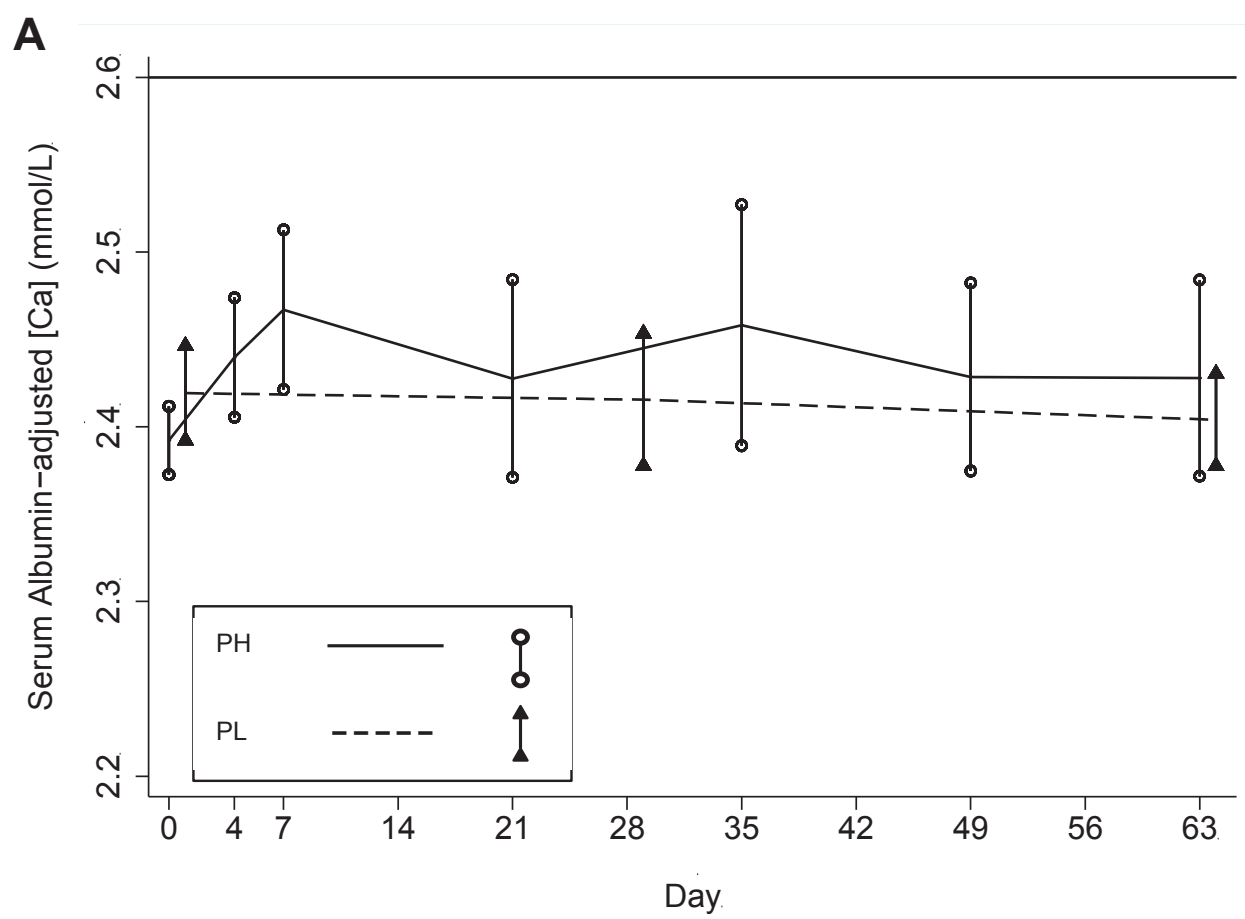

B

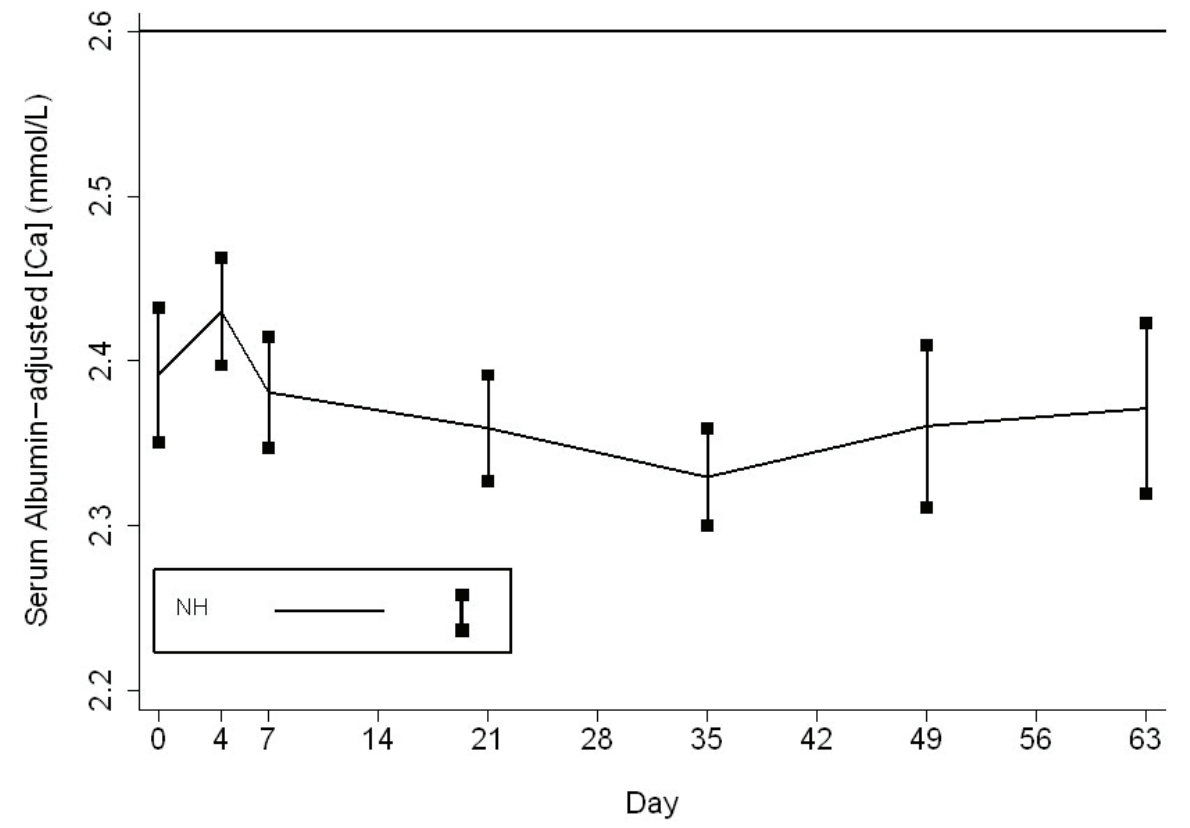


Urine ca:cr rose initially during follow-up in all groups but appeared to plateau in PL and decline in $\mathrm{PH}$ and $\mathrm{NH}$ during the latter half of the follow-up period (Table 5; Figure 7). There were five episodes of ca:cr $>1.0 \mathrm{mmol} / \mathrm{mmol}$ (Table 5). One participant in group PL had two consecutive episodes on days 42 and 44 and thus met the definition for persistent hypercalciuria by study criteria; however, [Ca] was normal and despite continued supplementation, the ca:cr was within the normal limits thereafter. The higher-dose intervention (PH) suppressed the average PTH concentration, which was significantly different from the increase observed in PL $(p=0.011)$ (Table 2).

Table 5. Urine calcium:creatinine ratio in random spot urine specimens collected at baseline, 1st to 5th weeks of follow-up, and 6th week to the end of the supplementation period in non-pregnant women who received an initial dose of 70,000 IU vitamin D3 and $35,000 \mathrm{IU} /$ week $(\mathrm{NH})$, pregnant women who received an initial dose of 70,000 IU vitamin $\mathrm{D} 3$ and 35,000 IU/week $(\mathrm{PH})$, and pregnant women who received 14,000 IU/week (PL).

\begin{tabular}{|c|c|c|c|c|c|c|c|c|c|c|c|}
\hline \multirow[b]{3}{*}{ Follow-up period } & \multirow[b]{3}{*}{ NH } & \multirow{3}{*}{$\begin{array}{c}n^{1} \\
\text { PH }\end{array}$} & \multirow[b]{3}{*}{ PL } & \multicolumn{5}{|c|}{ Urinary calcium-creatinine ratio (mmol/mmol) } & \multirow{2}{*}{\multicolumn{3}{|c|}{$\begin{array}{c}\text { \# Episodes } \\
>\mathbf{1 . 0} \mathbf{~ m m o l} / \mathbf{m m o l} \\
(\# \text { Participants ever having } \\
>1.0 \mathrm{mmol} / \mathrm{mmol} \text { ) }\end{array}$}} \\
\hline & & & & \multicolumn{3}{|c|}{$\begin{array}{c}\text { Mean }^{2} \\
\text { Minimum, Maximum }\end{array}$} & \multirow[t]{2}{*}{$\begin{array}{c}p \text { value } \\
\text { PH vs. } \\
\text { NH }^{3}\end{array}$} & \multirow[t]{2}{*}{$\begin{array}{c}p \text { value } \\
\text { PH vs. } \\
\text { PL }^{3}\end{array}$} & & & \\
\hline & & & & NH & PH & PL & & & NH & PH & PL \\
\hline \multirow{2}{*}{ Baseline } & \multirow{2}{*}{16} & \multirow{2}{*}{14} & \multirow{2}{*}{14} & 0.23 & 0.10 & 0.21 & \multirow[b]{2}{*}{-} & \multirow[b]{2}{*}{-} & 0 & 0 & 0 \\
\hline & & & & $0.04,0.58$ & $0.01,0.44$ & $0.06,0.91$ & & & $(0)$ & $(0)$ & $(0)$ \\
\hline \multirow{2}{*}{ 1st to 5 th weeks } & \multirow{2}{*}{49} & \multirow{2}{*}{36} & \multirow{2}{*}{12} & $0.36^{4}$ & $0.24^{5}$ & 0.24 & \multirow{2}{*}{0.164} & \multirow{2}{*}{0.105} & 3 & 0 & 0 \\
\hline & & & & $0.04,1.47$ & $0.02,0.95$ & $0.07,0.64$ & & & $(2)$ & $(0)$ & $(0)$ \\
\hline \multirow{2}{*}{ 6th week to end } & \multirow{2}{*}{62} & \multirow{2}{*}{53} & \multirow{2}{*}{33} & 0.26 & $0.18^{6}$ & 0.30 & \multirow{2}{*}{0.164} & \multirow{2}{*}{0.500} & 0 & 0 & 2 \\
\hline & & & & $0.03,0.91$ & $0.01,0.96$ & $0.05,1.05$ & & & $(0)$ & $(0)$ & (1) \\
\hline \multirow{2}{*}{ Total } & \multirow{2}{*}{127} & 10 & \multirow{2}{*}{59} & 0.29 & 0.19 & 0.26 & \multirow{2}{*}{0.014} & \multirow{2}{*}{0.047} & 3 & 0 & 2 \\
\hline & & 3 & & $0.03,1.47$ & $0.01,0.96$ & $0.05,1.05$ & & & (2) & $(0)$ & (1) \\
\hline
\end{tabular}

\footnotetext{
${ }^{1}$ Number of specimens (there may have been multiple specimens from a single participant during a given follow-up period). ${ }^{2}$ Geometric means. ${ }^{3}$ Group by time interactions using GEE with robust standard errors. ${ }^{4}$ The $p$ value for the test of the difference from baseline was 0.018 ; however, this was not statistically significant after adjustment for multiple testing (adjusted critical $p$ value of 0.017$){ }^{5}$ The increase from baseline was statistically significant $(p<0.001)$ and remained so after adjustment for multiple testing (adjusted critical $p$ value of 0.025 ). ${ }^{6}$ Not significantly different from baseline after adjustment for multiple testing ( $p=0.042$, adjusted critical $p$ of 0.025$)$; and, not significantly different from the period of 1 st to 5 th weeks $(p=0.136)$.
} 
Figure 7. Mean urine calcium:creatinine ratio in the three participant groups.(A) Mean urine calcium:creatinine ratio in pregnant participants who received an initial dose of 70,000 IU and then 35,000 IU/week (PH) and pregnant participants who received a weekly dose of 14,000 IU/week (PL), and (B) Mean urine calcium:creatinine ratio in non-pregnant participants who received an initial dose of 70,000 IU and then 35,000 IU/week (NH). Vertical bars represent the $95 \%$ confidence intervals of the means at each scheduled follow-up time.
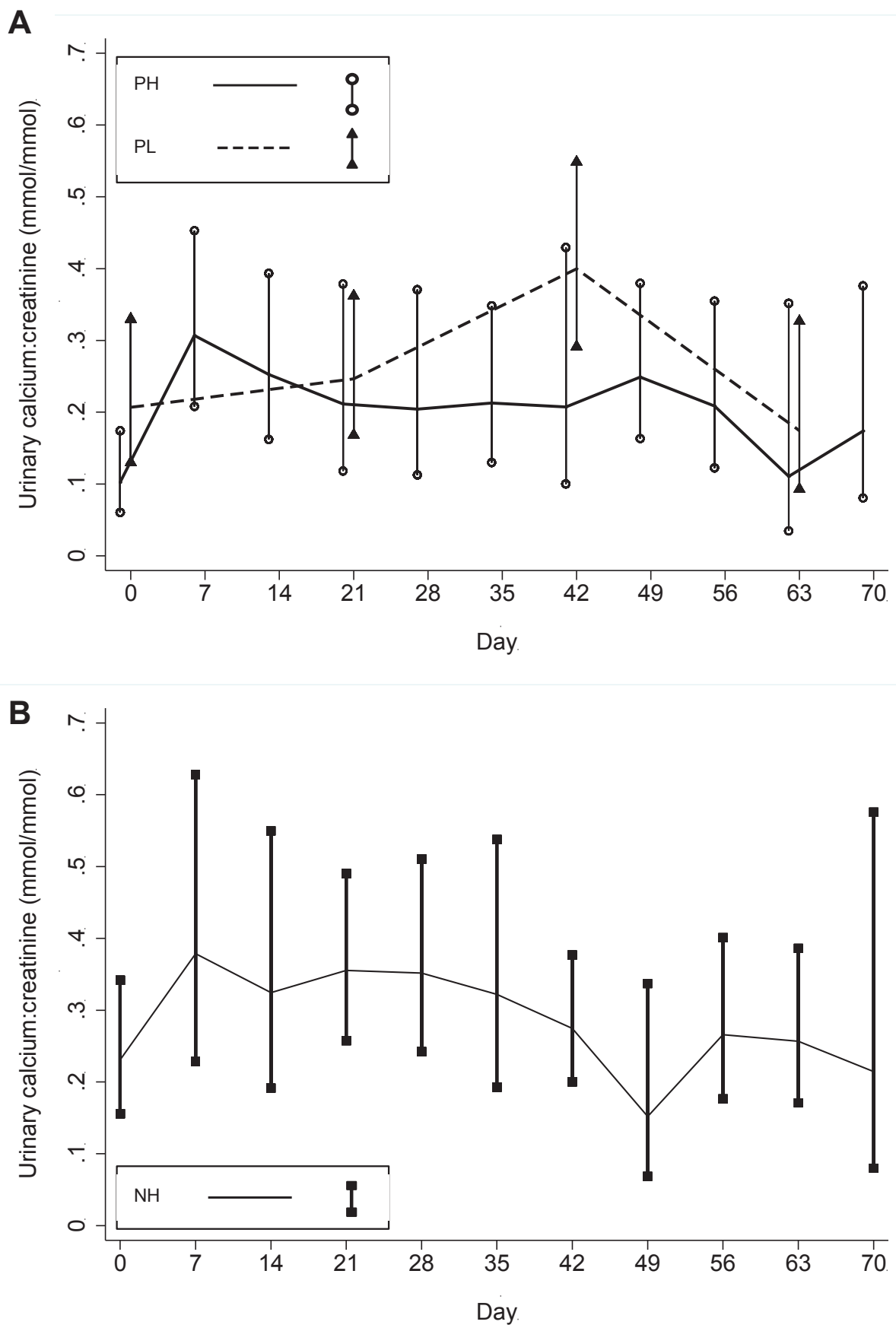


\subsection{Clinical Outcomes}

There were no known supplement-related clinical adverse events. One pregnant participant in the lower-dose group (PL) developed new-onset mild hypertension unassociated with any significant morbidity or biochemical abnormalities (highest serum [25(OH)D] was $86 \mathrm{nmol} / \mathrm{L}$ ); her pregnancy ended in an uncomplicated term delivery. The frequency of possible hypercalcemia symptoms was similar during follow-up in PH when compared to PL (odds ratio, $0.82 ; 95 \%$ CI, 0.35 to 1.92; $p=0.65$ ). Groups PH and PL were similar with respect to pregnancy and newborn outcomes (Table 6). Anthropometric measures at birth did not significantly differ between the two groups (data not shown).

Table 6. Pregnancy and newborn outcomes among women who received an initial dose of 70,000 IU vitamin D3 and 35,000 IU/week (PH) or 14,000 IU/week (PL) during the third trimester.

\begin{tabular}{|c|c|c|c|}
\hline & PH & PL & $\begin{array}{c}p \text { value } \\
\text { (for between-group difference) }\end{array}$ \\
\hline$n$ & 12 & 12 & \\
\hline \multicolumn{4}{|l|}{ Gestational age at birth, weeks (by LMP) ${ }^{1}$} \\
\hline Mean $( \pm$ SD) & $39.2( \pm 2.3)$ & $38.5( \pm 2.7)$ & 0.512 \\
\hline Range & $33.6-42.3$ & $32.7-43.2$ & \\
\hline Preterm, \# (\%) & $1(8 \%)$ & $3(25 \%)$ & 0.590 \\
\hline \multicolumn{4}{|l|}{ Birth weight (g) } \\
\hline Mean $( \pm \text { SD })^{2}$ & $2774( \pm 456)$ & $2604( \pm 379)$ & 0.332 \\
\hline Range & $2210-4000$ & $2020-3120$ & \\
\hline$\#(\%)$ SGA $^{3}$ & $9(75 \%)$ & $8(67 \%)$ & 1.000 \\
\hline \# (\%) LBW & $2(17 \%)$ & $4(33 \%)$ & 0.640 \\
\hline Delivery mode, \# (\%) Cesarean section ${ }^{4}$ & $6(50 \%)$ & $6(50 \%)$ & 1.000 \\
\hline Sex, \# $(\%)$ female & $6(50 \%)$ & $6(50 \%)$ & 1.000 \\
\hline Live births ${ }^{5}, \#(\%)$ & $12(100 \%)$ & $12(100 \%)$ & - \\
\hline Alive at 1 month of age, \# (\%) & $12(100 \%)$ & $12(100 \%)$ & - \\
\hline $\begin{array}{l}{ }^{1} \text { In a sample of } 113 \text { deliveries at the study } \\
\text { of last menstrual period, the mean gestatic } \\
\text { liveborn infants delivered at the study site } \\
{ }^{3} \text { Based on US newborn birthweight refer } \\
\text { (October } 2009 \text { to January 2010), there were } \\
\text { site (Oct } 2009 \text { to Jan 2010), there were } 7 \text { st }\end{array}$ & $\begin{array}{l}\text { October } 2009 \text { to } \\
\text { age at birth was } \\
\text { ober } 2009 \text { to Jan } \\
\text { [19]. }{ }^{4} \text { In a co } \\
\text { cesarean delive } \\
\text { rths }(2 \%) \text {. }\end{array}$ & $\begin{array}{l}\text { nuary } 2010) \text { for } \\
.7 \text { weeks }( \pm 2.2) \\
\text { ry } 2010) \text {, the me } \\
\text { ecutive sample } \\
\text { ( } 54 \%) .{ }^{5} \text { In a sa }\end{array}$ & $\begin{array}{l}\text { ich there was a recalled first day } \\
\text { In a consecutive sample of } 362 \\
\text { birth weight was } 2780 \mathrm{~g}( \pm 440) \text {. } \\
369 \text { deliveries at the study site } \\
\text { ole of } 369 \text { deliveries at the study }\end{array}$ \\
\hline
\end{tabular}

\section{Discussion}

This study demonstrated the biochemical dose response to third-trimester high-dose weekly antenatal vitamin D3 supplementation. Among Bangladeshi women with a mean $[25(\mathrm{OH}) \mathrm{D}]$ of $33 \mathrm{nmol} / \mathrm{L}, 70,000 \mathrm{IU}$ followed by 35,000 IU/week of vitamin D3 until delivery yielded an average $[25(\mathrm{OH}) \mathrm{D}]$ that was about $20 \mathrm{nmol} / \mathrm{L}$ higher than an antenatal dose of 14,000 IU/week (the IOM vitamin D upper limit at the time the study was conducted). Similar to our conclusions from analyses of single-dose vitamin D3 pharmacokinetics in the same study setting (and involving an overlapping 
group of participants) [13], we found that the minor differences between pregnant vs. non-pregnant participants receiving the same dose were within the margins of error given the small sample size. However, based on the present analysis, we could not exclude the possibility of a slightly diminished $25(\mathrm{OH}) \mathrm{D}$ response to a weekly dose of vitamin D during the third trimester of pregnancy.

To our knowledge, the 35,000 IU/week regimen used in this study is the highest vitamin D3 maintenance dose studied in pregnancy under controlled conditions. Devlin et al. (1986) reported that a daily dose of 1000 IU vitamin D3 administered to 15 French women during the third trimester modestly raised mean maternal serum $[25(\mathrm{OH}) \mathrm{D}]$ from $55 \mathrm{nmol} / \mathrm{L}$ to $65 \mathrm{nmol} / \mathrm{L}[20]$. The largest published study of vitamin D3 supplementation in pregnancy was conducted by Bruce Hollis and colleagues in South Carolina, in which 502 pregnant women at 12 to 16 weeks gestation were randomized to $400 \mathrm{IU} /$ day, $2000 \mathrm{IU} /$ day, or $4000 \mathrm{IU} /$ day vitamin D3 [21]. This population was more vitamin D-replete at baseline (mean $[25(\mathrm{OH}) \mathrm{D}]=60 \mathrm{nmol} / \mathrm{L}$ ) compared to the present study. Based on data from the 350 participants (70\%) followed until delivery, the $2000 \mathrm{IU} /$ day and $4000 \mathrm{IU} /$ day regimens raised [25(OH)D] to means of $105 \mathrm{nmol} / \mathrm{L}$ (rise of $47 \mathrm{nmol} / \mathrm{L}$ ) and $119 \mathrm{nmol} / \mathrm{L}$ (rise of $60 \mathrm{nmol} / \mathrm{L})$, respectively, at one month before delivery [21]. The $\Delta[25(\mathrm{OH}) \mathrm{D}]$ in the $2000 \mathrm{IU} / \mathrm{day}$ group in the Hollis study was similar to the response we observed in the 14,000 IU/day group (equivalent regimen) in the present study, substantiating the consistency of vitamin D3 dose-response modeling across diverse populations of pregnant women. In a separate trial in South Carolina, Wagner et al. reported comparatively less robust responses to $2000 \mathrm{IU} /$ day and $4000 \mathrm{IU} /$ day during pregnancy, which may have been attributable to non-adherence to the supplementation regimen [22].

The lower dose produced a more efficient $25(\mathrm{OH}) \mathrm{D}$ response per mcg of vitamin D3 when compared to the high-dose regimen: 0.73 vs. $0.46 \mathrm{nmol} / \mathrm{L} / \mathrm{mcg} /$ day in the empiric estimates, and 0.90 versus $0.49 \mathrm{nmol} / \mathrm{L} / \mathrm{mcg} /$ day based on the pharmacokinetic model. These estimates, as well as those from the non-pregnant cohort that received the higher-dose regimen $(0.61 \mathrm{nmol} / \mathrm{L} / \mathrm{mcg} / \mathrm{day}$ based on 10th-week data, and $0.63 \mathrm{nmol} / \mathrm{L} / \mathrm{mcg} /$ day based on the parametric model), were similar to the values conventionally cited for non-pregnant adults: $\sim 0.70 \mathrm{nmol} / \mathrm{L} / \mathrm{mcg} /$ day $[23,24]$. However, analyses by Barger-Lux et al. (1998) [25] and Aloia et al. (2008) [24], as well the recent IOM report (2010) [1], have demonstrated that the $\Delta[25(\mathrm{OH}) \mathrm{D}]$ per mcg is a curvilinear inverse function of vitamin $\mathrm{D}$ intake at doses $<50 \mathrm{mcg} /$ day, but nearly proportional to intake at $>50 \mathrm{mcg} /$ day [24], which may explain the greater observed efficiency of the lower dose.

A unique aspect of this study was the measurement of biochemical parameters between weekly doses at the end of the supplementation period. These data showed an absence of inter-dose perturbations in calcium homeostasis that might have otherwise been missed by sampling serum only at the time of the "trough" $[25(\mathrm{OH}) \mathrm{D}]$ (i.e., immediately preceding administration of a weekly dose). Although the study may have been too small to detect minor inter-dose fluctuations in $[25(\mathrm{OH}) \mathrm{D}]$, the data supported the appropriateness of administering weekly doses of 35,000 IU instead of daily administration of 5000 IU.

In pregnant participants, the higher-dose vitamin $\mathrm{D}$ regimen had a significant suppressive effect on maternal PTH secretion, relative to the lower dose, as indicated by the change in average PTH concentrations from baseline to delivery, similar to previous observations by Wagner et al. in South Carolina [22]. However, since the role of PTH as a vitamin D status biomarker during pregnancy is 
unclear [26], the clinical significance of the apparent dose-response effect of vitamin D on PTH requires further study.

Both the higher and lower vitamin D3 regimens administered to pregnant women attained fetal $[25(\mathrm{OH}) \mathrm{D}] \geq 50 \mathrm{nmol} / \mathrm{L}$. Therefore, in this small sample, we did not observe a clear benefit of the higher-dose over the lower-dose regimen with respect to neonatal vitamin D status. In a related study at the same study site, we observed a mean cord [25(OH)D] of $50 \mathrm{nmol} / \mathrm{L}$ (range of 29 to $80 \mathrm{nmol} / \mathrm{L}$ ) in a group of neonates born to women who had received a single vitamin D3 dose of 70,000 IU at 30 weeks gestation [13], and previous studies in South Asia have found cord serum [25(OH)D] ranging from 17 to $59 \mathrm{nmol} / \mathrm{L}$ [27-30].

Appreciable increases in serum calcium in the higher-dose relative to the lower-dose group highlighted a dose-dependent effect of vitamin D3 supplementation on calcium homeostasis. We previously reported that mean serum calcium concentrations rose slightly but significantly during the first week after administration of a single 70,000 IU dose of vitamin D3 in both pregnant and non-pregnant participant groups [13]. However, in the present analyses of weekly-dose vitamin D3, a significant increase in serum [Ca] from baseline was only observed in pregnant women who received the higher dose. Pregnancy is associated with an elevation in the maternal serum concentration of the active vitamin D metabolite, 1,25-dihydroxyvitamin D (1,25(OH)2D) [31,32], which appears to be primarily attributable to classic renal $1 \alpha$-hydroxylation of $25(\mathrm{OH}) \mathrm{D}$ [33]. However, placental trophoblasts and decidual cells [34] are capable of extra-renal $1 \alpha$-hydroxylation which could theoretically predispose the pregnant woman to exaggerated physiological responses to increases in [25(OH)D] [9]. Similar to the participants who received only a single dose of 70,000 IU [13], maternal serum calcium values in the weekly-dose participants were all below the threshold for defining hypercalcemia used by the IOM in setting the 1997 dietary reference intakes (DRIs) for vitamin D (2.75 mmol/L) [35] and in the revised DRIs in 2010 (2.63 mmol/L) [1]. Cord blood calcium concentrations were also within reference limits, and $[25(\mathrm{OH}) \mathrm{D}]$ were well below the range that has been associated with toxicity in adults [36] and older children [37]. Pregnancy and newborn clinical outcomes were within the expected range for the study population, but we were unable to draw conclusions from this study regarding clinical effects of vitamin D. Nonetheless, this study together with the recent findings of Hollis and Wagner and colleagues in South Carolina [21,22] demonstrate that vitamin D3 doses during pregnancy up to $25 \%$ above the current IOM UL of 4000 IU/day do not induce hypercalcemia, and have not led to any observed short-term clinical adverse effects.

There were several important limitations of this study. First, precision of estimates of pharmacokinetic parameters and between-group comparisons, as well as the generalizability of inferences regarding maternal-fetal safety of high-dose vitamin D supplementation, were limited by the small number of participants, stringent inclusion/exclusion criteria, and enrolment of pregnant and non-pregnant participants at one clinic site. Moreover, the lower-dose pregnancy group had less frequent blood sampling (a cost-savings measure given the relative lack of safety concerns for this group) and only 9 of 14 enrolled women contributed endpoint samples during the 10th week of supplementation. The supplementation period may not have been long enough to ensure that all participants reached a steady-state $[25(\mathrm{OH}) \mathrm{D}]$. Conclusions based on comparisons between pregnant and non-pregnant women were tempered by the differences in baseline characteristics, including 
season of enrolment and the relatively higher socioeconomic status of the non-pregnant participants. In addition, there were too few participants to consider modifiers of $\Delta[25(\mathrm{OH}) \mathrm{D}]$. Most importantly, the present results do not yet provide sufficient evidence that the regimens studied are beneficial or safe for use in clinical or public health practice; rather, they serve to inform application of these dose regimens in future research studies.

\section{Conclusions}

This detailed analysis of the response to high-dose weekly vitamin D3 administered during the third-trimester of pregnancy demonstrated a dose-responsiveness to oral vitamin D3 in Bangladeshi women that echoed observations in other settings, and was generally in accordance with established pharmacokinetic characteristics of vitamin D3. Nonetheless, increases in the mean calcium concentration (within the normal range) and suppression of PTH secretion among pregnant women receiving the higher-dose regimen (70,000 IU initial dose followed by weekly doses of 35,000 IU) highlighted the physiological impact of the intervention and the need to cautiously address potential pregnancy-specific sensitivities to vitamin D supplementation.

Prior to undertaking large trials to test the effects of prenatal micronutrient interventions on pregnancy and birth outcomes, preliminary dose-finding and safety studies are essential, particularly when the intervention is a fat-soluble vitamin at a dose above the conventional upper limit of tolerability (i.e., $4000 \mathrm{IU} /$ day for vitamin $\mathrm{D}$, as established by the Institute of Medicine [1]). The most direct application of the present observations is to guide the design of future trials of vitamin D3 (at doses up to 35,000 IU per week) aimed at confirming safety and establishing the health benefits of antenatal vitamin D supplementation in South Asia, where many potentially vitamin D-responsive outcomes (e.g., infant growth and infectious disease morbidity) are major public health priorities. Following from our preliminary pharmacokinetic studies, we have conducted a placebo-controlled trial of 35,000 IU/ week during the third trimester $(n=160)$, with follow-up of infants to monitor growth to one year of age (NCT01126528). Future trials in Dhaka will address the dose-dependency of the effects of prenatal vitamin D supplementation on infant growth and morbidity.

\section{Acknowledgments}

We appreciate the efforts of the following individuals: staff at icddr,b and Shimantik, including Taufiq Rahman, Sultana Mahabbat-e Khoda, Eliza Roy, Ashish Chowdhury, and Kazi Moksedur Rahman; Reinhold Vieth (Mount Sinai Hospital, Toronto) for performing the measurement of 25-hydroxyvitamin D concentrations and verifying the concentration of the vitamin D3 supplement; Brendon Pezzack for assistance with manuscript preparation. We also thank Diasorin Inc. (Stillwater, MN) for donating the kits used in the Liaison Total assay, and Popular Pharmaceuticals Inc. (Dhaka, Bangladesh) for supplying the Vigantol Oil. Daniel Roth was supported by The Alberta Heritage Foundation for Medical Research (Canada) and The Canadian Institutes of Health Research. The study was sponsored by the Center for Global Health, Johns Hopkins University and the Department of International Health at The Johns Hopkins Bloomberg School of Public Health. 


\section{Conflicts of Interest}

The authors declare no conflict of interest.

\section{Appendix}

Non-Linear Modeling of Change in 25[(OH)D] over Time

Mean changes in $[25(\mathrm{OH}) \mathrm{D}]$ over time in each group were modeled as continuous non-linear parametric functions. Consistent with the first-order process that characterizes 25(OH)D metabolism in the physiological range of vitamin D inputs, Heaney's group has shown empirically that a negative exponential growth function is well suited to model the gradual rise in $[25(\mathrm{OH}) \mathrm{D}]$ over time $(t)$ to a steady-state plateau in response to daily (Heaney et al., 2003 [23]) or weekly (Heaney et al., 2011 [38]) oral vitamin D3 supplementation:

$$
[25(O H) D]_{t}=[25(O H) D]_{t=0}+\alpha\left(1-\mathrm{e}^{-k t}\right)
$$

A particular advantage of this model is that despite its non-linearity, the coefficients are easily interpreted: $a$ is the $\Delta[25(\mathrm{OH}) \mathrm{D}]$ above baseline at steady-state and $k$ is the slope that defines the rate of the rise (the higher is $k$, the more rapidly the steady-state is reached). The steady-steady is the $[25(\mathrm{OH}) \mathrm{D}]$ at which the rate of $25(\mathrm{OH}) \mathrm{D}$ formation theoretically equals the rate of $25(\mathrm{OH}) \mathrm{D}$ utilization/catabolism. Furthermore, the model could be readily extended to permit comparisons between groups of participants (g), with the aim of estimating the average difference (d) between the groups' $[25(\mathrm{OH}) \mathrm{D}]$ at steady-state (see below for derivation of the extended model):

$$
[25(O H) D]_{t}=[25(O H) D]_{t=0}+\beta g+\left(g d+\alpha_{0}\right)\left(1-\mathrm{e}^{-k t}\right)
$$

Regression coefficients were estimated by a non-linear least-squares approach, assuming a log-normal error distribution of $[25(\mathrm{OH}) \mathrm{D}]$ and standard error estimation that accounted for the intra-subject correlation of repeated measures.

To derive an extended negative exponential growth function that enabled comparison of the steady-state concentrations between the two groups, we first considered a generic model for the negative exponential growth function, where $[25(\mathrm{OH}) \mathrm{D}]$ at time $t$ is a function of the baseline concentration $[25(\mathrm{OH}) \mathrm{D}]$ at $\mathrm{t}=0$, the slope of the exponential rise, $k$, and the asymptotic maximal rise above baseline, $a$, at steady-state $(t=$ infinity):

$$
[25(O H) D]_{t}=[25(O H) D]_{t=0}+\alpha\left(1-\mathrm{e}^{-k t}\right)
$$

We can consider Equation (1) with respect to two different groups, $g$, such that if:

$$
[25(\mathrm{OH}) D]_{t}=[25(\mathrm{OH}) D]_{t=0}+\alpha_{0}\left(1-\mathrm{e}^{-k t}\right) \text { if } g=0
$$

Then,

$$
[25(O H) D]_{t}=[25(O H) D]_{t=0}+\alpha_{0}(1-g)\left(1-\mathrm{e}^{-k t}\right)
$$

Similarly for group $g=1$, if:

$$
[25(O H) D]_{t}=[25(O H) D]_{t=0}+\alpha_{1}\left(1-\mathrm{e}^{-k t}\right) \text { if } g=1
$$


Then,

$$
[25(O H) D]_{t}=[25(O H) D]_{t=0}+\alpha_{1} g\left(1-\mathrm{e}^{-k t}\right)
$$

Combining Equation (3) and Equation (4) into one model, and allowing the intercept to vary by group:

$$
[25(O H) D]_{t}=[25(O H) D]_{t=0}+\beta g+\alpha_{1} g\left(1-\mathrm{e}^{-k t}\right)+\alpha_{0}(1-g)\left(1-\mathrm{e}^{-k t}\right)
$$

Solving further:

$$
\begin{aligned}
& {[25(O H) D]_{t}=[25(O H) D]_{t=0}+\beta g+\left[\alpha_{1} g+\alpha_{0}(1-g)\right]\left(1-\mathrm{e}^{-k t}\right)} \\
& \left.[25(O H) D]_{t}=[25(O H) D]_{t=0}+\beta g+\left[\alpha_{1} g+\alpha_{0}-\alpha_{0} g\right)\right]\left(1-\mathrm{e}^{-k t}\right) \\
& {[25(O H) D]_{t}=[25(O H) D]_{t=0}+\beta g+\left[g\left(\alpha_{1}-\alpha_{0}\right)+\alpha_{0}\right]\left(1-\mathrm{e}^{-k t}\right)}
\end{aligned}
$$

Since we are specifically interested in measuring the difference between $a_{1}$ and $a_{0}$, we can invoke a new coefficient $d$, whereby:

$$
d=\alpha_{1}-\alpha_{0}
$$

Then, substituting Equation (7) into Equation (6) yields:

$$
[25(O H) D]_{t}=[25(O H) D]_{t=0}+\beta g+\left(g d+\alpha_{0}\right)\left(1-\mathrm{e}^{-k t}\right)
$$

\section{References}

1. Ross, A.C.; Taylor, C.L.; Yaktine, A.L.; Del Valle, H.B. Committee to Review Dietary Reference Intakes for Vitamin D and Calcium, Institute of Medicine. Dietary Reference Intakes for Calcium and Vitamin D; The National Academies Press: Washington, DC, USA, 2010.

2. De-Regil, L.M.; Palacios, C.; Ansary, A.; Kulier, R.; Pena-Rosas, J.P. Vitamin D supplementation for women during pregnancy. Cochrane Database Syst. Rev. 2012, 2, doi:10.1002/14651858.CD008873.pub2.

3. Finkelstein, J.L.; Mehta, S.; Duggan, C.; Manji, K.P.; Mugusi, F.M.; Aboud, S.; Spiegelman, D.; Msamanga, G.I.; Fawzi, W.W. Maternal vitamin D status and child morbidity, anemia, and growth in human immunodeficiency virus-exposed children in Tanzania. Pediatr. Infect. Dis. J. 2012, 31, 171-175.

4. Belderbos, M.E.; Houben, M.L.; Wilbrink, B.; Lentjes, E.; Bloemen, E.M.; Kimpen, J.L.; Rovers, M.; Bont, L. Cord blood vitamin D deficiency is associated with respiratory syncytial virus bronchiolitis. Pediatrics 2011, 127, e1513-e1520.

5. Morales, E.; Guxens, M.; Llop, S.; Rodriguez-Bernal, C.L.; Tardon, A.; Riano, I.; Ibarluzea, J.; Lertxundi, N.; Espada, M.; Rodriguez, A.; et al. Circulating 25-hydroxyvitamin D3 in pregnancy and infant neuropsychological development. Pediatrics 2012, 130, e913-e920.

6. Bischoff-Ferrari, H.; Shao, A.; Dawson-Hughes, B.; Hathcock, J.; Giovannucci, E.; Willett, W. Benefit-risk assessment of vitamin D supplementation. Osteoporosis Int. 2010, 21, 1121-1132.

7. Vieth, R. What is the optimal vitamin D status for health? Prog. Biophys. Mol. Biol. 2006, 92, 26-32. 
8. Hollis, B.W. Circulating 25-hydroxyvitamin D levels indicative of vitamin D sufficiency: Implications for establishing a new effective dietary intake recommendation for vitamin D. J. Nutr. 2005, 135, 317-322.

9. Roth, D.E. Vitamin D supplementation during pregnancy: Safety considerations in the design and interpretation of clinical trials. J. Perinatol. 2011, 31, 449-459.

10. Black, R.E.; Allen, L.H.; Bhutta, Z.A.; Caulfield, L.E.; de Onis, M.; Ezzati, M.; Mathers, C.; Rivera, J. Maternal and child undernutrition: Global and regional exposures and health consequences. Lancet 2008, 371, 243-260.

11. Islam, M.Z.; Lamberg-Allardt, C.; Karkkainen, M.; Outila, T.; Salamatullah, Q.; Shamim, A.A. Vitamin D deficiency: A concern in premenopausal Bangladeshi women of two socio-economic groups in rural and urban region. Eur. J. Clin. Nutr. 2002, 56, 51-56.

12. Roth, D.E.; Shah, M.R.; Black, R.E.; Baqui, A.H. Vitamin D status of infants in northeastern rural Bangladesh: Preliminary observations and a review of potential determinants. J. Health Popul. Nutr. 2010, 28, 458-469.

13. Roth, D.E.; Mahmud, A.; Raqib, R.; Black, R.E.; Baqui, A.H. Pharmacokinetics of a single oral dose of vitamin D3 (70,000 IU) in pregnant and non-pregnant women. Nutr. J. 2012, 11, doi:10.1186/1475-2891-11-114.

14. Wagner, D.; Hanwell, H.E.; Vieth, R. An evaluation of automated methods for measurement of serum 25-hydroxyvitamin D. Clin. Biochem. 2009, 42, 1549-1556.

15. Carter, G.D.; Berry, J.L.; Gunter, E.; Jones, G.; Jones, J.C.; Makin, H.L.; Sufi, S.; Wheeler, M.J. Proficiency testing of 25-hydroxyvitamin D (25-OHD) assays. J. Steroid. Biochem. Mol. Biol. 2010, 121, 176-179.

16. Perkins, S.L.; Livesey, J.F.; Belcher, J. Reference intervals for 21 clinical chemistry analytes in arterial and venous umbilical cord blood. Clin. Chem. 1993, 39, 1041-1044.

17. Vieth, R.; Chan, P.C.; MacFarlane, G.D. Efficacy and safety of vitamin D3 intake exceeding the lowest observed adverse effect level. Am. J. Clin. Nutr. 2001, 73, 288-294.

18. Gordon, A.Y.; Salzman, P. Optimality of the holm procedure among general step-down multiple testing procedures. Stat. Probab. Lett. 2008, 78, 1878-1884.

19. Oken, E.; Kleinman, K.P.; Rich-Edwards, J.; Gillman, M.W. A nearly continuous measure of birth weight for gestational age using a United States national reference. BMC Pediatr. 2003, 3, doi:10.1186/1471-2431-3-6.

20. Delvin, E.E.; Salle, B.L.; Glorieux, F.H.; Adeleine, P.; David, L.S. Vitamin D supplementation during pregnancy: Effect on neonatal calcium homeostasis. J. Pediatr. 1986, 109, 328-334.

21. Hollis, B.W.; Johnson, D.; Hulsey, T.C.; Ebeling, M.; Wagner, C.L. Vitamin D supplementation during pregnancy: Double blind, randomized clinical trial of safety and effectiveness. J. Bone Miner Res. 2011, 26, 2341-2357.

22. Wagner, C.L.; McNeil, R.; Hamilton, S.A.; Winkler, J.; Rodriguez Cook, C.; Warner, G.; Bivens, B.; Davis, D.J.; Smith, P.G.; Murphy, M.; et al. A randomized trial of vitamin D supplementation in 2 community health center networks in south carolina. Am. J. Obstet. Gynecol. 2013, 208, 137.e1-137.e13. 
23. Heaney, R.P.; Davies, K.M.; Chen, T.C.; Holick, M.F.; Barger-Lux, M.J. Human serum 25-hydroxycholecalciferol response to extended oral dosing with cholecalciferol. Am. J. Clin. Nutr. 2003, 77, 204-210.

24. Aloia, J.F.; Patel, M.; Dimaano, R.; Li-Ng, M.; Talwar, S.A.; Mikhail, M.; Pollack, S.; Yeh, J.K. Vitamin D intake to attain a desired serum 25-hydroxyvitamin D concentration. Am. J. Clin. Nutr. 2008, 87, 1952-1958.

25. Barger-Lux, M.J.; Heaney, R.P.; Dowell, S.; Chen, T.C.; Holick, M.F. Vitamin D and its major metabolites: Serum levels after graded oral dosing in healthy men. Osteoporos. Int. 1998, 8, 222-230.

26. Wagner, C.L.; Hollis, B.W. Beyond PTH: Assessing vitamin D status during early pregnancy. Clin. Endocrinol. 2011, 75, 285-286.

27. Goswami, R.; Gupta, N.; Goswami, D.; Marwaha, R.K.; Tandon, N.; Kochupillai, N. Prevalence and significance of low 25-hydroxyvitamin D concentrations in healthy subjects in delhi. Am. J. Clin. Nutr. 2000, 72, 472-475.

28. Sachan, A.; Gupta, R.; Das, V.; Agarwal, A.; Awasthi, P.K.; Bhatia, V. High prevalence of vitamin $\mathrm{D}$ deficiency among pregnant women and their newborns in northern India. Am. J. Clin. Nutr. 2005, 81, 1060-1064.

29. Bhalala, U.; Desai, M.; Parekh, P.; Mokal, R.; Chheda, B. Subclinical hypovitaminosis D among exclusively breastfed young infants. Indian Pediatr. 2007, 44, 897-901.

30. Doi, M.; Sultana Rekha, R.; Ahmed, S.; Okada, M.; Kumar Roy, A.; El Arifeen, S.; Ekstrom, E.C.; Raqib, R.; Wagatsuma, Y. Association between calcium in cord blood and newborn size in Bangladesh. Br. J. Nutr. 2011, 106, 1398-1407.

31. Kovacs, C.S. Vitamin D in pregnancy and lactation: Maternal, fetal, and neonatal outcomes from human and animal studies. Am. J. Clin. Nutr. 2008, 88, 520S-528S.

32. Papapetrou, P.D. The interrelationship of serum 1,25-dihydroxyvitamin D, 25-hydroxyvitamin $\mathrm{D}$ and 24,25-dihydroxyvitamin D in pregnancy at term: A meta-analysis. Hormones (Athens) 2010, 9, 136-144.

33. Kovacs, C.S.; Kronenberg, H.M. Maternal-fetal calcium and bone metabolism during pregnancy, puerperium, and lactation. Endocr. Rev. 1997, 18, 832-872.

34. Zehnder, D.; Evans, K.N.; Kilby, M.D.; Bulmer, J.N.; Innes, B.A.; Stewart, P.M.; Hewison, M. The ontogeny of 25-hydroxyvitamin $\mathrm{D}(3)$ 1alpha-hydroxylase expression in human placenta and decidua. Am. J. Pathol. 2002, 161, 105-114.

35. Standing Committee on the Scientific Evaluation of Dietary Reference Intakes; Food and Nutrition Board; Institute of Medicine. DRI: Dietary Reference Intakes for Calcium, Phosphorus, Magnesium, Vitamin D, and Fluoride; National Academy Press: Washington, DC, USA, 1997.

36. Hathcock, J.N.; Shao, A.; Vieth, R.; Heaney, R. Risk assessment for vitamin D. Am. J. Clin. Nutr. 2007, 85, 6-18.

37. Joshi, R. Hypercalcemia due to hypervitaminosis D: Report of seven patients.J. Trop. Pediatr. 2009, 55, 396-398.

38. Heaney, R.P.; Recker, R.R.; Grote, J.; Horst, R.L.; Armas, L.A. Vitamin D(3) is more potent than vitamin D(2) in humans. J. Clin. Endocrinol. Metab. 2011, 96, E447-E452. 



\section{Chapter 3}

\section{Health Impact}





\title{
Does Vitamin D Sufficiency Equate to a Single Serum 25-Hydroxyvitamin D Level or Are Different Levels Required for Non-Skeletal Diseases?
}

\section{Simon Spedding, Simon Vanlint, Howard Morris and Robert Scragg}

\begin{abstract}
Objective: Clarify the concept of vitamin D sufficiency, the relationship between efficacy and vitamin D status and the role of Vitamin D supplementation in the management of non-skeletal diseases. We outline reasons for anticipating different serum vitamin D levels are required for different diseases. Method: Review the literature for evidence of efficacy of supplementation and minimum effective 25-hydroxyvitamin D (25-OHD) levels in non-skeletal disease. Results: Evidence of efficacy of vitamin supplementation is graded according to levels of evidence. Minimum effective serum 25-OHD levels are lower for skeletal disease, e.g., rickets ( $25 \mathrm{nmol} / \mathrm{L})$, osteoporosis and fractures $(50 \mathrm{nmol} / \mathrm{L})$, than for premature mortality $(75 \mathrm{nmol} / \mathrm{L})$ or non-skeletal diseases, e.g., depression $(75 \mathrm{nmol} / \mathrm{L})$, diabetes and cardiovascular disease $(80 \mathrm{nmol} / \mathrm{L})$, falls and respiratory infections (95 nmol/L) and cancer $(100 \mathrm{nmol} / \mathrm{L})$. Conclusions: Evidence for the efficacy of vitamin D supplementation at serum 25-OHD levels ranging from 25 to $100 \mathrm{nmol} / \mathrm{L}$ has been obtained from trials with vitamin D interventions that change vitamin D status by increasing serum 25-OHD to a level consistent with sufficiency for that disease. This evidence supports the hypothesis that just as vitamin D metabolism is tissue dependent, so the serum levels of 25-OHD signifying deficiency or sufficiency are disease dependent.
\end{abstract}

Reprinted from Nutrients. Cite as: Spedding, S.; Vanlint, S.; Morris, H.; Scragg, R. Does Vitamin D Sufficiency Equate to a Single Serum 25-Hydroxyvitamin D Level or Are Different Levels Required for Non-Skeletal Diseases? Nutrients 2013, 5, 5127-5139.

\section{Introduction}

Vitamin D supplementation is established in both the prevention and treatment of bone disease [1] but its role in other clinical situations remains contentious. This article examines the role of vitamin $\mathrm{D}$ in the management of non-skeletal disease.

The term "vitamin D" is a misnomer. It is a secosteroid pro-hormone synthesized in response to sunshine exposure and has autocrine, paracrine and endocrine modes of action [2]. Vitamin D receptors (VDRs) occupy 2776 genomic positions and modulate 229 genes [3]. The wide distribution of VDRs suggests a role for vitamin D in most physiological systems of the body, implying that deficiency may lead to physiological changes predisposing to chronic diseases. Vitamin D deficiency is a public health concern as it is pandemic [4], affecting a third of the population, even in tropical and subtropical regions [5-10]. There is considerable debate about terminology and serum 25-hydroxyvitamin D (25-OHD) levels [11]. A 25-OHD concentration for vitamin D deficiency is defined as $<50 \mathrm{nmol} / \mathrm{L}$ and sufficiency as $>75 \mathrm{nmol} / \mathrm{L}$ in this paper, although the concept of a single level for sufficiency is not universal, one example being acetylsalicylic acid that requires different serum levels for analgesia and anticoagulation. 
There is substantial disagreement about what constitutes vitamin D sufficiency and the role of vitamin $\mathrm{D}$ in the management of non-skeletal disease, despite an exponential increase in vitamin D research and myriad of consensus statements and guidelines $[12,13]$. Thus clinicians still lack clear information about efficacy of vitamin D and the relationship between supplement dose or vitamin D status and clinical response in non-skeletal disease.

Whilst the influential Institute of Medicine (IOM) report on calcium and vitamin D asserted that the role for vitamin D was exclusive to bone health, based on null studies relating to non-skeletal disease [1], more than 12,000 vitamin D articles have been published since the IOM report [12]. This suggests it is timely to review the role of vitamin D in non-skeletal disease. Furthermore the view is gaining traction that these null studies relating to non-skeletal disease were due to methodological limitations of inadequate doses of vitamin D and inappropriate baseline levels of 25-OHD that led to inept conclusions about vitamin D efficacy $[13,14]$. Studies and reviews with these limitations were identified using the approach advocated by Lappe and Heaney [15]. These include studies that did not measure baseline 25OHD levels [16] or used doses of vitamin D that were unlikely to achieve sufficiency $[16,17]$ or whose participants who were already vitamin D replete $[18,19]$.

\section{Tissue Dependence of Vitamin D}

These design limitations have a profound effect on the results due to the pharmacokinetics of vitamin D. Unlike most vitamins and many nutrients, vitamin D activity is tissue-dependent. Different levels of 25-OHD have different therapeutic effects which vary according to the target tissue [2]. 25-OHD is not the active form of vitamin $\mathrm{D}$ and requires hydroxylation to the active form 1,25-dihydroxyvitamin $\mathrm{D}\left(1,25 \mathrm{OH}_{2} \mathrm{D}\right)$. This occurs either in the kidney, which accounts for circulating concentrations of $1,25 \mathrm{OH}_{2} \mathrm{D}$, and locally in specific tissues through the action of the enzyme 25-hydroxyvitamin $\mathrm{D}_{3}-1 \alpha$-hydroxylase. However the levels of this enzyme vary between tissues, therefore the serum $25-\mathrm{OHD}$ concentration required for the adequate synthesis of $1,25 \mathrm{OH}_{2} \mathrm{D}$ also varies between tissues [20].

It follows that each physiological or therapeutic effect may require a specific minimum serum 25-OHD concentration. Good evidence already exists for differential levels in bone disease, with most evidence suggesting that serum 25-OHD concentrations of at least 20-25 nmol/L are required to prevent rickets, but that higher concentrations of $50-60 \mathrm{nmol} / \mathrm{L}$ are required to prevent osteoporosis and fractures [21].

We explore the evidence for therapeutic levels in non-skeletal disease, and hypothesise that tissue dependence may be responsible for the limitations of some studies included in previous reviews. 


\section{Aims of This Paper}

1. To examine trials and reviews of vitamin D supplementation in non-skeletal diseases.

2. To examine the minimum effective levels of 25-OHD that are associated with non-skeletal diseases.

3. To assess the level of evidence for the efficacy of vitamin D supplementation in non-skeletal disease.

\section{Methods}

This review focuses on studies with a primary endpoint of a non-skeletal disease using therapeutic doses that were shown to improve participants' vitamin D status. Studies were collected through Medline searches and by examining the publications of two US groups: the IOM [1] and the Vitamin D Council [12].

The authors reviewed evidence for vitamin $\mathrm{D}$ in the management of non-skeletal diseases. Studies and reviews with significant limitations due to design flaws were identified, using the approach advocated by Lappe and Heaney [15], which emphasises the value of RCTs, where baseline vitamin D status was known and where the supplemental dose of vitamin D administered could therefore be shown to have improved vitamin D status. However, excluding studies which did not satisfy all of these criteria was not always possible due to limitations of the current evidence base.

Consensus statements will be presented for:

1. The highest level of evidence for each non-skeletal disease, evaluated according to the Australian National Health and Medical Research Council levels of evidence (Table 1) [22];

2. The minimum effective serum level of 25-OHD in non-skeletal disease.

Table 1. Australian National Health and Medical Research Council (Australia) Evidence Hierarchy: designations of "levels of evidence" [22].

\begin{tabular}{cl}
\hline Level & \multicolumn{1}{c}{ Intervention } \\
\hline I & Systematic review of Level II studies \\
II & Randomised controlled trial \\
III-1 & Pseudo-randomised controlled trial \\
III-2 & Comparative study with concurrent controls: non-randomised, experimental \\
& trial, cohort study, case-control study, or interrupted time series with a \\
& control group \\
III-3 & A comparative study without concurrent controls: historical control study, \\
& or \\
& two or more single arm study, Interrupted time series without a parallel \\
IV & control group \\
& Case series with either post-test or pre-test/post-test outcomes \\
\hline
\end{tabular}




\section{Findings}

Premature mortality - There is Level I evidence that vitamin D supplementation reduces premature mortality. A Cochrane review of randomized controlled trials (RCTs) showed a 6\% reduction in all-cause mortality [23], with observational data from the NHANES and ESTHER studies suggesting a minimum 25-OHD concentration of $75 \mathrm{nmol} / \mathrm{L}$ for preventing premature mortality [24,25].

Cancer-There is Level II evidence from one RCT that vitamin D supplementation leads to a substantial reduction in overall risk of internal cancers [26]. The reported minimum 25-OHD concentration for cancer prevention is $100 \mathrm{nmol} / \mathrm{L}$ [27] whilst a review shows an inverse, non-linear relationship between 25-OHD levels and cancer risk [28].

Muscle function and falls - There is Level I evidence from a meta-analysis that demonstrated a $26 \%$ reduction in falls with vitamin D supplementation [29]. Other meta-analyses [30-32] that included RCTs with design limitations reached a null conclusion [33]. The optimal 25-OHD concentration for lowest risk of falls is $95 \mathrm{nmol} / \mathrm{L}$ [34].

There is Level II evidence for the efficacy of vitamin D supplementation in vitamin D deficiency for lower limb muscle strength [35].

Cardiovascular disease-There is Level III-2 evidence that vitamin D protects against cardiovascular disease from three meta-analyses of cohort studies [36,37]. One study of 300,000 person years demonstrated a 39\% reduction in IHD in people with high 25-OHD concentrations in comparison with those with severe deficiency and a similar effect on incidence of ischaemic stroke [38]. In another study, Vitamin D supplementation conferred substantial survival benefit (OR for death $0.39 p<0.0001)$ in cardiac patients [39]. These estimates are very similar to those proposed in an earlier systematic review and meta-analysis by Parker et al. [40]. The reported minimum 25-OHD concentration associated with lowest CVD risk is $80 \mathrm{nmol} / \mathrm{L} \mathrm{[41].}$

Respiratory infections - There is Level II evidence that vitamin D supplementation reduces rates of respiratory infections including pneumonia [42], influenza [43] and upper respiratory infections [44]. This is supported by epidemiological studies for tuberculosis $[45,46]$ and other respiratory infections [47-49]. The reported minimum 25OH-D concentration associated with a reduced incidence of viral respiratory infections is $95 \mathrm{nmol} / \mathrm{L}$ [50].

Diabetes - There is Level II evidence from two randomized controlled trials that vitamin D supplementation improves insulin sensitivity and decreases insulin resistance [51,52]. This is supported by three meta-analysis of cohort studies, which found low 25-OHD concentrations predicted increased risk of type 2 diabetes [53-55]. The reported minimum vitamin D concentration for reducing insulin resistance is $80 \mathrm{nmol} / \mathrm{L}$ [56].

Depression-There is Level II evidence that vitamin D supplementation is effective in treating depression from four RCTs in which supplementation significantly reduced depression in vitamin D deficient depressed patients [56-59]. The reported minimum 25OH-D concentration associated with reduced depression is $95 \mathrm{nmol} / \mathrm{L}$ [58].

Dental disease - There is Level II evidence from a RCT for dental caries [60]. There is also evidence from the NHANES database showing that attachment loss (AL) is significantly and 
inversely correlated with serum 25-OHD concentrations in those aged over 50 years AL was lowest in individuals whose 25-OHD levels exceeded $84 \mathrm{nmol} / \mathrm{L}$ [61].

Pain-There is Level II evidence for the efficacy of vitamin D supplementation in chronic musculoskeletal pain patients who are also vitamin D deficient [62]. An RCT in vitamin D insufficient non-Western immigrants in the Netherlands showed a clinically meaningful positive effect on pain 6 weeks after high-dose vitamin D supplementation [63]. No threshold level has been proposed.

Health service utilisation and costs - There is Level III-2 evidence that vitamin D deficiency increases health care utilisation and cost. A large study of health care costs in US veterans found that vitamin D deficiency predicted increased health care costs at all six participating sites [64]. Another study estimated that health care utilisation was 39\% higher in vitamin D deficient patients [65]. Vitamin D supplementation may be more cost effective than currently funded prevention programs; single studies show that supplementation appears to be more cost effective than screening programs for bowel cancer [66] and more cost effective than multifactorial interventions for preventing falls in the elderly [67]. Considering the economic burden of disease due to vitamin D deficiency, the estimated benefit of increasing vitamin D status across Western Europe was said to be $€ 187 \mathrm{~b}$ per year [68], calculated on a population basis the equivalent figure for Australia was $\$ 14 \mathrm{~b}$ per year.

\section{Summary of the Evidence}

There is evidence for the efficacy of vitamin D (Table 2):

Level I for prevention of falls and premature mortality;

Level II for prevention of diabetes, respiratory infections, cancer and for managing musculoskeletal pain and depression;

Level III for health service utilisation, prevention of cardiovascular disease, musculoskeletal strength and dental disease.

Evidence supports the contention that specific 25-OHD levels may be required to prevent disease, with higher concentrations required for non-skeletal disease. Table 2 shows the levels associated with lowest risk of the following diseases:

20-25 nmol/L for osteomalacia, and rickets,

50-60 nmol/L for osteoporosis, and fractures,

75-85 nmol/L for premature mortality cardiovascular disease, diabetes, and for treatment of depression and dental disease,

95-100 nmol/L for falls, cancer, and respiratory infections. 
Table 2. Proposed vitamin D concentrations in the management of non-skeletal diseases.

\begin{tabular}{lll}
\hline & $\begin{array}{l}\text { Level of evidence } \\
\text { NHMRC }[\mathbf{2 4}]\end{array}$ & $\begin{array}{l}\text { Minimum effective serum 25-OHD } \\
\text { concentration in } \text { nmol/L [24] }\end{array}$ \\
\hline Premature mortality & Level 1 & 75 \\
Falls prevention & Level 1 & 95 \\
Cancer prevention & Level ll & 100 \\
Respiratory infection prevention & Level ll & 95 \\
Diabetes prevention & Level ll & 80 \\
Depression treatment & Level ll & 75 \\
Musculoskeletal pain management & Level ll & \\
$\begin{array}{l}\text { Dental disease } \\
\text { Musculoskeletal strength }\end{array}$ & Level ll1-2 & $>84$ \\
Cardiovascular disease & Level lll-1 & \\
Health service utilisation & Level lll-2 & 80 \\
\hline
\end{tabular}

\section{Discussion}

The evidence supports the following conclusions:

- The use of vitamin D supplements in the management of non-skeletal diseases is supported by; High level of evidence for reducing premature mortality, falls (Level I), Moderate evidence for reducing diabetes, respiratory infections, depression and some cancers (Level II), and a Lower level of evidence for preventing cardiovascular disease, and treating musculoskeletal pain and dental disease(Level III).

- The minimum level of 25-OHD required for preventing non-skeletal disease is higher than for skeletal disease.

- Economic evaluations indicate vitamin D supplementation reduces health service costs.

However there is still skepticism about the role of vitamin D in non-skeletal disease based on the IOM report [1]. Since this report there have been 12,000 vitamin D publications, including reviews showing increasing consistency in the evidence about efficacy and the therapeutic levels in non-skeletal disease [69-72]. This evidence comes from research in different disciplines, study designs, and countries. Beyond this evidence, there are publications showing how the Bradford Hill criteria are satisfied in non-skeletal diseases [73], and genetic evidence from the first randomized trial on gene expression demonstrating a "molecular fingerprint that help explain the non-skeletal health benefits of vitamin D" [74].

There is currently considerable concern regarding the accuracy of serum 25-OHD measurements. While international efforts towards a standardisation program for this assay are making considerable progress, we have worked with the best available evidence that is currently available. We recognize that these ranges of 25-50 nmol/L for skeletal disease and 75-100 $\mathrm{nmol} / \mathrm{L}$ for non-skeletal disease may change slightly when a standardisation program is fully implemented. However it is likely these diverse ranges for skeletal and non-skeletal disease will remain quite separate even after standardisation. This would be consistent with the hypothesis that the different ranges reflect different mechanisms or tissue dependence, as seen with other medicines, such as acetylsalicylic acid. 
It is likely that an improved community vitamin D status would reduce health care utilisation and costs, and thus the economic burden of a wide range of chronic diseases. Adequately-powered, purpose-designed, randomised studies may provide greater certainty if baseline 25OHD is measured and adequate doses of vitamin D are used. Currently clinicians may accept the information provided pathology companies as to what constitutes a "target" 25-OHD level. Pathology companies generally suggest a wide target range, for example $60-160 \mathrm{nmol} / \mathrm{L}$ rather than specifically designate levels for deficiency and sufficiency, whilst the Royal College of Pathologists of Australasia is less specific, suggesting a level greater than $50 \mathrm{nmol} / \mathrm{L}$ for "optimal health".

There is concern about morbidity at high levels of 25-OHD raised by epidemiological studies, a retrospective cohort in general practice (CopD) [75] and a prospective community cohort study (NHANES III) [76]. They support the hypothesis of a reverse J curve indicating morbidity increases at higher levels of 25-OHD (140 nmol/L). The authors grant that these studies "did not allow inference of causality". Whereas meta-analysis of prospective cohort studies do provide Level I evidence of causality according to the NHMRC [22]. Three meta-analyses did not demonstrate increased mortality at higher levels of 25OHD [77-79]. A 28\% reduction in mortality in the highest category was demonstrated in the largest study and largest meta-analysis performed to date [78]. These studies indicate there is conflicting evidence for an upper inflection point in a $J$ shaped curve at "high" 25-OHD levels.

We hypothesise that just as vitamin D metabolism is tissue dependent, so the levels of 25-OHD that signify sufficiency are disease dependent. Thus the response to vitamin D supplementation is dependent on the baseline 25-OHD level, the dose of vitamin D and dose-response characteristics.

In the field of research, trials can be designed to minimise limitations of study design that lead to null outcomes knowing the range of serum 25-OHD in which changes in serum levels will lead to responses in specific body systems. This will also provide more clarity when assessing the effectiveness of vitamin D in different diseases, greater uniformity in the results of meta-analyses and less confusing guidelines.

In the field of health care, knowledge of the minimum therapeutic level for vitamin D will ensure clinicians can provide the correct dose and monitor it appropriately for treatment of specific diseases, and that policy makers have the opportunity to make evidence-based decisions about nutritional advice, supplementation, and fortification of food with vitamin D.

Further research to clarify the specific therapeutic levels for vitamin D sufficiency in different diseases and the dose response characteristics of vitamin D will ensure progress in research, clinical medicine, and public health.

\section{Conflicts of Interest}

The authors declare no conflict of interest.

\section{References}

1. Ross, A.; Taylor, C.; Yaktine, A.; del Valle, H. Dietary reference intakes for calcium and vitamin D. The National Academies Press: Washington, DC, USA, 2010. 
2. Morris, H.; Anderson, P. Autocrine and paracrine actions of vitamin D. Clin. Biochem. Rev. 2010, 31, 129-138.

3. Ramagopalan, S.V.; Heger, A.; Berlanga, A.J.; Maugeri, N.J.; Lincoln, M.R.; Burrell, A.; Handunnetthi, L.; Handel, A.E.; Disanto, G.; Orton, S.-M.; et al. A ChIP-seq defined genomewide map of vitamin D receptor binding: Associations with disease and evolution. Genome Res. 2010, 20, 1352-1360.

4. Holick, M. The vitamin D deficiency pandemic: A forgotten hormone important for health. Pubic Health Rev. 2010, 32, 267-283.

5. Daly, R.M.; Gagnon, C.; Lu, Z.X.; Magliano, D.J.; Dunstan, D.W.; Sikaris, K.A.; Zimmet, P.Z.; Ebeling, P.R.; Shaw, J.E. Prevalence of vitamin D deficiency and its determinants in Australian adults aged 25 years and older: A national, population-based study. Clin. Endocrinol. 2012, 77, 26-35.

6. Van der Mei, I.; Ponsonby, A.; Engelsen, O.; Pasco, J.; McGrath, J.; Eyles, D.; Blizzard, L.; Dwyer, T.; Lucas, R.; Jones, G. The high prevalence of vitamin D insufficiency across Australian populations is only partly explained by season and latitude. Environ. Health Perspect. 2007, 115, 1132-1139.

7. Sud, S.R.; Montenegro-Bethancourt, G.; Bermúdez, O.I.; Heaney, R.P.; Armas, L.; Solomons, N.W. Older Mayan residents of the western highlands of Guatemala lack sufficient levels of vitamin D. Nutr. Res. 2010, 30, 739-746.

8. Sheikh, A.; Saeed, Z.; Jafri, S.A.D.; Yazdani, I.; Hussain, S.A. Vitamin D levels in asymptomatic adults-A population survey in Karachi, Pakistan. PLoS One 2012, 7, e33452.

9. Arabi, A.; El Rassi, R.; El-Hajj Fuleihan, G. Hypovitaminosis D in developing countries - Prevalence, risk factors and outcomes. Nat. Rev. Endocrinol. 2010, 6, 550-561.

10. Lips, P. Worldwide status of vitamin D nutrition. J. Steroid Biochem. Mol. Biol. 2010, 121, 297-300.

11. Scragg, R. Vitamin D and public health: An overview of recent research on common diseases and mortality in adulthood. Pubic Health Nutr. 2011, 14, 1515-1532.

12. Exponential Growth in Vitamin D Publications. Available online: http://www.vitamindwiki. com/tikiindex.php?page=Expodential+growth+in+vitamin $+\mathrm{D}+$ publications $\quad($ accessed on 2 November 2013).

13. Heaney, R. Vitamin D-Baseline status and effective dose. N. Engl. J. Med. 2012, 367, 77-78.

14. Heaney, R. Nutrients, endpoints, and the problem of proof. J. Nutr. 2008, 138, 1591-1595.

15. Lappe, J.; Heaney, R. Why randomized controlled trials of calcium and vitamin D sometimes fail. Dermato-Endocrinology 2012, 4, 95-100.

16. Jackson, R.D.; LaCroix, A.Z.; Gass, M.; Wallace, R.B.; Robbins, J.; Lewis, C.E.; Bassford, T.; Beresford, S.A.A.; Black, H.R.; Blanchette, P.; et al. Calcium plus vitamin D supplementation and the risk of fractures. N. Engl. J. Med. 2006, 354, 669-683.

17. Wactawski-Wende, J.; Kotchen, J.M.; Anderson, G.L.; Assaf, A.R.; Brunner, R.L.; O’Sullivan, M.J.; Margolis, K.L.; Ockene, J.K.; Phillips, L.; Pottern, L.; et al. Calcium plus vitamin D supplementation and the risk of colorectal cancer. N. Engl. J. Med. 2006, 354, 684-696. 
18. Dean, A.; Bellgrove, M.; Hall, T.; Phan, W.; Eyles, D.; Kvaskoff, D.; McGrath, J. Effects of vitamin D supplementation on cognitive and emotional functioning in young adults-A randomised controlled trial. PLoS One 2011, 61, e25966.

19. Jorde, R.; Figenschau, Y. Supplementation with cholecalciferol does not improve glycaemic control in diabetic subjects with normal serum 25-hydroxyvitamin D levels. Eur. J. Nutr. 2009, 48, 349-354.

20. Hendrix, I.; Anderson, P.; May, B.; Morris, H. Regulation of gene expression by the CYP27B1 promoter-Study of a transgenic mouse model. J. Steroid Biochem. Mol. Biol. 2004, 89-90, 139-142.

21. Turner, A.G.; Anderson, P.H.; Morris, H.A. Vitamin D and bone health. Scand. J. Clin. Lab. Investig. 2012, 72, 65-72.

22. NHMRC Additional Levels of Evidence and Grades for Recommendations for Developers of Guidelines. Available online: https:/www.nhmrc.gov.au/_files_nhmrc/file/guidelines/developers/ nhmrc_levels_grades_evidence_120423.pdf (accessed on 2 November 2013).

23. Bjelakovic, G.; Gluud Lise, L.; Nikolova, D.; Whitfield, K.; Wetterslev, J.; Simonetti Rosa, G.; Bjelakovic, M.; Gluud, C. Vitamin D Supplementation for Prevention of Mortality in Adults. In Cochrane Database of Systematic Reviews; John Wiley \& Sons, Ltd., New York, NY, USA, 2011.

24. Ford, E.S.; Zhao, G.; Tsai, J.; Li, C. Vitamin D and all-cause mortality among adults in USA: Findings from the national health and nutrition examination survey linked mortality study. Int. J. Epidemiol. 2011, 40, 998-1005.

25. Schöttker, B.; Haug, U.; Schomburg, L.; Köhrle, J.; Perna, L.; Müller, H.; Holleczek, B.; Brenner, H. Strong associations of 25-hydroxyvitamin D concentrations with all-cause, cardiovascular, cancer, and respiratory disease mortality in a large cohort study. Am. J. Clin. Nutr. 2013, 97, 782-793.

26. Lappe, J.M.; Travers-Gustafson, D.; Davies, K.M.; Recker, R.R.; Heaney, R.P. Vitamin D and calcium supplementation reduces cancer risk: Results of a randomized trial. Am. J. Clin. Nutr. 2007, 85, 1586-1591.

27. Garland, C.; Gorham, E.D.; Mohr, S.B.; Garland, F.C. Vitamin D for cancer prevention: Global perspective. Ann. Epidemiol. 2009, 19, 468-483.

28. Grant, W.B. Relation between prediagnostic serum 25-hydroxyvitamin D level and incidence of breast, colorectal, and other cancers. J. Photochem. Photobiol. 2010, 101, 130-136.

29. Bischoff-Ferrari, H.A.; Dawson-Hughes, B.; Staehelin, H.B.; Orav, J.E.; Stuck, A.E.; Theiler, R.; Wong, J.B.; Egli, A.; Kiel, D.P.; Henschkowski, J. Fall prevention with supplemental and active forms of vitamin D: A meta-analysis of randomised controlled trials. BMJ 2009, 339, b3692.

30. Cranney, A.; Weiler, H.A.; O'Donnell, S.; Puil, L. Summary of evidence-based review on vitamin D efficacy and safety in relation to bone health. Am. J. Clin. Nutr. 2008, 88, 513S-519S.

31. Gillespie, L.; Robertson, M.; Gillespie, W. Interventions for preventing falls in older people living in the community. Cochrane Database Syst. Rev. 2009, doi:10.1002/14651858. CD007146.pub2. 
32. Murad, M.H.; Elamin, K.B.; Abu Elnour, N.O.; Elamin, M.B.; Alkatib, A.A.; Fatourechi, M.M.; Almandoz, J.P.; Mullan, R.J.; Lane, M.A.; Liu, H.; et al. The effect of vitamin D on falls: A systematic review and meta-analysis. J. Clin. Endocrinol. Metab. 2011, 96, 2997-3006.

33. Scragg, R. Do we need to take calcium with vitamin D supplements to prevent falls, fractures, and death? Curr. Opin. Clin. Nutr. Metab. Care 2012, 15, 614-624.

34. Bischoff-Ferrari, H.A.; Dietrich, T.; Orav, E.J.; Hu, F.; Zhang, Y.; Karlson, E.; Dawson-Hughes, B. Higher 25-hydroxyvitamin D concentrations are associated with better lower-extremity function in both active and inactive persons aged $>$ or $=60$ y. Am. J. Clin. Nutr. 2004, 80, 752-758.

35. Stockton, K.; Mengersen, K.; Paratz, J.; Kandiah, D.; Bennell, K. Effect of vitamin D supplementation on muscle strength: A systematic review and meta-analysis. Osteoporos. Int. 2011, 22, 859-871.

36. Grandi, N.C.; Breitling, L.P.; Brenner, H. Vitamin D and cardiovascular disease: Systematic review and meta-analysis of prospective studies. Prev. Med. 2010, 51, 228-233.

37. Sokol, S.I.; Tsang, P.; Aggarwal, V.; Melamed, M.L.; Srinivas, V.S. Vitamin D status and risk of cardiovascular events: Lessons learned via systematic review and meta-analysis. Cardiol. Rev. 2011, 19, 192-201.

38. Brøndum-Jacobsen, P.; Benn, M.; Jensen, G.B.; Nordestgaard, B.G. 25-Hydroxyvitamin D levels and risk of ischemic heart disease, myocardial infarction, and early death. Arterioscler. Thromb. Vasc. Biol. 2012, 32, 2794-2802.

39. Vacek, J.L.; Vanga, S.R.; Good, M.; Lai, S.M.; Lakkireddy, D.; Howard, P.A. Vitamin D deficiency and supplementation and relation to cardiovascular health. Am. J. Cardiol. 2012, 109, 359-363.

40. Parker, J.; Hashmi, O.; Dutton, D.; Mavrodaris, A.; Stranges, S.; Kandala, N.-B.; Clarke, A.; Franco, O.H. Levels of vitamin D and cardiometabolic disorders: Systematic review and meta-analysis. Maturitas 2010, 65, 225-236.

41. Ginde, A.A.; Scragg, R.; Schwartz, R.S.; Camargo, C.A. Prospective study of serum 25-hydroxyvitamin D level, cardiovascular disease mortality, and all-cause mortality in older U.S. adults. J. Am. Geriatr. Soc. 2009, 57, 1595-1603.

42. Manaseki-Holland, S.; Qader, G.; Isaq Masher, M.; Bruce, J.; Zulf Mughal, M.; Chandramohan, D.; Walraven, G. Effects of vitamin D supplementation to children diagnosed with pneumonia in Kabul: A randomised controlled trial. Trop. Med. Int. Health 2010, 15, 1148-1155.

43. Urashima, M.; Segawa, T.; Okazaki, M.; Kurihara, M.; Wada, Y.; Ida, H. Randomized trial of vitamin D supplementation to prevent seasonal influenza A in schoolchildren. Am. J. Clin. Nutr. 2010, 91, 1255-1260.

44. Camargo, C.A.; Ganmaa, D.; Frazier, A.L.; Kirchberg, F.F.; Stuart, J.J.; Kleinman, K.; Sumberzul, N.; Rich-Edwards, J.W. Randomized trial of vitamin D supplementation and risk of acute respiratory infection in mongolia. Pediatrics 2012, 130, e561-e567.

45. Martineau, A.R.; Honecker, F.U.; Wilkinson, R.J.; Griffiths, C.J. Vitamin D in the treatment of pulmonary tuberculosis. J. Steroid Biochem. Mol. Biol. 2007, 103, 793-798. 
46. Gibney, K.B.; MacGregor, L.; Leder, K.; Torresi, J.; Marshall, C.; Ebeling, P.R.; Biggs, B.-A. Vitamin D deficiency is associated with tuberculosis and latent tuberculosis infection in immigrants from sub-saharan Africa. Clin. Infect. Dis. 2008, 46, 443-446.

47. Ginde, A.A.; Mansbach, J.M.; Camargo, C.A., Jr. Association between serum 25-hydroxyvitamin $\mathrm{D}$ level and upper respiratory tract infection in the third national health and nutrition examination survey. Arch. Intern. Med. 2009, 169, 384-390.

48. Wayse, V.; Yousafzai, A.; Mogale, K.; Filteau, S. Association of subclinical vitamin D deficiency with severe acute lower respiratory infection in Indian children under 5 y. Eur. J. Clin. Nutr. 2004, 58, 563-567.

49. Aloia, J.; Li-Ng, M. Re: Epidemic influenza and vitamin D. Epidemiol. Infect. 2007, 135, 1095-1098.

50. Sabetta, J.R.; DePetrillo, P.; Cipriani, R.J.; Smardin, J.; Burns, L.A.; Landry, M.L. Serum 25-hydroxyvitamin $\mathrm{D}$ and the incidence of acute viral respiratory tract infections in healthy adults. PLoS One 2010, 5, e11088.

51. Mitri, J.; Dawson-Hughes, B.; Hu, F.; Pittas, A. Effects of vitamin D and calcium supplementation on pancreatic $\beta$ cell function, insulin sensitivity, and glycemia in adults at high risk of diabetes: The Calcium and Vitamin D for Diabetes Mellitus (CaDDM) randomized controlled trial. Am. J. Clin. Nutr. 2011, 94, 486-494.

52. Von Hurst, P.R.; Stonehouse, W.; Coad, J. Vitamin D supplementation reduces insulin resistance in South Asian women living in New Zealand who are insulin resistant and vitamin D deficient - A randomised, placebo-controlled trial. Br. J. Nutr. 2010, 103, 549-555.

53. Mitri, J.; Muraru, M.D.; Pittas, A.G. Vitamin D and type 2 diabetes: A systematic review. Eur. J. Clin. Nutr. 2011, 65, 1005-1015.

54. Forouhi, N.G.; Ye, Z.; Rickard, A.P.; Khaw, K.T.; Luben, R.; Langenberg, C.; Wareham, N.J. Circulating 25-hydroxyvitamin D concentration and the risk of type 2 diabetes: Results from the European Prospective Investigation into Cancer (EPIC)-Norfolk cohort and updated meta-analysis of prospective studies. Diabetologia 2012, 55, 2173-2182.

55. Afzal, S.; Bojesen, S.E.; Nordestgaard, B.G. Low 25-hydroxyvitamin D and risk of type 2 diabetes: A prospective cohort study and metaanalysis. Clin. Chem. 2013, 59, 381-391.

56. Gloth, F.; Alam, W.; Hollis, B. Vitamin D vs. broad spectrum phototherapy in the treatment of seasonal affective disorder. J. Nutr. Health Aginig 1999, 3, 5-7.

57. Jorde, R.; Sneve, M.; Figenschau, Y.; Svartberg, J.; Waterloo, K. Effects of vitamin D supplementation on symptoms of depression in overweight and obese subjects: Randomized double blind trial. J. Intern. Med. 2008, 264, 599-609.

58. Vieth, R.; Kimball, S.; Hu, A.; Walfish, P. Randomized comparison of the effects of the vitamin D3 adequate intake versus $100 \mathrm{mcg}$ (4000 IU) per day on biochemical responses and the wellbeing of patients. Nutr. J. 2004, 3, 1-10.

59. Lansdowne, A.; Provost, S. Vitamin D3 enhances mood in healthy subjects during winter. Psychopharmacology 1998, 135, 319-323.

60. Hujoel, P.P. Vitamin D and dental caries in controlled clinical trials: Systematic review and meta-analysis. Nutr. Rev. 2013, 71, 88-97. 
61. Dietrich, T.; Joshipura, K.J.; Dawson-Hughes, B.; Bischoff-Ferrari, H.A. Association between serum concentrations of 25-hydroxyvitamin D3 and periodontal disease in the US population. Am. J. Clin. Nutr. 2004, 80, 108-113.

62. Heath, K.M.; Elovic, E.P. Vitamin D deficiency: Implications in the rehabilitation setting. Am. J. Phys. Med. Rehab. 2006, 85, 916-923.

63. Schreuder, F.; Bernsen, R.M.D.; van der Wouden, J.C. Vitamin D supplementation for nonspecific musculoskeletal pain in non-western immigrants: A randomized controlled trial. Ann. Fam. Med. 2012, 10, 547-555.

64. Bailey, B.A.; Manning, T.; Peiris, A.N. Vitamin D Testing Patterns among Six Veterans Medical Centers in the Southeastern United States: Links with Medical Costs. Military Med. 2012, 177, 70-76.

65. Peiris, A.; Bailey, B.; Manning, T. The Relationship of Vitamin D Deficiency to Health Care Costs in Veterans. Military Med. 2008, 173, 1214-1218.

66. Genuis, S. What's out there making us sick? J. Environ. Pubic Health 2012, 2012, doi:10.1155/2012/605137

67. Church, J.; Goodall, S.; Norman, R.; Haas, M. An economic evaluation of community and residential aged care falls prevention strategies in NSW. NSW Public Health Bull. 2011, 22, $60-68$.

68. Grant, W.B.; Cross, H.S.; Garland, C.F.; Gorham, E.D.; Moan, J.; Peterlik, M.; Porojnicu, A.C.; Reichrath, J.; Zittermann, A. Estimated benefit of increased vitamin D status in reducing the economic burden of disease in western Europe. Prog. Biophys. Mol. Biol. 2009, 99, 104-113.

69. Holick, M.F.; Binkley, N.C.; Bischoff-Ferrari, H.A.; Gordon, C.M.; Hanley, D.A.; Heaney, R.P.; Murad, M.H.; Weaver, C.M. Evaluation, treatment, and prevention of vitamin D deficiency: An endocrine society clinical practice guideline. J. Clin. Endocrinol. Metab. 2011, 96, 1911-1930.

70. Holick, M.F.; Binkley, N.C.; Bischoff-Ferrari, H.A.; Gordon, C.M.; Hanley, D.A.; Heaney, R.P.; Murad, M.H.; Weaver, C.M. Guidelines for preventing and treating vitamin D deficiency and insufficiency revisited. J. Clin. Endocrinol. Metab. 2012, 97, 1153-1158.

71. Pludowski, P.; Holick, M.F.; Pilz, S.; Wagner, C.L.; Hollis, B.W.; Grant, W.B.; Shoenfeld, Y.; Lerchbaum, E.; Llewellyn, D.J.; Kienreich, K.; Soni, M. Vitamin D effects on musculoskeletal health, immunity, autoimmunity, cardiovascular disease, cancer, fertility, pregnancy, dementia and mortality_A review of recent evidence. Autoimmun. Rev. 2013, 12, 976-989.

72. Souberbielle, J.C.; Body, J.J.; Lappe, J.M.; Plebani, M.; Shoenfeld, Y.; Wang, T.J.; Bischoff-Ferrari, H.A.; Cavalier, E.; Ebeling, P.R.; Fardellone, P.; et al. Vitamin D and musculoskeletal health, cardiovascular disease, autoimmunity and cancer: Recommendations for clinical practice. Autoimmun. Rev. 2010, 9, 709-715.

73. Grant, W.B.; Boucher, B.J. Are Hill's criteria for causality satisfied for vitamin D and periodontal disease. Dermato-Endocrinology 2010, 2, 30-36.

74. Hossein-nezhad, A.; Spira, A.; Holick, M.F. Influence of vitamin D status and vitamin D3 supplementation on genome wide expression of white blood cells: A randomized double-blind clinical trial. PLoS One 2013, 8, e58725. 
75. Durup, D.; Jørgensen, H.L.; Christensen, J.; Schwarz, P.; Heegaard, A.M.; Lind, B. A reverse J-shaped association of all-cause mortality with serum 25 -hydroxyvitamin D in general practice: The CopD study. J. Clin. Endocrinol. Metab. 2012, 97, 2644-2652.

76. Sempos, C.T.; Durazo-Arvizu, R.A.; Dawson-Hughes, B.; Yetley, E.A.; Looker, A.C.; Schleicher, R.L.; Cao, G.; Burt, V.; Kramer, H.; Bailey, R.L.; et al. Is there a reverse J-shaped association between 25-hydroxyvitamin D and all-cause mortality? J. Clin. Endocrinol. Metab. 2013, 98, 3001-3009.

77. Rush, L.; McCartney, G.; Walsh, D.; MacKay, D. Vitamin D and subsequent all-age and premature mortality: A systematic review. BMC Public Health 2013, 13, 679.

78. Tomson, J.; Emberson, J.; Hill, M.; Gordon, A.; Armitage, J.; Shipley, M.; Collins, R.; Clarke, R. Vitamin D and risk of death from vascular and non-vascular causes in the Whitehall study and meta-analyses of 12,000 deaths. Eur. Heart J. 2013, 34, 1365-1374.

79. Zittermann, A.; Iodice, S.; Pilz, S.; Grant, W.B.; Bagnardi, V.; Gandini, S. Vitamin D deficiency and mortality risk in the general population: A meta- analysis of prospective cohort studies. Am. J. Clin. Nutr. 2012, 95, 91-100. 



\section{Chapter 4}

Neoplastic Disorders 



\title{
Molecular Link between Vitamin D and Cancer Prevention
}

\author{
Meis Moukayed and William B. Grant
}

\begin{abstract}
The metabolite of vitamin D, 1 $\alpha, 25$-dihydroxyvitamin $\mathrm{D}_{3}$ (also known as calcitriol), is a biologically active molecule required to maintain the physiological functions of several target tissues in the human body from conception to adulthood. Its molecular mode of action ranges from immediate nongenomic responses to longer term mechanisms that exert persistent genomic effects. The genomic mechanisms of vitamin $\mathrm{D}$ action rely on cross talk between 1 $\alpha, 25$-dihydroxyvitamin $\mathrm{D}_{3}$ signaling pathways and that of other growth factors or hormones that collectively regulate cell proliferation, differentiation and cell survival. In vitro and in vivo studies demonstrate a role for vitamin D (calcitriol) in modulating cellular growth and development. Vitamin D (calcitriol) acts as an antiproliferative agent in many tissues and significantly slows malignant cellular growth. Moreover, epidemiological studies have suggested that ultraviolet-B exposure can help reduce cancer risk and prevalence, indicating a potential role for vitamin $\mathrm{D}$ as a feasible agent to prevent cancer incidence and recurrence. With the preventive potential of this biologically active agent, we suggest that countries where cancer is on the rise-yet where sunlight and, hence, vitamin D may be easily acquired - adopt awareness, education and implementation strategies to increase supplementation with vitamin $\mathrm{D}$ in all age groups as a preventive measure to reduce cancer risk and prevalence.
\end{abstract}

Reprinted from Nutrients. Cite as: Moukayed, M.; Grant, W.B. Molecular Link between Vitamin D and Cancer Prevention. Nutrients 2013, 5, 3993-4021.

\begin{abstract}
Abbreviations
WSTF: Williams syndrome transcription factor; WINAC: WSTF including nucleosome assembly complex; MCF-7: Michigan Cancer Foundation-7 human breast adenocarcinoma cell line; MART-10: 19-nor-2 $\alpha$-(3-hydroxypropyl)-1 $\alpha, 25$-Dihydroxyvitamin D3; EB1089: Seocalcitol; SW620: Human colorectal adenocarcinoma cell line; PC/JW: Human colorectal adenoma-derived epithelial cell line derived from adenomatous polyposis; HT29: Human colorectal adenocarcinoma cell line HT29; SW-480-ADH: malignant colon cancer subline of human Dukes' type B colorectal adenocarcinoma cell line SW-480; LNCaP: Human prostatic carcinoma cell line LNCaP; CL-1: Human prostate cancer cell line derived from LNCaP; IGFBP3: IGF-binding protein 3; MCF10CA: Human metastatic breast cancer cell line MCF10CA; HL60: Human promyelocytic leukemia cells; mTOR: mammalian target of rapamycin; CDK: Cyclin-dependent kinase; APC: Adenomatous polyposis coli; LPB-Tag: Long Probasin Promoter-Large T Antigen.
\end{abstract}




\section{Vitamin D: Introduction, Function and Metabolism}

\subsection{Introduction to Vitamin D: History and Physiological Roles}

Vitamin $\mathrm{D}$ refers to two fat soluble substances, vitamin $\mathrm{D}_{3}$ (cholecalciferol) and vitamin $\mathrm{D}_{2}$ (ergocalciferol), and their metabolites, which are considered to be important nutrients for human health. Dietary vitamin $\mathrm{D}_{3}$ sources include dairy, eggs, fish and meat [1], while dietary vitamin $\mathrm{D}_{2}$ (ergocalciferol) sources are UVB-irradiated yeast and fungi [2,3]. However, there seem to be some unaccounted for dietary sources [4]. In humans, vitamin $\mathrm{D}_{3}$, made naturally by the body following exposure to ultraviolet light, acts as an important endocrine hormone precursor.

Mellanby [5] and McCollum [6] first identified vitamin D's effects in experiments that investigated the chemical components of cod liver oil that could prevent rickets in animals. Later studies by Hess [7], McCollum [8], Steenbock [9], Askew [9] and Windaus [10] helped isolate, identify and determine the structure and function of this hormone and its essential role in skeletal health. In 1939, Windhaus determined the structure and initial pathways by which vitamin $\mathrm{D}_{3}$ was synthesized from 7-dehydrocholesterol [11,12]. His biochemical investigations were the basis for Holick and colleagues [13-16] to later elucidate the biochemical pathways and physiological mechanisms that regulated the formation of the active vitamin $\mathrm{D}$ hormone, $1 \alpha, 25$-dihydroxyvitamin $\mathrm{D}_{3}$ [17]. Vitamin $D_{3}$ is initially synthesized via the initial conversation of 7-dehydrocholesterol upon UV irradiation of the skin [13-16]. This provitamin $\mathrm{D}_{3}$ is further metabolized in the liver and kidneys to produce the active hormone, 1 1 ,25-dihydroxyvitamin $\mathrm{D}_{3}$, often mentioned in the literature simply as vitamin $\mathrm{D}$ [18-21] (Figure 1). However, extrarenal production of 1,25-dihydroxyvitamin D also occurs in many organs [22]. 25-hydroxyvitamin $\mathrm{D}[25(\mathrm{OH}) \mathrm{D}]$ is the circulating metabolite of vitamin $\mathrm{D}$ that is routinely measured.

In the 90 years since the above discovery to date, scientists have demonstrated unequivocally that vitamin D exerts a spectrum of biological effects well beyond its classical role in calcium and phosphate homeostasis. The cloning of the vitamin D receptor (VDR) in 1987 and the detection of VDRs in almost all tissues of the body spurred wide interest in its physiological functions [23]. Vitamin $\mathrm{D}_{3}$ has important homeostatic functions in fetal and adult development and differentiation in endocrine, metabolic, neurological, epidermal and immunological systems of the human body [24-26] (Table 1). Moreover, several studies support an essential role for vitamin D in regulating mechanisms controlling cell proliferation, differentiation and growth. These bodies of evidence reveal protective functions for vitamin D against carcinogen-induced neoplasia and recurrent secondary metastasis [27]. 
Table 1. Vitamin D can exert its action in several organ systems and tissues of the body. This occurs in a paracrine, autocrine, intracrine or endocrine manner [24-26].

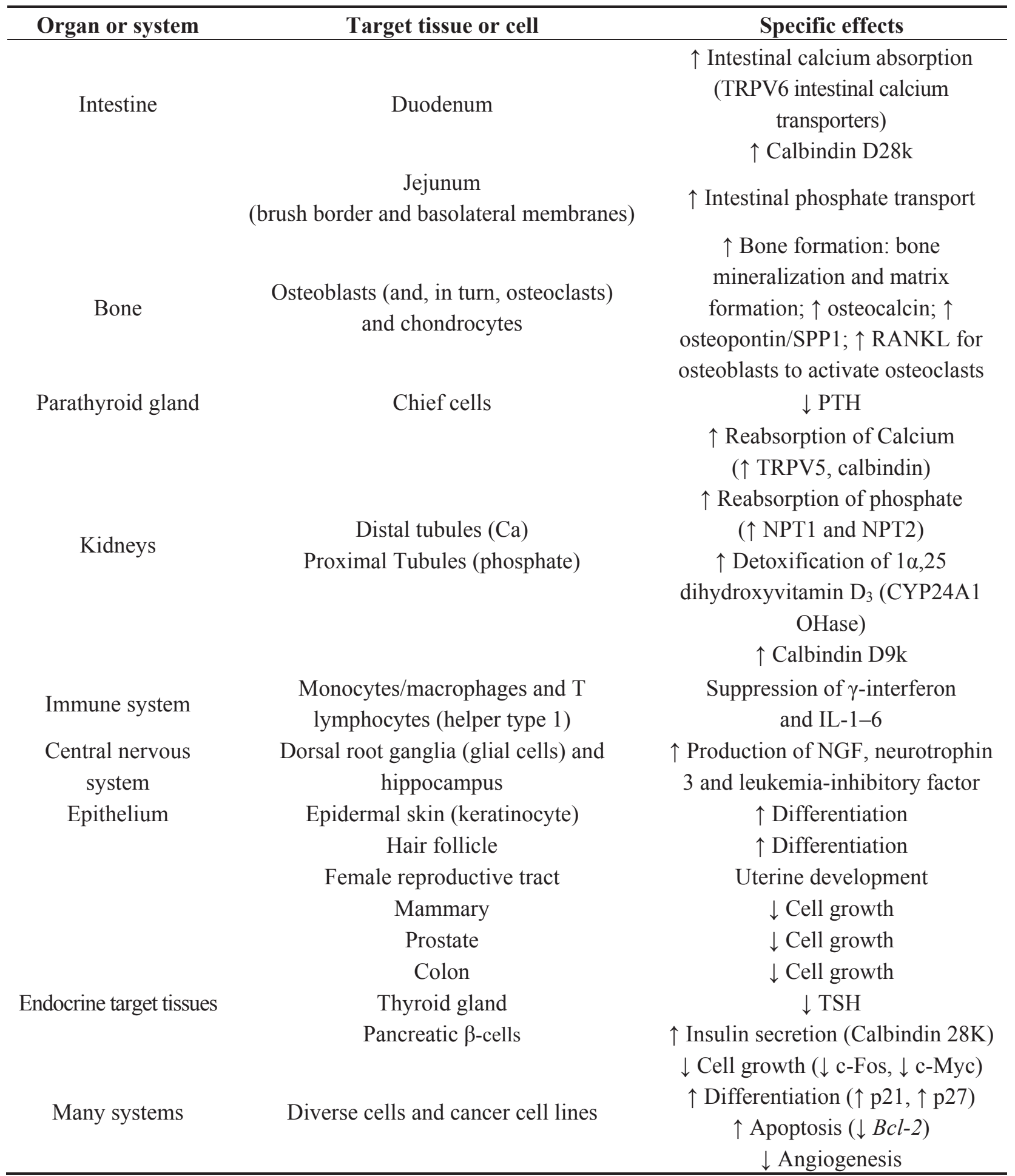

$\uparrow=$ upregulates/increases; $\downarrow=$ downregulates/decreases. CYP24A1 OHase $=1,25$-dihydroxyvitamin $\mathrm{D}_{3}$ 24-hydroxylase; IL = interleukin; NGF = nerve growth factor; NPT1 = sodium-dependent phosphate transporter 1; NPT2 = sodium-dependent phosphate transporter 2; PTH = parathyroid hormone; RANKL $=$ receptor activator of nuclear factor kappa-B ligand; SPP-1 = secreted phosphoprotein 1; TRPV5 = transient receptor potential cation channel subfamily V member 5; TRPV6 = transient receptor potential cation channel subfamily $\mathrm{V}$ member 6 ; $\mathrm{TSH}=$ thyroid stimulating hormone. 
Figure 1. Ultraviolet radiation $(290-330 \mathrm{~nm})$ from the sun and body heat convert 7-dehydrocholesterol in the skin to cholecalciferol (vitamin $\mathrm{D}_{3}$ ). Vitamin $\mathrm{D}_{3}$ is further metabolized in the liver and kidneys to yield the active metabolite $1,25(\mathrm{OH})_{2} \mathrm{D}_{3}$.
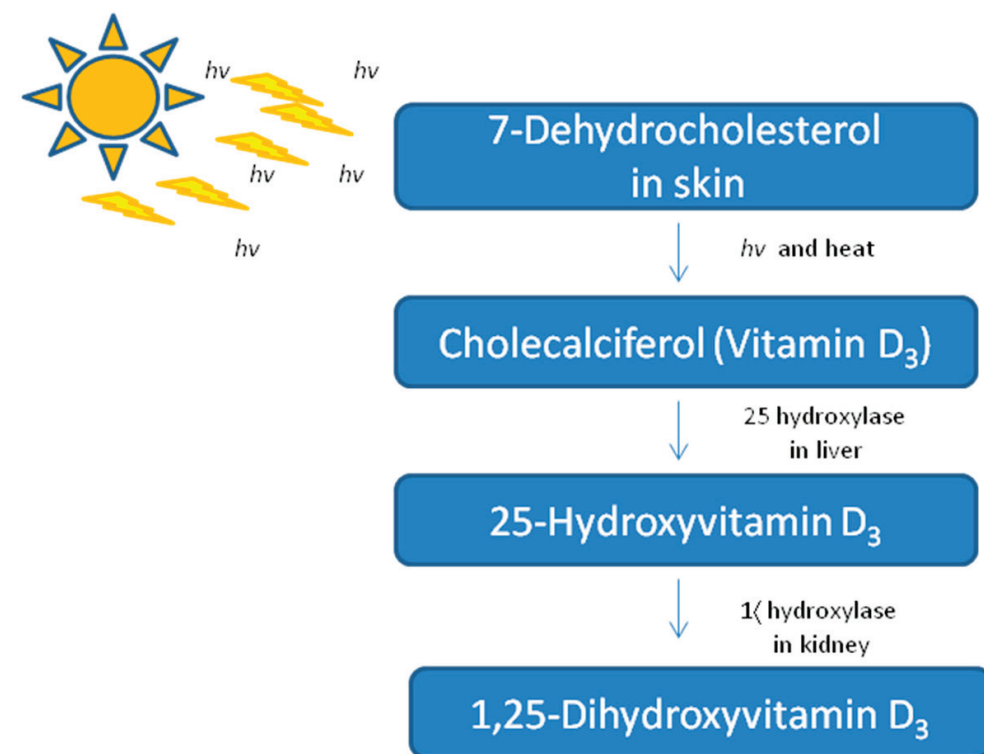

Biological actions

\subsection{Vitamin D Genomic Action via the VDR}

\subsubsection{VDR Distribution, Dimerization and Function}

Vitamin D acts as a steroid hormone by regulating gene transcription. The effect of the bioactive hormone 1 $\alpha, 25$-dihydroxyvitamin $\mathrm{D}_{3}$ occurs via the VDR. The VDR was identified in 1969 [28] and was cloned in 1987 [29]; its crystal structure and binding to its natural ligand were characterized in 2000 [30]. Since its discovery, researchers have detected the VDR in many tissues of the body, including bone, pancreatic $\beta$ cells, parathyroid gland, brain, skin, prostate, testes, heart, skeletal muscle tissue, breast, liver, lung, intestine, kidneys, adipose cells and immune response cells, such as macrophages, dendritic cells and activated B- and T-cells [31-36]. The receptor is widely distributed in the body, indicating physiological roles in homeostatic regulation beyond bone tissue mineralization. Ligand binding by $1 \alpha, 25$-dihydroxyvitamin $\mathrm{D}_{3}$ to its receptor can either activate or repress genes [37-41]. The VDR exerts its genomic effects by binding to regulatory vitamin D response elements (VDREs) present in the promoter regions of target genes in these tissues. The most prevalent motif for the VDRE sequence consists of two half-sites, each with the six-nucleotide consensus sequence, GGTCCA, separated by three other nucleotides of any sequence [37]. This motif is known as direct repeat 3 (DR3), although other configurations of VDRE-binding sites, including DR6 and DR4, exist in some vitamin D-regulated genes [26,37,42,43].

Ligand-bound VDR-dependent transcription regulation of genes occurs via the dimerization of the receptor with the retinoic X receptor (RXR). Although VDR preferentially binds to RXR, creating a VDR-RXR dimer, VDR can also bind other receptors of the nuclear receptor superfamily, which include thyroid, vitamin A, PPAR- $\gamma$ and other orphan receptors [44]. The VDR-RXR dimer can regulate genes in several systems and tissues [26]. 


\subsubsection{VDR Structure and Role of Cofactors}

The VDR has two variant isoforms, one with 424 amino acids (aa) and the other with 427 aa $[45,46]$. The complete functional protein contains several distinct domains: a DNA-binding domain (aa 24-115), a nuclear localization sequence (aa 44-55, 79-105), a hinge domain (aa 116-226), a dimerization domain (aa 37, 91-92, 244-263, 317-395) that overlaps with the ligand-binding domain (LBD) (aa 227-244, 268-326, 396-422) and the sequences of the activation domain (AF2; aa $246,416-422)[47,48]$.

As with other nuclear hormone receptors, VDR has a conserved DNA-binding domain that comprises two zinc fingers necessary for target gene VDRE binding and dimerization of the activated receptor. The first zinc finger (proximal to the $\mathrm{N}$ terminus of the protein) is required for docking at the VDRE, whereas the second zinc finger is required for binding dimerization with RXR. A nuclear localization sequence is located within the DNA-binding region and just upstream of the $C$ terminal to this binding domain. The nuclear localization sequence directs localization of the activated receptor to the nucleus to regulate gene transcription. The LBD, which consists of $12 \alpha$-helices joined by $\beta$-sheets, has regions required for $1 \alpha, 25$-dihydroxyvitamin $\mathrm{D}_{3}$ binding at helix 2 and two further regions required for RXR heterodimerization (see [30,37] for key references on LBD crystal structure and properties and functions). Within this $C$-terminus region of the LBD lies the major activation domain, AF2, required for transcription coactivation. Ligand binding by the active hormone creates a conformational change that releases transcription repressors such as WSTF and WINAC and enables attachment of coactivators [49-51]. AF2 is bound by cofactors of the steroid receptor coactivator (SRC) family, NCoA62-SKIP, CBP/p300 and p/CAF, and proteins of the VDR-interacting protein complex (DRIP/Mediator) [52,53]. CBP/p300 and p/CAF have histone acetyl transferase ability, which unravels the chromatin of the target gene [54]. This unwinding of the chromatin enables the transcription machinery, such as TF2B and RNA polymerase II, to bind and subsequently initiate transcription [55]. The DRIP complex, made up of approximately 15 leucine-rich proteins, is unique in its function in that it creates a bridge between the SRC coactivator proteins lodged at the VDRE of the gene and the transcription machinery at the start site of the regulated gene. DRIP 205 specifically is required in this vitamin D-induced transcription activation to enable an open position of the transcribed chromatin $[56,57]$.

\subsubsection{Nongenomic Cytoplasmic Action}

Vitamin D can also function via nongenomic mechanisms without exerting transcriptional effects or requiring additional protein synthesis. Such nongenomic effects have previously been reported for other nuclear hormones, such as estrogen, thyroid hormone and corticosteroids [58,59]. Vitamin D is thought to regulate rapid cellular calcium flux and calcium-activated chloride channel activity via a membrane-bound receptor protein known as 1,25- $(\mathrm{OH})_{2} \mathrm{D}$ membrane-associated rapid response steroid-binding protein (1,25 D-MARRSBP), also known as endoplasmic reticulum stress protein 57. MARRSBP was identified, purified and cloned from chick intestinal epithelium [60]. MARRSBP has also been found in tissues, including osteoblasts, liver, adipocytes and muscle [61-67]. MARRSBP acts via a $\mathrm{G}$ protein-coupled process that activates phospholipase C. Phospholipase C 
hydrolyzes membrane-bound phosphoinositol bisphosphate $\left(\mathrm{PIP}_{2}\right)$ to release inositol trisphosphate $\left(\mathrm{IP}_{3}\right)$ and diacylglycerol. In turn, these cellular messengers enable the release of calcium from endoplasmic stores and mediate the acute quick release of calcium in the cell [68]. This interaction, however, cannot occur without nuclear-targeted VDR, indicating that a cooperative mechanism of action between membrane-bound MARRSBP and VDR mediates rapid responses in some tissue types [35,69]. This interaction between the membrane-bound and nucleus-bound receptors can occur in cancer cell lines. In MCF-7 breast cancer cells, MARRSBP antagonizes VDR, thus implicating the membrane-bound MARRSBP in the modulation of cellular proliferation and malignancy. However, the full purpose of this interaction is yet to be elucidated [70].

\section{Vitamin D and Cancer}

\subsection{Vitamin D Mechanisms Regulating Cellular Proliferation and Growth}

Through the genomic actions of vitamin $\mathrm{D}_{3}$ via VDR regulation of several genes containing VDREs, $1 \alpha, 25(\mathrm{OH})_{2}$ vitamin $\mathrm{D}_{3}$ and its analogues inhibit cell cycle progression and tumor cell growth in several cancer cell lines. Such mechanisms range from preventing cell proliferation (cell cycle arrest) to inducing apoptosis to inducing or suppressing cell adhesion molecules and growth factors that promote cellular homing and metastasis.

\subsection{Cell Cycle and Apoptosis}

The cell cycle is regulated by a complex network of interlinked regulators that in concert govern cellular proliferation. Vitamin D may exert growth inhibitory effects through repression of different key molecules involved in cell cycle regulation. For example, Jensen and colleagues [71] demonstrated that $1, \mathrm{a}, 25-(\mathrm{OH})_{2} \mathrm{D}_{3}$ treatment of human breast cancer cell line MCF-7 resulted in repression of c-Myc, a known proto-oncogene in the cell cycle regulatory machinery. Salehi-Tabar and colleagues [72] demonstrated that $1, \mathrm{a}, 25-(\mathrm{OH})_{2} \mathrm{D}_{3}$ can suppress expression of the oncogene c-Myc and, thus, promotes the increased expression of its antagonist, the transcriptional repressor, MAD1/MXD1. This effect is mediated by other cellular regulators, such as F-box protein (FBW7). Meyer and colleagues [73] also demonstrated that in colonic cells of colorectal tumors, treatment with 1,a,25-(OH $)_{2} \mathrm{D}_{3}$ can suppress c-Fos and c-Myc gene expression in a manner that involves B-catenin signaling interactions. In prostate cancer cells, Washington and colleagues [74] showed that this repression is independent of retinoblastoma protein $(\mathrm{Rb})$. Li and colleagues [75] showed that treatment of ovarian cancer cells with 1,25-dihydroxyvitamin $\mathrm{D}_{3}$ results in $\mathrm{p} 27$ (Kip1) stabilization and G(1) arrest through downregulation of cyclin E/cyclin-dependent kinase 2 and Skp1-Cullin-F-box protein/Skp2 ubiquitin ligase. Collectively, these studies confirm that vitamin D can suppress cell proliferation through inhibitory effects on several regulators in the network of cell cycle control machinery.

Akutsu and colleagues [76] reported that treating human head and neck squamous cell carcinoma cells SCC25 with vitamin D arrested cell proliferation at the $\mathrm{G}_{0} / \mathrm{G}_{1}$ phase. Vitamin $\mathrm{D}$ also upregulated the growth-arrest DNA damage repair factor, GADD45a, at both the mRNA and protein levels. Cyclin-dependent kinase inhibitor p21 was also induced at the mRNA level, but not at the protein level. Chiang and colleagues showed that both vitamin D and its analogue, MART-10, were potent 
chemicals that could arrest the cell cycle at the $\mathrm{G}_{0} / \mathrm{G}_{1}$ transition phase in MCF-1 breast cancer cell lines. This process occurred through the expression of BAX/BCL proapoptotic proteins and the initiation of apoptosis via release of cytochrome $\mathrm{C}$ from mitochondria. Moreover, MART-10 induced no observed hypercalcemic effects (which are sometimes observed with vitamin D treatment), suggesting that such analogues would be good agents to deter cancer progression without detrimental side effects ([77]). In adenoma and carcinoma colorectal cell lines (SW620, PC/JW and HT29), treatment with vitamin D or its analogue, EB1089, induced apoptosis independent of p53, increased the number of cells in the $\mathrm{G}_{1}$ phase and increased levels of the proapoptotic protein, Bak (a member of the $\mathrm{BCl}-2$ gene family), in all cell lines tested. This finding suggested that vitamin $\mathrm{D}$ and its analogues are clinically effective for treating colorectal cancers. In rat glioma C6.9 cell lines, vitamin D treatment induced apoptosis via DNA fragmentation and upregulation of p53 and GADD45 genes [78]. In prostate cancer cell lines, vitamin D treatment inhibited cyclin-dependent kinase 2 activity and induced $\mathrm{G}_{0} / \mathrm{G}_{1}$ cell cycle arrest [79]. Therefore, several lines of evidence from in vitro studies support the role of vitamin D in promoting cell cycle arrest and promoting apoptosis in malignant transformed cells.

\subsection{Hypoxia, Oxidative Stress, HIF-1 and Angiogenesis}

Hypoxia and oxidative stress are often associated with cancer progression. Oxidative stress often induces DNA damage and loss of DNA-repair ability [80,81]. Hypoxia also promotes hypoxia-inducible factor 1 (HIF-1)-dependent angiogenesis essential for tumor growth [82]. Treatment of several cell lines, such as SW-480-ADH, LNCaP (a prostate cancer cell line) and MCF-7, with vitamin D activates cellular signaling cascades that reduce thioredoxin and promote antioxidant responses, induce mRNA expression of superoxide dismutase (in prostate epithelial cells) and downregulate glutathione levels by increasing glucose-6-phosphate dehydrogenase expression [83-85].

Vitamin $D_{3}$ inhibits initiators of cellular angiogenesis in several cancer cell lines. Adding vitamin $\mathrm{D}_{3}$ to the highly aggressive androgen-insensitive prostate cancer cell line, CL-1, inhibits proliferation of these cells both in normoxia and in hypoxic environments that resemble those in cancer tissues. This effect is also observed in $\mathrm{LNCaP}$ and in SW-480 colon cancer cell lines. Vitamin $\mathrm{D}_{3}$ also inhibits secretion of vascular endothelial growth factor (VEGF) in these cell lines, as well as in the MCF-7 breast cancer cell line. Furthermore, vitamin D treatment downregulates endothelin 1 (ET-1) and glucose transporter 1 (Glut-1). VEGF-1, ET-1 and Glut-1 are essential for inducing angiogenesis. This molecular effect is mediated via significant downregulation of HIF-1 transcription and translation [86].

A study by Chung and colleagues [87] showed that in tumor-derived endothelial cells from VDR knockout mice, loss of VDR resulted in an increase in HIF-1 $\alpha$, VEGF, angiopoietin 1 and platelet-derived growth factor levels. Moreover, in vivo, mice lacking VDR exhibited enlarged blood vessels to perfuse tumor lesions. This clearly implicates VDR in the control of tumor-associated angiogenesis. 


\subsection{Interactions with Growth Factors that Mediate Transformation, Cell Adhesion and Metastasis}

Vitamin D3 strongly inhibits the Sonic Hedgehog signaling cascade in human renal cell carcinoma. Mice xenografted with renal cell carcinoma cells yet treated with vitamin D exhibit the absence of tumor development or substantial growth inhibition, suggesting the vitamin $\mathrm{D}_{3}$ can be used prophylactically to prevent tumor development or regression [88].

Insulin-like growth factors (IGFs) and their binding proteins have been implicated in the development of several tumors [89]. In prostate cancer, for example, IGFBP3 protein levels decrease when cells progress from benign to malignant metastasis [90,91]. Microarray analysis has shown that vitamin D3 regulates IGFBP3, which sequesters and modulates levels of IGF-I, in LNCaP human prostate cancer cell lines [92]. Upon treatment with vitamin $\mathrm{D}_{3}$, IGFBP3 mRNA is highly upregulated in LNCaP cells. In malignant and metastatic MCF10CA breast cancer cells, IGFBP3 transcripts were upregulated after treatment with a vitamin D analogue, Gemini [93]. IGFBP5 gene expression was induced after treatment with vitamin $\mathrm{D}_{3}$ in MCF-7 breast cancer cell lines [94].

An interaction in cellular signaling that is required for vitamin D's growth-inhibitory role in prostate cancer cells occurs between vitamin D and androgens [79]. Without androgen receptors, vitamin $\mathrm{D}$ is ineffective in its protective action, indicating that the protective function of the hormone requires signaling cross talk between both molecules.

Similarly, in the colon cancer cell line, Caco-2, vitamin D signaling is thought to modulate the apoptotic effects of transforming growth factor $\beta 1$ (TGF- $\beta 1$ ). Unlike its effect on normal epithelial cells, TGF $\beta$ cannot inhibit cell growth in Caco-2 (and other colon cancer-derived cell lines, such as SW-480). However, this resistance is reverted upon treatment of Caco-2 cell lines with vitamin D [95]. The effect of vitamin $\mathrm{D}_{3}$ appears to be via upregulation of IGF-II receptors and increased expression of TFG- $\beta 1$ itself in these cell lines. Furthermore, the apparent sensitization of cells to apoptosis by vitamin $\mathrm{D}$ in otherwise apoptosis-resistant malignant cell lines indicates an important role for vitamin $\mathrm{D}$ in the treatment of resistant refractory tumors. Vitamin D interacts with TGF- $\beta$-SMAD1 signaling, blocks transcriptional expression of cell cycle proteins and inhibits the action of cell cycle protein cyclins $\mathrm{D}_{1}, \mathrm{D}_{2}, \mathrm{D}_{3}$ and $\mathrm{E}$. Vitamin $\mathrm{D}$ can also inhibit epidermal growth factor signaling and its mitogenic Ras signaling [40,96,97].

Wnt signaling activation is a key culprit in the pathogenesis of several cancers [98-101]. Wnt acts through the nuclear localization of $\beta$-catenin and its association with downstream transcription factors, such as TCF1 (also known as TCF7) and TCF4 (also known as TCF7L2). Upon nuclear localization, $\beta$-catenin can activate several genes involved in tumor growth, metastasis or angiogenesis. Wnt can upregulate cell cycle progression genes, such as cyclin D, c-Myc and c-jun, matrix metalloproteinase MMP-7 [102], and limb, bud and heart (LBH) transcription factor, which promote cell malignancy and metastasis. Wnt also upregulates endothelin 1, VEGF and interleukin 8 (IL-8), all of which promote angiogenesis, required to feed aggressive tumors [103-105]. Wnt also downregulates E-cadherin, required for cellular adhesion [106]. This downregulation may modulate the metastatic transformation associated with Wnt-activated tumor cells [107]. The antitumor effects of vitamin D that antagonize Wnt signaling action have been best demonstrated in breast, colorectal and prostate cancer cells. Vitamin D treatment downregulates several of the above 
tumorigenesis-promoting genes. This action is modulated by the genomic effects of VDR interaction with $\beta$-catenin and, therefore, suppression of Wnt-activated transcription induction of the above-mentioned genes [85,94,107-112]. Vitamin D also upregulates the Wnt antagonist, DKK-1, thus suppressing Wnt activation and associated transformation [112,113].

Vitamin D treatment blocks production of IL-1 $\beta$ in macrophages [114] and, hence, blocks inflammation associated with colon carcinoma progression. This inactivation of IL-1 $\beta$ suppresses inflammation and, in turn, Wnt signaling activation in colon cancer epithelial cells required for the progression of colon tumors [115]. The suppressive effects of vitamin D on inflammation have also been confirmed through studies that have shown that vitamin D can suppress IL-1 $\beta$, IL-6 and IL-17 and NF- $\kappa \mathrm{B}$ in inflammation associated with breast and prostate cancer cells $[107,116,117]$.

\subsection{Autophagy}

Vitamin D regulates cancer-associated autophagy. Autophagy is important in the prevention of tumor progression in vivo $[118,119]$. Autophagy allows cells to survive in conditions of stress, such as low oxygen or nutrient deprivation, through digestion of cellular debris or accumulated damaged organelles. Such debris may damage the cell and, hence, affect viability and survival. Although it may be considered a contradiction to accept that promoting cell survival in tumor cells would be warranted, studies in HL60 myeloid leukemia cells and in MCF-7 breast cancer cell lines indicate that activating autophagy pathways by the hormone, vitamin $\mathrm{D}$, is important in exciting signaling pathways mediating the tumor-suppressive role of vitamin D. Vitamin D treatment of tumor cells seems to mediate upregulation of a protein known as beclin-1, which interacts with PI3 kinase (PI3K), which, in turn, inhibits mTOR, responsible for promoting tumor growth and progression. The specific inhibition of mTOR signaling is one that suppresses molecules, such as CDK inhibitors, p19 and p27, but is independent of p53-mediated mechanisms. Vitamin D treatment is crucial for maintaining cell viability and survival via signaling pathways that require PI3K and mitogen-activated protein kinase signaling and that are independent of pathways eliciting apoptosis. Such signaling activation is effective at early stages of cellular damage, which is associated with carcinogenesis (and cancer therapy), and where neovascularization of tissues is absent. Treatment with vitamin D in such tumors at early stages of cellular transformation would induce autophagy with the aim of clearing damaged chromosomes and organelle debris [120]. After autophagy, p53 and other tumor-suppressor genes can either initiate cellular repair cascades if tumorigenesis is in its earlier stages or induce apoptosis in cells sensitized for treatment with radiotherapy or chemotherapy $[119,121]$ Moreover, the beneficial effects of vitamin D-induced autophagy have been postulated to be exerted via attenuation of inflammation associated with tumorigenesis. However, because autophagy effects can promote both cell survival and inhibition of tumor progression, albeit under different cellular environments, the balance between both these opposing mechanisms on vitamin $\mathrm{D}$ treatment needs further study. Such studies will help determine the exact sequence and dose of vitamin D treatment relevant as an adjuvant anticancer drug therapy in combination with tumor treatments [36,122-124]. 


\subsection{Lessons from Animal Models}

Animal models offer additional supportive evidence that demonstrates the effective role of vitamin D in preventing uncontrollable hyperplasia and neoplasia leading to tumor formation [24]. Several kinds of animal models support this notion:

- VDR-knockout mouse models of VDR (VDRKO or $\mathrm{VDR}^{-/-}$) vs. wild-type counterparts (VDRWT)

- Animals supplemented with vitamin D

- Animal models in which tumorigenesis is chemically induced before animals are supplemented with vitamin $\mathrm{D}$ or its analogues

- VDRKO models where tumors are implanted or transfected

- knockout animal or heterozygotes of other genes (e.g., $\mathrm{APC}^{\mathrm{min} /+}$ or nude mice) subsequently supplemented with vitamin D

Two initial VDRKO models have been established and subsequently reproduced by others to characterize the in vivo role of VDR in animal models [125,126]. In vivo, the effects of vitamin D on cellular proliferation and terminal differentiation differed between tissues; some tissue types showed hypoplasia and impaired development, whereas other tissues displayed clear hyperplasia. For example, the knockout mice, $\mathrm{VDR}^{-/-}$, exhibit growth retardation, impairment of bone formation, osteomalacia, alopecia, impaired folliculogenesis and uterine hypoplasia. Furthermore, transfection of mice with Lewis lung carcinoma cells shows that $\mathrm{VDR}^{-/-}$mice display significantly reduced metastasis of lung cancer cells compared with VDRWT animals [127].

In contrast, $\mathrm{VDR}^{-/-}$mice show hyperproliferation in mammary glands, the parathyroid gland and the descending colon. $\mathrm{VDR}^{-/-}$mice displayed colonic cell division and increased expression of cell proliferation markers, PCNA (proliferating cell nuclear antigen) and cyclin D1. Scientists in the Cross lab later confirmed the $\mathrm{VDR}^{-/-}$model as a suitable model of colorectal hyperproliferation and DNA damage $[128,129]$.

Using the $\mathrm{VDR}^{-1-}$ mouse, Zinser and colleagues [130] demonstrated the role of vitamin $\mathrm{D}$ and its receptor in mammary gland morphogenesis. Comparing $\mathrm{VDR}^{-/-}$mice with VDRWT, they showed that mammary glands from developing VDR mice at puberty were heavier and exhibited faster growth and ductal branching than their VDRWT counterparts. In organ culture, mammary tissues from $\mathrm{VDR}^{-/-}$treated with estrogen or progesterone exhibited greater hormonal sensitivity than VDRWT. After pregnancy and lactation, $\mathrm{VDR}^{-/-}$mice showed delayed breast gland involution after offspring weaning compared with VDRWT. Mehta and colleagues [131,132] demonstrated in rodent mammary organ culture that treatment with a noncalcemic vitamin D analogue significantly reduces tumor lesion incidence at both the early initiation and promotion stages. In dietary supplementation studies, Jacobson and colleagues [133] demonstrated in dimethylbenzanthracene (DMBA)-treated rats that rats fed on low vitamin D diets had higher tumor incidence than those fed vitamin D-replete diets. VanWeelden and colleagues transplanted nude mice (nu/nu mice) with MCF-7 breast cancer xenografts and, then, followed the treatment with supplementation. Mice were either supplemented with the low-calcemic vitamin D analogue (EB1089) or placebo pellets given to corresponding control subjects. This supplementation lasted for five weeks. In animals administered the vitamin D 
analogue, cell proliferation in tumors was reduced two-fold compared with control mice. Moreover, the rate of apoptosis was enhanced in EB1089-treated mice, which also experienced significant regression of implanted breast tumors compared with control subjects [134]. These studies indicate that vitamin D action via the VDR is essential to modulate mammary gland proliferation and induce differentiation [130,135,136]. Moreover, such studies indicate an in vivo cytoprotective role for vitamin D (and its analogues) in cancer prevention [134,137]. Xu and colleagues suggest through their studies on $\mathrm{APC}^{\mathrm{min} /+}$ heterozygous mouse models that the in vivo growth-modulating effect of vitamin D might be a result of a signaling interplay that allows vitamin D to block the mitogenic effects of growth factors implicated in neoplasty [138,139].

Moreover, deletion of the VDR gene in $\mathrm{VDR}^{-/-}$mice predisposes these mice to higher risk and susceptibility to chemically-induced cancers of the skin, prostate, blood, lymph and breast. For example, treatment of VDRKO mice with DMBA dysregulates skin proliferation and hyperproliferates both basal and epidermal cells with reduced epidermal differentiation. DMBA induced higher incidence of cutaneous lesions in $\mathrm{VDR}^{-/}$mice than in VDRWT [140-142]. However, $\mathrm{VDR}^{-/}$mice do not spontaneously develop skin tumors without predisposition to carcinogens. Upon DMBA treatment, VDRKO mice also exhibit higher incidence of alveolar and ductal hyperplasias and 16\% higher lymphoblastic and thymic lymphomas than VDRWT counterparts. No observed tumor development occurred in tissues, such as ovary, uterus, lung and liver [130,142]. Similarly, in LPB-Tag transgenic mice, pRB and p53 tumor-suppressor genes are inactivated, causing prostate cancer, cellular proliferation and tumor progression much faster in VDRKO mice than in VDRWT. These differences are abolished upon treatment with testosterone. This finding indicated that vitamin D has a growth-modulating effect on cell growth through measured signaling interplay with other growth factors [143].

\subsection{Evidence for Vitamin D and Cancer}

The solar ultraviolet-B (UVB)-vitamin D-cancer hypothesis was proposed by the brothers Cedric and Frank Garland after seeing the atlas of colon cancer mortality rates (MRs) in the United States as beginning graduate students at Johns Hopkins University in 1974. They recognized a general inverse correlation between the regional doses of sunlight and colon cancer MRs. Their seminal paper was published in 1980 [144]. They later added breast [145] and ovarian [146] cancer to the list of cancers with reduced MRs in regions of higher solar radiation.

They used the ecological approach, which compares disease outcomes averaged by geographical units with risk-modifying factors, providing perhaps the strongest evidence that solar UVB and vitamin D reduce the risk of cancer. The evidence has been reviewed in recent papers $[147,148]$. The reasons the ecological approach is strong for cancer include: that many cases are included, that solar UVB, through production of vitamin $\mathrm{D}$, is a significant risk reduction factor for many types of cancer, that other risk-modifying factors, such as ethnic background and smoking, can be used in the analyses and that cancers develop slowly.

Single-country ecological studies have provided strong and relatively consistent findings for many types of cancer. Single countries have the advantages for ecological studies that the populations are reasonably homogeneous, that they have similar diets and smoking habits, etc., but if not, the 
differences can be easily modeled, as has been done for the United States in an ecological study that used solar UVB doses for July, lung cancer MRs as the index of smoking and alcohol consumption, those of Hispanic heritage included in the category "white Americans", poverty level and urban/rural residence [149]. The findings for UVB were similar to those found in an earlier study that used only UVB doses, but omitted several states with high Hispanic population rates [150]. The results for alcohol consumption, Hispanic heritage and smoking were in good agreement with the journal literature.

There have been several solar UVB indices used in single-country ecological studies: annual solar radiation doses [144,151], solar UVB from the Total Ozone Monitoring System (TOMS) operated by NASA $[149,150,152-154]$ and latitude [155-160]. A new set of solar UVB dose by month for North America is also available [161]. In some studies, indices of personal UVB irradiance were used: non-melanoma skin cancer in Spain [157] and California [159] and lip cancer less lung cancer in Nordic countries [162]. No factor other than vitamin D production has been proposed to explain the inverse correlations between the solar UVB indices and cancer incidence and/or MRs except for prostate cancer. It was noticed that the geographical variation of prostate cancer MR in the United States shared many features with the atlas of greatest ancestry by county for 2000 [163]. That finding inspired a multi-country ecological study involving genetics, diet and socioeconomic status. The hypothesized genetic link was to the apolipoprotein E epsilon4 (ApoE4) allele, an important risk factor for Alzheimer's disease [164]. Two important functions of this allele are to increase the production of insulin and cholesterol in order for those with this allele to be able to store more of the excess energy consumed in order to last between feasts. This allele is more common among hunter-gatherer people and those living at high northern latitudes. Several dietary supply factors were considered, and the energy derived from cereals/grains was found to be inversely correlated with prostate cancer incidence and MRs. The index of socioeconomic status used was gross national product per capita. Multiple linear regression analyses using these three factors found an adjusted $\mathrm{R}$ of about 0.5 , with all three factors having significant correlations with prostate cancer, ApoE4 and gross national product (GNP)/capita as risk factors [164]. Numerous studies have investigated the role of genes in the risk of prostate cancer. Only one other study identified ApoE4 as a risk factor, however [165]. Observational studies generally do not find that serum 25-hydroxyvitmain D (25(OH)D) levels are associated with the risk of prostate cancer [166].

Table 2 summarizes the findings from various single-country ecological studies. 
Table 2. Cancers for which inverse correlations between incidence and/or mortality rates (MRs) were found with respect to indices of solar UVB dose in single-country studies (references to be supplied).

\begin{tabular}{|c|c|c|c|c|c|c|c|c|}
\hline Cancer & $\begin{array}{c}\text { MR } \\
\text { (deaths/100,000/ } \\
\text { Year) * } \\
\end{array}$ & $\begin{array}{c}\text { US } \\
{[149,152]}\end{array}$ & $\begin{array}{c}\text { Australia } \\
\text { [155] }\end{array}$ & $\begin{array}{c}\text { China } \\
{[153,156]}\end{array}$ & $\begin{array}{c}\text { France } \\
{[158]}\end{array}$ & $\begin{array}{r}\text { Japan } \\
{[151]}\end{array}$ & $\begin{array}{c}\text { Nordic } \\
\text { countries } \\
{[162]} \\
\end{array}$ & $\begin{array}{l}\text { Spain } \\
{[157]}\end{array}$ \\
\hline Lung & 69.4 & & & $\mathrm{X}$ & & & $\mathrm{X}$ & $\mathrm{X}$ \\
\hline Breast & 26.9 & $\mathrm{X}$ & $\mathrm{X}$ & $\mathrm{X}$ & $\mathrm{X}$ & & $\mathrm{X}$ & $\mathrm{X}$ \\
\hline Colorectal & 24.5 & $\mathrm{X}$ & $\mathrm{X}$ & $\mathrm{X}$ & $\mathrm{X}$ & $\mathrm{X}$ & $\mathrm{X}$ & $\mathrm{X}$ \\
\hline Prostate & 22.0 & $\mathrm{X}$ & $\mathrm{X}$ & & & & $\mathrm{X}$ & \\
\hline Colon & 20.1 & $\mathrm{X}$ & $\mathrm{X}$ & $\mathrm{X}$ & $\mathrm{X}$ & $\mathrm{X}$ & $\mathrm{X}$ & $\mathrm{X}$ \\
\hline Pancreatic & 10.2 & $\mathrm{X}$ & $\mathrm{X}$ & & & $\mathrm{X}$ & $\mathrm{X}$ & $\mathrm{X}$ \\
\hline Leukemia & 8.8 & $\mathrm{X}$ & $\mathrm{X}$ & $\mathrm{X}$ & & $\mathrm{X}$ & & \\
\hline Ovarian & 8.4 & $\mathrm{X}$ & $\mathrm{X}$ & & & & & $\mathrm{X}$ \\
\hline Gastric & 7.3 & $\mathrm{X}$ & $\mathrm{X}$ & $\mathrm{X}$ & & $\mathrm{X}$ & $\mathrm{X}$ & $\mathrm{X}$ \\
\hline $\begin{array}{l}\text { non-Hodgkin's } \\
\text { lymphoma (NHL) }\end{array}$ & 7.0 & $\mathrm{X}$ & $\mathrm{X}$ & & & & & $\mathrm{X}$ \\
\hline Bladder & 6.6 & $\mathrm{X}$ & & $\mathrm{X}$ & & & $\mathrm{X}$ & $\mathrm{X}$ \\
\hline Brain & 5.2 & & & & & & & $\mathrm{X}$ \\
\hline Renal & 4.9 & $\mathrm{X}$ & & & & & $\mathrm{X}$ & \\
\hline Esophageal & 4.8 & $\mathrm{X}$ & $\mathrm{X}$ & $\mathrm{X}$ & $\mathrm{X}$ & $\mathrm{X}$ & $\mathrm{X}$ & $\mathrm{X}$ \\
\hline Rectal & 4.4 & $\mathrm{X}$ & & $\mathrm{X}$ & & $\mathrm{X}$ & $\mathrm{X}$ & $\mathrm{X}$ \\
\hline Oral, pharyngeal & 4.0 & $\mathrm{X}$ & & & & & $\mathrm{X}$ & \\
\hline Endometrial & 3.7 & $\mathrm{X}$ & & & $\mathrm{X}$ & & & $\mathrm{X}$ \\
\hline Cervical & 3.2 & & & $\mathrm{X}$ & $\mathrm{X}$ & & & \\
\hline Gallbladder & 1.1 & $\mathrm{X}$ & & & & $\mathrm{X}$ & $\mathrm{X}$ & $\mathrm{X}$ \\
\hline $\begin{array}{l}\text { Hodgkin's } \\
\text { lymphoma }\end{array}$ & 1.1 & $\mathrm{X}$ & & & & & & $\mathrm{X}$ \\
\hline Thyroid & 0.4 & $\mathrm{X}$ & & & & & & $\mathrm{X}$ \\
\hline Vulvar & 0.3 & $\mathrm{X}$ & & & & & & \\
\hline
\end{tabular}

The types of cancer are ordered by the MR for males (females for female cancers) for the United States for the period 1970-1994 [167]. The reason for doing so is that the more common a type of cancer is, the easier it is to include enough cases in the study to find significant correlations with solar UVB or vitamin D [168]. As expected, the types of cancer with the higher MRs generally have more findings of inverse correlation with solar UVB doses. For those with fewer significant findings relative to others with similar MRs, this suggests that the beneficial effect of UVB and vitamin D is weak or nonexistent, such as for brain cancer. For those with a relatively higher number of findings for its MR range, it is likely that the effects of UVB and vitamin D are strong, such as for esophageal and gallbladder cancer.

These findings are discussed in greater detail in Grant [147]. Several things can be inferred from the table. One is that the types of cancer with the higher MRs are generally more likely to have been found inversely correlated with the solar UVB indices. This is reasonable, since the uncertainty of 
each value is reduced [168]. There is little support for vitamin D reducing the risk of brain cancer, as it is a relatively common cancer, but with only one supporting study. On the other hand, the findings for thyroid and vulvar cancers are considered good evidence of the protection by vitamin D, since the MRs are so low.

While ecological studies may provide the earliest and, in some respects, best evidence for a role of solar UVB and vitamin D in reducing the risk of cancer, such findings should be supported by other types of studies in order to evaluate the findings from ecological studies. The next most common type of epidemiological study used to evaluate the role of solar UVB and vitamin D in reducing the risk of cancer is the observational study. There are two types of observational studies: case-control studies in which serum the $25(\mathrm{OH}) \mathrm{D}$ level is determined near the time of cancer diagnosis; and nested case-control studies from cohort studies. The perceived advantages of the nested case-control studies include unbiased matching of controls and using 25(OH)D levels, UVB doses or irradiances not affected by the health outcome. Case-control studies could have serum $25(\mathrm{OH}) \mathrm{D}$ levels affected by the disease state. However, there does not appear to be any evidence that the existence of cancer per se affects serum 25(OH)D levels or behavior, since most people do not know they have cancer until it is diagnosed. The primary problem of the cohort study approach is that serum $25(\mathrm{OH}) \mathrm{D}$ levels from blood drawn at time of enrollment are generally used in the analysis, no matter how many years have elapsed since enrollment. As shown in a pair of papers that examined health outcome with respect to years after enrollment, linear declines were found in risk reduction with respect to follow-up time for cancers [169] and all-cause MR [170].

Table 3 presents an overview of the findings from observational studies supporting the UVB-vitamin D-cancer hypothesis.

Table 3. Results from observational studies of cancer incidence with respect to UVB irradiance or serum 25(OH)D levels.

\begin{tabular}{|c|c|c|c|c|}
\hline Cancer & [171] & [154] & [172] & Others \\
\hline Bladder & $X^{*}$ & $\mathrm{X}$ & $\mathrm{X}$ & {$[173]$} \\
\hline \multicolumn{5}{|l|}{ Brain } \\
\hline Breast & & & & Case-control \\
\hline Colon & & $\mathrm{X}$ & & Cohort \\
\hline Colorectal & $\mathrm{X}$ & & & Cohort \\
\hline Endometrial & & & & [174] \\
\hline Esophageal & $\mathrm{X}$ & & & \\
\hline Esophageal, squamous cell & & $X^{*}$ & & \\
\hline Gastric & $X^{*}$ & & $\mathrm{X}^{*}$ & \\
\hline Head and neck & & & $\mathrm{X}$ & [175] \\
\hline Hepatoblastoma & & & & [160] \\
\hline Leukemia & $\mathrm{X}$ & & & \\
\hline Leukemia, acute lymphoblastic & & & & [160] \\
\hline Liver & & & $\mathrm{X}^{*}$ & \\
\hline Lung & $X^{*}$ & & $\mathrm{X}$ & \\
\hline Lung, adeno, squamous cell & & $\mathrm{X}$ & & \\
\hline
\end{tabular}


Table 3. Cont.

\begin{tabular}{ccccc}
\hline NHL & X* & X & & {$[160,176]$} \\
Oral/pharyngeal & $\mathrm{X}$ & & & \\
Ovarian & & & & {$[177]$} \\
Pancreatic & $\mathrm{X}$ & $\mathrm{X}$ & $\mathrm{X} *$ & {$[178]$} \\
Pleura & & $\mathrm{X}$ & & \\
Prostate & $\mathrm{X} *$ & $\mathrm{X}$ & & \\
Rectal & & $\mathrm{X}$ & & Cohort \\
Renal & $\mathrm{X} *$ & $\mathrm{X}$ & $\mathrm{X}$ & {$[179]$} \\
Thyroid & & $\mathrm{X}$ & & {$[180]$} \\
\hline \multicolumn{5}{c}{ * Not statistically significant. }
\end{tabular}

It is noted that the study from the Health Professionals Follow-up Study [171] and the National Institutes of Health-American Association of Retired Persons (NIH-AARP) Diet and Health study [154] were based, in large part, on solar UVB doses. That was explicit in Lin [154] and implicit in Giovannucci [171] in that it used a modeled serum 25(OH)D level based on measurements of serum 25(OH)D levels in 1000 men with respect to such factors as geographical location, skin pigmentation and leisure time spent out of doors. Other studies based on UVB were those for endometrial cancer [181], hepatoblastoma, acute lymphoblastic leukemia and NHL $[160,176]$.

For colorectal cancer, cohort studies with follow-up times out to 12 years found a significantly reduced risk for higher serum 25(OH)D levels [158]. However, for breast cancer, significantly reduced risk for higher serum 25(OH)D levels was reported only for follow-up times less than three years [169]. The reason for the different findings is that breast cancer is a rapidly developing cancer, while colorectal cancer is not. For example, breast cancer is diagnosed more frequently in spring and fall [182]. The authors suggested that vitamin D reduces breast cancer risk in summer, while melatonin does in winter, due to low sunlight levels.

\subsection{Clinical Trials}

There have been two randomized controlled trials (RCT) using vitamin D and calcium that found a beneficial effect in reducing cancer incidence. The first one was one conducted on post-menopausal women in Nebraska [183]. Those in the treatment arms took $1450 \mathrm{mg} / \mathrm{day}$ calcium or $1450 \mathrm{mg} / \mathrm{day}$ calcium plus $1100 \mathrm{IU} /$ day vitamin $\mathrm{D}_{3}$. At the time of enrollment, the mean serum 25(OH)D level was $72 \mathrm{nmol} / \mathrm{L}$. At the end of the first year, those taking vitamin D plus calcium had a mean serum $25(\mathrm{OH}) \mathrm{D}$ level of $96 \mathrm{nmol} / \mathrm{L}$, while those in the other two arms had $71 \mathrm{nmol} / \mathrm{L}$. Between the ends of the first and fourth years, those taking only calcium had a $44 \%$ reduction in all-cancer incidence, while those taking calcium plus vitamin D had a 77\% reduction. Based on the relationship between breast cancer incidence rates from case-control studies $v s$. serum $25(\mathrm{OH}) \mathrm{D}$ level, the expected reduction would be $18 \%$. The difference between $44 \%$ and $77 \%$ is $33 \%$. Given the uncertainty in both values, and the confounding of vitamin $\mathrm{D}$ with calcium, the finding in the RCT is in reasonable agreement with the cancer risk-25(OH)D relation. Calcium has been found to reduce the risk of cancer in a number of studies [184]. 
The second successful vitamin D/calcium RCT was from a reanalysis of a subset of data from the Women's Health Initiative study. Participants were given $400 \mathrm{IU} /$ day vitamin D 3 and $1500 \mathrm{mg} /$ day calcium, but many did not comply and take all of the supplements. For those participants who had not been taking vitamin $\mathrm{D}$ or calcium supplements prior to enrolling, there were significant decreases of total, breast and invasive breast cancer of $14 \%-20 \%$ and, nonsignificantly, a reduced risk of colorectal cancer of $17 \%$ [185]. For those taking vitamin $\mathrm{D}$ and/or calcium prior to enrollment, there was no beneficial effect of taking them during the study. This reduction is about twice that expected from vitamin $\mathrm{D}$ alone, so it probably includes a contribution from calcium.

There are a number of reasons why there are so few vitamin D RCTs reporting a reduced risk of cancer. One is that for many years, researchers used $400 \mathrm{IU} /$ day vitamin $\mathrm{D}_{3}$. This amount reduces the risk of rickets, but not much else. It is only in the past decade that many of the non-calcemic benefits of vitamin D were reported. For non-calcemic benefits, higher serum 25(OH)D levels are required. A second reason is that most of the vitamin D RCTs treated vitamin D as a drug, assuming that other sources of vitamin $\mathrm{D}$ were unimportant and that there was a simple dose-response relation between oral vitamin D and the serum 25(OH)D level. In fact, the vitamin D-serum 25(OH)D level relation is nonlinear and eventually saturates [186]. Proper vitamin D RCTs should have a model relationship between serum 25(OH)D level and health outcome, generally from observational studies, enroll people at the lower end of this relationship, supplement them with sufficient vitamin $\mathrm{D}_{3}$ to raise their serum 25(OH)D level to the upper end and measure serum 25(OH)D levels at the time of enrollment and after a year of supplementing, as well as consider other sources of vitamin D, such as solar UVB irradiance. The guidelines for conducting vitamin D RCTs were outlined in a recent paper [187].

\subsection{Cancer Survival}

Another way to examine whether vitamin D reduces the risk of cancer is to investigate whether those with higher serum $25(\mathrm{OH}) \mathrm{D}$ levels at the time of cancer diagnosis have better survival rates than those with lower levels. Those diagnosed with colorectal cancer with serum 25(OH)D levels in the upper quartile had half the MR of those in the lower quartile [188]. Such findings led to a study on the disparities in cancer survival rates for black Americans compared to white Americans [189]. It was found that there are disparities for 13 types of cancer after consideration of socioeconomic status, stage at diagnosis and treatment: bladder, breast, colon, endometrial, lung, ovarian, pancreatic, prostate, rectal, testicular and vaginal cancer, Hodgkin lymphoma and melanoma. Solar UVB doses and/or serum 25(OH)D levels have been reported to be inversely correlated with incidence and/or MRs for all of these cancers. The disparities not accounted for by socioeconomic status, stage at diagnosis and treatment ranged from $0 \%$ to $50 \%$, with a mean value near $25 \%$. This value is consistent with the disparities in serum 25(OH)D levels for black and white Americans: $40 \mathrm{nmol} / \mathrm{L}$ for black Americans and $65 \mathrm{nmol} / \mathrm{L}$ for white Americans [190]. 


\subsection{Hill's Criteria for Causality}

A. Bradford Hill laid down the criteria for causality in a biological system in 1965 [191]. The primary criteria appropriate for UVB irradiance and vitamin D include strength of association, consistent findings in different populations, temporality, biological gradient, plausibility (e.g., mechanisms), experiment (e.g., RCT) and analogy. As seen from the information presented in this work, most of the criteria are satisfied to a reasonable extent, especially for breast and colorectal cancer. By invoking analogy, many of the other cancers also qualify based on the findings in ecological studies. Hill's criteria for causality have been applied to many types of cancer, finding them largely satisfied for breast and colorectal cancer and somewhat satisfied for several other types of cancer [192], as well as for breast cancer [193].

\section{Recommendations/Conclusions}

Based on the evidence reviewed in this paper, there is very good-to-excellent scientific evidence that solar UVB irradiance and vitamin D reduce the risk of many types of cancer. However, acceptance by public policy review boards has not yet accepted the evidence. Part of the reason is that they generally require well-conducted randomized controlled trials rather than ecological or observational studies before making recommendations. Another reason is that UV irradiance is considered the most important risk factor for melanoma and non-melanoma skin cancer, so many public health bodies are reluctant to recommend more sun exposure.

\section{Conflicts of Interest}

WBG receives funding from Bio-Tech Pharmacal (Fayetteville, AR) and the Sunlight Research Forum (Veldhoven) and has received funding from the UV Foundation (McLean, VA), the Vitamin D Council (San Luis Obispo, CA), and the Vitamin D Society (Canada).

\section{References}

1. Severo, M.; Lopes, C.; Lucas, R.; Barros, H. Development of a tool for the assessment of calcium and vitamin D intakes in clinical settings. Osteoporos. Int. 2009, 20, 231-237.

2. Calvo, M.S.; Babu, U.S.; Garthoff, L.H.; Woods, T.O.; Dreher, M.; Hill, G.; Nagaraja, S. Vitamin D2 from light-exposed edible mushrooms is safe, bioavailable and effectively supports bone growth in rats. Osteoporos. Int. 2013, 24, 197-207.

3. Hohman, E.E.; Martin, B.R.; Lachcik, P.J.; Gordon, D.T.; Fleet, J.C.; Weaver, C.M. Bioavailability and efficacy of vitamin D2 from UV-irradiated yeast in growing, vitamin D-deficient rats. J. Agric. Food Chem. 2011, 59, 2341-2346.

4. Heaney, R.P.; Armas, L.A.; French, C. All-Source basal vitamin D inputs are greater than previously thought and cutaneous inputs are smaller. J. Nutr. 2013, 143, 571-575.

5. Mellanby, E. An experimental investigation on rickets. Lancet 1919, 1, 407-412.

6. McCollum, E.V.; Simmonds, N.; Becker, J.E.; Shipley, P.G. An experimental demonstration of the existence of a vitamin which promotes calcium deposition. J. Biol. Chem. 1922, 53, 293-298. 
7. Hess, A.F.; Unger, L.F. Cure of infantile rickets by sunlight. J. Am. Med. Assoc. 1921, 77, 39.

8. Steenbock, H.; Black, A. A fat-soluble vitamins. XVII. The induction of growth-promoting and clcifying properties in a ration by exposure to ultraviolet light. J. Biol. Chem. 1924, 61, 405-422.

9. Askew, F.A.; Bourdillon, B.R.; Bruce, H.M.; Jenkins, R.G.C. The distillation of vitamin D. Proc. R. Soc. 1931, B107, 76-90.

10. Windaus, A.; Schenck, F.; von Werder, F. Uber das antirachitisch wirksame bestrahlungsproduct aus 7-dehydro-cholesterin. Hoppe-Seylers Z. Physiol. Chem. 1936, 241, 100-103 (in German).

11. McCollum, E.V. The paths to the discovery of vitamins A and D. J. Nutr. 1967, 91, 11-16.

12. Wolf, G. The discovery of vitamin D: The contribution of adolf windaus. J. Nutr. 2004, 134, 1299-1302.

13. Holick, M.F.; McNeill, S.C.; MacLaughlin, J.A.; Holick, S.A.; Clark, M.B.; Potts, J.T., Jr. Physiologic implications of the formation of previtamin D3 in skin. Trans. Assoc. Am. Phys. 1979, 92, 54-63.

14. Holick, M.F.; Richtand, N.M.; McNeill, S.C.; Holick, S.A.; Frommer, J.E.; Henley, J.W.; Potts, J.T., Jr. Isolation and identification of previtamin D3 from the skin of rats exposed to ultraviolet irradiation. Biochemistry 1979, 18, 1003-1008.

15. Holick, S.A.; Holick, M.F.; MacLaughlin, J.A. Chemical synthesis of [1 beta-3H] 1 alpha, 25-dihydroxyvitamin D3 and [1 alpha-3H] 1 beta, 25-dihydroxyvitamin D2: Biological activity of 1 beta, 25-dihydroxyvitamin D3. Biochem. Biophys. Res. Commun. 1980, 97, 1031-1037.

16. Holick, M.F.; MacLaughlin, J.A.; Clark, M.B.; Holick, S.A.; Potts, J.T., Jr.; Anderson, R.R.; Blank, I.H.; Parrish, J.A.; Elias, P. Photosynthesis of previtamin D3 in human skin and the physiologic consequences. Science 1980, 210, 203-205.

17. Zusman, I.; Hirsh, B.E.; Edelstein, S.; Ornoy, A. Transplacental effects of 1,25-dihydroxycholecalciferol and of 24,25-dihydroxycholecalciferol on the limb skeleton of fetuses and offspring rats. Acta Anat. (Basel) 1981, 111, 343-351.

18. Guo, Y.D.; Strugnell, S.; Back, D.W.; Jones, G. Transfected human liver cytochrome p-450 hydroxylates vitamin D analogs at different side-chain positions. Proc. Natl. Acad. Sci. USA 1993, 90, 8668-8672.

19. Cheng, J.B.; Levine, M.A.; Bell, N.H.; Mangelsdorf, D.J.; Russell, D.W. Genetic evidence that the human cyp2r1 enzyme is a key vitamin D 25-hydroxylase. Proc. Natl. Acad. Sci. USA 2004, 101, 7711-7715.

20. Takeyama, K.; Kitanaka, S.; Sato, T.; Kobori, M.; Yanagisawa, J.; Kato, S. 25-Hydroxyvitamin D3 1alpha-hydroxylase and vitamin D synthesis. Science 1997, 277, 1827-1830.

21. Dilworth, F.J.; Scott, I.; Green, A.; Strugnell, S.; Guo, Y.D.; Roberts, E.A.; Kremer, R.; Calverley, M.J.; Makin, H.L.; Jones, G. Different mechanisms of hydroxylation site selection by liver and kidney cytochrome p450 species (cyp27 and cyp24) involved in vitamin D metabolism. J. Biol. Chem. 1995, 270, 16766-16774.

22. Zehnder, D.; Bland, R.; Williams, M.C.; McNinch, R.W.; Howie, A.J.; Stewart, P.M.; Hewison, M. Extrarenal expression of 25-hydroxyvitamin D(3)-1 alpha-hydroxylase. J. Clin. Endocrinol. Metab. 2001, 86, 888-894. 
23. Baker, A.R.; McDonnell, D.P.; Hughes, M.; Crisp, T.M.; Mangelsdorf, D.J.; Haussler, M.R.; Pike, J.W.; Shine, J.; O’Malley, B.W. Cloning and expression of full-length cdna encoding human vitamin D receptor. Proc. Natl. Acad. Sci. USA 1988, 85, 3294-3298.

24. Bouillon, R.; Carmeliet, G.; Verlinden, L.; van Etten, E.; Verstuyf, A.; Luderer, H.F.; Lieben, L.; Mathieu, C.; Demay, M. Vitamin d and human health: Lessons from vitamin D receptor null mice. Endocr. Rev. 2008, 29, 726-776.

25. Bikle, D.D. Protective actions of vitamin D in UVB induced skin cancer. Photochem. Photobiol. Sci. 2012, 11, 1808-1816.

26. Rosen, C.J.; Adams, J.S.; Bikle, D.D.; Black, D.M.; Demay, M.B.; Manson, J.E.; Murad, M.H.; Kovacs, C.S. The nonskeletal effects of vitamin D: An endocrine society scientific statement. Endocr. Rev. 2012, 33, 456-492.

27. Mohr, S.B. A brief history of vitamin D and cancer prevention. Ann. Epidemiol. 2009, 19, 79-83.

28. Haussler, M.R.; Norman, A.W. Chromosomal receptor for a vitamin D metabolite. Proc. Natl. Acad. Sci. USA 1969, 62, 155-162.

29. McDonnell, D.P.; Mangelsdorf, D.J.; Pike, J.W.; Haussler, M.R.; O’Malley, B.W. Molecular cloning of complementary DNA encoding the avian receptor for vitamin D. Science 1987, 235, 1214-1217.

30. Rochel, N.; Wurtz, J.M.; Mitschler, A.; Klaholz, B.; Moras, D. The crystal structure of the nuclear receptor for vitamin D bound to its natural ligand. Mol. Cell 2000, 5, 173-179.

31. Lee, S.; Clark, S.A.; Gill, R.K.; Christakos, S. 1,25-Dihydroxyvitamin D3 and pancreatic beta-cell function: Vitamin D receptors, gene expression, and insulin secretion. Endocrinology 1994, 134, 1602-1610.

32. McGrath, J.J.; Feron, F.P.; Burne, T.H.; Mackay-Sim, A.; Eyles, D.W. Vitamin D3-implications for brain development. J. Steroid Biochem. Mol. Biol. 2004, 89-90, 557-560.

33. De Paula, F.J.; Dick-de-Paula, I.; Bornstein, S.; Rostama, B.; Le, P.; Lotinun, S.; Baron, R.; Rosen, C.J. VDR haploinsufficiency impacts body composition and skeletal acquisition in a gender-specific manner. Calcif. Tissue Int. 2011, 89, 179-191.

34. DeLuca, H.F. Overview of general physiologic features and functions of vitamin D. Am. J. Clin. Nutr. 2004, 80, 1689S-1696S.

35. Zanatta, L.; Zamoner, A.; Zanatta, A.P.; Bouraima-Lelong, H.; Delalande, C.; Bois, C.; Carreau, S.; Silva, F.R. Nongenomic and genomic effects of 1alpha, $25(\mathrm{OH}) 2$ vitamin D3 in rat testis. Life Sci. 2011, 89, 515-523.

36. Wang, Y.; Zhu, J.; DeLuca, H.F. Where is the vitamin D receptor? Arch. Biochem. Biophys. 2012, 523, 123-133.

37. Haussler, M.R.; Whitfield, G.K.; Haussler, C.A.; Hsieh, J.C.; Thompson, P.D.; Selznick, S.H.; Dominguez, C.E.; Jurutka, P.W. The nuclear vitamin D receptor: Biological and molecular regulatory properties revealed. J. Bone Miner. Res. 1998, 13, 325-349.

38. Rachez, C.; Suldan, Z.; Ward, J.; Chang, C.P.; Burakov, D.; Erdjument-Bromage, H.; Tempst, P.; Freedman, L.P. A novel protein complex that interacts with the vitamin D3 receptor in a ligand-dependent manner and enhances VDR transactivation in a cell-free system. Genes Dev. 1998, 12, 1787-1800. 
39. Christakos, S.; Dhawan, P.; Liu, Y.; Peng, X.; Porta, A. New insights into the mechanisms of vitamin D action. J. Cell. Biochem. 2003, 88, 695-705.

40. Deeb, K.K.; Trump, D.L.; Johnson, C.S. Vitamin D signalling pathways in cancer: Potential for anticancer therapeutics. Nat. Rev. Cancer 2007, 7, 684-700.

41. Teichert, A.; Arnold, L.A.; Otieno, S.; Oda, Y.; Augustinaite, I.; Geistlinger, T.R.; Kriwacki, R.W.; Guy, R.K.; Bikle, D.D. Quantification of the vitamin D receptor-coregulator interaction. Biochemistry 2009, 48, 1454-1461.

42. Gill, R.K.; Christakos, S. Identification of sequence elements in mouse calbindin-d28k gene that confer 1,25-dihydroxyvitamin D3- and butyrate-inducible responses. Proc. Natl. Acad. Sci. USA 1993, 90, 2984-2988.

43. Xie, Z.; Bikle, D.D. Cloning of the human phospholipase C-gamma1 promoter and identification of a DR6-type vitamin D-responsive element. J. Biol. Chem. 1997, 272, 6573-6577.

44. Kurokawa, R.; Yu, V.C.; Naar, A.; Kyakumoto, S.; Han, Z.; Silverman, S.; Rosenfeld, M.G.; Glass, C.K. Differential orientations of the DNA-binding domain and carboxy-terminal dimerization interface regulate binding site selection by nuclear receptor heterodimers. Genes Dev. 1993, 7, 1423-1435.

45. Crofts, L.A.; Hancock, M.S.; Morrison, N.A.; Eisman, J.A. Multiple promoters direct the tissue-specific expression of novel $n$-terminal variant human vitamin $\mathrm{D}$ receptor gene transcripts. Proc. Natl. Acad. Sci. USA 1998, 95, 10529-10534.

46. Sunn, K.L.; Cock, T.A.; Crofts, L.A.; Eisman, J.A.; Gardiner, E.M. Novel $n$-terminal variant of human VDR. Mol. Endocrinol. 2001, 15, 1599-1609.

47. Hsieh, J.C.; Shimizu, Y.; Minoshima, S.; Shimizu, N.; Haussler, C.A.; Jurutka, P.W.; Haussler, M.R. Novel nuclear localization signal between the two DNA-binding zinc fingers in the human vitamin D receptor. J. Cell. Biochem. 1998, 70, 94-109.

48. Michigami, T.; Suga, A.; Yamazaki, M.; Shimizu, C.; Cai, G.; Okada, S.; Ozono, K. Identification of amino acid sequence in the hinge region of human vitamin $\mathrm{D}$ receptor that transfers a cytosolic protein to the nucleus. J. Biol. Chem. 1999, 274, 33531-33538.

49. Kato, S.; Fujiki, R.; Kitagawa, H. Vitamin D receptor (VDR) promoter targeting through a novel chromatin remodeling complex. J. Steroid Biochem. Mol. Biol. 2004, 89-90, 173-178.

50. Fujiki, R.; Kim, M.S.; Sasaki, Y.; Yoshimura, K.; Kitagawa, H.; Kato, S. Ligand-Induced transrepression by VDR through association of WSTF with acetylated histones. EMBOJ. 2005, 24, 3881-3894.

51. Kato, S.; Fujiki, R.; Kim, M.S.; Kitagawa, H. Ligand-Induced transrepressive function of VDR requires a chromatin remodeling complex, WINAC. J. Steroid Biochem. Mol. Biol. 2007, 103, 372-380.

52. Oda, Y.; Sihlbom, C.; Chalkley, R.J.; Huang, L.; Rachez, C.; Chang, C.P.; Burlingame, A.L.; Freedman, L.P.; Bikle, D.D. Two distinct coactivators, DRIP/mediator and SRC/p160, are differentially involved in VDR transactivation during keratinocyte differentiation. J. Steroid Biochem. Mol. Biol. 2004, 89-90, 273-276. 
53. Oda, Y.; Chalkley, R.J.; Burlingame, A.L.; Bikle, D.D. The transcriptional coactivator drip/mediator complex is involved in vitamin $\mathrm{D}$ receptor function and regulates keratinocyte proliferation and differentiation. J. Investig. Dermatol. 2010, 130, 2377-2388.

54. Garcia-Bassets, I.; Kwon, Y.S.; Telese, F.; Prefontaine, G.G.; Hutt, K.R.; Cheng, C.S.; Ju, B.G.; Ohgi, K.A.; Wang, J.; Escoubet-Lozach, L.; et al. Histone methylation-dependent mechanisms impose ligand dependency for gene activation by nuclear receptors. Cell 2007, $128,505-518$.

55. Li, B.; Carey, M.; Workman, J.L. The role of chromatin during transcription. Cell 2007, 128, 707-719.

56. Rachez, C.; Lemon, B.D.; Suldan, Z.; Bromleigh, V.; Gamble, M.; Naar, A.M.; Erdjument-Bromage, H.; Tempst, P.; Freedman, L.P. Ligand-dependent transcription activation by nuclear receptors requires the DRIP complex. Nature 1999, 398, 824-828.

57. Rachez, C.; Gamble, M.; Chang, C.P.; Atkins, G.B.; Lazar, M.A.; Freedman, L.P. The DRIP complex and SRC-1/p160 coactivators share similar nuclear receptor binding determinants but constitute functionally distinct complexes. Mol. Cell Biol. 2000, 20, 2718-2726.

58. Segal, J. Action of the thyroid hormone at the level of the plasma membrane. Endocr. Res. 1989, 15, 619-649.

59. Morley, P.; Whitfield, J.F.; Vanderhyden, B.C.; Tsang, B.K.; Schwartz, J.L. A new, nongenomic estrogen action: The rapid release of intracellular calcium. Endocrinology 1992, $131,1305-1312$.

60. Nemere, I.; Safford, S.E.; Rohe, B.; DeSouza, M.M.; Farach-Carson, M.C. Identification and characterization of 1,25D3-membrane-associated rapid response, steroid (1,25d3-MARRS) binding protein. J. Steroid Biochem. Mol. Biol. 2004, 89-90, 281-285.

61. Caffrey, J.M.; Farach-Carson, M.C. Vitamin D3 metabolites modulate dihydropyridine-sensitive calcium currents in clonal rat osteosarcoma cells. J. Biol. Chem. 1989, 264, 20265-20274.

62. Baran, D.T.; Sorensen, A.M.; Honeyman, T.W.; Ray, R.; Holick, M.F. 1 alpha,25-dihydroxyvitamin D3-induced increments in hepatocyte cytosolic calcium and lysophosphatidylinositol: Inhibition by pertussis toxin and 1 beta,25-dihydroxyvitamin D3. J. Bone Miner. Res. 1990, 5, 517-524.

63. Wali, R.K.; Baum, C.L.; Sitrin, M.D.; Brasitus, T.A. 1,25(OH)2 vitamin D3 stimulates membrane phosphoinositide turnover, activates protein kinase $\mathrm{C}$, and increases cytosolic calcium in rat colonic epithelium. J. Clin. Investig. 1990, 85, 1296-1303.

64. Morelli, S.; de Boland, A.R.; Boland, R.L. Generation of inositol phosphates, diacylglycerol and calcium fluxes in myoblasts treated with 1,25-dihydroxyvitamin D3. Biochem. J. 1993, 289, 675-679.

65. Baran, D.T.; Sorensen, A.M. Rapid actions of 1 alpha-25-dihydroxyvitamin D3 physiologic role. Proc. Soc. Exp. Biol. Med. 1994, 207, 175-179.

66. Sitrin, M.D.; Bissonnette, M.; Bolt, M.J.; Wali, R.; Khare, S.; Scaglione-Sewell, B.; Skarosi, S.; Brasitus, T.A. Rapid effects of 1,25(OH)2 vitamin D3 on signal transduction systems in colonic cells. Steroids 1999, 64, 137-142. 
67. Norman, A.W.; Ishizuka, S.; Okamura, W.H. Ligands for the vitamin D endocrine system: Different shapes function as agonists and antagonists for genomic and rapid response receptors or as a ligand for the plasma vitamin D binding protein. J. Steroid Biochem. Mol. Biol. 2001, $76,49-59$.

68. Nemere, I.; Garbi, N.; Hammerling, G.; Hintze, K.J. Role of the 1,25D3-MARRS receptor in the 1,25(OH)2D3-stimulated uptake of calcium and phosphate in intestinal cells. Steroids 2012, 77, 897-902.

69. Shi, H.; Norman, A.W.; Okamura, W.H.; Sen, A.; Zemel, M.B. 1alpha,25-Dihydroxyvitamin D3 modulates human adipocyte metabolism via nongenomic action. FASEB J. 2001, 15, 2751-2753.

70. Richard, C.L.; Farach-Carson, M.C.; Rohe, B.; Nemere, I.; Meckling, K.A. Involvement of 1,25D3-MARRS (membrane associated, rapid response steroid-binding), a novel vitamin D receptor, in growth inhibition of breast cancer cells. Exp. Cell Res. 2010, 316, 695-703.

71. Jensen, S.S.; Madsen, M.W.; Lukas, J.; Binderup, L.; Bartek, J. Inhibitory effects of 1alpha,25-dihydroxyvitamin D(3) on the G(1)-S phase-controlling machinery. Mol. Endocrinol. 2001, 15, 1370-1380.

72. Salehi-Tabar, R.; Nguyen-Yamamoto, L.; Tavera-Mendoza, L.E.; Quail, T.; Dimitrov, V.; An, B.S.; Glass, L.; Goltzman, D.; White, J.H. Vitamin D receptor as a master regulator of the c-MYC/MXD1 network. Proc. Natl. Acad. Sci. USA 2012, 109, 18827-18832.

73. Meyer, M.B.; Goetsch, P.D.; Pike, J.W. VDR/RXR and TCF4/ $\beta$-catenin cistromes in colonic cells of colorectal tumor origin: Impact on c-FOS and c-MYC gene expression. Mol. Endocrinol. 2012, 26, 37-51.

74. Washington, M.N.; Kim, J.S.; Weigel, N.L. 1 1 ,25-dihydroxyvitamin D3 inhibits C4-2 prostate cancer cell growth via a retinoblastoma protein ( $\mathrm{Rb}$ )-independent G1 arrest. Prostat 2011, 71, 98-110.

75. Li, P.; Li, C.; Zhao, X.; Zhang, X.; Nicosia, S.V.; Bai, W. p27(Kip1) stabilization and G(1) arrest by 1,25 -dihydroxyvitamin $\mathrm{D}(3)$ in ovarian cancer cells mediated through down-regulation of cyclin E/cyclin-dependent kinase 2 and Skp1-Cullin-F-box protein/Skp2 ubiquitin ligase. J. Biol. Chem. 2004, 279, 25260-25267.

76. Akutsu, N.; Lin, R.; Bastien, Y.; Bestawros, A.; Enepekides, D.J.; Black, M.J.; White, J.H. Regulation of gene expression by 1alpha,25-dihydroxyvitamin D3 and its analog EB1089 under growth-inhibitory conditions in squamous carcinoma cells. Mol. Endocrinol. 2001, 15, $1127-1139$.

77. Chiang, K.C.; Yeh, C.N.; Chen, S.C.; Shen, S.C.; Hsu, J.T.; Yeh, T.S.; Pang, J.H.; Su, L.J.; Takano, M.; Kittaka, A.; et al. MART-10, a new generation of vitamin D analog, is more potent than 1alpha,25-dihydroxyvitamin $\mathrm{D}(3)$ in inhibiting cell proliferation and inducing apoptosis in ER+ MCF-7 breast cancer cells. Evid. Based Complement. Alternat. Med. 2012, 2012, 310872.

78. Baudet, C.; Chevalier, G.; Chassevent, A.; Canova, C.; Filmon, R.; Larra, F.; Brachet, P.; Wion, D. 1,25-Dihydroxyvitamin D3 induces programmed cell death in a rat glioma cell line. J. Neurosci. Res. 1996, 46, 540-550. 
79. Bao, B.Y.; Hu, Y.C.; Ting, H.J.; Lee, Y.F. Androgen signaling is required for the vitamin D-mediated growth inhibition in human prostate cancer cells. Oncogene 2004, 23, 3350-3360.

80. Valko, M.; Rhodes, C.J.; Moncol, J.; Izakovic, M.; Mazur, M. Free radicals, metals and antioxidants in oxidative stress-induced cancer. Chem. Biol. Interact. 2006, 160, 1-40.

81. Valko, M.; Leibfritz, D.; Moncol, J.; Cronin, M.T.; Mazur, M.; Telser, J. Free radicals and antioxidants in normal physiological functions and human disease. Int. J. Biochem. Cell. Biol. 2007, 39, 44-84.

82. Losso, J.N.; Bawadi, H.A. Hypoxia inducible factor pathways as targets for functional foods. J. Agric. Food Chem. 2005, 53, 3751-3768.

83. Peehl, D.M.; Shinghal, R.; Nonn, L.; Seto, E.; Krishnan, A.V.; Brooks, J.D.; Feldman, D. Molecular activity of 1,25-dihydroxyvitamin D3 in primary cultures of human prostatic epithelial cells revealed by cDNA microarray analysis. J. Steroid Biochem. Mol. Biol. 2004, 92, 131-141.

84. Bao, B.Y.; Ting, H.J.; Hsu, J.W.; Lee, Y.F. Protective role of 1 alpha, 25-dihydroxyvitamin D3 against oxidative stress in nonmalignant human prostate epithelial cells. Int. J. Cancer 2008, 122, 2699-2706.

85. Fleet, J.C.; DeSmet, M.; Johnson, R.; Li, Y. Vitamin D and cancer: A review of molecular mechanisms. Biochem. J. 2012, 441, 61-76.

86. Ben-Shoshan, M.; Amir, S.; Dang, D.T.; Dang, L.H.; Weisman, Y.; Mabjeesh, N.J. 1alpha,25Dihydroxyvitamin D3 (calcitriol) inhibits hypoxia-inducible factor-1/vascular endothelial growth factor pathway in human cancer cells. Mol. Cancer Ther. 2007, 6, 1433-1439.

87. Chung, I.; Han, G.; Seshadri, M.; Gillard, B.M.; Yu, W.D.; Foster, B.A.; Trump, D.L.; Johnson, C.S. Role of vitamin D receptor in the antiproliferative effects of calcitriol in tumor-derived endothelial cells and tumor angiogenesis in vivo. Cancer Res. 2009, 69, 967-975.

88. Dormoy, V.; Beraud, C.; Lindner, V.; Coquard, C.; Barthelmebs, M.; Brasse, D.; Jacqmin, D.; Lang, H.; Massfelder, T. Vitamin D3 triggers antitumor activity through targeting hedgehog signaling in human renal cell carcinoma. Carcinogenesis 2012, 33, 2084-2093.

89. Yu, H.; Rohan, T. Role of the insulin-like growth factor family in cancer development and progression. J. Natl. Cancer Inst. 2000, 92, 1472-1489.

90. Figueroa, J.A.; de Raad, S.; Tadlock, L.; Speights, V.O.; Rinehart, J.J. Differential expression of insulin-like growth factor binding proteins in high versus low gleason score prostate cancer. J. Urol. 1998, 159, 1379-1383.

91. Kriebitzsch, C.; Verlinden, L.; Eelen, G.; Tan, B.K.; van Camp, M.; Bouillon, R.; Verstuyf, A. The impact of $1,25(\mathrm{OH}) 2 \mathrm{D} 3$ and its structural analogs on gene expression in cancer cells-A microarray approach. Anticancer Res. 2009, 29, 3471-3483.

92. Krishnan, A.V.; Shinghal, R.; Raghavachari, N.; Brooks, J.D.; Peehl, D.M.; Feldman, D. Analysis of vitamin D-regulated gene expression in LNCaP human prostate cancer cells using cDNA microarrays. Prostate 2004, 59, 243-251.

93. Lee, H.J.; Liu, H.; Goodman, C.; Ji, Y.; Maehr, H.; Uskokovic, M.; Notterman, D.; Reiss, M.; Suh, N. Gene expression profiling changes induced by a novel gemini vitamin D derivative during the progression of breast cancer. Biochem. Pharmacol. 2006, 72, 332-343. 
94. Swami, S.; Raghavachari, N.; Muller, U.R.; Bao, Y.P.; Feldman, D. Vitamin D growth inhibition of breast cancer cells: Gene expression patterns assessed by cDNA microarray. Breast Cancer Res. Treat. 2003, 80, 49-62.

95. Chen, A.; Davis, B.H.; Sitrin, M.D.; Brasitus, T.A.; Bissonnette, M. Transforming growth factor-beta 1 signaling contributes to caco-2 cell growth inhibition induced by 1,25(OH)(2)D(3). Am. J. Physiol. Gastrointest Liver Physiol. 2002, 283, G864-G874.

96. Tong, W.M.; Hofer, H.; Ellinger, A.; Peterlik, M.; Cross, H.S. Mechanism of antimitogenic action of vitamin D in human colon carcinoma cells: Relevance for suppression of epidermal growth factor-stimulated cell growth. Oncol. Res. 1999, 11, 77-84.

97. Yanagisawa, J.; Yanagi, Y.; Masuhiro, Y.; Suzawa, M.; Watanabe, M.; Kashiwagi, K.; Toriyabe, T.; Kawabata, M.; Miyazono, K.; Kato, S. Convergence of transforming growth factor-beta and vitamin D signaling pathways on SMAD transcriptional coactivators. Science 1999, 283, 1317-1321.

98. Schlange, T.; Matsuda, Y.; Lienhard, S.; Huber, A.; Hynes, N.E. Autocrine WNT signaling contributes to breast cancer cell proliferation via the canonical WNT pathway and EGFR transactivation. Breast Cancer Res. 2007, 9, R63.

99. Rosenbluh, J.; Nijhawan, D.; Cox, A.G.; Li, X.; Neal, J.T.; Schafer, E.J.; Zack, T.I.; Wang, X.; Tsherniak, A.; Schinzel, A.C.; et al. Beta-Catenin-Driven cancers require a yap 1 transcriptional complex for survival and tumorigenesis. Cell 2012, 151, 1457-1473.

100. Behrens, J. Everything you would like to know about wnt signaling. Sci. Signal. 2013, 6, pe17.

101. Vadlakonda, L.; Pasupuleti, M.; Pallu, R. Role of pi3k-akt-mtor and wnt signaling pathways in transition of g1-s phase of cell cycle in cancer cells. Front. Oncol. 2013, 3, 85.

102. Brabletz, T.; Jung, A.; Dag, S.; Hlubek, F.; Kirchner, T. Beta-Catenin regulates the expression of the matrix metalloproteinase-7 in human colorectal cancer. Am. J. Pathol. 1999, 155, 1033-1038.

103. Nusse, R.; Varmus, H.E. Wnt genes. Cell 1992, 69, 1073-1087.

104. Nusse, R. The wnt gene family in tumorigenesis and in normal development. J. Steroid Biochem. Mol. Biol. 1992, 43, 9-12.

105. Nusse, R. Wnt signaling and stem cell control. Cell Res. 2008, 18, 523-527.

106. Jamora, C.; DasGupta, R.; Kocieniewski, P.; Fuchs, E. Links between signal transduction, transcription and adhesion in epithelial bud development. Nature 2003, 422, 317-322.

107. Kovalenko, P.L.; Zhang, Z.; Cui, M.; Clinton, S.K.; Fleet, J.C. 1,25 dihydroxyvitamin d-mediated orchestration of anticancer, transcript-level effects in the immortalized, non-transformed prostate epithelial cell line, rwpe1. BMC Genomics 2010, 11, 26.

108. Palmer, H.G.; Gonzalez-Sancho, J.M.; Espada, J.; Berciano, M.T.; Puig, I.; Baulida, J.; Quintanilla, M.; Cano, A.; de Herreros, A.G.; Lafarga, M.; et al. Vitamin d(3) promotes the differentiation of colon carcinoma cells by the induction of e-cadherin and the inhibition of beta-catenin signaling. J. Cell Biol. 2001, 154, 369-387.

109. Pendas-Franco, N.; Aguilera, O.; Pereira, F.; Gonzalez-Sancho, J.M.; Munoz, A. Vitamin d and wnt/beta-catenin pathway in colon cancer: Role and regulation of dickkopf genes. Anticancer Res. 2008, 28, 2613-2623. 
110. Beildeck, M.E.; Islam, M.; Shah, S.; Welsh, J.; Byers, S.W. Control of tcf-4 expression by vdr and vitamin $\mathrm{d}$ in the mouse mammary gland and colorectal cancer cell lines. PLoS One 2009, 4, e7872.

111. Rohan, J.N.; Weigel, N.L. 1alpha,25-dihydroxyvitamin d3 reduces c-myc expression, inhibiting proliferation and causing g1 accumulation in c4-2 prostate cancer cells. Endocrinology 2009, 150, 2046-2054.

112. Rawson, J.B.; Sun, Z.; Dicks, E.; Daftary, D.; Parfrey, P.S.; Green, R.C.; Gallinger, S.; McLaughlin, J.R.; Wang, P.P.; Knight, J.A.; et al. Vitamin d intake is negatively associated with promoter methylation of the wnt antagonist gene dkk1 in a large group of colorectal cancer patients. Nutr. Cancer 2012, 64, 919-928.

113. Aguilera, O.; Pena, C.; Garcia, J.M.; Larriba, M.J.; Ordonez-Moran, P.; Navarro, D.; Barbachano, A.; Lopez de Silanes, I.; Ballestar, E.; Fraga, M.F.; et al. The wnt antagonist dickkopf-1 gene is induced by 1 alpha,25-dihydroxyvitamin $\mathrm{d} 3$ associated to the differentiation of human colon cancer cells. Carcinogenesis 2007, 28, 1877-1884.

114. Villaggio, B.; Soldano, S.; Cutolo, M. 1,25-Dihydroxyvitamin D3 downregulates aromatase expression and inflammatory cytokines in human macrophages. Clin. Exp. Rheumatol. 2012, 30, 934-938.

115. Kaler, P.; Augenlicht, L.; Klampfer, L. Macrophage-derived il-1beta stimulates wnt signaling and growth of colon cancer cells: A crosstalk interrupted by vitamin d3. Oncogene 2009, 28, 3892-3902.

116. Bao, B.Y.; Yao, J.; Lee, Y.F. 1alpha,25-Dihydroxyvitamin d3 suppresses interleukin-8-mediated prostate cancer cell angiogenesis. Carcinogenesis 2006, 27, 1883-1893.

117. Tse, A.K.; Zhu, G.Y.; Wan, C.K.; Shen, X.L.; Yu, Z.L.; Fong, W.F. 1 alpha,25-Dihydroxyvitamin $\mathrm{d} 3$ inhibits transcriptional potential of nuclear factor kappa $\mathrm{b}$ in breast cancer cells. Mol. Immunol. 2010, 47, 1728-1738.

118. Hoyer-Hansen, M.; Jaattela, M. Autophagy: An emerging target for cancer therapy. Autophagy 2008, 4, 574-580.

119. Bristol, M.L.; Di, X.; Beckman, M.J.; Wilson, E.N.; Henderson, S.C.; Maiti, A.; Fan, Z.; Gewirtz, D.A. Dual functions of autophagy in the response of breast tumor cells to radiation: Cytoprotective autophagy with radiation alone and cytotoxic autophagy in radiosensitization by vitamin d 3. Autophagy 2012, 8, 739-753.

120. Mathew, R.; Kongara, S.; Beaudoin, B.; Karp, C.M.; Bray, K.; Degenhardt, K.; Chen, G.; Jin, S.; White, E. Autophagy suppresses tumor progression by limiting chromosomal instability. Genes Dev. 2007, 21, 1367-1381.

121. Karantza-Wadsworth, V.; Patel, S.; Kravchuk, O.; Chen, G.; Mathew, R.; Jin, S.; White, E. Autophagy mitigates metabolic stress and genome damage in mammary tumorigenesis. Genes Dev. 2007, 21, 1621-1635.

122. Wang, J. Beclin 1 bridges autophagy, apoptosis and differentiation. Autophagy 2008, 4, 947-948.

123. Wang, J.; Lian, H.; Zhao, Y.; Kauss, M.A.; Spindel, S. Vitamin d3 induces autophagy of human myeloid leukemia cells. J. Biol. Chem. 2008, 283, 25596-25605. 
124. Lozy, F.; Karantza, V. Autophagy and cancer cell metabolism. Semin. Cell Dev. Biol. 2012, 23, 395-401.

125. Kato, S.; Takeyama, K.; Kitanaka, S.; Murayama, A.; Sekine, K.; Yoshizawa, T. In vivo function of VDR in gene expression-VDR knock-out mice. J. Steroid Biochem. Mol. Biol. 1999, 69, 247-251.

126. Li, Y.C.; Pirro, A.E.; Amling, M.; Delling, G.; Baron, R.; Bronson, R.; Demay, M.B. Targeted ablation of the vitamin d receptor: An animal model of vitamin d-dependent rickets type ii with alopecia. Proc. Natl. Acad. Sci. USA 1997, 94, 9831-9835.

127. Nakagawa, K.; Kawaura, A.; Kato, S.; Takeda, E.; Okano, T. Metastatic growth of lung cancer cells is extremely reduced in vitamin d receptor knockout mice. J. Steroid Biochem. Mol. Biol. 2004, 89-90, 545-547.

128. Kallay, E.; Pietschmann, P.; Toyokuni, S.; Bajna, E.; Hahn, P.; Mazzucco, K.; Bieglmayer, C.; Kato, S.; Cross, H.S. Characterization of a vitamin d receptor knockout mouse as a model of colorectal hyperproliferation and DNA damage. Carcinogenesis 2001, 22, 1429-1435.

129. Kallay, E.; Bareis, P.; Bajna, E.; Kriwanek, S.; Bonner, E.; Toyokuni, S.; Cross, H.S. Vitamin $\mathrm{d}$ receptor activity and prevention of colonic hyperproliferation and oxidative stress. Food Chem. Toxicol. 2002, 40, 1191-1196.

130. Zinser, G.; Packman, K.; Welsh, J. Vitamin d(3) receptor ablation alters mammary gland morphogenesis. Development 2002, 129, 3067-3076.

131. Mehta, R.G.; Moriarty, R.M.; Mehta, R.R.; Penmasta, R.; Lazzaro, G.; Constantinou, A.; Guo, L. Prevention of preneoplastic mammary lesion development by a novel vitamin $\mathrm{d}$ analogue, 1alpha-hydroxyvitamin d5. J. Natl. Cancer Inst. 1997, 89, 212-218.

132. Mehta, R.; Hawthorne, M.; Uselding, L.; Albinescu, D.; Moriarty, R.; Christov, K. Prevention of $N$-methyl- $N$-nitrosourea-induced mammary carcinogenesis in rats by 1alpha-hydroxyvitamin d(5). J. Natl. Cancer Inst. 2000, 92, 1836-1840.

133. Jacobson, E.A.; James, K.A.; Newmark, H.L.; Carroll, K.K. Effects of dietary fat, calcium, and vitamin $\mathrm{d}$ on growth and mammary tumorigenesis induced by 7,12-dimethylbenz(a)anthracene in female sprague-dawley rats. Cancer Res. 1989, 49, 6300-6303.

134. VanWeelden, K.; Flanagan, L.; Binderup, L.; Tenniswood, M.; Welsh, J. Apoptotic regression of MCF-7 xenografts in nude mice treated with the vitamin d3 analog, Eb1089. Endocrinology 1998, 139, 2102-2110.

135. Welsh, J. Vitamin d and breast cancer: Insights from animal models. Am. J. Clin. Nutr. 2004, $80,1721 \mathrm{~S}-1724 \mathrm{~S}$.

136. Zinser, G.M.; Welsh, J. Accelerated mammary gland development during pregnancy and delayed postlactational involution in vitamin d3 receptor null mice. Mol. Endocrinol. 2004, 18, $2208-2223$.

137. Welsh, J. Cellular and molecular effects of vitamin d on carcinogenesis. Arch. Biochem. Biophys. 2012, 523, 107-114.

138. Xu, H.; McCann, M.; Zhang, Z.; Posner, G.H.; Bingham, V.; El-Tanani, M.; Campbell, F.C. Vitamin $\mathrm{d}$ receptor modulates the neoplastic phenotype through antagonistic growth regulatory signals. Mol. Carcinog. 2009, 48, 758-772. 
139. Xu, H.; Posner, G.H.; Stevenson, M.; Campbell, F.C. Apc(min) modulation of vitamin d secosteroid growth control. Carcinogenesis 2010, 31, 1434-1441.

140. Xie, Z.; Komuves, L.; Yu, Q.C.; Elalieh, H.; Ng, D.C.; Leary, C.; Chang, S.; Crumrine, D.; Yoshizawa, T.; Kato, S.; et al. Lack of the vitamin d receptor is associated with reduced epidermal differentiation and hair follicle growth. J. Investig. Dermatol. 2002, 118, 11-16.

141. Zinser, G.M.; Sundberg, J.P.; Welsh, J. Vitamin d(3) receptor ablation sensitizes skin to chemically induced tumorigenesis. Carcinogenesis 2002, 23, 2103-2109.

142. Zinser, G.M.; Tribble, E.; Valrance, M.; Urben, C.M.; Knutson, J.C.; Mazess, R.B.; Strugnell, S.A.; Welsh, J. 1,24(s)-dihydroxyvitamin d2, an endogenous vitamin d2 metabolite, inhibits growth of breast cancer cells and tumors. Anticancer Res. 2005, 25, 235-241.

143. Mordan-McCombs, S.; Brown, T.; Wang, W.L.; Gaupel, A.C.; Welsh, J.; Tenniswood, M. Tumor progression in the lpb-tag transgenic model of prostate cancer is altered by vitamin $\mathrm{d}$ receptor and serum testosterone status. J. Steroid Biochem. Mol. Biol. 2010, 121, 368-371.

144. Garland, C.F.; Garland, F.C. Do sunlight and vitamin d reduce the likelihood of colon cancer? Int. J. Epidemiol. 1980, 9, 227-231.

145. Garland, F.C.; Garland, C.F.; Gorham, E.D.; Young, J.F. Geographic variation in breast cancer mortality in the united states: A hypothesis involving exposure to solar radiation. Prev. Med. 1990, 19, 614-622.

146. Lefkowitz, E.S.; Garland, C.F. Sunlight, vitamin d, and ovarian cancer mortality rates in US women. Int. J. Epidemiol. 1994, 23, 1133-1136.

147. Grant, W.B. Ecological studies of the UVB-vitamin d-cancer hypothesis. Anticancer Res. 2012, $32,223-236$.

148. Grant, W.B. Update on evidence that support a role of solar ultraviolet-b irradiance in reducing cancer risk. Anti-Cancer Agents Med. Chem. 2013, 13, 140-146.

149. Grant, W.B.; Garland, C.F. The association of solar ultraviolet b (UVB) with reducing risk of cancer: Multifactorial ecologic analysis of geographic variation in age-adjusted cancer mortality rates. Anticancer Res. 2006, 26, 2687-2699.

150. Grant, W.B. An estimate of premature cancer mortality in the U.S. Due to inadequate doses of solar ultraviolet-b radiation. Cancer 2002, 94, 1867-1875.

151. Mizoue, T. Ecological study of solar radiation and cancer mortality in japan. Health Phys. 2004, $87,532-538$.

152. Boscoe, F.P.; Schymura, M.J. Solar ultraviolet-b exposure and cancer incidence and mortality in the united states, 1993-2002. BMC Cancer 2006, 6, 264.

153. Chen, W.; Clements, M.; Rahman, B.; Zhang, S.; Qiao, Y.; Armstrong, B.K. Relationship between cancer mortality/incidence and ambient ultraviolet $\mathrm{b}$ irradiance in china. Cancer Causes Control. 2010, 21, 1701-1709.

154. Lin, S.W.; Wheeler, D.C.; Park, Y.; Cahoon, E.K.; Hollenbeck, A.R.; Freedman, D.M.; Abnet, C.C. Prospective study of ultraviolet radiation exposure and risk of cancer in the united states. Int. J. Cancer 2012, 131, E1015-E1023.

155. Astbury, A. Non Uniformity in Cancer Mortality in the USA and Australia Appears to Share a Common Origin; TRIUMF: Vancouver, BC, USA, 2005. 
156. Grant, W.B. Does solar ultraviolet irradiation affect cancer mortality rates in china? Asian Pac. J. Cancer Prev. 2007, 8, 236-242.

157. Grant, W.B. An ecologic study of cancer mortality rates in spain with respect to indices of solar UVB irradiance and smoking. Int. J. Cancer 2007, 120, 1123-1128.

158. Grant, W.B. An ecological study of cancer incidence and mortality rates in france with respect to latitude, an index for vitamin d production. Deramato-Endocrinology 2010, 2, 62-67.

159. Grant, W.B. An ecological study of cancer mortality rates in california, 1950-64, with respect to solar UVB and smoking indices. Dermato-Endocrinology 2012, 4, 176-182.

160. Lombardi, C.; Heck, J.E.; Cockburn, M.; Ritz, B. Solar UV radiation and cancer in young children. Cancer Epidemiol. Biomark. Prev. 2013, 22, 1118-1128.

161. Fioletov, V.E.; McArthur, L.J.; Mathews, T.W.; Marrett, L. Estimated ultraviolet exposure levels for a sufficient vitamin d status in north america. J. Photochem. Photobiol. B 2010, 100, $57-66$.

162. Grant, W.B. Role of solar UVB irradiance and smoking in cancer as inferred from cancer incidence rates by occupation in nordic countries. Dermato-Endocrinology 2012, 4, 203-211.

163. Brittingham, A.; de la Cruz, G.P. Ancestry 2000. Census 2000 Brief ck2br-35; U.S. Dept. of Commerce: Washington, DC, USA, 2004.

164. Grant, W.B. A multicountry ecological study of risk-modifying factors for prostate cancer: Apolipoprotein e epsilon 4 as a risk factor and cereals as a risk reduction factor. Anticancer Res. 2010, 30, 189-199.

165. Lehrer, S. Possible relationship of the apolipoprotein e (apoe) epsilon4 allele to prostate cancer. Br. J. Cancer 1998, 78, 1398.

166. Grant, W.B. Relation between prediagnostic serum 25-hydroxyvitamin d level and incidence of breast, colorectal, and other cancers. J. Photochem. Photobiol. B 2010, 101, 130-136.

167. Devesa, S.S.; Grauman, D.J.; Blot, W.J.; Pennello, G.A.; Hoover, R.N.; Fraumeni, J.F.J. Atlas of Cancer Mortality in the United States, 1950-1994. In NIH Publication No. 99-4564; National Institute of Health: Rockville, MD, USA, 1999.

168. Grant, W.B. An ecological study of cancer mortality rates in the united states with respect to solar ultraviolet-b doses, smoking, alcohol consumption and urban/rural residence. Dermato-Endocrinology 2010, 2, 68-76.

169. Grant, W.B. Effect of interval between serum draw and follow-up period on relative risk of cancer incidence with respect to 25-hydroxyvitamin d level: Implications for meta-analyses and setting vitamin d guidelines. Dermato-Endocrinology 2011, 3, 199-204.

170. Grant, W.B. Effect of follow-up time on the relation between prediagnostic serum 25-hydroxyvitamin d and all-cause mortality rate. Dermato-Endocrinology 2012, 4, 198-202.

171. Giovannucci, E.; Liu, Y.; Rimm, E.B.; Hollis, B.W.; Fuchs, C.S.; Stampfer, M.J.; Willett, W.C. Prospective study of predictors of vitamin d status and cancer incidence and mortality in men. J. Natl. Cancer Inst. 2006, 98, 451-459.

172. Afzal, S.; Bojesen, S.E.; Nordestgaard, B.G. Low plasma 25-hydroxyvitamin d and risk of tobacco-related cancer. Clin. Chem. 2013, 59, 771-780. 
173. Mondul, A.M.; Weinstein, S.J.; Mannisto, S.; Snyder, K.; Horst, R.L.; Virtamo, J.; Albanes, D. Serum vitamin $d$ and risk of bladder cancer. Cancer Res. 2010, 70, 9218-9223.

174. Epstein, E.; Lindqvist, P.G.; Geppert, B.; Olsson, H. A population-based cohort study on sun habits and endometrial cancer. Br. J. Cancer 2009, 101, 537-540.

175. Orell-Kotikangas, H.; Schwab, U.; Osterlund, P.; Saarilahti, K.; Makitie, O.; Makitie, A.A. High prevalence of vitamin $\mathrm{d}$ insufficiency in patients with head and neck cancer at diagnosis. Head Neck 2012, 34, 1450-1455.

176. Kricker, A.; Armstrong, B.K.; Hughes, A.M.; Goumas, C.; Smedby, K.E.; Zheng, T.; Spinelli, J.J.; de Sanjose, S.; Hartge, P.; Melbye, M.; et al. Personal sun exposure and risk of non hodgkin lymphoma: A pooled analysis from the interlymph consortium. Int. J. Cancer 2008, $122,144-154$.

177. Toriola, A.T.; Surcel, H.M.; Calypse, A.; Grankvist, K.; Luostarinen, T.; Lukanova, A.; Pukkala, E.; Lehtinen, M. Independent and joint effects of serum 25-hydroxyvitamin $\mathrm{d}$ and calcium on ovarian cancer risk: A prospective nested case-control study. Eur. J. Cancer 2010, 46, 2799-2805.

178. Wolpin, B.M.; Ng, K.; Bao, Y.; Kraft, P.; Stampfer, M.J.; Michaud, D.S.; Ma, J.; Buring, J.E.; Sesso, H.D.; Lee, I.M.; et al. Plasma 25-hydroxyvitamin d and risk of pancreatic cancer. Cancer Epidemiol. Biomark. Prev. 2012, 21, 82-91.

179. Joh, H.K.; Giovannucci, E.L.; Bertrand, K.A.; Lim, S.; Cho, E. Predicted plasma 25-hydroxyvitamin d and risk of renal cell cancer. J. Natl. Cancer Inst. 2013, 105, 726-732.

180. Roskies, M.; Dolev, Y.; Caglar, D.; Hier, M.P.; Mlynarek, A.; Majdan, A.; Payne, R.J. Vitamin $\mathrm{d}$ deficiency as a potentially modifiable risk factor for thyroid cancer. J. Otolaryngol. Head Neck Surg. 2012, 41, 160-163.

181. Shimada, T.; Urakawa, I.; Isakova, T.; Yamazaki, Y.; Epstein, M.; Wesseling-Perry, K.; Wolf, M.; Salusky, I.B.; Juppner, H. Circulating fibroblast growth factor 23 in patients with end-stage renal disease treated by peritoneal dialysis is intact and biologically active. J. Clin. Endocrinol. Metab. 2010, 95, 578-585.

182. Oh, E.Y.; Ansell, C.; Nawaz, H.; Yang, C.H.; Wood, P.A.; Hrushesky, W.J. Global breast cancer seasonality. Breast Cancer Res. Treat. 2010, 123, 233-243.

183. Lappe, J.M.; Travers-Gustafson, D.; Davies, K.M.; Recker, R.R.; Heaney, R.P. Vitamin d and calcium supplementation reduces cancer risk: Results of a randomized trial. Am. J. Clin. Nutr. 2007, 85, 1586-1591.

184. Peterlik, M.; Grant, W.B.; Cross, H.S. Calcium, vitamin d and cancer. Anticancer Res. 2009, 29, 3687-3698.

185. Bolland, M.J.; Grey, A.; Gamble, G.D.; Reid, I.R. Calcium and vitamin d supplements and health outcomes: A reanalysis of the women's health initiative (whi) limited-access data set. Am. J. Clin. Nutr. 2011, 94, 1144-1149.

186. Garland, C.F.; French, C.B.; Baggerly, L.L.; Heaney, R.P. Vitamin d supplement doses and serum 25-hydroxyvitamin $d$ in the range associated with cancer prevention. Anticancer Res. 2011, 31, 607-611. 
187. Lappe, J.M.; Heaney, R.P. Why randomized controlled trials of calcium and vitamin d sometimes fail. Dermato-Endocrinology 2012, 4, 95-100.

188. Ng, K.; Meyerhardt, J.A.; Wu, K.; Feskanich, D.; Hollis, B.W.; Giovannucci, E.L.; Fuchs, C.S. Circulating 25-hydroxyvitamin d levels and survival in patients with colorectal cancer. J. Clin. Oncol. 2008, 26, 2984-2991.

189. Grant, W.B.; Peiris, A.N. Differences in vitamin d status may account for unexplained disparities in cancer survival rates between african and white americans. Dermato-Endocrinology 2012, 4, 85-94.

190. Ginde, A.A.; Liu, M.C.; Camargo, C.A., Jr. Demographic differences and trends of vitamin d insufficiency in the us population, 1988-2004. Arch. Intern. Med. 2009, 169, 626-632.

191. Hill, A.B. The environment and disease: Association or causation? Proc. R. Soc. Med. 1965, 58, 295-300.

192. Grant, W.B. How strong is the evidence that solar ultraviolet $b$ and vitamin $d$ reduce the risk of cancer? An examination using hill's criteria for causality. Dermato-Endocrinology 2009, 1, 17-24.

193. Mohr, S.B.; Gorham, E.D.; Alcaraz, J.E.; Kane, C.I.; Macera, C.A.; Parsons, J.K.; Wingard, D.L.; Garland, C.F. Does the evidence for an inverse relationship between serum vitamin $\mathrm{d}$ status and breast cancer risk satisfy the hill criteria? Dermato-Endocrinology 2012, 4, 152-157. 


\title{
The Role of Vitamin D in Hematologic Disease and Stem Cell Transplantation
}

\author{
Aric C. Hall and Mark B. Juckett
}

\begin{abstract}
Vitamin D is a steroid hormone with a broad range of biological effects ranging from the classical role as a mediator of calcium and phosphate balance to cellular differentiation and immune modulation. These effects impact normal and dysfunctional hematopoietic and immune function, which may allow an avenue for improved treatment and support of patients suffering from hematologic disorders. In this review, we will summarize the role of vitamin D in normal hematopoiesis, discuss ways in which vitamin D may improve outcomes, and discuss a potential role of vitamin D for treating hematologic disorders and modulating the immune system to improve the outcome of allogeneic stem cell transplant.
\end{abstract}

Reprinted from Nutrients. Cite as: Hall, A.C.; Juckett, M.B. The Role of Vitamin D in Hematologic Disease and Stem Cell Transplantation. Nutrients 2013, 5, 2206-2221.

\section{Introduction}

Vitamin D is a steroid hormone whose classical role is as a regulator of calcium and phosphate metabolism. This action, carried out through the renal, skeletal, and gastrointestinal systems has accumulated additional attention over the last several years with osteoporosis and bone health becoming an increasingly important concern for an aging population. Further investigation has discovered high rates of vitamin D deficiency in the United States population leading to a proliferation of recommendations for supplementation and investigation into the activity of the hormone. As investigators pursued the biochemical targets of vitamin D that influenced calcium homeostasis, multiple other roles of vitamin D signaling were discovered in various tissues that express vitamin D receptors (VDRs). VDRs have been identified on hematopoietic and lymphoid cells, leading to efforts to understand the role of vitamin D in blood cell development and immune system function. In this review we will attempt to give an overview of the role of vitamin D in normal hematopoietic and immune function, explore experience at exploiting vitamin D receptor signaling to treat hematologic disorders, discuss roles of the vitamin D receptor in immune recovery following hematopoietic stem cell transplant, and finally discuss the use of vitamin D in hematology and stem cell transplant patients.

\section{Vitamin D in Normal Hematopoiesis}

\subsection{Vitamin D Production and Mechanism of Biological Action}

Human vitamin D production requires the skin, liver, and kidney to convert 7-dehydrocholesterol in the skin to 1,25-dihydroxycholecalciferol, the active form of the hormone. Dehydroxycholesterol is converted to cholecalciferol (Vitamin D3) with UV light as a cofactor and then goes through two hydroxylation steps to 25-hydroxycholecalciferol and then 1,25-dihydroxycholecalciferol in the liver 
and kidney [1]. Vitamin D2 (ergocalciferol) is produced in some fungi and invertebrates in an analogous manner, as ergosterol is converted to ergocalciferol with UVB light as a necessary cofactor [2]. Ergocalciferol can be absorbed from the intestines when taken as a supplement and is similarly hydroxylated for activity so that, like 1,25 dihydroxyvitamin $\mathrm{D}$, it can activate the vitamin $\mathrm{D}$ receptor (VDR). Vitamins D2 and D3 can both be referred to as vitamin D and references differ as to their biological equivalence. In general, authors who contend a difference in biological activity suggest vitamin D3 is more biologically potent [3]. The VDR is the mediator of vitamin D activity. It is a receptor in the family of steroid/thyroid hormone-activated transcription factors. The gene for VDR is encoded on chromosome 12 , and is subject to significant variability among individuals due to numerous polymorphisms [4]. Several germ line variants of the vitamin D receptor gene (alleles) are known to exist, explaining at least part of the variability in VDR activity among individuals [5]. These polymorphisms have been linked to such diverse biological effects as adult height, bone mineral density, and susceptibility to early periodontal disease and tuberculosis [6,7].

After being bound by activated vitamin D, VDR can form a homodimer or form a heterodimer with a retinoid $\mathrm{X}$ receptor. Those dimers can then binds to vitamin $\mathrm{D}$ response elements (VDREs) in promoters of target genes eventually leading to target gene transcription [1,4]. Screens for VDREs have revealed them in multiple growth, differentiation, and apoptosis governing genes including some known to be involved in human malignancy such as cyclin D1; Cyclin dependent kinases 2,4,6; PTEN; P53; and PI3 Kinase [8].

\subsection{Vitamin D Role in Myeloid Differentiation}

Aside from its ubiquity in affecting the transcription of many genes, VDR is present on a wide variety of tissues outside the intestine, bones, and kidneys, which are the organs most involved in the classical role of vitamin D. In the hematopoietic system the VDR receptor is expressed on various hematopoietic precursors as well as monocytes, some thymocytes, and activated $\mathrm{B}$ and $\mathrm{T}$ lymphocytes [8]. Studies with knock-out (KO) animals show vitamin D signaling does not appear to be necessary for differentiation of the normal hematopoietic cell repertoire, as mice with vitamin $\mathrm{D}$ receptor knock-out produce normal numbers and proportions of blood cells [8-10]. Still, vitamin D stimulation can influence hematopoietic development as experiments treating both normal hematopoietic stem cell lines and leukemic cell lines with the active form of vitamin D led to increased monocyte/macrophage differentiation and increased numbers of those mature cells-an effect that is not observed in VDR KO mice [11,12]. After being bound by a vitamin D analogue, the VDR forms a homodimer or binds the retinoid $\mathrm{X}$ receptor and proceeds to interact with a VDRE and signal transcription of various effector RNAs [9,11]. The retinoic acid receptor (RAR) is an alternative dimerization partner for the retinoid X receptor [13]. Just as VDR activity seems to drive monocytic differentiation, the RAR activity drives differentiation toward mature granulocytes [11]. Experiments in cell cultures suggest that activated RAR and VDR compete for binding of RXR and the relative balance between RAR/RXR vs. VDR/RXR dimers influence the relative activity of monopoiesis and granulopoiesis [11,14]. A double knock-out animal for VDR and RAR create appropriate colony forming units for monocytes and granulocytes so it is suspected that the primary effect of vitamin D is on cytokine signaling and the final steps in differentiation in these two cell types [11]. 
The complexity of the interaction between VDR and RXR is highlighted by a recent finding that retinoic acid and vitamin D can potentiate the other's activity as VDR stimulation seems to be augmented in the presence of RAR:RXR binding [11].

\subsection{Vitamin D Role in Immune Modulation}

In addition to the role of vitamin D and the VDR in normal hematopoiesis and leukocyte differentiation the presence of the VDR on activated lymphocytes suggests a role in immune modulation on differentiated cells. VDR knock-out (KO) mice have a normal number of $\mathrm{T}$ and $\mathrm{B}$ lymphocytes but there are changes in the cytokine profile in VDR knock-out mice that affect the T-helper (Th) immune response. Inflammatory cytokines can elicit a cellular immune response termed "Th1" or a humoral immune response termed "Th2". The Th1 response is stimulated by IL2 and IFN- $\gamma$ as major mediators, while the Th2 response is driven by IL-4, IL-6, and IL-10 [9]. In general, stimulation of the VDR receptor has been shown to favor the Th2 response by suppressing IFN $-\gamma$ and this underlies the immune-modulatory effects of Vitamin D $[8,9]$. This is somewhat confounded, however, by the fact that in the context of complete vitamin D knock-out, the Th1 response is actually impaired, possibly through inhibition of IL-18 [9]. The VDR also seems to be crucial for proper development of invariant natural killer (iNK) cells, a subset of lymphoid cells involved in the most basic immune responses and also in restricting autoimmunity $[15,16]$. This was noted in the rodent VDR KO model in which iNKT cells were reduced. Reduced iNKT may promote autoimmunity but also blunted airway response to inflammation $[15,16]$. VDR receptor may also have a role in the homing of lymphoid cells to specific tissues and in attenuating inflammation. This has been shown by $\mathrm{Yu}$ and colleagues who studied a mouse model of inflammatory bowel disease. They were able to show that VDR KO animals developed an autoimmune inflammatory bowel disease in response to commensal flora, but the effect could be blunted with wild type CD4 cells [17]. Ultimately, they demonstrated that intraepithelial lymphocytes (with a pattern of CD4 $\alpha \alpha+$ ) were dramatically reduced in VDR KO mice due to lower production and impaired tissue homing. CD4 $\alpha \alpha+\mathrm{T}$ lymphocytes are T-regulatory cells that help suppress inflammation by secretion of IL-10 and thus their reduced presence led to a fulminant colitis [17]. This provides an example of vitamin $\mathrm{D}$ mediated attenuation of an immune response suggesting that vitamin $\mathrm{D}$ may have a role in immune tolerance, the process by which autoimmunity is prevented.

\section{Utilizing Vitamin D in Antineoplastic Therapy}

\subsection{Vitamin D as Differentiation Therapy for Myeloid Malignancies}

In vitro studies show that vitamin $\mathrm{D}$ promotes differentiation of normal hematopoietic precursors and malignant myeloblasts, which has led to significant interest in studying vitamin D analogues as treatment for myeloid malignancies, particularly myelodysplastic syndrome (MDS) and acute myeloid leukemia (AML). MDS is a hematologic condition that typically manifests as cytopenias (low numbers of normal blood cells) and is due to acquired mutations in the DNA of hematopoietic stem cells causing difficulties in differentiation and production of mature cell types. Some forms of MDS may cause increased numbers of myeloblasts or "blasts," which are immature, undifferentiated 
precursors of blood cells found circulating in the blood or present in the marrow and indicative of flawed differentiation of hematopoietic stem cells. These forms of MDS are considered higher risk as they are more likely to accumulate additional mutations that may lead to AML. The blast percentage, severity of cytopenias, age, and cytogenetics (chromosomal abnormalities found in tissue culture) are also used to risk stratify patients with MDS using tools such as the International Prognostic Scoring System (IPSS). AML is an aggressive blood cancer defined by replacement of normal hematopoietic cells with rapidly replicating myeloblasts, which are unable to undergo differentiation and thus accumulate in the marrow and peripheral blood. It may evolve spontaneously or as a secondary process from MDS. Much of the early research into therapy with vitamin D analogues paralleled the discovery that all-trans retinoic acid (ATRA), a ligand for RXR, could produce deep, durable remissions for acute promyelocytic leukemia (APML), a particular form of AML. This discovery supported the idea of differentiation therapy as a viable and exciting treatment for myeloid malignancies and an alternative to the classic approach using cytotoxic chemotherapy. Specific pre-clinical experience with HL-60 and other leukemic lines, such as U-937 and THP-1 have all shown differentiation and apoptosis of neoplastic myeloblasts with vitamin $\mathrm{D}$, which would seem to suggest these compounds can, like ATRA in APML, convert differentiation-arrested myeloblasts into a mature blood cell $[1,11,18]$. The exact mechanisms by which this change is induced by Vitamin D receptor activation is not fully understood and investigations have revealed complex cross-signaling involving PI3 Kinase, activation of multiple pro-differentiation steps in the MAPK pathway, and likely upregulation of pro-apoptotic factors such as p53 [1]. Of note, early preclinical trials used supraphysiological doses of vitamin $\mathrm{D}$ to induce differentiation, raising concern therapeutic doses would necessarily cause hypercalcemia.[11,19] Early efforts, however, showed that fractionated dosing seemed to offer some promise for achieving differentiation with lower doses within physiological ranges [12].

\subsection{Vitamin D as Therapy for Myelodysplastic Syndrome}

There are seven studies of single agent vitamin D therapy for MDS. Koeffler and colleagues performed the first study of such a regimen in 1985, when they reported on 18 patients with MDS treated with single agent 1,25-dihydroxycholecalciferol up to a dose of $2 \mu \mathrm{g}$ per day [19]. Although $8 / 18$ patients had minor hematologic responses, no response persisted over the full 12 weeks of the study and hypercalcemia was a common toxicity of treatment [19]. It is important to note that this was a population at high-risk for death or the development of AML. Seven individuals had disease that transformed to AML over the study period [19]. Takahashi and colleagues treated 11 patients with MDS with 1-hydroxycholecalciferol and similarly saw partial or minor and transient responses in five of the 11 [20]. They were able to minimize hypercalcemia with intermittent dosing of the vitamin D, but this may have reduced efficacy [20]. Other studies of vitamin D analogues alone for MDS include Molnar and colleagues, who in 2007 reported treating 23 patients with low or intermediate risk MDS with 2000-4000 international units of cholecalciferol daily. They failed to show any hypercalcemia, but there were no hematologic responses [21]. Mellibovsky and colleagues reported a positive single arm study of 19 patients with low or intermediate risk MDS by IPSS treated with either 25-hydroxycholecalciferol (five patients) or 1,25 dihydroxycholecalciferol (14 patients) 
of whom 11/19 had significant hematologic response [22]. Of note, there were no cases of hypercalcemia in this study.

There are two studies looking at vitamin D2 analogues for MDS. Koeffler and colleagues treated 12 patients with low to high risk disease with a vitamin D2 analogue (Paracalcitol) with no responses noted; one patient had a doubling of his platelet count but succumbed to fatal fungal infection shortly afterward [23]. In 2008 Petrich and colleagues reported a phase II study with 15 MDS patients, 12 with low risk disease, who were treated with doxercalciferol, another D2 analogue with possibly lower risk of hypercalcemia [24]. Nine of 14 patients completed the full planned 12 weeks of treatment with five stopping early due to progressive disease and one due to hypercalcemia [24]. Six patients had stable disease and ultimately eight progressed while on study leading to a conclusion that doxercalciferol had at best minimal activity. Notably, both patients with CMML experienced worsening monocytosis that reversed upon discontinuation of doxercalciferol, which may suggest an in vivo differentiation effect [24].

One notable study is a series of 30 patients treated by Motomura and colleagues in which patients were randomized to supportive care or 1-hydroxyvitamin D3 starting at $1 \mu \mathrm{g}$ and titrated up to 4-6 $\mu \mathrm{g}$ if tolerated [25]. The two groups were matched for diagnoses and had a reasonable balance of higher and lower risk patients. The patients allotted to vitamin D3 received on average 17 months of therapy. Two patients developed hypercalcemia on the treatment arm and only one had a transient hematologic improvement with the rest failing to improve. Nevertheless, only one of the 15 patients on vitamin $\mathrm{D}$ analogue therapy and $7 / 15$ on the control arm progressed to AML leading to a statistically significant benefit in survival in the treatment group [25].

There have been efforts to combine vitamin D with other differentiation and cytotoxic agents. Blazsek and colleagues reported a case in 1991 of a patient with MDS who responded to a combination of cis-retinoic acid and vitamin D therapy, leading to the hypothesis that this combination could be useful for non-APML myeloid disorders [26]. Siitonen and colleagues reported the results of a series of 19 patients with all types and risk categories of MDS treated with a combination of 13-cis-retinoic acid, 1,25-dihydroxycholecalciferol (1 $\mu \mathrm{g}$ daily), and valproic acid [27]. Valproic acid in this case was being used as a histone deacetylase (HDAC) inhibitor. Overall 3/19 patients had minor hematologic improvement, but toxicity, general linked to valproic acid (fatigue, liver function changes) and retinoids (skin problems), led 8/19 patients to be intolerant of the regimen [27]. The authors ultimately concluded that the addition of vitamin D did not seem to add significant synergistic activity to the valproic acid regimen already known to have modest activity.

One promising path is the use of growth factors with differentiating agents. Ferrero and colleagues reported in 2008 on a study of 63 patients with MDS, excluding high risk patients with high numbers of blasts, treated with a combination of recombinant human erythropoietin as well as cis-retinoic acid; 1,25 dihydroxycholecalciferol; and 6-thioguanine if blasts were present [28]. The purpose of this study was primarily to assess erythroid response and compare it to the rates seen with erythropoietin alone or the combination of the two differentiating agents and thioguanine alone, which was approximately $30 \%$ for each. Overall, there was an erythroid response rate of $60 \%$ overall, which was better in the low risk patients than those with excess blasts [28]. There was also data for improved survival for the low risk patients who had a response, with $93 \%$ of responders $v s$. $58 \%$ of 
nonresponders alive at three years [28]. Though presented as a positive study, the lack of erythropoietin dose standardization or control arm makes the benefits from the new agents difficult to appreciate. Toxicity was generally mild and primarily related to the retinoid agents. Surprisingly, there was little emphasis on the cardiovascular mortality rate of 8/63 patients, which could have been associated with the use of the erythroid stimulating agent [28].

Another group of studies has looked at combinations of vitamin D analogues as differentiating agents with cytotoxic chemotherapy. Ferrero and colleagues reported on experience treating 53 MDS patients with 13-cis-retinoic acid (20-40 mg/day) and 1,25-dihydroxycholecalciferol (1-1.5 $\mu \mathrm{g} /$ day) with or without intermittent thioguanine, a cytotoxic agent [29]. Thioguanine was utilized three out of every six weeks for subjects with a marrow blast count of $>5 \%$ or those who failed to respond to the dual differentiating agent therapy [29]. Ultimately, 40/53 patients ended up receiving thioguanine. Overall response rate was $60 \%$ but rates of complete response (CR) were low with only two patients in the entire cohort achieving that outcome. Still, 50\% of patients had a decline in transfusion requirement, which is a clinically relevant outcome and could significantly improve quality of life [29].

\subsection{Vitamin D as Therapy for Acute Myeloid Leukemia}

MDS, particularly in its lower risk forms, may be an indolent disease with a natural history extending over years. AML, on the other hand, tends to be fatal over weeks to a short number of months without effective treatment. Therefore, therapeutic plans that include only differentiating agents such as vitamin D have tended to be used only in patients with very treatment resistant disease or those at high risk for side effects of conventional cytotoxic chemotherapy. The only studies of vitamin D monotherapy in AML consists of a total of five patients treated in Japan in the mid 1980s of whom $4 / 5$ had transient improvements in blasts and 1/5 had reported brief normalization of the bone marrow as reported by Irino, Takahashi, and Nakayama [20].

There are at least two series of elderly AML patients treated with low dose cytarabine regimens (subcutaneous in Europe and low dose IV in the US) combined with vitamin D analogue and another agent. Low dose cytarabine is a relatively well-tolerated cytotoxic regimen that had been the standard of care for elderly or unfit patients with AML. Slapak and colleagues in 1992 reported the use of cytarabine at continuous infusion $\left(20 \mathrm{mg} / \mathrm{m}^{2} /\right.$ day) for 21 days with oral hydroxyurea (500 $\mathrm{mg}$ twice daily) starting the day preceding cytarabine and continuing for 22 days, and 1,25 dihydroxyvitamin $\mathrm{D}(0.5 \mu \mathrm{g}$ twice daily) from the initiation of therapy until relapse or end of study [30]. Of the 29 patients (all older than 62 years) there was an overall response rate $79 \%$ of which $13(45 \%)$ were in CR [30]. Toxicity was primarily hematologic with severe neutropenia and thrombocytopenia common, though this is essentially an unavoidable side effect of all effective therapies for AML. Only two patients developed hypercalcemia, which was asymptomatic and required no further treatment than holding the vitamin D analogue. Median remission duration was 9.8 months with overall survival of 12 and 14 months for non-responders and responders, respectively [30]. These results are considered at least not inferior to those seen with low dose cytarabine alone. Ferrero and colleagues in 2004 report a similar low dose cytarabine $\left(8 \mathrm{mg} / \mathrm{m}^{2}\right.$ subcutaneously twice daily) and 1,25-dihydroxyvitamin $\mathrm{D}(1 \mu \mathrm{g}$ daily) regimen with 13 cis-retinoic acid (20-40 mg daily) and 
thioguanine (40 mg daily). Thioguanine and cytarabine were given for the first two to three weeks with 1,25-dihydroxyvitamin D3 and 13 cis-retinoic acid for a five week course. Those who responded received dual differentiating agent therapy continuously with either daily thioguanine, one out of every three weeks, or 6-mercaptopurine + cytarabine for two weeks out of every five or six [31]. Thirty total patients were treated (24 with AML and 6 with MDS) and using similar response criteria to the Slapak study, this regimen showed a 50\% response rate with 8 having a CR (27\%) and seven having a partial response (PR) [31]. Once again, toxicity was primarily related to cytopenias and is difficult to separate from disease effect. Median survival was 7.5 months for the entire treatment arm but significantly better at 16.5 months in responders [31]. Toxicity was primarily related to profound cytopenias with no hypercalcemia reported [31]. As in other studies with retinoic acid derivatives, dry mouth and lips were a common side effect.

The only study of vitamin D analogue combined with chemotherapy with a control group in the published literature is that reported by Hellstrom and colleagues in Sweden [32]. Seventy-eight total patients were treated, 68 with MDS and 15 with AML (either proceeding from MDS or with blast count of $<30 \%$ ). Cytarabine was given in a divided dose of $15 \mathrm{mg} / \mathrm{m}^{2} /$ day subcutaneously and continued until bone marrow cellularity was $\leq 50 \%$ of that at study initiation or until "unacceptable" peripheral cytopenias developed. At that point cytarabine was held to allow counts to stabilize then reintroduced unless there was failure for count recovery, clear signs of progression, intolerance, or response. Half of the patients received dual differentiating agent therapy in addition to cytarabine with 13 cis-retinoic acid at $1 \mathrm{mg} / \mathrm{kg} /$ day and $1 \alpha \mathrm{OH}$-vitamin D at a starting dose of $1 \mu \mathrm{g} /$ day and increasing until mild hypercalcemia developed [32]. In this study there was no significant difference in response rate between the two arms with response rates of $26.1 \%$ overall for the study [32]. Median overall survival was 10.5 months and not different between the arms [32]. Rate of transformation to AML in the MDS cohort was similarly unchanged. Given increased toxicity in the experimental arm, this was felt to suggest no additive benefit of differentiation agents with low dose cytarabine in the management of high risk MDS/AML [32].

Unfortunately, the studies of vitamin D monotherapy for myeloid disorders suffer from limitations including lack of control group, variable response criteria, and unclear end-points, which makes drawing conclusions difficult. Ultimately, it seems a portion of patients with myeloid disorders may respond to vitamin D monotherapy but the numbers are likely small and the responses transient. Patients with lower grade disease and without blasts may have a higher likelihood of response. Combination therapies may show more promise but few of these include a control group and the use of other active agents makes it difficult to determine the activity of the vitamin analogues. The only vitamin $\mathrm{D}$ and chemotherapy combination regimen studied in a controlled trial failed to show benefit from the addition of vitamin D. It is possible that hypercalcemia in vivo, which was seen in almost all studies, limits the maximally tolerated dose, significantly diminishing the therapeutic potential of vitamin D based therapies. Further studies are needed to define a role of vitamin D therapy in the treatment of myeloid malignancies. 


\subsection{Vitamin D as Therapy for Non-Myeloid Hematologic Cancers}

There are few studies examining the role of vitamin D in non-myeloid blood disorders. Multiple pre-clinical studies have shown activity of the vitamin D analogue EB1089 in the myeloma H929 cell line [33-35]. In this case, the agent appears to promote apoptosis, and induce cell cycle arrest by down regulation of cyclin dependent kinases, an activity that is augmented by transforming growth factor beta (TGF- $\beta$ ) [33]. There are pre-clinical studies demonstrating that vitamin D has an inhibitory effect on lymphoid neoplastic cells, but to date, no studies in humans have been performed [8].

\section{Vitamin D as a Modulatory of Immune Response in Allogeneic Transplant}

There is considerable interest in vitamin D analogues for their immune-modulatory effects. The last decade has brought a renaissance of immune therapies in oncology with the approval of rituximab, a humanized IgG1 targeting CD20 on lymphoma cells; ipalimumab, a CTLA-4 inhibitor that upregulates the immune response against melanoma; and promising results with PD-1 inhibitors, which also increase immune responses against many other malignancies. Hematologic malignancies have long been known to be susceptible to immune surveillance and allogeneic hematopoietic stem cell transplantation (HSCT) has been established as a curative therapy for many hematologic malignancies since the 1980s. HSCT is performed by replacing an individual's entire hematopoietic system, and hence his or her immune system, with that of another person. HSCT is the only potential curative therapy for several hematological disorders but presents two major barriers associated with the immune system. To allow a donor immune system to persist without being rejected, the recipient must receive immune suppressive chemotherapy, which causes severe reduction in immune cells and a significantly increased risk of infection. Post-transplant, when the donor immune system has grown, it may attack or reject the recipient's tissues in a process called graft versus host disease (GVHD). Treatment of GVHD requires immune suppressive medications, thus leading to a delicate interplay between the dangers of immune stimulation and immune suppression. Optimal therapy would involve stimulation of the donor immune system to react and destroy the blood disease, without driving the immune response to healthy recipient tissues or organs. This process of immune modulation is mediated by a complex system of lymphocytes and associated cytokine regulators and agents that can modulate this system are attractive to improve outcomes.

Vitamin D via the VDR has a role in immune regulation and vitamin D analogues are well-established therapies for some autoimmune conditions such as psoriasis. This effect is likely due to the known effects of vitamin D on activated $\mathrm{T}$ and $\mathrm{B}$ cells that may affect signaling, tissue targeting, or immune regulation. Of course, given their roles in monopoiesis and macrophage activity, VDR stimulation appears to be crucial to the activity of antigen presenting cells and this is felt to be responsible for the role VDR activity seems to play in response to infections. For example, in tuberculosis infection decreased vitamin D levels appear to increase the risk of tuberculosis disease and supplementation may help resolve infection [7,36]. The immunomodulatory role of vitamin D suggests possible importance in the outcome following HSCT. VDR genes are polymorphic in the human population and the genetic variation in VDR has been a subject of investigation in patients undergoing HSCT. These polymorphisms were discovered by variation in restriction enzyme cleavage sites and thus are 
defined and named for these enzymes (i.e., TaqI, ApaI) with two different allele possibilities with different cleavage patterns. Though the exact ways in which various polymorphisms change vitamin $D$ receptor activity are often unknown these genetic variations have been associated with variability in immune function and other vitamin D activities such as growth, bone formation, and susceptibility to infectious diseases [6].

Cho et al. reported an analysis of VDR polymorphisms in 147 Korean patients who underwent matched related donor HSCT. They analyzed recipient VDR polymorphisms and retrospectively evaluated the association with patient outcomes including infection, GVHD, overall survival (OS) and disease free survival (DFS) [37]. The most significant findings were a correlation between polymorphisms at the Taq1 cleavage site where heterozygotes, (those possessing at least one copy of the $\mathrm{C}$ allele), had better disease free survival (DFS) and overall survival than TT homozygotes [37]. The functional significance of this allelic variation is unknown and no direct link to high or low VDR activity is reported. This study also found that recipients having two copies of the "A" allele for the ApaI polymorphism experienced decreased rates of acute GVHD and infection [37]. Polymorphisms of ApaI have been correlated to VDR activity with homozygosity for the "a" allele associated with higher VDR activity [4].

Middleton and colleagues reported a cohort of 88 patients with myeloid malignancies whose VDR gene polymorphisms and those of their 80 sibling donors were analyzed and correlated to outcomes [6]. Like Cho and colleagues, they noted a marked trend toward diminished acute GVHD in recipients with the AA (low VDR activity) genotype. Donor results for ApaI polymorphisms were somewhat more confusing. Recipients of donors with high VDR activity genotype (aa) showed a trend toward more GVHD, though not statistically significant $(p=0.065)$, but recipients of donors with the low VDR activity genotype (AA) had a statistically significant increased rate of death (HR 2.027, $p=0.0232$ ) [6]. This is somewhat unusual as GVHD is a major contributor of mortality from HSCT and typically increased rates of GVHD will lead to increased mortality. This decreased survival was not statistically significant in patients who received cyclosporine GVHD prophylaxis alone $(p=0.83)$ but was marked for those patients who received multiagent GVHD prophylaxis with cyclosporine and the addition of methotrexate $(n=17)$, ATG $(n=4)$, corticosteroids $(n=4)$, or multiple additional agents $(n=2)(\mathrm{HR}>12, p<0.0 .0001)$. Primary causes of death in this cohort were infection, relapse, and interstitial pneumonitis [6].

Finally, Bogunia-Kubik and colleagues published an analysis of 123 Polish patients with the inclusion of those receiving unrelated donors [4]. They found an association between the FF genotype, which is associated with high VDR activity, and outcomes following HSCT. If both the donor and recipient possessed the FF genotype, the recipient experienced higher risk of GVHD [4]. As in the other studies ApaI genotype also impacted risks of GVHD. Interestingly, different genotypes corresponded to higher GVHD risks depending on whether they were present in the donor or recipient. In this analysis, unlike the previous one by Middleton, donor AA (low VDR activity) genotype correlated with a higher risk for GVHD than donor genotypes with at least one "a" allele [4]. At the same time, recipient aa (high VDR activity) genotype had a higher risk of GVHD and death than the low VDR activity genotype, which is consistent with what has been seen in other studies [4]. 
Clearly, in its mediation of immune signaling the vitamin D receptor appears to have an impact on immune reconstitution after HSCT and subsequent risks of infection, graft versus host disease, and graft versus disease effect. This would suggest a wide range of therapeutic potential if these relationships can be better understood. Despite the fact that vitamin D through the VDR usually plays an immune modulatory role, it appears that the most vigorous phenotype of the vitamin $\mathrm{D}$ receptor, when present in the recipients of HSCT leads to worse GVHD and worse outcomes [4,6,37]. This is suggested to be due to a particular role of stimulating the immediate immune response and cytokine storm that initiates GVHD, which is likely through a paracrine effect with its presence in recipient tissue [4]. One could also see vigorous VDR activity as promoting antigen presentation and thus a vigorous immune response though there is less speculation on this mechanism. Regarding donor VDR genotype, results are contradictory. The idea of vigorous VDR signaling in donors being favorable (as seen in the study of Bogunia-Kubik and colleagues) could suggest VDR mediated immune activation could promote more aggressive immune surveillance of infection and preclinical disease relapse. In addition, the role of VDR in production and homing of T regulatory cells, such as the CD $4 \alpha \alpha+$ cells seen in the rodent model, could lead to improved modulation of the reconstituted immune system and improve outcomes. This data is compelling in suggesting that VDR and likely vitamin D play a role in immune reconstitution and immune surveillance in transplant patients and the findings may lead to opportunities to improve outcome following HSCT by the use of vitamin D analogues. A better understanding of how vitamin D status may affect the immune milieu in the setting of different polymorphisms could be useful for making recommendations about vitamin D supplementation. Given the general recommendations to supplement vitamin D after transplantation, it may be important to study the role of supplementation on outcome particularly in recipients with vigorous VDR phenotypes. Such a relationship is suggested by unpublished data from Bogunia-Kubik and colleagues, which showed higher rates of GVHD in recipients with the high VDR activity (aa) genotype treated with supplementary vitamin D [4]. Similarly, these findings suggest that in some recipients of HSCT, supplementation could promote better immune function in the reconstituted immune system and help prevent infections or late forms of GVHD [38].

\section{Vitamin D Status in Supportive Care for Treatment of Hematologic Malignancies}

Finally, at the same time vitamin D status is examined as a factor in disease outcomes and the non-classical roles are explored, the classical role of vitamin $\mathrm{D}$ in regulating bone calcium deposition and bone density remains critical. Two populations in which significant attention has been paid to vitamin D status are patients after allogeneic stem cell transplant, who are known to have significant endocrinologic and nutritional risk factors for deficiency related to their treatment, and patients with multiple myeloma, a disease intimately related to bone health.

Pediatric allogeneic stem cell transplant recipients are at particular vulnerability to bone loss due to the high risks of total body irradiation and conditioning chemotherapy to cause endocrine failure syndromes prior to final adult growth and the deposition of primary bone mass in the $20 \mathrm{~s}$. Moustoufi-Moab and colleagues looked at 55 patients who received allogeneic transplant between the ages of 5-26 and who were at least three years from the procedure and compared their bone mineral density scores to healthy controls [39]. Eighty-nine percent of patients did have 
endocrinopathies, and post-transplant patients were found to have significantly lower bone mineral density than healthy controls. Rates of vitamin D deficiency were high but not significantly different between patients and controls in this analysis [39].

Studies of adults have been contradictory in regards to assessing vitamin D status of allogeneic stem cell transplant survivors, though timing at which assessment is done likely has a significant effect. Joseph and colleagues assessing patients on day 0 and day +100 found $70 \%$ and $58 \%$ of patients to be vitamin D deficient, respectively [40]. Of note, this was an observational study and the different time points were assessed in different cohorts of patients. Fourty-six of the 72 patients in the post-transplant cohort had bone mineral density measurements with high rates of osteopenia $(83 \%)$ and osteoporosis $(22 \%)$ but these values were not significantly different in the vitamin D deficient and non-vitamin D deficient patients [39]. Still, the small sample size makes this lack of difference difficult to confirm. Robien and colleagues looked at a mixed cohort of 95 adults and children after at least one-year post transplant and found a only $10 \%$ were frankly vitamin D deficient with an additional $24 \%$ being insufficient [41]. Of note, greater than $60 \%$ of the patients in the study were taking vitamin D supplementation and oral intake was the greatest factor related to adequate levels. A daily intake of 400-600 IU corresponded with adequate levels of serum vitamin D and, as anticipated, prednisone diminished serum vitamin D concentrations [41]. No analysis of bone mineral density was done in this study.

Given these findings, there are clear recommendations on management of vitamin D status and bone health for patients after allogeneic stem cell transplant. UK guidelines recommend DEXA scanning for all patients expected to be on chronic ( $>3$ months) of corticosteroid therapy as well as measurement for and correction of vitamin D deficiency [42]. ASBMT guidelines recommend DEXA for older women, all allogeneic stem cell transplant recipients, or those on chronic steroids or calcineurin inhibitors [43]. Both recommend assessment for vitamin D deficiency, and correction if present.

Myeloma is a plasma cell malignancy that causes lytic bone disease as one of its hallmarks. Given the importance of vitamin D in bone formation, the role of vitamin D supplementation in myeloma has gained some attention. Myeloma is known to promote osteoclastic over osteoblastic activity as a factor in causing lytic bone lesions. This activity can be reversed by one of our newer and best agents for myeloma, bortezomib. It has been shown that vitamin D significantly promotes the ability of bortezomib to inhibit osteoclast proliferation and heal bone lesions [44]. This would suggest an important role for supplementation, or at least adequate repletion, during treatment in order promote healing of bone disease.

At the same time the role of vitamin D has been examined clinically and observationally. Badros and colleagues surveyed 100 consecutive patients seen in University of Maryland cancer center with multiple myeloma and found $75 \%$ of patients were frankly deficient or insufficient [45]. They used this information to emphasize the need for checking and supplementation, particularly as bisphosphonates that are widely recommended in MM have never been shown to be effective without adequate calcium and vitamin D levels [45]. Additionally, attempts have been made to correlate vitamin D levels with myeloma behavior. Two observational studies from the Mayo clinic and Australia look at the clinical characteristics of vitamin D deficient myeloma patients $[46,47]$. 
Diamond and colleagues in New South Wales followed a group of myeloma patients for 10 years and stratified them into quartiles by serum vitamin D concentration. Statistically significant differences in paraprotein level and albumin concentration were seen with vitamin D quartile with lower quartiles associated with worse myeloma markers [47]. The lowest quartile also had significantly worse bone mineral density compared to the other quartiles. On the other hand, $\mathrm{Ng}$ and colleagues at the Mayo clinic looked at 148 newly diagnosed patients and were able to correlate 1,25-dihydroxyvitamin D levels of $<50 \mathrm{nmol} / \mathrm{L}$ with lower albumin and higher CRP [46]. There was also a trend toward higher levels of vitamin D deficiency with higher staged myeloma, but there was no difference in skeletal events between the groups [46]. It should be noted that as observational studies both of these analyses cannot show causation, as it is impossible to know whether bad myeloma influences vitamin D status or vitamin D status influences myeloma behavior. Still, given the role of vitamin $\mathrm{D}$ in helping repair bone damage repletion is likely prudent to assure adequate repletion during treatment.

Ultimately, studies have revealed that post-allogeneic stem cell patients are vulnerable to endocrinopathies and bone mineral loss. Supplementation of fairly low doses seems to correspond to adequate levels and despite somewhat unclear direct relationships between vitamin $\mathrm{D}$ level and bone density at one point in time it seems prudent to maintain normal levels in patients particularly vulnerable to bone disease. Similarly, data that vitamin D is required for bisphosphonate activity and may help bortezomib, an important agent used for treatment of myeloma, suggests that those with lytic skeletal disease should at least receive replacement to assure a normal vitamin D level.

\section{Summary and General Conclusions}

Ultimately, the ubiquity of the vitamin D receptor and the myriad physiologic effects that have been found suggest multiple mechanisms of potential benefit from the use of Vitamin D in the treatment of hematologic disease. In the hematopoietic system there is evidence that the vitamin D pathway affects both differentiation of cells and their ultimate activation once differentiated, although the importance in various disease states remains poorly understood. It can be said with some level of certainty that vitamin D promotes differentiation of monocytes and macrophages under certain conditions, and there is a suggestion that at least a fraction of cases of myeloid disorders (i.e., CMML) may respond to vitamin D supplementation. Similarly, immune-modulatory effects of vitamin D almost certainly affect the complicated immune environment in patients who have had allogeneic stem cell transplants. As the modulation of their immune system is a major factor in the clinical outcomes of these patients, understanding vitamin D signaling better in this situation may be helpful. Well-controlled trials will likely be necessary to confirm any anti-leukemic or anti-MDS benefits of vitamin D therapy. Continued scientific investigation of immune modulation and the role of vitamin $\mathrm{D}$ in that process is still necessary to understand proper mediation of immune function with vitamin $\mathrm{D}$.

\section{Acknowledgments}

The authors would like to thank Howard Bailey for his advice regarding direction of this manuscript. 


\section{Conflicts of Interest}

The authors declare no conflict of interest.

\section{References}

1. Kim, M.; Mirandola, L.; Pandey, A.; Nguyen, D.D.; Jenkins, M.R.; Turcel, M.; Cobos, E.; Chiriva-Internati, M. Application of vitamin D and derivatives in hematological malignancies. Cancer Lett. 2012, 319, 8-22.

2. Shoback, D.; Sellmeyer, D. Metabolic Bone Disease. In Greenspan's Basic and Clinical Endocrinology, 9th ed.; Gardner, D., Shoback, D., Eds.; McGraw-Hill: New York, NY, USA, 2011.

3. Li, B.; Byrjalsen, I.; Glendenning, P.; Henriksen, D.B.; Hoeck, H.C.; Taranto, M.; Vasikaran, S.; Fraser, W.D.; Christiansen, C.; Qvist, P. Selective monitoring of vitamin D2 and D3 supplementation with a highly specific 25-hydroxyvitamin D3 immunoassay with negligible cross-reactivity to 25-hydroxyvitamin D2. Clin. Chim. Acta 2009, 404, 144-148.

4. Bogunia-Kubik, K.; Middleton, P.; Norden, J.; Dickinson, A.; Lange, A. Association of vitamin D receptor polymorphisms with the outcome of allogeneic haematopoietic stem cell transplantation. Int. J. Immunogenet. 2008, 35, 207-213.

5. Bogunia-Kubik, K.; Lange, A. Development of the hla proficiency testing for central and east europe. Int. J. Immunogenet. 2008, 35, 409-416.

6. Middleton, P.G.; Cullup, H.; Dickinson, A.M.; Norden, J.; Jackson, G.H.; Taylor, P.R.; Cavet, J. Vitamin D receptor gene polymorphism associates with graft-versus-host disease and survival in hla-matched sibling allogeneic bone marrow transplantation. Bone Marrow Transplant. 2002, 30, 223-228.

7. Rathored, J.; Sharma, S.K.; Singh, B.; Banavaliker, J.N.; Sreenivas, V.; Srivastava, A.K.; Mohan, A.; Sachan, A.; Harinarayan, C.V.; Goswami, R. Risk and outcome of multidrugresistant tuberculosis: Vitamin D receptor polymorphisms and serum 25(OH)D. Int. J. Tuberc. Lung Dis. 2012, 16, 1522-1528.

8. Luong, Q.T.; Koeffler, H.P. Vitamin D compounds in leukemia. J. Steroid Biochem. Mol. Biol. 2005, 97, 195-202.

9. O’Kelly, J.; Hisatake, J.; Hisatake, Y.; Bishop, J.; Norman, A.; Koeffler, H.P. Normal myelopoiesis but abnormal t lymphocyte responses in vitamin D receptor knockout mice. $J$. Clin. Investig. 2002, 109, 1091-1099.

10. Jeanson, N.T.; Scadden, D.T. Vitamin D receptor deletion leads to increased hematopoietic stem and progenitor cells residing in the spleen. Blood 2010, 116, 4126-4129.

11. Bunce, C.M.; Brown, G.; Hewison, M. Vitamin D and hematopoiesis. Trends Endocrinol. Metab. 1997, 8, 245-251.

12. Grande, A.; Montanari, M.; Tagliafico, E.; Manfredini, R.; Zanocco Marani, T.; Siena, M.; Tenedini, E.; Gallinelli, A.; Ferrari, S. Physiological levels of 1alpha, 25 dihydroxyvitamin D3 induce the monocytic commitment of CD34+ hematopoietic progenitors. J. Leukoc. Biol. 2002, $71,641-651$. 
13. Friedman, A.D. Transcriptional control of granulocyte and monocyte development. Oncogene 2007, 26, 6816-6828.

14. Labrecque, J.; Allan, D.; Chambon, P.; Iscove, N.N.; Lohnes, D.; Hoang, T. Impaired granulocytic differentiation in vitro in hematopoietic cells lacking retinoic acid receptors alpha1 and gamma. Blood 1998, 92, 607-615.

15. Yu, S.; Cantorna, M.T. Epigenetic reduction in invariant nkt cells following in utero vitamin D deficiency in mice. J. Immunol. 2011, 186, 1384-1390.

16. Yu, S.; Zhao, J.; Cantorna, M.T. Invariant NKT cell defects in vitamin D receptor knockout mice prevents experimental lung inflammation. J. Immunol. 2011, 187, 4907-4912.

17. Yu, S.; Bruce, D.; Froicu, M.; Weaver, V.; Cantorna, M.T. Failure of T cell homing, reduced CD4/CD8alphaalpha intraepithelial lymphocytes, and inflammation in the gut of vitamin D receptor ko mice. Proc. Natl. Acad. Sci. USA 2008, 105, 20834-20839.

18. Hughes, P.J.; Marcinkowska, E.; Gocek, E.; Studzinski, G.P.; Brown, G. Vitamin D3-driven signals for myeloid cell differentiation-Implications for differentiation therapy. Leuk. Res. 2010, 34, 553-565.

19. Koeffler, H.P.; Hirji, K.; Itri, L. 1,25-dihydroxyvitamin D3: In vivo and in vitro effects on human preleukemic and leukemic cells. Cancer Treat. Rep. 1985, 69, 1399-1407.

20. Harrison, J.S.; Bershadskiy, A. Clinical experience using vitamin D and analogs in the treatment of myelodysplasia and acute myeloid leukemia: A review of the literature. Leuk. Res. Treat. 2012, 2012, 125814.

21. Molnar, I.; Stark, N.; Lovato, J.; Powell, B.L.; Cruz, J.; Hurd, D.D.; Mathieu, J.S.; Chen, T.C.; Holick, M.F.; Cambra, S.; et al. Treatment of low-risk myelodysplastic syndromes with highdose daily oral cholecalciferol (2000-4000 IU vitamin D(3)). Leukemia 2007, 21, 1089-1092.

22. Mellibovsky, L.; Diez, A.; Perez-Vila, E.; Serrano, S.; Nacher, M.; Aubia, J.; Supervia, A.; Recker, R.R. Vitamin D treatment in myelodysplastic syndromes. Br. J. Haematol. 1998, 100, $516-520$.

23. Koeffler, H.P.; Aslanian, N.; O’Kelly, J. Vitamin D(2) analog (paricalcitol; zemplar) for treatment of myelodysplastic syndrome. Leuk. Res. 2005, 29, 1259-1262.

24. Petrich, A.; Kahl, B.; Bailey, H.; Kim, K.; Turman, N.; Juckett, M. Phase II study of doxercalciferol for the treatment of myelodysplastic syndrome. Leuk. Lymphoma 2008, 49, 57-61.

25. Motomura, S.; Kanamori, H.; Maruta, A.; Kodama, F.; Ohkubo, T. The effect of 1-hydroxyvitamin D3 for prolongation of leukemic transformation-free survival in myelodysplastic syndromes. Am. J. Hematol. 1991, 38, 67-68.

26. Blazsek, I.; Farabos, C.; Musset, M.; Goldschmidt, E.; Comisso, M.; Benavides, M.; Wang, Z.Y.; Labat, M.L.; Mathe, G.; Misset, J.L. Retinoic acid in mono- or combined differentiation therapy of myelodysplasia and acute promyelocytic leukemia. Biomed. Pharmacother. 1991, 45, 169-177.

27. Siitonen, T.; Timonen, T.; Juvonen, E.; Terava, V.; Kutila, A.; Honkanen, T.; Mikkola, M.; Hallman, H.; Kauppila, M.; Nylanden, P.; et al. Valproic acid combined with 13-cis retinoic acid and 1,25-dihydroxyvitamin D3 in the treatment of patients with myelodysplastic syndromes. Haematologica 2007, 92, 1119-1122. 
28. Ferrero, D.; Darbesio, A.; Giai, V.; Genuardi, M.; Dellacasa, C.M.; Sorasio, R.; Bertini, M.; Boccadoro, M. Efficacy of a combination of human recombinant erythropoietin + 13-cis-retinoic acid and dihydroxylated vitamin D3 to improve moderate to severe anaemia in low/intermediate risk myelodysplastic syndromes. Br. J. Haematol. 2009, 144, 342-349.

29. Ferrero, D.; Bruno, B.; Pregno, P.; Stefani, S.; Larizza, E.; Ciravegna, G.; Luraschi, A.; Vietti-Ramus, G.; Schinco, P.; Bazzan, M.; et al. Combined differentiating therapy for myelodysplastic syndromes: A phase II study. Leuk. Res. 1996, 20, 867-876.

30. Slapak, C.A.; Desforges, J.F.; Fogaren, T.; Miller, K.B. Treatment of acute myeloid leukemia in the elderly with low-dose cytarabine, hydroxyurea, and calcitriol. Am. J. Hematol. 1992, 41, $178-183$.

31. Ferrero, D.; Campa, E.; Dellacasa, C.; Campana, S.; Foli, C.; Boccadoro, M. Differentiating agents + low-dose chemotherapy in the management of old/poor prognosis patients with acute myeloid leukemia or myelodysplastic syndrome. Haematologica 2004, 89, 619-620.

32. Hellstrom, E.; Robert, K.H.; Samuelsson, J.; Lindemalm, C.; Grimfors, G.; Kimby, E.; Oberg, G.; Winqvist, I.; Billstrom, R.; Carneskog, J.; et al. Treatment of myelodysplastic syndromes with retinoic acid and 1 alpha-hydroxy-vitamin D3 in combination with low-dose ara-c is not superior to ara-c alone. Results from a randomized study. The scandinavian myelodysplasia group (smg). Eur. J. Haematol. 1990, 45, 255-261.

33. Park, W.H.; Seol, J.G.; Kim, E.S.; Binderup, L.; Koeffler, H.P.; Kim, B.K.; Lee, Y.Y. The induction of apoptosis by a combined 1,25(OH)2D3 analog, EB1089 and TGF-beta1 in NCI-H929 multiple myeloma cells. Int. J. Oncol. 2002, 20, 533-542.

34. Park, W.H.; Seol, J.G.; Kim, E.S.; Jung, C.W.; Lee, C.C.; Binderup, L.; Koeffler, H.P.; Kim, B.K.; Lee, Y.Y. Cell cycle arrest induced by the vitamin D(3) analog EB1089 in NCI-H929 myeloma cells is associated with induction of the cyclin-dependent kinase inhibitor p27. Exp. Cell Res. 2000, 254, 279-286.

35. Puthier, D.; Bataille, R.; Barille, S.; Mellerin, M.P.; Harousseau, J.L.; Ponzio, A.; Robillard, N.; Wijdenes, J.; Amiot, M. Myeloma cell growth arrest, apoptosis, and interleukin-6 receptor modulation induced by EB1089, a vitamin D3 derivative, alone or in association with dexamethasone. Blood 1996, 88, 4659-4666.

36. Salahuddin, N.; Ali, F.; Hasan, Z.; Rao, N.; Aqeel, M.; Mahmood, F. Vitamin D accelerates clinical recovery from tuberculosis: Results of the succinct study [supplementary cholecalciferol in recovery from tuberculosis]. A randomized, placebo-controlled, clinical trial of vitamin D supplementation in patients with pulmonary tuberculosis'. BMC Infect. Dis. 2013, 13, 22.

37. Cho, H.J.; Shin, D.Y.; Kim, J.H.; Bae, J.Y.; Lee, K.H.; See, C.J.; Kim, N.; Park, E.K.; Ra, E.K.; Lee, J.E.; et al. Impact of vitamin D receptor gene polymorphisms on clinical outcomes of HLA-matched sibling hematopoietic stem cell transplantation. Clin. Transplant. 2012, 26, 476-483.

38. Pakkala, I.; Taskinen, E.; Pakkala, S.; Raisanen-Sokolowski, A. Mc1288, a vitamin D analog, prevents acute graft-versus-host disease in rat bone marrow transplantation. Bone Marrow Transplant. 2001, 27, 863-867. 
39. Mostoufi-Moab, S.; Ginsberg, J.P.; Bunin, N.; Zemel, B.; Shults, J.; Leonard, M.B. Bone density and structure in long-term survivors of pediatric allogeneic hematopoietic stem cell transplantation. J. Bone Miner. Res. 2012, 27, 760-769.

40. Joseph, R.W.; Alousi, A.; Konda, B.; Komanduri, K.; Neumann, J.; Trevino, C.; Stolar, K.; Qazilbash, M.; Hosing, C.; Kebriaei, P.; et al. High incidence of vitamin D deficiency in patients undergoing allogeneic stem cell transplantation. Am. J. Hematol. 2011, 86, 954-956.

41. Robien, K.; Strayer, L.G.; Majhail, N.; Lazovich, D.; Baker, K.S.; Smith, A.R.; Mulrooney, D.A.; Burns, L.J. Vitamin D status among long-term survivors of hematopoietic cell transplantation. Bone Marrow Transplant. 2011, 46, 1472-1479.

42. Dignan, F.L.; Scarisbrick, J.J.; Cornish, J.; Clark, A.; Amrolia, P.; Jackson, G.; Mahendra, P.; Taylor, P.C.; Shah, P.; Lightman, S.; et al. Organ-specific management and supportive care in chronic graft-versus-host disease. Br. J. Haematol. 2012, 158, 62-78.

43. Majhail, N.S.; Rizzo, J.D.; Lee, S.J.; Aljurf, M.; Atsuta, Y.; Bonfim, C.; Burns, L.J.; Chaudhri, N.; Davies, S.; Okamoto, S.; et al. Recommended screening and preventive practices for long-term survivors after hematopoietic cell transplantation. Hematol. Oncol. Stem Cell Ther. 2012, 5, 1-30.

44. Kaiser, M.F.; Heider, U.; Mieth, M.; Zang, C.; von Metzler, I.; Sezer, O. The proteasome inhibitor bortezomib stimulates osteoblastic differentiation of human osteoblast precursors via upregulation of vitamin D receptor signalling. Eur. J. Haematol. 2013, 90, 263-272.

45. Badros, A.; Goloubeva, O.; Terpos, E.; Milliron, T.; Baer, M.R.; Streeten, E. Prevalence and significance of vitamin D deficiency in multiple myeloma patients. Br. J. Haematol. 2008, 142, 492-494.

46. Ng, A.C.; Kumar, S.K.; Rajkumar, S.V.; Drake, M.T. Impact of vitamin D deficiency on the clinical presentation and prognosis of patients with newly diagnosed multiple myeloma. Am. J. Hematol. 2009, 84, 397-400.

47. Diamond, T.; Golombick, T.; Manoharan, A. Vitamin D status may effect the skeletal complications of multiple myeloma. Am. J. Hematol. 2010, 85, 302-303. 


\title{
A Novel Role for a Major Component of the Vitamin D Axis: Vitamin D Binding Protein-Derived Macrophage Activating Factor Induces Human Breast Cancer Cell Apoptosis through Stimulation of Macrophages
}

\author{
Lynda Thyer, Emma Ward, Rodney Smith, Maria Giulia Fiore, Stefano Magherini, \\ Jacopo J. V. Branca, Gabriele Morucci, Massimo Gulisano, Marco Ruggiero and Stefania Pacini
}

\begin{abstract}
The role of vitamin D in maintaining health appears greater than originally thought, and the concept of the vitamin $\mathrm{D}$ axis underlines the complexity of the biological events controlled by biologically active vitamin $\mathrm{D}(1,25(\mathrm{OH})(2) \mathrm{D} 3)$, its two binding proteins that are the vitamin $\mathrm{D}$ receptor (VDR) and the vitamin D-binding protein-derived macrophage activating factor (GcMAF). In this study we demonstrate that GcMAF stimulates macrophages, which in turn attack human breast cancer cells, induce their apoptosis and eventually phagocytize them. These results are consistent with the observation that macrophages infiltrated implanted tumors in mice after GcMAF injections. In addition, we hypothesize that the last 23 hydrophobic amino acids of VDR, located at the inner part of the plasma membrane, interact with the first 23 hydrophobic amino acids of the GcMAF located at the external part of the plasma membrane. This allows 1,25(OH)(2)D3 and oleic acid to become sandwiched between the two vitamin D-binding proteins, thus postulating a novel molecular mode of interaction between GcMAF and VDR. Taken together, these results support and reinforce the hypothesis that GcMAF has multiple biological activities that could be responsible for its anti-cancer effects, possibly through molecular interaction with the VDR that in turn is responsible for a multitude of non-genomic as well as genomic effects.
\end{abstract}

Reprinted from Nutrients. Cite as: Thyer, L.; Ward, E.; Smith, R.; Fiore, M.G.; Magherini, S.; Branca, J.J.V.; Morucci, G.; Gulisano, M.; Ruggiero, M.; Pacini, S. A Novel Role for a Major Component of the Vitamin D Axis: Vitamin D Binding Protein-Derived Macrophage Activating Factor Induces Human Breast Cancer Cell Apoptosis through Stimulation of Macrophages. Nutrients 2013, 5, $2577-2589$.

\section{Introduction}

The so-called vitamin D axis is involved in various aspects of human breast cancer, the most common human tumor. The vitamin D axis is composed of the biologically active form of vitamin D $(1,25(\mathrm{OH})(2) \mathrm{D} 3)$, and by two proteins that specifically bind it. These proteins are the vitamin $\mathrm{D}$ receptor (VDR) and the vitamin $\mathrm{D}$ binding protein that is the precursor of the vitamin $\mathrm{D}$ binding protein-derived macrophage activating factor, also termed GcMAF [1]. The role of vitamin D in human breast cancer is witnessed by the number of studies that have been published on the subject [2]. More intriguing, however, is the relative lack of information about GcMAF and human breast cancer; in fact, in the peer-reviewed literature, as of today (May 2013), there are only four studies on this subject. In two of these studies, the effects of GcMAF were observed on the human breast cancer cell line MCF-7 in vitro [3,4]. Another study examined the glycosylation status of vitamin D binding protein in cancer patients 
including breast cancer patients [5], whereas a less recent study reported the effects of administering GcMAF to metastatic breast cancer patients [6].

It is interesting to notice that no studies have, so far, been performed in order to assess whether GcMAF, which is a known powerful activator of macrophages, was indeed capable of activating macrophages that could in turn "attack" human breast cancer cells. There is indirect evidence suggesting that GcMAF activates macrophages that infiltrate experimental tumors in animal models [7,8]. This evidence, however, is indirect and, most important, refers to experimental tumors other than human breast cancer. In addition, since the observations quoted above were performed in experimental animals, the presence of confounding factors associated with the complexity of the responses of the whole organism to the presence of transplanted or advanced tumors, limits the possibility of interpretation of the presented results.

Therefore, in order to fill this gap of knowledge, we performed experiments to provide clear-cut evidence that GcMAF, as part of the vitamin D axis, activates normal macrophages that in turn exert a tumoricidal action against human breast cancer cells without the presence of confounding factors.

\section{Experimental Section}

Purified, activity-tested GcMAF was obtained from Immuno Biotech Ltd., Guernsey, Channel Islands. Paricalcitol was from Abbott, Roma, Italy. All other reagents were from Sigma Aldrich, Milano, Italy.

\subsection{Cell Lines}

Human breast cancer cells (cell line MCF-7) were obtained from the Istituto Zooprofilattico Sperimentale della Lombardia e dell'Emilia-Romagna, Brescia, Italy. Cells were routinely maintained at $37{ }^{\circ} \mathrm{C}$ in a humidified atmosphere of $5 \% \mathrm{CO}_{2}$ in Eagle's minimum essential medium in Earle's Balanced salt solution, supplemented with $1 \mathrm{mM}$ sodium pyruvate, $10 \%$ fetal bovine serum (FBS), $100 \mathrm{U} / \mathrm{mL}$ penicillin, and $100 \mu \mathrm{g} / \mathrm{mL}$ streptomycin (Invitrogen, Carlsbad, CA, USA). No 1,25(OH)(2)D3 was present in the culture medium. In experiments of co-cultures, macrophages (cell line Raw 264.7, HPA Culture Collection) were activated by culturing them in the same medium of MCF-7 cells and in the presence of $100 \mathrm{ng} / \mathrm{mL}$ GcMAF for $72 \mathrm{~h}$ prior to addition to the MCF-7 cell culture. GcMAF concentration was established by preliminary experiments showing a linear dose-response curve. The initial response was observed at $1 \mathrm{ng} / \mathrm{mL}$ and a plateau was reached at $100 \mathrm{ng} / \mathrm{mL}$. These concentrations were consistent with the results previously reported $[3,4]$. Before addition to the MCF-7 cell culture, the macrophages were gently centrifuged and re-suspended in fresh medium in order to avoid transferring GcMAF to the co-culture. In this way, we could rule out direct effects of GcMAF on MCF-7 cells. The macrophages were added at a ratio of 1:1 to the MCF-7 cell culture. The cells were then allowed to settle for $1 \mathrm{~h}$ before time-lapse photography. Photography was taken over a 7-day period using an Olympus CK2 microscope and a GXCAM-3 with NCH Debut capture software. In the experiments described in Figures $1 \mathrm{~A}$ and 2 , the cells were fixed and stained as described below $40 \mathrm{~h}$ after co-culturing them. 


\subsection{Study of Cell Morphology}

Cell morphology was studied by phase-contrast microscopy using an Optika inverted microscope (Model XDS-2; Optika Microscopes, Bergamo, Italy). This microscope had a positive-phase plate for phase-contrast imaging below a long working distance condenser lens, and an $8 \mathrm{Mp}$ digital camera with LCD Screen (Optika Microscopes, Bergamo, Italy). The light source was a $6 \mathrm{~V} / 30 \mathrm{~W}$ halogen pre-centered illuminator, with adjustable intensity. Phase-contrast imaging was performed on living cells without any fixation or treatment. A series of digital images of living cells were recorded for each experimental point and the most representative were chosen.

Haematoxylin-eosin and Papanicolaou staining were also performed. This last staining results in very transparent cells, such that even thicker specimens with overlapping cells could be recorded. Briefly, cells were stained with Harris haematoxylin as nuclear stain. Orange G and EA-65 (Light Green, Bismarck Brown, and Eosin) were used for cytoplasmic staining (Sigma Aldrich, Milano, Italy). Slides were mounted with permanent mounting medium and observed under light microscopy (Nikon Instruments SpA, Milano, Italy). Pictures shown are representative of typical experimental data. Each experiment was performed with quadrupled samples and was replicated three times.

\subsection{Study of Cell Proliferation}

Assessment of cell proliferation was determined by a Calbiochem Rapid Cell Proliferation Kit (Calbiochem, D.B.A., Milano, Italy) [9]. Each condition was replicated with quadrupled samples and each experiment was replicated three times. Differences between experimental values were evaluated by the Student's $t$-test.

\subsection{Study of Amino Acid Alignments and Functions}

Analyses were carried out on the nucleotide and amino-acid sequences of the genes coding for vitamin D binding protein/GcMAF (isoform 1 precursor; gi|324021743|ref|NP_001191235.1) and VDR (gi|38511972|gb|AAH60832.1) in Homo sapiens. In reference to the protein alignments, three parameters have been taken into account:

1. sequence identity

2. sequence similarity

3. hydrophobic profile

These criteria were evaluated because they determine the quality of the alignments. In addition, we evaluated the functional value of the amino acids replaced, i.e., the importance that any divergence assumes within the sequence. The values obtained have allowed the scores to be added, rather than multiplied, in the global calculation of alignment scores. Information concerning the selected genes was obtained from the database at the University of California, Santa Cruz [10] referring to the latest published version of the human genome [11]. In particular, we used the table refGene, containing all gene coding and non-coding for proteins. In this way, it was possible to obtain detailed information on human genes, such as: chromosome, position of the start and the end of transcription, position of the 
start and the end of coding part, and the number and the positions of exons. The annotations for the genes were obtained using the algorithm liftOver [12].

The presence of conserved elements within the alignment was verified by using the information contained in the phastConsElements28way table of the UCSC database. This table contains the predictions of conserved elements produced by the phastCons program. The positions were reported on the alignment. All operations, from the search of genomic information to the creation of the alignments, were made using R Statistical Mathematical Software. Once the sequences were aligned, the columns of residues were taken into consideration. Any lined-up residue is to be considered implicitly related to evolution. The hydrophobic profile was obtained using software on the website [13]. Among the several systems that can be used for the calculation of the index of the amino acid sequence hydrophobicity/hydrophilicity, we selected the Kyte and Doolittle's method [14]. The three-dimensional protein structures of vitamin D-binding protein and VDR were obtained through the use of the PDB archive [15]. Superposition between the two structures was possible through the use of the Swiss Pdb Viewer software [16]. The PDB archive contains information about experimentally-determined structures of proteins, nucleic acids, and complex assemblies. SwissPdb Viewer is an application that provides an interface allowing analysis of several proteins at the same time. The proteins can be superimposed in order to deduce structural alignments and compare their active sites or any other relevant parts. Amino acid mutations, H-bonds, angles, and distances between atoms are easy to obtain thanks to the intuitive graphic and menu interface.

\section{Results}

When co-cultured with human breast cancer cells in the absence of GcMAF, macrophages did not interact with human breast cancer cells and their characteristically irregular morphology was maintained (Figure 1A). Little or no vacuoles could be observed in macrophage cytoplasm, indirect evidence of a lack of activation. As described before, human breast cancer cells exhibited their typically non-homogeneous morphology, with some cells larger than other. The morphology of the cells was irregularly polygonal. As expected, human breast cancer cells tended to grow, one on top of the other, forming clusters that reflected the characteristic loss of contact inhibition. Figure 1B depicts phase contrast microphotography of a cluster of human breast cancer cells cultured in the absence of macrophages or any other addition. Cancer cells are visible as cords of cells growing in multi-layers in the center of the Figure. At higher magnification (Figure 1C), the cells appeared densely packed, with linear, non-fragmented, margins, and with a clearly recognizable organization of chromatin inside the nucleus, indicating a strong synthetic activity compatible with the high rate of proliferation of these cells. The nucleoli are clearly visible. Figure 1D, shows Papanicolau staining of only human breast cancer cells; a significant cluster can be observed in the left lower side of the image. The nuclei appear heavily stained as expected in growing cancer cells. The perimeter of the cells is linear with no indents or signs of fragmentation. Empty (white) areas in the well are also clearly observable. These represent naked areas of the plastic well that reflect the loss of adherence typical of cancer cells. Loss of adherence is a pre-requisite for cellular detachment, invasiveness, and metastatic potential. 
Figure 1. (A) Haematoxylin-eosin staining (magnification 300×); in the absence of GcMAF, small macrophages do not appear to interact with MCF-7 human breast cancer cells. The picture refers to $40 \mathrm{~h}$ co-culture. (B) Phase contrast microphotography $(300 \times)$ of a cluster of cancer cells in the center. (C) At higher magnification (1200×) the cells appear densely packed. (D) Papanicolau staining (1200×); a cluster in the left lower side of the image. The nuclei are heavily stained and the perimeter of the cells is linear with no indents or signs of fragmentation.
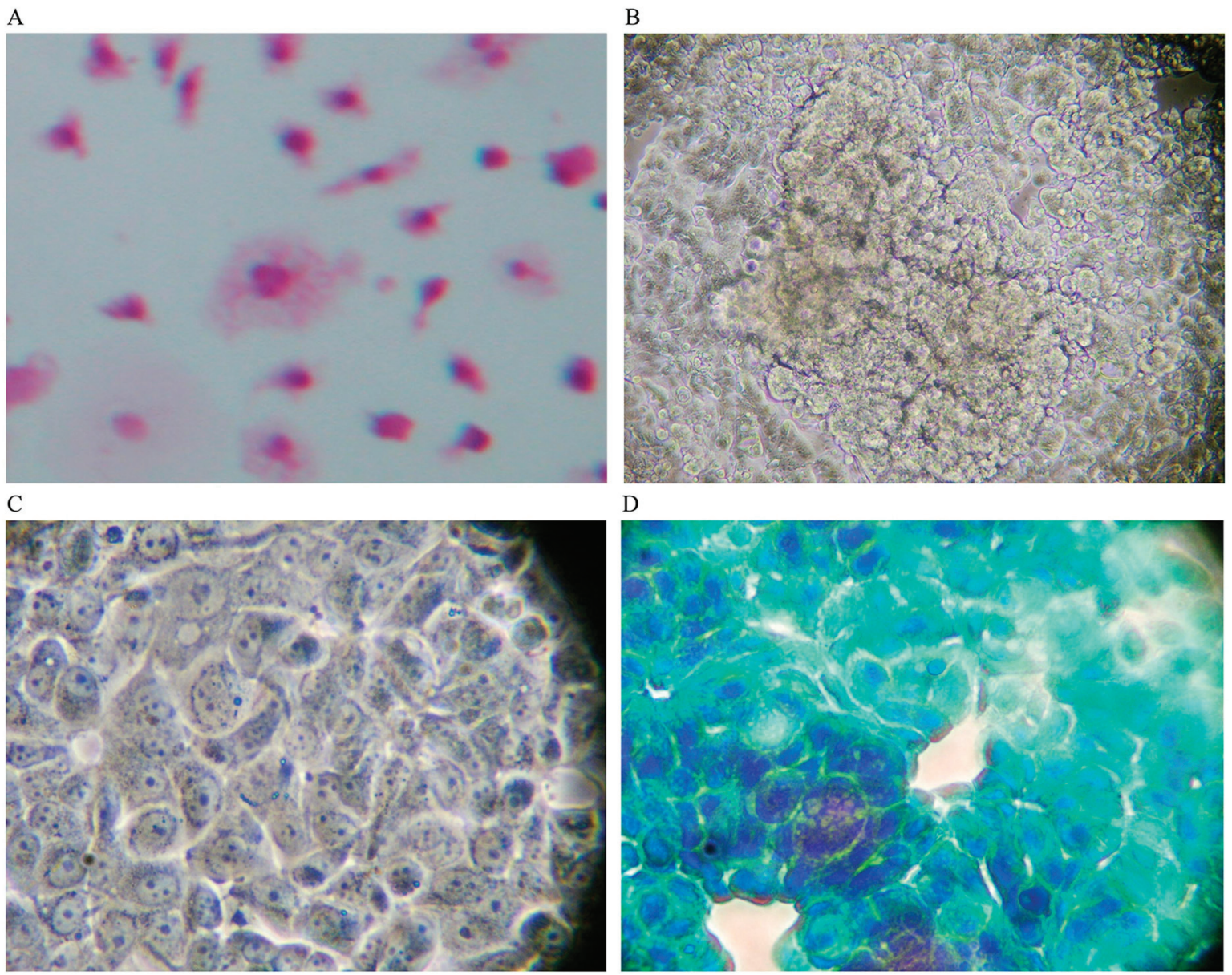

However, when human breast cancer cells were co-cultured with macrophages that had been previously activated by GcMAF $(100 \mathrm{ng} / \mathrm{mL})$ for $72 \mathrm{~h}$, the picture was completely different as shown in Figures 2 and 3. The pictures show co-culture of GcMAF-activated macrophages and human breast cancer cells after $40 \mathrm{~h}$ incubation. GcMAF-activated macrophages appeared as small cells that surrounded human breast cancer cells. Figure 2A (Papanicolau staining, ) clearly shows a group of human breast cancer cell in the center of the image surrounded by hundreds of small macrophages. At higher magnification, (Figure 2B) one human breast cancer cell appears completely surrounded by macrophages that are also observable on top of the cell. The nucleus of the macrophages is well stained, whereas the chromatin in the nucleus of the cancer cell appears fragmented and disorganized. The nucleoli, however, are still recognizable; this phenomenon can be interpreted as an index of remaining synthetic activity as expected in cells undergoing active apoptosis. The cytoplasm of macrophages appears vacuolated thus suggesting active phagocytosis. Figure $2 \mathrm{C}$ shows another field where two large human breast cancer 
cells are surrounded by GcMAF-activated macrophages that appear to emit cytoplasmic extrusions that search for contact with the membrane of cancer cells. The cell in the center of Figure $2 \mathrm{C}$, at higher magnification (Figure 2D), shows a peculiar aspect; the chromatin in the nucleus appears fragmented and, in the lower right corner, the cytoplasm appears to be indented as if the two macrophages in that region were actively deconstructing the cytoplasmic assembly of the cancer cell. A similar phenomenon can be observed on the left where two macrophages indent the cytoplasmic profile of the cancer cell.

It is worth noticing that all these morphological changes are consistent with the induction of apoptosis of human breast cancer cells by activated macrophages [17]. In particular, some of the morphological changes were consistent with the early phases of apoptosis and the morphology of the nucleus of human breast cancer cells shown in Figure 2 is almost superimposable to that represented in Figure 1 (left panel) of Hacker, 2000 [17]. Even the changes in the morphology of the cytoplasm were consistent with the induction of apoptosis by GcMAF-activated macrophages and the cytoplasm of human breast cancer cells showed the typical pattern of disintegration that precedes the formation of apoptotic bodies. In addition, in this case, the morphology of the cytoplasm of the cancer cells appears remarkably similar to that presented in Figure 1 (middle panel) of Hacker, 2000 [17]. Although the morphological features observed here are suggestive of active apoptosis, further studies using ELISA tests to quantify the level of human active caspase-3 protein, the major executioner protease in apoptosis, will determine quantitatively the degree of apoptosis induced by GcMAF-activated macrophages.

Figure 2. Co-culture of GcMAF-activated macrophages and human breast cancer cells; Papanicolau staining. (A) Cancer cells in the center are surrounded by hundreds of small macrophages $(100 \times)$. (B) One human breast cancer cell is completely surrounded by macrophages that are also observable on top of the cell $(200 \times)$. (C) Two large cancer cells are surrounded by GcMAF-activated macrophages $(100 \times)$. (D) The same cell $(200 \times)$; the chromatin in the nucleus is fragmented and, in the lower right corner, the cytoplasm is to be indented as if the two macrophages in that region were actively deconstructing the cytoplasm of the cancer cell.
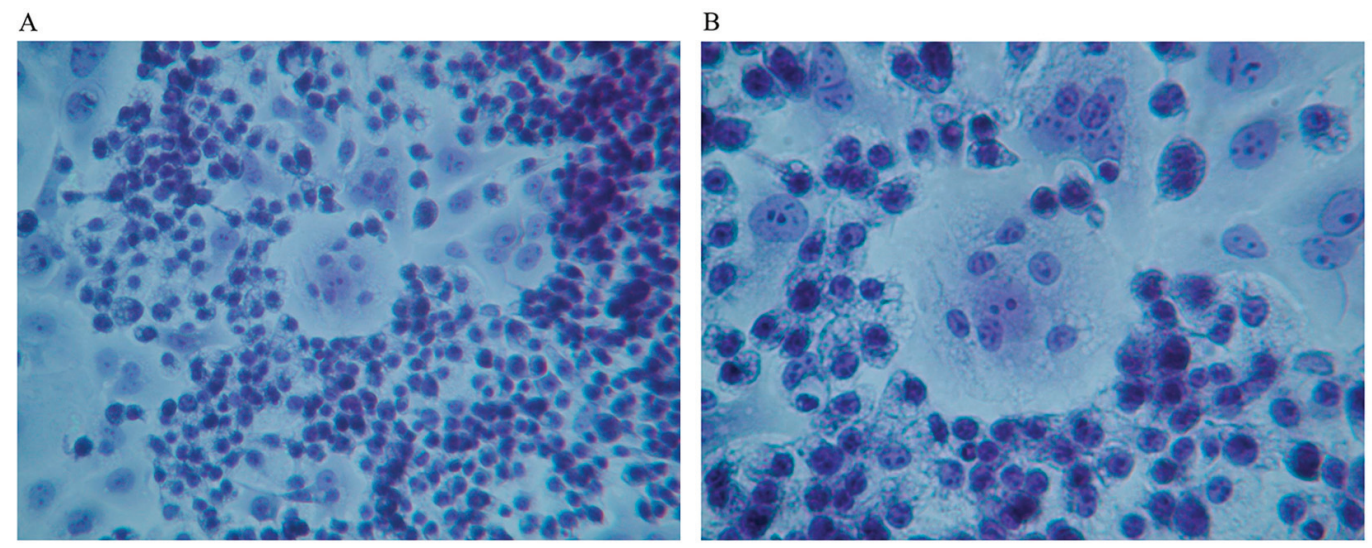
Figure 2. Cont.
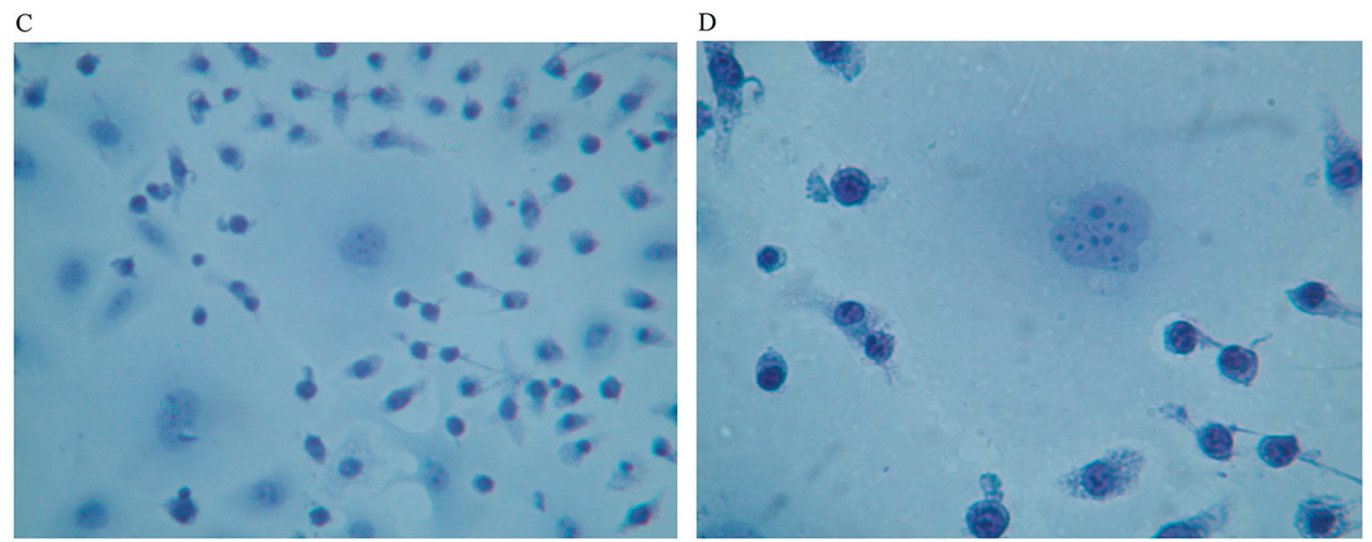

Time-lapse micro-photography shows that after about seven days of co-culture of GcMAF-activated macrophages with human breast cancer cells, the irregular growth of the breast carcinoma cells was arrested and the large protruding cell biomass was reduced. Figure 3A shows the human breast cancer cells and the GcMAF-activated macrophages at day one; the cancer cells, as expected, form an irregular layer that covers the field of observation. Individual cancer cells can be recognized as well as the naked areas of the plate as described above. GcMAF-activated macrophages appear as small cells that are attached to the cancer cells, in most cases, above them. It is interesting to notice that almost no macrophages can be observed in the naked areas of the plate, thus confirming the observation that GcMAF-activated macrophages seek for contact with the cancer cells. After seven days of co-incubation (Figure 3B), no individual cancer cell can be recognized. After macrophage-induced apoptosis, their apoptotic bodies are all grouped together in the center of the field of observation, and most of the field is empty of cancer cells. Most GcMAF-activated macrophages surround and infiltrate the mass of cancer cell debris in the center.

Figure 3. Phase contrast microphotography from time-lapse recording of co-culture of GcMAF-activated macrophages and human breast cancer cells. (A) Day one of co-culture; the cancer cells form an irregular layer. Individual cancer cells can be recognized. GcMAF-activated macrophages appear as small cells that are attached to the cancer cells, in most cases above them. (B) Day seven of co-culture. No individual cancer cell can be recognized. Their apoptotic bodies are grouped together in the center of the field, and most of the field is empty of cancer cells. Most GcMAF-activated macrophages surround and infiltrate the mass of cancer cell debris in the center.
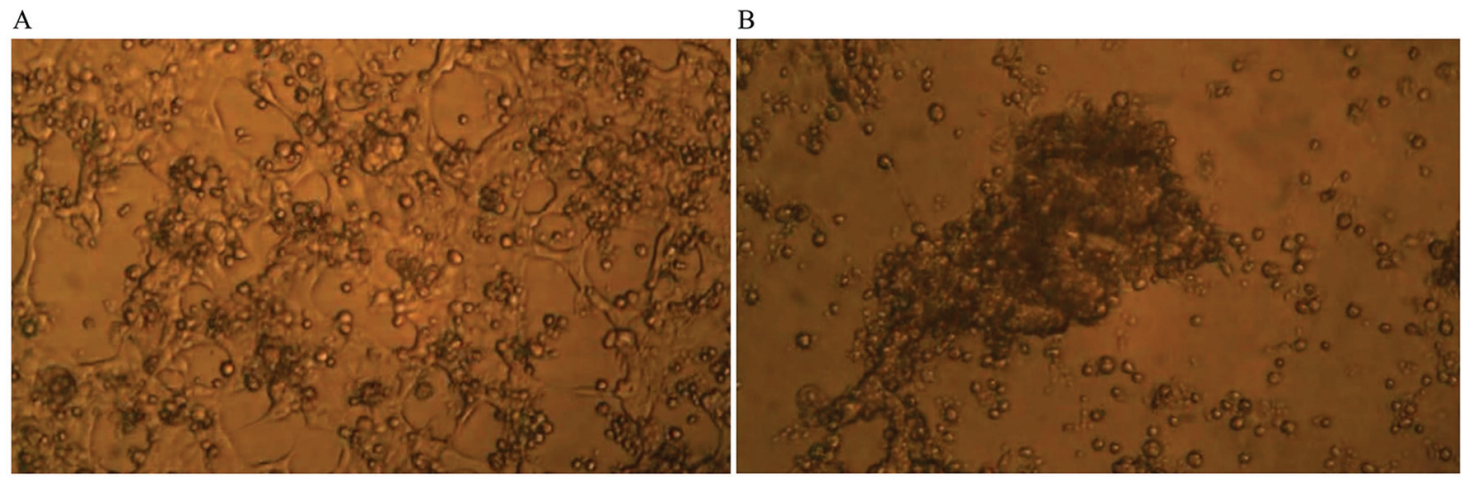
Taken together these results demonstrate for the first time that GcMAF-activated macrophages induce human breast cancer cell apoptosis and the subsequent reduction of the cancer cell mass following phagocytosis of apoptotic cancer cells by macrophages.

\section{Discussion}

It is long considered that the role of vitamin D in maintaining health is much greater than originally supposed, up to the point that some authors jokingly wonder whether "does vitamin D make the world go 'round'?" [18]. The emergence of the concept of the vitamin D axis [1,19] further underlines the complexity of the biological events controlled by $1,25(\mathrm{OH})(2) \mathrm{D} 3$ through its two binding proteins (VDR and vitamin D-binding protein/GcMAF) that interfere with a growing number of events at the cellular and molecular level. In this study we focused our attention on the product of deglycosylation of the vitamin D-binding protein that is GcMAF, probably one of the most potent macrophage activators so far discovered [20]. Our results demonstrate that GcMAF stimulates macrophages that in turn attack human breast cancer cells, possibly induce their apoptosis and eventually phagocytise them. These results are consistent with the observation that macrophages infiltrated experimental tumors implanted in severely immunodeficient mice after GcMAF injections [8]. However, at variance with the observation reported above, in our experiments we could rule out indirect effects due to the adaptive response of the whole organism to the presence of an advanced tumor and to the GcMAF-induced inhibition of angiogenesis with consequent tumor hypoxia and necrosis [8]. A limitation of the present study is represented by the use of only two cell lines, which are human breast cancer cell line MCF-7, and mouse Raw 264.7 macrophages. It should be noticed, however, that GcMAF exerted qualitatively superimposable effects on primary human mononuclear cells [21] and in the human monocytoid cell line, MonoMac 6 [22]. Future experiments will elucidate whether the effects observed in this study can be extrapolated to other human breast cancer cell lines challenged with GcMAF-activated human macrophages.

The observation that GcMAF, a component of the vitamin D axis, exerts tumoricidal effects on human breast cancer cells through macrophage activation raises the question of whether there is any interaction between GcMAF and the VDR. Such a type of interaction would be critical to understand the effects of 1,25(OH)(2)D3 and GcMAF at the molecular level. This question might appear odd at first, as, for many years, it had been thought that VDR was localized in the cytoplasm and in the nucleus, and GcMAF could not cross the plasma membrane and therefore had to be recognized by a surface receptor, possibly a lectin-type receptor [23]. However, the observation of an association between the polymorphisms of the gene coding for VDR, and differential responses to GcMAF in human monocytes [21], as well as with metastatic breast cancer [24], raises the apparently odd issue of a molecular interaction between GcMAF and the VDR. In support for this hypothesis there is the observation that the VDR translocates to the plasma membrane [25], and plasma membrane associated VDR is responsible for the rapid, nongenomic effects of vitamin D [26]. Thus, in order to verify the possibility of a molecular interaction between GcMAF and VDR, we compared the amino acid sequences corresponding to their respective 1,25(OH)(2)D3 binding sites. There are 23 hydrophobic amino acids near the amino terminus of GcMAF (----MKRVLVLLLAVAFGHALERGRDY) and 23 amino acids near the carboxyl terminus of the VDR (SFQPECSMKLTPLVLEVFGNEIS-----). If these two sequences are aligned (Figure 4A), it is possible to observe, not only that in both proteins there is a long stretch $[21,24]$ of hydrophobic amino acids 
(highlighted in green in Figure 4A, upper insert), but that four hydrophobic amino acids are identical (L L FG; indicated in yellow and in green above and under the alignment. The sequence of GcMAF is above). In addition, 11 amino acids have similar functional valence as indicated by the conventional symbols (*), (.) and (:). Therefore, in the 1,25(OH)(2)D3 binding domains of GcMAF and VDR there are in total 11 out of 23 amino acids that show functional identity or similarity and 13-14 that are hydrophobic. A molecular interaction between the two proteins can therefore be proposed (Figure 4A). According to this model, the last 23 hydrophobic amino acids of VDR (VDR is on the right of Figure 4A), located at the inner part of the plasma membrane (represented as a dotted line), could interact with the first 23 hydrophobic amino acids of the GcMAF (GcMAF is on the left of the Figure 4A) located at the external part of the plasma membrane, with 1,25(OH)(2)D3 (represented in yellow) sandwiched between the two vitamin D-binding proteins. Oleic acid, taken as an example of an unsaturated fatty acid bound to GcMAF [27], could stabilize the complex at the level of the plasma membrane. In fact, both $1,25(\mathrm{OH})(2) \mathrm{D} 3$ and oleic acid in GcMAF are located in a shallow cleft of the GcMAF protein that makes them accessible to the plasma membrane. In addition to the mode of interaction proposed in Figure 4A, there could be further additional interaction that takes into consideration just the fact that vitamin $\mathrm{D}$ binding-protein (and therefore also GcMAF) binds unsaturated fatty acids as demonstrated by Williams et al., 1998 [27]. The fatty acid binding site is located between domains II and III, which is between positions 304 and 387. When we aligned the 23 hydrophobic amino acids of the VDR quoted above (represented in the insert in Figure 4B; also in this case, the sequence of GcMAF is represented above that of VDR) and the corresponding hydrophobic amino acids of the unsaturated fatty acid binding site of GcMAF (in particular, those in position 356-386), we observed that there was a significant degree of functional homology; in fact there are eight amino acids with similar functional valence in a long stretch of hydrophobic amino acids (highlighted in blue). 
Figure 4. Amino acid alignments and three-dimensional protein structures of vitamin D-binding protein/GcMAF and VDR. (A) 23 hydrophobic amino acids of VDR (on the right), located at the inner part of the plasma membrane (dotted line), interact with 23 hydrophobic amino acids of the GcMAF (on the left of the Figure) located at the external part of the plasma membrane. In the insert the hydrophobic amino acids are highlighted in green and the four hydrophobic amino acids that are identical (L L FG) are highlighted in yellow and in green above and under the alignment. Vitamin D indicates 1,25(OH)(2)D3. (B) 23 hydrophobic amino acids of the VDR interact with a stretch of hydrophobic amino acids of the unsaturated fatty acid binding site of GcMAF. In the insert, eight amino acids with similar functional valence in a long stretch of hydrophobic amino acids highlighted in blue.
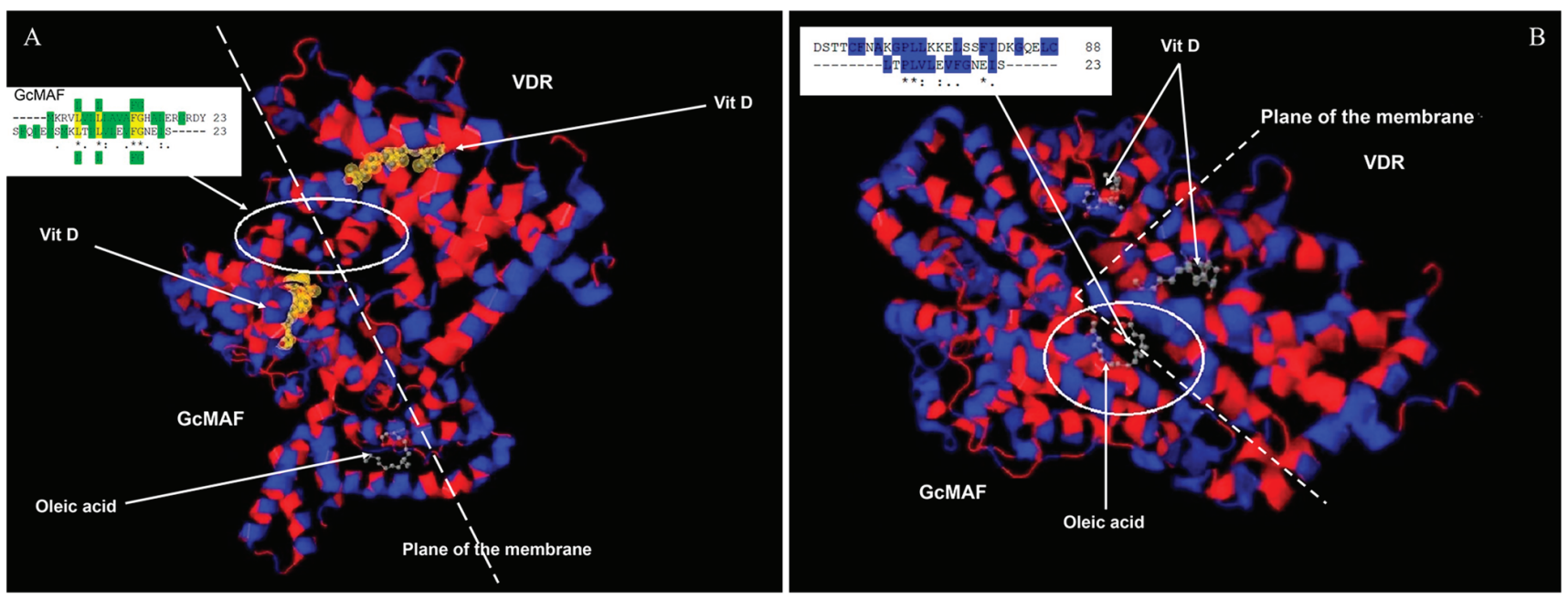

Therefore, it can be hypothesized that GcMAF and the VDR have multiple sites of interaction at the level of the plasma membrane. According to this model, the presence of $1,25(\mathrm{OH})(2) \mathrm{D} 3$, in the culture medium should increase the effects of GcMAF by facilitating the interaction between GcMAF and VDR. Consistent with this model, we previously demonstrated that the effects of 1,25(OH)(2)D3 and GcMAF were synergistic in inhibiting MCF-7 cell proliferation [4], and the preliminary results reported in Table 1 indicate that GcMAF and paricalcitol, a non-hypercalcemic VDRagonist, also have synergistic effects. In the experiment described in Table 1, we chose to use paricalcitol instead of 1,25(OH)(2)D3 in order to determine whether the synergism between 1,25(OH)(2)D3 and GcMAF that we had previously observed [4], was to be ascribed exclusively to $1,25(\mathrm{OH})(2) \mathrm{D} 3$, or could also be obtained with other VDR agonists. From the results presented in Table 1, it appears that paricalcitol, and, possibly, other VDR agonists, could fit the molecular model proposed in Figure 4. 
Table 1. Effects of GcMAF and paricalcitol on Raw 264.7 macrophages. Raw 264.7 cells were incubated for 30 min with indicated additions. The effects of GcMAF on macrophage activation were assessed by determining cell proliferation. In fact, it was demonstrated that monocytes/macrophages activated by GcMAF administration immediately stop DNA replication and rapidly synthesize a large amount of Fc-receptors as well as an enormous variation of receptors [28]. Paricalcitol was added at the concentration of $300 \mathrm{fg} / \mathrm{mL}$. At this concentration, paricalcitol did not exert any effect. In the presence of paricalcitol ( $300 \mathrm{fg} / \mathrm{mL}$ ), the effect of $4 \mathrm{ng} / \mathrm{mL}$ GcMAF was identical to that of $40 \mathrm{ng} / \mathrm{mL}$ GcMAF in the absence of paricalcitol. These results demonstrate that the presence of a selective VDR agonist at a concentration that is not sufficient to activate VDR per se increases by an order of magnitude the response to GcMAF. Data are presented as means \pm S.E.M. $(n=12)$. $* p<0.02$ vs. control.

\begin{tabular}{cc}
\hline Treatment & Absorbance units $\left(\times \mathbf{1 0}^{\mathbf{3}}\right)$ \\
\hline Control (no addition) & $390 \pm 11$ \\
Paricalcitol & $450 \pm 10$ \\
GcMAF 40 ng/mL & $379 \pm 9 *$ \\
GcMAF 4 ng/mL + paricalcitol & $327 \pm 10^{*}$ \\
\hline
\end{tabular}

Taken together, these results support the hypothesis that the interaction between GcMAF and VDR might be facilitated by VDR agonists. This hypothesis is further strengthened by the recent observation that activated macrophages are able to generate enough biologically active vitamin D so as to be detectable in the general circulation [29], thus suggesting a paracrine/autocrine positive feedback loop.

\section{Conclusions}

The results presented in this study suggest that the role of vitamin D in physiology and pathology is far more complex than previously envisaged. Thus, in addition to $1,25(\mathrm{OH})(2) \mathrm{D} 3$ itself, at least another component of the vitamin D axis, GcMAF, exerts significant effects at the cellular level and it appears that the effects of GcMAF are interconnected with VDR activation. Therefore, it can be hypothesized that these interconnections between 1,25(OH)(2)D3, GcMAF and VDR will be instrumental in devising new therapeutic usages for the components of the vitamin $\mathrm{D}$ axis.

\section{Acknowledgments}

Marco Ruggiero, Stefania Pacini and Massimo Gulisano received grants from the University of Firenze and the Project PRIN 2009.

\section{Conflict of Interest}

All authors declare no conflicts of interest. 


\section{References}

1. Ruggiero, M.; Pacini, S. The vitamin D axis in chronic kidney disease-State of the art and future perspectives. Eur. Nephrol. 2011, 5, 15-19.

2. Shao, T.; Klein, P.; Grossbard, M.L. Vitamin D and breast cancer. Oncologist 2012, 17, 36-45.

3. Pacini, S.; Morucci, G.; Punzi, T.; Gulisano, M.; Ruggiero, M. Gc protein-derived macrophage-activating factor (GcMAF) stimulates cAMP formation in human mononuclear cells and inhibits angiogenesis in chick embryo chorionallantoic membrane assay. Cancer Immunol. Immunother. 2011, 60, 479-485.

4. Pacini, S.; Punzi, T.; Morucci, G.; Gulisano, M.; Ruggiero, M. Effects of vitamin D-binding protein-derived macrophage-activating factor on human breast cancer cells. Anticancer Res. 2012, $32,45-52$.

5. Rehder, D.S.; Nelson, R.W.; Borges, C.R. Glycosylation status of vitamin D binding protein in cancer patients. Protein Sci. 2009, 18, 2036-2042.

6. Yamamoto, N.; Suyama, H.; Yamamoto, N.; Ushijima, N. Immunotherapy of metastatic breast cancer patients with vitamin D-binding protein-derived macrophage activating factor (GcMAF). Int. J. Cancer 2008, 122, 461-467.

7. Toyohara, Y.; Hashitani, S.; Kishimoto, H.; Noguchi, K.; Yamamoto, N.; Urade, M. Inhibitory effect of vitamin D-binding protein-derived macrophage activating factor on DMBA-induced hamster cheek pouch carcinogenesis and its derived carcinoma cell line. Oncol. Lett. 2011, 2, 685-691.

8. Nonaka, K.; Onizuka, S.; Ishibashi, H.; Uto, Y.; Hori, H.; Nakayama, T.; Matsuura, N.; Kanematsu, T.; Fujioka, H. Vitamin D binding protein-macrophage activating factor inhibits HCC in SCID mice. J. Surg. Res. 2012, 172, 116-122.

9. Hayon, T.; Dvilansky, A.; Shpilberg, O.; Nathan, I. Appraisal of the MTT-based assay as a useful tool for predicting drug chemosensitivity in leukemia. Leuk. Lymphoma 2003, 44, 1957-1962.

10. UCSC Genome Bioinformatics. Available online: http://genome.ucsc.edu (accessed on 22 January 2013).

11. Index of/goldenPath/hg18. Available online: http://hgdownload.cse.ucsc.edu/goldenPath/hg18 (accessed on 25 January 2013).

12. Lift Genome Annotations. Available online: http://genome.ucsc.edu/cgi-bin/hgLiftOver (accessed on 26 January 2013).

13. ProtScale. Available online: http://web.expasy.org/protscale/ (accessed on 6 February 2013).

14. ProtScale Tool. Available online: http://web.expasy.org/protscale/pscale/Hphob.Doolittle.html (accessed on 6 February 2013).

15. Biological Macromolecular Resource. Available online: http://www.rcsb.org/pdb/home/home.do (accessed on 11 February 2013).

16. DeepView-Swiss-PdbViewer. Available online: http://spdbv.vital-it.ch/ (accessed on 13 February 2013).

17. Häcker, G. The morphology of apoptosis. Cell Tissue Res. 2000, 301, 5-17.

18. Wagner, C.L.; Taylor, S.N.; Hollis, B.W. Does vitamin D make the world go "round"? Breastfeed. Med. 2008, 3, 239-250. 
19. Chishimba, L.; Thickett, D.R.; Stockley, R.A.; Wood, A.M. The vitamin D axis in the lung: A key role for vitamin D-binding protein. Thorax 2010, 65, 456-462.

20. Uto, Y.; Yamamoto, S.; Mukai, H.; Ishiyama, N.; Takeuchi, R.; Nakagawa, Y.; Hirota, K.; Terada, H.; Onizuka, S.; Hori, H. $\beta$-Galactosidase treatment is a common first-stage modification of the three major subtypes of Gc protein to GcMAF. Anticancer Res. 2012, 32, 2359-2364.

21. Pacini, S.; Morucci, G.; Punzi, T.; Gulisano, M.; Ruggiero, M.; Amato, M.; Aterini, S. Effect of paricalcitol and GcMAF on angiogenesis and human peripheral blood mononuclear cell proliferation and signaling. J. Nephrol. 2012, 25, 577-581.

22. Pacini, S.; Branca, J.J.V.; Morucci, G.; Gulisano, M.; Ruggiero, M. Effects of GcMAF on monocyte-macrophage cells. 2013, to be submitted for publication.

23. Iida, S.; Yamamoto, K.; Irimura, T. Interaction of human macrophage C-type lectin with $O$-linked $\mathrm{N}$-acetylgalactosamine residues on mucin glycopeptides. J. Biol. Chem. 1999, 274, 10697-10705.

24. Ruggiero, M.; Pacini, S.; Aterini, S.; Fallai, C.; Ruggiero, C.; Pacini, P. Vitamin D receptor gene polymorphism is associated with metastatic breast cancer. Oncol. Res. 1998, 10, 43-46.

25. Capiati, D.; Benassati, S.; Boland, R.L. 1,25(OH)2-vitamin D3 induces translocation of the vitamin D receptor (VDR) to the plasma membrane in skeletal muscle cells. J. Cell. Biochem. 2002, 86, 128-135.

26. Ceglia, L.; Harris, S.S. Vitamin d and its role in skeletal muscle. Calcif. Tissue Int. 2013, 92, 151-162.

27. Williams, M.H.; van Alstyne, E.L.; Galbraith, R.M. Evidence of a novel association of unsaturated fatty acids with Gc (vitamin D-binding protein). Biochem. Biophys. Res. Commun. 1988, 153, 1019-1024.

28. Yamamoto, N.; Ushijima, N.; Koga, Y. Immunotherapy of HIV-infected patients with Gc protein-derived macrophage activating factor (GcMAF). J. Med. Virol. 2008, 81, 16-26.

29. Adams, J.S.; Hewison, M. Extrarenal expression of 25-hydroxyvitamin D-1-hydroxylase. Arch. Biochem. Biophys. 2012, 523, 95-102. 

Chapter 5

Cardiovascular Disorders 



\title{
Does Sufficient Evidence Exist to Support a Causal Association between Vitamin D Status and Cardiovascular Disease Risk? An Assessment Using Hill's Criteria for Causality
}

\author{
Patricia G. Weyland, William B. Grant and Jill Howie-Esquivel
}

\begin{abstract}
Serum 25-hydroxyvitamin D (25(OH)D) levels have been found to be inversely associated with both prevalent and incident cardiovascular disease (CVD) risk factors; dyslipidemia, hypertension and diabetes mellitus. This review looks for evidence of a causal association between low 25(OH)D levels and increased CVD risk. We evaluated journal articles in light of Hill's criteria for causality in a biological system. The results of our assessment are as follows. Strength of association: many randomized controlled trials (RCTs), prospective and cross-sectional studies found statistically significant inverse associations between 25(OH)D levels and CVD risk factors. Consistency of observed association: most studies found statistically significant inverse associations between $25(\mathrm{OH}) \mathrm{D}$ levels and CVD risk factors in various populations, locations and circumstances. Temporality of association: many RCTs and prospective studies found statistically significant inverse associations between 25(OH)D levels and CVD risk factors. Biological gradient (dose-response curve): most studies assessing 25(OH)D levels and CVD risk found an inverse association exhibiting a linear biological gradient. Plausibility of biology: several plausible cellular-level causative mechanisms and biological pathways may lead from a low 25(OH)D level to increased risk for CVD with mediators, such as dyslipidemia, hypertension and diabetes mellitus. Experimental evidence: some well-designed RCTs found increased CVD risk factors with decreasing 25(OH)D levels. Analogy: the association between serum 25(OH)D levels and CVD risk is analogous to that between $25(\mathrm{OH}) \mathrm{D}$ levels and the risk of overall cancer, periodontal disease, multiple sclerosis and breast cancer. Conclusion: all relevant Hill criteria for a causal association in a biological system are satisfied to indicate a low $25(\mathrm{OH}) \mathrm{D}$ level as a CVD risk factor.
\end{abstract}

Reprinted from Nutrients. Cite as: Weyland, P.G.; Grant, W.B.; Howie-Esquivel, J. Does Sufficient Evidence Exist to Support a Causal Association between Vitamin D Status and Cardiovascular Disease Risk? An Assessment Using Hill's Criteria for Causality. Nutrients 2014, 6, 3403-3430.

\section{Introduction}

Cardiovascular disease (CVD) is the leading cause of death in the United States and has been since the early 1900s [1]. CVD incidence peaked in the 1960s and then gradually declined over the next 50 years. From 1980 to 2000, the death rate for coronary heart disease (CHD) for men, adjusted for age, decreased from 543 to 267 per 100,000, and for women, the death rate decreased from 263 to 134 per 100,000. Almost half of the decline can be attributed to decreasing CVD risk factors, including hypertension (HTN), smoking and dyslipidemia [2]. The CVD death rate has now plateaued, but, alarmingly, may be increasing [1], reducing life expectancy for the first time [3]. To decrease CVD morbidity and mortality, we must identify and effectively treat all risk factors and their causes.

Robert Scragg [4] first hypothesized that increasing ultra-violet (UV)-related vitamin D status affords protection against CVD. The serum 25-hydroxyvitamin D $(25(\mathrm{OH}) \mathrm{D})$ level is the most widely used 
measurement to assess overall vitamin D status [5]. Serum 25(OH)D levels are inversely associated with several CVDs, including myocardial infarction (MI) [6,7], coronary artery disease (CAD), heart failure, atrial fibrillation, ventricular tachycardia [8], peripheral vascular disease (PVD) [8-11], stroke [8,12], incident coronary artery calcium (CAC) [13-16], cardiac valve and vascular calcification [17] and all CVDs [18].

Study findings have inversely associated risk factors for CVD with serum 25(OH)D levels, including lower serum high-density lipoprotein cholesterol (HDL-C) levels, higher serum triglyceride (TG) levels [15], diabetes mellitus (DM) [8,19], increased blood pressure (BP) [15,20-25], dysfunctional changes in the characteristics of plasma lipids [26-28], inflammation [29] and increased serum parathyroid hormone (PTH) levels [30].

Isolating primary risk factors that cause CVD is challenging, because the human body responds to disrupted homeostasis by up- and down-regulating cellular function. Multiple pathways may exist between a low serum 25(OH)D level and increased CVD risk. Some pathways may be direct and not include any intermediate factors, whereas others may be indirect and include an intermediate factor(s). Moreover, CVD is not a single diagnosis, but rather, according to the National Center for Health Statistics, a group of diagnoses, including CAD, heart failure, essential HTN, hypertensive renal disease, cardiac dysrhythmias, rheumatic heart disease, cardiomyopathy, pulmonary heart disease and cerebrovascular disease [31].

The level of sufficiency for serum $25(\mathrm{OH}) \mathrm{D}$ is still being debated. Two schools of thought exist regarding what constitutes a sufficient level: $20 \mathrm{ng} / \mathrm{mL}$ [32,33] and $30 \mathrm{ng} / \mathrm{mL}$ [34-37]. Approximately $32 \%$ of the U.S. population has a deficient serum $25(\mathrm{OH}) \mathrm{D}$ level (defined as $<20 \mathrm{ng} / \mathrm{mL}$ ) [38]. The worldwide prevalence of deficient serum 25(OH)D levels is approximately one billion [39]. The primary causes of low serum $25(\mathrm{OH}) \mathrm{D}$ levels are strict sun protection and inadequate dietary or supplemental vitamin D intake [40]. Levels are easily elevated by oral vitamin D supplementation [41]. A daily intake of 10,000-20,000 IU of cholecalciferol (vitamin $\mathrm{D}_{3}$ ) per day is unlikely to result in vitamin D toxicity [42]. Results from epidemiological studies suggest that if a low serum $25(\mathrm{OH}) \mathrm{D}$ level is a primary risk factor for CVD and then corrected, all-cause mortality could decrease significantly, both in the United States [43] and worldwide [44].

\section{Approach and Rationale}

The research studies used for this evaluation were located in the PubMed database by using the following search terms: Hill's criteria for causality, vitamin D, cardiovascular disease, randomized controlled trial, seasonality, hypertension, dyslipidemia, coronary artery calcium, parathyroid hormone, inflammation, diabetes mellitus and high-density lipoprotein cholesterol. Studies were also sought in the references of the preceding studies. We evaluated studies for relevance to this assessment and being representative of current research. We included them regardless of whether they supported criteria for a causal association between serum $25(\mathrm{OH}) \mathrm{D}$ levels and CVD risk.

We evaluated the likelihood of a causal association between a low serum 25(OH)D level and increased risk for CVD by applying Sir Austin Bradford Hill's criteria for causality in a biological system [45] (see Table 1). Causality is multifaceted, and certain conditions must be met to determine that a causal association is likely. Hill stated that the criteria are useful, as we most often depend on observed 
events to detect relationships between sickness and its antecedents. Waiting to take action until research results explain the entire chain of events that lead to disease may not be necessary when discovering a few links in the chain may suffice.

Table 1. Hill's criteria for causality in a biological system.

\begin{tabular}{ll}
\hline \multicolumn{1}{c}{ Criterion } & \multicolumn{1}{c}{ Defining Question } \\
\hline Strength of the association & $\begin{array}{l}\text { Is there a large difference in the outcome between } \\
\text { exposed and non-exposed persons? }\end{array}$ \\
\hline Consistency of the observed association & $\begin{array}{l}\text { Has the outcome been observed by multiple researchers, } \\
\text { in various circumstances, places and at different times? }\end{array}$ \\
\hline Specificity of the association & $\begin{array}{l}\text { Are there specific persons or geographic locations } \\
\text { associated with specific outcomes? }\end{array}$ \\
\hline $\begin{array}{l}\text { Temporality (temporal relationship of } \\
\text { the association) }\end{array}$ & Does the cause always precede the effect? \\
\hline Biological gradient & Is there a dose-response curve? \\
\hline Plausibility of the biology & $\begin{array}{l}\text { Is the suspected causation consistent with current } \\
\text { knowledge of biology? }\end{array}$ \\
\hline Coherence & $\begin{array}{l}\text { Are there any serious conflicts with the biology or } \\
\text { natural history of the disease? }\end{array}$ \\
\hline $\begin{array}{l}\text { Experiment (experimental or } \\
\text { quasi-experimental evidence) }\end{array}$ & $\begin{array}{l}\text { Has an observed association led to a preventive action } \\
\text { that has prevented the outcome? }\end{array}$ \\
\hline Analogy & Is there an analogous exposure and outcome? \\
\hline
\end{tabular}

The criteria relevant to this evaluation include all, except specificity and coherence. This evaluation does not include specificity, because evidence supports low serum 25(OH)D levels and increased risk of several other disease processes [43]. This evaluation does not include coherence, because of its similarity to plausibility (see Table 2), and the information would be redundant. Hill's criteria have been used to assess a causal association between serum 25(OH)D levels and cancer risk [46], periodontal disease [37], multiple sclerosis (MS) [47], breast cancer risk [48] and the most prevalent cancers [49].

To arrive at the most accurate conclusions and to intervene with the most effective treatments, a thorough understanding of causality and of the limitations inherent in how we determine whether a causal association exists is essential. No single type of study, including randomized controlled trials (RCTs), can evaluate each of Hill's criteria. This evaluation used Hill's criteria, because it can consider the results of RCTs, prospective, cross-sectional and epidemiological studies. 
Table 2. Studies used to evaluate causality between low vitamin D and increased risk of CVD. HTN, hypertension; DM, diabetes mellitus; PWV, pulse wave velocity.

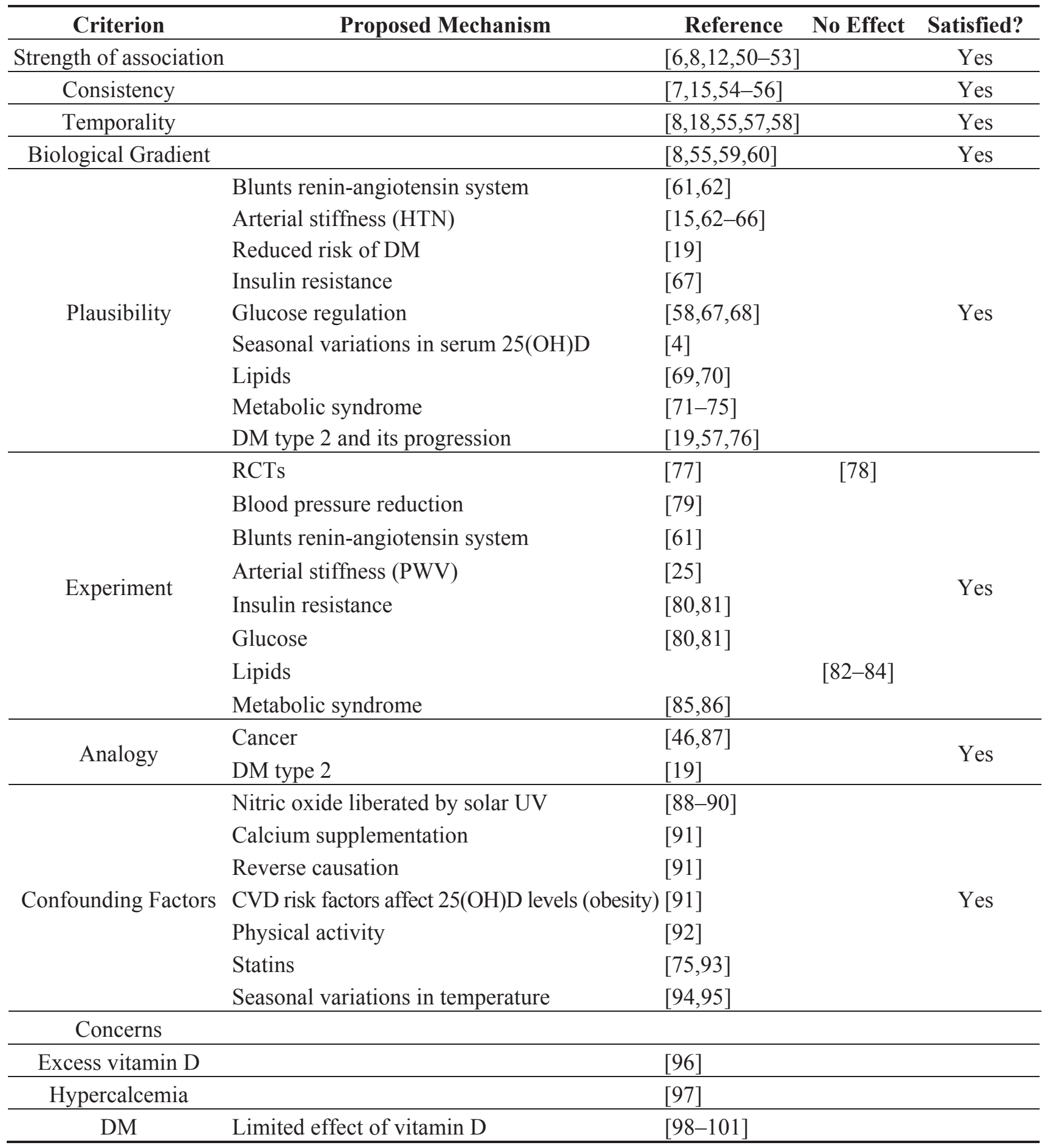

\section{Findings: Evaluation Using Hill's Criteria for Causality}

The studies included in this criteria section and all of the studies in the subsequent criteria sections are ordered by design; first are the meta-analyses, then prospective, retrospective, cross-sectional, case-control and lastly ecological studies. They are then ordered from the highest to the lowest relative risk ration (RR), hazards ratio (HR) or odds ratio (OR) when available. 


\subsection{Strength of the Association}

The stronger the positive or negative association between two variables, the more likely the association is causal. However, this may not always be true. One must consider all that is known about the two variables before concluding that an association is causal. For example, a very strong association may exist between an exposure and a disease, but another unknown variable may mediate the two. Alternatively, an exposure may directly cause a disease, but only under certain, sometimes very limited, circumstances; therefore, the association between the exposure and the disease would be weak. Therefore, a strong association is neither necessary nor sufficient to determine the likelihood of a causal association.

Satisfying the strength of association criterion requires a thorough evaluation of the correlation between vitamin D status and CVD risk. To come as close as possible to determining the true strength of an association, one must determine and then consistently use the most accurate and precise measures of the exposure and the disease [102]. Most researchers agree that the serum 25(OH)D level is the most accurate measure of overall vitamin D status. Several investigators have found statistically significant associations between serum 25(OH)D levels and CVD risk factors or CVDs.

Correia and colleagues [51] performed a prospective study in which they examined the association between serum 25(OH)D levels and the incidence of CVD-related mortalities during hospitalization. Ten percent of their 206 participants were severely deficient, defined as serum 25(OH)D levels $\leq 10 \mathrm{ng} / \mathrm{mL}$. Incident CVD-related mortality was much higher at $24 \%$ for the group of patients with severe serum $25(\mathrm{OH}) \mathrm{D}$ deficiency versus $4.9 \%$ in the group of patients with levels $>10 \mathrm{ng} / \mathrm{mL}$ (RR $4.3,95 \% \mathrm{CI}, 1.8,10, p=0.001)$. These results are impressive, but the authors acknowledge that the CIs were very wide. Anderson and colleagues [8] completed a study with both cross-sectional and prospective data, which offered support for an association between serum 25(OH)D levels and CVD risk. The researchers examined 41,504 electronic health records and concluded from the cross-sectional data that there is an inverse association between prevalence of CVD risk factors and serum 25(OH)D levels. A significant increase in the prevalence of HTN (30\% relative increase RI), DM $(90 \% \mathrm{RI}), \mathrm{PVD}(53 \% \mathrm{RI})$ and hyperlipidemia (9\% RI) was present in the group with serum 25(OH)D levels $\leq 15 \mathrm{ng} / \mathrm{mL}$ compared with the group with levels $>30 \mathrm{ng} / \mathrm{mL}$ ( $p<0.0001$ for all, significant after Bonferroni correction for multiple comparisons). The authors acknowledge that selection bias may have been present, because only individuals who had serum 25(OH)D levels in their record were included in the study.

Researchers outside North America have also found inverse associations between serum 25(OH)D levels and risk factors for CVD, although sun exposure and diet may differ. Jang and colleagues [50] performed a cross-sectional study with 320 Korean girls whose average age was 13 years, 63.8\% of whom had serum $25(\mathrm{OH}) \mathrm{D}$ levels $<20 \mathrm{ng} / \mathrm{mL}$. After adjusting for physical activity and BMI $Z$-score, the researchers found that serum $25(\mathrm{OH}) \mathrm{D}$ levels were negatively associated with fasting blood glucose levels $(r=-0.1748, p=0.0033)$ and insulin resistance $(r=-0.1441, p=0.0154)$, both risk factors for metabolic disorders.

The 2013 study by Deleskog and colleagues [53] had mixed results. The researchers performed a cross-sectional study with 3430 participants, $8 \%$ of whom had deficient serum 25(OH)D levels defined as $<51 \mathrm{nmol} / \mathrm{L}(<20 \mathrm{ng} / \mathrm{mL}), 82 \%$ had insufficient levels defined as $51-75 \mathrm{nmol} / \mathrm{L}(20-30 \mathrm{ng} / \mathrm{mL})$ and $10 \%$ had sufficient levels defined as $>75 \mathrm{nmol} / \mathrm{L}(>30 \mathrm{ng} / \mathrm{mL})$. No independent association emerged 
between serum 25(OH)D level insufficiency and carotid intima media thickness. However, those with deficient levels were more likely to have CVD risk factors, including higher BP, blood glucose, TG levels and lower serum HDL-C levels. Additionally, they were more likely to have DM.

Sun and colleagues [12] performed a case-control study in which they examined the association between ischemic stroke risk and serum 25(OH)D levels in 464 females with ischemic stroke and 464 female matched controls. The researchers compared participants in the lowest versus highest tertiles of serum $25(\mathrm{OH}) \mathrm{D}$ levels after adjusting for dietary and lifestyle covariates. Lower serum 25(OH)D levels were associated with an increased risk for ischemic stroke (OR 1.49, 95\% CI, 1.01, 2.18, $p<0.04$ ).

Scragg and colleagues [6] were one of the first research teams to examine the association between serum 25(OH)D levels and CVD. The researchers performed a case-control study with 179 MI cases with controls matched for age, sex and date of blood collection. They found an RR for MI of $0.43(95 \% \mathrm{CI}, 0.27,0.69)$ for participants with serum $25(\mathrm{OH}) \mathrm{D}$ levels at or above their study median value of $32 \mathrm{nmol} / \mathrm{L}(12.8 \mathrm{ng} / \mathrm{mL})$ versus below the median.

Deleskog and colleagues [52] included 774 participants in a case-control study to evaluate the association between serum 25(OH)D levels and premature MI (younger than 60 years). Serum 25(OH)D levels were analyzed twice as a categorical variable; insufficiency was defined as $<50 \mathrm{nmol} / \mathrm{L}$ (20 ng/mL) and was compared with levels $\geq 50 \mathrm{nmol} / \mathrm{L}$; a separate analysis defined insufficiency as $<75$ $\mathrm{nmol} / \mathrm{L}(30 \mathrm{ng} / \mathrm{mL})$, which was compared with levels $\geq 75 \mathrm{nmol} / \mathrm{L}$. Neither of the definitions of serum 25(OH)D level insufficiency were independently associated with premature MI. Therefore, the results do not support the criterion. The researchers concluded that the serum 25(OH)D level insufficiency may promote risk factors that are already established and known to promote atherothrombosis.

The criterion strength of the association has thus been met for 25(OH)D levels and CVD or CVD risk factors, including MI, CVD-related mortality, ischemic stroke risk, HTN, DM, PVD, hyperlipidemia, elevated blood glucose and increased insulin resistance.

\subsection{Consistency of the Association}

An association is consistent if it is observed under different circumstances, at different times, in various places and by various researchers [45]. Consistency is also confirmed if the results of a study can be replicated with a different sample of participants with the same study design and analytic methods. Inconsistent study results may occur when differences exist in study design, lab assays, definitions of serum 25(OH)D level deficiency, insufficiency versus sufficiency and statistical methods. Confidence in the results of meta-analyses depends on an assessment of the comparability of all studies included in the analysis [103].

Parker and colleagues [54] carried out the study with the strongest support for the criterion of consistency. In their meta-analysis, they systematically reviewed 28 studies with a total of 99,745 participants. The researchers reported important variations among studies included in their review, including categories of serum 25(OH)D levels, study design and analyses. Despite these differences, 29 of 33 ORs from the 28 studies showed an inverse association between serum 25(OH)D levels and the prevalence of cardio-metabolic disorders. One study demonstrated no effect, and three studies showed a positive association. Parker and colleagues [54] found a 43\% reduction in cardio-metabolic disorders with the highest levels of serum 25(OH)D (OR 0.57, 95\% CI, 0.48, 0.68). 
The meta-analysis by Wang and colleagues [55] offers additional strong support. They included 19 prospective studies with a total of 65,994 participants, of whom 6123 developed CVD. The 19 studies included CVD, CVD mortality, CHD and stroke as outcomes. Wang and colleagues found an inverse linear association between serum $25(\mathrm{OH}) \mathrm{D}$ in the range $20-60 \mathrm{nmol} / \mathrm{L}(8-24 \mathrm{ng} / \mathrm{mL})$ and the risk of CVD (RR, 1.03, 95\% CI, 1.00, 1.06).

Giovannucci and colleagues [7] found results consistent with the previous studies. This prospective, nested, case-control study included 454 male participants who were CHD cases and 900 male controls matched for age, HTN, aspirin use, physical activity, serum TG and low-density lipoprotein cholesterol (LDL-C) levels, as well as alcohol use. The median values for each of the four categories of serum $25(\mathrm{OH}) \mathrm{D}$ levels were entered as continuous variables in a regression model. The researchers found a two-fold increase in risk for $\mathrm{MI}$ if the serum 25(OH)D level was less than $16 \mathrm{ng} / \mathrm{mL}$ compared with those with a level of at least $30 \mathrm{ng} / \mathrm{mL}$ (RR, 2.42, 95\% CI, 1.53, 3.84; $p<0.001$ ). They also found a $2.1 \%$ decreased risk of $\mathrm{MI}$ for every $1 \mathrm{ng} / \mathrm{mL}$ increase in serum $25(\mathrm{OH}) \mathrm{D}$ levels. Only including males in the study prevents the generalizability of the results to females.

Support for the consistency criterion is also evident in the prospective study by de Boer and colleagues [15] $(N=1370)$. At baseline, $723(53 \%)$ had CAC. Over a three-year period, 135 participants developed CAC. The researchers adjusted for gender, age, ethnicity/race, location, season, activity level, smoking status, body mass index (BMI), DM, BP and serum lipid and C-reactive protein (CRP) levels. They found that serum $25(\mathrm{OH}) \mathrm{D}$ levels were inversely associated with incident, but not prevalent, CAC; for every $10 \mathrm{ng} / \mathrm{mL}$ decrease in the serum $25(\mathrm{OH}) \mathrm{D}$ level, the risk of developing CAC increased by $23 \%$ (RR, 1.23, 95\% CI, 1.00, 1.52, $p=0.049)$.

Finally, a cross-sectional study by Kendrick and colleagues [56] found similar supporting results by using data from 16,603 participants of the Third National Health and Nutrition Examination Survey (NHANES III). Serum 25(OH)D level deficiency, defined as $<20 \mathrm{ng} / \mathrm{mL}$, was associated with a $57 \%$ increased odds for prevalent CVD. After adjusting for gender, age, ethnicity/race, season, activity level, smoking status, HTN, DM, BMI, dyslipidemia, chronic kidney disease and vitamin D use, the odds decreased to $20 \%$ (OR, 1.20, 95\% CI, 1.01, 1.36, $p=0.03$ ).

A study-participant characteristic that should be included in the evaluation of the consistency criterion is ethnicity. A prospective study by Michos and colleagues [82] found that serum 25(OH)D levels less than $15 \mathrm{ng} / \mathrm{mL}$ were not associated with fatal stroke in blacks, but were associated with fatal stroke in whites. One limitation of this study is that because the median time to fatal stroke was 14.1 years and the serum $25(\mathrm{OH}) \mathrm{D}$ levels were only drawn once at baseline, there could have been undetected significant changes in serum 25(OH)D levels during the study. Differences in CHD events, including angina, MI, cardiac arrest or CHD death, by ethnicity were found in a prospective study by Robinson-Cohen and colleagues [66]. The researchers found an association between lower serum 25(OH)D levels and incident CHD events for white or Chinese, but not black or Hispanic participants. The same limitation is present in this study; only a baseline serum $25(\mathrm{OH}) \mathrm{D}$ level was drawn, and there was a median follow-up period of 8.5 years.

An unexplained difference by ethnicity was found by Gupta and colleagues [104], who performed a cross-sectional study. The researchers found significant associations between both pre-diabetes and pre-hypertension and gender, age and BMI in Mexican-Americans. However, they did not find an association between either pre-diabetes or pre-hypertension and serum $25(\mathrm{OH}) \mathrm{D}$ levels, as has been 
found for both non-Hispanic whites and non-Hispanic blacks. The authors stated that the reason for these results was unclear.

Results from a study performed by Rezai and colleagues [14] found that there was an association between serum 25(OH)D levels and left ventricular end-diastolic volumes for men of all ethnicities. The results of this cross-sectional study add support to the criterion of consistency, because low 25(OH)D levels showed the same association with poorer CV status for all ethnicities. This may mean that disparities in the prevalence of low vitamin D status among ethnicities may cause the disparities among ethnicities in the prevalence of CVD. Webb and colleagues [12] found that the pulse wave velocity (PWV) was higher in British South Asians of Indian descent than in white Europeans $(9.32 \mathrm{~m} / \mathrm{s} v s .8 .68 \mathrm{~m} / \mathrm{s}$, $p=0.001)$ using a cross-sectional design. They also found that the serum $25(\mathrm{OH}) \mathrm{D}$ level was independently associated with PWV, when adjusted for age, mean arterial pressure, sex, glucose, heart rate, vasoactive medications and South Asian ethnicity $\left(R^{2}=0.73, p=0.004\right)$. The researchers concluded that vitamin D insufficiency may mediate an increase in aortic stiffness without a difference in the risk profile, including vascular disease.

The preceding studies have shown mixed results, and the reasons for the differences are multifaceted. One reason for the disparity in serum $25(\mathrm{OH}) \mathrm{D}$ levels among different ethnic groups is that vitamin $\mathrm{D}$ production is inversely proportional to skin pigmentation [105]. Skin pigmentation varies among members of the same ethnic group, and designing a study in which skin pigmentation is objectively quantified and included as a variable may help to clarify differences between individuals versus groups. Studies that use ethnicity self-reporting or that have the investigator determine the ethnicity of the participants can also decrease the validity of the findings.

The consistency of the association criterion has thus been met due to the research results regarding the systematic review by Parker and colleagues and the smaller described supporting studies. The studies regarding ethnicity have mixed results. Parker and colleagues in their meta-analysis found overall associations between serum 25(OH)D levels and MI, stroke, ischemic heart disease, PVD, DM and metabolic syndrome.

\subsection{Temporality}

Temporality refers to the direction of influence in a sequence of events. An event or phenomenon cannot cause another event or phenomenon if the presumed cause does not precede the presumed effect. Determining whether a potential risk factor precedes a disease process is particularly difficult when the disease is chronic and progresses slowly [45]. Determining the temporal direction of influence of low serum $25(\mathrm{OH}) \mathrm{D}$ levels in relation to CVD risk by examining the results of prospective studies or meta-analyses that have included only prospective studies will help determine if the criterion of temporality has been met.

DM is a well-established risk factor for CVD, and the association between serum 25(OH)D levels and DM has been prospectively studied. Song and colleagues [57] included 21 prospective studies with 76,220 participants, 4996 incident type $2 \mathrm{DM}$ cases and serum 25(OH)D in a meta-analysis. The researchers compared the highest to lowest serum 25(OH)D levels using categories and found that the summary RR for type $2 \mathrm{DM}$ was $0.62(95 \% \mathrm{CI}, 0.54,0.70)$. The statistical significance of the inverse association between DM risk and serum 25(OH)D levels remained after controlling for sex, criteria for 
DM diagnosis, follow-up time, sample size and 25(OH)D assay type. Each $10 \mathrm{nmol} / \mathrm{mL}(4 \mathrm{ng} / \mathrm{mL})$ increase in the serum $25(\mathrm{OH}) \mathrm{D}$ level was associated with a $4 \%$ lower risk of type $2 \mathrm{DM}(95 \% \mathrm{CI}, 3,6$; $p$ linear trend $=0.0001)$. Therefore, low $25(\mathrm{OH}) \mathrm{D}$ levels may be a risk factor for CVD with type $2 \mathrm{DM}$ as the mediator.

Wang and colleagues [55] examined the association between CVD mortality along with CVD risk and serum 25(OH)D levels in a meta-analysis of 19 prospective studies. Collectively, these studies had 65,994 participants, of whom 6123 developed CVD. The researchers used the median serum 25(OH)D levels, or if unavailable, they compared the mean or the midpoint of the upper and lower bounds in each of the $25(\mathrm{OH}) \mathrm{D}$ categories from each of the 19 studies to the category of the risk of CVD. Being in the lowest category was associated with a higher risk for all CVDs (pooled RR 1.52, 95\% CI, 1.30, 1.77), for CVD mortality (pooled RR 1.42, 95\% CI, 1.19, 1.71), for CHD (pooled RR 1.38, 95\% CI, 1.21, 1.57) and for stroke (pooled RR 1.64, 95\% CI, 1.27, 2.10) than the highest category.

Although the study by Anderson and colleagues [8] was included in both the Song and colleagues and Wang and colleagues meta-analyses, it is a landmark study, and the results are important to cite. This study offers strong support for temporality. The prospective study using electronic health records monitored participants for an average of 1.3 years and a maximum of 9.3 years. The prevalence of serum $25(\mathrm{OH}) \mathrm{D}$ levels $\leq 30 \mathrm{ng} / \mathrm{mL}$ was $63.6 \%$. Participants without risk factors for CVD with serum $25(\mathrm{OH}) \mathrm{D}$ levels $\leq 15 \mathrm{ng} / \mathrm{mL}$ had a higher risk of incident HTN, dyslipidemia and DM than those with levels $>30$ $\mathrm{ng} / \mathrm{mL}$. Adjusted relative rates for death increased by $20 \%$ for serum $25(\mathrm{OH}) \mathrm{D}$ levels of $16-30 \mathrm{ng} / \mathrm{mL}$ and increased by $77 \%$ for serum $25(\mathrm{OH}) \mathrm{D}$ levels $\leq 15 \mathrm{ng} / \mathrm{mL}$. The researchers concluded that these data provide support for low serum $25(\mathrm{OH}) \mathrm{D}$ level as a primary risk factor for CVD. Schöttker and colleagues [18], in a prospective study with 9578 participants, found an increased risk of cardiovascular mortality associated with decreased serum 25(OH)D levels (hazards ratio (HR) 1.39, 95\% CI, 1.02, 1.89).

Tsur and colleagues [58] conducted a prospective cohort study over a two-year period that assessed incident impaired fasting glucose (IFG) and DM type 2 in 117,960 participants. The researchers adjusted for several variables, including sex, age, BMI, serum LDL-C, HDL-C, TG levels, history of HTN, smoking status and CVD. Participants with a serum $25(\mathrm{OH}) \mathrm{D}$ level $\leq 25 \mathrm{nmol} / \mathrm{L}(10 \mathrm{ng} / \mathrm{mL})$ had an OR for progression from normoglycemia to IFG of 1.13 (95\% CI, 1.03, 1.24), from normoglycemia to DM of $1.77(95 \% \mathrm{CI}, 1.11,2.83)$ and from IFG to DM of 1.43 (95\% CI, 1.16, 1.76), compared with a serum $25(\mathrm{OH}) \mathrm{D}$ level $>75 \mathrm{nmol} / \mathrm{L}(30 \mathrm{ng} / \mathrm{mL})$. The researchers concluded that a low serum $25(\mathrm{OH}) \mathrm{D}$ level may be an independent risk factor for IFG and DM that can eventually lead to CVD.

The previously described meta-analyses of prospective studies and the additional prospective studies

offer evidence that temporality is satisfied, because they all use serum $25(\mathrm{OH}) \mathrm{D}$ levels taken at the time of enrollment, which precedes the incident event or death. Furthermore, most reviewed individual prospective studies, and a meta-analysis of prospective studies showed an increased incidence of CVD or CVD risk factors with decreasing serum 25(OH)D. The CVDs or risk factors for CVD included CVD mortality, CHD, stroke, dyslipidemia, HTN, type 2 DM and IFG.

\subsection{Biological Gradient (Dose-Response Relation)}

In the context of this assessment, the biological gradient, or dose-response relation, refers to the change in the prevalence or incidence rate of CVD or risk factors for CVD as serum 25(OH)D levels 
change. The biological gradient criterion is satisfied when the value of the dependent variable (effect) can be predicted, with some degree of confidence, when the value of the independent variable (cause) is known. Hill [45] states that securing a satisfactory quantitative measure to use for this purpose is often difficult.

Wang and colleagues [55] showed a biological gradient effect in their 2012 meta-analysis. They found a linear (graded) and inverse association between serum 25(OH)D levels of 20-60 nmol/L $(8-24 \mathrm{ng} / \mathrm{mL})$ and the risk of CVD. They found a linear trend for the RR $=1.03(95 \% \mathrm{CI}, 1.00,1.06)$ for every $25 \mathrm{nmol} / \mathrm{L}(10 \mathrm{ng} / \mathrm{mL})$ decrease in 25(OH)D ([55]; Figure 3). Wang and colleagues had similar results in an earlier study [60]. They examined low serum 25(OH)D levels and incident CVD prospectively in 1739 participants from the Framingham Offspring Study. A serum 25(OH)D level $<15 \mathrm{ng} / \mathrm{mL}$ was associated with a two-fold increase in an age and sex-adjusted five-year incident rate for CVD compared with those with a level of $\geq 15 \mathrm{ng} / \mathrm{mL}$ (multivariable-adjusted HR $=1.62$, $95 \% \mathrm{CI}, 1.11,2.36 ; p=0.01)$. The researchers also found a graded increase in CVD risk for serum 25(OH)D levels of 10-14 ng/mL (multivariable-adjusted $\mathrm{HR}=1.53,95 \% \mathrm{CI}, 1.00,2.36 ; p=0.01$ ) versus levels $<10 \mathrm{ng} / \mathrm{mL}$ (multivariable-adjusted $\mathrm{HR}=1.80,95 \% \mathrm{CI}, 1.05,3.08 ; p=0.01$ ).

Anderson and colleagues [8] performed a prospective study, which was included in the Wang and colleagues meta-analysis. The researchers found statistically significant and biologically-graded inverse associations between serum 25(OH)D levels and the prevalence of CVD and CVD risk factors, including PVD, HTN, DM and hyperlipidemia (all $p<0.0001$ ). The researchers categorized serum 25(OH)D levels; levels of serum $25(\mathrm{OH}) \mathrm{D} \leq 15 \mathrm{ng} / \mathrm{mL}$ versus those $>30 \mathrm{ng} / \mathrm{mL}$ were associated with increased prevalence of DM ( $90 \%$ relative and $14 \%$ absolute) and HTN (30\% relative and $12 \%$ absolute) ( $p$ trend for both $<0.0001)$.

Vacek and colleagues [59] performed a retrospective study $(n=10,899)$ for a 68 -month period. Using univariate analysis, the researchers found statistically significant ORs for vitamin D deficiency, defined as $<30 \mathrm{ng} / \mathrm{mL}$, and CAD (OR, 1.16, 95\% CI, 1.012, 1.334, $p=0.03$ ), cardiomyopathy (OR, 1.29, 95\% CI, $1.019,1.633, p=0.03)$ and HTN (OR, 1.40, 95\% CI, 1.285, 1.536, $p \leq 0.0001)$.

The criterion, biological gradient, or dose-response curve, has thus been met. Most reviewed studies used serum 25(OH)D levels as categorical or continuous variables and found strong evidence for a graded association between levels and CVD/CVD risk factors, including nonspecific CVD, PVD, HTN, DM, hyperlipidemia, elevated BMI, elevated serum LDL-C and TG levels and decreased serum HDL-C levels.

\subsection{Plausibility}

Biological plausibility can be confirmed when the suspected causation mechanism is consistent with the current knowledge of biology. The actual physiological pathway of the hypothesized causal association between low serum 25(OH)D levels and increased risk for CVD may include mediators that are known CVD risk factors or other unknown factors. Specific cellular-level causative mechanisms that explain the increase in CVD associated with low vitamin D status need to be identified in order to definitively state that the criterion, biological plausibility, has been met.

Several cellular-level causative mechanisms have been proposed. It should be taken into consideration that, in contrast to the causative agent of an infectious disease, these proposed causative mechanisms do 
not necessarily compete with one another and are not mutually exclusive. Some or all of the proposed mechanisms may be accurate. This is because CVD is a broad category of diseases, and each of the diseases has multiple causes.

An in vitro study by $\mathrm{Oh}$ and colleagues [17] found an inhibition of foam cell formation when macrophages from persons with type 2 DM exposed to modified LDL were cultured in the bio-active form of vitamin $\mathrm{D} ; 1 \alpha, 25$-dihydroxyvitamin $\mathrm{D}_{3}\left(1 \alpha, 25(\mathrm{OH})_{2} \mathrm{D}_{3}\right)$. They also found accelerated foam cell formation when the vitamin D receptors (VDRs) were deleted from the macrophages. A reduction in the formation of atherosclerotic lesions in mice with the administration of the vitamin D analog, calcitriol $\left(1 \alpha, 25(\mathrm{OH})_{2} \mathrm{D}_{3}\right)$, was seen by Takeda and colleagues [32]. They hypothesize that calcitriol modulates the systemic and intestinal immune systems by inducing immunologically-tolerant dendritic cells and T-cells, both of which are anti-atherogenic.

Additionally, an in vitro study by Riek and colleagues [106] was performed in order to determine if vitamin D plays a role in monocyte migration and adhesion. The researchers examined monocytes from study participants $(n=12)$ with type $2 \mathrm{DM}$ and obesity who were vitamin $\mathrm{D}$ deficient. The researchers found a $20 \%$ reduction in monocyte migration in monocytes incubated with $25(\mathrm{OH}) \mathrm{D}_{3}$ compared to vitamin D-deficient conditions $(p<0.005)$. They also found that, compared to monocytes maintained in vitamin D-deficient conditions, incubation with $25(\mathrm{OH}) \mathrm{D}_{3}$ also significantly decreased adhesion $(p<0.05)$. The researchers concluded that hydroxylation of $25(\mathrm{OH}) \mathrm{D}_{3}$ to $1,25(\mathrm{OH})_{2} \mathrm{D}_{3}$ at the cellular level may play a role in vitamin D anti-atherogenic effects.

VDRs were also found in human coronary artery smooth muscle cells (CASMC) by Wu-Wong and colleagues [107]. When CASMC were treated with the vitamin D analogs, calcitriol or paricalcitol (19-nor-1 $\left.\alpha, 25(\mathrm{OH})_{2} \mathrm{D}_{2}\right)$ there was an upregulation of 24-hydroxylase and also an upregulation of thrombomodulin (TM) mRNA. Downregulation of TM mRNA has been associated with atherosclerosis and thrombosis. Finding that upregulation occurred led the researchers to hypothesize that this is the mechanism that leads to a decrease in morbidity and mortality with vitamin D analog use in persons with chronic kidney disease.

Many studies have shown inverse associations between established CVD risk factors, such as dyslipidemia, HTN and DM [108] and serum 25(OH)D levels (see Table 2). The following research studies further assist in evaluating the plausibility of a causal association.

\subsubsection{Dyslipidemia}

A proposed causal mechanism for the association between low serum 25(OH)D levels and increased risk for CVD involves dysfunctional changes in the characteristics of plasma lipids, including metabolism or transport [26], the ability to promote macrophage efflux, [27] and changes in serum levels of total cholesterol (total-C), HDL-C, LDL-C and TGs [15,28].

Skaaby and colleagues [70] investigated the association between serum 25(OH)D levels at baseline and incident dyslipidemia over five years in a prospective study with 4330 participants. A serum $25(\mathrm{OH}) \mathrm{D}$ level of $10 \mathrm{nmol} / \mathrm{L}(4 \mathrm{ng} / \mathrm{mL})$ higher at baseline was associated with decreased serum TG levels $(\beta=-0.52,95 \% \mathrm{CI},-0.99,-0.05, p=0.03)$ and decreased serum very-low-density lipoprotein cholesterol (VLDL-C) levels ( $\beta=-0.66,95 \% \mathrm{CI},-1.1,-0.2, p=0.005)$. With the same higher serum $25(\mathrm{OH}) \mathrm{D}$ level at baseline, the OR for incident hypercholesterolemia was 0.94 (95\% CI, 0.90, 0.99 , 
$p=0.01)$. The researchers concluded that higher serum $25(\mathrm{OH}) \mathrm{D}$ levels may favorably change lipid profiles and therefore positively influence cardiovascular health.

Karhapää and colleagues [109] performed a cross-sectional study in which they examined the relationship between serum 25(OH)D levels and total-C, LDL-C, HDL-C and TG levels in a study that included 909 male participants. The researchers found a significant inverse association between serum $25(\mathrm{OH}) \mathrm{D}$ levels and total-C, LDL-C and TG levels $(\beta=-0.15,-0.13$ and -0.17 , respectively; $p<0.001)$, which supports lower serum 25(OH)D levels leading to a less favorable lipid profile. However, they found no association between serum 25(OH)D and HDL-C levels, which does not support an association between lower serum $25(\mathrm{OH}) \mathrm{D}$ levels and a more favorable lipid profile.

Jorde and colleagues [28] also examined the association between serum 25(OH)D levels and serum lipid levels by using both cross-sectional and longitudinal data collected over 14 years. The cross-sectional study included 10,105 participants, and the researchers found that with increasing quartiles of serum 25(OH)D levels, serum HDL-C and LDL-C levels increased and serum TG levels decreased. In the longitudinal study with 2159 participants, the researchers found that increasing quartiles of serum $25(\mathrm{OH}) \mathrm{D}$ levels were associated with decreased serum TG levels. These results, except for the increase in serum LDL-C levels, support associating higher serum 25(OH)D levels with a more favorable lipid profile.

Researchers have also conducted genomic and cytochrome P450 enzyme studies to determine mechanisms that cause low serum 25(OH)D levels to lead to dysfunctional changes in lipids. Shirts and colleagues [69], in a cross-sectional study with 1060 participants, investigated the influence of single-nucleotide polymorphisms on serum HDL-C, LDL-C and TG levels for gene-25(OH)D interactions. Participants with deficient levels of serum 25(OH)D were more likely to also have lower serum HDL-C levels $(p=0.0003)$. Chow and colleagues [110] incubated human hepatocytes with $1,25(\mathrm{OH})_{2} \mathrm{D}_{3}$ and found a reduction in cholesterol production due to an increase in cytochrome $\mathrm{P} 450$ enzyme 7A1 activation of the VDR.

Guasch and colleagues [29] found an association between low plasma 25(OH)D levels and atherogenic dyslipidemia after adjusting for BMI in a cross-sectional study with 316 participants. When the researchers introduced serum-ultrasensitive CRP levels as a covariable, an association was no longer present. They suggested that inflammation may mediate the effect of serum $25(\mathrm{OH}) \mathrm{D}$ levels on lipid profiles.

\subsubsection{Hypertension}

The cause of HTN is usually unknown. Researchers have investigated the association between serum 25(OH)D levels and both prevalent and incident idiopathic HTN and pre-HTN [20,22-25]. Carrara and colleagues [61] conducted a prospective interventional trial in which they administered 25,000 IU of oral cholecalciferol (vitamin $D_{3}$ ) weekly over two months to 15 participants with essential HTN. There was neither randomization to different interventions or a placebo group. Because the researchers found reduced aldosterone $(p<0.05)$ and renin plasma levels $(p<0.05)$ after supplementation, they concluded that for persons with essential HTN and a low serum 25(OH)D level, vitamin D supplementation may help decrease BP.

Forman and colleagues [21] performed a prospective study with 1811 participants with measured plasma $25(\mathrm{OH}) \mathrm{D}$ levels. The researchers found that incident HTN was greater for participants with a 
plasma 25(OH)D level of $<15 \mathrm{ng} / \mathrm{mL}$ compared to those with a level $\geq 30 \mathrm{ng} / \mathrm{mL}$ (RR $3.18,95 \% \mathrm{CI}, 1.39$, 7.29). For men only $(n=613)$, the RR for the same comparison was much greater (RR 6.13, $95 \% \mathrm{CI}, 1.0,37.8)$ compared to women only $(n=1198)(\mathrm{RR} 2.67,95 \% \mathrm{CI}, 1.05,6.79)$. Forman and colleagues [62] also conducted a prospective study that included only women participants aged 32-52 years. The researchers found that incident HTN increased for the lowest quartile $(6.2-21.0 \mathrm{ng} / \mathrm{mL})$ versus the highest quartile $(32.3-89.5 \mathrm{ng} / \mathrm{mL})$ for $25(\mathrm{OH}) \mathrm{D}$ levels $(\mathrm{OR}, 1.66,95 \% \mathrm{CI}, 1.11,2.48, p=0.01)$.

Increased arterial stiffness may be an effect of low serum $25(\mathrm{OH}) \mathrm{D}$ level. Giallauria and colleagues [63], in a cross-sectional study with 1228 participants, found a statistically significant inverse association between serum 25(OH)D levels and arterial stiffness, measured with PWV (adjusted $R^{2}=0.27$, $\beta=-0.43 ; p=0.001)$. Furthermore, measuring PWV, Mayer and colleagues [64] performed a cross-sectional study and found a negative association with serum $25(\mathrm{OH}) \mathrm{D}$ level quartiles. The lowest serum 25(OH)D level quartile $(<20 \mathrm{ng} / \mathrm{mL})$ had the highest PWV score compared with the second, third or fourth quartile $(p=0.0001)$.

Three studies with only female participants had similar results. Pirro and colleagues conducted a cross-sectional study with 150 postmenopausal and serum $25(\mathrm{OH}) \mathrm{D}$-insufficient $(<30 \mathrm{ng} / \mathrm{mL})$ participants [65]. The researchers found a significant association between arterial stiffness, measured with PWV and serum 25(OH)D levels, but not after controlling for logarithmically-transformed serum PTH levels. Serum PTH levels were associated with arterial stiffness $(\beta=0.23, p=0.007)$. Reynolds and colleagues [66] in a cross-sectional study found a similar association between serum $25(\mathrm{OH}) \mathrm{D}$ levels and aortic stiffness (PWV scores) $(\beta=-0.0217,95 \% \mathrm{CI},-0.038,-0.005, p=0.010$ ) for 75 female participants with systemic lupus erythematosus. The authors did not state that serum PTH levels were measured and controlled for, and therefore, PTH levels may have mediated the association.

\subsubsection{Diabetes Mellitus}

DM is an important risk factor for CVD. Several studies have associated serum 25(OH)D levels and both prevalent and incident DM. Afzal and colleagues [19], in a prospective study with 9841 white participants, found an increased risk of type $2 \mathrm{DM}$ for study participants with plasma $25(\mathrm{OH}) \mathrm{D}$ levels $<5 \mathrm{ng} / \mathrm{mL}$ versus $\geq 20 \mathrm{ng} / \mathrm{mL}$ (HR, 1.22, 95\% CI, 0.85, 1.74). The researchers also performed a meta-analysis of 13 studies and found a greater prevalence of type $2 \mathrm{DM}$ for those in the lowest versus highest quartile for the serum 25(OH)D level (cut-points for the quartiles varied among the 13 studies) (OR, 1.39, 95\% CI, 1.21, 1.58). Anderson and colleagues [8], found an adjusted RI in incident DM of $89 \%$ for very low $(\leq 15 \mathrm{ng} / \mathrm{mL})$ versus sufficient $(>30 \mathrm{ng} / \mathrm{mL})$ categories of serum $25(\mathrm{OH}) \mathrm{D}$ levels (HR, 1.89, 95\% CI, 1.54, 2.33, $p<0.0001$ ). Forouhi and colleagues [67] found in a prospective study with 524 participants that baseline $25(\mathrm{OH}) \mathrm{D}$ levels were inversely associated with the 10 -year risk of hyperglycemia (fasting glucose: $\beta=-0.002, p=0.02$ ) and insulin resistance (fasting insulin $\beta=-0.15$, $p=0.01)$.

\subsubsection{Metabolic Syndrome}

Studies have been conducted to assess the association between both incident and prevalent metabolic syndrome and serum 25(OH)D levels. A prospective study by Gagnon and colleagues [74] found that $12.7 \%$ of 4164 participants developed metabolic syndrome over a five-year follow-up period. A higher 
risk of metabolic syndrome was present for those with serum $25(\mathrm{OH}) \mathrm{D}$ levels in the first quintile $(<18 \mathrm{ng} / \mathrm{mL})(\mathrm{OR}=1.41,95 \% \mathrm{CI}, 1.02,1.95)$ and second quintile $(18-23 \mathrm{ng} / \mathrm{mL})(\mathrm{OR}=1.74,95 \% \mathrm{CI}$, $1.28,2.37)$ compared with the highest quintile $(\geq 34 \mathrm{ng} / \mathrm{mL})$. Serum 25(OH)D levels were inversely associated with fasting glucose $(p<0.01)$, homeostasis model assessment for insulin resistance $(p<0.001)$, TG $(p<0.01)$ and waist circumference $(p<0.001)$. No association with two-hour plasma glucose $(p=0.29)$, HDL-C $(p=0.70)$ or BP $(p=0.46)$ was evident at the five-year follow-up.

Another cross-sectional study conducted by Brenner and colleagues [72] with 1818 participants found an $8.9 \%$ prevalence of metabolic syndrome. The researchers found an inverse association between plasma $25(\mathrm{OH}) \mathrm{D}$ levels and the number of components for metabolic syndrome $(\beta=-0.1$, $p<0.0001$ ). Components of metabolic syndrome included serum HDL-C level $<40 \mathrm{mg} / \mathrm{dL}$ (males) or $<50 \mathrm{mg} / \mathrm{dL}$ (females), serum $\mathrm{TG}$ level $>1.7 \mathrm{mmol} / \mathrm{L}$, fasting plasma glucose $>110 \mathrm{mg} / \mathrm{dL}$, $\mathrm{BP}>130 / 85 \mathrm{mmHg}$ and waist circumference $>102 \mathrm{~cm}$ (males) or $>88 \mathrm{~cm}$ (females). A lower OR $(0.50$, $95 \%$ CI, 0.24, 1.06) for metabolic syndrome was evident for study participants whose plasma 25(OH)D level was in the highest versus lowest quartile. After adjusting for age, sex, ethnicity, smoking status, physical activity and month of interview, researchers found that a $10-\mathrm{nmol} / \mathrm{mL}(4 \mathrm{ng} / \mathrm{mL})$ increase in the plasma $25(\mathrm{OH}) \mathrm{D}$ level was inversely associated with the homeostasis model assessment for insulin resistance score $(\beta=-0.08, p=0.006)$. Another cross-sectional study by Reis and colleagues [71] that included 1654 participants with DM assessed the prevalence of metabolic syndrome. The researchers divided serum 25(OH)D levels into quintiles and found an OR of $0.27(\mathrm{CI}, 0.15,0.46 ; p$ trend $<0.001)$ for metabolic syndrome for the highest quintile (median $=88 \mathrm{nmol} / \mathrm{L}(35 \mathrm{ng} / \mathrm{mL})$ ) versus the lowest quintile $($ median $=26.8 \mathrm{nmol} / \mathrm{L}(10.7 \mathrm{ng} / \mathrm{mL}))$.

In a case-control study by Makariou and colleagues, 52 participants with metabolic syndrome had lower serum 25(OH)D levels (mean $=11.8 \mathrm{ng} / \mathrm{mL}$, range $=0.6-48.3 \mathrm{ng} / \mathrm{mL}$ ) than 58 controls $($ mean $=17.2 \mathrm{ng} / \mathrm{mL}$, range $=4.8-62.4 \mathrm{ng} / \mathrm{mL} ; p=0.027)$ [75]. Serum 25(OH)D levels were inversely associated with serum TG levels $(r=-0.42, p=0.003)$ and small dense LDL-C $(r=-0.31, p=0.004)$.

The criterion for plausibility has thus been satisfied. There are several proposed biologically-plausible cellular-level mechanisms for the increase in CVD associated with low vitamin D status. Studies involving the assessment of an association between serum 25(OH)D levels and dyslipidemia, HTN, DM and metabolic syndrome have also been evaluated. Dyslipidemia, HTN, DM and metabolic syndrome are all plausible mediators between low serum 25(OH)D levels and increased risk of CVD. Specifically, the studies support increased serum LDL-C, VLDL-C and TG levels, decreased serum HDL-C levels, increased arterial stiffness, increased insulin resistance, hyperglycemia and increased incident metabolic syndrome as potentially plausible mediators.

\subsection{Experiment}

Researchers have conducted RCTs to assess the effect of serum 25(OH)D levels on CVD risk factors. However, vitamin D RCTs conducted to date have mixed results. The main reason is that vitamin D RCTs have been designed largely on the model used for pharmaceutical drugs, which assumes that the agent used in the trial is the only source of the agent and that a linear dose-response relation exists. Neither assumption is valid for vitamin D. 
Another consideration is that chronic disease is caused by more than one risk factor and may occur only after long-term versus short-term vitamin deficiency. Vitamin supplementation studies are usually designed to assess the decrease in risk due to increasing vitamin intake to meet the minimum sufficiency level. Additional information would be gained from studies that also test the effects of supplementation on levels beyond those previously established for disease risk [111].

Robert Heaney [112] recently outlined the steps to design and conduct vitamin D RCTs: (1) start with the 25(OH)D level-health outcome relation; (2) measure the 25(OH)D levels of prospective participants; (3) enroll only those with low $25(\mathrm{OH}) \mathrm{D}$ levels; (4) supplement with enough vitamin $\mathrm{D}_{3}$ to increase $25(\mathrm{OH}) \mathrm{D}$ levels to the upper end of the quasi-linear region of the $25(\mathrm{OH}) \mathrm{D}$ level-health outcome relation; and (5) re-measure $25(\mathrm{OH}) \mathrm{D}$ levels after supplementation. For CVD, these recommendations would translate to enrolling people with $25(\mathrm{OH}) \mathrm{D}$ levels below about $15 \mathrm{ng} / \mathrm{mL}$ and then supplementing with 2000-4000 IU of vitamin $\mathrm{D}_{3}$ per day to raise 25(OH)D levels to $>30-40 \mathrm{ng} / \mathrm{mL}$.

The effect of vitamin D supplementation on CVD risk factors for women with polycystic ovarian syndrome was investigated by Rahimi-Ardabili and colleagues [113]. The study participants taking the vitamin D supplement had a statistically significant increase in serum vitamin D level and statistically significant decreases in serum total-C, TGs and VLDL-C levels (all $p<0.05$ ). They did not have any changes in serum levels of HDL-C, LDL-C, apolipoprotein-A1 (Apo-A1) or high-sensitivity C-reactive protein (hs-CRP). The placebo group had no changes.

Schnatz and colleagues [77] supplemented participants $(n=600)$ with $1000 \mathrm{mg}$ of elemental calcium and $400 \mathrm{IU}$ of vitamin D per day. The researchers found a $1.28 \mathrm{mg} / \mathrm{dL}$ decrease in LDL-C $(p=0.04)$ with a $38 \%$ increase in the $25(\mathrm{OH}) \mathrm{D}$ level. The researchers also found an increase in HDL-C and a decrease in TGs.

Breslavsky and colleagues [68] conducted an RCT, including 47 participants with type $2 \mathrm{DM}$, who were randomized into two groups. One group received cholecalciferol (vitamin $\mathrm{D}_{3}$ ) at 1000 IU per day for 12 months, whereas the other group received a placebo. After being similar at baseline, the group receiving cholecalciferol had significantly decreased hemoglobin A1c levels $(p<0.0001)$, but no change occurred in the placebo group.

Grimnes and colleagues [114] performed an RCT with 94 participants with low serum 25(OH)D levels. The participants were randomly assigned to receive a 20,000 IU supplement of oral $\mathrm{D}_{3}$ or a placebo twice weekly for six months. The supplement did not improve the lipid profile, which included total-C, LDL-C, HDL-C and TGs.

Ponda and colleagues [82] conducted a randomized, placebo-controlled, double-blinded trial. They randomized 151 vitamin D-deficient participants to receive oral $\mathrm{D}_{3}$ at 50,000 IU weekly for eight weeks or placebo and then examined the effect on serum cholesterol levels. In the supplemented group, serum 25(OH)D levels increased, serum PTH levels decreased and serum calcium levels increased. When participants were stratified by the change in serum $25(\mathrm{OH}) \mathrm{D}$ level and the serum calcium level, those whose response was greater than the median response had an increase in serum LDL-C of $15.4 \mathrm{mg} / \mathrm{dL}$ compared with those who had lower than the median response. The analysis of the group receiving placebo did not show this relationship. Table 3 shows results from RCTs in order of serum 25(OH)D level at time of enrollment. This RCT does not support a beneficial effect on lipid status.

In a manuscript under preparation, it was found that for vitamin D RCTs related to CVD risk factors, the median baseline serum $25(\mathrm{OH}) \mathrm{D}$ level for the RCT with significant beneficial effects was $15 \mathrm{ng} / \mathrm{mL}$, 
while the median baseline serum 25(OH)D for those without beneficial effects was $19 \mathrm{ng} / \mathrm{mL}$ (Grant, in preparation). This finding underscores the importance of having a low baseline serum 25(OH)D level when designing and conducting vitamin D RCTs to evaluate the findings of observational studies, as proposed by Heaney [112].

The criterion for experiment has thus been met. We reviewed RCTs that supplemented participants with vitamin D and found that most well-designed RCTs supported a causal association between serum 25(OH)D levels and CVD risk. 


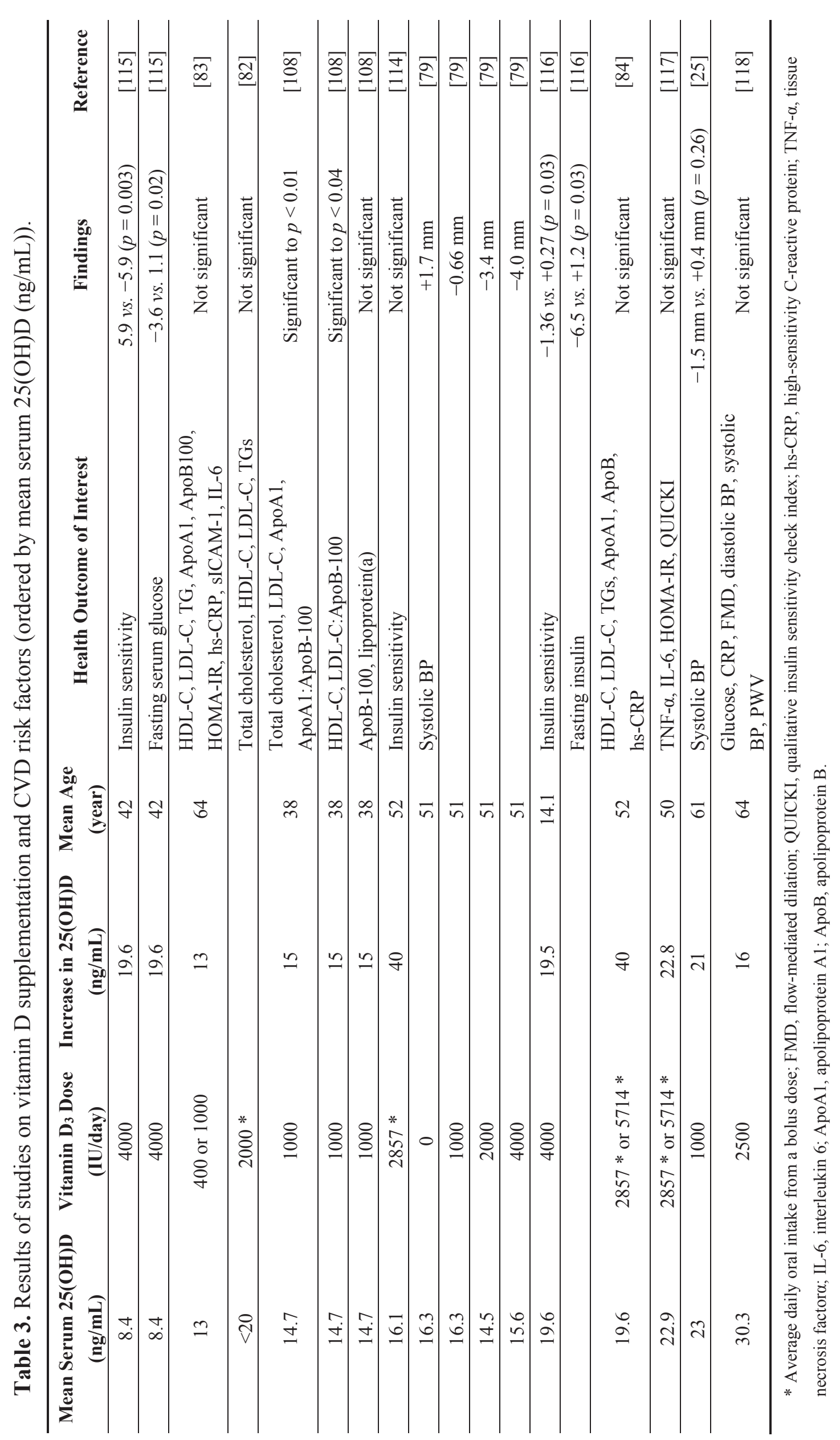

193 


\subsection{Analogy}

The likelihood of a causal association between low vitamin D status and several diseases has been evaluated using Hill's criteria for causality in a biological system. Hill's criteria were met when Grant [46] evaluated overall cancer risk, when breast cancer risk was evaluated by Mohr and colleagues [48], when Grant and Boucher [37] evaluated periodontal disease and when Hanwell and Banwell [47] evaluated multiple sclerosis. Hanwell and Banwell found that all of the criteria were satisfied, except the criterion for disease prevention by intervention (experiment). The researchers state that fulfilling this criterion will be difficult because multiple sclerosis has a low incidence, the age of onset is highly variable and there is a lack of consensus regarding optimal vitamin D dose and the timing of treatment.

The criterion, analogy, has thus been met. Several assessments with various diseases have shown an analogous association to low serum 25(OH)D levels and CVD risk.

\subsection{Confounding Factors}

Potischman and colleagues [119] discussed the inadequacies of traditional causal criteria for assessing nutrients, but they acknowledged that they are necessary for public health recommendations. The authors stated that additional important considerations exist, such as confounding, errors in measurement and dose-response curves for nutrients.

Opländer and colleagues [88] discovered a potentially confounding factor for the association between production of vitamin D in the skin and a decrease in BP. UVB irradiation is responsible for vitamin D production and is associated with a decrease in BP, but UVA irradiation was found to also decrease BP. The effect was attributed to UVA irradiation-induced release of nitric oxide.

Beveridge and colleagues identified other confounders [91]. Associations in vitamin D studies may be confounded by the effects of other CVD risk factors in addition to those being studied, and confounding related to the possibility of reverse causality may also occur. Liberopoulos and colleagues [93] found that statins have different effects on the increase of serum 25(OH)D levels. Woodhouse and colleagues [94] found a seasonal variation in serum total-C, HDL-C and TG levels. These confounders can be controlled for with the use of appropriate statistical analyses, just as age, gender, ethnicity, BMI and smoking status are often controlled for in research studies.

Essential to the credibility of study results is the measurement and reporting of adherence to the intervention. The evaluation of adherence to oral vitamin D supplements given in a study may be either absent or inadequate. Furthermore, an inquiry about concurrent use of personal oral vitamin D supplementation may differ across studies. Negative study results may simply be attributed to a lack of adherence to the intervention, because it leads to bias and a decrease in the statistical power.

\section{Conclusions}

Despite the identification and treatment of currently recognized CVD risks, CVD remains the leading cause of death. The focus of vitamin D research has recently expanded to include the effects of vitamin D status on CVD and CVD risk factors. Low serum 25(OH)D levels are associated with 
increased incidence [8], prevalence [56] and risk factors for CVD [15]. This assessment demonstrates that Hill's criteria were satisfied.

Potential benefits of decreasing the impact of a risk factor for CVD should outweigh potential risks. Repletion of vitamin D stores with a supplemental dose of 10,000 IU per day or less is unlikely to lead to toxic effects [39]. Repletion can be accomplished by a sensible increase in sun exposure [37] or by consuming vitamin D-rich foods, but this goal is most easily accomplished with oral supplementation. Furthermore, more severe deficiencies in serum 25(OH)D levels show a more rapid increase than less severe deficiencies [103]. Treatment for some CVD risk factors is expensive and may be difficult to access, but oral vitamin D supplements are readily accessible and reasonably priced. Other considerations for individualized treatment should include attention to skin melanin content, latitude and altitude of residence, dietary habits and amount of sun exposure.

The physiological mechanisms hypothesized to cause low vitamin D status to increase CVD risk have not yet been confirmed. Nearly all research studies regarding low vitamin D status and increased risk of CVD use observational study designs. More RCTs are needed that incorporate the complex pharmacokinetic and pharmacodynamic properties of vitamin D in the study design: dose-response curve, half-life, avoidance of toxicity and use of the most accurate and precise serum assays.

Exposure to sunlight or vitamin D supplementation may be used in an RCT, although having a control group with a zero serum $25(\mathrm{OH}) \mathrm{D}$ level would not be possible. This approach is possible only in drug studies [120]. Nutrients are more appropriately studied in the context of proving negative causation: the absence of an antecedent caused the consequence. This study design would be consistent with research involving preventive healthcare strategies.

Current scientific evidence supports a causal association between serum 25(OH)D levels and increased risk for CVD on the basis of Hill's criteria for causality in a biological system. Only RCTs starting with low serum 25(OH)D levels found significant beneficial effects of vitamin D supplementation in reducing risk factors associated with CVD. However, evidence to date suggests that raising serum $25(\mathrm{OH}) \mathrm{D}$ levels to at least $30 \mathrm{ng} / \mathrm{mL}$ will reduce the risk of CVD.

Whether it is ethical to design a study in which a group of people is deprived of a known essential nutrient to measure an endpoint should be carefully determined. Furthermore, waiting for completion of long-term RCTs to change treatment recommendations, especially when risks are minimal, may adversely affect the health of countless individuals. According to Hill [45]: "All scientific work is incomplete - whether it be observational or experimental. All scientific work is liable to be upset or modified by advancing knowledge. That does not confer upon us a freedom to ignore the knowledge we already have, or to postpone the action that it appears to demand at a given time."

\section{Acknowledgments}

The authors would like to acknowledge and thank Steven M. Paul, Principal Statistician, School of Nursing, UCSF, for his expert assistance with the interpretation of statistical analyses in the research studies we included in this review. 


\section{Author Contributions}

All of the authors contributed to the conception and design of this review as well as the analyses performed in order to determine if the criteria had been met. Patricia G. Weyland drafted the review with considerable assistance with the review of literature, organization and editing from Jill Howie-Esquivel and William B. Grant. All of the authors have approved all of the manuscript revisions as well as the final version prior to submission for publication.

\section{Conflicts of Interest}

The authors declare no conflicts of interest. Patricia G. Weyland receives funding from Bio-Tech Pharmacal (Fayetteville, AR, USA) and the Sunlight Research Forum (Veldhoven, The Netherlands).

\section{References}

1. Jones, D.S.; Podolsky, S.H.; Greene, J.A. The burden of disease and the changing task of medicine. N. Engl. J. Med. 2012, 366, 2333-2338.

2. Ford, E.S.; Ajani, U.A.; Croft, J.B.; Critchley, J.A.; Labarthe, D.R.; Kottke, T.E.; Giles, W.H.; Capewell, S. Explaining the decrease in U.S. deaths from coronary disease, 1980-2000. N. Engl. J. Med. 2007, 356, 2388-2398.

3. Olshansky, S.J.; Passaro, D.J.; Hershow, R.C.; Layden, J.; Carnes, B.A.; Brody, J.; Hayflick, L.; Butler, R.N.; Allison, D.B.; Ludwig, D.S. A potential decline in life expectancy in the United States in the 21st century. N. Engl. J. Med. 2005, 352, 1138-1145.

4. Scragg, R. Seasonality of cardiovascular disease mortality and the possible protective effect of ultra-violet radiation. Int. J. Epidemiol. 1981, 10, 337-341.

5. DeLuca, H.F. Overview of general physiologic features and functions of vitamin D. Am. J. Clin. Nutr. 2004, 80, 1689S-1696S.

6. Pludowski, P.; Grant, W.B.; Bhattoa, H.P.; Bayer, M.; Povoroznyuk, V.; Rudenka, E.; Ramanau, H.; Varbiro, S.; Rudenka, A.; Karczmarewicz, E.; et al. Vitamin D status in Central Europe. Int. J. Endocrinol. 2014, 2014, 589587, doi:10.1155/2014/589587.

7. Giovannucci, E.; Liu, Y.; Hollis, B.W.; Rimm, E.B. 25-Hydroxyvitamin D and risk of myocardial infarction in men: A prospective study. Arch. Intern. Med. 2008, 168, 1174-1180.

8. Anderson, J.L.; May, H.T.; Horne, B.D.; Bair, T.L.; Hall, N.L.; Carlquist, J.F.; Lappe, D.L.; Muhlestein, J.B. Relation of vitamin D deficiency to cardiovascular risk factors, disease status, and incident events in a general healthcare population. Am. J. Cardiol. 2010, 106, 963-968.

9. Zagura, M.; Serg, M.; Kampus, P.; Zilmer, M.; Eha, J.; Unt, E.; Lieberg, J.; Cockcroft, J.R.; Kals, J. Aortic stiffness and vitamin D are independent markers of aortic calcification in patients with peripheral arterial disease and in healthy subjects. Eur. J. Vasc. Endovasc. Surg. 2011, 42, 689-695.

10. Gaddipati, V.C.; Bailey, B.A.; Kuriacose, R.; Copeland, R.J.; Manning, T.; Peiris, A.N. The relationship of vitamin $\mathrm{D}$ status to cardiovascular risk factors and amputation risk in veterans with peripheral arterial disease. J. Am. Med. Dir. Assoc. 2011, 12, 58-61. 
11. Van de Luijtgaarden, K.M.; Voute, M.T.; Hoeks, S.E.; Bakker, E.J.; Chonchol, M.; Stolker, R.J.; Rouwet, E.V.; Verhagen, H.J. Vitamin D deficiency may be an independent risk factor for arterial disease. Eur. J. Vasc. Endovasc. Surg. 2012, 44, 301-306.

12. Webb, D.R.; Khunti, K.; Lacy, P.; Gray, L.J.; Mostafa, S.; Talbot, D.; Williams, B.; Davies, M.J. Conduit vessel stiffness in British South Asians of Indian descent relates to 25-hydroxyvitamin D status. J. Hypertens. 2012, 30, 1588-1596.

13. Pletcher, M.J.; Tice, J.A.; Pignone, M.; Browner, W.S. Using the coronary artery calcium score to predict coronary heart disease events: A systematic review and meta-analysis. Arch. Intern. Med. 2004, 164, 1285-1292.

14. Rezai, M.R.; Balakrishnan Nair, S.; Cowan, B.; Young, A.; Sattar, N.; Finn, J.D.; Wu, F.C.; Cruickshank, J.K. Low vitamin D levels are related to left ventricular concentric remodelling in men of different ethnic groups with varying cardiovascular risk. Int. J. Cardiol. 2012, 158, 444-447.

15. De Boer, I.H.; Kestenbaum, B.; Shoben, A.B.; Michos, E.D.; Sarnak, M.J.; Siscovick, D.S. 25-Hydroxyvitamin D levels inversely associate with risk for developing coronary artery calcification. J. Am. Soc. Nephrol. 2009, 20, 1805-1812.

16. Taylor, A.J.; Fiorilli, P.N.; Wu, H.; Bauer, K.; Bindeman, J.; Byrd, C.; Feuerstein, I.M.; O’Malley, P.G. Relation between the Framingham risk score, coronary calcium, and incident coronary heart disease among low-risk men. Am. J. Cardiol. 2010, 106, 47-50.

17. Oh, J.; Weng, S.; Felton, S.K.; Bhandare, S.; Riek, A.; Butler, B.; Proctor, B.M.; Petty, M.; Chen, Z.; Schechtman, K.B.; et al. 1,25(OH)2 vitamin D inhibits foam cell formation and suppresses macrophage cholesterol uptake in patients with type 2 diabetes mellitus. Circulation 2009, 120, 687-698.

18. Grant, W.B.; Mascitelli, L.; Goldstein, M.R. Comment on Ryan; et al. An investigation of association between chronic musculoskeletal pain and cardiovascular disease in the Health Survey for England (2008). Eur. J. Pain 2014, 18, 893-894.

19. Afzal, S.; Bojesen, S.E.; Nordestgaard, B.G. Low plasma 25-hydroxyvitamin D and risk of tobacco-related cancer. Clin. Chem. 2013, 59, 771-780.

20. Krause, R.; Buhring, M.; Hopfenmuller, W.; Holick, M.F.; Sharma, A.M. Ultraviolet B and blood pressure. Lancet 1998, 352, 709-710.

21. Forman, J.P.; Giovannucci, E.; Holmes, M.D.; Bischoff-Ferrari, H.A.; Tworoger, S.S.; Willett, W.C.; Curhan, G.C. Plasma 25-hydroxyvitamin D levels and risk of incident hypertension. Hypertension 2007, 49, 1063-1069.

22. Zhao, G.; Ford, E.S.; Li, C.; Kris-Etherton, P.M.; Etherton, T.D.; Balluz, L.S. Independent associations of serum concentrations of 25-hydroxyvitamin D and parathyroid hormone with blood pressure among US adults. J. Hypertens. 2010, 28, 1821-1828.

23. Goel, R.K.; Lal, H. Role of vitamin D supplementation in hypertension. Indian J. Clin. Biochem. 2011, 26, 88-90.

24. Gupta, A.K.; Brashear, M.M.; Johnson, W.D. Prediabetes and prehypertension in healthy adults are associated with low vitamin D levels. Diabetes Care 2011, 34, 658-660. 
25. Larsen, T.; Mose, F.H.; Bech, J.N.; Hansen, A.B.; Pedersen, E.B. Effect of cholecalciferol supplementation during winter months in patients with hypertension: A randomized, placebo-controlled trial. Am. J. Hypertens. 2012, 25, 1215-1222.

26. Schwartz, J.B. Effects of vitamin D supplementation in atorvastatin-treated patients: A new drug interaction with an unexpected consequence. Clin. Pharmacol. Ther. 2009, 85, 198-203.

27. De la Llera-Moya, M.; Drazul-Schrader, D.; Asztalos, B.F.; Cuchel, M.; Rader, D.J.; Rothblat, G.H. The ability to promote efflux via ABCA1 determines the capacity of serum specimens with similar high-density lipoprotein cholesterol to remove cholesterol from macrophages. Arterioscler. Thromb. Vasc. Biol. 2010, 30, 796-801.

28. Jorde, R.; Figenschau, Y.; Hutchinson, M.; Emaus, N.; Grimnes, G. High serum 25-hydroxyvitamin D concentrations are associated with a favorable serum lipid profile. Eur. J. Clin. Nutr. 2010, 64, 1457-1464.

29. Guasch, A.; Bullo, M.; Rabassa, A.; Bonada, A.; del Castillo, D.; Sabench, F.; Salas-Salvado, J. Plasma vitamin $\mathrm{D}$ and parathormone are associated with obesity and atherogenic dyslipidemia: A cross-sectional study. Cardiovasc. Diabetol. 2012, 11, 149.

30. Van Ballegooijen, A.J.; Snijder, M.B.; Visser, M.; van den Hurk, K.; Kamp, O.; Dekker, J.M.; Nijpels, G.; Stehouwer, C.D.; Henry, R.M.; Paulus, W.J.; et al. Vitamin D in relation to myocardial structure and function after eight years of follow-up: The Hoorn Study. Ann. Nutr. Metab. 2012, 60, 69-77.

31. Minino, A. Mortality among teenagers aged 12-19 years: United States, 1999-2006. NCHS Data Brief 2010, 37, 1-8.

32. Takeda, M.; Yamashita, T.; Sasaki, N.; Nakajima, K.; Kita, T.; Shinohara, M.; Ishida, T.; Hirata, K. Oral administration of an active form of vitamin $\mathrm{D}_{3}$ (calcitriol) decreases atherosclerosis in mice by inducing regulatory $\mathrm{T}$ cells and immature dendritic cells with tolerogenic functions. Arterioscler. Thromb. Vasc. Biol. 2010, 30, 2495-2503.

33. Bouillon, R.; van Schoor, N.M.; Gielen, E.; Boonen, S.; Mathieu, C.; Vanderschueren, D.; Lips, P. Optimal vitamin D status: A critical analysis on the basis of evidence-based medicine. J. Clin. Endocrinol. Metab. 2013, 98, E1283-E1304.

34. Grant, W.B. Using findings from observational studies to guide vitamin D randomized controlled trials. J. Intern. Med. 2014, 59, 1-4.

35. Holick, M.F.; Binkley, N.C.; Bischoff-Ferrari, H.A.; Gordon, C.M.; Hanley, D.A.; Heaney, R.P.; Murad, M.H.; Weaver, C.M. Evaluation, treatment, and prevention of vitamin D deficiency: An endocrine society clinical practice guideline. J. Clin. Endocrinol. Metab. 2011, 96, 1911-1930.

36. Holick, M.F.; Binkley, N.C.; Bischoff-Ferrari, H.A.; Gordon, C.M.; Hanley, D.A.; Heaney, R.P.; Murad, M.H.; Weaver, C.M. Guidelines for preventing and treating vitamin D deficiency and insufficiency revisited. J. Clin. Endocrinol. Metab. 2012, 97, 1153-1158.

37. Grant, W.B.; Boucher, B.J. Are Hill's criteria for causality satisfied for vitamin D and periodontal disease? Derm. Endocrinol. 2010, 2, 30-36.

38. Looker, A.C.; Johnson, C.L.; Lacher, D.A.; Pfeiffer, C.M.; Schleicher, R.L.; Sempos, C.T. Vitamin D status: United States, 2001-2006. NCHS Data Brief 2011, 59, 1-8.

39. Holick, M.F. Vitamin D deficiency. N. Engl. J. Med. 2007, 357, 266-281. 
40. Vieth, R. What is the optimal vitamin D status for health? Prog. Biophys. Mol. Biol. 2006, 92, 26-32.

41. Heaney, R.P.; Recker, R.R.; Grote, J.; Horst, R.L.; Armas, L.A. Vitamin $\mathrm{D}_{3}$ is more potent than vitamin $\mathrm{D}_{2}$ in humans. J. Clin. Endocrinol. Metab. 2011, 96, E447-E452.

42. Garland, C.F.; French, C.B.; Baggerly, L.L.; Heaney, R.P. Vitamin D supplement doses and serum 25-hydroxyvitamin D in the range associated with cancer prevention. Anticancer Res. 2011, 31, 607-611.

43. Grant, W.B. In defense of the sun: An estimate of changes in mortality rates in the United States if mean serum 25-hydroxyvitamin D levels were raised to $45 \mathrm{ng} / \mathrm{mL}$ by solar ultraviolet-B irradiance. Derm. Endocrinol. 2009, 1, 207-214.

44. Grant, W.B. An estimate of the global reduction in mortality rates through doubling vitamin D levels. Eur. J. Clin. Nutr. 2011, 65, 1016-1026.

45. Hill, A.B. The environment and disease: Association or causation? Proc. R. Soc. Med. 1965, $58,295-300$.

46. Grant, W.B. How strong is the evidence that solar ultraviolet B and vitamin D reduce the risk of cancer? An examination using Hill's criteria for causality. Derm. Endocrinol. 2009, 1, 14-21.

47. Hanwell, H.E.; Banwell, B. Assessment of evidence for a protective role of vitamin D in multiple sclerosis. Biochim. Biophys. Acta 2011, 1812, 202-212.

48. Mohr, S.B.; Gorham, E.D.; Alcaraz, J.E.; Kane, C.I.; Macera, C.A.; Parsons, J.K.; Wingard, D.L.; Garland, C.F. Does the evidence for an inverse relationship between serum vitamin D status and breast cancer risk satisfy the Hill criteria? Derm. Endocrinol. 2012, 4, 152-157.

49. Robsahm, T.E.; Schwartz, G.G.; Tretli, S. The inverse relationship between 25-hydroxyvitamin D and cancer survival: Discussion of causation. Cancers 2013, 5, 1439-1455.

50. Jang, H.B.; Lee, H.J.; Park, J.Y.; Kang, J.H.; Song, J. Association between serum vitamin D and metabolic risk factors in Korean schoolgirls. Osong Public Health Res. Perspect. 2013, 4, 179-186.

51. Correia, L.C.; Sodre, F.; Garcia, G.; Sabino, M.; Brito, M.; Kalil, F.; Barreto, B.; Lima, J.C.; Noya-Rabelo, M.M. Relation of severe deficiency of vitamin D to cardiovascular mortality during acute coronary syndromes. Am. J. Cardiol. 2013, 111, 324-327.

52. Deleskog, A.; Piksasova, O.; Silveira, A.; Samnegard, A.; Tornvall, P.; Eriksson, P.; Gustafsson, S.; Ostenson, C.G.; Ohrvik, J.; Hamsten, A. Serum 25-hydroxyvitamin D concentration, established and emerging cardiovascular risk factors and risk of myocardial infarction before the age of 60 years. Atherosclerosis 2012, 223, 223-229.

53. Deleskog, A.; Piksasova, O.; Silveira, A.; Gertow, K.; Baldassarre, D.; Veglia, F.; Sennblad, B.; Strawbridge, R.J.; Larsson, M.; Leander, K.; et al. Serum 25-hydroxyvitamin D concentration in subclinical carotid atherosclerosis. Arterioscler. Thromb. Vasc. Biol. 2013, 33, 2633-2638.

54. Parker, J.; Hashmi, O.; Dutton, D.; Mavrodaris, A.; Stranges, S.; Kandala, N.B.; Clarke, A.; Franco, O.H. Levels of vitamin D and cardiometabolic disorders: Systematic review and meta-analysis. Maturitas 2010, 65, 225-236. 
55. Wang, L.; Song, Y.; Manson, J.E.; Pilz, S.; Marz, W.; Michaelsson, K.; Lundqvist, A.; Jassal, S.K.; Barrett-Connor, E.; Zhang, C.; et al. Circulating 25-hydroxy-vitamin D and risk of cardiovascular disease: A meta-analysis of prospective studies. Circ. Cardiovasc. Qual. Outcomes 2012, 5, 819-829.

56. Kendrick, J.; Targher, G.; Smits, G.; Chonchol, M. 25-Hydroxyvitamin D deficiency is independently associated with cardiovascular disease in the Third National Health and Nutrition Examination Survey. Atherosclerosis 2009, 205, 255-260.

57. Song, Y.; Wang, L.; Pittas, A.G.; del Gobbo, L.C.; Zhang, C.; Manson, J.E.; Hu, F.B. Blood 25-hydroxy vitamin D levels and incident type 2 diabetes: A meta-analysis of prospective studies. Diabetes Care 2013, 36, 1422-1428.

58. Tsur, A.; Feldman, B.S.; Feldhammer, I.; Hoshen, M.B.; Leibowitz, G.; Balicer, R.D. Decreased serum concentrations of 25-hydroxycholecalciferol are associated with increased risk of progression to impaired fasting glucose and diabetes. Diabetes Care 2013, 36, 1361-1367.

59. Vacek, J.L.; Vanga, S.R.; Good, M.; Lai, S.M.; Lakkireddy, D.; Howard, P.A. Vitamin D deficiency and supplementation and relation to cardiovascular health. Am. J. Cardiol. 2012, 109, 359-363.

60. Wang, T.J.; Pencina, M.J.; Booth, S.L.; Jacques, P.F.; Ingelsson, E.; Lanier, K.; Benjamin, E.J.; D’Agostino, R.B.; Wolf, M.; Vasan, R.S. Vitamin D deficiency and risk of cardiovascular disease. Circulation 2008, 117, 503-511.

61. Carrara, D.; Bernini, M.; Bacca, A.; Rugani, I.; Duranti, E.; Virdis, A.; Ghiadoni, L.; Taddei, S.; Bernini, G. Cholecalciferol administration blunts the systemic renin-angiotensin system in essential hypertensives with hypovitaminosis D. J. Renin Angiotensin Aldosterone Syst. 2014, $15,82-87$.

62. Forman, J.P.; Curhan, G.C.; Taylor, E.N. Plasma 25-hydroxyvitamin D levels and risk of incident hypertension among young women. Hypertension 2008, 52, 828-832.

63. Giallauria, F.; Milaneschi, Y.; Tanaka, T.; Maggio, M.; Canepa, M.; Elango, P.; Vigorito, C.; Lakatta, E.G.; Ferrucci, L.; Strait, J. Arterial stiffness and vitamin D levels: The Baltimore Longitudinal Study of Aging. J. Clin. Endocrinol. Metab. 2012, 97, 3717-3723.

64. Mayer, O., Jr.; Filipovsky, J.; Seidlerova, J.; Vanek, J.; Dolejsova, M.; Vrzalova, J.; Cifkova, R. The association between low 25-hydroxyvitamin D and increased aortic stiffness. J. Hum. Hypertens. 2012, 26, 650-655.

65. Pirro, M.; Manfredelli, M.R.; Helou, R.S.; Scarponi, A.M.; Schillaci, G.; Bagaglia, F.; Melis, F.; Mannarino, E. Association of parathyroid hormone and 25-OH-vitamin D levels with arterial stiffness in postmenopausal women with vitamin D insufficiency. J. Atheroscler. Thromb. 2012, 19, 924-931.

66. Robinson-Cohen, C.; Hoofnagle, A.N.; Ix, J.H.; Sachs, M.C.; Tracy, R.P.; Siscovick, D.S.; Kestenbaum, B.R.; de Boer, I.H. Racial differences in the association of serum 25-hydroxyvitamin D concentration with coronary heart disease events. JAMA 2013, 310, 179-188. 
67. Forouhi, N.G.; Luan, J.; Cooper, A.; Boucher, B.J.; Wareham, N.J. Baseline serum 25-hydroxyvitamin D is predictive of future glycemic status and insulin resistance: The Medical Research Council Ely Prospective Study 1990-2000. Diabetes 2008, 57, 2619-2625.

68. Breslavsky, A.; Frand, J.; Matas, Z.; Boaz, M.; Barnea, Z.; Shargorodsky, M. Effect of high doses of vitamin D on arterial properties, adiponectin, leptin and glucose homeostasis in type 2 diabetic patients. Clin. Nutr. 2013, 32, 970-975.

69. Grant, W.B.; Garland, C.F. Vitamin D has a greater impact on cancer mortality rates than on cancer incidence rates. Br. Med. J. 2014, 348, g2862, doi:http://dx.doi.org/10.1136/bmj.g2862.

70. Grant, W.B. Primary malignancy in patients with nonmelanoma skin cancer-letter. Cancer Epidemiol. Biomark. Prev. 2014, 23, 1438, doi:10.1158/1055-9965.

71. Savic, L.; Mrdovic, I.; Perunicic, J.; Asanin, M.; Lasica, R.; Marinkovic, J.; Vasiljevic, Z.; Ostojic, M. Impact of the combined left ventricular systolic and renal dysfunction on one-year outcomes after primary percutaneous coronary intervention. J. Interv. Cardiol. 2012, 25, 132-139.

72. Brenner, D.R.; Arora, P.; Garcia-Bailo, B.; Wolever, T.M.; Morrison, H.; El-Sohemy, A.; Karmali, M.; Badawi, A. Plasma vitamin D levels and risk of metabolic syndrome in Canadians. Clin. Investig. Med. 2011, 34, E377.

73. Boucher, B.J. Is vitamin D status relevant to metabolic syndrome? Derm. Endocrinol. 2012, 4, 212-224.

74. Gagnon, C.; Lu, Z.X.; Magliano, D.J.; Dunstan, D.W.; Shaw, J.E.; Zimmet, P.Z.; Sikaris, K.; Ebeling, P.R.; Daly, R.M. Low serum 25-hydroxyvitamin D is associated with increased risk of the development of the metabolic syndrome at five years: Results from a national, population-based prospective study (The Australian Diabetes, Obesity and Lifestyle Study: Ausdiab). J. Clin. Endocrinol. Metab. 2012, 97, 1953-1961.

75. Makariou, S.; Liberopoulos, E.; Florentin, M.; Lagos, K.; Gazi, I.; Challa, A.; Elisaf, M. The relationship of vitamin $\mathrm{D}$ with non-traditional risk factors for cardiovascular disease in subjects with metabolic syndrome. Arch. Med. Sci. 2012, 8, 437-443.

76. De Boer, I.H.; Katz, R.; Chonchol, M.; Ix, J.H.; Sarnak, M.J.; Shlipak, M.G.; Siscovick, D.S.; Kestenbaum, B. Serum 25-hydroxyvitamin D and change in estimated glomerular filtration rate. Clin. J. Am. Soc. Nephrol. 2011, 6, 2141-2149.

77. Schnatz, P.F.; Jiang, X.; Vila-Wright, S.; Aragaki, A.K.; Nudy, M.; O’Sullivan, D.M.; Jackson, R.; Leblanc, E.; Robinson, J.G.; Shikany, J.M.; et al. Calcium/vitamin D supplementation, serum 25-hydroxyvitamin D concentrations, and cholesterol profiles in the Women's Health Initiative calcium/vitamin D randomized trial. Menopause 2014, 8, 823-833.

78. Bolland, M.J.; Grey, A.; Gamble, G.D.; Reid, I.R. The effect of vitamin D supplementation on skeletal, vascular, or cancer outcomes: A trial sequential meta-analysis. Lancet Diabetes Endocrinol. 2014, 2, 307-320.

79. Forman, J.P.; Scott, J.B.; Ng, K.; Drake, B.F.; Suarez, E.G.; Hayden, D.L.; Bennett, G.G.; Chandler, P.D.; Hollis, B.W.; Emmons, K.M.; et al. Effect of vitamin D supplementation on blood pressure in blacks. Hypertension 2013, 61, 779-785. 
80. George, P.S.; Pearson, E.R.; Witham, M.D. Effect of vitamin D supplementation on glycaemic control and insulin resistance: A systematic review and meta-analysis. Diabet. Med. 2012, 29, e142-e150.

81. Talaei, A.; Mohamadi, M.; Adgi, Z. The effect of vitamin D on insulin resistance in patients with type 2 diabetes. Diabetol. Metab. Syndr. 2013, 5, 8, doi:10.1186/1758-5996-5-8.

82. Michos, E.D.; Reis, J.P.; Post, W.S.; Lutsey, P.L.; Gottesman, R.F.; Mosley, T.H.; Sharrett, A.R.; Melamed, M.L. 25-Hydroxyvitamin D deficiency is associated with fatal stroke among whites but not blacks: The NHANES-III linked mortality files. Nutrition 2012, 28, 367-371.

83. Wood, A.D.; Secombes, K.R.; Thies, F.; Aucott, L.; Black, A.J.; Mavroeidi, A.; Simpson, W.G.; Fraser, W.D.; Reid, D.M.; Macdonald, H.M. Vitamin D3 supplementation has no effect on conventional cardiovascular risk factors: A parallel-group, double-blind, placebo-controlled RCT. J. Clin. Endocrinol. Metab. 2012, 97, 3557-3568.

84. Jorde, R.; Strand Hutchinson, M.; Kjaergaard, M.; Sneve, M.; Grimnes, G. Supplementation with high doses of vitamin D to subjects without vitamin D deficiency may have negative effects: Pooled data from four intervention trials in Tromsø. ISRN Endocrinol. 2013, 2013, 348705, doi:10.1155/2013/348705.

85. Liu, S.; Song, Y.; Ford, E.S.; Manson, J.E.; Buring, J.E.; Ridker, P.M. Dietary calcium, vitamin $\mathrm{D}$, and the prevalence of metabolic syndrome in middle-aged and older U.S. women. Diabetes Care 2005, 28, 2926-2932.

86. Fung, G.J.; Steffen, L.M.; Zhou, X.; Harnack, L.; Tang, W.; Lutsey, P.L.; Loria, C.M.; Reis, J.P.; van Horn, L.V. Vitamin D intake is inversely related to risk of developing metabolic syndrome in African American and white men and women over $20 \mathrm{y}$ : The Coronary Artery Risk Development in Young Adults study. Am. J. Clin. Nutr. 2012, 96, $24-29$.

87. Moukayed, M.; Grant, W.B. Molecular link between vitamin D and cancer prevention. Nutrients 2013, 5, 3993-4021.

88. Oplander, C.; Volkmar, C.M.; Paunel-Gorgulu, A.; van Faassen, E.E.; Heiss, C.; Kelm, M.; Halmer, D.; Murtz, M.; Pallua, N.; Suschek, C.V. Whole body UVA irradiation lowers systemic blood pressure by release of nitric oxide from intracutaneous photolabile nitric oxide derivates. Circ. Res. 2009, 105, 1031-1040.

89. Andrukhova, O.; Slavic, S.; Zeitz, U.; Riesen, S.C.; Heppelmann, M.S.; Ambrisko, T.D.; Markovic, M.; Kuebler, W.M.; Erben, R.G. Vitamin D is a regulator of endothelial nitric oxide synthase and arterial stiffness in mice. Mol. Endocrinol. 2014, 28, 53-64.

90. Jamaluddin, S.; Liang, Z.; Lu, J.M.; Yao, Q.; Chen, C. Roles of cardiovascular risk factors in endothelial nitric oxide synthase regulation: An update. Curr. Pharm. Des. 2013, 22, 3563-3578.

91. Beveridge, L.A.; Witham, M.D. Vitamin D and the cardiovascular system. Osteoporos. Int. 2013, 24, 2167-2180.

92. Zittermann, A.; Iodice, S.; Pilz, S.; Grant, W.B.; Bagnardi, V.; Gandini, S. Vitamin D deficiency and mortality risk in the general population: A meta-analysis of prospective cohort studies. Am. J. Clin. Nutr. 2012, 95, 91-100. 
93. Liberopoulos, E.N.; Makariou, S.E.; Moutzouri, E.; Kostapanos, M.S.; Challa, A.; Elisaf, M. Effect of simvastatin/ezetimibe $10 / 10 \mathrm{mg}$ versus simvastatin $40 \mathrm{mg}$ on serum vitamin D levels. J. Cardiovasc. Pharmacol. Ther. 2013, 18, 229-233.

94. Woodhouse, P.R.; Khaw, K.T.; Plummer, M. Seasonal variation of blood pressure and its relationship to ambient temperature in an elderly population. J. Hypertens. 1993, 11, 1267-1274.

95. Burkart, K.; Schneider, A.; Breitner, S.; Khan, M.H.; Kramer, A.; Endlicher, W. The effect of atmospheric thermal conditions and urban thermal pollution on all-cause and cardiovascular mortality in Bangladesh. Environ. Pollut. 2011, 159, 2035-2043.

96. Brandenburg, V.M.; Vervloet, M.G.; Marx, N. The role of vitamin D in cardiovascular disease: From present evidence to future perspectives. Atherosclerosis 2012, 225, 253-263.

97. Hathcock, J.N.; Shao, A.; Vieth, R.; Heaney, R. Risk assessment for vitamin D. Am. J. Clin. Nutr. 2007, 85, 6-18.

98. Gradinaru, D.; Borsa, C.; Ionescu, C.; Margina, D.; Prada, G.I.; Jansen, E. Vitamin D status and oxidative stress markers in the elderly with impaired fasting glucose and type 2 diabetes mellitus. Aging Clin. Exp. Res. 2012, 24, 595-602.

99. Alkharfy, K.M.; Al-Daghri, N.M.; Sabico, S.B.; Al-Othman, A.; Moharram, O.; Alokail, M.S.; Al-Saleh, Y.; Kumar, S.; Chrousos, G.P. Vitamin D supplementation in patients with diabetes mellitus type 2 on different therapeutic regimens: A one-year prospective study. Cardiovasc. Diabetol. 2013, 12, 113, doi:10.1186/1475-2840-12-113.

100. Wilmot, E.G.; Edwardson, C.L.; Biddle, S.J.; Gorely, T.; Henson, J.; Khunti, K.; Nimmo, M.A.; Yates, T.; Davies, M.J. Prevalence of diabetes and impaired glucose metabolism in younger "at risk" UK adults: Insights from the STAND programme of research. Diabet. Med. 2013, 30, 671-675.

101. Yiu, Y.F.; Yiu, K.H.; Siu, C.W.; Chan, Y.H.; Li, S.W.; Wong, L.Y.; Lee, S.W.; Tam, S.; Wong, E.W.; Lau, C.P.; et al. Randomized controlled trial of vitamin D supplement on endothelial function in patients with type 2 diabetes. Atherosclerosis 2013, 227, 140-146.

102. DeKeyser, F.G.; Pugh, L.C. Assessment of the reliability and validity of biochemical measures. Nurs. Res. 1990, 39, 314-317.

103. Lappe, J.M.; Heaney, R.P. Why randomized controlled trials of calcium and vitamin D sometimes fail. Derm. Endocrinol. 2012, 4, 95-100.

104. Gupta, A.K.; Brashear, M.M.; Johnson, W.D. Predisease conditions and serum vitamin D levels in healthy Mexican American adults. Postgrad. Med. 2012, 124, 136-142.

105. Harris, S.S. Vitamin D and African Americans. J. Nutr. 2006, 136, 1126-1129.

106. Riek, A.E.; Oh, J.; Darwech, I.; Moynihan, C.E.; Bruchas, R.R.; Bernal-Mizrachi, C. 25(OH) vitamin D suppresses macrophage adhesion and migration by downregulation of ER stress and scavenger receptor A1 in type 2 diabetes. J. Steroid Biochem. Mol. Biol. 2013, 144, 172-179, doi:10.1016/j.jsbmb.2013.10.016.

107. Wu-Wong, J.R.; Nakane, M.; Ma, J.; Ruan, X.; Kroeger, P.E. VDR-mediated gene expression patterns in resting human coronary artery smooth muscle cells. J. Cell. Biochem. 2007, 100, $1395-1405$. 
108. Yusuf, S.; Hawken, S.; Ounpuu, S.; Dans, T.; Avezum, A.; Lanas, F.; McQueen, M.; Budaj, A.; Pais, P.; Varigos, J.; et al. Effect of potentially modifiable risk factors associated with myocardial infarction in 52 countries (the INTERHEART study): Case-control study. Lancet 2004, 364, 937-952.

109. Karhapaa, P.; Pihlajamaki, J.; Porsti, I.; Kastarinen, M.; Mustonen, J.; Niemela, O.; Kuusisto, J. Diverse associations of 25-hydroxyvitamin D and 1,25-dihydroxy-vitamin D with dyslipidaemias. J. Intern. Med. 2010, 268, 604-610.

110. Chow, E.C.; Magomedova, L.; Quach, H.P.; Patel, R.; Durk, M.R.; Fan, J.; Maeng, H.J.; Irondi, K.; Anakk, S.; Moore, D.D.; et al. Vitamin D receptor activation down-regulates the small heterodimer partner and increases CYP7A1 to lower cholesterol. Gastroenterology 2014, $146,1048-1059$.

111. Moser, U. Vitamins - Wrong approaches. Int. J. Vitam. Nutr. Res. 2012, 82, 327-332.

112. Heaney, R.P. Guidelines for optimizing design and analysis of clinical studies of nutrient effects. Nutr. Rev. 2014, 72, 48-54.

113. Rahimi-Ardabili, H.; Pourghassem Gargari, B.; Farzadi, L. Effects of vitamin D on cardiovascular disease risk factors in polycystic ovary syndrome women with vitamin D deficiency. J. Endocrinol. Investig. 2013, 36, 28-32.

114. Grimnes, G.; Figenschau, Y.; Almas, B.; Jorde, R. Vitamin D, insulin secretion, sensitivity, and lipids: Results from a case-control study and a randomized controlled trial using hyperglycemic clamp technique. Diabetes 2011, 60, 2748-2757.

115. Von Hurst, P.R.; Stonehouse, W.; Coad, J. Vitamin D supplementation reduces insulin resistance in South Asian women living in New Zealand who are insulin resistant and vitamin D deficient-A randomised, placebo-controlled trial. Br. J. Nutr. 2010, 103, 549-555.

116. Belenchia, A.M.; Tosh, A.K.; Hillman, L.S.; Peterson, C.A. Correcting vitamin D insufficiency improves insulin sensitivity in obese adolescents: A randomized controlled trial. Am. J. Clin. Nutr. 2013, 97, 774-781.

117. Beilfuss, J.; Berg, V.; Sneve, M.; Jorde, R.; Kamycheva, E. Effects of a 1-year supplementation with cholecalciferol on interleukin-6, tumor necrosis factor-alpha and insulin resistance in overweight and obese subjects. Cytokine 2012, 60, 870-874.

118. Gepner, A.D.; Ramamurthy, R.; Krueger, D.C.; Korcarz, C.E.; Binkley, N.; Stein, J.H. A prospective randomized controlled trial of the effects of vitamin D supplementation on cardiovascular disease risk. PLoS One 2012, 7, e36617, doi:10.1371/journal.pone.0036617.

119. Potischman, N.; Weed, D.L. Causal criteria in nutritional epidemiology. Am. J. Clin. Nutr. 1999, 69, 1309S-1314S.

120. Biesalski, H.K.; Aggett, P.J.; Anton, R.; Bernstein, P.S.; Blumberg, J.; Heaney, R.P.; Henry, J.; Nolan, J.M.; Richardson, D.P.; van Ommen, B.; et al. 26th Hohenheim Consensus Conference, September 11, 2010 Scientific substantiation of health claims: Evidence-based nutrition. Nutrition 2011, 27, S1-S20. 


\title{
Vitamin D Levels Are Associated with Cardiac Autonomic Activity in Healthy Humans
}

\author{
Michelle C. Mann, Derek V. Exner, Brenda R. Hemmelgarn, Darlene Y. Sola, Tanvir C. Turin, \\ Linda Ellis and Sofia B. Ahmed
}

\begin{abstract}
Vitamin D deficiency ( $\leq 50 \mathrm{nmol} / \mathrm{L} 25$-hydroxy vitamin D) is a cardiovascular $(\mathrm{CV})$ risk factor that affects approximately one billion people worldwide, particularly those affected by chronic kidney disease (CKD). Individuals with CKD demonstrate abnormal cardiac autonomic nervous system activity, which has been linked to the significant rates of CV-related mortality in this population. Whether vitamin $\mathrm{D}$ deficiency has a direct association with regulation of cardiac autonomic activity has never been explored in humans. Methods: Thirty-four (34) healthy, normotensive subjects were studied and categorized based on 25-hydroxy vitamin D deficiency (deficient $v s$. non-deficient, $n=7 v$ s. 27), as well as 1,25-dihydroxy vitamin D levels (above vs. below 25 th percentile, $n=8 v$ s. 26). Power spectral analysis of electrocardiogram recordings provided measures of cardiac autonomic activity across low frequency (LF) and high frequency (HF, representative of vagal contribution) bands, representative of the sympathetic and vagal limbs of the autonomic nervous system when transformed to normalized units (nu), respectively, as well as overall cardiosympathovagal balance (LF:HF) during graded angiotensin II (AngII) challenge ( $3 \mathrm{ng} / \mathrm{kg} / \mathrm{min} \times 30 \mathrm{~min}, 6 \mathrm{ng} / \mathrm{kg} / \mathrm{min} \times 30 \mathrm{~min}$ ). Results: At baseline, significant suppression of sympathovagal balance was observed in the 25-hydroxy vitamin D-deficient participants (LF:HF, $p=0.02$ vs. non-deficient), although no other differences were observed throughout AngII challenge. Participants in the lowest 1,25-dihydroxy VD quartile experienced significant withdrawal of inhibitory vagal control, as well as altered overall sympathovagal balance throughout AngII challenge (HF, mean difference $=-6.98 \pm 3 \mathrm{nu}, p=0.05$; $\mathrm{LF}: \mathrm{HF}$, mean difference $=0.34 \pm 0.1, p=0.043 v$ s. above 25 th percentile). Conclusions: Vitamin D deficiency is associated with suppression of resting cardiac autonomic activity, while low 1,25-dihydroxy vitamin D levels are associated with unfavourable cardiac autonomic activity during an acute AngII stressor, offering a potential pathophysiological mechanism that may be acting to elevate $\mathrm{CV}$ risk in in populations with low vitamin D status.
\end{abstract}

Reprinted from Nutrients. Cite as: Mann, M.C.; Exner, D.V.; Hemmelgarn, B.R.; Sola, D.Y.; Turin, T.C.; Ellis, L.; Ahmed, S.B. Vitamin D Levels Are Associated with Cardiac Autonomic Activity in Healthy Humans. Nutrients 2013, 5, 2114-2127.

\section{Introduction}

Vitamin D deficiency is influenced by dietary habits, body composition, ethnicity, as well as sun exposure relative to geographical location and altitude, and has been shown to be associated with poor cardiovascular (CV) outcomes and risk [1-3]. Vitamin D deficiency has been shown to alter activity of the cardiovascular system and related pressor responses [4] and dysfunction of the cardiac autonomic nervous system (ANS) is predictive of CV risk [5-11], specifically risk of sudden cardiac death $(\mathrm{SCD})[8,10]$, in humans. These observations suggest a potential link between low vitamin D 
and unfavourable alterations in cardiac autonomic tone. The cardiac sympathetic and parasympathetic (vagal) limbs of the ANS act in concert with one another to stimulate and inhibit heart rate and contractility, respectively. Though the association between vitamin D and cardiac autonomic tone has not specifically previously been studied in humans, patients with impaired vitamin D synthesis such as those with chronic kidney disease (CKD) demonstrate unfavourable cardiosympathovagal activity characterized by a significant withdrawal of inhibitory vagal activity [5]. Together, these observations suggest a potential link between vitamin D and cardiac autonomic nervous system (ANS) control and therefore CV risk; though whether vitamin D deficiency has a direct influence on cardiac autonomic activity remains unknown in humans. As such, we sought to evaluate the impact of low serum levels of vitamin D metabolites: (a) vitamin D deficiency, defined as $<50 \mathrm{nmol} / \mathrm{L}$ (or $20 \mathrm{ng} / \mathrm{mL}: 1 \mathrm{nmol} / \mathrm{L}=0.4 \mathrm{ng} / \mathrm{mL}$ ) 25-hydroxy vitamin D [12]; and (b) low 1,25-dihydroxy vitamin D, defined as within the lowest quartile of 1,25-dihydroxy vitamin D [2] on cardiac autonomic activity in healthy humans.

\section{Methods}

Thirty-four (34) healthy, normotensive volunteer subjects underwent graded angiotensin II (AngII) challenge $(3 \mathrm{ng} / \mathrm{kg} / \mathrm{min} \times 30 \mathrm{~min}, 6 \mathrm{ng} / \mathrm{kg} / \mathrm{min} \times 30 \mathrm{~min})$. This challenge involved infusion of exogenous AngII, the effector hormone of the renin-angiotensin system (RAS) within the kidney which induces a slight increase in blood pressure (BP) and thus acts as an acute physiological stressor in healthy subjects $[13,14]$. This study was approved by the Conjoint Health Research Ethics Board (CHREB) at the University of Calgary (project ID \#22859) and was conducted in accordance with the principles outlined in the Declaration of Helsinki.

Subjects were recruited year-round via posters on the University of Calgary Health Sciences and Main campuses. Exclusion criteria were hypertension (BP $>140 / 90$ or the use of antihypertensive medications), diabetes (fasting glucose $\geq 7 \mathrm{mmol} / \mathrm{L}$ or the use of hypoglycemic medications), smoking and use of any regular prescription medications. Following collection of verbal and written informed consent, subjects consumed $>150 \mathrm{mmol} \mathrm{Na}^{+} /$day for three days prior to the study to ensure maximal RAS suppression [15]. All women were studied mid-menstrual cycle, confirmed by counting days from the last menstrual period and measurement of sex hormone levels. All studies were conducted at the University of Calgary Human Physiology Laboratory and commenced at 08:00 with fasting subjects lying supine in a quiet, temperature-controlled room. An 18-gauge peripheral venous cannula was then inserted into each antecubital vein for blood sampling and infusions. BP was monitored throughout the study by an automated sphygmomanometer cuff (GE Healthcare, Dinamap) at 15 min intervals. Following a 90-min equilibration period, subjects underwent graded AngII infusion.

\subsection{Cardiac Autonomic Activity}

Electrocardiogram (ECG) data were collected during spontaneous, natural breathing with a 3-lead ambulatory Holter monitor (GE Healthcare; Milwaukee, WI, USA) at a sampling frequency of $125 \mathrm{~Hz}$. ECG data was collected continuously throughout the duration of the $180 \mathrm{~min}$ study period (baseline $\times 90 \mathrm{~min}, 3 \mathrm{ng} / \mathrm{kg} / \mathrm{min}$ AngII $\times 30 \mathrm{~min}, 6 \mathrm{ng} / \mathrm{kg} / \mathrm{min}$ AngII $\times 30 \mathrm{~min}$ ). ECG tracings were 
screened by a qualified technician for artefacts and irregular QRS complexes in order to exclude any abnormal rhythms. Power spectral density analysis was then calculated to include rhythms with activity within the bandwidth frequency of 0.003-1.7 Hz (MARS v. 7; GE Healthcare; Milwaukee, WI, USA). Absolute values of cardiac autonomic activity (in milliseconds) in the total power (TP), very-low frequency (VLF, 0.003-0.04 Hz), low-frequency (LF, 0.04-0.15 Hz) and high-frequency (HF, 0.15-0.4 Hz) bands were recorded. Both absolute LF and HF parameters were then squared and log-transformed $\left(\ln \mathrm{ms}^{2}\right)$, as well as converted to normalized units (nu) to account for changes in total autonomic power [16]. Overall cardiosympathovagal balance (LF:HF) was derived automatically within the MARS software by comparing the LF/LF + HF and HF/HF + LF ratios.

While some methodological variation exists within the literature [17], it is widely accepted that power spectral density analysis measurements within the HF frequency bandwidth represents independent contribution from the vagal limb of the cardiac autonomic nervous system $[5,16]$. While some controversy exists with regards to the remaining frequency domain measures, it has been speculated that activity within the VLF frequency bandwidth may represent contribution influenced by both the RAS [18], and more recently, the vagal limb of the cardiac ANS [19]. Previously, the LF bandwidth has been attributed to the sympathetic limb of the cardiac ANS [16], however this view has been challenged and interpreted as being largely influenced by vagal outflow generated by the baroreceptor reflex [20]. The LF:HF measure is thought to be an overall estimate of the relative balance of autonomic activity within the entire cardiac ANS [16].

\subsection{Biochemical Analysis}

Serum 25-hydroxy vitamin D and parathyroid hormone (PTH) were quantified using chemiluminescence immunoassay techniques (Liaison 25-hydroxyVitamin D Total Assay, Liaison N-TACT PTH, DiaSorin Clinical Assays; Stillwater, MN, USA). Serum 1,25-dihydroxy vitamin D level was determined by a two-step assay involving extraction of vitamin D metabolites followed by competitive radioimmunoassay (1,25-dihydroxy vitamin D 125I RIA kit, DiaSorin Clinical Assays; Stillwater, MN, USA). Plasma renin activity (PRA) was determined by quantification of plasma angiotensin I, the primary product of renin activation, using radioimmunoassay (Plasma Renin Activity 125I, DiaSorin Clinical Assays; Stillwater, MN, USA). AngII plasma levels were measured by standard immunoassay techniques (Quest Diagnostics; San Juan Capistrano, CA, USA). Serum aldosterone levels were determined by radioimmunoassay (Aldosterone Coat a Count Kit, Intermedico; Markham, Ontario, Canada). Serum creatinine and cholesterol were quantified by enzymatic colorimetric assay (Roche/Hitachi Creatinine Plus, CHOD-PAP, HDL-C Plus, and Triglycerides GPO-PAP Kits, Roche Diagnostics; Indianapolis, IN, USA).

\subsection{Statistical Analysis}

Data are expressed as mean \pm SE. The primary outcome was the association between 25 -hydroxy vitamin D status (deficient vs. non-deficient) and cardiac autonomic activity differences at baseline and in response to AngII challenge. The secondary outcome was the association between active 1,25-dihydroxy vitamin D status (below 25 th percentile $v s$. above 25 th percentile) [2] and changes 
in cardiac autonomic activity at baseline and in response to AngII challenge. Spearman's correlational analyses were employed to evaluate the various relationships between relevant variables and outcomes. Differences between the vitamin D groups at specific time points were tested using non-parametric Mann-Whitney U. Linear trends for responses over time were calculated using the non-parametric one-way analysis of variance (Kruskal-Wallis). Within-subject and between-group responses were also analyzed with two-way repeated measures analysis of variance (ANOVA) to account for both the dose of AngII infusion and vitamin D group allocation, with race and gender [21] included as covariates within the model and multiple comparison corrections used as appropriate. All test assumptions were tested and verified before conducting analyses utilizing SPSS (IBM; v. 19) with a significance level of $\alpha \leq 0.05$.

\section{Results}

\subsection{Subject Characteristics}

Subject characteristics are described in Table 1. All subjects were normotensive irrespective of 25-hydroxy or 1,25-dihydroxy vitamin D serum concentration, though vitamin D deficient subjects demonstrated greater parathyroid hormone levels $(p=0.046)$ and lower epinephrine levels $(p=0.019)$. There was a trend towards lower self-reported Caucasian race and lower 1,25-dihydroxy vitamin D levels in the Vitamin D deficient group but this did not achieve statistical significance. When subjects were stratified by 1,25-dihydroxy vitamin D status, similar findings were observed.. No association between 25-hydroxy vitamin D and 1,25-dihydroxy vitamin $\mathrm{D}$ was observed $(r=0.061, p=0.73)$.

Table 1. Baseline characteristics.

\begin{tabular}{|c|c|c|c|c|c|c|c|}
\hline & \multirow[b]{2}{*}{$\begin{array}{l}\text { All Subjects } \\
\quad(n=34)\end{array}$} & \multicolumn{3}{|c|}{$\begin{array}{c}\text { Stratification by } \\
25 \text {-hydroxy } \\
\text { vitamin D status }\end{array}$} & \multicolumn{3}{|c|}{$\begin{array}{l}\text { Stratification by } \\
1,25 \text {-dihydroxy } \\
\text { vitamin D status }\end{array}$} \\
\hline & & $\begin{array}{c}\text { Deficient } \\
<50 \mathrm{nmol} / \mathrm{L} \\
\quad(n=7)\end{array}$ & $\begin{array}{l}\text { Non-Deficient } \\
\begin{array}{c}>50 \mathrm{nmol} / \mathrm{L} \\
(n=27)\end{array}\end{array}$ & $p$-value & $\begin{array}{l}\text { Below 25th } \\
\text { percentile } \\
<76 \mathrm{pmol} / \mathrm{L} \\
(n=8)\end{array}$ & $\begin{array}{l}\text { Above 25th } \\
\text { percentile } \\
>76 \mathrm{pmol} / \mathrm{L} \\
(n=26)\end{array}$ & $p$-value \\
\hline Age (years) & $38 \pm 2$ & $37 \pm 5$ & $38 \pm 3$ & 0.96 & $37 \pm 4$ & $38 \pm 3$ & 0.92 \\
\hline Caucasian (\%) & $25(74 \%)$ & $4(57 \%)$ & $21(77 \%)$ & 0.08 & $7(88 \%)$ & $18(69 \%)$ & 0.56 \\
\hline Female (\%) & $22(65 \%)$ & $5(71 \%)$ & $17(63 \%)$ & 0.69 & $6(75 \%)$ & $16(62 \%)$ & 0.22 \\
\hline BMI $\left(\mathrm{kg} / \mathrm{m}^{2}\right)$ & $26 \pm 1$ & $27 \pm 1$ & $25 \pm 1$ & 0.43 & $27 \pm 2$ & $25 \pm 1$ & 0.21 \\
\hline SBP (mmHg) & $115 \pm 2$ & $112 \pm 4$ & $115 \pm 3$ & 0.63 & $115 \pm 2$ & $115 \pm 3$ & 0.37 \\
\hline DBP (mmHg) & $68 \pm 1$ & $64 \pm 5$ & $69 \pm 2$ & 0.28 & $70 \pm 3$ & $68 \pm 2$ & 0.44 \\
\hline MAP (mmHg) & $84 \pm 2$ & $80 \pm 4$ & $84 \pm 2$ & 0.37 & $85 \pm 2$ & $83 \pm 2$ & 0.41 \\
\hline $\begin{array}{c}\text { 25-hydroxy } \\
\text { vitamin } \mathrm{D}(\mathrm{nmol} / \mathrm{L})\end{array}$ & $71 \pm 4$ & $38 \pm 2$ & $81 \pm 4$ & $<0.001$ & $61 \pm 7$ & $74 \pm 5$ & 0.16 \\
\hline $\begin{array}{c}1,25 \text {-dihydroxy } \\
\text { vitamin D (pmol/L) }\end{array}$ & $105 \pm 6$ & $86 \pm 9$ & $107 \pm 7$ & 0.15 & $64 \pm 3$ & $117 \pm 6$ & $<0.001$ \\
\hline PTH (ng/L) & $37 \pm 2$ & $45 \pm 9$ & $34 \pm 2$ & 0.046 & $33 \pm 6$ & $38 \pm 3$ & 0.29 \\
\hline
\end{tabular}


Table 1. Cont.

\begin{tabular}{|c|c|c|c|c|c|c|c|}
\hline & \multirow[b]{2}{*}{$\begin{array}{l}\text { All Subjects } \\
\qquad(n=34)\end{array}$} & \multicolumn{3}{|c|}{$\begin{array}{c}\text { Stratification by } \\
25 \text {-hydroxy } \\
\text { vitamin D status }\end{array}$} & \multicolumn{3}{|c|}{$\begin{array}{c}\text { Stratification by } \\
1,25 \text {-dihydroxy } \\
\text { vitamin D status }\end{array}$} \\
\hline & & $\begin{array}{c}\text { Deficient } \\
<50 \mathrm{nmol} / \mathrm{L} \\
\quad(n=7)\end{array}$ & $\begin{array}{l}\text { Non-Deficient } \\
\begin{array}{c}>50 \mathrm{nmol} / \mathrm{L} \\
(n=27)\end{array}\end{array}$ & $p$-value & $\begin{array}{l}\text { Below 25th } \\
\text { percentile } \\
<76 \mathrm{pmol} / \mathrm{L} \\
(n=8)\end{array}$ & $\begin{array}{c}\text { Above 25th } \\
\text { percentile } \\
>76 \mathrm{pmol} / \mathrm{L} \\
(n=26)\end{array}$ & $p$-value \\
\hline $\begin{array}{l}\text { Serum calcium } \\
\quad(\mathrm{mmol} / \mathrm{L})\end{array}$ & $2.2 \pm 0.01$ & $2.3 \pm 0.04$ & $2.2 \pm 0.01$ & 0.47 & $2.2 \pm 0.03$ & $2.2 \pm 0.02$ & 0.49 \\
\hline $\begin{array}{l}\text { Serum phosphate } \\
\quad(\mathrm{mmol} / \mathrm{L})\end{array}$ & $1.0 \pm 0.03$ & $0.9 \pm 0.08$ & $1.01 \pm 0.03$ & 0.35 & $1.03 \pm 0.04$ & $0.97 \pm 0.04$ & 0.29 \\
\hline HDL (mmol/L) & $1.4 \pm 0.05$ & $1.3 \pm 0.1$ & $1.5 \pm 0.05$ & 0.21 & $1.45 \pm 0.07$ & $1.4 \pm 0.06$ & 0.62 \\
\hline $\mathrm{LDL}(\mathrm{mmol} / \mathrm{L})$ & $2.2 \pm 0.1$ & $1.8 \pm 0.2$ & $2.3 \pm 0.2$ & 0.17 & $2.1 \pm 0.4$ & $2.2 \pm 0.2$ & 0.65 \\
\hline $\mathrm{NE}(\mathrm{nmol} / \mathrm{L})$ & $1.3 \pm 0.2$ & $1.2 \pm 0.06$ & $1.3 \pm 0.2$ & 0.79 & $1.5 \pm 0.3$ & $1.3 \pm 0.2$ & 0.37 \\
\hline Epi $(\mathrm{pmol} / \mathrm{L})$ & $97 \pm 13$ & $69 \pm 11$ & $117 \pm 17$ & 0.019 & $76 \pm 13$ & $103 \pm 15$ & 0.64 \\
\hline $\begin{array}{l}\text { Urinary sodium } \\
\text { (mmol/day) }\end{array}$ & $373 \pm 20$ & $355 \pm 48$ & $379 \pm 25$ & 0.65 & $348 \pm 25$ & $380 \pm 27$ & 0.40 \\
\hline $\begin{array}{l}\text { Serum creatinine } \\
\quad(\mathrm{umol} / \mathrm{L})\end{array}$ & $69 \pm 4$ & $69 \pm 7$ & $70 \pm 3$ & 0.92 & $70 \pm 5$ & $67 \pm 4$ & 0.98 \\
\hline PRA (ng/L/s) & $0.21 \pm 0.03$ & $0.23 \pm 0.04$ & $0.20 \pm 0.03$ & 0.48 & $0.15 \pm 0.03$ & $0.23 \pm 0.03$ & 0.19 \\
\hline Ang II (ng/L) & $18 \pm 1$ & $18 \pm 3$ & $16 \pm 1$ & 0.38 & $18 \pm 2$ & $18 \pm 1$ & 0.81 \\
\hline Aldo (pmol/L) & $106 \pm 10$ & $90 \pm 8$ & $110 \pm 12$ & 0.61 & $99 \pm 14$ & $109 \pm 12$ & 0.91 \\
\hline
\end{tabular}

BMI, body mass index; SBP, systolic blood pressure; DBP, diastolic blood pressure; MAP, mean arterial pressure; PTH, parathyroid hormone; HDL, high-density lipoprotein (cholesterol); LDL, low-density lipoprotein (cholesterol); NE, norepinephrine; Epi, epinephrine; PRA, plasma renin activity Ang II, angiotensin II; Aldo, aldosterone. All data are expressed as mean $\pm \mathrm{SE}$ unless otherwise indicated.

\subsection{Cardiac Autonomic Responses in 25-Hydroxy Vitamin D Deficient vs. Non-Vitamin D} Deficient Subjects

At baseline, significant suppression of overall resting sympathovagal balance was observed in the 25-hydroxy vitamin D deficient participants (LF:HF, $p=0.02$ ) (Figure 1). During AngII infusion, no significant differences between groups at a specific time point were noted. Further, no significant trends in responses over time were observed within either group (Table 2). Two-way repeated measures ANOVA revealed no significant within-subject or between-group contrasts despite controlling for potential confounders, suggesting that differences in 25-hydroxy vitamin D did not alter cardiac autonomic responses to AngII. 
Figure 1. Cardiosympathovagal modulation during angiotensin II challenge, by vitamin D status LF:HF, low- to high-frequency ratio representative of cardiosympathovagal balance.

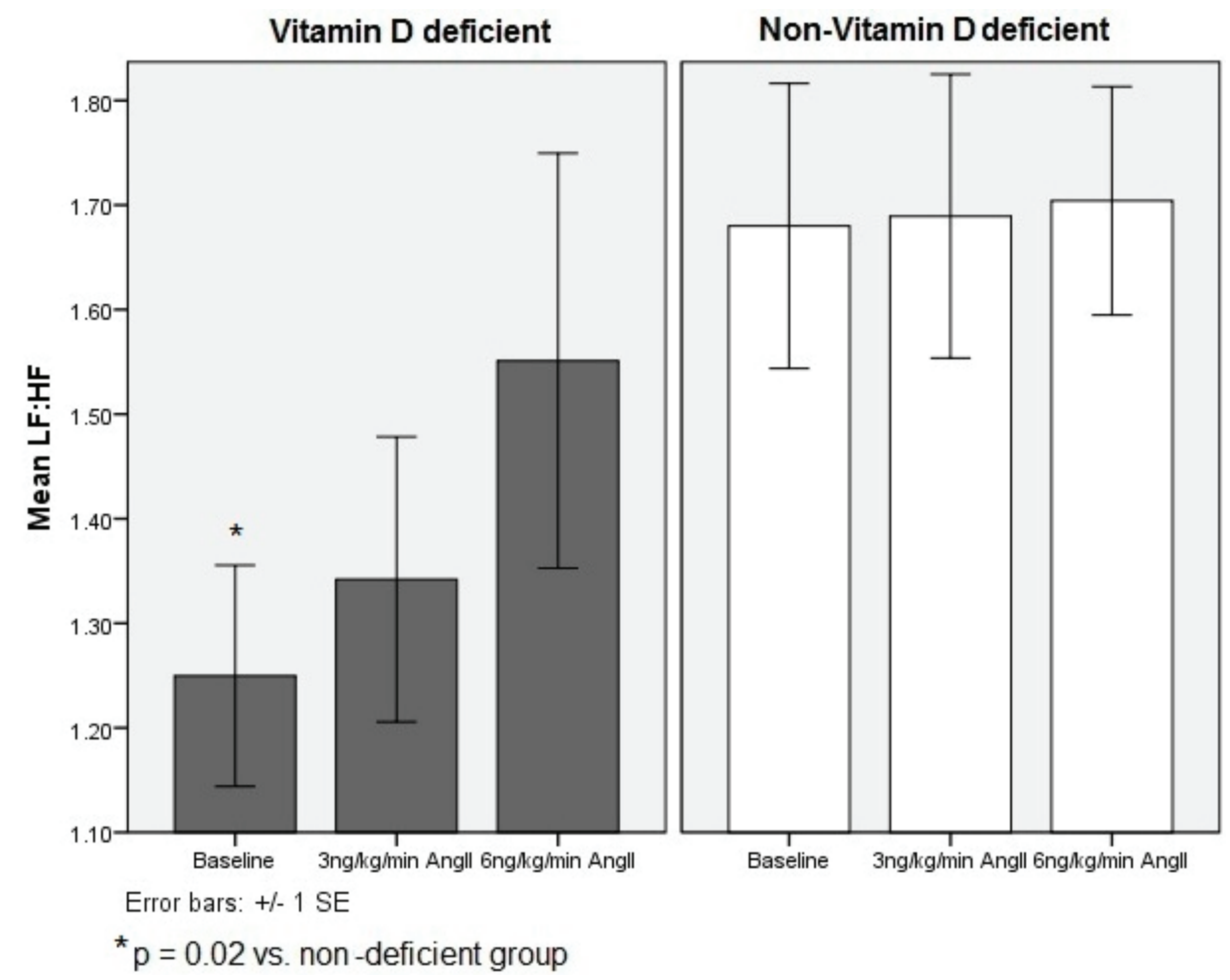

Table 2. Cardiac autonomic response to angiotensin II challenge by 25 -hydroxy vitamin D status.

\begin{tabular}{cccc}
\hline & Baseline & $\mathbf{3 ~ n g} / \mathbf{k g} / \mathbf{m i n}$ AngII & $\mathbf{6 ~ n g} / \mathbf{k g} / \mathbf{m i n}$ AngII \\
\hline Heart rate (bpm) & & & \\
Vitamin D deficient & $62 \pm 3$ & $56 \pm 3$ & $65 \pm 4$ \\
Non-vitamin D deficient & $57 \pm 2$ & $56 \pm 3$ & $58 \pm 2$ \\
TP (ms) & & & \\
Vitamin D deficient & $56 \pm 7$ & $66 \pm 7$ & $63 \pm 8$ \\
Non-vitamin D deficient & $69 \pm 6$ & $74 \pm 7$ & $73 \pm 7$ \\
VLF (ms) & & & \\
Vitamin D deficient & $39 \pm 6$ & $48 \pm 6$ & $47 \pm 6$ \\
Non-vitamin D deficient & $51 \pm 5$ & $55 \pm 7$ & $56 \pm 5$ \\
LF (ms) & & & \\
Vitamin D deficient & $31 \pm 4$ & $34 \pm 4$ & $37 \pm 5$ \\
Non-vitamin D deficient & $37 \pm 4$ & $37 \pm 3$ & \\
LF (ln ms $\mathbf{2}$ & & & $6.84 \pm 0.3$ \\
Vitamin D deficient & $6.76 \pm 0.3$ & $6.93 \pm 0.3$ & $7.00 \pm 0.2$ \\
Non-vitamin D deficient & $6.99 \pm 0.2$ & $6.97 \pm 0.2$ & $62 \pm 4$ \\
LF (nu) & & & $68 \pm 2$ \\
Vitamin D deficient & $61 \pm 5$ & $58 \pm 3$ & \\
Non-vitamin D deficient & $67 \pm 3$ & $66 \pm 3$ & \\
\hline
\end{tabular}


Table 2. Cont.

\begin{tabular}{cccc}
\hline HF $(\mathbf{m s})$ & & \\
Vitamin D deficient & $25 \pm 3$ & $26 \pm 3$ & $24 \pm 4$ \\
Non-vitamin D deficient & $25 \pm 3$ & $24 \pm 3$ & $23 \pm 2$ \\
HF $\left(\boldsymbol{l n} \mathbf{~ m s}^{2}\right)$ & & & \\
Vitamin D deficient & $6.36 \pm 0.3$ & $6.40 \pm 0.3$ & $6.06 \pm 0.6$ \\
Non-vitamin D deficient & $6.12 \pm 0.2$ & $6.02 \pm 0.2$ & $5.98 \pm 0.2$ \\
HF (nu) & & & \\
Vitamin D deficient & $31 \pm 7$ & $37 \pm 4$ & $32 \pm 5$ \\
Non-vitamin D deficient & $33 \pm 3$ & $28 \pm 3$ & $26 \pm 2$ \\
LF:HF & $1.25 \pm 0.1$ & $1.34 \pm 0.2$ & $1.55 \pm 0.2$ \\
Vitamin D deficient & $1.66 \pm 0.1^{\dagger}$ & $1.72 \pm 0.1$ & $1.74 \pm 0.1$ \\
Non-vitamin D deficient & & & \\
\hline
\end{tabular}

TP, total power; VLF, very-low frequency; LF, low-frequency; HF, high-frequency. All data are expressed as mean \pm SE unless otherwise indicated. Vitamin D deficient $(n=7)$, non-vitamin D deficient $(n=27)$.

$* p<0.05 v s$. response from baseline; ${ }^{\dagger} p<0.05 v s$. vitamin $\mathrm{D}$ deficient value at this time point.

\subsection{Cardiac Autonomic Responses below vs. above 25th Percentile 1,25-Dihydroxy}

\section{Vitamin D Subjects}

At baseline, there were no significant differences in cardiac autonomic activity between groups. In response to 30 min of AngII challenge, subjects with 1,25-dihydroxy vitamin D levels below the 25 th percentile demonstrated loss of vagal tone as demonstrated by a decrease in HF (nu) compared to subjects with 1,25-dihydroxy vitamin $\mathrm{D}$ levels above the 25 th percentile $(p=0.05)$. As a consequence of the loss of vagal tone in response to the stressor, subjects with 1,25-dihydroxy vitamin D levels below the 25th percentile were unable to maintain overall sympathovagal balance compared to those with 1,25-dihydroxy vitamin D levels above the 25th percentile $(p=0.043)$ (Table 3 ). Comparison of the higher and lower 1,25-dihydroxy vitamin D groups revealed that 1,25-hydroxy vitamin D status was associated with differences in the LF, HF, and LF:HF responses across AngII doses, specifically at the $3 \mathrm{ng} / \mathrm{kg} / \mathrm{min}$ dose (LF nu, mean difference $=8.84 \pm 4 \mathrm{nu}, p=0.034$; HF nu, mean difference $=-6.98 \pm 3 \mathrm{nu}, p=0.049$ (Figure 2); LF:HF, mean difference $=0.34 \pm 0.1$, $p=0.080)$. 
Table 3. Cardiac autonomic response to angiotensin II challenge by 1,25-dihydroxy vitamin D status.

\begin{tabular}{|c|c|c|c|}
\hline & Baseline & 3 ng/kg/min AngII & $6 \mathrm{ng} / \mathrm{kg} / \mathrm{min}$ AngII \\
\hline $\begin{array}{c}\text { Heart rate (bpm) } \\
\text { Below 25th percentile } \\
\text { Above 25th percentile }\end{array}$ & $\begin{array}{l}59 \pm 3 \\
58 \pm 2\end{array}$ & $\begin{array}{l}60 \pm 2 \\
56 \pm 2\end{array}$ & $\begin{array}{l}62 \pm 3 \\
58 \pm 2\end{array}$ \\
\hline $\begin{array}{l}\quad \mathbf{T P}(\mathbf{m s}) \\
\text { Below } 25 \text { th percentile } \\
\text { Above 25th percentile }\end{array}$ & $\begin{array}{c}70 \pm 15 \\
66 \pm 5\end{array}$ & $\begin{array}{c}83 \pm 16 \\
69 \pm 6\end{array}$ & $\begin{array}{c}85 \pm 18 \\
67 \pm 5\end{array}$ \\
\hline $\begin{array}{l}\quad \mathbf{V L F}(\mathbf{m s}) \\
\text { Below 25th percentile } \\
\text { Above 25th percentile }\end{array}$ & $\begin{array}{c}50 \pm 12 \\
48 \pm 4\end{array}$ & $\begin{array}{c}66 \pm 13 \\
50 \pm 5\end{array}$ & $\begin{array}{c}58 \pm 11 \\
53 \pm 4\end{array}$ \\
\hline $\begin{array}{l}\quad \mathbf{L F}(\mathbf{m s}) \\
\text { Below } 25 \text { th percentile } \\
\text { Above 25th percentile }\end{array}$ & $\begin{array}{l}40 \pm 8 \\
35 \pm 3\end{array}$ & $\begin{array}{l}43 \pm 8 \\
34 \pm 3\end{array}$ & $\begin{array}{c}40 \pm 10 \\
35 \pm 2\end{array}$ \\
\hline $\begin{array}{l}\quad \mathbf{L F}\left(\boldsymbol{l n} \mathbf{m s}^{\mathbf{2}}\right) \\
\text { Below 25th percentile } \\
\text { Above 25th percentile }\end{array}$ & $\begin{array}{l}7.04 \pm 0.4 \\
6.91 \pm 0.2\end{array}$ & $\begin{array}{l}7.28 \pm 0.4 \\
6.87 \pm 0.2\end{array}$ & $\begin{array}{l}6.89 \pm 0.5 \\
6.99 \pm 0.2\end{array}$ \\
\hline $\begin{array}{l}\quad \mathbf{L F}(\mathbf{n u}) \\
\text { Below } 25 \text { th percentile } \\
\text { Above 25th percentile }\end{array}$ & $\begin{array}{l}67 \pm 4 \\
65 \pm 3\end{array}$ & $\begin{array}{l}72 \pm 4 \\
62 \pm 3\end{array}$ & $\begin{array}{l}69 \pm 4 \\
66 \pm 2\end{array}$ \\
\hline $\begin{array}{l}\quad \mathbf{H F}(\mathbf{m s}) \\
\text { Below } 25 \text { th percentile } \\
\text { Above 25th percentile }\end{array}$ & $\begin{array}{l}25 \pm 5 \\
25 \pm 3\end{array}$ & $\begin{array}{l}23 \pm 4 \\
25 \pm 2\end{array}$ & $\begin{array}{l}21 \pm 5 \\
24 \pm 2\end{array}$ \\
\hline $\begin{array}{l}\text { HF }\left(\boldsymbol{l n} \mathbf{~ m s}^{\mathbf{2}}\right) \\
\text { Below 25th percentile } \\
\text { Above 25th percentile }\end{array}$ & $\begin{array}{l}6.15 \pm 0.4 \\
6.17 \pm 0.2\end{array}$ & $\begin{array}{l}6.04 \pm 0.3 \\
6.12 \pm 0.2\end{array}$ & $\begin{array}{l}5.79 \pm 0.4 \\
6.06 \pm 0.2\end{array}$ \\
\hline $\begin{array}{l}\quad \mathbf{H F}(\mathbf{n u}) \\
\text { Below 25th percentile } \\
\text { Above 25th percentile }\end{array}$ & $\begin{array}{l}37 \pm 7 \\
34 \pm 3\end{array}$ & $\begin{array}{c}23 \pm 3 \\
32 \pm 3^{\dagger}\end{array}$ & $\begin{array}{l}25 \pm 3 \\
28 \pm 3\end{array}$ \\
\hline $\begin{array}{c}\text { LF:HF } \\
\text { Below 25th percentile } \\
\text { Above 25th percentile }\end{array}$ & $\begin{array}{l}1.6 \pm 0.1 \\
1.6 \pm 0.1\end{array}$ & $\begin{array}{c}1.9 \pm 0.2 \\
1.6 \pm 0.1^{\dagger}\end{array}$ & $\begin{array}{l}1.8 \pm 0.2 \\
0.7 \pm 0.1\end{array}$ \\
\hline
\end{tabular}

TP, total power; VLF, very-low frequency; LF, low-frequency; HF, high-frequency. All data are expressed as mean \pm SE unless otherwise indicated. Below 25th percentile $(n=8)$, above 25th percentile $(n=26)$. $* p<0.05 v s$. response from baseline; ${ }^{\dagger} p<0.05 v s$. below 25 th percentile at this time point. 
Figure 2. Comparison of cardiac vagal autonomic activity between 1,25-dihydroxy vitamin D groups during angiotensin II challenge HF, high-frequency representative of cardiac parasympathetic activity; nu, normalized units.

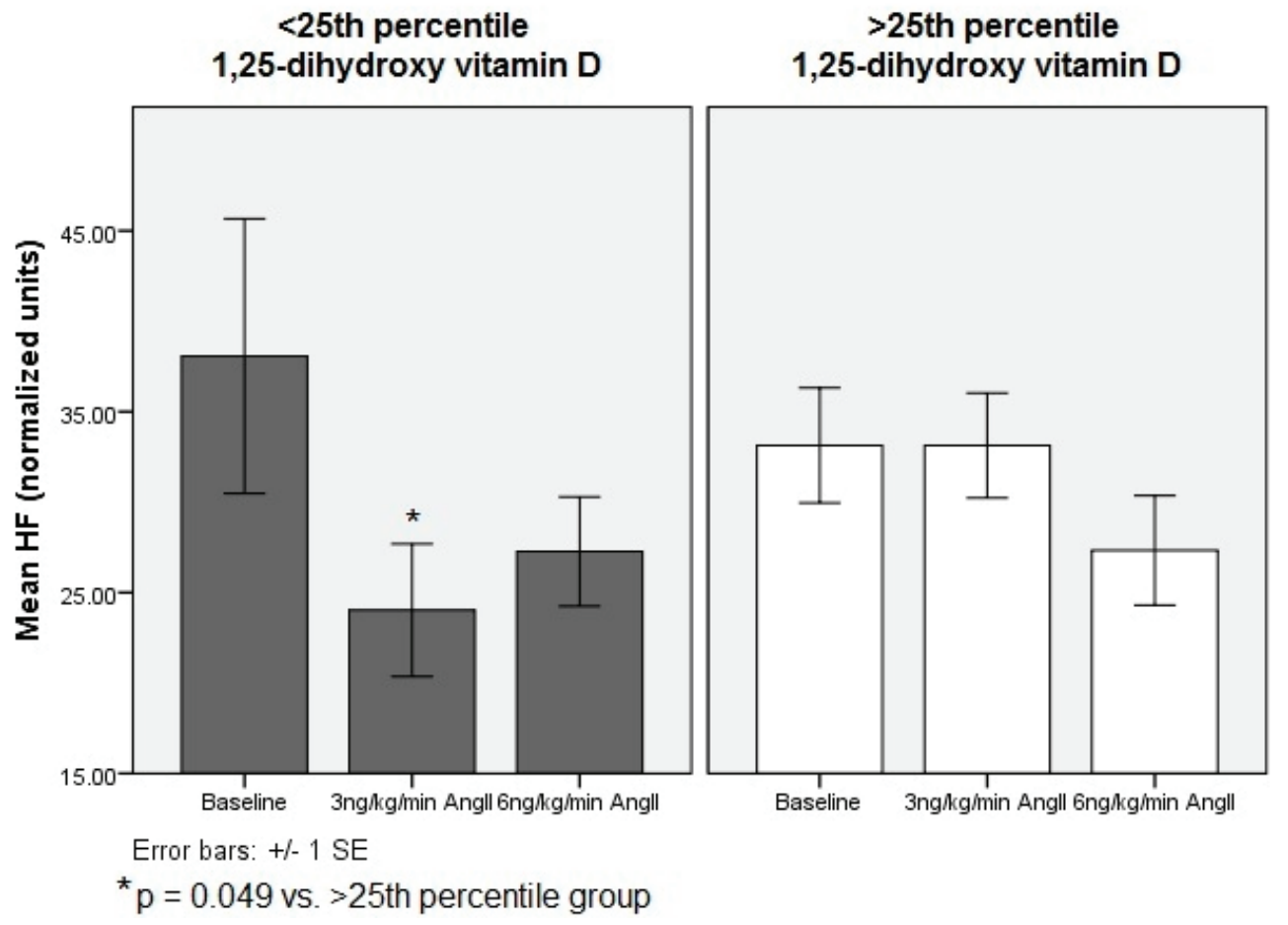

\subsection{Systemic Responses to Angiotensin II Challenge}

Throughout the AngII challenge, BP parameters increased similarly in all subjects regardless of vitamin D stratification. Further, PRA decreased from baseline while aldosterone levels increased from baseline (Table 1) as expected throughout the AngII challenge, with no significant differences in PRA or aldosterone activity between either vitamin D stratification groups. No significant trends or repeated measures comparisons were observed in any BP or RAS parameter.

\section{Discussion}

This is the first study to examine the relationship between 25-hydroxy vitamin D, the barometer of vitamin D status, as well as 1,25-dihydroxy vitamin D, the activated form of vitamin D, with cardiac autonomic activity at baseline and in response to a vascular stressor in humans. Our key findings were: (1) vitamin D deficiency as determined by 25-hydroxy vitamin D levels in healthy humans were associated only with a suppression of resting overall cardiosympathovagal balance; and (2) lower levels of 1,25-dihydroxy vitamin D, the activated form of vitamin D, were associated with unfavourable shifts in cardiosympathovagal balance, driven by exaggerated withdrawal of cardioprotective vagal tone, in response to AngII challenge. 


\subsection{Vitamin D and Cardiovascular Risk}

Vitamin D deficiency ( $<50 \mathrm{nmol} / \mathrm{L} 25$-hydroxy vitamin D) has been highlighted in numerous studies involving high-risk CV disease populations [22,23], suggesting that low vitamin D status may be a potentially significant and treatable risk factor. In an observational study by Wang and colleagues [24], normotensive subjects with 25-hydroxy vitamin D serum concentrations of $<37.5 \mathrm{nmol} / \mathrm{L}$ demonstrated a $62 \%$ increase in $\mathrm{CV}$ disease risk over a 5.4-year follow-up compared to those with higher levels. Similarly, Dobnig et al. reported that in 3258 patients referred for coronary angiography, those within the lowest quartiles of 25 -hydroxy $(<35 \mathrm{nmol} / \mathrm{L})$ or 1,25-dihydroxy vitamin $\mathrm{D}(<51 \mathrm{pmol} / \mathrm{L})$ were at highest risk of sudden cardiac death [2], hence the rationale to also stratify subjects by the lowest 1,25-dihydroxy vitamin D quartile in the present study. Moreover, recent studies of healthy adults have shown that lower 25-hydroxy vitamin D were independently associated with an increased risk of SCD [3,24], suggesting that vitamin D status is important even in populations without established pathologies.

\subsection{Vitamin D and Cardiac Autonomic Activity}

Our results suggest that low serum levels of vitamin D may be associated with a decline in cardioprotective vagal tone in response to an acute vascular stressor, largely through the action of the active 1,25-dihydroxy vitamin D metabolite. Active 1,25-dihydroxy vitamin $\mathrm{D}$ has been identified in some mammalian studies as having the potential to influence cardiac autonomic activity [25-27]. These studies demonstrate that cardiac myocytes isolated from vitamin D receptor knockout mice display accelerated rates of contraction as compared with wild type. Further, exposure to 1,25-dihydroxy vitamin $\mathrm{D}$ directly attenuated this rapid contractility in the wild-type but not the knockout cardiac myocyte [27], suggesting a relationship between 1,25-dihydroxy vitamin D and vagal inhibitory outflow. Moreover, activated vitamin D is thought to have the ability to diffuse across the blood-brain barrier, implicating a role for 1,25-dihydroxy vitamin $\mathrm{D}$ in augmenting autonomic vagal control by binding directly to nuclear vitamin $\mathrm{D}$ receptors in the adrenergic neurons located centrally in the spinal cord and brain tissue [26].

In support of our novel observations in healthy humans, patients with chronic kidney disease (CKD) demonstrate chronic RAS upregulation [28,29], as well as altered cardiac autonomic activity defined primarily by extreme vagal insufficiency [5]. This same population has a diminished capacity to convert 25-hydroxy vitamin $\mathrm{D}$ to 1,25 -dihydroxy vitamin $\mathrm{D}$ due to decreased renal 1- $\alpha$ hydroxylase capacity [1]; however, CV mortality in this population declines following treatment with exogenous 1,25-dihydroxy vitamin D supplementation [30]. Moreover, a recent study by Kendrick et al. demonstrates that both $\mathrm{CV}$ death and time to initiation of dialysis in individuals with advanced stages of CKD are independently associated with serum 1,25-dihydroxy vitamin D levels. The authors did not find these associations when stratifying subjects by 25-hydroxy vitamin D status [31]. As such, the withdrawal of vagal tone observed in low 1,25-dihydroxy vitamin D subjects during AngII challenge may mimic the loss of vagal tone that has been noted in the end-stage renal disease population [5]. These patients are characterized by severe vitamin D deficiency [1] — particularly low 
serum concentrations of 1,25-dihydroxy vitamin D due to compromised 1-alpha hydroxylase activity housed within the functional renal tissue - as well as chronically upregulated RAS activity.

Further, dysfunction of cardiac autonomic tone, specifically vagal tone, has been shown to be predictive of sudden cardiac death [5,8-10]. Wolf and colleagues have demonstrated a survival advantage and specifically a decrease in cardiovascular death in hemodialysis patients taking 1,25-dihydroxy vitamin D supplementation irrespective of 25-hydroxy vitamin D level [30]. These observations support our findings which suggest that the activated 1,25-dihydroxy vitamin D metabolite may be capable of altering cardiac autonomic tone in individuals experiencing upregulation of the RAS and that low 1,25-dihydroxy vitamin D levels contribute to the loss of vagal outflow, and therefore high CV risk, observed in these populations.

\subsection{Challenges and Limitations}

In humans, vitamin D is part of a complex mineral metabolism involving additional measures that have been shown to influence CV risk, including PTH, phosphate, and calcium [32]. While PTH levels were significantly elevated in the 25-hydroxy vitamin D deficient group, PTH and other mineral metabolism variables were within narrow, healthy ranges. Further, our study was performed over different seasons, though the season of study was not found to be correlated to vitamin D levels. Salt intake has been shown to influence cardiac ANS activity [33]. However, all subjects were in high-salt balance to ensure a maximal suppression of basal RAS levels, allowing for meaningful comparisons between subjects and direct observation of the impact of exogenous AngII infusion on cardiac autonomic activity. The study sample size was limited and only included non-smoking healthy subjects who were non-obese, normotensive, non-diabetic with normal kidney function, limiting the generalizability of our study results to the general population. However, by studying a healthier population, we aimed to examine the impact of various vitamin D metabolites on cardiac autonomic tone at baseline and in response to AngII while minimizing confounding factors. Furthermore, this group reflects the population referenced by the Endocrine Society which defines vitamin D deficiency as $<50 \mathrm{nmol} / \mathrm{L} 25$-hydroxy vitamin $\mathrm{D}$ [12]. We found that allocation to the 25-hydroxy vitamin D deficiency group was not correlated with allocation to the low 1,25-dihydroxy vitamin D group, a finding that is consistent with previous reports, thereby suggesting that the potential influences of individual dietary calcium consumption, PTH levels, or individual 1- $\alpha$ hydroxylase capacity may play a role in determining an individual's 25-hydroxy and 1,25-dihydroxy vitamin D levels [1,32]. Next, our results appeared most significant in response to the first graded dose of AngII, perhaps representing a threshold effect that persisted through the second dose. Lastly, while we cannot comment on the role of vitamin D supplementation in improving cardiac responses to AngII challenge or other stressors, our study adds to the growing body of literature supporting a link between vitamin D metabolite levels and overall CV outcomes.

\subsection{Implications}

To our knowledge, there have been no previous studies investigating the potential link between vitamin D metabolites and modulation of the cardiac ANS, a risk factor for poor CV outcomes in 
healthy and diseased populations $[2-4,7-11,16]$. Our study illustrates a unique relationship between low 25-hydroxy vitamin D levels and depressed baseline cardiac autonomic activity, as well as low 1,25-dihydroxy vitamin $\mathrm{D}$ and unfavourable cardiosympathovagal shifts during acute AngII challenge, which allows for unique insight into a pathophysiological mechanism that may be acting to elevate $\mathrm{CV}$ risk, particularly in renal populations with chronic upregulation of the RAS, in addition to impaired 1- $\alpha$ hydroxylase activity [22-31]. While larger studies involving supplementation are required, dysregulation of vitamin $\mathrm{D}$ metabolism remains a potentially treatable condition that warrants further investigation in high-risk populations.

\section{Funding Sources}

This work was supported by an Establishment Grant from Alberta Innovates-Health Solutions. MC Mann is supported by the Queen Elizabeth II Doctoral Scholarship and the Achievers in Medical Science Graduate Recruitment Scholarship. DV Exner, BR Hemmelgarn, and SB Ahmed are supported by Alberta Innovates-Health Solutions and the Canadian Institute of Health Research. BR Hemmelgarn and SB Ahmed are supported by a joint initiative between Alberta Health and Wellness and the Universities of Alberta and Calgary. DV Exner is Canada Research Chair in Cardiovascular Clinical Trials and receives unrestricted support from GE Healthcare. Funding sources had no role in the design, conduct, or reporting of this study.

\section{Conflicts of Interest}

The authors declare no conflict of interest.

\section{Reference}

1. Holick, M.F. Vitamin D. N. Engl. J. Med. 2007, 357, 266-281.

2. Dobnig, H.; Pilz, S.; Scharnagl, H.; Renner, W.; Seelhorst, U.; Wellnitz, B.; Kinkeldi, J.; Boehm, B.O.; Weihrauch, G.; Maerz, W. Independent association of low serum 25-hydroxyvitamin D and 1,25-dihydroxyvitamin D levels with all-cause and cardiovascular mortality. Arch. Intern. Med. 2008, 168, 1340-1349.

3. Deo, R.; Katz, R.; Shlipk, M.G.; Sotoodehnia, N.; Psaty, B.M.; Sarnak, M.J.; Fried, L.F.; Chonchol, M.; de Boer, I.H.; Enquobahrie, D.; et al. Vitamin D, paraythyroid hormone and sudden cardiac death: Results from the Cardiovascular Health Study. Hypertension 2011, 58, 1021-1028.

4. De Novellis, V.; Loffreda, A.; Vitagliano, S. Effects of dietary vitamin D deficiency on the cardiovascular system. Res. Commun. Chem. Pathol. Pharmacol. 1994, 83, 125-144.

5. Chan, C.T.; Levin, N.W.; Chertow, G.M.; Larive, B.; Schulman, G.; Kotanko, P.; The Frequent Hemodialysis Network Daily Trial Group. Determinants of cardiac autonomic dysfunction in ESRD. J. Am. Soc. Nephrol. 2010, 5, 1821-1827.

6. Kneip, C.F.; Mallet, R.T.; Williams, A.G.; Hamdan, M.H.; Smith, M.L. Vagal modulation of heart rate variability during atrial fibrillation in pigs. Exp. Bil. Med. (Maywood) 2010, 235, 1007-1014. 
7. Tsuji, H.; Venditti, F.J.; Manders, E.S.; Evans, J.C.; Feldman, C.L.; Levy, D. Impact of reduced heart rate variability on risk for cardiac events: The framingham heart study. Circulation 1996, 94, 2850-2855.

8. La Rovere, M.T.; Pinna, G.D.; Maestri, R.; Mortara, A.; Capomolla, S.; Febo, O.; Ferrari, R.; Franchini, M.; Gnemmi, M.; Opasich, C.; et al. Short-term heart rate variability strongly predicts sudden cardiac death in chronic heart failure patients. Circulation 2003, 107, 565.

9. Ranpuria, R.; Hall, M.; Chan, C.; Unruh, M. Heart rate variability (HRV) in kidney failure: Measurement and consequences of HRV. Neph. Dial. Transplant. 2007, 23, 444-449.

10. Goldberger, J.J.; Cain, M.E.; Hohnloser, S.H.; Kadish, A.H.; Knight, B.P.; Lauer, M.S.; Maron, B.J.; Page, R.L.; Passman, R.S.; Siscovick, D.; et al. American Heart Association/American College of Cardiology Foundation/Heart Rhythm Society Scientific Statement on noninvasive risk stratification techniques for identifying patients at risk for sudden cardiac death. Circulation 2008, 118, 1497-1518.

11. Lauer, M.S. Autonomic function and prognosis. Cleve Clin. J. Med. 2009, 76, S18-S22.

12. Holick, M.F.; Binkley, N.C.; Bischoff-Ferrari, H.A.; Gordon, C.M.; Hanley, D.A.; Heaney, R.P.; Murad, M.H.; Weaver, C.M. Evaluation, treatment, and prevention of vitamin D deficiency: An Endocrine Society Clinical Practice Guideline. J. Clin. Endocrinol. Metab. 2011, 96, 1911-1930.

13. Forman, J.P.; Williams, J.S.; Fisher, N.D. Plasma 25-hydroxyvitamin D and regulation of the renin-angiotensin system. Hypertension 2010, 55, 1283-1288.

14. Vaidya, A.; Forman, J.P.; Williams, J.S. Vitamin D and the vascular sensitivity to angiotensin II in obese Caucasians with hypertension. J. Hum. Hypertens. 2011, 25, 672-678.

15. Shoback, D.M.; Williams, G.H.; Swartz, S.L.; Davies, R.O.; Hollenberg, N.K. Time course and effect of sodium intake on vascular and hormonal responses to enalapril (MK 421) in normal subjects. J. Cardiovasc. Pharmacol. 1983, 5, 1010-1018.

16. Task Force of the European Society of Cardiology; the North American Society of Pacing Electrophysiology. Heart rate variability: Standards of measurement, physiological interpretation, and clinical use. Circulation 1996, 93, 1043-1065.

17. Zhong, Y.; Jan, K.M.; Ju, K.H.; Chon, K.H. Quantifying cardiac sympathetic and parasympathetic nervous activities using principal dynamic modes analysis of heart rate variability. Am. J. Physiol. Heart Circ. Physiol. 2006, 291, H1475-H1483.

18. Taylor, J.A.; Carr, D.L.; Myers, C.W.; Eckberg, D.L. Mechanisms underlying very-low frequency RR-interval oscillations in humans. Circulation 1998, 98, 547-555.

19. Tripathi, K.K. Very low frequency oscillations in the power spectra of heart rate variability during dry supine immersion and exposure to non-hypoxic hypobaria. Physiol. Meas. 2011, 32, $717-729$.

20. Grasso, R.; Schena, F.; Gulli, G.; Cevese, A. Does low-frequency variability of heart period reflect a specific parasympathetic mechanism? J. Auton. Nerv. Syst. 1997, 63, 30-38.

21. Mann, M.C.; Exner, D.V.; Hemmelgarn, B.R.; Sola, D.Y.; Turin, T.C.; Ahmed, S.B. Impact of gender on the cardiac autonomic response to angiotensin II in healthy humans. J. Appl. Physiol. 2012, 112, 1001-1007. 
22. Dreschler, C.; Pilz, S.; Obermayer-Pietsch, B.; Verduijn, M.; Tomaschitz, A.; Krane, V.; Espe, K.; Dekker, F.; Brandenburg, V.; Marz, W.; et al. Vitamin D deficiency is associated with sudden cardiac death, combined cardiovascular events, and mortality in hemodialysis patients. Eur. Heart J. 2010, 31, 2253-2261.

23. Pilz, S.; Iodice, S.; Zittermann, A.; Grant, W.B.; Gandini, S. Vitamin D status and mortality risk in CKD: A meta-analysis of prospective studies. Am. J. Kidney Dis. 2011, 58, 374-382.

24. Wang, T.; Pencina, M.; Booth, S.; Jacques, P.; Ingelsson, E.; Lanier, K.; Benjamin, E.; D'Agostino, R.; Wolf, M.; Vasan, R. Vitamin D deficiency and risk of cardiovascular disease. Circulation 2008, 117, 503-511.

25. Scorza, F.A.; Albuquerque, M.; Arida, R.M.; Tera, V.C.; Machado, H.R.; Cavalheiro, E.A. Benefits of sunlight: Vitamin D deficiency might increase the risk of sudden unexpected death in epilepsy. Med. Hypotheses 2010, 74, 158-161.

26. Sternberg, Z. Autonomic dysfunction: A unifying multiple sclerosis theory, linking chronic cerebrospinal venous insufficiency, vitamin D3 and Epstein-Barr virus. Autoimmun. Rev. 2012, $12,250-259$.

27. Tishkoff, D.X.; Nibbelink, K.A.; Holmberg, K.H.; Dandu, L.; Simpson, R.U. Functional vitamin D receptor (VITAMIN DR) in the t-tubules of cardiac myocytes: VITAMIN DR knockout cardiomyocyte contractility. Endocrinology 2008, 149, 558-564.

28. Brewster, U.C.; Setaro, J.F.; Perazella, M.A. The renin-angiotensin-aldosterone system: Cardiorenal effects and implications for renal and cardiovascular disease states. Am. J. Med Sci. 2003, 326, 15-24.

29. Perazella, M.A.; Setaro, J.F. Renin-angiotensin-aldosterone system: Fundamental aspects and clinical implications in renal and cardiovascular disorders. J. Nucl. Cardiol. 2003, 10, 184-196.

30. Wolf, M.; Shah, A.; Gutierrez, O.; Ankers, E.; Monroy, M.; Tamez, H.; Steele, D.; Chang, Y.; Camargo, C.A., Jr.; Tonelli, M.; et al. Vitamin D levels and early mortality among incident hemodialysis patients. Kidney Int. 2007, 72, 1004-1013.

31. Kendrick, J.; Cheung, A.K.; Kaufman, J.S.; Green, T.; Roberta, W.L.; Smits, G.; Chonchol, M.; HOST Study Inventigators. Associations of plasma 25-hydroxyvitamin D and 1,25-dihydroxyvitamin $\mathrm{D}$ concentrations with death and progression to maintenance dialysis in patients with advanced kidney disease. Am. J. Kidney Dis. 2012, 60, 567-575.

32. Palmer, S.C.; Hayen, A.; Macaskill, P.; Pellegrini, F.; Craig, J.C.; Elder, G.J.; Strippoli, G.F. Serum levels of phosphorus, parathyroid hormone, and calcium and risks of death and cardiovascular disease in individuals with chronic kidney disease: A systematic review and meta-analysis. JAMA 2011, 305, 1119-1127.

33. McNeely, J.D.; Windham, B.G.; Anderson, D.E. Dietary sodium effects on heart rate variability in salt-sensitivity of blood pressure. Psychophysiology 2007, 45, 405-411. 


\title{
Effects of Vitamin $D_{3}$ and Paricalcitol on Immature Cardiomyocytes: A Novel Role for Vitamin D Analogs in the Prevention of Cardiovascular Diseases
}

\author{
Stefania Pacini, Gabriele Morucci, Jacopo J. V. Branca, Stefano Aterini, Marcello Amato, \\ Massimo Gulisano and Marco Ruggiero
}

\begin{abstract}
Cardiovascular diseases are more prevalent in patients with chronic kidney disease than in the general population and they are considered the leading cause of death in patients with end-stage renal disease. The discovery that vitamin $\mathrm{D}_{3}$ plays a considerable role in cardiovascular protection has led, in recent years, to an increase in the administration of therapies based on the use of this molecule; nevertheless, several studies warned that an excess of vitamin $\mathrm{D}_{3}$ may increase the risk of hypercalcemia and vascular calcifications. In this study we evaluated the effects of vitamin $\mathrm{D}_{3}$, and of its selective analog paricalcitol, on immature cardiomyocytes. Results show that vitamin $\mathrm{D}_{3}$ induces cAMP-mediated cell proliferation and significant intracellular calcification. Paricalcitol, however, induces cell differentiation, morphological modifications in cell shape and size, and no intracellular calcification. Furthermore, vitamin $\mathrm{D}_{3}$ and paricalcitol differently affect cardiomyoblasts responses to acetylcholine treatment. In conclusion, our results demonstrate that the effects of vitamin $\mathrm{D}_{3}$ and paricalcitol on cardiomyoblasts are different and, if these in vitro observations could be extrapolated in vivo, they suggest that paricalcitol has the potential for cardiovascular protection without the risk of inducing intracellular calcification.
\end{abstract}

Reprinted from Nutrients. Cite as: Pacini, S.; Morucci, G.; Branca, J.J.V.; Aterini, S.; Amato, M.; Gulisano, M.; Ruggiero, M. Effects of Vitamin $\mathrm{D}_{3}$ and Paricalcitol on Immature Cardiomyocytes: A Novel Role for Vitamin D Analogs in the Prevention of Cardiovascular Diseases. Nutrients 2013, 5, 2076-2092.

\section{Introduction}

The National Kidney Foundation Task Force on Cardiovascular Disease recommends that chronic kidney disease (CKD) patients be considered among the highest risk group for developing cardiovascular (CV) disease [1]. In fact, CV disease is more prevalent in patients with CKD than in the general population [2] and it is the leading cause of death in patients with end-stage renal disease [1]. It has been reported that nearly $30 \%$ of deaths among patients with CKD are to be attributed to cardiovascular causes [3].

CKD associated hyperparathyroidism and mineral metabolism disorders, such as hyperphosphatemia, have been significantly correlated with vascular and visceral calcifications, and consequently with increased risk of CV disease [4].

In addition to vascular problems, left ventricular hypertrophy, subclinical systolic dysfunction, and diastolic dysfunction have been consistently observed in subjects with $\mathrm{CKD}$ [5]. It is conjectured that abnormal diastolic function is an independent predictor of decreased aerobic capacity during the early stages of CKD [6]. 
Nevertheless, even though numerous factors have been implicated in the aetiology of the cardiac abnormalities observed in patients with CKD, the exact aetiology of the observed cardiac changes still remains unclear. A number of cardiac pathologies, including heart failure, are associated with alterations in myocardial energy metabolism [7] as well as with the activation of different intracellular signal transduction pathways.

Vitamin $\mathrm{D}_{3}$ (in its active form, i.e., calcitriol or 1,25-dihydroxyvitamin $\mathrm{D}_{3}$ ), as many other direct-acting positive inotropic agents, stimulates cyclic AMP (cAMP) formation [8], a major mediator of the amplitude and time course of cardiac contraction [8]. This cAMP-mediated inotropic effect occurs through activation of a variety of cAMP-dependent protein kinases that are capable of phosphorylating a series of proteins that affect the energetic status of the cells and alter the flux of calcium in the sarcoplasm [8].

In fact, calcium release and energetic systems are strictly and subtly modulated by different molecules, and an imbalance of sympathetic and parasympathetic drive to the heart represents an important risk factor for cardiac death in patients with renal insufficiency [9].

Since vitamin $\mathrm{D}_{3}$, together with dietary restrictions and phosphate binders, represents the primary medication to treat secondary hyperparathyroidism and the associated calcium and phosphate metabolic alterations in $\mathrm{CKD}$, interest in the role of vitamin $\mathrm{D}_{3}$ axis in the cardiovascular system recently increased. Thus, the vitamin D axis, which plays a critical role in the development of CKD, includes vitamin D, the polymorphic vitamin D receptor (VDR), and the vitamin D-binding protein (Gc-globulin) [10]. That is the precursor of a potent macrophage activating factor (GcMAF), which has potent effects on the immune system and angiogenesis [11].

In parallel, with this interest for beneficial effects [12], however, other studies warned that an excess of vitamin $\mathrm{D}_{3}$ increases the risk of hypercalcemia and vascular calcifications thus increasing the risk for CV and reducing survival in patients with CKD [13,14].

In this study we investigated the effect of vitamin $\mathrm{D}_{3}$ as well as of one of its non-hypercalcemic analogs, paricalcitol, on cultured cardiomyocytes. Paricalcitol (19-nor-1,25-dihydroxyvitamin $\mathrm{D}_{2}$ ) is a vitamin $\mathrm{D}_{3}$ analog, acting as a selective VDR activator; for this reason it might provide a vitamin $\mathrm{D}_{3}$-like protective efficacy without the hypercalcemic and hypophosphatemic side effects of vitamin $\mathrm{D}_{3}$, thus representing a potentially useful tool against the cardio-renal syndrome [12,15]. In particular, to better evaluate the modifications of the intracellular energy status as well as the modifications in signal transduction, in this study we compared the effects of vitamin $\mathrm{D}_{3}$, to those of paricalcitol in a myoblastic cell line H9c2, not completely differentiated showing electrophysiological and biochemical properties of cardiac muscle tissue [16]. Proliferation, mitochondrial activity, morphological alterations, calcification, cAMP pathway activation, and response to acetylcholine were thus investigated.

\section{Experimental Section}

\subsection{Pharmacological Agents}

Vitamin $D_{3}$ and Paricalcitol were respectively obtained from Sigma Aldrich, Milano, Italy and from Abbott, Roma, Italy. Due to differences in test compounds, two different treatment vehicles 
were used during the study: $100 \%$ ethanol (for vitamin $\mathrm{D}_{3}$ ) and $30 \%$ polyethylene glycol $/ 20 \%$ ethanol in water (for paricalcitol). Preliminary experiments did not show significant differences among different concentrations of vehicles in cell responses (not shown). Therefore, the different vehicle concentrations data were pooled for the analysis in this report. Vitamin D binding protein-derived macrophage activating factor (GcMAF) was obtained from Immuno Biotech Ltd. (Guernsey, Channel Islands). $\beta$-Glycerol-phosphate ( $\beta$-GP), ethanol, polyethylene glycol, and acetylcholine were obtained from Sigma Aldrich, Milano, Italy.

\subsection{Cell Cultures}

H9c2 cells, immortalized ventricular myocytes derived from rat (Rattus norvegicus) embryos, were obtained from the "Istituto Zooprofilattico della Lombardia e dell'Emilia Romagna", Brescia, Italy. H9c2 is a myoblast cell line not yet completely differentiated into non-proliferating myocytes/ myotubes and with electrophysiological and biochemical properties of both skeletal and cardiac muscle tissue. Cell were grown in a monolayer culture at $37{ }^{\circ} \mathrm{C}$ in a $5 \% \mathrm{CO}_{2}$ humidified atmosphere in Dulbecco's modified eagle's medium (DMEM) supplemented with $10 \%$ foetal bovine serum (FBS). The medium was renewed every two to three days, when the cells reached sub-confluence (70\%-80\%). Vitamin $\mathrm{D}_{3}$ and paricalcitol were added to the cells at the following concentrations: [1 nM], [10 nM], [100 nM], and [300 nM]. Treatment with acetylcholine was performed with and without vitamin $\mathrm{D}_{3}$ and paricalcitol $[300 \mathrm{nM}]$ at the concentration of $[10 \mu \mathrm{M}]$ for $1 \mathrm{~h}$.

\subsection{Cell Viability and Proliferation}

The effects of vitamin $\mathrm{D}_{3}$ and paricalcitol on $\mathrm{H}_{9} 2$ cell lines, were evaluated by the cell viability assay using the 2-(2-methoxy-4-nitrophenyl)-3-(4-nitrophenyl)-5-(2,4-disulf-ophenyl)-2 $H$ tetrazolium monosodium salt (WST-8) reagent (Sigma Aldrich, Milano, Italy), according to the manufacturer's protocol. This test is based on a colorimetric conversion of a tetrazolium salt (WST-8) to water-soluble formazan products as previously described [17]. H9c2 cells were plated at density of $2 \times 10^{4}$ per well in a 96-well plate with fresh growth medium for one day. Then, vitamin $\mathrm{D}_{3}$ and paricalcitol were diluted to appropriate concentration and added to the culture medium for $48 \mathrm{~h}$. After the exposure-time, WST- 8 solution was added to each well and the micro-plate incubated for $4 \mathrm{~h}$ at $37^{\circ} \mathrm{C}$. Cell viability was measured by a micro-plate reader (Multiscan FC/FL6111900 model, Thermo Fisher Scientific, M-Medical, Milano, Italy) at $450 \mathrm{~nm}$ after incubation-time. To directly evaluate cell proliferation, a cell count by a haemocytometer (Housser Scientific, Horsham, PA, USA) was also performed. Also the evaluation of cardiomyocytes response to acetylcholine in the presence of vitamin $\mathrm{D}_{3}$ and paricalcitol was evaluated by cell viability assay. In each experiment, at least seven wells were used for each experimental point.

\subsection{Cell Morphology}

To evaluate changes in $\mathrm{H} 9 \mathrm{c} 2$ cell morphology before and after vitamin $\mathrm{D}_{3}$ and paricalcitol exposure, contrast phase microscopy and Haematoxylin-Eosin staining were performed.

Briefly, H9c2 cells were seeded on a cover slip at the density of $10 \times 10^{4}$ cells/cover slip. 
Cells were grown in culture medium with different concentrations of vitamin $\mathrm{D}_{3}$ and paricalcitol for $48 \mathrm{~h}$. For the Haematoxylin-Eosin staining, H9c2 cells were fixed with paraformaldehyde $0.5 \%$ in $[0.1 \mathrm{M}]$ PBS (phosphate buffer saline) and dried for $2 \mathrm{~h}$. Then, cells were stained with Haematoxylin-Eosin dye following a standard protocol.

Cell morphology was evaluated by optical microscope (XDS-2 + M-795 model, Optika Microscope, Milano, Italy) and digital images were captured.

After the treatment at different concentration of vitamin $\mathrm{D}_{3}$ and paricalcitol for $48 \mathrm{~h}$, cell size of H9c2 was measured by Adobe Pohotoshop CS2 software (9.0 version, Adobe System Incorporated 1990-2005; Adobe Systems Inc., San Jose, CA, USA) and Scion Image software (Beta 3b freeware version; based on NIH Image for Macintosh by Wayne Rasband, National Institute of Health, USA and modified for Windows by Scion Corporation; July, the 23rd 1998; Scion Co., Frederick, MD, USA). Images of $\mathrm{H} 9 \mathrm{c} 2$ in Haematoxylin-Eosin staining were captured using a digital camera (DIGI-full HD video/photo camera, Optika Microscopes, Milano, Italy). Digital images were adjusted by Adobe Photoshop CS2 to remove from the picture everything (background) except the cells and then $\mathrm{H} 9 \mathrm{c} 2$ cells size was measured by Scion Image.

\section{5. cAMP Assay}

$\mathrm{H} 9 \mathrm{c} 2$ cells were cultured at density of $1 \times 10^{4}$ cells per well in a 6-well plate with DMEM supplemented with $10 \%$ FBS and exposed at [1 nM], [10 nM], [100 nM], and [300 nM] vitamin $\mathrm{D}_{3}$ and paricalcitol for $48 \mathrm{~h}$. The cAMP intracellular level was evaluated by a direct competitive immunoassay for sensitive and quantitative determination using a cAMP assay kit (Abnova, Heidelberg, Germany) according to the Manufacturer's instructions. After the treatments, the experiment was blocked adding $[0.1 \mathrm{M}] \mathrm{HCl}$. Then, after scraping cells from the substrate, the suspension was centrifuged for $10 \mathrm{~min}$ at $2340 \mathrm{rpm}$. The supernatant was assayed for cAMP concentration by a micro-plate reader (Multiscan FC/FL6111900 model, Thermo Fisher Scientific, M-Medical, Milano, Italy) at $450 \mathrm{~nm}$ after incubation-time. cAMP values were normalized to the assayed proteins.

\subsection{Analysis of H9c2 Calcification}

To evaluate the deposits of calcium in $\mathrm{H} 9 \mathrm{c} 2$ after vitamin $\mathrm{D}_{3}$ and Paricalcitol exposure, cells were seeded on cover slip at density $10 \times 10^{4}$ per cover slip and cultured in their culture medium (DMEM $+10 \%$ FBS). The medium was substituted with fresh culture medium every two days. After five days from confluence, fresh medium containing different concentration of vitamin $\mathrm{D}_{3}$ and paricalcitol was used for a further seven days. To amplify the calcification effects of vitamin $\mathrm{D}_{3}$ and paricalcitol, the medium was enriched with [4 mM] $\beta$-GP (calcification media). Quantification of calcium deposits was performed by von Kossa staining (von Kossa Kit, Bio Optica, Milano, Italy); cells were soaked in a lithium carbonate solution (for $10 \mathrm{~min}$ ) to prevent false results caused by the presence of uric acid and urates in the samples; then, cells were treated with a silver nitrate solution, left in dark for $1 \mathrm{~h}$, and then a reduction solution was applied for $5 \mathrm{~min}$; then, samples were treated with a sodium sulphite solution for 5 min and with a Mayer's Carmalum solution to contrast the calcium deposits. The intracellular calcification was evaluated by optical microscope (XDS-2 + M-795 model, Optika Microscope, 
Milano, Italy) and images of H9c2 cell line in von Kossa staining were captured using a digital camera (DIGI-full HD video/photo camera, Optika Microscopes, Milano, Italy) and then analyzed.

\subsection{Statistics}

All values are means \pm standard error (SE) for at least three determinations. Differences between experimental points were evaluated by Student's $t$-test. $p$ was considered statistically significant when $p<0.05$.

\section{Results}

\subsection{Cell Viability and Proliferation}

Data obtained from cell viability assay show that treatment for $48 \mathrm{~h}$ with vitamin $\mathrm{D}_{3}$ or paricalcitol induces a similar dose-dependent response. In particular, [1 nM], [10 nM], and [100 nM] vitamin $\mathrm{D}_{3}$ and paricalcitol induce a statistically significant increase in cell viability in comparison to untreated control cells, as well as in comparison to cells treated with appropriate vehicles alone (Figure 1).

Figure 1. Cell viability assay in $\mathrm{H} 9 \mathrm{c} 2$ cells shows that $[1 \mathrm{nM}],[10 \mathrm{nM}]$, and $[100 \mathrm{nM}]$ Vitamin $\mathrm{D}_{3}$ and Paricalcitol induce no significant differences in cell viability in comparison to control cells (both untreated and treated with appropriate vehicles). On the contrary, a significant difference $(p<0.05)$ is observed when H9c2 are treated with $[300 \mathrm{nM}]$ vitamin $\mathrm{D}_{3}$. Data are expressed as means \pm S.E.M. (standard error of the mean) vs. control. * Statistically significant difference $(p<0.05)$ of vitamin $\mathrm{D}_{3}$ (or paricalcitol) vs. controls (untreated cells and vehicles). Black dot indicates a statistically significant difference between vitamin $\mathrm{D}_{3}$ and paricalcitol.

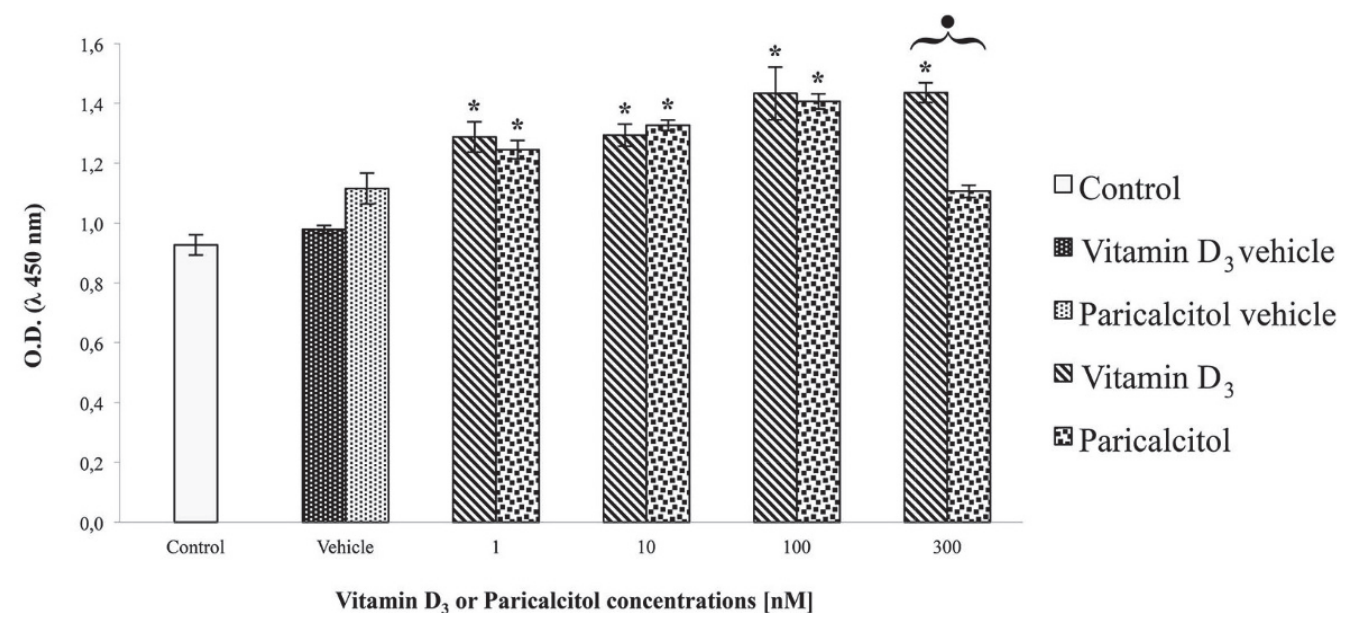

However, when cells are treated with $[300 \mathrm{nM}]$ vitamin $\mathrm{D}_{3}$ or $[300 \mathrm{nM}]$ paricalcitol a significant difference $(p<0.05)$ in cell viability occurs (Figure 1); vitamin $\mathrm{D}_{3}$ induces an increase in cell viability while paricalcitol induces a significant decrease.

Since cell viability is correlated, but not completely superimposable to cell proliferation, we tested the effects of vitamin $\mathrm{D}_{3}$ and paricalcitol on cell proliferation by direct cell count. Results show that 
only when cells are treated with $[300 \mathrm{nM}]$ vitamin $\mathrm{D}_{3}$ a significant increase in cell number and density occurs (Figure 2). No significant increases in cell number and density are observed comparing controls (untreated or treated with appropriate vehicles) to exposed cells with all the other experimental concentrations and stimuli (Figure 2).

Figure 2. A significant increase in cell density is observed when cells are treated with $[300 \mathrm{nM}]$ Vitamin $\mathrm{D}_{3}$ in comparison to controls. Data are expressed as means \pm S.E.M. vs. control. * Statistically significant difference $(p<0.05)$ of vitamin $\mathrm{D}_{3} v s$. controls (untreated cells and vehicles).

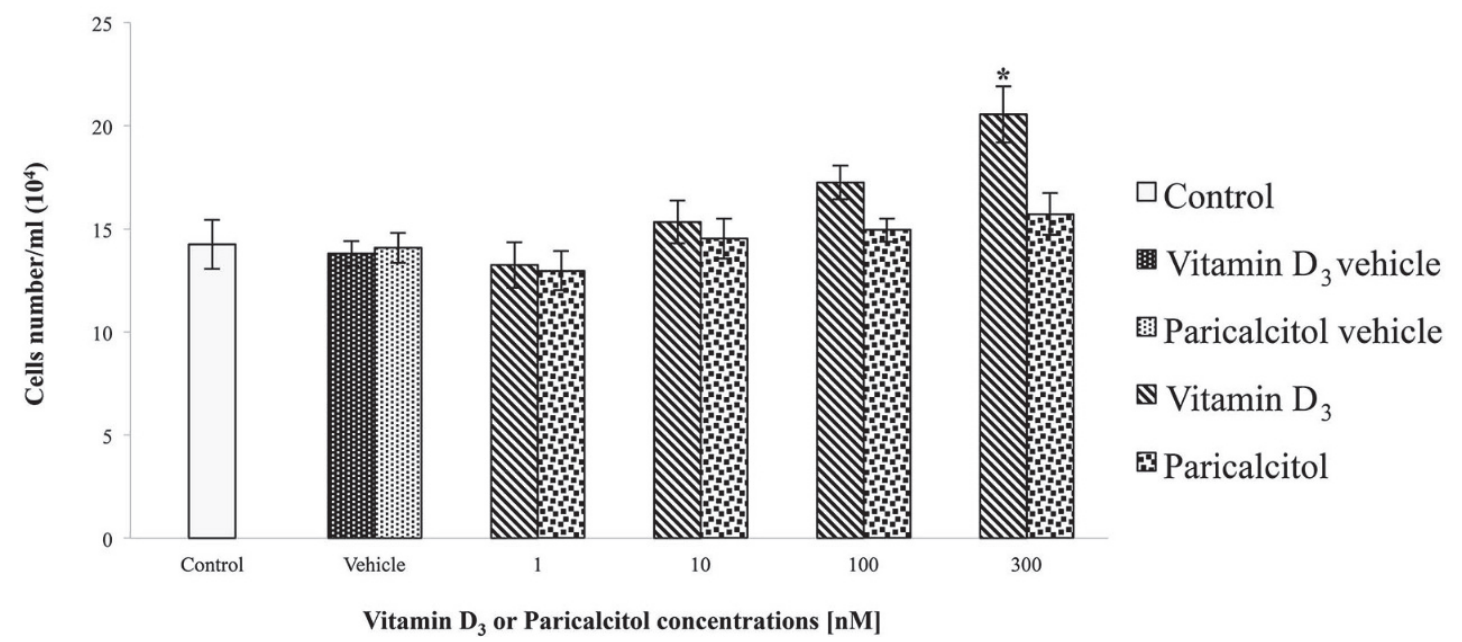

\section{2. cAMP Levels}

Data obtained after treatment of $\mathrm{H} 9 \mathrm{c} 2$ cells for $48 \mathrm{~h}$, with vitamin $\mathrm{D}_{3}$ and paricalcitol at different concentrations, show different trends for the two molecules: vitamin $\mathrm{D}_{3}$ induces a dose-dependent increase in cAMP level (even though not statistically significant), while paricalcitol induces a dose-dependent decreases in comparison to controls (Figure 3). For [10 nM], [100 nM], and [300 $\mathrm{nM}$ ] paricalcitol, differences in cAMP level are statistically significant in comparison to controls. At the same concentrations the differences in cAMP levels induced by treatment with vitamin $\mathrm{D}_{3}$ and paricalcitol are also statistically significant. 
Figure 3. A different pattern in cAMP levels can be observed when cells are treated with vitamin D3 or Paricalcitol. Data are expressed as means \pm S.E.M. vs. control. * Statistically significant difference $(p<0.05)$ of vitamin $\mathrm{D}_{3}$ (or paricalcitol) vs. controls (untreated cells and vehicles). Black dots indicate statistically significant differences between vitamin $\mathrm{D}_{3}$ and paricalcitol.

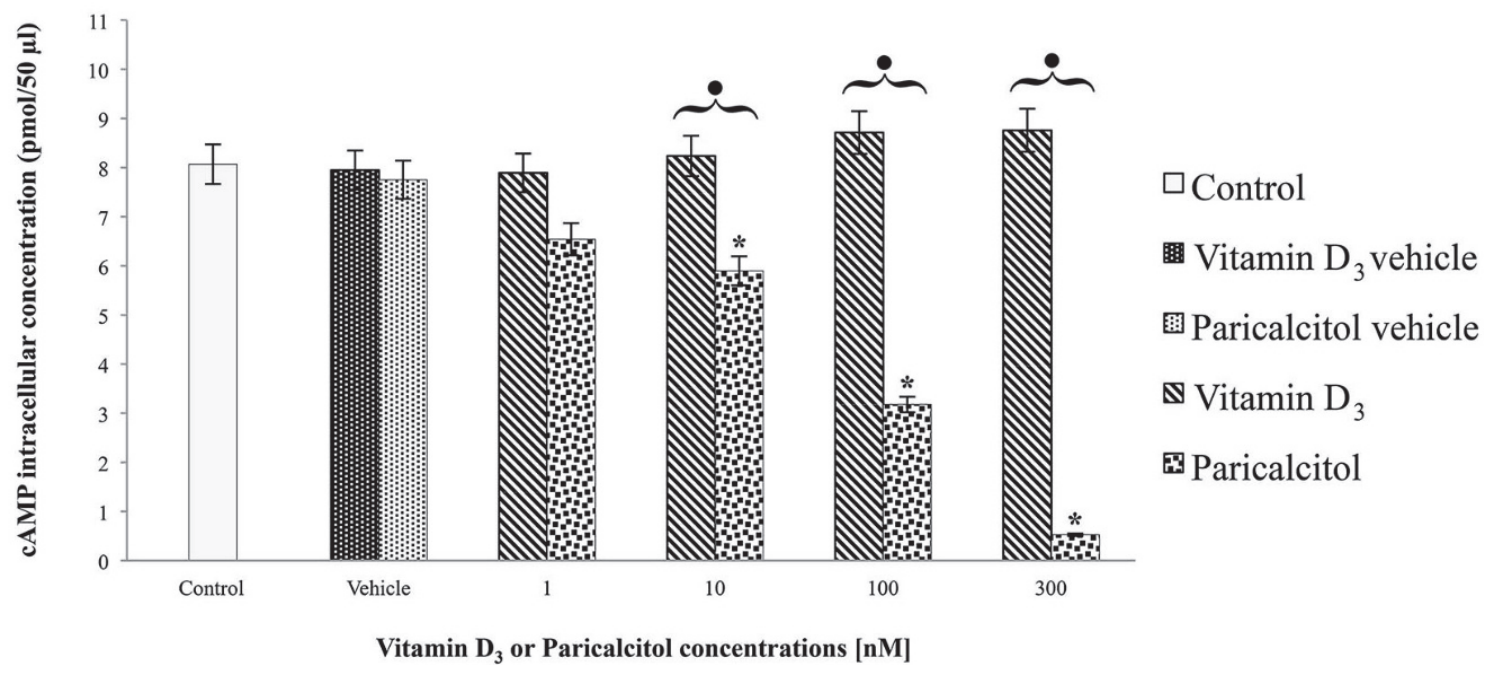

\subsection{Cell Morphology}

Significant changes in cell morphology are observed when cell are treated with $[300 \mathrm{nM}]$ paricalcitol. No significant changes are observed when cells are treated both with lower concentrations of paricalcitol, and with vitamin $\mathrm{D}_{3}$. Direct observation by contrast light microscopy, as well as after Haematoxilyn-Eosin staining, reveals relevant modifications in cell shape between treated and control cells (Figure 4). Control cells show a spindle-like shape with an oval nucleus, localized in the central part of the cell, and several elongations arising from the cell body. Cells tend to establish contact with each other so that cytoplasmic elongations appear sometimes very long and thin (Figure 4A). 
Figure 4. H9c2 cell morphology analysis by contrast phase microscopy and after Haematoxylin-Eosin staining. Panels A: untreated and vehicles treated cells observed by contrast phase microscopy (upper panel) and after Haematoxylin-Eosin staining (lower panel). Cells are spindle-like and with evidently thin and numerous cytoplasmic elongations (arrows). Panels B: cells treated with [300 nM] vitamin $\mathrm{D}_{3}$. Cells do not show significant morphological changes in comparison to controls both when observed by contrast phase microscopy (upper panel) and after staining (lower panel). Panels C: cells treated with $[300 \mathrm{nM}]$ paricalcitol in some cases appear roundish and with a reduced number of cytoplasmic elongations (arrows). Upper panels: total magnification $150 \times$. Lower panels: total magnification 100×. Bars: $100 \mu \mathrm{m}$.

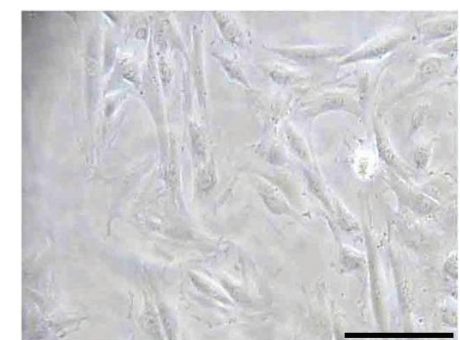

A

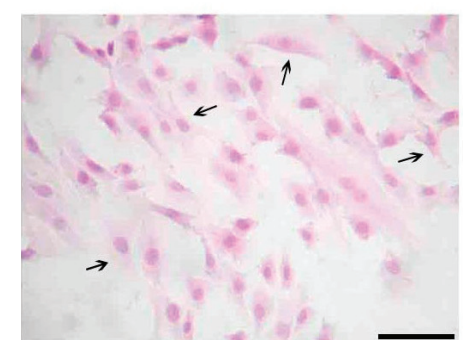

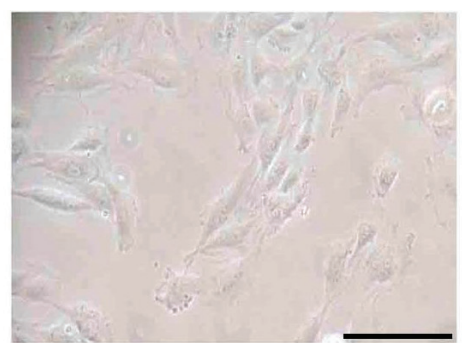

B

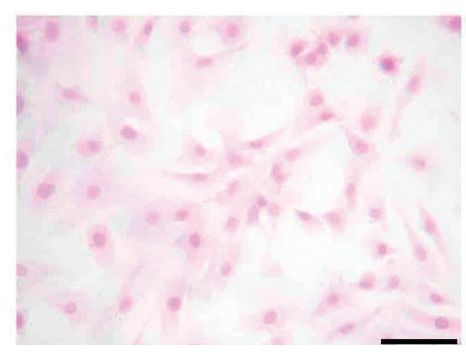

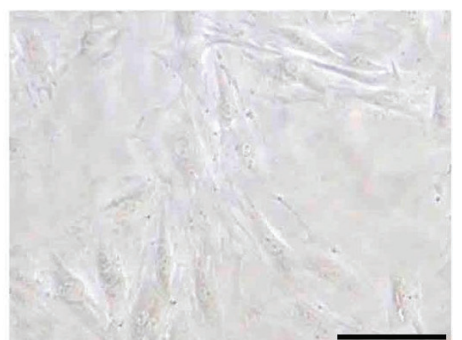

C

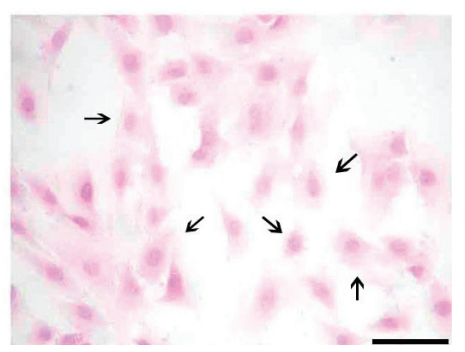

Treatment of $\mathrm{H} 9 \mathrm{c} 2$ cells with $[300 \mathrm{nM}]$ vitamin $\mathrm{D}_{3}$ does not induce significant changes in cell morphology (Figure 4B), whereas the same concentration of paricalcitol induce significant modification (Figure 4C): a great number of cells appear roundish instead of spindle-like and the number of cytoplasmic elongations appears greatly reduced. The changes in cell morphology are associated with an increase in cell size. In fact, when cells are treated with [300 nM] paricalcitol but not with the same concentration of vitamin $\mathrm{D}_{3}$ a significant difference in cell size can be observed (Figure 5). After treatment with $[300 \mathrm{nM}]$ paricalcitol, cell diameter increases from $15 \pm 1.2 \mu \mathrm{m}$ (mean \pm S.E.M.) to $20 \pm 1.0 \mu \mathrm{m}$ (mean \pm S.E.M.). Cell size, expressed as cell area and calculated by appropriate software as described in the Section 2 Materials and Methods, appears about 40\% wider: after the treatment with $[300 \mathrm{nM}]$ paricalcitol cell surface increases from $106.96 \pm 7.36 \mu^{2}$ (mean area $\pm \mathrm{SEM}$ ) to $211.16 \pm 6.12 \mu \mathrm{m}^{2}$ (mean area \pm S.E.M.). 
Figure 5. A significant difference in cell size is observed when cells are treated with $[300 \mathrm{nM}]$ paricalcitol. Data are expressed as means \pm S.E.M. vs. control. * Statistically significant difference $(p<0.05)$ of paricalcitol vs. controls (untreated cells and vehicles). Black dot indicates a statistically significant difference between vitamin $\mathrm{D}_{3}$ and paricalcitol.

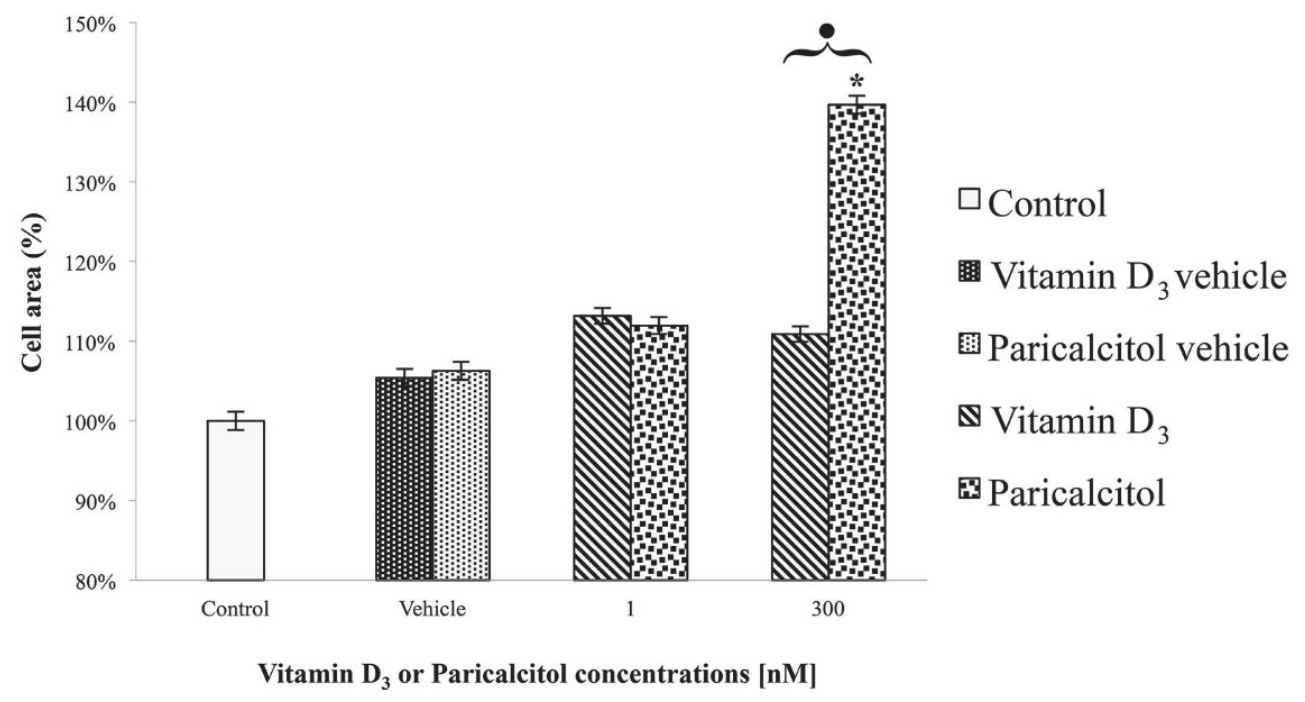

\subsection{Acetylcholine and Cell Viability}

Treatment with $[10 \mu \mathrm{M}]$ acetylcholine induces, as expected, a significant decrease in the mitochondrial activity of $\mathrm{H} 9 \mathrm{c} 2$ whereas stimulation with [300 nM] vitamin $\mathrm{D}_{3}$ (or [300 nM] paricalcitol) induces a significant increase in mitochondrial activity in comparison to control, untreated cells (Figure 6). 
Figure 6. $[10 \mu \mathrm{M}]$ acetylcholine, acting on $\mathrm{H} 9 \mathrm{c} 2$ by interaction with muscarinic M2 receptors, induces a significant decrease in mitochondrial activation in comparison to untreated cells. When $[300 \mathrm{nM}]$ vitamin $\mathrm{D}_{3}$ and $[10 \mu \mathrm{M}]$ acetylcholine are at the same time present in the medium, no changes are observed; on the contrary, when $[300 \mathrm{nM}]$ paricalcitol and $[10 \mu \mathrm{M}]$ acetylcholine are present in the medium, the effect of acetylcholine is abolished. Data are expressed as means \pm S.E.M. vs. control. * Statistically significant difference $(p<0.05)$ of paricalcitol $v s$. controls (untreated cells and vehicles). ** Statistically significant difference $(p<0.05)$ of paricalcitol + acetylcholine $v s$. acetylcholine. Black dot indicates a statistically significant difference between vitamin $\mathrm{D}_{3}$ and vitamin $\mathrm{D}_{3}+$ acetylcholine.

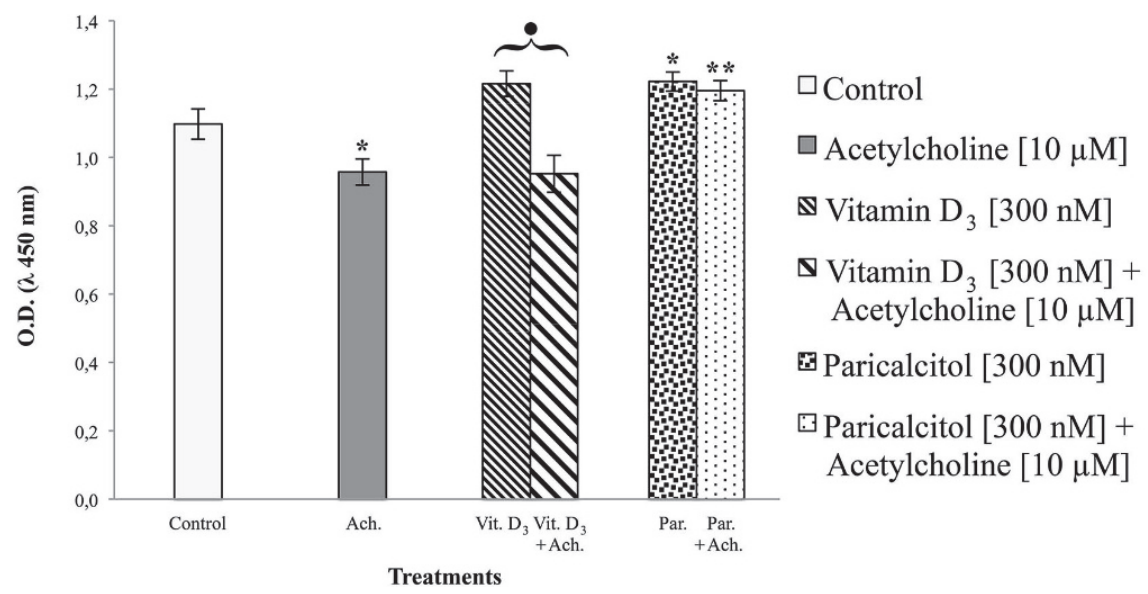

When $[10 \mu \mathrm{M}]$ acetylcholine and $[300 \mathrm{nM}]$ vitamin $\mathrm{D}_{3}$ are simultaneously present in the culture medium, no significant changes are observed in comparison to treatment with acetylcholine alone, but when $[10 \mu \mathrm{M}]$ acetylcholine and $[300 \mathrm{nM}]$ paricalcitol are used (instead of vitamin $\mathrm{D}_{3}$ ) the effect of the neurotransmitter is completely counteracted.

\subsection{Cell Calcification}

Figure 7 shows the effect of Vitamin $\mathrm{D}_{3}$ and Paricalcitol on intracellular calcification occurring in $\mathrm{H} 9 \mathrm{c} 2$ cardiomyocytes. [300 $\mathrm{nM}$ ] Vitamin $\mathrm{D}_{3}$ induces a significant increase in calcium incorporation in the cells in comparison to control cells (Figure 7A,B). [300 nM] Paricalcitol does not have a statistically significant effect on calcium content in $\mathrm{H} 9 \mathrm{c} 2$ cardiomyocytes in comparison to control, untreated cells (Figure 7C). 
Figure 7. Effect of vitamin $\mathrm{D}_{3}$ and paricalcitol on intracellular $\mathrm{H} 9 \mathrm{c} 2$ calcification. Panels A: control and vehicles treated cells. A background weak intracellular calcification is present: colored dots (dark pink) are evident into the cell cytoplasm. Panels B: [300 nM] vitamin $\mathrm{D}_{3}$ treatment induces a strong intracellular calcification in H9c2. Panels $\mathbf{C}$ : [300 $\mathrm{nM}$ ] paricalcitol induces an intracellular calcification at the same extent of that observed in controls. Upper panels: total magnification 100×. Lower panels: total magnification 200×. Bars: $100 \mu \mathrm{m}$. Panel D: percentage of calcification area. * Statistically significant difference $(p<0.01)$ in calcification induced by vitamin $\mathrm{D}_{3} v s$. controls (untreated cells). Black dot indicates a statistically significant difference $(p<0.01)$ in calcification induced by vitamin $\mathrm{D}_{3}$ vs. paricalcitol.
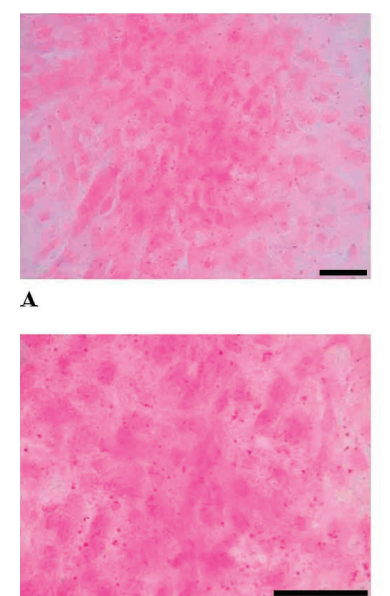

D
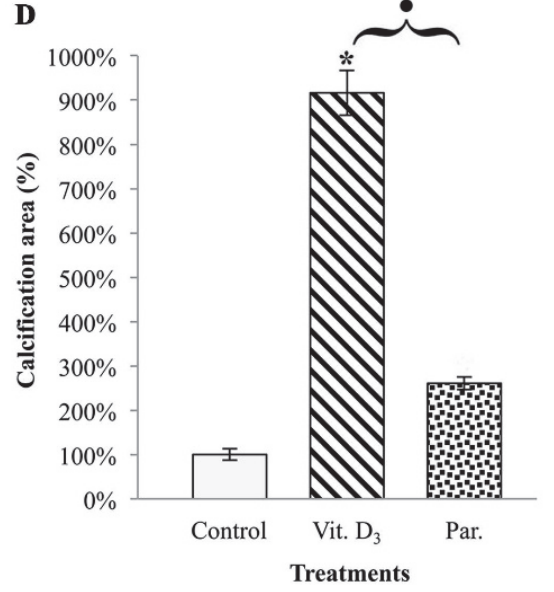

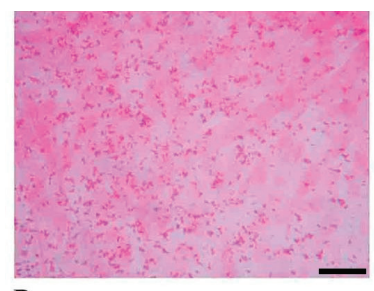

B

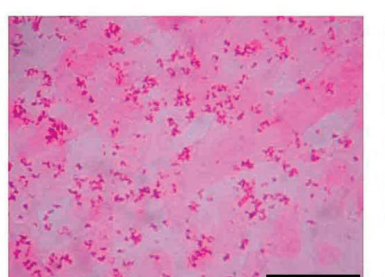

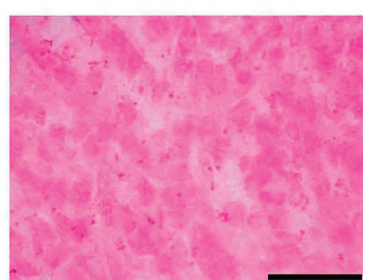

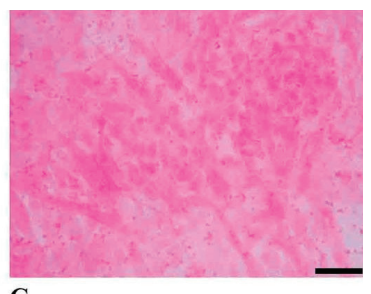

$\square$ Control

$\mathbb{\nabla}$ Vitamin $\mathrm{D}_{3}[300 \mathrm{nM}]$

옹 Paricalcitol $[300 \mathrm{nM}]$

\section{Discussion}

Recent observational studies provided support for a possible protective role for vitamin $\mathrm{D}_{3}$ in the $\mathrm{CKD}$ population $[1,2]$. Recent scientific evidence suggests that vitamin $\mathrm{D}_{3}$ may negatively regulate the renin-angiotensin system, inhibit cardiomyocyte hypertrophy and proliferation, as well as modulate and suppress the inflammatory response to blood vessel injury [18]. Cardiovascular diseases, especially atherosclerosis, have been reported to be the main causes of dialysis-related morbidity and mortality [2]. In the development of these vasculopathies, not only are traditional risk factors involved, but also CKD-related biochemical changes such as hypocalcemia, vitamin D deficiency and hyperparathyroidism, which are considered promoting factors. Previous in vitro 
studies [19] have demonstrated that endothelial cells stimulated with low $\mathrm{Ca}^{2+}$, high advanced glycation end products (AGEs), and parathyroid hormone (PTH), responded by increasing the expression and production of pro-inflammatory and atherosclerotic parameters such as interleukin 6 (IL6), nuclear factor kappa B (NFKB), and endothelial nitric oxide synthase (eNOS). These CKD-related biochemical changes are significantly counteracted by vitamin $\mathrm{D}_{3}$ treatment, which induces a decrease in the elevated IL-6 mRNA expression, positively affects the activity of NFKB, and normalizes the parameters associated with the eNOS system.

On the other hand, other studies highlighted that an excess of vitamin $\mathrm{D}_{3}$ increases the risk of hypercalcemia and vascular calcification, thus worsening CV risk and reducing survival in patients with CKD [4,20]. In fact, vitamin $\mathrm{D}_{3}$ belongs to the category of general VDR activators, having a wide range of affinity for the components of the vitamin $\mathrm{D}_{3}$ system, both for the vitamin $\mathrm{D}_{3}$-binding protein and for the nuclear VDR. Among the VDR activators, selective activators such as Paricalcitol play a major role in controlling mineral metabolism. These selective molecules act more efficiently on parathyroid glands rather than on intestine and bone; this leads both to a lower serum calcium and phosphorus increases and to an improvement of hyperplasia of the parathyroid gland and secondary hyperparathyroidism [15]. For these reasons, selective VDR activators could provide a vitamin D-like protective efficacy without the hypercalcemic and hypophosphatemic collateral effects, thus representing a potential therapeutic tool against the cardio-renal syndrome.

From data presented in this study, it emerges that selective activators of the VDR such as Paricalcitol are not only associated with less side effects in comparison to vitamin $\mathrm{D}_{3}$ but they also can also act directly on cardiomyocytes inducing morphological changes, differentiation, and variations in cell ability to respond to acetylcholine. The most notable differences in vitro were observed at high doses; this phenomenon could be interpreted considering the peculiar mode interaction between VDR and the genes that it regulates. A differential occupancy of the receptor might in fact be responsible for the difference in the array of genes that are being regulated by VDR [21].

In fact, one of the most potentially dangerous side effects associated with long term therapy with vitamin $\mathrm{D}_{3}$, i.e., intracellular calcification, appears to be much less relevant when the selective activator of vitamin $\mathrm{D}_{3}$ axis paricalcitol is used instead. From our results it appears that vitamin $\mathrm{D}_{3}$ and its analog paricalcitol activate different signal transduction pathways in H9c2 cells: vitamin $\mathrm{D}_{3}$ induces cell proliferation associated with an increase of cAMP, whereas paricalcitol induces, at the same concentration, cell differentiation, associated with cAMP decreases. In fact, low levels of intracellular cAMP are necessary to induce muscle cell differentiation of different myogenic cell lines [16] such as H9c2. It is worth remembering that these cells derive from embryonic rat ventricle; thus, they are not yet completely differentiated into non-proliferating myocytes/myotubes and they maintain the electrophysiological and biochemical properties of both skeletal and cardiac muscle tissue [16]. Data in literature demonstrate that H9c2 cell differentiation is associated with an increase of myogenin expression, a typical skeletal muscle protein [16], and with an increase of myotrophin, a protein associated with normal cardiomyocyte development [22].

Treatment with high concentration of paricalcitol also induces a significant increase in cell size. Cardiomyocyte hypertrophy occurs in response to numerous agonists [23] and growth factor signaling pathways that induce hypertrophy are intimately interconnected with intracellular pathways 
that link changes in calcium homeostasis with the reprogramming of cardiac gene expression [24]. The calcium, calmodulin-dependent protein, phosphatase calcineurin, serves as a point of convergence of these different intracellular pathways [25] and has been shown to be necessary and sufficient for hypertrophy in response to a wide range of signals in vivo and in vitro [26]. Therefore, cardiomyocyte hypertrophy in response to paricalcitol has to be interpreted as an adaptive response that parallels and accompanies cell differentiation. Future studies will assess whether this adaptive responses will occur also in fully differentiated cardiomyocytes.

Given the recent interest in the role of vitamin $\mathrm{D}_{3}$ deficiency in CV system and heart functionality and integrity, we investigated the effects on heart diastolic function of the components of the vitamin $\mathrm{D}_{3}$ axis that are known to be associated with VDR, that are vitamin $\mathrm{D}_{3}$, paricalcitol, and GcMAF, and preliminary data demonstrated that the components of the vitamin $\mathrm{D}_{3}$ axis differently affect the diastolic function. Diastolic function has been evaluated by a rapid and non-invasive method, based on ultrasounds and measuring the interval in milliseconds between aortic closure and mitral opening; this interval of time is known as iso-volumetric relaxation time (IVRT) [27]. Physiologically, it is the time that it takes to pump free calcium out of the myocardium, to produce relaxation of the myofibrils and to allow ventricular filling, and takes all the available free energy in the heart to do so. IVRT is inversely related to the cellular free energy so the higher the IVRT is, the lower the cellular free energy is. From our data emerged that, while vitamin $\mathrm{D}_{3}$ increased IVRT, paricalcitol significantly decreased it, thus demonstrating a positive inotropic effects on the levels of cellular free energy. In fact, these results can be interpreted as that paricalcitol increased the cellular free energy, a novel positive feature of this analog that had not been described before. The effects of GcMAF were comparable to those obtained with paricalcitol. These results can be interpreted considering that paricalcitol and GcMAF showed similar, although not superimposable, effects at the cellular and organism level in other model systems. In fact, both compounds stimulated cAMP formation in human mononuclear cells with the highest effect on the "bb" genotype of the VDR. Both paricalcitol and GcMAF inhibited the angiogenesis induced by prostaglandin E1 in the chick embryo chorionallantoic membrane [28]. This unexpected effect of paricalcitol suggests that its advantage over vitamin $\mathrm{D}_{3}$ might be far more ranging than simply being non-hypercalcemic. In fact, the decrease of cellular free energy in a chronic condition is at the basis of a number of signs and symptoms that are negatively associated with the prognosis. Our observation that paricalcitol and GcMAF show an inotropic effect at variance with vitamin $\mathrm{D}_{3}$ opens the perspective of administering this molecule in a variety of chronic conditions where the use of vitamin $\mathrm{D}_{3}$, although potentially beneficial, has been discouraged by the concomitant and potentially harmful side effects of vitamin $\mathrm{D}_{3}$. Since the majority of the results presented in this study have been conducted in vitro in a rat myoblast cell line, not yet completely differentiated, the results cannot be directly extrapolated to clinical recommendations. Nevertheless, such a model system could provide novel indications that can be applied to the clinic. Thus, paricalcitol is a molecule that has been safely used for years and this study demonstrates that it shows some novel effects that could be exploited in order to maximize therapeutic effects while, at the same time, minimizing side effects. The stimulation of cardiomyocyte viability and differentiation without inducing intracellular calcification, and the positive inotropic effect in the absence of hypercalcemia, might therefore represent novel fields of application of this molecule. 


\section{Conclusions}

Biochemical modifications, promoting factors and pro-inflammatory interleukins play a crucial role in the development of cardiovascular diseases in CKD patients as well as in the increase of mortality in most of the chronic diseases. Vitamin $\mathrm{D}_{3}$ treatment counteracts most of the CKD related modifications in cellular homeostasis and signaling decreasing the elevated IL-6 mRNA expression, positively affecting the activity of NFKB and normalizing the eNOS system. However it has been demonstrated that vitamin $\mathrm{D}_{3}$ long-term treatment is strongly related to vascular calcification with consequent reduction of patients' survival. For this reason selective activators of VDR (such as paricalcitol) and factors involved in the vitamin $\mathrm{D}_{3}$ axis (as GcMAF) might represent new therapeutic tools both for treatment of CKD and for treatment of chronic conditions where the use of vitamin $\mathrm{D}_{3}$, although potentially beneficial, has been discouraged for the harmful side effects.

\section{Acknowledgments}

Fondi di Ricerca di Ateneo and PRIN 2009.

\section{Conflicts of Interest}

All the Authors declare no conflicts of interest.

\section{References}

1. Levey, A.S.; Beto, J.A.; Coronado, B.E.; Eknoyan, G.; Foley, R.N.; Kasiske, B.L.; Klag, M.J.; Mailloux, L.U.; Manske, C.L.; Meyer, K.B.; et al. Controlling the epidemic of cardiovascular disease in chronic renal disease: What do we know? What do we need to learn? Where do we go from here? National Kidney Foundation Task Force on Cardiovascular Disease. Am. J. Kidney Dis. 1998, 32, 853-906.

2. Foley, R.N.; Parfrey, P.S.; Sarnak, M.J. Clinical epidemiology of cardiovascular disease in chronic renal disease. Am. J. Kidney Dis. 1998, 32, S112-S119.

3. Hojs, R.; Bevc, S.; Ekart, R. Biomarkers in hemodialysis patients. Adv. Clin. Chem. 2012, 57, 29-56.

4. Blacher, J.; Guerin, A.P.; Pannier, B.; Marchais, S.J.; London, G.M. Arterial calcifications, arterial stiffness, and cardiovascular risk in end-stage renal disease. Hypertension 2001, 38, 938-942.

5. Artaza, J.N.; Mehrotra, R.; Norris, K.C. Vitamin D and the cardiovascular system. Clin. J. Am. Soc. Nephrol. 2009, 4, 1515-1522.

6. Weaver, D.J., Jr.; Kimball, T.R.; Knilans, T.; Mays, W.; Knecht, S.K.; Gerdes, Y.M.; Witt, S.; Glascock, B.J.; Kartal, J.; Khoury, P.; Mitsnefes, M.M. Decreased maximal aerobic capacity in pediatric chronic kidney disease. J. Am. Soc. Nephrol. 2008, 19, 624-630.

7. Ventura-Clapier, R.; Garnier, A.; Veksler, V. Energy metabolism in heart failure. J. Physiol. 2004, 555, 1-13. 
8. Feldman, M.D.; Copelas, L.; Gwathmey, J.K.; Phillips, P.; Warren, S.E.; Schoen, F.J.; Grossman, W.; Morgan, J.P. Deficient production of cyclic AMP: Pharmacologic evidence of an important cause of contractile dysfunction in patients with end-stage heart failure. Circulation 1987, 75, 331-339.

9. Oberhauser, V.; Schwertfeger, E.; Rutz, T.; Beyersdorf, F.; Rump, L.C. Acetylcholine release in human heart atrium: Influence of muscarinic autoreceptors, diabetes, and age. Circulation 2001, 103, 1638-1643.

10. Chishimba, L.; Thickett, D.R.; Stockley, R.A.; Wood, A.M. The vitamin D axis in the lung: A key role for vitamin D-binding protein. Thorax 2010, 65, 456-462.

11. Yamamoto, N.; Naraparaju, V.R. Immunotherapy of BALB/c mice bearing Ehrlich ascites tumor with vitamin D-binding protein-derived macrophage activating factor. Cancer Res. 1997, 57, 2187-2182.

12. Cozzolino, M.; Ronco, C. The impact of paricalcitol on left ventricular hypertrophy. Contrib. Nephrol. 2011, 171, 161-165.

13. Goldsmith, D.J.; Covic, A.; Sambrook, P.A.; Ackrill, P. Vascular calcification in long-term haemodialysis patients in a single unit: A retrospective analysis. Nephron 1997, 77, 37-43.

14. Milliner, D.S.; Zinsmeister, A.R.; Lieberman, E.; Landing, B. Soft tissue calcification in pediatric patients with end-stage renal disease. Kidney Int. 1990, 38, 931-936.

15. Cozzolino, M.; Bruschetta, E.; Stucchi, A.; Ronco, C.; Cusi, D. Role of vitamin D receptor activators in cardio-renal syndromes. Semin. Nephrol. 2012, 32, 63-69.

16. Pagano, M.; Naviglio, S.; Spina, A.; Chiosi, E.; Castoria, G.; Romano, M.; Sorrentino, A.; Illiano, F.; Illiano, G. Differentiation of H9c2 cardiomyoblasts: The role of adenylate cyclase system. J. Cell. Physiol. 2004, 198, 408-416.

17. Morgan, D.M. Tetrazolium (MTT) assay for cellular viability and activity. Methods Mol. Biol. 1998, 79, 179-183.

18. Gonzalez-Parra, E.; Rojas-Rivera, J.; Tuñón, J.; Praga, M.; Ortiz, A.; Egido, J. Vitamin D receptor activation and cardiovascular disease. Nephrol. Dial. Transplant. 2012, 27, 17-21.

19. Talmor-Barkan, Y.; Rashid, G.; Weintal, I.; Green, J.; Bernheim, J.; Benchetrit, S. Low extracellular $\mathrm{Ca}^{2+}$ : A mediator of endothelial inflammation. Nephrol. Dial. Transplant. 2009, 24, 3306-3312.

20. Teng, M.; Wolf, M.; Lowrie, E.; Ofsthun, N.; Lazarus, J.M.; Thadhani, R. Survival of patients undergoing hemodialysis with paricalcitol or calcitriol therapy. N. Engl. J. Med. 2003, 349, 446-456.

21. Heikkinen, S.; Väisänen, S.; Pehkonen, P.; Seuter, S.; Benes, V.; Carlberg, C. Nuclear hormone 1 $\alpha, 25$-dihydroxyvitamin D3 elicits a genome-wide shift in the locations of VDR chromatin occupancy. Nucleic Acids Res. 2011, 39, 9181-9193.

22. Nibbelink, K.A.; Tishkoff, D.X.; Hershey, S.D.; Rahman, A.; Simpson, R.U. 1,25(OH)2-vitamin D3 actions on cell proliferation, size, gene expression, and receptor localization, in the HL-1 cardiac myocyte. J. Steroid Biochem. Mol. Biol. 2007, 103, 533-537.

23. Liu, Z.P.; Olson, E.N. Suppression of proliferation and cardiomyocyte hyperthrophy by CHAMP, a cardiac-specific RNA helicase. Proc. Natl. Acad. Sci. USA 2002, 99, 2043-2048. 
24. Lim, H.W.; New, L.; Han, J.; Molkentin, J.D. Calcineurin enhances MAPK phosphatase-1 expression and p38 MAPK inactivation in cardiac myocytes. J. Biol. Chem. 2001, 276, $15913-15919$.

25. Frey, N.; McKinsey, T.A.; Olson, E.N. Decoding calcium signals involved in cardiac growth and function. Nat. Med. 2000, 6, 1221-1227.

26. Murat, A.; Pellieux, C.; Brunner, H.R.; Pedrazzini, T. Calcineurin blockade prevents cardiac mitogen-activated protein kinase activation band hypertrophy in renovascular hypertension. J. Biol. Chem. 2000, 275, 40867-40873.

27. Weidemann, F.; Niemann, M.; Herrmann, S.; Ertl, G.; Störk, S. Assessment of diastolic heart failure: Current role of echocardiography. Herz 2013, 38, 18-25.

28. Pacini, S.; Morucci, G.; Punzi, T.; Gulisano, M.; Ruggiero, M.; Amato, S.; Aterini, S. Effect of paricalcitol and GcMAF on angiogenesis and human peripheral blood mononuclear cell proliferation and signaling. J. Nephrol. 2012, 25, 577-581. 


\title{
The Role of Vitamin D in Blood Pressure, Endothelial and Renal Function in Postmenopausal Women
}

\author{
Zhao-Min Liu, Jean Woo, Sheng-Hui Wu and Suzanne C. Ho
}

\begin{abstract}
Background: Vitamin D is a pro-hormone that plays an essential role in the vasculature and in kidney function. Aims: To review the extra-skeletal effects of vitamin D on blood pressure, endothelial and renal function with emphasis on recent findings in postmenopausal women. Methods: Included in this review was a PubMed database search for English language articles through March 2013. This review discussed the physiology and definition of vitamin D deficiency, the recent evidence for the role vitamin D in blood pressure, vascular and renal function. Results: Experimental and epidemiological data suggest that vitamin D plays an important role in the vasculature and in kidney function. Low vitamin D concentrations appear to significantly associate with hypertension, endothelial and renal dysfunction. However, the results of clinical trials have generally been mixed. Studies specifically conducted among postmenopausal women are limited and findings are still inconsistent. Conclusions: Definitive studies are warranted to elucidate the effects of vitamin D supplementation on vascular and renal function and a more detailed work is needed to outline the route, duration and optimal dose of supplementation. It is premature to recommend vitamin D as a therapeutic option in the improvement of vascular and renal function at the current stage.
\end{abstract}

Reprinted from Nutrients. Cite as: Liu, Z.-M.; Woo, J.; Wu, S.-H.; Ho, S.C. The Role of Vitamin D in Blood Pressure, Endothelial and Renal Function in Postmenopausal Women. Nutrients 2013, 5, 2590-2610.

\section{Introduction}

Vitamin D is a pro-hormone and plays an essential role in a vast number of physiologic processes and clinical consequences [1]. In addition to its traditional effects on calcium homeostasis and bone health, vitamin D receptors (VDR) exist on a very wide range of tissues, including the endothelium, vascular smooth muscle, and cardiomyocytes, suggesting a much wider range of biological functions [1]. However, the association between vitamin D deficiency and non-musculoskeletal conditions, as well as the efficacy of vitamin D supplementation on vascular and renal function, is not adequately characterized. The evidence of causality is inconsistent and inconclusive.

Vitamin D inadequacy is a primary concern for post-menopausal women as they are already predisposed to the osteoporosis and cardiovascular diseases due to decreased oestrogen levels. Menopause represents an important transition in vitamin D requirements due to the dependence of the VDR on oestrogen [2]. In this review, we summarize recent clinical evidence addressing potential mechanisms, epidemiologic associations between vitamin D status and vascular health and renal function, and the effects of supplementation, especially among postmenopausal women.

Studies were identified by searching PubMed for English-language articles through March 2013 by using keywords such as "vitamin D, hypertension, blood pressure, endothelial function, vascular health, chronic kidney disease, renal or kidney function", alone or in combination. The reference lists of published reports were also searched. 


\subsection{Epidemiology of Vitamin D Status}

Vitamin D deficiency is an increasingly recognized public health problem. The direct comparison of data on vitamin D status from various studies are limited by the varying population characteristics, assay methods and the differing thresholds of vitamin D inadequacy. However, all the data suggest that there is a high rate of vitamin D insufficiency. A 2009 study of global vitamin D status reported that serum levels below $30 \mathrm{ng} / \mathrm{mL}(75 \mathrm{nmol} / \mathrm{L})$ prevailed in every region studied [3]. It has been estimated that 1 billion individuals worldwide are vitamin D insufficient or deficient [4]. Vitamin D insufficiency (serum $25(\mathrm{OH}) \mathrm{D}$ level $<30 \mathrm{ng} / \mathrm{mL})$ affects almost $50 \%$ of the population worldwide and the prevalence of deficiency (serum $25(\mathrm{OH}) \mathrm{D}$ level $<10 \mathrm{ng} / \mathrm{mL}$ ) ranging from $5 \%$ to $25 \%$ in the general population $[5,6]$.

The inadequacy of vitamin D affects an even larger proportion of postmenopausal women, particularly those with osteoporosis and a history of fracture [7]. One large international investigation indicated $71 \%$ of postmenopausal women with osteoporosis in Eastern Asia had vitamin D inadequacy $(25(\mathrm{OH}) \mathrm{D}<30 \mathrm{ng} / \mathrm{mL})$. Prevalence rates using this cut-off level were $47 \%$ in Thailand, $49 \%$ in Malaysia, 90\% in Japan, 92\% in South Korea and 62.3\% in Hong Kong (in adults aged > 50 year) [8]. Very deficient levels $(25(\mathrm{OH}) \mathrm{D}<10 \mathrm{ng} / \mathrm{mL})$ are most prevalent in South Asia and the Middle East $[6,9]$, possibly due to the wearing of traditional costumes that limit sun exposure, and also extended periods of breastfeeding without vitamin D supplementation. A telephone survey in mid-life Hong Kong Chinese women also showed that $62.3 \%$ women actively avoided sunlight exposure by staying indoors or using sunscreen products and parasols [10].

\subsection{Vitamin D Measurement and Classification}

No universal consensus has been reached on which level of serum 25(OH)D reflects optimum vitamin D status. In addition to the large inter-laboratory differences in assays for serum $25(\mathrm{OH}) \mathrm{D}[11]$, there are differing recommendations on the serum concentration of $25(\mathrm{OH}) \mathrm{D}$ required to maintain the general health [12]. Current International Osteoporosis Foundation guidelines [13] recommended a target level of $30 \mathrm{ng} / \mathrm{mL}(75 \mathrm{nmol} / \mathrm{L})$, which is associated with maximal suppression of parathyroid hormone $(\mathrm{PTH})$, and defined vitamin D insufficiency as $25(\mathrm{OH}) \mathrm{D}$ levels less than $20 \mathrm{ng} / \mathrm{mL}(50 \mathrm{nmol} / \mathrm{L})$ and deficiency as levels less than $10 \mathrm{ng} / \mathrm{mL}(25 \mathrm{nmol} / \mathrm{L})$. However, a report from the Institute of Medicine recommended that a serum 25(OH)D level of $20 \mathrm{ng} / \mathrm{mL}(50 \mathrm{nmol} / \mathrm{L})$ was sufficient to ensure bone health. The Institute of Medicine report does not support the recommendation that all adults should have levels of $25(\mathrm{OH}) \mathrm{D}$ greater than $30 \mathrm{ng} / \mathrm{mL}(75 \mathrm{nmol} / \mathrm{L})$. The report also notes that higher levels of vitamin D may lead to adverse health outcomes, including kidney stones and renal impairment [14]. Although there is still debate on how to classify vitamin D status, it is generally accepted that serum 25(OH)D levels of 10 and $30 \mathrm{ng} / \mathrm{mL}$ are the cut-off values for deficiency and insufficiency, respectively [15].

Several commercial methods are available for serum 25(OH)D measurements. The international Vitamin D Quality Assessment Scheme [16] demonstrated that most commercial 25(OH)D measurements were capable of producing reliable results, but the results were operator-dependent and most methods had notable bias compared with HPLC methods. The variation between laboratories may be as high as $30 \%$. Liquid chromatography tandem mass spectroscopy (LC-MS) is considered the "gold standard" [17]. 
Other simpler methods, such as radioimmunoassay (RIA), enzyme-linked immunoassay (ELISA), and chemiluminescence assay may not measure all circulating forms of vitamin D.

\subsection{Vitamin D Metabolism}

In humans, skin synthesis of vitamin D from sunlight exposure is the major source of vitamin D $(80 \%-90 \%)$ in humans under natural conditions [18]. The dietary supply of vitamin D is minor $(10 \%-20 \%)$ compared to skin formation but can become an important source of vitamin D with supplementation [4].

Vitamin D is a collection of fat-soluble steroids. There are two types of physiologically important vitamin D: cholecalciferol (vitamin $\mathrm{D}_{3}$ ) and ergocalciferol (vitamin $\mathrm{D}_{2}$ ). $\mathrm{D}_{2}$ is typically obtained from fortified foods and vitamin sources [19], while $\mathrm{D}_{3}$ is mainly synthesised in the skin from 7-dehydrocholesterol upon exposure to UVB $(290-320 \mathrm{~nm})$ [20]. In the circulation, vitamin D is metabolised to 25-hydroxyvitamin D [25(OH)D] in the liver and further metabolised to its biologically active form, 1,25-dihydroxyvitamin $\mathrm{D}\left[1,25(\mathrm{OH})_{2} \mathrm{D}\right]$ in the kidney by 25 -hydroxyvitamin $\mathrm{D}$-1 $\alpha$-hydroxylase (CYP27B1).

The concentration of $1,25(\mathrm{OH})_{2} \mathrm{D}$ is regulated by a variety of factors including serum $\mathrm{PTH}$, calcium, and phosphate [20,21]. PTH increases the activity of CYP27B1, resulting in increased production of $1,25(\mathrm{OH})_{2} \mathrm{D}$. The higher $1,25(\mathrm{OH})_{2} \mathrm{D}_{3}$ level can also inhibit PTH secretion, completing the feedback loop. $25(\mathrm{OH}) \mathrm{D}$ is the major circulating form of vitamin $\mathrm{D}$. The majority of circulating $25(\mathrm{OH}) \mathrm{D}$ and $1,25(\mathrm{OH})_{2} \mathrm{D}$ is bound to vitamin D binding protein (DBP) $(80 \%-90 \%)$ and albumin $(10 \%-20 \%)$, while a small fraction of both $25(\mathrm{OH}) \mathrm{D}(0.02 \%-0.05 \%)$ and $1,25(\mathrm{OH})_{2} \mathrm{D}(0.2 \%-0.6 \%)$ is free [17].

The serum 25(OH)D level is the best indicator of overall vitamin D status since its long half-life (10-27 days following oral or intravenous administration and 1-3 months based on pharmacodynamic response, a more clinically-oriented half-life $[22,23]$ ) and its metabolism reflects total vitamin D from dietary intake and sunlight exposure, as well as the conversion of vitamin D from adipose stores in the liver $[24,25]$. 1,25(OH $)_{2} \mathrm{D}$ circulates in much lower concentrations than $25(\mathrm{OH}) \mathrm{D}$ but has much greater affinity for the vitamin D receptor (VDR) and is biologically more potent. Although $25(\mathrm{OH}) \mathrm{D}$ requires additional hydroxylation in the kidney to become its active form, serum concentrations of $1,25(\mathrm{OH})_{2} \mathrm{D}$ should never be used to determine vitamin $\mathrm{D}$ status. This is because of the short half-life of $1,25(\mathrm{OH})_{2} \mathrm{D}$ in the circulation $(<4 \mathrm{~h})$, its concentrations are $\sim 1000$-fold less than those of $25(\mathrm{OH}) \mathrm{D}$ and most importantly, the $1,25(\mathrm{OH})_{2} \mathrm{D}$ level would be normal or even elevated as a result of vitamin $\mathrm{D}$ deficiency or secondary hyperparathyroidism [4].

The active form of 1,25(OH)2D binds to VDR. VDR is nearly ubiquitously expressed. In addition to its traditional effects on bone health, there is accumulating evidence to suggest that the VDR has a broad spectrum of effects on various cell types including the endothelium [26], vascular smooth muscle [27,28], and cardiomyocytes [29]. Approximately $3 \%$ of the human genome is directly or indirectly regulated by the vitamin D endocrine system, which supports the idea that vitamin D insufficiency has widespread adverse consequences for human health [30]. 


\subsection{Risk Factors with Vitamin D Deficiency}

The principal causes of low vitamin D levels are limited cutaneous synthesis due to inadequate sun exposure (sunscreen use, institutionalized or homebound status) or combined with low dietary intake of vitamin D rich foods [7] including fortified milk [4]. Other risk factors include aging, pigmented skin, smoking, obesity, air pollution, abnormal intestinal function, malabsorption, or reduced synthesis or increased degradation of vitamin D due to chronic liver or renal disease [4,7].

Older people have lower dermal synthesis of vitamin D. Even similar exposure to sunlight, a person aged 70 y produces $75 \%$ less vitamin D than a person aged 20 [31]. Persons with a high body mass index are also susceptible to low vitamin D levels because of the decreased bioavailability of vitamin $\mathrm{D}$ that is stored in excess adipose tissue. Gender disparities exist with women having lower 25(OH)D levels than men [32]. Vitamin D inadequacy could also be affected by the ethnic and culture factors. Asian women often have a lactase deficiency and lower fortified milk consumption, and often avoid the sun exposure and skin pigmentation by using sunscreening cosmetics and parasols [10], which increase their risk of vitamin D insufficiency. Urbanization is also an important risk factor for an inadequate vitamin D status, which often leads to insufficient outdoor activities [33] and is associated with highly polluted air in some cities [34].

\subsection{Vitamin D Supplementation Guidelines}

Foods naturally containing rich vitamin D are limited. Oily fish (salmon, mackerel, and sardines) and cod liver oil are good sources of vitamin $\mathrm{D}_{3}$. Other food source includes egg yolk, fortified milk and orange juice, some cereals, mushrooms and cheese [20,35]. It has been estimated that for every $100 \mathrm{IU}$ of vitamin D ingested, the blood level of $25(\mathrm{OH}) \mathrm{D}$ increases by around $1 \mathrm{ng} / \mathrm{mL}$ $(2.5 \mathrm{nmol} / \mathrm{L})[36]$. The estimate could be varied with starting level of vitamin $\mathrm{D}$, body composition, dose quantity and frequency, or the supplemental form of vitamin D [37] Data from NHANES-III indicate a "J-shaped" association between $25(\mathrm{OH}) \mathrm{D}$ levels and mortality, with slightly increasing mortality in those with supraphysiological 25(OH)D levels. However, other data indicate that particularly high levels of vitamin D are optimal for cancer prevention [38,39].

The US Institute of Medicine concluded that serum 25(OH)D of $20 \mathrm{ng} / \mathrm{mL}$ or more will cover the requirements of $97.5 \%$ of the population and recommended Dietary Allowance at 600 IU per day for people aged 1-70 year and 800 IU per day for older adults [40]. The US Endocrine Society's Clinical Practice Guideline suggested that 600-1000 IU per day for children aged one year or more, and 1500-2000 IU per day for adults aged 19 years or more to maintain 25(OH)D above the optimal level of $30 \mathrm{ng} / \mathrm{mL}$ [41]. The guideline also recommended screening for vitamin D deficiency in individuals at risk for deficiency and concluded that there was not sufficient evidence to recommend screening individuals who are not at risk for deficiency or to prescribe vitamin D to attain the non-calcaemic benefit for cardiovascular protection [41]. Difference in the recommendations reflects different goals and views on current evidence. 


\section{Vitamin D and Blood Pressure}

The effect of vitamin D on blood pressure could be one of the potential mechanisms underlying the link between vitamin $\mathrm{D}$ and cardiovascular diseases. The $1 \alpha$-hydroxylase enzyme that converts $25(\mathrm{OH}) \mathrm{D}$ to $1,25(\mathrm{OH})_{2} \mathrm{D}$ is expressed in human endothelial and vascular smooth muscle cells which have special relevance in the genesis of hypertension. In vitro and animal studies demonstrated that vitamin $\mathrm{D}$ appears to have antihypertensive and vasculoprotective effects via multiple pathways [42]. The antihypertensive properties of vitamin D include renoprotective effects, suppression of the rennin-angiotensin-aldosterone system (RAS), direct effects on vascular cells, and effects on calcium metabolism, including prevention of secondary hyperparathyroidism and hypocalcemia [43]. Vitamin D might also act as a negative regulator of the renin gene, and low vitamin D may increase the expression of the RAS [44].

\subsection{Cross Sectional Data}

The association between 25(OH)D levels and arterial hypertension has been assessed in a number of cross-sectional studies [45-53]. Most, though not all, of the observational data support the links between low 25-(OH)-D levels and a higher risk of hypertension [54,55].

Several large scale cross-sectional investigations revealed an inverse association of vitamin D levels with prevalence of hypertension. The Third National Health and Nutrition Examination Survey (NHANES-III) [45] among 12,644 non-institutionalized US civilians showed that systolic BP was inversely and significantly correlated with $25(\mathrm{OH}) \mathrm{D}$ levels. The mean systolic and diastolic BP reduced 3.0 and $1.6 \mathrm{~mm} \mathrm{Hg}(p<0.05)$ for participants in the highest quintile compared with the lowest after adjusting for potential cofounders. Subgroup analyses also confirmed that age adjusted systolic BP was significantly lower in individuals with vitamin D sufficiency and the impact of vitamin D deficiency was highly significant in the elderly (age $>50$ years) relative to younger individuals $[46,47]$. The German National Health Interview and Examination Survey [48], 1958 British Birth Cohort [49] and US adolescent population (NHANES 2001 and 2006) [56] all demonstrated that 25(OH) D was inversely associated with the prevalence of hypertension.

A large cohort study [57] in Shanghai, China also reported an association between a lower risk of hypertension and the highest quintile of $25(\mathrm{OH}) \mathrm{D}(\mathrm{OR}=0.16$ for vitamin $\mathrm{D} \geq 50.6 \mathrm{nmol} / \mathrm{L}$ compared with $<23.5 \mathrm{nmol} / \mathrm{L} ; p$ for trend 0.02 ). However, among women, no significant associations were found for BP parameters and hypertension with $25(\mathrm{OH}) \mathrm{D}$ level. Another two observational studies among older populations, the Longitudinal Aging Study Amsterdam [58] and the Ranch Bernardo Study [51] reported the lack of a significant association between vitamin D status and BP. The lack of association might be attributable to the relatively high baseline levels of $25(\mathrm{OH}) \mathrm{D}$ among the study participants. Despite the inconsistent findings from some cross-sectional investigations, the majority of studies with large sample sizes have demonstrated an inverse relationship between 25(OH)D levels and BP [43].

\subsection{Cohort Studies}

A few prospective studies [51,59-62] have addressed the association of 25(OH) D and change in blood pressure or new-onset hypertension. The findings are still inconsistent. 
A 5-year prospective study among 1471 older women (mean age 74 year) fail to show a significant association between initial levels of $25(\mathrm{OH}) \mathrm{D}$ and change of BP. However, when the analyses were adjusted for vitamin D supplement use, greater initial levels of $25(\mathrm{OH}) \mathrm{D}(\geq 20 \mathrm{ng} / \mathrm{mL}$ compared to $<20 \mathrm{ng} / \mathrm{mL}$ ) were paradoxically associated with larger increases in BP [59]. In a cohort [60] of 4863 postmenopausal women who were recruited into the Women's Health Initiative between 1993 and 1998 , serum levels of $25(\mathrm{OH}) \mathrm{D}$ were also not related to changes in $\mathrm{BP}$, and evidence for an association with lower risk of incident hypertension was weak.

Wang et al. [61] studied more than 1700 Framingham Offspring Study participants (mean age 59 years; 55\% women; all white) and suggested that low serum vitamin D levels appear to interact with pre-existing hypertension to dramatically raise the risk of future cardiovascular events. In another large cohort [62] including 77,531 women who were followed for 18 years, the multivariable relative risks comparing the lowest to highest deciles were 1.57 (95\% CI: 1.44 to 1.72) in women. However, this study was limited since the $25(\mathrm{OH}) \mathrm{D}$ levels of study populations were predicted using sun exposure or nutritional vitamin D intake as a surrogate.

\subsection{Clinical Trials on Vitamin D Supplementation and BP}

A number of randomized controlled trials (RCT) evaluated the impact of vitamin D supplementation on BP, however the results are inconclusive. Several large scale trials specifically conducted among postmenopausal women also reported inconsistent findings.

In the largest trial [63] in this field - the Women's Health initiative (WHI) - 36,282 postmenopausal women were randomly assigned to receive either $400 \mathrm{IU}$ vitamin D plus $1000 \mathrm{mg}$ calcium daily or placebo. After seven years' follow-up, changes in systolic and diastolic BP, as well as the frequency of incident hypertension, were not significantly different between the intervention and placebo groups. However, the findings may not rule out an effect of vitamin D on blood pressure since $80 \%$ of patients enrolled in this trial were normotensive at baseline, the dose (400 IU/day) of vitamin $\mathrm{D}_{3}$ used was relatively low to sufficiently increase the $25(\mathrm{OH}) \mathrm{D}$ concentrations, and adherence to treatment was also low with only $60 \%-63 \%$ in the first three years and $59 \%$ at the end of the trial.

Similarly, a one year RCT in UK among 305 healthy postmenopausal women aged 60-70 years also reported that daily doses of vitamin D3 at 400 or 1000 IU/day for one year did not affect conventional markers of cardiovascular disease (CVD) risk including lipids, blood pressure and endothelial markers etc. [64]. However, another RCT among 148 elderly women (aged 70 year or above) with vitamin D deficiency $(25[\mathrm{OH}] \mathrm{D}$ levels $<25 \mathrm{ng} / \mathrm{mL})$ demonstrated a significant antihypertensive effect of vitamin D [65]. In this study, subjects were assigned to receive either $1200 \mathrm{mg}$ of calcium or $1200 \mathrm{mg}$ of calcium plus 800 IU of vitamin D daily. After eight weeks of treatment, a significant reduction in SBP (7.4 $\mathrm{mmHg}, p=0.02$ ) was observed in the vitamin $\mathrm{D}$ plus calcium group, but no significant difference in diastolic BP [65]. Another trial included 34 vitamin-D deficient patients with type 2 diabetes also reported positive findings. Subjects were randomly assigned to receive a single dose of 100,000 IU vitamin D or placebo [66]. After eight weeks of follow-up, the mean office systolic BP was $14 \mathrm{mmHg}$ lower in the vitamin D group ( $p=0.001)$.

A meta-analysis including 11 RCT provided little support for a positive effect of vitamin D supplementation on blood pressure [67]. Most of these studies were small of variable methodological 
quality and indicated a significant heterogeneity. Only a small effect of vitamin D on diastolic BP (3.1 $\mathrm{mmHg}, 95 \% \mathrm{CI}$ : 5.5 to 0.6) was found with no significant fall in systolic BP in studies of hypertensive patients [67]. The inconclusive findings could be because the majority of trials included were not primarily or adequately designed to evaluate the antihypertensive effects of vitamin D and participants had $25(\mathrm{OH}) \mathrm{D}$ levels within the normal range and were largely free from arterial hypertension at baseline. In addition, BP was generally measured in the office, and more sophisticated BP measurements such as ambulatory blood pressure, were not used.

In summary, although experimental and observational studies favor the hypothesis that vitamin D sufficiency promotes the lowering of arterial BP, findings from clinical trials are still inconclusive, especially among postmenopausal women. In general, the antihypertensive effects of vitamin D seem to be particularly prominent in vitamin-D-deficient patients with elevated blood pressure. Further large and well designed randomized, placebo-controlled trials are needed to clarify the effect of vitamin D on blood pressure.

\section{Vitamin D and Endothelial Function}

Endothelial dysfunction is an early vascular pathology and characterized by a change in the properties of the endothelium toward decreased vasodilatation and the creation of a proinflammatory and prothrombotic state [68]. Endothelial function is a valuable surrogate marker of cardiovascular risk [68]. It plays an important role in the pathogenesis of atherosclerosis contributing to plaque initiation and progression [69], as well as increasing arterial stiffness [70].

There are many potential mechanisms mediating a vitamin D effect on endothelial function. Vitamin D may improve endothelial function indirectly by reducing BP, which may in turn be due to its suppressing renin-angiotensin system [42] and/or to its decreasing vascular resistance [71]. Vascular smooth muscle and endothelial cells express VDR as well as $1 \alpha$-hydoxylase [28], allowing for autocrine production of $1,25(\mathrm{OH})_{2} \mathrm{D}$, which may act at the local level to modulate the effects of inflammatory cytokines on the vasculature [72], such as decreasing endothelial adhesion molecules, increasing nitric oxide production [73] and reducing platelet aggregation [74,75]. Other potential mechanisms linking vitamin $\mathrm{D}$ to vascular health include the decrease in oxidative stress [76], attenuation of NF- $\mathrm{KB}$ activation [77] and reduction of PTH [78]. Vitamin D deficiency is also associated with higher circulating concentrations of matrix metalloproteinase-9 which controls vascular wall remodeling [79].

\subsection{Observational Studies}

Most observational studies have shown associations between low circulating levels of vitamin D and endothelial dysfunction. In a large study of 554 healthy individuals, serum vitamin D levels were independently associated with brachial artery flow mediated vasodilatation (FMD) and arterial stiffness after adjusting for age, sex, race, BMI, serum lipid levels, plasma C-reactive protein and medications [80]. A small study involving 23 asymptomatic subjects demonstrated that subjects with significant vitamin D deficiency have impaired brachial artery FMD, which improved after vitamin D replacement therapy [76]. Recently, a step-wise change in FMD according to vitamin D status was demonstrated and an inverse association between serum 25(OH)D levels and vascular inflammatory markers observed [77]. 
In addition to healthy individuals, vitamin D levels can influence endothelial function in disease states. Impaired brachial artery FMD was documented in 280 type 2 diabetic individuals who had low serum vitamin D concentrations [80]. Another study [81] among 66 obese Caucasian children aged 7-14 years indicated that Vitamin D status is linked to biomarkers of oxidative stress, inflammation, and endothelial activation, among which the obese children with vitamin D insufficiency had substantially elevated malondialdehyde, myeloperoxidase, 3-nitrotyrosine, interleukin-6, and sVCAM-1 levels.

\subsection{Clinical Trials on Vitamin D and Endothelial Function}

Clinical trials using vitamin D as supplement produced inconsistent findings (Table 1) [66,76,82-90]. Several RCTs [76,86-89] have shown vitamin D supplementation improves endothelial function while others not $[82-84,90]$. The discrepancies may be due to various vitamin D dosages or dosing interval, study duration, outcome measures, sample size and participant features etc. 


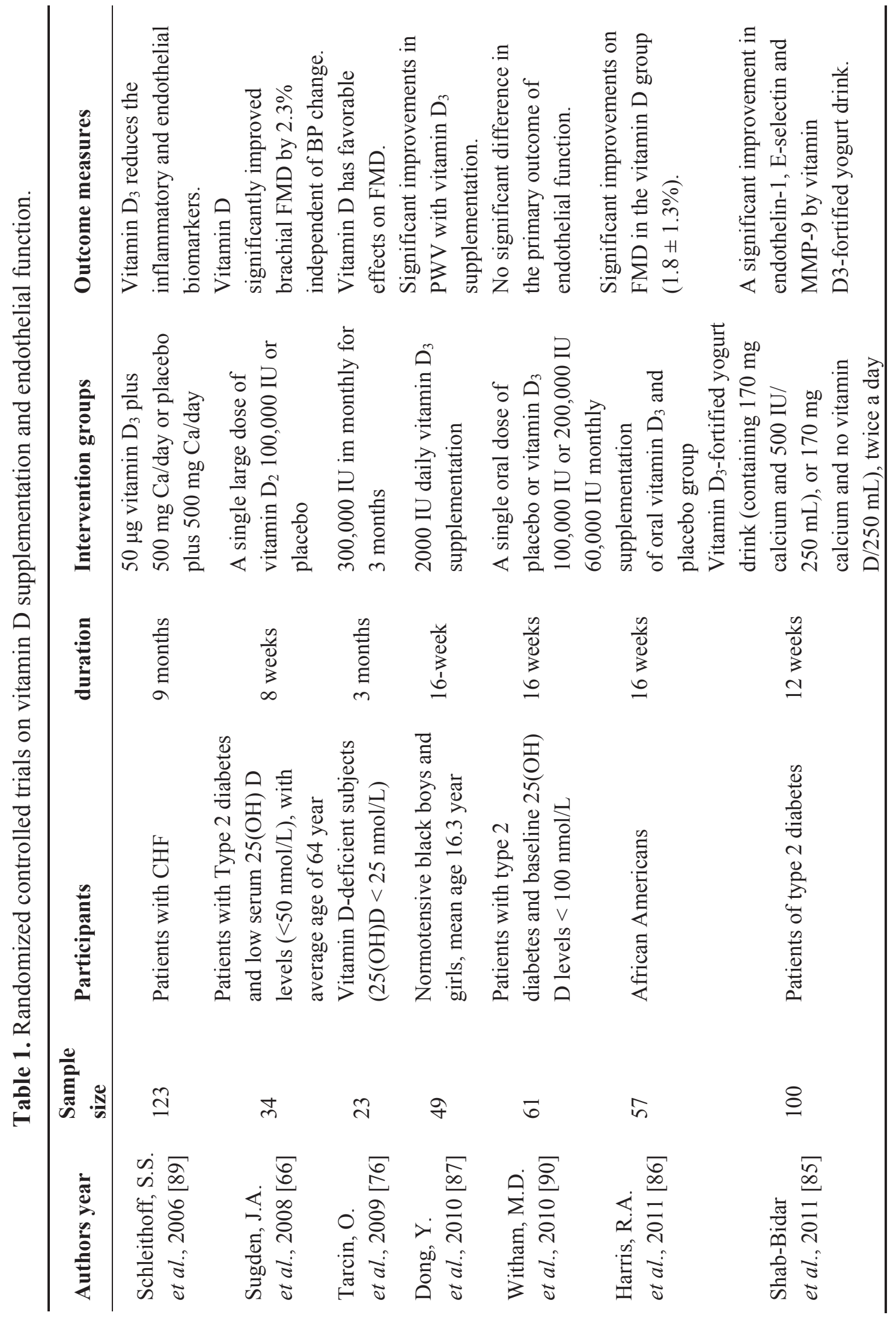




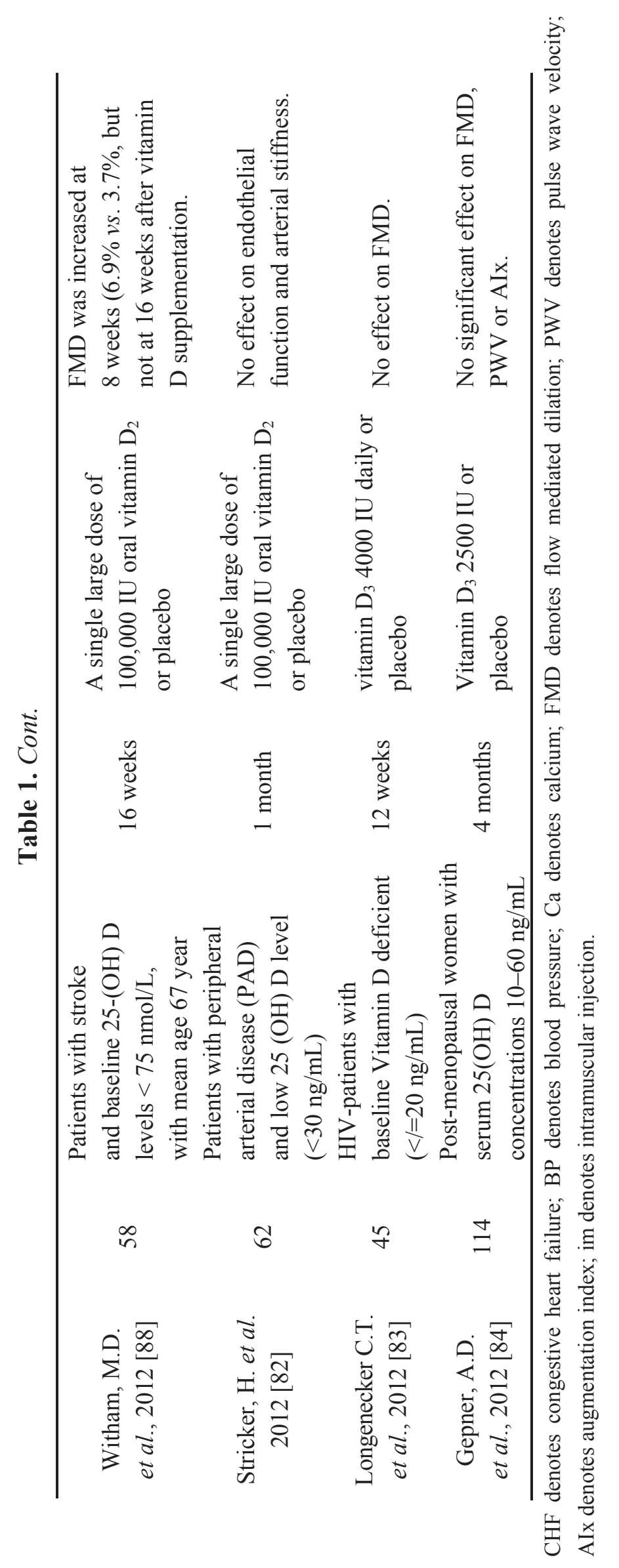


A study by Harris et al. [86] reported that supplementation with 60,000 IU per month of oral $\mathrm{D}_{3}$ for 16 weeks is effective in improving vascular endothelial function in African-American adults with significant improvements in FMD $(1.8 \pm 1.3 \%)$. Another study in 42 subjects with vitamin D insufficiency, normalization of serum $25(\mathrm{OH}) \mathrm{D}$ was associated with increases in reactive hyperemia index $(0.38 \pm 0.14, p=0.009)$ and a decrease in mean arterial pressure $(4.6 \pm 2.3 \mathrm{mmHg}, p=0.02)$ after 6 months of supplementation [91]. A 16 week RCT [87] indicated that vitamin D supplementation (2000 IU/day) decreased arterial stiffness by the mean pulse wave velocity reduced from $5.41 \mathrm{~m} / \mathrm{s}$ at baseline to $5.33 \mathrm{~m} / \mathrm{s}(p=0.031)$. In another RCT [66], which was conducted among diabetic patients with baseline $25(\mathrm{OH}) \mathrm{D}$ insufficiency, a single dose of 100,000 IU vitamin $\mathrm{D}_{2}$ significantly improved FMD of the brachial artery by $2.3 \%$ at 8 weeks. A 9 -month RCT [89] among 123 patients with congestive heart failure also indicated that $50 \mu \mathrm{g}$ vitamin $\mathrm{D}_{3}$ plus $500 \mathrm{mg}$ Ca per day notably improves cytokine profiles and decreases PTH level compared with calcium alone.

In contrast, a 16-week RCT [90] of vitamin D replacement among 61 diabetic subjects, both low- and high-dose vitamin D3 supplementation (100,000 and 200,000 IU) failed to modulate FMD, although an effect on BP was noted. A one month pilot study [82] among 62 patients with peripheral arterial disease, a single large dosage of $100,000 \mathrm{IU}$ oral vitamin $\mathrm{D}_{2}$ indicated nil effect on endothelial function and arterial stiffness. The non-significant finding of the pilot trial might be due to the short duration or underpowered study participants. Clinical trials specifically conducted among postmenopausal women are limited. One RCT among 114 post-menopausal women with serum low vitamin D status $(25(\mathrm{OH}) \mathrm{D}>10$ and $<60 \mathrm{ng} / \mathrm{mL})$, reported that $2500 \mathrm{IU}$ Vitamin $\mathrm{D}_{3}$, daily for 4 months did not improve endothelial function, arterial stiffness, or inflammation [84]. Multivariable models showed no significant interactions between treatment group and Vitamin D status $(<30 \mathrm{ng} / \mathrm{mL})$. Further studies applying different dosages of vitamin D, with adequate sample size and study duration, among postmenopausal women with confirmed vitamin D deficiency are necessary to elaborate the effectiveness of vitamin D on vascular function in women after menopause.

\section{Vitamin D and Renal Function}

The kidney plays an essential role in vitamin D metabolism in circulation [92]. Chronic kidney disease (CKD) is a condition characterized by a gradual loss of kidney function over time. The abnormalities in vitamin D metabolism could contribute to the development of mineral and skeletal disorders, elevations in PTH, hypertension, systemic inflammation, and finally result in renal and cardiovascular damage [93]. The 2009 KDIGO (Kidney Diseases: Improving Global Outcomes) clinical practice guidelines recommended correcting $25(\mathrm{OH}) \mathrm{D}$ deficiency and insufficiencies for the general population [94].

The reasons for this marked vitamin D deficiency in CKD are multi-factorial. CKD can induce a progressive loss of the capacity of the kidney not only to convert $25(\mathrm{OH}) \mathrm{D}$ to circulating calcitriol (the vitamin D hormone), but also to maintain serum 25(OH)D levels for non-renal calcitriol synthesis. The resulting calcitriol and $25(\mathrm{OH}) \mathrm{D}$ deficiency associates directly with accelerated disease progression and death [93,95]. Another interesting hypothesis is that urinary loss of $25(\mathrm{OH}) \mathrm{D}-\mathrm{VDBP}$ (the main plasma carrier of vitamin $\mathrm{D}$ in circulation) associated with proteinuria and reduced megalin-mediated uptake might result in vitamin D deficiency. Alternatively, reduced 
levels of $25(\mathrm{OH}) \mathrm{D}$ might be a result of compromised endogenous pre-vitamin $\mathrm{D}$ production in the skin due to severe renal dysfunction [96] or simply lack of outdoor sunlight exposure due to morbidity.

Most [97-99], though not all [75], the observational studies have demonstrated 25(OH)D deficiency is independently associated with impaired renal function. A cross-sectional analysis of the NHANES III data [100] revealed an association between vitamin D deficiency and increased risk of albuminuria in the US adult population. The possible explanation could be that vitamin D may have an intrinsic antiproteinuric activity, or the fraction of vitamin D which is bound to albumin is lost during albuminuria. Cohort studies among patients with end stage renal disease (ESRD) also indicated higher $25(\mathrm{OH}) \mathrm{D}$ or $1,25(\mathrm{OH})_{2} \mathrm{D}$ levels were associated with decreased overall mortality [101-103]. Most of the current observational findings are from patients with CKD or severe kidney dysfunction; studies among individuals with mildly or moderately declined renal function especially among postmenopausal women are few.

Results from animal experiments demonstrated reno-protective effects of both active vitamin D and its analogues [104]. The favourable effects are mediated by the vitamin D receptor (VDR) and appear to act by regulating multiple pathways including the renin-angiotensin system (RAS), NF- $\kappa B, W n t / \beta$-catenin and some key structural proteins [105]. Therefore, addition of vitamin D to conventional therapy may present a promising treatment modality that extends its classical role in the maintenance of mineral homeostasis [106,107].

Randomized, controlled trials addressing renoprotective potential of vitamin D are not adequately studied. Indirect evidence suggests that treatment with vitamin D receptor activators confers a considerable survival advantage in hemodialysis patients [108-110]. A large scale cohort study [110] among chronic hemodialysis patients demonstrated that patients who received injectable vitamin D had a significant survival advantage (20\%) than the non-vitamin D group. The incidence of cardiovascular-related mortality was significantly reduced with vitamin D injection from 14.6/100 to 7.6/100 person-years $(p<0.001)$. Another 16-month trial [108] among Latin America countries also showed that the 7203 patients who received oral active vitamin D had significant reductions in overall, cardiovascular, infectious and neoplastic mortality compared to the 8801 patients that had not received vitamin D. Several trials using vitamin D analog paricalcitol in patients with CKD have noted significant reduction in albuminuria and the effects were independent of hemodynamics or parathyroid hormone suppression [111-113]. In a multinational, 24-week RCT [111] among patients with type 2 diabetes and albuminuria, intake of 2 mug paricalcitol showed a safe and sustained reduction in urinary albumin-to-creatinine ratio (UACR) ranging from $-18 \%$ to $-28 \%$ ( $p=0.014 v s$. placebo). Another pilot trial [113] also showed that ingestion of paricalcitol for 1 month significantly reduces albuminuria and inflammation levels in 24 patients with CKD and the effect was independent of hemodynamics or parathyroid hormone suppression. Large, randomized, controlled clinical trials to assess the renoprotective potential of vitamin D are expected in the near future.

Although no consensus exists on the optimal levels of $25(\mathrm{OH}) \mathrm{D}$ measured in the serum, the US Institute of Medicine recommended that mean 25(OH)D levels should be $>20 \mathrm{ng} / \mathrm{mL}$ in the general population. It might be reasonable to suggest slightly higher levels (up to $30 \mathrm{ng} / \mathrm{mL}$ ) in patients with CKD [114] for extra renal production of $1,25(\mathrm{OH})_{2} \mathrm{D}_{3}$ and regulation of PTH secretion. In CKD, 
supplementation with vitamin $\mathrm{D}$ is recommended at the inception of the disease, with the addition of 25(OH)D beginning in Stage 3 [115].

\section{Vitamin D Intoxication (VDI)}

In clinical trials, vitamin D toxicity was not observed with doses of up to $10,000 \mathrm{IU}$ per day [116]. Serum 25(OH)D levels above $150 \mathrm{ng} / \mathrm{mL}$ are considered as VDI [117]. There is a wide margin between the level of $25(\mathrm{OH}) \mathrm{D}$ required for vitamin D adequacy $(>30 \mathrm{ng} / \mathrm{mL})$ and the level of toxicity $(>150 \mathrm{ng} / \mathrm{mL})$. The adverse effects with VDI are mainly related to serum hypercalcemia and its duration, which can cause reversible hypertension [4]. When the calcium concentration exceeds $14 \mathrm{mg} / \mathrm{dL}$, emergency intervention is necessary since hypercalcemia will cause organ dysfunction. Since vitamin D is stored in fat tissues, effects of toxicity may last for months despite the removal of the exogenous source of vitamin D.

\section{Conclusions}

The observational data presented in this review suggest an association between low vitamin $\mathrm{D}$ status and increased blood pressure, endothelial and renal dysfunction; however, clinical trials have reported inconsistent findings. The discordant findings of RCTs could be due to the differences in features of participants, their adherence to the supplements, study duration, various dosage regimes or dosing interval of vitamin $\mathrm{D}$, the concomitant calcium intake, or the primary outcome that was measured [118]. Moreover, a number of RCTs have paid little attention to the baseline vitamin D status and dose adequacy and studies tested the dose-response relationship were few [119].

Although a single hormone like vitamin D seems unlikely to play a substantial role in preventing or ameliorating the diverse range of diseases [120], future studies from appropriately powered and controlled clinical trials to examine the dose-response effect, selecting patients at risk for vascular and renal dysfunction in the context of initial vitamin D deficiency, and defining the appropriate product regimes and dosing interval, are much needed before vitamin $\mathrm{D}$ can be recommended as an auxiliary or therapeutic option for the improvement of vascular and renal function.

\section{Acknowledgments}

The present research received no specific grant from any funding agency in the public, commercial or not-for-profit sectors.

\section{Conflicts of Interest}

The author declares no conflict of interest.

\section{References}

1. Perez-Lopez, F.R.; Chedraui, P.; Fernandez-Alonso, A.M. Vitamin d and aging: Beyond calcium and bone metabolism. Maturitas 2011, 69, 27-36. 
2. Duque, G.; El Abdaimi, K.; Macoritto, M.; Miller, M.M.; Kremer, R. Estrogens (e2) regulate expression and response of 1,25-dihydroxyvitamin $\mathrm{D}_{3}$ receptors in bone cells: Changes with aging and hormone deprivation. Biochem. Biophys. Res. Commun. 2002, 299, 446-454.

3. Mithal, A.; Wahl, D.A.; Bonjour, J.P.; Burckhardt, P.; Dawson-Hughes, B.; Eisman, J.A.; El-Hajj Fuleihan, G.; Josse, R.G.; Lips, P.; Morales-Torres, J. Global vitamin D status and determinants of hypovitaminosis d. Osteoporos. Int. 2009, 20, 1807-1820.

4. Holick, M.F. Vitamin D deficiency. N. Engl. J. Med. 2007, 357, 266-281.

5. Sai, A.J.; Walters, R.W.; Fang, X.; Gallagher, J.C. Relationship between vitamin D, parathyroid hormone, and bone health. J. Clin. Endocrinol. Metab. 2011, 96, E436-E446.

6. Howe, W.R.; Dellavalle, R. Vitamin D deficiency. N. Engl. J. Med. 2007, 357,266-281.

7. Gaugris, S.; Heaney, R.P.; Boonen, S.; Kurth, H.; Bentkover, J.D.; Sen, S.S. Vitamin D inadequacy among post-menopausal women: A systematic review. Q. J. Med. 2005, 98, 667-676.

8. Wat, W.Z.; Leung, J.Y.; Tam, S.; Kung, A.W. Prevalence and impact of vitamin D insufficiency in southern chinese adults. Ann. Nutr. Metab. 2007, 51, 59-64.

9. Lim, S.K.; Kung, A.W.; Sompongse, S.; Soontrapa, S.; Tsai, K.S. Vitamin D inadequacy in postmenopausal women in Eastern Asia. Curr. Med. Res. Opin. 2008, 24, 99-106.

10. Kung, A.W.; Lee, K.K. Knowledge of vitamin d and perceptions and attitudes toward sunlight among chinese middle-aged and elderly women: A population survey in hong kong. $B M C$ Public Health 2006, 6, 226.

11. Lips, P.; Duong, T.; Oleksik, A.; Black, D.; Cummings, S.; Cox, D.; Nickelsen, T. A global study of vitamin D status and parathyroid function in postmenopausal women with osteoporosis: Baseline data from the multiple outcomes of raloxifene evaluation clinical trial. J. Clin. Endocrinol. Metab. 2001, 86, 1212-1221.

12. Baz-Hecht, M.; Goldfine, A.B. The impact of vitamin D deficiency on diabetes and cardiovascular risk. Curr. Opin. Endocrinol. Diabetes Obes. 2010, 17, 113-119.

13. Dawson-Hughes, B.; Mithal, A.; Bonjour, J.P.; Boonen, S.; Burckhardt, P.; Fuleihan, G.E.; Josse, R.G.; Lips, P.; Morales-Torres, J.; Yoshimura, N. Iof position statement: Vitamin D recommendations for older adults. Osteoporos. Int. 2010, 21, 1151-1154.

14. Ross, A.C.; Manson, J.E.; Abrams, S.A.; Aloia, J.F.; Brannon, P.M.; Clinton, S.K.; Durazo-Arvizu, R.A.; Gallagher, J.C.; Gallo, R.L.; Jones, G.; et al. The 2011 report on dietary reference intakes for calcium and vitamin $\mathrm{D}$ from the institute of medicine: What clinicians need to know. J. Clin. Endocrinol. Metab. 2011, 96, 53-58.

15. Dawson-Hughes, B.; Heaney, R.P.; Holick, M.F.; Lips, P.; Meunier, P.J.; Vieth, R. Estimates of optimal vitamin D status. Osteoporos. Int. 2005, 16, 713-716.

16. Carter, G.D.; Carter, R.; Jones, J.; Berry, J. How accurate are assays for 25-hydroxyvitamin D? Data from the international vitamin D external quality assessment scheme. Clin. Chem. 2004, 50, 2195-2197.

17. Zerwekh, J.E. Blood biomarkers of vitamin d status. Am. J. Clin. Nutr. 2008, 87, 1087S-1091S.

18. Calvo, M.S.; Whiting, S.J.; Barton, C.N. Vitamin D fortification in the united states and canada: Current status and data needs. Am. J. Clin. Nutr. 2004, 80, 1710S-1716S. 
19. Dusso, A.S.; Brown, A.J.; Slatopolsky, E. Vitamin D. Am. J. Physiol. Ren. Physiol. 2005, 289, F8-F28.

20. Holick, M.F. Sunlight and vitamin D for bone health and prevention of autoimmune diseases, cancers, and cardiovascular disease. Am. J. Clin. Nutr. 2004, 80, 1678S-1688S.

21. Wolpowitz, D.; Gilchrest, B.A. The vitamin D questions: How much do you need and how should you get it? J. Am. Acad. Dermatol. 2006, 54, 301-317.

22. Chel, V.; Wijnhoven, H.A.; Smit, J.H.; Ooms, M.; Lips, P. Efficacy of different doses and time intervals of oral vitamin D supplementation with or without calcium in elderly nursing home residents. Osteoporos. Int. 2008, 19, 663-671.

23. Wu, F.; Staykova, T.; Horne, A.; Clearwater, J.; Ames, R.; Mason, B.; Orr-Walker, B.; Gamble, G.; Scott, M.; Reid, I. Efficacy of an oral, 10-day course of high-dose calciferol in correcting vitamin D deficiency. N. Z. Med. J. 2003, 116, U536.

24. DeLuca, H.F. Overview of general physiologic features and functions of vitamin D. Am. J. Clin. Nutr. 2004, 80, 1689S-1696S.

25. Heaney, R.P. The vitamin D requirement in health and disease. J. Steroid Biochem. Mol. Biol. 2005, 97, 13-19.

26. Merke, J.; Milde, P.; Lewicka, S.; Hugel, U.; Klaus, G.; Mangelsdorf, D.J.; Haussler, M.R.; Rauterberg, E.W.; Ritz, E. Identification and regulation of 1,25-dihydroxyvitamin $\mathrm{D}_{3}$ receptor activity and biosynthesis of 1,25-dihydroxyvitamin $\mathrm{D}_{3}$. Studies in cultured bovine aortic endothelial cells and human dermal capillaries. J. Clin. Investig. 1989, 83, 1903-1915.

27. Merke, J.; Hofmann, W.; Goldschmidt, D.; Ritz, E. Demonstration of 1,25(OH)2 vitamin D 3 receptors and actions in vascular smooth muscle cells in vitro. Calcif. Tissue Int. 1987, 41, $112-114$.

28. Somjen, D.; Weisman, Y.; Kohen, F.; Gayer, B.; Limor, R.; Sharon, O.; Jaccard, N.; Knoll, E.; Stern, N. 25-Hydroxyvitamin $\mathrm{D}_{3}$-1alpha-hydroxylase is expressed in human vascular smooth muscle cells and is upregulated by parathyroid hormone and estrogenic compounds. Circulation 2005, 111, 1666-1671.

29. Wu-Wong, J.R. Potential for vitamin D receptor agonists in the treatment of cardiovascular disease. Br. J. Pharmacol. 2009, 158, 395-412.

30. Zittermann, A. Vitamin D and disease prevention with special reference to cardiovascular disease. Prog. Biophys. Mol. Biol. 2006, 92, 39-48.

31. Hagenau, T.; Vest, R.; Gissel, T.N.; Poulsen, C.S.; Erlandsen, M.; Mosekilde, L.; Vestergaard, P. Global vitamin D levels in relation to age, gender, skin pigmentation and latitude: An ecologic meta-regression analysis. Osteoporos. Int. 2009, 20, 133-140.

32. Ginde, A.A.; Liu, M.C.; Camargo, C.A., Jr. Demographic differences and trends of vitamin D insufficiency in the US population, 1988-2004. Arch. Intern. Med. 2009, 169, 626-632.

33. Godar, D.E.; Landry, R.J.; Lucas, A.D. Increased uva exposures and decreased cutaneous vitamin $\mathrm{D}_{3}$ levels may be responsible for the increasing incidence of melanoma. Med. Hypotheses 2009, 72, 434-443.

34. Zittermann, A.; Schleithoff, S.S.; Koerfer, R. Putting cardiovascular disease and vitamin D insufficiency into perspective. Br. J. Nutr. 2005, 94, 483-492. 
35. Tangpricha, V.; Koutkia, P.; Rieke, S.M.; Chen, T.C.; Perez, A.A.; Holick, M.F. Fortification of orange juice with vitamin D: A novel approach for enhancing vitamin D nutritional health. Am. J. Clin. Nutr. 2003, 77, 1478-1483.

36. Holick, M.F.; Biancuzzo, R.M.; Chen, T.C.; Klein, E.K.; Young, A.; Bibuld, D.; Reitz, R.; Salameh, W.; Ameri, A.; Tannenbaum, A.D. Vitamin $\mathrm{D}_{2}$ is as effective as vitamin $\mathrm{D}_{3}$ in maintaining circulating concentrations of 25-hydroxyvitamin D. J. Clin. Endocrinol. Metab. 2008, 93, 677-681.

37. Bacon, C.J. High-Dose Vitamin D Supplementation in the Elderly. In Handbook of Vitamin D in Human Health; Watson, R.R., Ed.; Wageningen Academic Publishers: Wageningen, the Netherlands, 2013.

38. Melamed, M.L.; Michos, E.D.; Post, W.; Astor, B. 25-Hydroxyvitamin D levels and the risk of mortality in the general population. Arch. Intern. Med. 2008, 168, 1629-1637.

39. Cannell, J.J.; Hollis, B.W. Use of vitamin D in clinical practice. Altern. Med. Rev. 2008, 13, 6-20.

40. Bischoff-Ferrari, H.A.; Giovannucci, E.; Willett, W.C.; Dietrich, T.; Dawson-Hughes, B. Estimation of optimal serum concentrations of 25-hydroxyvitamin D for multiple health outcomes. Am. J. Clin. Nutr. 2006, 84, 18-28.

41. Holick, M.F.; Binkley, N.C.; Bischoff-Ferrari, H.A.; Gordon, C.M.; Hanley, D.A.; Heaney, R.P.; Murad, M.H.; Weaver, C.M. Evaluation, treatment, and prevention of vitamin D deficiency: An endocrine society clinical practice guideline. J. Clin. Endocrinol. Metab. 2011, 96, 1911-1930.

42. Li, Y.C.; Kong, J.; Wei, M.; Chen, Z.F.; Liu, S.Q.; Cao, L.P. 1,25-Dihydroxyvitamin D3 is a negative endocrine regulator of the renin-angiotensin system. J. Clin. Investig. 2002, 110, 229-238.

43. Pilz, S.; Tomaschitz, A.; Ritz, E.; Pieber, T.R. Vitamin D status and arterial hypertension: A systematic review. Nat. Rev. Cardiol. 2009, 6, 621-630.

44. Rammos, G.; Tseke, P.; Ziakka, S. Vitamin D, the renin-angiotensin system, and insulin resistance. Int. Urol. Nephrol. 2008, 40, 419-426.

45. Scragg, R.; Sowers, M.; Bell, C. Serum 25-hydroxyvitamin D, ethnicity, and blood pressure in the third national health and nutrition examination survey. Am. J. Hypertens. 2007, 20, 713-719.

46. Judd, S.E.; Nanes, M.S.; Ziegler, T.R.; Wilson, P.W.; Tangpricha, V. Optimal vitamin D status attenuates the age-associated increase in systolic blood pressure in white americans: Results from the third national health and nutrition examination survey. Am. J. Clin. Nutr. 2008, 87, 136-141.

47. Martins, D.; Wolf, M.; Pan, D.; Zadshir, A.; Tareen, N.; Thadhani, R.; Felsenfeld, A.; Levine, B.; Mehrotra, R.; Norris, K. Prevalence of cardiovascular risk factors and the serum levels of 25-hydroxyvitamin D in the united states: Data from the third national health and nutrition examination survey. Arch. Intern. Med. 2007, 167, 1159-1165.

48. Hintzpeter, B.; Mensink, G.B.; Thierfelder, W.; Muller, M.J.; Scheidt-Nave, C. Vitamin D status and health correlates among german adults. Eur. J. Clin. Nutr. 2008, 62, 1079-1089.

49. Hypponen, E.; Boucher, B.J.; Berry, D.J.; Power, C. 25-hydroxyvitamin d, igf-1, and metabolic syndrome at 45 years of age: A cross-sectional study in the 1958 british birth cohort. Diabetes 2008, 57, 298-305. 
50. Forouhi, N.G.; Luan, J.; Cooper, A.; Boucher, B.J.; Wareham, N.J. Baseline serum 25-hydroxy vitamin $\mathrm{D}$ is predictive of future glycemic status and insulin resistance: The medical research council ely prospective study 1990-2000. Diabetes 2008, 57, 2619-2625.

51. Reis, J.P.; von Muhlen, D.; Kritz-Silverstein, D.; Wingard, D.L.; Barrett-Connor, E. Vitamin D, parathyroid hormone levels, and the prevalence of metabolic syndrome in community-dwelling older adults. Diabetes Care 2007, 30, 1549-1555.

52. Gannage-Yared, M.H.; Chedid, R.; Khalife, S.; Azzi, E.; Zoghbi, F.; Halaby, G. Vitamin D in relation to metabolic risk factors, insulin sensitivity and adiponectin in a young Middle-Eastern population. Eur. J. Endocrinol. 2009, 160, 965-971.

53. Pasco, J.A.; Henry, M.J.; Nicholson, G.C.; Brennan, S.L.; Kotowicz, M.A. Behavioural and physical characteristics associated with vitamin D status in women. Bone 2009, 44, 1085-1091.

54. Rueda, S.; Fernandez-Fernandez, C.; Romero, F.; Martinez de Osaba, J.; Vidal, J. Vitamin D, PTH, and the metabolic syndrome in severely obese subjects. Obes. Surg. 2008, 18, 151-154.

55. Chan, R.; Chan, D.; Woo, J.; Ohlsson, C.; Mellstrom, D.; Kwok, T.; Leung, P. Serum 25-hydroxyvitamin D and parathyroid hormone levels in relation to blood pressure in a cross-sectional study in older chinese men. J. Hum. Hypertens. 2012, 26, 20-27.

56. Williams, D.M.; Fraser, A.; Lawlor, D.A. Associations of vitamin D, parathyroid hormone and calcium with cardiovascular risk factors in US adolescents. Heart 2011, 97, 315-320.

57. Dorjgochoo, T.; Shu, X.O.; Xiang, Y.B.; Yang, G.; Cai, Q.; Li, H.; Ji, B.T.; Cai, H.; Gao, Y.T.; Zheng, W. Circulating 25-hydroxyvitamin D levels in relation to blood pressure parameters and hypertension in the shanghai women's and men's health studies. Br. J. Nutr. 2012, 108, 449-458.

58. Snijder, M.B.; Lips, P.; Seidell, J.C.; Visser, M.; Deeg, D.J.; Dekker, J.M.; van Dam, R.M. Vitamin D status and parathyroid hormone levels in relation to blood pressure: A population-based study in older men and women. J. Intern. Med. 2007, 261, 558-565.

59. Bolland, M.J.; Bacon, C.J.; Horne, A.M.; Mason, B.H.; Ames, R.W.; Wang, T.K.; Grey, A.B.; Gamble, G.D.; Reid, I.R. Vitamin D insufficiency and health outcomes over 5 year in older women. Am. J. Clin. Nutr. 2010, 91, 82-89.

60. Margolis, K.L.; Martin, L.W.; Ray, R.M.; Kerby, T.J.; Allison, M.A.; Curb, J.D.; Kotchen, T.A.; Liu, S.; Wassertheil-Smoller, S.; Manson, J.E. A prospective study of serum 25-hydroxyvitamin D levels, blood pressure, and incident hypertension in postmenopausal women. Am. J. Epidemiol. 2012, 175, 22-32.

61. Wang, T.J.; Pencina, M.J.; Booth, S.L.; Jacques, P.F.; Ingelsson, E.; Lanier, K.; Benjamin, E.J.; D’Agostino, R.B.; Wolf, M.; Vasan, R.S. Vitamin D deficiency and risk of cardiovascular disease. Circulation 2008, 117, 503-511.

62. Forman, J.P.; Giovannucci, E.; Holmes, M.D.; Bischoff-Ferrari, H.A.; Tworoger, S.S.; Willett, W.C.; Curhan, G.C. Plasma 25-hydroxyvitamin D levels and risk of incident hypertension. Hypertension 2007, 49, 1063-1069. 
63. Margolis, K.L.; Ray, R.M.; van Horn, L.; Manson, J.E.; Allison, M.A.; Black, H.R.; Beresford, S.A.; Connelly, S.A.; Curb, J.D.; Grimm, R.H., Jr.; et al. Effect of calcium and vitamin D supplementation on blood pressure: The women's health initiative randomized trial. Hypertension 2008, 52, 847-855.

64. Wood, A.D.; Secombes, K.R.; Thies, F.; Aucott, L.; Black, A.J.; Mavroeidi, A.; Simpson, W.G.; Fraser, W.D.; Reid, D.M.; Macdonald, H.M. Vitamin $\mathrm{D}_{3}$ supplementation has no effect on conventional cardiovascular risk factors: A parallel-group, double-blind, placebo-controlled RCT. J. Clin. Endocrinol. Metab. 2012, 97, 3557-3568.

65. Pfeifer, M.; Begerow, B.; Minne, H.W.; Nachtigall, D.; Hansen, C. Effects of a short-term vitamin $\mathrm{D}_{3}$ and calcium supplementation on blood pressure and parathyroid hormone levels in elderly women. J. Clin. Endocrinol. Metab. 2001, 86, 1633-1637.

66. Sugden, J.A.; Davies, J.I.; Witham, M.D.; Morris, A.D.; Struthers, A.D. Vitamin D improves endothelial function in patients with type 2 diabetes mellitus and low vitamin D levels. Diabet. Med. 2008, 25, 320-325.

67. Witham, M.D.; Nadir, M.A.; Struthers, A.D. Effect of vitamin d on blood pressure: A systematic review and meta-analysis. J. Hypertens. 2009, 27, 1948-1954.

68. Widlansky, M.E.; Gokce, N.; Keaney, J.F., Jr.; Vita, J.A. The clinical implications of endothelial dysfunction. J. Am. Coll. Cardiol. 2003, 42, 1149-1160.

69. Nadar, S.; Blann, A.D.; Lip, G.Y. Endothelial dysfunction: Methods of assessment and application to hypertension. Curr. Pharm. Des. 2004, 10, 3591-3605.

70. Endemann, D.H.; Schiffrin, E.L. Endothelial dysfunction. J. Am. Soc. Nephrol. 2004, 15, 1983-1992.

71. Norman, P.E.; Powell, J.T. Vitamin D, shedding light on the development of disease in peripheral arteries. Arterioscler. Thromb. Vasc. Biol. 2005, 25, 39-46.

72. Zehnder, D.; Bland, R.; Chana, R.S.; Wheeler, D.C.; Howie, A.J.; Williams, M.C.; Stewart, P.M.; Hewison, M. Synthesis of 1,25-dihydroxyvitamin $\mathrm{D}_{3}$ by human endothelial cells is regulated by inflammatory cytokines: A novel autocrine determinant of vascular cell adhesion. $J$. Am. Soc. Nephrol. 2002, 13, 621-629.

73. Molinari, C.; Uberti, F.; Grossini, E.; Vacca, G.; Carda, S.; Invernizzi, M.; Cisari, C. 1 $\alpha, 25$-dihydroxycholecalciferol induces nitric oxide production in cultured endothelial cells. Cell. Physiol. Biochem. 2011, 27, 661-668.

74. Aihara, K.; Azuma, H.; Akaike, M.; Ikeda, Y.; Yamashita, M.; Sudo, T.; Hayashi, H.; Yamada, Y.; Endoh, F.; Fujimura, M.; et al. Disruption of nuclear vitamin D receptor gene causes enhanced thrombogenicity in mice. J. Biol. Chem. 2004, 279, 35798-35802.

75. Urena-Torres, P.; Metzger, M.; Haymann, J.P.; Karras, A.; Boffa, J.J.; Flamant, M.; Vrtovsnik, F.; Gauci, C.; Froissart, M.; Houillier, P.; et al. Association of kidney function, vitamin D deficiency, and circulating markers of mineral and bone disorders in CKD. Am. J. Kidney Dis. 2011, 58, 544-553.

76. Tarcin, O.; Yavuz, D.G.; Ozben, B.; Telli, A.; Ogunc, A.V.; Yuksel, M.; Toprak, A.; Yazici, D.; Sancak, S.; Deyneli, O.; et al. Effect of vitamin D deficiency and replacement on endothelial function in asymptomatic subjects. J. Clin. Endocrinol. Metab. 2009, 94, 4023-4030. 
77. Jablonski, K.L.; Chonchol, M.; Pierce, G.L.; Walker, A.E.; Seals, D.R. 25-hydroxyvitamin D deficiency is associated with inflammation-linked vascular endothelial dysfunction in middle-aged and older adults. Hypertension 2011, 57, 63-69.

78. Pilz, S.; Tomaschitz, A.; Drechsler, C.; Ritz, E.; Boehm, B.O.; Grammer, T.B.; Marz, W. Parathyroid hormone level is associated with mortality and cardiovascular events in patients undergoing coronary angiography. Eur. Heart J. 2010, 31, 1591-1598.

79. Momiyama, Y.; Ohmori, R.; Tanaka, N.; Kato, R.; Taniguchi, H.; Adachi, T.; Nakamura, H.; Ohsuzu, F. High plasma levels of matrix metalloproteinase- 8 in patients with unstable angina. Atherosclerosis 2010, 209, 206-210.

80. Yiu, Y.F.; Chan, Y.H.; Yiu, K.H.; Siu, C.W.; Li, S.W.; Wong, L.Y.; Lee, S.W.L.; Tam, S.; Wong, E.W.K.; Cheung, B.M.Y.; et al. Vitamin D deficiency is associated with depletion of circulating endothelial progenitor cells and endothelial dysfunction in patients with type 2 diabetes. J. Clin. Endocrinol. Metab. 2011, 96, E830-E835.

81. Codoner-Franch, P.; Tavarez-Alonso, S.; Simo-Jorda, R.; Laporta-Martin, P.; Carratala-Calvo, A.; Alonso-Iglesias, E. Vitamin D status is linked to biomarkers of oxidative stress, inflammation, and endothelial activation in obese children. J. Pediatr. 2012, 161, 848-854.

82. Stricker, H.; Tosi Bianda, F.; Guidicelli-Nicolosi, S.; Limoni, C.; Colucci, G. Effect of a single, oral, high-dose vitamin D supplementation on endothelial function in patients with peripheral arterial disease: A randomised controlled pilot study. Eur. J. Vasc. Endovasc. Surg. 2012, 44, 307-312.

83. Longenecker, C.T.; Hileman, C.O.; Carman, T.L.; Ross, A.C.; Seydafkan, S.; Brown, T.T.; Labbato, D.E.; Storer, N.; Tangpricha, V.; McComsey, G.A. Vitamin D supplementation and endothelial function in vitamin D deficient hiv-infected patients: A randomized placebo-controlled trial. Antivir. Ther. 2012, 17, 613-621.

84. Gepner, A.D.; Ramamurthy, R.; Krueger, D.C.; Korcarz, C.E.; Binkley, N.; Stein, J.H. A prospective randomized controlled trial of the effects of vitamin D supplementation on cardiovascular disease risk. PLoS One 2012, 7, e36617.

85. Shab-Bidar, S.; Neyestani, T.R.; Djazayery, A.; Eshraghian, M.R.; Houshiarrad, A.; Gharavi, A.; Kalayi, A.; Shariatzadeh, N.; Zahedirad, M.; Khalaji, N.; et al. Regular consumption of vitamin D-fortified yogurt drink (doogh) improved endothelial biomarkers in subjects with type 2 diabetes: A randomized double-blind clinical trial. BMC Med. 2011, 9, 125.

86. Harris, R.A.; Pedersen-White, J.; Guo, D.H.; Stallmann-Jorgensen, I.S.; Keeton, D.; Huang, Y.; Shah, Y.; Zhu, H.; Dong, Y. Vitamin D 3 supplementation for 16 weeks improves flow-mediated dilation in overweight african-american adults. Am. J. Hypertens. 2011, 24, 557-562.

87. Dong, Y.; Stallmann-Jorgensen, I.S.; Pollock, N.K.; Harris, R.A.; Keeton, D.; Huang, Y.; Li, K.; Bassali, R.; Guo, D.H.; Thomas, J.; et al. A 16-week randomized clinical trial of 2000 international units daily vitamin $\mathrm{D}_{3}$ supplementation in black youth: 25-Hydroxyvitamin $\mathrm{D}$, adiposity, and arterial stiffness. J. Clin. Endocrinol. Metab. 2010, 95, 4584-4591.

88. Witham, M.D.; Dove, F.J.; Sugden, J.A.; Doney, A.S.; Struthers, A.D. The effect of vitamin D replacement on markers of vascular health in stroke patients - A randomised controlled trial. Nutr. Metab. Cardiovasc. Dis. 2012, 22, 864-870. 
89. Schleithoff, S.S.; Zittermann, A.; Tenderich, G.; Berthold, H.K.; Stehle, P.; Koerfer, R. Vitamin D supplementation improves cytokine profiles in patients with congestive heart failure: A double-blind, randomized, placebo-controlled trial. Am. J. Clin. Nutr. 2006, 83, 754-759.

90. Witham, M.D.; Dove, F.J.; Dryburgh, M.; Sugden, J.A.; Morris, A.D.; Struthers, A.D. The effect of different doses of vitamin $\mathrm{D}_{3}$ on markers of vascular health in patients with type 2 diabetes: A randomised controlled trial. Diabetologia 2010, 53, 2112-2119.

91. Al Mheid, I.; Patel, R.; Murrow, J.; Morris, A.; Rahman, A.; Fike, L.; Kavtaradze, N.; Uphoff, I.; Hooper, C.; Tangpricha, V.; et al. Vitamin D status is associated with arterial stiffness and vascular dysfunction in healthy humans. J. Am. Coll. Cardiol. 2011, 58, 186-192.

92. Levin, A.; Bakris, G.L.; Molitch, M.; Smulders, M.; Tian, J.; Williams, L.A.; Andress, D.L. Prevalence of abnormal serum vitamin D, PTH, calcium, and phosphorus in patients with chronic kidney disease: Results of the study to evaluate early kidney disease. Kidney Int. 2007, 71, 31-38.

93. Dusso, A.; Gonzalez, E.A.; Martin, K.J. Vitamin d in chronic kidney disease. Best Pract. Res. Clin. Endocrinol. Metab. 2011, 25, 647-655.

94. Moe, S.; Drueke, T.; Cunningham, J.; Goodman, W.; Martin, K.; Olgaard, K.; Ott, S.; Sprague, S.; Lameire, N.; Eknoyan, G. Definition, evaluation, and classification of renal osteodystrophy: A position statement from kidney disease: Improving global outcomes (kdigo). Kidney Int. 2006, 69, 1945-1953.

95. Andress, D.L. Vitamin D in chronic kidney disease: A systemic role for selective vitamin d receptor activation. Kidney Int. 2006, 69, 33-43.

96. Jacob, A.I.; Sallman, A.; Santiz, Z.; Hollis, B.W. Defective photoproduction of cholecalciferol in normal and uremic humans. J. Nutr. 1984, 114, 1313-1319.

97. Barreto, D.V.; Barreto, F.C.; Liabeuf, S.; Temmar, M.; Boitte, F.; Choukroun, G.; Fournier, A.; Massy, Z.A. Vitamin D affects survival independently of vascular calcification in chronic kidney disease. Clin. J. Am. Soc. Nephrol. 2009, 4, 1128-1135.

98. Inaguma, D.; Nagaya, H.; Hara, K.; Tatematsu, M.; Shinjo, H.; Suzuki, S.; Mishima, T.; Kurata, K. Relationship between serum 1,25-dihydroxyvitamin D and mortality in patients with pre-dialysis chronic kidney disease. Clin. Exp. Nephrol. 2008, 12, 126-131.

99. Ravani, P.; Malberti, F.; Tripepi, G.; Pecchini, P.; Cutrupi, S.; Pizzini, P.; Mallamaci, F.; Zoccali, C. Vitamin D levels and patient outcome in chronic kidney disease. Kidney Int. 2009, $75,88-95$.

100. De Boer, I.H.; Ioannou, G.N.; Kestenbaum, B.; Brunzell, J.D.; Weiss, N.S. 25-hydroxyvitamin $\mathrm{D}$ levels and albuminuria in the third national health and nutrition examination survey (NHANES iii). Am. J. Kidney Dis. 2007, 50, 69-77.

101. Tentori, F.; Hunt, W.C.; Stidley, C.A.; Rohrscheib, M.R.; Bedrick, E.J.; Meyer, K.B.; Johnson, H.K.; Zager, P.G. Mortality risk among hemodialysis patients receiving different vitamin D analogs. Kidney Int. 2006, 70, 1858-1865.

102. Shoben, A.B.; Rudser, K.D.; de Boer, I.H.; Young, B.; Kestenbaum, B. Association of oral calcitriol with improved survival in nondialyzed ckd. J. Am. Soc. Nephrol. 2008, 19, 1613-1619. 
103. Wolf, M.; Shah, A.; Gutierrez, O.; Ankers, E.; Monroy, M.; Tamez, H.; Steele, D.; Chang, Y.; Camargo, C.A., Jr.; Tonelli, M.; et al. Vitamin D levels and early mortality among incident hemodialysis patients. Kidney Int. 2007, 72, 1004-1013.

104. Mirkovic, K.; van den Born, J.; Navis, G.; de Borst, M.H. Vitamin D in chronic kidney disease: New potential for intervention. Curr. Drug Targets 2011, 12, 42-53.

105. Li, Y.C. Vitamin D: Roles in renal and cardiovascular protection. Curr. Opin. Nephrol. Hypertens. 2012, 21, 72-79.

106. Dusso, A.S.; Tokumoto, M. Defective renal maintenance of the vitamin D endocrine system impairs vitamin d renoprotection: A downward spiral in kidney disease. Kidney Int. 2011, 79, $715-729$.

107. Gal-Moscovici, A.; Sprague, S.M. Use of vitamin D in chronic kidney disease patients. Kidney Int. 2010, 78, 146-151.

108. Naves-Diaz, M.; Alvarez-Hernandez, D.; Passlick-Deetjen, J.; Guinsburg, A.; Marelli, C.; Rodriguez-Puyol, D.; Cannata-Andia, J.B. Oral active vitamin D is associated with improved survival in hemodialysis patients. Kidney Int. 2008, 74, 1070-1078.

109. Teng, M.; Wolf, M.; Lowrie, E.; Ofsthun, N.; Lazarus, J.M.; Thadhani, R. Survival of patients undergoing hemodialysis with paricalcitol or calcitriol therapy. N. Engl. J. Med. 2003, 349, 446-456.

110. Teng, M.; Wolf, M.; Ofsthun, M.N.; Lazarus, J.M.; Hernan, M.A.; Camargo, C.A., Jr.; Thadhani, R. Activated injectable vitamin D and hemodialysis survival: A historical cohort study. J. Am. Soc. Nephrol. 2005, 16, 1115-1125.

111. De Zeeuw, D.; Agarwal, R.; Amdahl, M.; Audhya, P.; Coyne, D.; Garimella, T.; Parving, H.H.; Pritchett, Y.; Remuzzi, G.; Ritz, E.; et al. Selective vitamin D receptor activation with paricalcitol for reduction of albuminuria in patients with type 2 diabetes (vital study): A randomised controlled trial. Lancet 2010, 376, 1543-1551.

112. Agarwal, R.; Acharya, M.; Tian, J.; Hippensteel, R.L.; Melnick, J.Z.; Qiu, P.; Williams, L.; Batlle, D. Antiproteinuric effect of oral paricalcitol in chronic kidney disease. Kidney Int. 2005, 68, 2823-2828.

113. Alborzi, P.; Patel, N.A.; Peterson, C.; Bills, J.E.; Bekele, D.M.; Bunaye, Z.; Light, R.P.; Agarwal, R. Paricalcitol reduces albuminuria and inflammation in chronic kidney disease: A randomized double-blind pilot trial. Hypertension 2008, 52, 249-255.

114. Verstuyf, A.; Carmeliet, G.; Bouillon, R.; Mathieu, C. Vitamin D: A pleiotropic hormone. Kidney Int. 2010, 78, 140-145.

115. Jones, G. Expanding role for vitamin D in chronic kidney disease: Importance of blood 25-(OH)-D levels and extra-renal 1alpha-hydroxylase in the classical and nonclassical actions of 1alpha,25-dihydroxyvitamin $\mathrm{D}_{3}$. Semin. Dial. 2007, 20, 316-324.

116. Hathcock, J.N.; Shao, A.; Vieth, R.; Heaney, R. Risk assessment for vitamin D. Am. J. Clin. Nutr. 2007, 85, 6-18.

117. Ozkan, B.; Hatun, S.; Bereket, A. Vitamin D intoxication. Turk. J. Pediatr. 2012, 54, 93-98. 
118. Sanders, K.M.; Stuart, A.L.; Williamson, E.J.; Simpson, J.A.; Kotowicz, M.A.; Young, D.; Nicholson, G.C. Annual high-dose oral vitamin D and falls and fractures in older women: A randomized controlled trial. JAMA 2010, 303, 1815-1822.

119. Heaney, R.P. Vitamin D-Baseline status and effective dose. N. Engl. J. Med. 2012, 367, 77-78.

120. Grey, A.; Bolland, M. Vitamin D: A place in the sun? Arch. Intern. Med. 2010, 170, 1099-1100. 


\section{Chapter 6}

Metabolic Disorders 



\title{
Vitamin D Intake and Risk of Type 1 Diabetes: A Meta-Analysis of Observational Studies
}

\author{
Jia-Yi Dong, Weiguo Zhang, Jiong Jack Chen, Zeng-Li Zhang, Shu-Fen Han and Li-Qiang Qin
}

\begin{abstract}
Vitamin D is suggested to have protective effects against type 1 diabetes. However, the results from observational studies have been inconsistent. We aimed to examine their association by conducting a meta-analysis of observational studies. Multiple databases were searched in June 2013 to identify relevant studies including both case-control and cohort studies. Either a fixed- or random-effects model was used to calculate the pooled risk estimate. We identified eight studies (two cohort studies and six case-control studies) on vitamin D intake during early life and three studies (two cohort studies and one case-control study) on maternal vitamin D intake during pregnancy. The pooled odds ratio for type 1 diabetes comparing vitamin D supplementation with non-supplementation during early life was 0.71 (95\% confidence interval [CI], 0.51-0.98). Similar results were observed in the case-control subgroup analysis but not in the cohort subgroup analysis. The pooled odds ratio with maternal intake of vitamin D during pregnancy was 0.95 (95\% CI, 0.66-1.36). In conclusion, vitamin D intake during early life may be associated with a reduced risk of type 1 diabetes. However, there was not enough evidence for an association between maternal intake of vitamin D and risk of type 1 diabetes in the offspring.
\end{abstract}

Reprinted from Nutrients. Cite as: Dong, J.-Y.; Zhang, W.; Chen, J.J.; Zhang, Z.-L.; Han, S.-F.; Qin, L.-Q. Vitamin D Intake and Risk of Type 1 Diabetes: A Meta-Analysis of Observational Studies. Nutrients 2013, 5, 3551-3562.

\section{Introduction}

Type 1 diabetes is an immune-mediated disease characterized by the loss of the insulin-producing $\beta$-cells, which eventually results in high levels of glucose in the blood. The incidence of type 1 disease is increasing all over the world $[1,2]$. Ecological study has shown an association between ultraviolet B irradiance, the primary source of circulating vitamin D in humans, and high incidence of type 1 diabetes in children, leading to the hypothesis that vitamin D may have a role in the disease [3]. The prevalent vitamin D deficiency/insufficiency, for example in the Chinese population, represents an important public health issue [4].

Indeed, recent cross-sectional studies consistently suggested a high vitamin D deficiency in patients with type 1 diabetes [5-7]. EURODIAB, the first case-control study on this topic, showed a reduced risk of developing type 1 diabetes by vitamin D supplementation during the first year of life [8]. However, subsequent observational studies (case-control and cohort studies) yielded inconsistent results [9-15]. The protective effect of vitamin D against type 1 diabetes was observed in some studies $[8,10,11,13,14]$ but not all $[9,12,15]$. The small sample size may have led to the null associations in some studies. On the other hand, there is evidence that the destruction of $\beta$-cells could be initiated in utero [16]. Growing attention therefore has been paid to the role of maternal vitamin D status in type 1 diabetes in the offspring. Yet the results from observational studies $[9,11,17]$ were also inconclusive. 
Thus, the aim of this study was to examine the association between vitamin D intake and risk of type 1 diabetes by conducting a meta-analysis of relevant studies. In addition, we examined the association between maternal intake of vitamin D during pregnancy and risk of type 1 diabetes in the offspring.

\section{Methods}

\subsection{Literature Search}

We generally followed the Meta-Analysis of Observational Studies in Epidemiology (MOOSE) guidelines [18] in the design, analysis, and reporting of this study. We searched PubMed, Web of Science, and Cochrane Library databases in June 2013 to identify relevant studies that assessed the association between intake of vitamin $\mathrm{D}$ and risk of type 1 diabetes. The literature search was supplemented through the manual review of reference lists of retrieved articles and recent reviews. Search terms included "vitamin D”, "25-hydroxyvitamin D”, “type 1 diabetes”. No restriction was imposed.

\subsection{Study Selection}

Studies were eligible for analysis if they met the following criteria: the study design was a casecontrol study or cohort study; the outcome was type 1 diabetes; and risk estimates of diabetes related to vitamin D intake during early life or maternal intake of vitamin D and associated $95 \%$ CIs (or data to calculate them) were reported. Initially, we reviewed titles and abstracts to ascertain the potential fit to the inclusion criteria. In the presence of uncertainty regarding the relevancy, a subsequent full-text assessment was conducted.

\subsection{Data Extraction}

We extracted the following information: the first author's name, publication year, study population, location, characteristics of the study population (age, sex, and sample size), assessment of exposure and outcome, length of follow-up (for cohort studies), most fully adjusted risk estimates (with corresponding 95\% CI) from a multivariable model, and statistical adjustment for the potential confounding factors. For two studies $[15,17]$ that examined different sources of vitamin D (i.e., total vitamin D, vitamin D from food only, and vitamin D from supplementation only), the risk estimates for total vitamin D intake were used. Two authors (Dong, J.Y. and Qin, L.Q.) independently performed data extraction, with disagreements resolved by discussion.

\subsection{Statistical Analysis}

Odds ratio (OR) was used as a measure of the association between vitamin D intake and risk of type 1 diabetes. For cohort studies, incidence rate, rate ratio, and relative risk were considered as a surrogate measure of OR. Because the absolute risk of type 1 diabetes is low, the relative risks approximate OR values. We calculated the $Q$ and $I^{2}$ statistics [19] to examine statistical heterogeneity across studies. $I^{2}$ is the proportion of total variation contributed by between-study variation. Either a fixed-effects model or, in the presence of heterogeneity, random-effects model was used to combine the study-specific ORs and compute the summary one. Subgroup analysis by study design (i.e., case-control vs. cohort studies) 
was performed. We also conducted sensitivity analysis to investigate the influence of a single study on the overall risk estimates by omitting one study in each turn. Potential publication bias was assessed by Egger test [20]. All analyses were performed using STATA version 11.0 [21]. A $P$ value $<0.05$ was considered statistically significant.

\section{Results}

\subsection{Literature Search}

Figure 1 presents a flow chart showing the study selection process. We initially identified 1588 potential articles from three electronic databases; most were excluded because they were not case-control or cohort studies or because the exposure or endpoint was not relevant to our analysis, leaving 11 potentially eligible papers for full-text review. One study [22] was excluded because the outcome was immune-mediated diseases but not type 1 diabetes. Another study [23] was excluded because the risk estimate for the association of interest was not available. Finally, nine articles [8-15,17] with 12 risk estimates on vitamin D and type 1 diabetes were included in the final analysis. The EURODIAB [8] that combined the results of seven case-control studies across European countries was considered as one multi-center, case-control study. Among the nine articles, eight studies [8-15] reported results on vitamin D intake during early life (e.g., the first year of life or childhood period) and three studies $[9,11,17]$ reported results on maternal vitamin $\mathrm{D}$ intake during pregnancy. 
Figure 1. Flow chart of literature search and study selection.

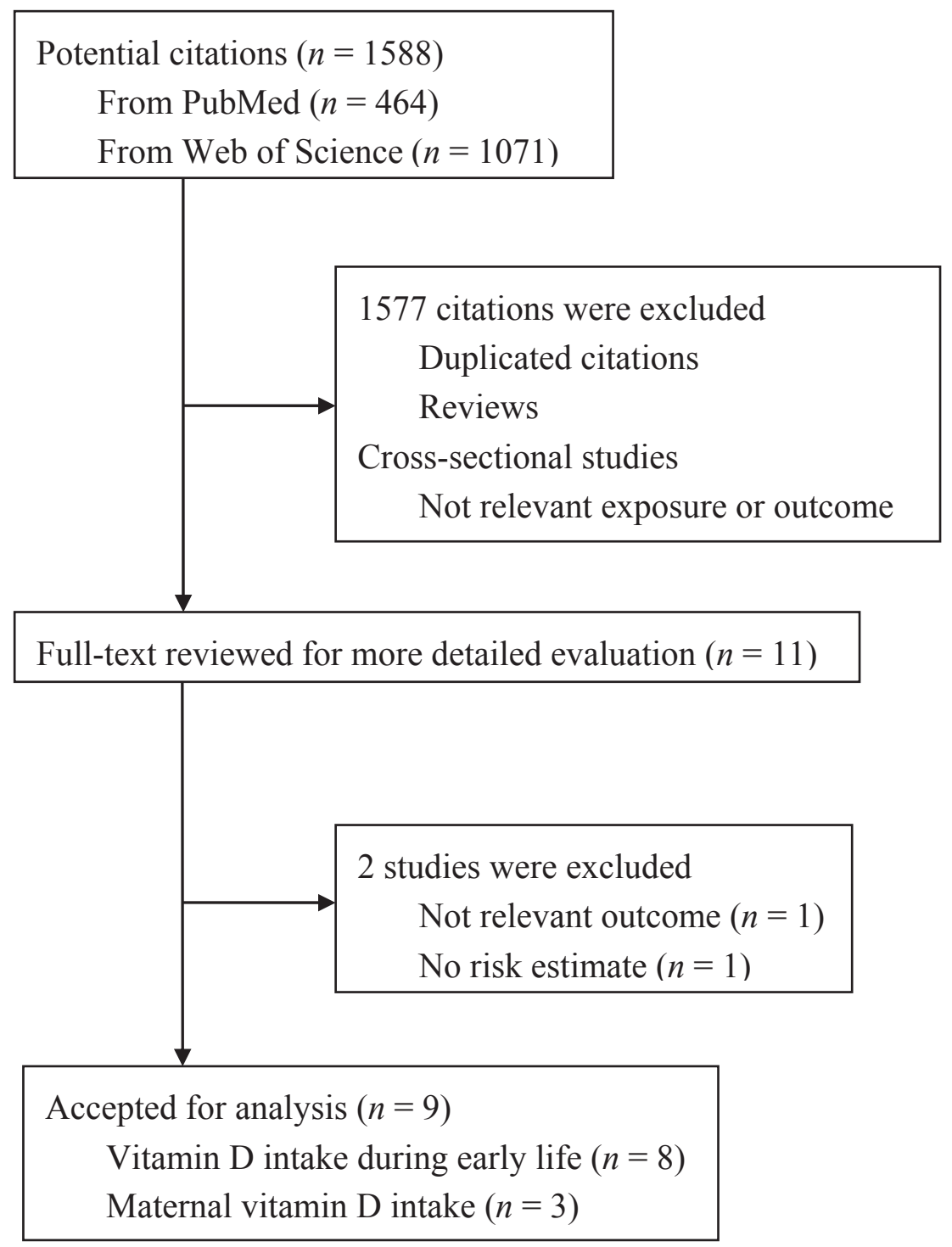

\subsection{Study Characteristics}

Characteristics of the selected studies are presented in Table 1. These studies were published between 1999 and 2011. Among them, seven studies were conducted in Europe, and one each in the United States and Iran. Six studies had a case-control design, comprising 1860 cases and 6243 controls; the other three had a cohort design, comprising 171 cases and 14,264 participants. The length of follow-up for cohort studies ranged from 5 to 31 years. Most selected/incident cases aged $\leq 18$ years old, whereas two studies had cases with a wider age range between 0 and 30 years old. All studies included both male and female participants. Two studies used food-frequency questionnaires for assessment of vitamin D intake and the remaining used other questionnaires or interview. Type 1 diabetes cases were mainly ascertained on the basis of WHO criteria or EURODIAB criteria. The adjusted covariates in individual studies differed from each other. 


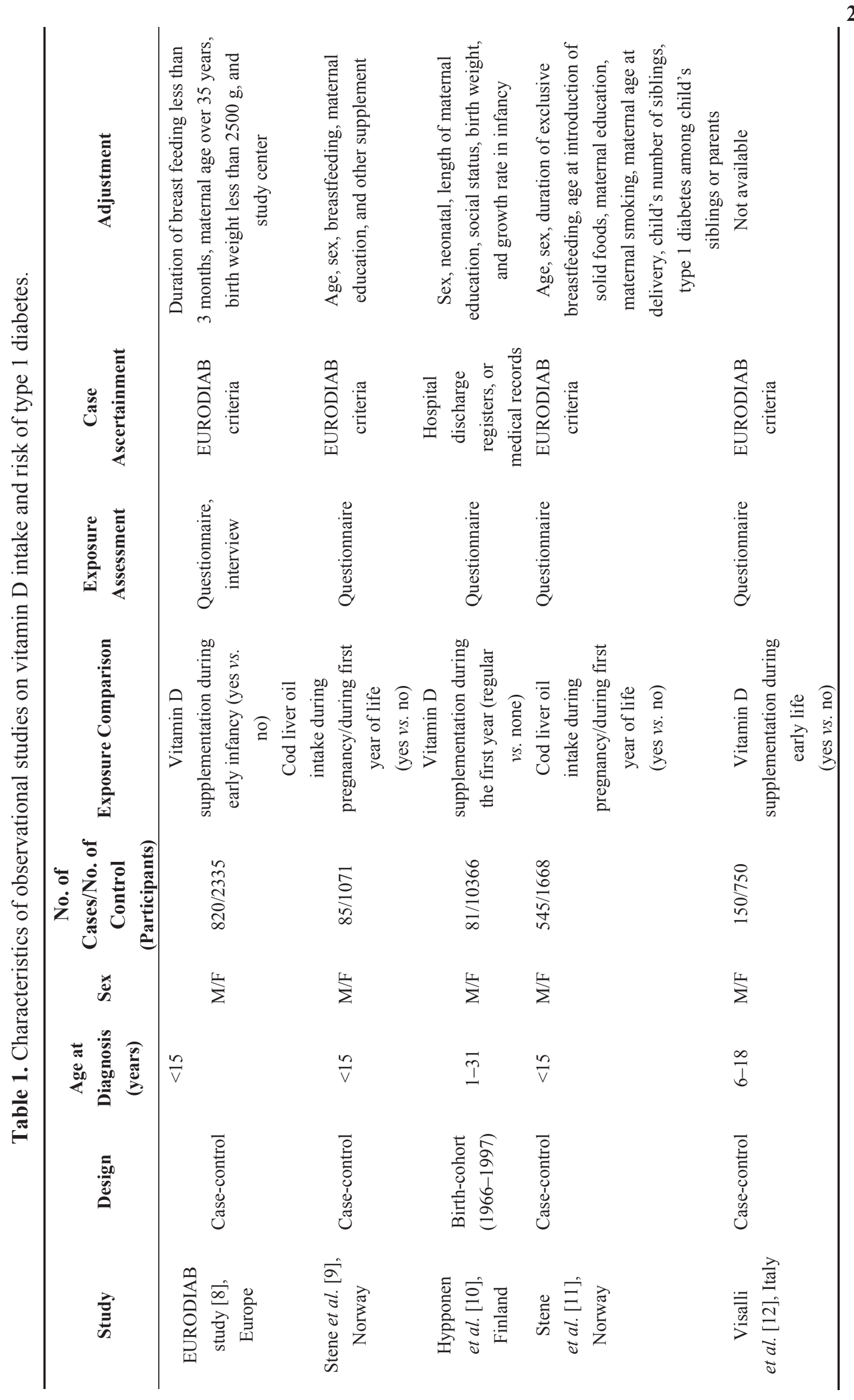




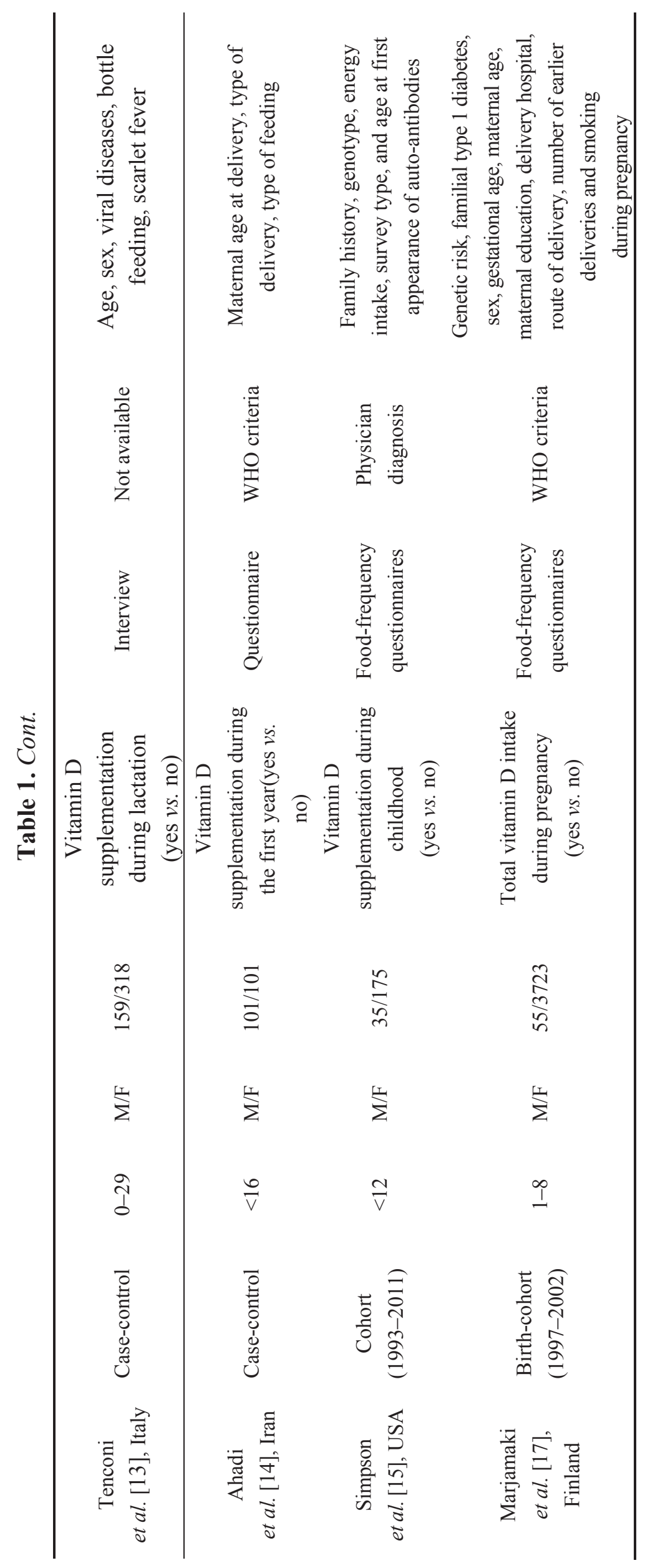




\subsection{Vitamin D Intake and Risk of Type 1 Diabetes}

There were eight epidemiologic studies (six case-control studies and two cohort studies) reporting the association between vitamin D intake and risk of type 1 diabetes. All of them focused on vitamin $\mathrm{D}$ intake during early life. The ORs from individual studies varied from 0.22 to 1.30 . Of the eight included studies, five reported a significant inverse association between vitamin D intake and risk of type 1 diabetes, while the other three found null associations. The pooled OR was 0.71 (95\% CI, 0.51-0.98; Figure 2), with evidence of heterogeneity across studies $\left(P<0.001, I^{2}=74.9 \%\right)$. We further conducted subgroup analysis by study design (case-control studies $v s$. cohort studies). The pooled risk estimate for six case-control studies was similar to the overall one ( $\mathrm{OR}=0.68 ; 95 \% \mathrm{CI}, 0.49-0.94)$, while the pooled risk estimate for two cohort studies was non-significant with a wide CI (relative risk $=0.62$; $95 \%$ CI, 0.11-3.45). The sensitivity analysis in which one study at a time was omitted generally showed statistically significant or marginally significant results; the pooled ORs ranged from 0.64 (95\% CI, 0.46-0.89) to 0.78 (95\% CI, 0.57-1.07). The Egger test suggested no evidence of publication bias $(P=0.28)$.

Three studies (two case-control studies and one cohort study) examined the association between maternal intake of vitamin D and risk of type 1 diabetes in the offspring. All of them reported nonsignificant associations. The pooled OR was 0.95 (95\% CI, 0.66-1.36; Figure 2), with little evidence of heterogeneity $\left(P=0.27, I^{2}=23.7 \%\right)$. Because of the small number of studies, subgroup analysis, sensitivity analysis, and Egger test were not performed.

Figure 2. Meta-analysis of observational studies on vitamin D intake and risk of type 1 diabetes.

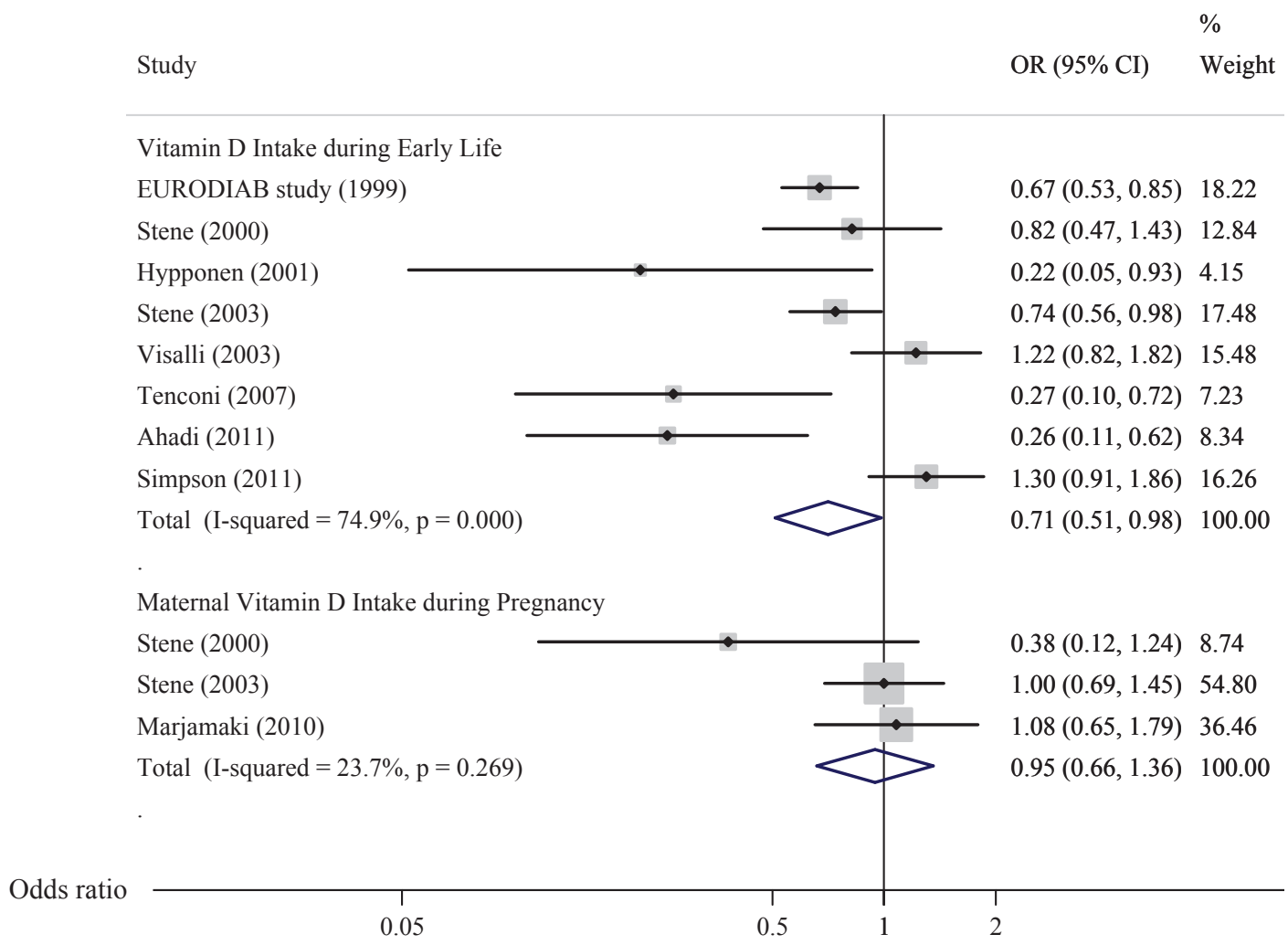




\section{Discussion}

The present meta-analysis aimed to assess the association between vitamin D intake and risk of type 1 diabetes. By combining the results from published case-control and cohort studies to date, our findings suggested vitamin D intake during early life was associated with a reduced risk of type 1 diabetes. However, current evidence does not support an association between maternal intake of vitamin $\mathrm{D}$ and risk of type 1 diabetes in the offspring.

Complex mechanisms (which are beyond our scope) may be involved in the potentially protective effects of vitamin D against type 1 diabetes. The beneficial effects of vitamin D are proposed to be related to the regulation of the immune system, including its actions in monocytes, macrophages, dendritic cells, $\mathrm{T}$ and $\mathrm{B}$ lymphocytes. In fact, experimental studies and clinical trials have shown beneficial effects of vitamin D supplementation on immune function [24]. Another possible mechanism is that vitamin $\mathrm{D}$ may have direct effects on $\beta$-cells, including improving insulin secretion, enhancing expression of vitamin D receptor, and improving islet morphology [24,25]. In addition, vitamin D may have effects on regulation of inflammatory responses [24].

To date, no randomized controlled trials directly look at the effects of vitamin D supplementation on incidence of type 1 diabetes. Several trials tested the effects of vitamin D supplementation on residual $\beta$-cell function in recent-onset type 1 diabetes [26-29]. However, the results were somewhat mixed. Three trials [26-28] reported that calcitriol (the active form of vitamin D) had no protective effects or only modest effects on $\beta$-cell function, while one trial [29] suggested cholecalciferol (vitamin D3) used as adjunctive therapy with insulin slowed decline of residual $\beta$-cell function. Interestingly, in one trial conducted in healthy subjects, vitamin D3 supplementation did not improve $\beta$-cell function but significantly increased the frequency of regulatory $\mathrm{T}$ cells [30]. Such protective immunologic effect, which was also observed in an above mentioned trial conducted in type 1 diabetes [29], indicated a possible mechanistic link between vitamin D and the disease.

Measurement of vitamin D intake using questionnaires is inaccurate due to recall bias and other measurement errors; moreover, sun exposure was not taken into account. Therefore, some studies assessed blood 25-hydroxyvitamin D [25(OH)D] level as a reliable measure of total vitamin D status. One case-cohort study found plasma $25(\mathrm{OH}) \mathrm{D}$ level was not associated with type 1 diabetes risk in islet autoimmunity-positive children (adjusted hazard ratio $=0.91,95 \%$ CI 0.68-1.22) [15]. The small number of identified cases $(n=35)$ may be responsible for the null association. More recently, one nested case-control study among US military personnel with more cases $(n=310)$ reported that higher blood $25(\mathrm{OH}) \mathrm{D}$ levels was associated with a significantly lower risk of type 1 diabetes among non-Hispanic whites, although such association was not observed in non-Hispanic blacks or Hispanics [31].

Whether vitamin D deficiency in the fetal period contributes to increased risk of type 1 diabetes is of additional interest. Previous study indicated maternal intake of vitamin D had a protective effect on preventing diabetes-associated autoimmunity in the children [32]. Yet, our meta-analysis of three studies $[9,11,17]$ provided no evidence for the association between maternal intake of vitamin $\mathrm{D}$ and diabetes risk in the offspring. In line with our finding, one Finnish study also found no difference in serum 25(OH)D levels during early pregnancy between case and control mothers [33]. By contrast, another study in Norway suggested lower maternal level of $25(\mathrm{OH}) \mathrm{D}$ during pregnancy was associated 
with a significantly higher risk of type 1 diabetes in the offspring $(\mathrm{OR}=2.38,95 \% \mathrm{CI} 1.12-5.07)$ [34]. Given the limited and inconsistent evidence at present, more studies are needed to determine the role of vitamin $\mathrm{D}$ in the fetal period in the development of type 1 diabetes.

Compared with a previous meta-analysis of only five studies [35], we added more evidence linking vitamin $\mathrm{D}$ and type 1 diabetes up to date. We were also able to examine the association between maternal vitamin D intake during pregnancy and diabetes risk in the offspring, which was not measured in that meta-analysis. However, limitations of the present study should be considered. First, because the findings were mainly based on case-control studies, recall bias and selection bias were inevitable. Also, the adjusted covariates in individual studies differed and the lack of control for important confounding factors increased the risk of confounding bias. Second, there was considerable heterogeneity among studies. Study design did not seem to explain much between-study variation as heterogeneity remained in both case-control and cohort subgroups (data not shown). Third, measurement errors could influence our findings as assessments of vitamin D intake largely depended upon questionnaires. Moreover, whether the questionnaires were validated was not reported in several studies. Fourth, we included two studies $[9,11]$ using cod liver oil as vitamin D supplementation, we therefore could not rule out the possibility that $n-3$ fatty acids in cod liver oil may, at least in part, account for the observed benefits on type 1 diabetes risk. Finally, although there was no evidence of publication bias, we could not completely rule out such bias because of the limited number of studies.

\section{Conclusions}

Our findings suggest that vitamin D intake during early life is associated with a reduced risk of type 1 diabetes. There was not enough evidence supporting an association between maternal intake of vitamin D and type 1 diabetes risk in the offspring. Because these findings were largely based upon case-control studies, well-designed cohort studies are still needed to determine the role of vitamin $\mathrm{D}$ in the prevention of type 1 diabetes.

\section{Acknowledgments}

The study was supported by 2012 Chinese Nutrition Society (CNS) Nutrition Research Foundation-DSM Research Fund.

\section{Conflicts of Interest}

The authors declare no conflict of interest.

\section{References}

1. DIAMOND Project Group. Incidence and trends of childhood type 1 diabetes worldwide 1990-1999. Diabet. Med. 2006, 23, 857-866. 
2. Onkamo, P.; Vaananen, S.; Karvonen, M.; Tuomilehto, J. Worldwide increase in incidence of Type I diabetes - The analysis of the data on published incidence trends. Diabetologia 1999, 42, 1395-1403.

3. Mohr, S.B.; Garland, C.F.; Gorham, E.D.; Garland, F.C. The association between ultraviolet B irradiance, vitamin $\mathrm{D}$ status and incidence rates of type 1 diabetes in 51 regions worldwide. Diabetologia 2008, 51, 1391-1398.

4. Zhang, W.; Stoecklin, E.; Eggersdorfer, M. A glimpse of vitamin D status in Mainland China. Nutrition 2013, 29, 953-957.

5. Bin-Abbas, B.S.; Jabari, M.A.; Issa, S.D.; Al-Fares, A.H.; Al-Muhsen, S. Vitamin D levels in Saudi children with type 1 diabetes. Saudi Med. J. 2011, 32, 589-592.

6. Janner, M.; Ballinari, P.; Mullis, P.E.; Fluck, C.E. High prevalence of vitamin D deficiency in children and adolescents with type 1 diabetes. Swiss Med. Wkly. 2010, 140, w13091.

7. Mutlu, A.; Mutlu, G.Y.; Ozsu, E.; Cizmecioglu, F.M.; Hatun, S. Vitamin D deficiency in children and adolescents with type 1 diabetes. J. Clin. Res. Pediatr. Endocrinol. 2011, 3, 179-183.

8. The EURODIAB Substudy 2 Study Group. Vitamin D supplement in early childhood and risk for type I (insulin-dependent) diabetes mellitus. Diabetologia 1999, 42, 51-54.

9. Stene, L.C.; Ulriksen, J.; Magnus, P.; Joner, G. Use of cod liver oil during pregnancy associated with lower risk of type I diabetes in the offspring. Diabetologia 2000, 43, 1093-1098.

10. Hypponen, E.; Laara, E.; Reunanen, A.; Jarvelin, M.R.; Virtanen, S.M. Intake of vitamin D and risk of type 1 diabetes: A birth-cohort study. Lancet 2001, 358, 1500-1503.

11. Stene, L.C.; Joner, G. Use of cod liver oil during the first year of life is associated with lower risk of childhood-onset type 1 diabetes: A large, population-based, case-control study. Am. J. Clin. Nutr. 2003, 78, 1128-1134.

12. Visalli, N.; Sebastiani, L.; Adorisio, E.; Conte, A.; De Cicco, A.L.; D’Elia, R.; Manfrini, S.; Pozzilli, P.; IMDIAB Group. Environmental risk factors for type 1 diabetes in Rome and province. Arch. Dis. Child. 2003, 88, 695-698.

13. Tenconi, M.T.; Devoti, G.; Comelli, M.; Pinon, M.; Capocchiano, A.; Calcaterra, V.; Pretti, G.; Pavia T1DM Registry Group. Major childhood infectious diseases and other determinants associated with type 1 diabetes: A case-control study. Acta Diabetol. 2007, 44, 14-19.

14. Ahadi, M.; Tabatabaeiyan, M.; Moazzami, K. Association between environmental factors and risk of type 1 diabetes-A case-control study. Endokrynol. Pol. 2011, 62, 134-137.

15. Simpson, M.; Brady, H.; Yin, X.; Seifert, J.; Barriga, K.; Hoffman, M.; Bugawan, T.; Barón, A.E.; Sokol, R.J.; Eisenbarth, G.; et al. No association of vitamin D intake or 25-hydroxyvitamin D levels in childhood with risk of islet autoimmunity and type 1 diabetes: The Diabetes Autoimmunity Study in the Young (DAISY). Diabetologia 2011, 54, 2779-2788.

16. Lindberg, B.; Ivarsson, S.A.; Landin-Olsson, M.; Sundkvist, G.; Svanberg, L.; Lernmark, A. Islet autoantibodies in cord blood from children who developed type I (insulin-dependent) diabetes mellitus before 15 years of age. Diabetologia 1999, 42, 181-187. 
17. Marjamaki, L.; Niinisto, S.; Kenward, M.G.; Uusitalo, L.; Uusitalo, U.; Ovaskainen, M.L.; Kronberg-Kippilä, C.; Simell, O.; Veijola, R.; Ilonen, J.; et al. Maternal intake of vitamin D during pregnancy and risk of advanced $\beta$ cell autoimmunity and type 1 diabetes in offspring. Diabetologia 2010, 53, 1599-1607.

18. Stroup, D.F.; Berlin, J.A.; Morton, S.C.; Olkin, I.; Williamson, G.D.; Rennie, D.; Moher, D.; Becker, B.J.; Sipe, T.A.; Thacker, S.B.; et al. Meta-analysis of observational studies in epidemiology. JAMA 2000, 283, 2008-2012.

19. Higgins, J.P.; Thompson, S.G. Quantifying heterogeneity in a meta-analysis. Stat. Med. 2002, $21,1539-1558$.

20. Egger, M.; Davey, S.G.; Schneider, M.; Minder, C. Bias in meta-analysis detected by a simple, graphical test. $B M J 1997,315,629-634$.

21. STATA, version 11.0; StataCrop: 4905 Lakeway Drive, College Station, TX, USA, 2009.

22. Disanto, G.; Chaplin, G.; Morahan, J.M.; Giovannoni, G.; Hypponen, E.; Ebers, G.C.; Ramagopalan, S.V. Month of birth, vitamin D and risk of immune mediated disease: A case control study. BMC Med. 2012, 10, 69.

23. Greer, R.M.; Portelli, S.L.; Hung, B.S.; Cleghorn, G.J.; McMahon, S.K.; Batch, J.A.; Conwell, L.S. Serum vitamin D levels are lower in Australian children and adolescents with type 1 diabetes than in children without diabetes. Pediatr. Diabetes 2013, 14, 31-41.

24. Prietl, B.; Treiber, G.; Pieber, T.R.; Amrein, K. Vitamin D and immune function. Nutrients 2013, 5, 2502-2521.

25. Wacker, M.; Holick, M.F. Vitamin D-effects on skeletal and extraskeletal health and the need for supplementation. Nutrients 2013, 5, 111-148.

26. Pitocco, D.; Crino, A.; Di Stasio, E.; Manfrini, S.; Guglielmi, C.; Spera, S.; Anguissola, G.B.; Visalli, N.; Suraci, C.; Matteoli, M.C.; et al. The effects of calcitriol and nicotinamide on residual pancreatic $\beta$-cell function in patients with recent-onset type 1 diabetes (IMDIAB XI). Diabet. Med. 2006, 23, 920-923.

27. Bizzarri, C.; Pitocco, D.; Napoli, N.; di Stasio, E.; Maggi, D.; Manfrini, S.; Suraci, C.; Cavallo, M.G.; Cappa, M.; Ghirlanda, G.; et al. No protective effect of calcitriol on $\beta$-cell function in recent-onset type 1 diabetes: The IMDIAB XIII trial. Diabetes Care 2010, 33, 1962-1963.

28. Walter, M.; Kaupper, T.; Adler, K.; Foersch, J.; Bonifacio, E.; Ziegler, A.G. No effect of the 1alpha,25-dihydroxyvitamin D3 on $\beta$-cell residual function and insulin requirement in adults with new-onset type 1 diabetes. Diabetes Care 2010, 33, 1443-1448.

29. Gabbay, M.A.; Sato, M.N.; Finazzo, C.; Duarte, A.J.; Dib, S.A. Effect of cholecalciferol as adjunctive therapy with insulin on protective immunologic profile and decline of residual $\beta$-cell function in new-onset type 1 diabetes mellitus. Arch. Pediatr. Adolesc. Med. 2012, 166, 601-607.

30. Bock, G.; Prietl, B.; Mader, J.K.; Holler, E.; Wolf, M.; Pilz, S.; Graninger, W.B.; Obermayer-Pietsch, B.M.; Pieber, T.R. The effect of vitamin D supplementation on peripheral regulatory $\mathrm{T}$ cells and $\beta$ cell function in healthy humans: a randomized controlled trial. Diabetes Metab. Res. Rev. 2011, 27, 942-945. 
31. Munger, K.L.; Levin, L.I.; Massa, J.; Horst, R.; Orban, T.; Ascherio, A. Preclinical serum 25-hydroxyvitamin D levels and risk of type 1diabetes in a cohort of US military personnel. Am. J. Epidemiol. 2013, 177, 411-419.

32. Fronczak, C.M.; Baron, A.E.; Chase, H.P.; Ross, C.; Brady, H.L.; Hoffman, M.; Eisenbarth, G.S.; Rewers, M.; Norris, J.M. In utero dietary exposures and risk of islet autoimmunity in children. Diabetes Care 2003, 26, 3237-3242.

33. Miettinen, M.E.; Reinert, L.; Kinnunen, L.; Harjutsalo, V.; Koskela, P.; Surcel, H.M.; Lamberg-Allardt, C.; Tuomilehto, J. Serum 25-hydroxyvitamin D level during early pregnancy and type 1 diabetes risk in the offspring. Diabetologia 2012, 55, 1291-1294.

34. Sorensen, I.M.; Joner, G.; Jenum, P.A.; Eskild, A.; Torjesen, P.A.; Stene, L.C. Maternal serum levels of 25-hydroxy-vitamin D during pregnancy and risk of type 1 diabetes in the offspring. Diabetes 2012, 61, 175-178.

35. Zipitis, C.S.; Akobeng, A.K. Vitamin D supplementation in early childhood and risk of type 1 diabetes: A systematic review and meta-analysis. Arch. Dis. Child. 2008, 93, 512-517. 


\title{
Vitamin D and Obesity
}

\section{Simon Vanlint}

\begin{abstract}
Obesity is a significant health problem world-wide, particularly in developed nations. Vitamin D deficiency is pandemic, and has been implicated in a wide variety of disease states. This paper seeks to examine the consistently reported relationship between obesity and low vitamin D concentrations, with reference to the possible underlying mechanisms. The possibility that vitamin D may assist in preventing or treating obesity is also examined, and recommendations for future research are made. There is a clear need for adequately-powered, prospective interventions which include baseline measurement of 25D concentrations and involve adequate doses of supplemental vitamin D. Until such studies have been reported, the role of vitamin D supplementation in obesity prevention remains uncertain.
\end{abstract}

Reprinted from Nutrients. Cite as: Hessel, V. Special Issue: Design and Engineering of Microreactor and Smart-Scaled Flow Processes. Nutrients 2013, 5, 949-956.

\section{Introduction}

Obesity, defined by the World Health Organisation as a body mass index (BMI) of $30 \mathrm{~kg} / \mathrm{m}^{2}$ or more, is pandemic, affecting at least five million Australians and substantial numbers in most developed nations [1]. If overweight (BMI 25-29.9) is included, then approximately 14 million Australians, and $70 \%$ of Americans aged over 60, are obese or overweight [2]. Older people who have excess body fat accumulation face increased risk for coronary heart disease, hypertension, metabolic syndrome, osteoarthritis, diabetes mellitus, and other co-morbidities [3-6]. It is imperative that modifiable risk factors for obesity be identified, particularly those which might be readily addressed.

There is a consistent association in the published literature between increasing BMI and lower serum 25-hydroxyvitamin D (25D) concentrations. Early, smaller studies $[7,8]$ reported an association between obesity and low serum 25D concentrations, as well as high concentrations of parathyroid hormone (PTH) and 1,25-dihydroxyvitamin D (1,25D). However, further, larger studies $[9,10]$ found obesity to be associated with lower 25D concentrations, high PTH concentrations and low 1,25D concentrations. It has also been reported that body fat content is inversely related to serum 25D concentration, and that this associations is stronger than those between 25D and BMI and body weight [11]. A bi-directional genetic study, which limits confounding, has suggested that higher BMI leads to lower 25D, with the effects of lower 25D on BMI likely to be small [12]. The association between reduced 25D concentrations and obesity is therefore well-established, although the mechanisms for the lower 25D concentrations are not fully described, and there is uncertainty as to what the health consequences of these lower concentrations might be. This paper attempts to summarise the current state of knowledge regarding the causes of these reduced 25D concentrations, as well as the possible effects of vitamin D supplementation on obesity. 


\section{Possible Mechanisms for Lower 25D Concentrations in Obese Individuals}

\subsection{Lower Dietary Intake}

Vitamin D intake has been reported as being lower in obese men, but not women, when compared to their non-obese counterparts [13]. Low calcium and D intake have also been associated with obesity in both men and women [14], but this association does not necessarily imply a causal relationship.

\subsection{Reduced Cutaneous Synthesis}

\subsubsection{Altered Behaviour}

It is possible that obese individuals expose less skin to the sun less often than non-obese individuals, resulting in reduced synthesis of vitamin D. BMI, \% body fat and sunbathing have been shown to be related in a population-based sample [15], although another study [16] found no relationship in a study of individuals aged over 65 years. This latter finding may be explained by the known decline in cutaneous vitamin D synthetic capacity associated with age [17]. It has also been noted that obesity results in larger body surface area [18], and thus could be expected to increase cutaneous vitamin D synthesis.

\subsubsection{Reduced Synthetic Capacity}

Concentrations of cutaneous 7-dehydrocholesterol (the substrate converted by ultraviolet light into previtamin D) appear not to vary between obese and non-obese individuals [19]. The existence of sufficient synthetic capacity is implied by the reduced risk of low serum 25D concentrations associated with outdoor exercise in obese individuals [20].

\subsection{Reduced Intestinal Absorption}

Hypovitaminosis D is well-documented in those who have had bariatric or gastric bypass procedures, in which a malabsorptive state is deliberately induced [21,22], but there is no evidence that obesity itself results in reduced absorption of dietary vitamin D. Given that vitamin D is fat-soluble, and that calcium absorption has been shown to be increased in diets high in fats [23], it is unlikely that obesity affects vitamin D-calcium homeostasis through altered gut absorption.

\subsection{Altered Metabolism}

\subsubsection{Reduced Activation and/or Increased Catabolism}

$1,25 \mathrm{D}$ acts to limit production of its precursor, 25D [24]. Because early studies suggested that $1,25 \mathrm{D}$ concentrations were elevated in obese individuals, it was thought that this may lower $25 \mathrm{D}$ levels. Given that further, larger studies have suggested that $1,25 \mathrm{D}$ concentrations tend to be lower in obese individuals, this feedback mechanism is unlikely to be relevant. Adipose tissue (AT) in obese women expresses the enzymes for both the formation of 25D and its active metabolite, 1,25D and for degradation of vitamin D [25]. Subcutaneous AT has also been found to have lower 
expression of one of the enzymes responsible for 25-hydroxylation of vitamin D (CYP2J2), as well as a tendency toward a decreased expression of the 1- $\alpha$ hydroxylase. These data suggest that both 25 -hydroxylation and 1- $\alpha$ hydroxylation are impaired in obesity. In vitro studies have demonstrated that $1,25 \mathrm{D}$ inhibits adipogenesis and induces adipocyte apoptosis [26,27]. Under normal physiological conditions the serum $1,25 \mathrm{D}$ concentration is tightly regulated, yet there can be significant differences between 1,25D concentrations within different tissues owing to in situ production. These factors make interpretation of the clinical significance of in vitro studies very difficult.

\subsubsection{Sequestration of $25 \mathrm{D}$ in Adipose Tissue}

Radio-labelling shows that $80 \%$ of vitamin $\mathrm{D}$ administered to rats is deposited rapidly in AT, from which it is then released very slowly [28]. Liquid chromatography/mass spectroscopy has shown a positive relationship between vitamin $\mathrm{D}$ in AT and serum 25D, consistent with AT being a storage site for 25D but not specifically implying sequestration [29]. These laboratory findings are consistent with clinical studies in which equal ultraviolet irradiation and also equal oral doses of vitamin D resulted in a $57 \%$ lower increase in serum $25 \mathrm{D}$ concentrations in obese individuals compared non-obese [19].

\subsection{A Much Simpler Explanation}

Despite the hypotheses and findings outlined above, an elegant study of 686 community-dwelling individuals showed that a volumetric dilutional model accounted for essentially all the variability in serum 25D concentrations attributable to obesity. Even though the factors described above may be operative, they are effectively "captured" by body weight. Once serum 25D concentrations in obese individuals are adjusted for body size, there is no longer a difference between obese and non-obese individuals [30]. The authors concluded that in obese individuals, vitamin D dosing for the treatment of deficiency should be based upon body weight, and calculated that an input of 70-80 IU/ kg/day would be expected to produce serum 25D concentrations in the $75-100 \mathrm{nmol} / \mathrm{L}$ range.

\section{Does Vitamin D Status Affect Obesity?}

\subsection{Background}

Nuclear and membrane vitamin D receptors (VDRs) have been demonstrated in adipocytes, suggesting that AT is responsive to vitamin D [31]. A variety of mechanisms by which vitamin D and/or calcium may influence adiposity and energy balance have been proposed [32], but interventional studies have thus far been inconclusive, at least in part due to methodological issues.

$1,25 \mathrm{D}$ has been shown to have an in vitro anti-inflammatory effect on adipocytes, but the same study failed to demonstrate any reduction in systemic inflammatory markers in vivo in participants receiving $7000 \mathrm{IU}$ oral vitamin D daily over an unspecified period [33]. Without knowing the pre-treatment 25D concentrations of the participants or the duration of oral supplementation it is difficult to interpret these findings. Baseline 25D concentrations were examined in relation to prevalent and cumulative incident obesity in a study of 2460 adults. In addition to prevalent obesity, serum 
25D concentrations below $50 \mathrm{nmol} / \mathrm{L}$ were significantly associated with new-onset obesity [34]. Although this does not prove a causative effect, it is highly suggestive and warrants further clinical trials.

\subsection{Co-Administered Vitamin D and Calcium}

Vitamin D is critical for calcium metabolism, and it is difficult to separate the effects of vitamin D from those of calcium, and of the two given in combination. Changes in weight, visceral fat mass and visceral fat area were compared in obese college students assigned to either a calorie-restricted diet with or without calcium $600 \mathrm{mg}$ plus vitamin D 125 IU daily for 12 weeks in an open-label study. There was no difference in weight change between the groups, although the supplementation group did exhibit significantly greater decreases in visceral fat mass and fat area [35]. This study was limited by the very modest vitamin $\mathrm{D}$ dose used and the lack of baseline $25 \mathrm{D}$ measurement. Another randomised, double-blind 16-week study of 171 overweight and obese adults found that the addition of $1050 \mathrm{mg}$ calcium with $300 \mathrm{IU}$ of vitamin D daily was associated with a significant reduction in visceral adiposity compared with placebo [36]. 25D concentrations were not measured. Secondary analysis of data from a population-based, double-blind, placebo-controlled, randomised trial of 1179 postmenopausal women designed to determine the effects of calcium and vitamin $\mathrm{D}$ on osteoporotic fractures concluded that calcium supplementation over a four year period had a beneficial effect on body composition, but with no additional effect of vitamin $\mathrm{D}$ in the presence of a high calcium intake [37].

\subsection{Vitamin D Alone}

Turning to vitamin D administered without calcium, one study randomised 77 overweight and obese women to receive either $1000 \mathrm{IU}$ of vitamin D daily or a placebo. In both groups the mean baseline 25D concentration was below $50 \mathrm{nmol} / \mathrm{L}$, indicating deficiency. Vitamin D supplementation caused a significant reduction in body fat mass compared with placebo, as well as a significant rise in $25 \mathrm{D}$ concentrations. However, neither body weight nor waist circumference changed significantly in either group [38]. Changes in 25D concentration and changes in fat mass were significantly inversely correlated. A randomised study of 445 overweight and obese adults to receive either 20,000 IU of vitamin D twice weekly (DD), 20,000 IU once weekly plus placebo once weekly (DP), or placebo twice weekly (PP) for 12 months. 25D concentrations rose significantly in both the DD and DP groups, but not the PP group. There was no difference in weight change between the three groups. However, mean 25D concentrations in all three groups were above $50 \mathrm{nmol} / \mathrm{L}$ at baseline [39], which substantially reduces the impact of these findings. The overall impression is that vitamin $\mathrm{D}$ with or without calcium appears not to have a definite effect on weight, but that it may affect fat mass and distribution. However, the evidence is not yet complete enough to be compelling, and further research is needed.

\subsection{Effects of Weight Loss on Vitamin D Concentration}

There is also evidence that weight loss leads to increased 25D concentrations, which may in turn provide additional protection against chronic disease. Data from 383 overweight or obese women 
who participated in a 2-year clinical trial of a weight-loss program showed that those who did not lose weight at 24 months had an increase in serum 25D of $1.9 \mathrm{ng} / \mathrm{mL}(4.8 \mathrm{nmol} / \mathrm{L})$. However, 25D increased by $2.7 \mathrm{ng} / \mathrm{mL}(6.8 \mathrm{nmol} / \mathrm{L})$ for those who lost $5 \%-10 \%$ of baseline weight, and by 5.0 $\mathrm{ng} / \mathrm{mL}(12.5 \mathrm{nmol} / \mathrm{L})$ for those who lost $>10 \%$ of baseline weight $(P=0.014)$ [40]. These findings suggest that weight loss is associated with increased serum $25 \mathrm{D}$ concentration in overweight or obese women. $49 \%$ of participants were deficient (25D below $20 \mathrm{ng} / \mathrm{mL}(50 \mathrm{nmol} / \mathrm{L})$ ) at baseline. By study end $36 \%$ of all participants were deficient, with $17 \%$ of those who achieved a normal BMI being deficient.

\section{Conclusions}

The association between reduced 25D concentrations and obesity is well established, and can be adequately accounted for by a volumetric, dilutional model. Correction of low 25D concentrations in obese individuals requires higher doses than those often advocated for the general population.

There are plausible mechanisms and some in vitro evidence supporting a role for vitamin D in weight reduction, with the proviso that it may be difficult to determine which effects are due to vitamin D itself and which are mediated via calcium. Clinical trials have not been conclusive, at least in part due to variable quality of study design. Some studies showing no effect of vitamin D supplementation on weight included participants who were vitamin D replete, and may thus have shown that giving supplemental vitamin $\mathrm{D}$ to those who are replete has no additional effect. There is a clear need for adequately-powered, prospective interventions which include baseline measurement of $25 \mathrm{D}$ concentrations and involve adequate doses of supplemental vitamin D. Until such studies have been reported, the role of vitamin D supplementation in obesity prevention remains uncertain.

\section{Conflicts of Interest}

The author declares no conflict of interest

\section{References}

1. Noncommunicable Diseases Country Profiles; World Health Organisation: Geneva, Switzerland, 2011; p. 209.

2. Pi-Sunyer, F.X. The obesity epidemic: Pathophysiology and consequences of obesity. Obes. Res. 2002, 10, 97S-104S.

3. Goodpaster, B.; Krishnaswami, S.; Harris, T.; Katsiaras, A.; Kritchevsky, S.; Simonsick, E.; Nevitt, M.; Holvoet, P.; Newman, A. Obesity, regional body fat distribution, and the metabolic syndrome in older men and women. Arch. Int. Med. 2005, 165, 777-783.

4. Stranges, S.; Trevisan, M.; Dorn, J.; Dmochowski, J.; Donahue, R. Body fat distribution, liver enzymes, and risk of hypertension: Evidence from the western New York study. Hypertension 2005, 46, 1186-1193.

5. Lementowski, P.; Zelicof, S. Obesity and osteoarthritis. Am. J. Orthop. 2008, 37, 148-151.

6. Must, A.; Spadano, J.; Coakley, E.; Field, A.; Colditz, G.; Dietz, W. The disease burden associated with overweight and obesity. JAMA 1999, 282, 1523-1529. 
7. Bell, N.H.; Epstein, S.; Greene, A.; Shary, J.; Oexmann, M.J.; Shaw, S. Evidence for alteration of the vitamin D-endocrine system in obese subjects. J. Clin. Invest. 1985, 76, 370-373.

8. Liel, Y.; Ulmer, E.; Shary, J.; Hollis, B.; Bell, N. Low circulating vitamin D in obesity. Calcif. Tissue Int. 1988, 43, 199-201.

9. Parikh, S.J.; Edelman, M.; Uwaifo, G.I.; Freedman, R.J.; Semega-Janneh, M.; Reynolds, J.; Yanovski, J.A. The relationship between obesity and serum 1,25-dihydroxy vitamin D concentrations in healthy adults. J. Clin. Endocrinol. Metab. 2004, 89, 1196-1199.

10. Lagunova, Z.; Porojnicu, A.; Lindberg, F.; Hexeberg, S.; Moan, J. The dependency of vitamin D status on body mass index, gender, age and season. Anticancer Res. 2009, 29, 3713-3720.

11. Arunabh, S.; Pollack, S.; Yeh, J.; Aloia, J.F. Body fat content and 25-hydroxyvitamin D levels in healthy women. J. Clin. Endocrinol. Metab. 2003, 88, 157-161.

12. Vimeswaran, K.; Berry, D.; Lu, C.; Pilz, S.; Hiraki, L.; Cooper, J.; Dastani, Z.; Li, R.; Houston, D.; Wood, A. Causal relationship between obesity and vitamin D status: Bi-directional mendelian randomization analysis of multiple cohorts. PLoS Med. 2013, 10, 1549-1676.

13. Kamycheva, E.; Sundsfjord, J.; Jorde, R. Serum parathyroid hormone level is associated with body mass index. The 5th tromso study. Eur. J. Endocrinol. 2004, 151, 167-172.

14. Kamycheva, E.; Joakimsen, R.M.; Jorde, R. Intakes of calcium and vitamin D predict body mass index in the population of northern norway. J. Nutr. 2003, 133, 102-106.

15. Kull, M.; Kallikorm, R.; Lember, M. Body mass index determines sunbathing habits: Implications on vitamin D levels. Intern. Med. J. 2009, 39, 256-258.

16. Harris, S.S.; Dawson-Hughes, B. Reduced sun exposure does not explain the inverse association of 25-hydroxyvitamin D with percent body fat in older adults. J. Clin. Endocrinol. Metab. 2007, 92, 3155-3157.

17. Holick, M.F.; Matsuoka, L.Y.; Wortsman, J. Age, vitamin D and solar ultraviolet. Lancet 1989, 334, 1104-1105.

18. Verbraecken, J.; van de Heyning, P.; De Backer, W.; van Gaal, L. Body surface area in normal-weight, overweight, and obese adults. A comparison study. Metabolism 2006, 55, $515-524$.

19. Wortsman, J.; Matsuoka, L.Y.; Chen, T.C.; Lu, Z.; Holick, M.F. Decreased bioavailability of vitamin D in obesity. Am. J. Clin. Nutr. 2000, 72, 690-693.

20. Florez, H.; Martinez, R.; Chacra, W.; Strickman-Stein, N.; Levis, S. Outdoor exercise reduces the risk of hypovitaminosis D in the obese. J. Steroid Biochem. Mol. Biol. 2007, 103, 679-681.

21. Hewitt, S.; Søvik, T.; Aasheim, E.; Kristinsson, J.; Jahnsen, J.; Birketvedt, G.; Bøhmer, T.; Eriksen, E.; Mala, T. Secondary hyperparathyroidism, vitamin D sufficiency, and serum calcium 5 years after gastric bypass and duodenal switch. Obes. Surg. 2013, 23, 384-390.

22. Aarts, E.; van Groningen, L.; Horst, R.; Telting, D.; van Sorge, A.; Janssen, I.; de Boer, H. Vitamin D absorption: Consequences of gastric bypass surgery. Eur. J. Endocrinol. 2011, 164, 827-832.

23. Shapses, S.A.; Sukumar, D.; Schneider, S.H.; Schlussel, Y.; Brolin, R.E.; Taich, L. Hormonal and dietary influences on true fractional calcium absorption in women: Role of obesity. Osteoporosis Int. 2012, 23, 2607-2614. 
24. Bell, N.H.; Shaw, S.; Turner, R.T. Evidence that 1,25-dihydroxyvitamin D3 inhibits the hepatic production of 25-hydroxyvitamin D in man. J. Clin. Invest. 1984, 74, 1540-1544.

25. Wamberg, L.; Christiansen, T.; Paulsen, S.K.; Fisker, S.; Rask, P.; Rejnmark, L.; Richelsen, B.; Pedersen, S.B. Expression of vitamin D-metabolizing enzymes in human adipose tissue-The effect of obesity and diet-induced weight loss. Int. J. Obes. 2012, doi:10.1038/ijo.2012.112.

26. Kong, J.; Li, Y.C. Molecular mechanism of 1,25-dihydroxyvitamin D3 inhibition of adipogenesis in 3t3-11 cells. Am. J. Physiol. Endocrinol. Metab. 2006, 290, E916-E924.

27. Sergeev, I. 1,25-dihydroxyvitamin D3 induces $\mathrm{Ca}^{2+}$-mediated apoptosis in adipocytes via activation of calpain and caspase-12. Biochem. Biophys. Res. Commun. 2009, 384, 18-21.

28. Rosenstreich, S.; Rich, C.; Volwiler, W. Deposition in and release of vitamin D3 from body fat: Evidence for a storage site in the rat. J. Clin. Invest. 1971, 50, 679-687.

29. Blum, M.; Dolnikowski, G.; Seyoum, E.; Harris, S.; Booth, S.; Peterson, J.; Saltzman, E.; Dawson-Hughes, B. Vitamin D3 in fat tissue. Endocrine 2008, 33, 90-94.

30. Drincic, A.T.; Armas, L.A.G.; van Diest, E.E.; Heaney, R.P. Volumetric dilution, rather than sequestration best explains the low vitamin D status of obesity. Obesity 2012, 20, 1444-1448.

31. Ding, C.; Gao, D.; Wilding, J.; Trayhurn, P.; Bing, C. Vitamin D signalling in adipose tissue. Br. J. Nutr. 2012, 108, 1915-1923.

32. Song, Q.; Sergeev, I.N. Calcium and vitamin D in obesity. Nutr. Res. Rev. 2012, 25, 130-141.

33. Wamberg, L.; Cullberg, Y.; Rejnmark, L.; Richelsen, B.; Pedersen, S. Investigations of the anti-inflammatory effects of vitamin D in adipose tissue: Results from an in vitro study and a randomized controlled trial. Horm. Metab. Res. 2013, in press.

34. Mai, X.-M.; Chen, Y.; Camargo, C.A.; Langhammer, A. Cross-sectional and prospective cohort study of serum 25-hydroxyvitamin D level and obesity in adults: The hunt study. Am. J. Epidemiol. 2012, 175, 1029-1036.

35. Zhu, W.; Cai, D.; Wang, Y.; Ning, L.; Hu, Q.; Qi, Y.; Ma, S.; Amarasekara, S. Calcium plus vitamin D3 supplementation facilitated fat loss in overweight and obese college students with very-low calcium consumption: A randomized controlled trial. Nutr. J. 2013, 12, 8-12.

36. Rosenblum, J.L.; Castro, V.M.; Moore, C.E.; Kaplan, L.M. Calcium and vitamin D supplementation is associated with decreased abdominal visceral adipose tissue in overweight and obese adults. Am. J. Clin. Nutr. 2012, 95, 101-108.

37. Zhou, J.; Zhou, L.-J.; Watson, P.; Zhang, Q.; Lappe, J. The effect of calcium and vitamin D supplementation on obesity in postmenopausal women: Secondary analysis for a large-scale, placebo controlled, double-blind, 4-year longitudinal clinical trial. Nutr. Metab. 2010, 7, 62.

38. Salehpour, A.; Hosseinpanah, F.; Shidfar, F.; Vafa, M.; Razaghi, M.; Dehghani, S.; Hoshiarrad, A.; Gohari, M. A 12-week double-blind randomized clinical trial of vitamin D3 supplementation on body fat mass in healthy overweight and obese women. Nutr. J. 2012, 11, 78.

39. Sneve, M.; Figenschau, Y.; Jorde, R. Supplementation with cholecalciferol does not result in weight reduction in overweight and obese subjects. Eur. J. Endocrinol. 2008, 159, 675-684.

40. Rock, C.L.; Emond, J.A.; Flatt, S.W.; Heath, D.D.; Karanja, N.; Pakiz, B.; Sherwood, N.E.; Thomson, C.A. Weight loss is associated with increased serum 25-hydroxyvitamin D in overweight or obese women. Obesity 2012, 20, 2296-2301. 


\title{
Association between Subcutaneous White Adipose Tissue and Serum 25-Hydroxyvitamin D in Overweight and Obese Adults
}

\author{
Brian D. Piccolo, Gregory Dolnikowski, Elias Seyoum, Anthony P. Thomas, Erik R. Gertz, \\ Elaine C. Souza, Leslie R. Woodhouse, John W. Newman, Nancy L. Keim, Sean H. Adams \\ and Marta D. Van Loan
}

\begin{abstract}
Cholecalciferol is known to be deposited in human adipose tissue, but it is not known whether 25-hydroxyvitamin $\mathrm{D}(25(\mathrm{OH}) \mathrm{D})$ is found in detectable concentrations. Therefore, our objective was to determine whether $25(\mathrm{OH}) \mathrm{D}$ is detectable in subcutaneous white adipose tissue (SWAT) in overweight and obese persons enrolled in a twelve week energy restricted diet. Baseline and post-intervention gluteal SWAT biopsies were collected from 20 subjects participating in a larger clinical weight loss intervention. LC-MS/MS was utilized to determine SWAT 25(OH)D concentrations. Serum $25(\mathrm{OH}) \mathrm{D}$ and $1,25(\mathrm{OH})_{2} \mathrm{D}$ were measured by RIA. Body composition was assessed by dual energy X-ray absorptiometry. SWAT 25(OH)D concentrations were $5.8 \pm 2.6 \mathrm{nmol} / \mathrm{kg}$ tissue and $6.2 \pm 2.7 \mathrm{nmol} / \mathrm{kg}$ tissue pre- and post-intervention SWAT, respectively. There was a significant positive association between SWAT 25(OH)D concentration and serum 25(OH)D concentration $(r=0.52, P<0.01)$. Both SWAT and serum $25(\mathrm{OH}) \mathrm{D}$ concentrations did not significantly change after a twelve-week period of energy restriction with approximately $5 \mathrm{~kg}$ of fat loss. In conclusion, we have demonstrated our LC-MS/MS method can detect $25(\mathrm{OH}) \mathrm{D}_{3}$ in human subcutaneous fat tissue from overweight and obese individuals and is consistent with previously reported concentrations in swine. Additionally, our findings of no significant changes in SWAT $25(\mathrm{OH}) \mathrm{D}_{3}$ or serum $25(\mathrm{OH}) \mathrm{D}$ after a $6 \%$ loss of total body weight and $13 \%$ reduction in total fat provides the first human evidence that adipose $25(\mathrm{OH}) \mathrm{D}$ does not likely contribute to serum $25(\mathrm{OH}) \mathrm{D}$ with moderate weight loss; whether this is also the case with larger amounts of weight loss is unknown. Weight loss alone is not sufficient to increase serum $25(\mathrm{OH}) \mathrm{D}$ and increases in dietary or dermal biosynthesis of vitamin D appear to be the most critical contributors to in vitamin D status.
\end{abstract}

Reprinted from Nutrients. Cite as: Piccolo, B.D.; Dolnikowski, G.; Seyoum, E.; Thomas, A.P.; Gertz, E.R.; Souza, E.C.; Woodhouse, L.R.; Newman, J.W.; Keim, N.L.; Adams, S.H.; Van Loan, M.D. Association between Subcutaneous White Adipose Tissue and Serum 25-Hydroxyvitamin D in Overweight and Obese Adults. Nutrients 2013, 5, 3352-3366.

\section{Introduction}

The analysis of vitamin D metabolites in adipose tissue is rare and likely due to difficulties in extracting and separating the metabolites from tissue matrices [1]. Nonetheless, there is a disparity in the literature between analyses of the parent compound, cholecalciferol, and the other vitamin D metabolites in adipose tissue. This would be expected since it has been well-established that cholecalciferol is the dominant metabolite distributed in adipose tissue [2]. In fact, there are only two reports of measurable concentrations of $25(\mathrm{OH}) \mathrm{D}$ in adipose tissue from human cadavers [2] and in swine [3]; suggesting a small yet quantifiable site of $25(\mathrm{OH}) \mathrm{D}$ deposition in adipose tissue. 
In a previous article, Heaney et al. [4] estimated adipose $25(\mathrm{OH}) \mathrm{D}$ content in a $70 \mathrm{~kg}$ woman with $24.5 \mathrm{~kg}$ fat mass and suggested a total of $114.5 \mathrm{nmol} 25(\mathrm{OH}) \mathrm{D}$ was distributed within this tissue compartment. However, the data used for this estimation were from swine adipose tissue [3]. To our knowledge, there have been no reports of adipose $25(\mathrm{OH}) \mathrm{D}$ analyses in healthy human subjects. Although the Heaney estimation was derived from a non-human source, the authors make the argument that the total content of $25(\mathrm{OH}) \mathrm{D}$ is widely distributed within other tissue compartments. It is widely known that the highest tissue concentration of $25(\mathrm{OH}) \mathrm{D}$ is found in serum, however, adipose tissue content of $25(\mathrm{OH}) \mathrm{D}$ could exceed serum content due to its large overall mass. Therefore, the content of adipose $25(\mathrm{OH}) \mathrm{D}$ may need to be considered when determining total body vitamin D status.

Thus, we analyzed samples of subcutaneous white adipose tissue (SWAT) obtained from overweight and obese individuals participating in controlled feeding weight loss intervention to determine whether $25(\mathrm{OH}) \mathrm{D}$ can be measured in human adipose tissue. Our objectives were to determine ranges of $25(\mathrm{OH}) \mathrm{D}_{3}$ in human SWAT, to test if SWAT concentrations of $25(\mathrm{OH}) \mathrm{D}_{3}$ are altered as a result of weight loss, and to assess whether or not SWAT 25(OH)D correlates with blood levels of this metabolite in humans.

\section{Materials and Methods}

\subsection{Study Design}

The current study was exploratory and part of a larger human clinical trial investigating the inclusion of 3-4 servings of dairy foods in an energy restricted diet; details of the parent project have been previously published [5]. The clinical intervention trial was registered at clinicaltrials.gov (NCT 00858312). Healthy women, aged 20-45 years, and healthy men, aged 20-50 years (lower age for women was used to avoid hormonal changes associated with the transition to menopause that might affect endocrine parameters and related study outcomes), were enrolled in a 15-week controlled feeding study in which all foods were provided or prepared by the Metabolic Food Laboratory (MFL) at the USDA, ARS, Western Human Nutrition Research Center (WHNRC). The 15-week study was divided into a 3-week "Run-In" period during which subjects were weighed daily and caloric intake adjusted to maintain body weight; thereby establishing energy requirements. The "Run-In" period was followed by a 12-week "Weight Loss Intervention" during which energy intake was reduced by $500 \mathrm{kcal} /$ day from the "Run-In" period. Therefore, pre-intervention measurements at the end of the "Run-In" period were considered baseline measures. Post-intervention measurements were made at the end of the 12-week "Weight Loss Intervention" period. Specific details regarding the dietary portion of the parent study can be found in the previously published manuscript [5]. The study and all procedures were approved by the Committee for Human Subjects Protection, Institutional Review Board of the University of California, Davis. Written informed consent was obtained from all research volunteers. 


\subsection{Subject Screening and Selection}

Overweight and obese adult females and males were recruited from the faculty, staff, and student population at University of California, Davis as well as the greater Davis and Sacramento, CA communities. Body mass index was between 28 and $37 \mathrm{~kg} / \mathrm{m}^{2}$. All participants were habitually low dairy consumers (defined as $\leq 1$ serving/day), and weight stable (no more than $3 \mathrm{~kg}$ weight loss during three months preceding the intervention). Exclusion was based on not meeting the age, not meeting the dairy and calcium criteria ( $\leq 1$ serving of dairy/day and a total calcium intake $\leq 600 \mathrm{mg} /$ day from all sources), diagnosis of type 2 diabetes, fasting glucose $\geq 110 \mathrm{mg} / \mathrm{dL}$, adverse response to study foods (lactose intolerance, dairy intolerance, dairy allergy determined by self-report), history or presence of significant metabolic disease (i.e., endocrine, hepatic, or renal), use of blood pressure or lipid-altering medications, resting blood pressure $\geq 160 / 100 \mathrm{mg} / \mathrm{Hg}$, triglyceride value $\geq 400 \mathrm{mg} / \mathrm{dL}$ or $\mathrm{LDL} \geq 160 \mathrm{mg} / \mathrm{dL}$, history of eating disorder, presence of active gastrointestinal disorders, pregnancy or lactation, use of obesity pharmacotherapeutic agents within the last 12 weeks, use of over-the-counter anti-obesity agents (e.g., those containing phenylpropanolamine, ephedrine and/or caffeine) within the last 12 weeks, use of calcium supplements in the past 12 weeks, recent (past four weeks) initiation of an exercise program, recent (past 12 weeks) initiation of hormonal birth control or change in hormonal birth control regimen, use of tobacco products, and exercise more than $30 \mathrm{~min} /$ day.

Body composition was assessed during the "Run-In" period and subjects were pair-matched based on percent body fat $(\% \mathrm{BF})$ and randomly assigned to one of two treatment groups: low dairy (LD, $\leq 1$ serving/day) or adequate dairy (AD, 3-4 servings/day). Enrollment was continuous with a new cohort starting approximately every 8 weeks. Consequently, volunteers were enrolled year-round. The Consort Diagram providing information on the number of eligible participants $v s$. enrolled participants has been previously published [5]. Forty percent of the participants were non-white and $25 \%$ of those were of Hispanic origin.

\subsection{Dietary Intake of Vitamin D}

Vitamin D content of the diet was determined by the Nutrition Data System for Research software (NDSR software version 2011, University of Minnesota, Minneapolis, MN) and expressed as daily IU intake. Daily dietary vitamin D intake varied based on diet group (AD, LD) and the menu day for the 7 day rotating diet. Average daily vitamin $\mathrm{D}$ intake is shown in Table 1.

Table 1. Subject characteristics at Baseline and Post-Intervention ${ }^{1}$.

\begin{tabular}{lcc}
\hline Variable & Baseline & Post-Intervention \\
\hline$N$ & 20 & 20 \\
Sex $(\mathrm{F} / \mathrm{M})$ & $15 / 5$ & \\
Age $(\mathrm{y})$ & $34.3(9.0)$ & \\
Height $(\mathrm{cm})$ & $170(1.0)$ & \\
Weight $(\mathrm{kg})$ & $93.1(10.9)$ & $87.3(10.3)^{*}$ \\
BMI $\left(\mathrm{kg} / \mathrm{m}^{2}\right)$ & $32.7(2.5)$ & $30.6(2.5)^{*}$ \\
Total Fat Mass $(\mathrm{kg})$ & $38.5(8.0)$ & $33.5(8.7)^{*}$ \\
Body Fat $(\%)$ & $43.5(6.0)$ & $40.9(6.7)$ \\
\hline
\end{tabular}


Table 1. Cont.

\begin{tabular}{|c|c|c|}
\hline Variable & Baseline & Post-Intervention \\
\hline Lean Mass (kg) & $49.3(10.0)$ & $48.3(9.6)$ \\
\hline Waist Circumference $(\mathrm{cm})^{2}$ & $94.2(8.3)$ & $89.2(7.2) *$ \\
\hline Intra-abdominal Adipose Tissue, cc & $41.7(20.0)$ & $31.5(15.9) *$ \\
\hline Android Fat (kg) & $3.8(0.7)$ & $3.3(0.7) *$ \\
\hline Gynoid Fat (kg) & $7.3(1.5)$ & $6.6(1.5) *$ \\
\hline Dietary Vitamin D intake (IU/day) & $190.2(18.2)$ & $216.5(83.8)$ \\
\hline
\end{tabular}

${ }^{1}$ Values expressed as mean (SD) (except $\mathrm{n}$ and Sex); * denotes significant difference $(P \leq 0.05)$ between baseline and post-intervention based on paired t-test; ${ }^{2} n=19$ post-intervention.

\subsection{Body Composition Measurements}

Body weight was measured on an electronic scale (Scale-tronic model 6002; Wheaton, IL, USA) to the nearest $0.1 \mathrm{~kg}$ with subjects in light clothing, all jewelry removed, pockets emptied, and shoes removed. Height was measured using a wall mounted stadiometer (Ayrton Stadiometer model S100; Prior Lake MN) and recorded to the nearest $0.1 \mathrm{~cm}$. Body mass index was calculated as $\mathrm{kg} / \mathrm{m}^{2}$. Waist circumference was measured with a metal non stretchable tape measure (Model Gluick, Lafayette Instrument, Lafayette, IN, USA) to the nearest $0.1 \mathrm{~cm}$ in the standing position against bare skin with the abdomen relaxed and arms at the sides. The average of two readings was reported for weight, height and waist circumference. Fat mass were assessed using dual energy x-ray absorptiometry (DXA, GE Lunar, Prodigy Model) during the "Run-In" period and at the end of the 12-week intervention. Daily calibration procedures were carried out per manufacturer instructions. To reduce the variance in the measurement data, all DXA scans were analyzed by a single technician. Intra-abdominal adipose tissue was measured using computed tomography (CT) trans-abdominal slices (Siemens Somaton 16 CT Scanner). Details of CT-scans have been previously published [5].

\subsection{Serum Vitamin D}

Fasting blood was drawn using sterile phlebotomony techniques at the end of the "Run-In" period and at the end of the 12 week intervention. Serum and plasma were stored at $-80{ }^{\circ} \mathrm{C}$ until analyzed. Serum vitamin D metabolites [25(OH)D and $\left.1,25(\mathrm{OH})_{2} \mathrm{D}\right]$ were analyzed using standard radioimmunoassay (RIA) procedures (DiaSorin, StillWater, MN and Immunodiagnostics Systems [IDS], Fountain Hills, AZ, respectively). Participants' baseline and post-intervention samples were run simultaneously in duplicate and processed in accordance with manufacturer's instructions. Interassay $\mathrm{CVs}$ were $6.0 \%$ and $6.3 \%$ for $25(\mathrm{OH}) \mathrm{D}$ and $1,25(\mathrm{OH})_{2} \mathrm{D}$, respectively. In regard to the DiaSorin RIA, both $25(\mathrm{OH}) \mathrm{D}_{2}$ and $25(\mathrm{OH}) \mathrm{D}_{3}$ have $100 \%$ cross-reactivity with the internal antibody. Therefore, reported serum $25(\mathrm{OH}) \mathrm{D}$ values herein represent cumulative $25(\mathrm{OH}) \mathrm{D}_{2}$ and $25(\mathrm{OH}) \mathrm{D}_{3}$ concentrations. The WHNRC participates in the Vitamin D External Quality Assessment Scheme (DEQAS) and calibration standards of $25(\mathrm{OH}) \mathrm{D}$ and $1,25(\mathrm{OH})_{2} \mathrm{D}$ from this program were analyzed in conjunction with participant samples. 


\subsection{Subcutaneous White Adipose Tissue (SWAT) Biopsies}

SWAT biopsies were obtained at the UC Davis Medical Center Clinical and Translation Science Center at "Run-In" and at the end of the 12-week intervention following a 12-h overnight fast. Biopsy samples from "Run-In" and at the end of the 12-week intervention were collected from opposite sides of the buttocks. Each sample was immediately rinsed in ice cold phosphate buffered saline (PBS) to minimize blood contamination. Samples were additionally rinsed in fresh ice cold PBS and aliquots were frozen in liquid nitrogen. Frozen samples were than stored at $-80{ }^{\circ} \mathrm{C}$ until analyzed.

SWAT biopsies from participants enrolled in cohorts beginning in November through January were chosen for analysis to minimize potential influences by endogenous vitamin D synthesis. Fourteen subjects from winter cohorts had complete SWAT biopsy sets (both baseline and post-intervention) available, therefore, six subjects were randomly chosen from spring cohorts (enrollment between February and April) to bring the total number of SWAT samples analyzed to twenty pairs. Biopsies used for this study were shipped overnight on dry ice to the Jean Mayer USDA Human Nutrition Research Center on Aging at Tufts University for analysis.

\subsection{Preparation and Analysis of SWAT 25(OH)D 3 by LC-MS/MS}

Subsamples of SWAT tissue (0.1-0.5 g) were added to a glass mortar filled with $2 \mathrm{~g}$ of $40 \mu \mathrm{m}$ Bondesil-C18. Tissue was blended with a glass pestle and spiked with $0.2 \mathrm{~mL}$ mixture of $25-\mathrm{OH}$ deuterated vitamin $\mathrm{D}_{3}-\mathrm{d}_{6}$ standards (Medical Isotopes, Inc., Pelham, NH, USA; purity 99.6\%), each at $111 \mathrm{ng} / \mathrm{mL}$. The mixture was further homogenized with a spatula and transferred to a $15 \mathrm{~mL}$ reservoir tube. A $20 \mu \mathrm{m}$ frit was placed on top of the reservoir. The reservoir was tightly compressed with a $10 \mathrm{cc}$ syringe plunger and $8 \mathrm{~mL}$ of water was added. The contents were collected and reduced to dryness using a Vac-Elute set to "Collect" mode. Residues were solubilized in acetone (4 mL), with supernatants being isolated, centrifuged at $3000 \mathrm{~g}$ for $11 \mathrm{~min}$ and filtered through a $0.2 \mu \mathrm{m}$ PTFE filter. The extract volume was reduced under $\mathrm{N}_{2}$ at $45{ }^{\circ} \mathrm{C}$ for $60 \mathrm{~min}$ and $200 \mu \mathrm{L}$ of the remaining extract was transferred to an autosampler vial insert. A $50 \mu \mathrm{L}$ aliquot was injected on a LC-MS/MS (Agilent 1100 HPLC-ABSciex 5500 QTRAP tandem mass spectrometer). The HPLC was equipped with a $4.6 \times 250 \mathrm{~mm}, 5-\mu \mathrm{m}$ ProntoSIL 200-5-C30 (MAC-MOD Analytical, Inc., Chadds Ford, PA, USA, manufactured by Bischoff Chromatography, Leonberg, Germany) C-30 column. The column was maintained at room temperature during the analyses. The mobile phase flow rate was $0.9 \mathrm{~mL} / \mathrm{min}$. Solvent A was methanol and solvent B was methylene chloride. The gradient was: $0-15 \mathrm{~min}, 100 \%$ solvent A, followed by $50 \%$ solvent A from 10.0 to 25.0 min and a 10 min re-equilibrated at $100 \%$ A. Post-column solvent flow was diverted to waste during the final $13 \mathrm{~min}$. The mass spectrometer employed an atmospheric pressure chemical ionization source operated in positive ion mode. The ion source temperature was $400^{\circ} \mathrm{C}$. The triple quadrupole MS/MS was operated in multiple reaction monitoring (MRM) mode to detect the mass transitions of the parent molecules after in source loss of water (i.e., $\left[\mathrm{M}-\mathrm{H}_{2} \mathrm{O}+\mathrm{H}\right]^{+}$) for 25-hydroxy vitamin $\mathrm{D}_{3} \mathrm{~m} / \mathrm{z}$ $383.4>211.3$ and 25-hydroxy vitamin $\mathrm{D}_{3}-\mathrm{d}_{6} \mathrm{~m} / z 389.4>211.3$, respectively. The collision gas was nitrogen and the pressure was set to medium. The collision energy for all MRM transitions was $40 \mathrm{~V}$ and the dwell time for each MRM transition was $1000 \mathrm{~ms}$. As described above, we utilized 
isotope dilution procedures to internalize standards within samples [6,7]. By adding a known quantities of isotopically labeled metabolites vitamin $\mathrm{D}_{3}-\mathrm{d}_{6}$ to each sample, the isotope ratio of the resulting solution can be deconvoluted using the known isotope ratio of the labeled compounds and the natural deuterium abundance in the sample $(<0.1 \%)$, to calculated the $\mathrm{D}_{3}$ metabolite concentrations in the samples. The average recovery was $72 \%$.

Based on the average variance of low abundance calibration standards $(2.5 \mathrm{nmol} / \mathrm{kg})$, method limit of detection and limit of quantification were estimated at 1.0 and $2.9 \mathrm{nmol} / \mathrm{kg}$, respectively. All reported data were greater than $2 x$ the limit of detection estimate.

\subsection{Statistical Methods}

All statistical analyses were performed using R version 2.15.1 (R Development Core Team 2012). Univariate analysis, including mean, range and standard deviation, was used for baseline and post-intervention physical and clinical characteristics. Associations among variables were determined using Pearson's correlation coefficients. Paired $t$-tests were used to determine differences between baseline and post-intervention measurements. All $P$-values $\leq 0.05$ were considered statistically significant. Graphical plots were constructed using the R package, ggplot2 [8].

To minimize any potential effects of sun exposure, biopsy samples were specifically chosen from winter and spring cohorts. Consequently, the treatment assignment became unbalanced with 7 samples assigned to the AD group and 13 samples to the LD group. Additionally, by chance, 2 samples within the AD group had undetectable $25(\mathrm{OH}) \mathrm{D}$, which reduced the number of paired baseline and post-intervention for the AD group to 5. Thus, treatment effect was not analyzed due to unequal and small sample sizes in each treatment group.

\section{Results}

\subsection{Physical Characteristics}

Physical characteristics of the subjects at pre- and post-intervention are shown in Table 1. The majority of subjects were female and $90 \%$ of the subjects were categorized as obese at baseline. Except for percent body fat, all adiposity markers significantly decreased by the end of the intervention. Participants lost an average of $6.1 \%$ total body weight and $12.8 \%$ total fat mass over the study period. Dietary vitamin D intakes were below current recommendations of $600 \mathrm{IU} / \mathrm{day}$; however, the study was conducted during the previous recommendations of $200 \mathrm{IU} /$ day. There was no statistical difference between baseline and intervention vitamin $\mathrm{D}$ intake. The large post-intervention standard deviation seen in vitamin D intake compared to baseline intakes was due to the inclusion of dairy foods for half of the subjects as part of the parent project intervention.

\subsection{Serum and SWAT Analyses}

Serum and SWAT concentrations of $25(\mathrm{OH}) \mathrm{D}$ and $1,25(\mathrm{OH})_{2} \mathrm{D}$ are shown in Table 2. Based on the guidelines suggested by the 2011 DRI for vitamin D, only two subjects had adequate serum $25(\mathrm{OH}) \mathrm{D}$ concentrations $(\geq 50 \mathrm{nmol} / \mathrm{L})$ at baseline. Of the remaining participants with sub-adequate 
serum 25(OH)D, eight had deficient serum 25(OH)D concentrations $(<30 \mathrm{nmol} / \mathrm{L})$. By the end of the intervention, the number of participants with adequate serum $25(\mathrm{OH}) \mathrm{D}$ concentrations improved in three participants, while the number of participants with deficient concentrations decreased by six participants. However, significant changes in serum vitamin D metabolites were not detected for the overall group.

Table 2. Serum and Subcutaneous White Adipose Tissue concentrations of 25(OH)D and $1,25(\mathrm{OH})_{2} \mathrm{D}$ at Baseline and Post-Weight Loss Intervention in adults ${ }^{1}$.

\begin{tabular}{lcc}
\hline Variable & Baseline & Post-Intervention \\
\hline Serum 25(OH)D,$(\mathrm{nmol} / \mathrm{L})$ & $33.5(12.0)$ & $38.6(10.3)$ \\
Serum 1,25(OH $)_{2} \mathrm{D}(\mathrm{pmol} / \mathrm{L})$ & $108.4(21.5)$ & $105.8(30.6)$ \\
SWAT 25(OH) $\mathrm{D}_{3}(\mathrm{nmol} / \mathrm{kg} \text { tissue })^{2}$ & $5.8(2.6)$ & $6.2(2.7)$ \\
\hline Values expressed as mean $(\mathrm{SD}){ }^{2} n=18$ & baseline, 2 SWAT & samples below detection; $n=19$ \\
pst-intervention, 1 SWAT sample below detection.
\end{tabular}

SWAT $25(\mathrm{OH}) \mathrm{D}_{3}$ concentrations were detected in 18 baseline samples and 19 post-intervention samples. Concentrations ranged from 2.3 to $12.8 \mathrm{nmol} / \mathrm{kg}$ SWAT $(0.9-4.9 \mathrm{ng} / \mathrm{g})$ at baseline and $2.8-13.1 \mathrm{nmol} / \mathrm{kg}$ SWAT $(1.1-5.03 \mathrm{ng} / \mathrm{g})$ post-intervention. There was no statistical difference between baseline and post-intervention SWAT 25(OH)D 3 concentrations (Table 2).

Individual changes in SWAT $25(\mathrm{OH}) \mathrm{D}_{3}$ concentrations varied (Figure 1) and ranged from $-3.4 \mathrm{nmol} / \mathrm{kg}$ to $6.0 \mathrm{nmol} / \mathrm{kg}$. Baseline and post-intervention SWAT 25(OH)D 3 concentrations were significantly correlated (Figure 2; $r=0.55, P=0.02$ ) and SWAT and serum 25(OH)D concentrations were significantly correlated at baseline $(r=0.55, P=0.02)$ and post-intervention $(r=0.48, P=0.04)$ respectively (Figure 3). The absolute change of serum and SWAT concentrations were not correlated $(r=-0.13, P=0.60)$. Additionally, serum $1,25(\mathrm{OH})_{2} \mathrm{D}$ concentrations and SWAT $25(\mathrm{OH}) \mathrm{D}_{3}$ concentrations were not significantly correlated $(r=-0.12, P=0.49)$.

Figure 1. Individual changes in Subcutaneous White Adipose Tissue $25(\mathrm{OH}) \mathrm{D}_{3}$ concentrations from Baseline to Post-Intervention in adults who participated in a 15 week weight loss trial. $n=18$ (Baseline) and 19 (Post-Intervention).

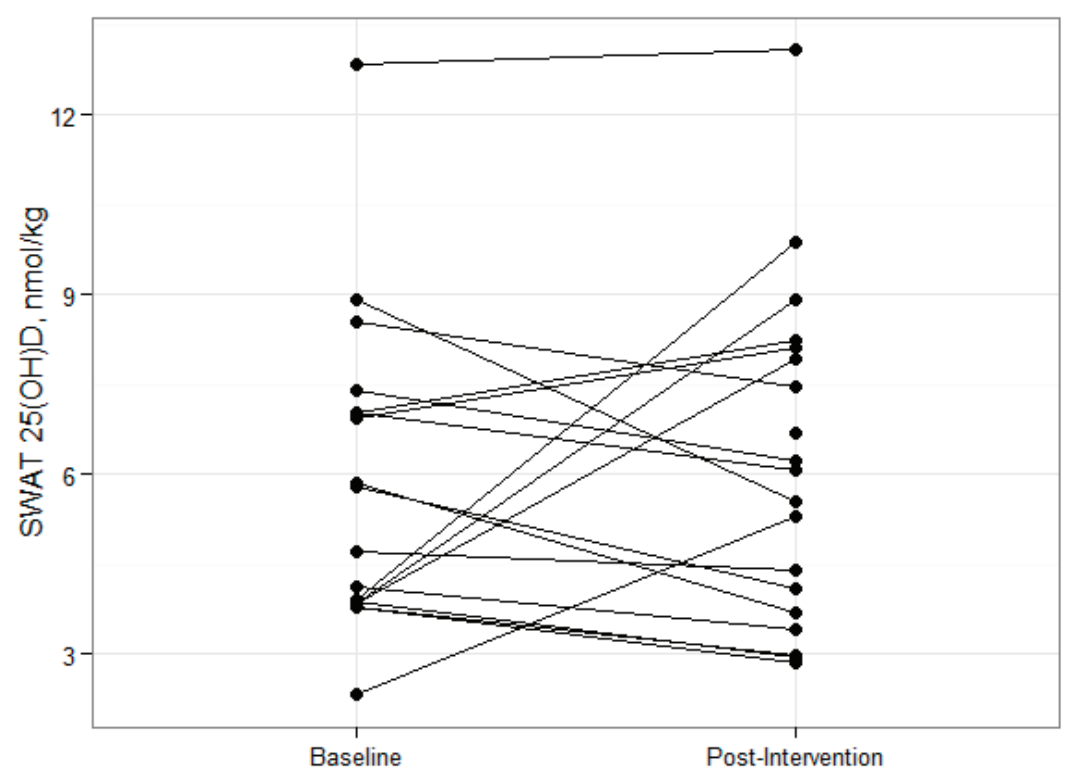


Figure 2. Association between Subcutaneous White Adipose Tissue 25(OH)D $\mathrm{D}_{3}$ at Baseline and Post-Intervention in adults who participated in a 15 week weight loss trial. Shaded line $=95 \%$ Confidence Interval.

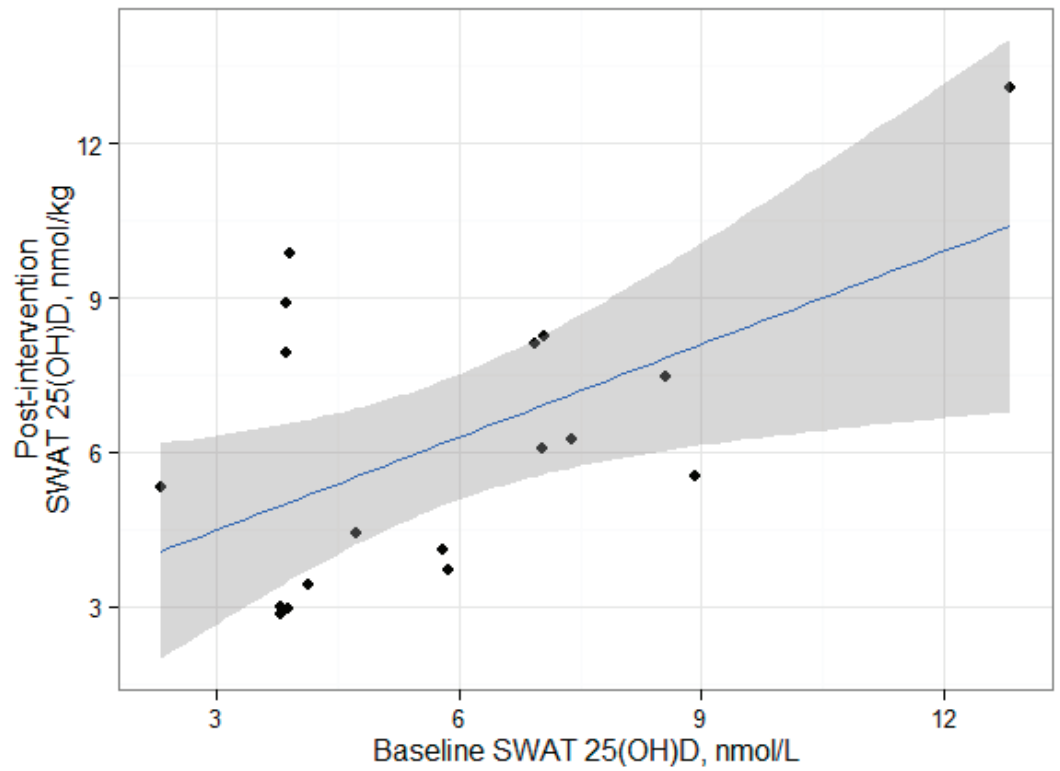

Figure 3. Association between Subcutaneous White Adipose Tissue and Serum $25(\mathrm{OH}) \mathrm{D}$ at (a) Baseline and (b) Post-Intervention following a 15 week weight loss regimen in adults. Shaded area $=95 \%$ Confidence Interval.
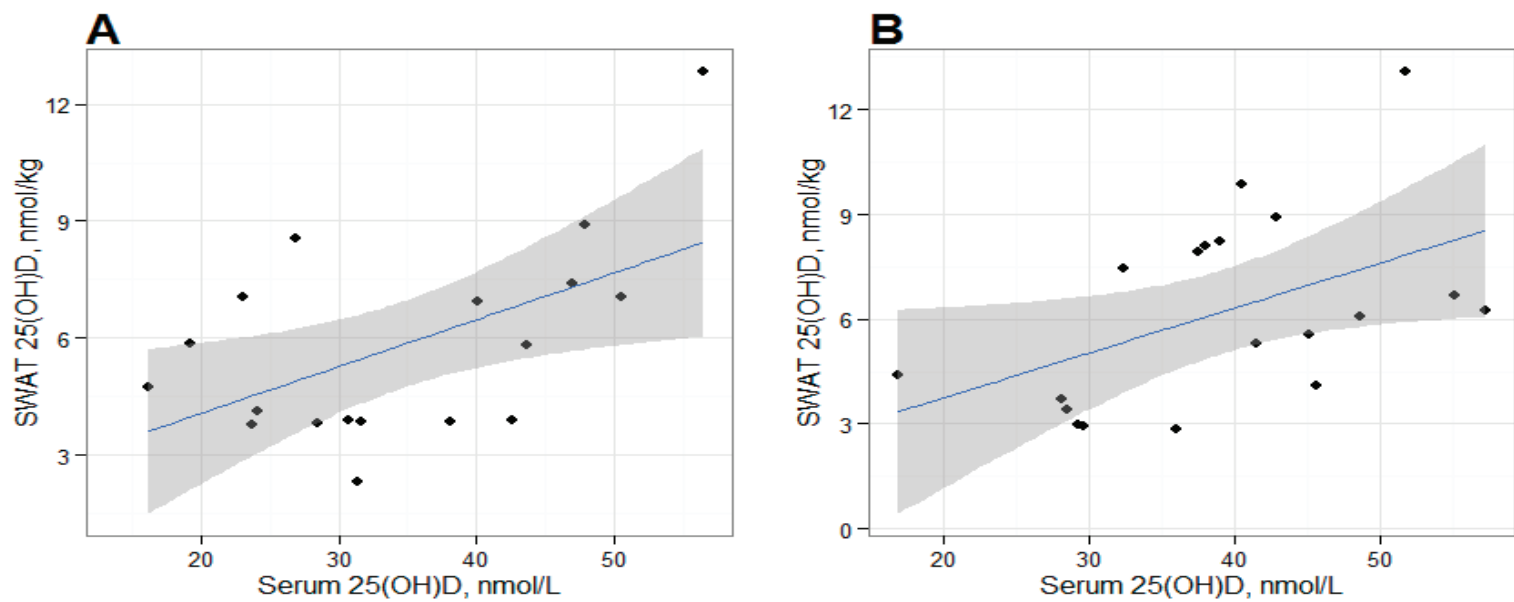

\section{Discussion}

Little is known about the content of $25(\mathrm{OH}) \mathrm{D}$ in human adipose tissue and whether weight loss or other conditions that impact adiposity influence fat tissue vitamin D metabolites. In swine, Jakobsen et al. found mean subcutaneous fat concentrations of $25(\mathrm{OH}) \mathrm{D}$ between $4.8 \mathrm{nmol} / \mathrm{kg}$ and $6.3 \mathrm{nmol} / \mathrm{kg}$ depending on the inclusion of vitamin D or 25(OH)D in their feed [3]. Our SWAT 25(OH)D3 concentrations of $5.8 \mathrm{nmol} / \mathrm{kg}$ at baseline and $6.2 \mathrm{nmol} / \mathrm{kg}$ post-intervention are in agreement with Jakobsen et al. and add to the few papers that have found measureable SWAT 25(OH)D concentrations [2]. However, it is acknowledged that other researchers have failed to detect $25(\mathrm{OH}) \mathrm{D}$ in 
adipose tissue [9]. This could be partly due to methodological differences. We quantified SWAT $25(\mathrm{OH}) \mathrm{D}_{3}$ using LC-MS/MS and acknowledge that at the time of our study there was no reference method to which our data could be compared. Considering the controversy regarding accurately assessing serum $25(\mathrm{OH}) \mathrm{D}$ concentrations [10-13], it would be presumptuous to relate our results to the general population. More analyses are needed to determine typical concentrations in wider populations of overweight and obese individuals and in different racial groups, and the current experiment provides a useful foundation for these efforts.

The majority of the subjects in our study had insufficient serum 25(OH)D concentrations according to the current IOM guidelines. This finding is consistent with other studies reporting an inverse relationship between measures of adiposity and serum 25(OH)D [14-18]. Deposition and sequestration of cholecalciferol in adipose tissue stores is an often cited mechanism behind this relationship, however there is very little experimental evidence to support this hypothesis. In this study, overweight and obese participants lost an average of $6 \%$ total body weight and $13 \%$ total fat. The fact that SWAT $25(\mathrm{OH}) \mathrm{D}_{3}$ concentrations remained unchanged after a significant decrease in adipose mass would suggest a release of $25(\mathrm{OH}) \mathrm{D}$ from adipose tissue. However, there were no changes in serum 25(OH)D, suggesting little contribution of released SWAT 25(OH)D to serum 25(OH)D concentrations. Drincic et al., [19] recently proposed that the inverse relationship between obesity and serum $25(\mathrm{OH}) \mathrm{D}$ is due to volumetric dilution of cholecalciferol in serum and adipose tissue. Perhaps, a similar mechanism exists between serum and adipose compartments of $25(\mathrm{OH}) \mathrm{D}$. The extra hydroxyl group on 25(OH)D would make it slightly less hydrophilic and less dilutable in adipose tissue than cholecalciferol resulting in a smaller diffusional equilibrium. Therefore, the release of $25(\mathrm{OH}) \mathrm{D}$ due to the volumetric decrease in adipose tissue would result in an attenuated effect on serum $25(\mathrm{OH}) \mathrm{D}$ as seen in our study.

The subjects in our study were overweight and obese, but did not have metabolic disorders associated with excess adiposity such as type 2 diabetes, metabolic syndrome and other metabolic diseases (i.e., renal disease). Therefore, we would not expect any perturbations in serum $1,25(\mathrm{OH})_{2} \mathrm{D}$ concentrations. Regulation of serum $1,25(\mathrm{OH})_{2} \mathrm{D}$ is tightly controlled and is generally not a good marker of vitamin D status [20]. Most of the serum $1,25(\mathrm{OH})_{2} \mathrm{D}$ is thought to be produced by renal 1-alpha-hydroxylase enzymes, but many non-renal tissues also contain 1-alpha-hydroxylase that convert $25(\mathrm{OH}) \mathrm{D}$ to $1,25(\mathrm{OH})_{2} \mathrm{D}$ for local gene regulation [21]. The supply of $25(\mathrm{OH}) \mathrm{D}$ is generally thought to be provided by the serum pool, but a small reservoir of $25(\mathrm{OH}) \mathrm{D}$ in the lipid droplets of adipocytes could supply the cell its functional need. In vitro evidence suggests that $1,25(\mathrm{OH})_{2} \mathrm{D}$ may regulate adipogenesis and lipid metabolism [22], however results from these studies have not been confirmed in human studies. It is an intriguing possibility to link $25(\mathrm{OH}) \mathrm{D}$ stored in lipid droplets to adipocyte gene regulation, but much more work still is needed.

We found positive and statistically significant relationships between serum $25(\mathrm{OH}) \mathrm{D}$ and SWAT $25(\mathrm{OH}) \mathrm{D}$ concentrations at baseline and post-intervention, suggesting that the deposition of $25(\mathrm{OH}) \mathrm{D}$ in SWAT could be dependent on serum $25(\mathrm{OH}) \mathrm{D}$ concentrations. Serum and adipose cholecalciferol concentrations have been previously reported to be positively correlated [23], but to our knowledge we are the first to report the relationship between serum $25(\mathrm{OH}) \mathrm{D}_{3}$ and SWAT $25(\mathrm{OH}) \mathrm{D}$ concentrations. The correlation between serum and SWAT 25(OH)D was similar at 
baseline and at post-intervention, suggesting an equilibrium between the two compartments. However, our results are based on alternative analytical methods for adipose tissue and serum 25(OH)D measurements which likely would affect the statistical interpretation. LC-MS/MS methods and the DiaSorin RIA generally produce consistent results in serum [24,25], but the DiaSorin RIA cannot distinguish between serum $25(\mathrm{OH}) \mathrm{D}_{2}$ and $25(\mathrm{OH}) \mathrm{D}_{3}$, while the LC-MS/MS adipose method was specific for only $25(\mathrm{OH}) \mathrm{D}_{3}$. Thus, we run the risk of under-reporting our SWAT $25(\mathrm{OH}) \mathrm{D}$ in comparison to serum concentrations. Serum concentrations of $25(\mathrm{OH}) \mathrm{D}_{2}$ are substantially lower than $25(\mathrm{OH}) \mathrm{D}_{3}[26-28]$ unless supplementing with vitamin $\mathrm{D}_{2}$ or consuming plant sources of vitamin $\mathrm{D}_{2}$. Based on the low 25(OH)D concentrations seen in our subjects, we would expect very little vitamin $\mathrm{D}_{2}$ consumption from supplements and, subsequently, very little $25(\mathrm{OH}) \mathrm{D}_{2}$ in adipose depots. Still, future studies should utilize analytical methods that can distinguish between both isoforms to accurately identify the relationship between the serum and adipose compartments.

It is important to recognize that the statistical significance of these correlations should be interpreted carefully due to the limited number of samples in this exploratory study. The statistical significance of each correlation appear to be leveraged by the participant with the greatest SWAT $25(\mathrm{OH}) \mathrm{D}_{3}$ measurement and removal of this datum results in non-significant correlations. It is unclear whether this participant is truly an outlier or would have been in the range of observed data from a larger sample. It should be noted that that our sample size is consistent with other studies that have measured cholecalciferol in humans [23,29,30], however, conclusions based on our results should be considered hypothesis generating data.

The changes in SWAT concentrations of vitamin D metabolites during energy restriction have not been reported. Our subjects lost an average of $5 \mathrm{~kg}$ of total fat mass after twelve weeks of reducing their energy requirements by $500 \mathrm{kcal}$, but had no significant change in SWAT, or serum, 25(OH)D concentrations. In contrast, serum cholecalciferol concentrations were shown to increase in fasted rats after the termination of a 14-day large daily vitamin D supplementation, [31], suggesting mobilization from adipose tissue stores. A recent study reported that women who lost more than $15 \%$ total body weight significantly increased serum $25(\mathrm{OH}) \mathrm{D}$ concentrations compared to women who were weight stable or lost $\leq 15 \%$ total body weight [32]. It is not definitively known in that particular study whether the increase in serum $25(\mathrm{OH}) \mathrm{D}$ concentration was the result of liberated cholecalciferol or $25(\mathrm{OH}) \mathrm{D}$ from adipose stores because no adipose biopsies were collected. Our subjects lost an average of only $6 \%$ total body weight after the intervention and we also failed to see any significant changes in serum $25(\mathrm{OH}) \mathrm{D}$. The data from these studies suggest that a substantial increase in adipocyte lipolysis is required to free stored cholecalciferol or perhaps, $25(\mathrm{OH}) \mathrm{D}$, to significantly increase serum $25(\mathrm{OH}) \mathrm{D}$ concentrations.

As discussed earlier, Heaney et al. hypothesized that a total of $114.5 \mathrm{nmol} 25(\mathrm{OH}) \mathrm{D}$ was distributed in $24.5 \mathrm{~kg}$ of total fat in a hypothetical $70 \mathrm{~kg}$ woman [4]. This is assuming that the concentration of $25(\mathrm{OH}) \mathrm{D}$ is equally distributed throughout the adipose mass. Using the same approach in our subjects, we estimate mean total adipose $25(\mathrm{OH}) \mathrm{D}$ content at baseline and post-intervention to be $212.2 \pm 93.7$ and $199.6 \pm 95.7 \mathrm{nmol}$, respectively. Additionally, estimated total fat 25(OH)D content ranged from 92.0 to $451.7 \mathrm{nmol}$, suggesting significant individual variability. Our samples were obtained from opposite gluteal sites and were relatively uniform between 
both time points. However, we cannot assume that the adipose $25(\mathrm{OH}) \mathrm{D}$ is distributed evenly in other adipose sites. Cholecalciferol deposition in adipose tissue has been shown to be quite variable among anatomical adipose sites $[9,30]$ and there is not enough evidence to suggest either a uniform or variable distribution of fat $25(\mathrm{OH}) \mathrm{D}$ at this time.

The strengths of our study were the longitudinal collection of SWAT, and thus measurement of within subject changes. To our knowledge, the current study is the first to report $25(\mathrm{OH}) \mathrm{D}$ concentrations in SWAT pre- and post-weight loss in humans. Additionally, we analyzed samples across a broad range of body weight, adiposity, and serum 25(OH)D concentrations. By leveraging samples from a larger controlled feeding trial, it enabled us to accurately control and assess vitamin $\mathrm{D}$ intake, caloric restriction, and other parameters.

Due to the exploratory, proof-of-principle nature of this investigation and the cost associated with the analyses, we restricted our first study to a limited number of samples. Additionally, our subjects were derived from a larger study that did not control for factors that could introduce inter-subject variability in our analysis. The parent project introduced dairy products that are fortified with vitamin $\mathrm{D}$, therefore subjects had variable intakes of vitamin D which could contribute to the variability in post-intervention measurements. We selected samples from winter and spring cohorts to minimize the possible effect of sun exposure, but inter-subject variability in serum and SWAT $25(\mathrm{OH}) \mathrm{D}_{3}$ measurements are likely attributed to seasonal differences. Also, serum analysis of 25(OH)D had previously been conducted for the parent study. Therefore, differences in methodological analyses could impact the accuracy of our measurements. Our subjects were considered overweight and obese yet generally healthy, so our findings may not apply to other overweight and obese individuals diagnosed with metabolic syndrome or other metabolic disturbances. Lastly, it is acknowledged that despite quite thorough biopsy washing steps, our samples may have minor contamination with blood, which could theoretically add variability to the outcome measurements and influence serum vs. SWAT correlations. Regardless, the current work provides a solid reference framework for understanding physiological concentrations of adipose $25(\mathrm{OH}) \mathrm{D}$ concentrations in humans.

\section{Conclusions and Implications}

In summary, we have demonstrated a LC-MS/MS method that can detect 25(OH)D in human adipose tissue collected from overweight and obese individuals enrolled in a clinical weight loss intervention. We found mean SWAT 25(OH)D concentrations of $5.8 \pm 2.6 \mathrm{nmol} / \mathrm{kg}$ tissue and $6.2 \pm 2.7 \mathrm{nmol} / \mathrm{kg}$ tissue pre- and post-intervention, respectively. Obese persons are thought to be at risk for vitamin D deficiency, which could contribute to the development or exacerbate other comorbidities associated with excessive adipose tissue. Although there are very little human data, the accumulation and release of vitamin D metabolites in excess adipose tissue is the mechanism often cited behind this relationship. Aside from the small $25(\mathrm{OH}) \mathrm{D}$ concentrations detected in SWAT, our results suggest that $25(\mathrm{OH}) \mathrm{D}$ is released from adipose tissue after a loss of $6 \%$ in body weight and a $13 \%$ reduction in total fat loss, but this release contributes little to serum $25(\mathrm{OH}) \mathrm{D}$ concentrations. Perhaps a greater loss of body weight or fat is needed to observe a significant contribution to serum $25(\mathrm{OH}) \mathrm{D}$ concentrations (i.e., $\geq 15 \%$ loss). Therefore, dietary intake and 
dermal biosynthesis should be paramount when devising strategies to improve serum $25(\mathrm{OH}) \mathrm{D}$ in obese individuals.

\section{Acknowledgments}

This study would not be possible without the efforts of a team of individuals starting with the research volunteers, the WHNRC metabolic food laboratory, human studies support and physiology and analytical laboratory staff members. Additional support was provided by the UC Davis Clinical and Translational Science Center nursing staff for assistance with blood draws and adipose biopsies. USDA is an equal opportunity provider and employer.

Financial Support: Major funding for this project was provided by the National Dairy Council administered by the Dairy Research Institute and the Dairy Council of California. Additional support was provided by the USDA-ARS Projects 5306-51530-006-00D and 5306-51530-016-00D, and the Clinical and Translational Science Center of the University of California, Davis, grant number UL1 RR024146 from the National Center for Research Resources (NCRR).

\section{Conflicts of Interest}

The authors declare no conflicts of interest.

\section{References}

1. Phillips, K.M.; Byrdwell, W.C.; Exler, J.; Harnly, J.M.; Holden, J.M.; Holick, M.F.; Hollis, B.W.; Horst, R.L.; Lemar, L.E.; Patterson, K.Y.; et al. Development and validation of control materials for the measurement of vitamin d-3 in selected us foods. J. Food Compos. Anal. 2008, 21, 527-534.

2. Mawer, E.B.; Backhouse, J.; Holman, C.A.; Lumb, G.A.; Stanbury, S.W. The distribution and storage of vitamin d and its metabolites in human tissues. Clin. Sci. 1972, 43, 413-431.

3. Jakobsen, J.; Maribo, H.; Bysted, A.; Sommer, H.M.; Hels, O. 25-Hydroxyvitamin d(3) affects vitamin $d$ status similar to vitamin d(3) in pigs-But the meat produced has a lower content of vitamin d. Br. J. Nutr. 2007, 98, 908-913.

4. Heaney, R.P.; Horst, R.L.; Cullen, D.M.; Armas, L.A. Vitamin d3 distribution and status in the body. J. Am. Coll. Nutr. 2009, 28, 252-256.

5. Van Loan, M.D.; Keim, N.L.; Adams, S.H.; Souza, E.; Woodhouse, L.R.; Thomas, A.; Witbracht, M.; Gertz, E.R.; Piccolo, B.; Bremer, A.A.; et al. Dairy foods in a moderate energy restricted diet do not enhance central rat, weight, and intra-abdominal adipose tissue losses nor reduce adipocyte size or inflammatory markers in overweight and obese adults: A controlled feeding study. J. Obes. 2011, 2011, 989657.

6. Fassett, J.D. Isotopic and nuclear analytical techniques in biological-systems-A critical-study. 10. Elemental isotope-dilution analysis with radioactive and stable isotopes. Pure Appl. Chem. 1995, 67, 1943-1949.

7. Friso, S.; Choi, S.W.; Dolnikowski, G.G.; Selhub, J. A method to assess genomic DNA methylation using high-performance liquid chromatography/electrospray ionization mass spectrometry. Anal. Chem. 2002, 74, 4526-4531. 
8. Wickham, H. Ggplot2: Elegant Graphics for Data Analysis; Springer: New York, NY, USA, 2009; p. viii.

9. Lawson, D.E.; Douglas, J.; Lean, M.; Sedrani, S. Estimation of vitamin d3 and 25-hydroxyvitamin d3 in muscle and adipose tissue of rats and man. Clin. Chim. Acta 1986, 157, 175-181.

10. Binkley, N.; Krueger, D.; Cowgill, C.S.; Plum, L.; Lake, E.; Hansen, K.E.; DeLuca, H.F.; Drezner, M.K. Assay variation confounds the diagnosis of hypovitaminosis d: A call for standardization. J. Clin. Endocrinol. Metab. 2004, 89, 3152-3157.

11. Singh, R.J.; Taylor, R.L.; Reddy, G.S.; Grebe, S.K. C-3 epimers can account for a significant proportion of total circulating 25-hydroxyvitamin $\mathrm{d}$ in infants, complicating accurate measurement and interpretation of vitamin d status. J. Clin. Endocrinol. Metab. 2006, 91, 3055-3061.

12. Roth, H.J.; Schmidt-Gayk, H.; Weber, H.; Niederau, C. Accuracy and clinical implications of seven 25-hydroxyvitamin d methods compared with liquid chromatography-tandem mass spectrometry as a reference. Ann. Clin. Biochem. 2008, 45, 153-159.

13. Lai, J.K.; Lucas, R.M.; Banks, E.; Ponsonby, A.L. Variability in vitamin d assays impairs clinical assessment of vitamin d status. Intern. Med. J. 2012, 42, 43-50.

14. Martins, D.; Wolf, M.; Pan, D.; Zadshir, A.; Tareen, N.; Thadhani, R.; Felsenfeld, A.; Levine, B.; Mehrotra, R.; Norris, K. Prevalence of cardiovascular risk factors and the serum levels of 25-hydroxyvitamin $\mathrm{d}$ in the united states - Data from the third national health and nutrition examination survey. Arch. Intern. Med. 2007, 167, 1159-1165.

15. Brock, K.; Huang, W.Y.; Fraser, D.R.; Ke, L.; Tseng, M.; Stolzenberg-Solomon, R.; Peters, U.; Ahn, J.; Purdue, M.; Mason, R.S.; et al. Low vitamin d status is associated with physical inactivity, obesity and low vitamin d intake in a large us sample of healthy middle-aged men and women. J. Steroid Biochem. Mol. Biol. 2010, 121, 462-466.

16. Arunabh, S.; Pollack, S.; Yeh, J.; Aloia, J.F. Body fat content and 25-hydroxyvitamin d levels in healthy women. J. Clin. Endocrinol. Metab. 2003, 88, 157-161.

17. Cheng, S.; Massaro, J.M.; Fox, C.S.; Larson, M.G.; Keyes, M.J.; McCabe, E.L.; Robins, S.J.; O’Donnell, C.J.; Hoffmann, U.; Jacques, P.F.; et al. Adiposity, cardiometabolic risk, and vitamin d status: The framingham heart study. Diabetes 2010, 59, 242-248.

18. Lagunova, Z.; Porojnicu, A.C.; Lindberg, F.; Hexeberg, S.; Moan, J. The dependency of vitamin d status on body mass index, gender, age and season. Anticancer Res. 2009, 29, 3713-3720.

19. Drincic, A.T.; Armas, L.A.; van Diest, E.E.; Heaney, R.P. Volumetric dilution, rather than sequestration best explains the low vitamin d status of obesity. Obesity (Silver Spring) 2012, 20, 1444-1448.

20. Zerwekh, J.E. Blood biomarkers of vitamin d status. Am. J. Clin. Nutr. 2008, 87, 1087S-1091S.

21. Bikle, D. Nonclassic actions of vitamin d. J. Clin. Endocrinol. Metab. 2009, 94, 26-34.

22. Ding, C.; Gao, D.; Wilding, J.; Trayhurn, P.; Bing, C. Vitamin d signalling in adipose tissue. Br. J. Nutr. 2012, 108, 1915-1923.

23. Blum, M.; Dolnikowski, G.; Seyoum, E.; Harris, S.S.; Booth, S.L.; Peterson, J.; Saltzman, E.; Dawson-Hughes, B. Vitamin d(3) in fat tissue. Endocrine 2008, 33, 90-94. 
24. Aronov, P.A.; Hall, L.M.; Dettmer, K.; Stephensen, C.B.; Hammock, B.D. Metabolic profiling of major vitamin $\mathrm{d}$ metabolites using diels-alder derivatization and ultra-performance liquid chromatography-tandem mass spectrometry. Anal. Bioanal. Chem. 2008, 391, 1917-1930.

25. Van den Ouweland, J.M.; Beijers, A.M.; Demacker, P.N.; van Daal, H. Measurement of 25-OH-vitamin $\mathrm{d}$ in human serum using liquid chromatography tandem-mass spectrometry with comparison to radioimmunoassay and automated immunoassay. J. Chromatogr. B 2010, 878, 1163-1168.

26. Fisk, C.M.; Theobald, H.E.; Sanders, T.A. Fortified malted milk drinks containing low-dose ergocalciferol and cholecalciferol do not differ in their capacity to raise serum 25-hydroxyvitamin $\mathrm{d}$ concentrations in healthy men and women not exposed to UV-b. J. Nutr. 2012, 142, 1286-1290.

27. Hall, L.M.; Kimlin, M.G.; Aronov, P.A.; Hammock, B.D.; Slusser, J.R.; Woodhouse, L.R.; Stephensen, C.B. Vitamin $d$ intake needed to maintain target serum 25-hydroxyvitamin d concentrations in participants with low sun exposure and dark skin pigmentation is substantially higher than current recommendations. J. Nutr. 2010, 140, 542-550.

28. Markestad, T.; Aksnes, L.; Ulstein, M.; Aarskog, D. 25-Hydroxyvitamin d and 1,25-dihydroxyvitamin $\mathrm{d}$ of $\mathrm{d} 2$ and $\mathrm{d} 3$ origin in maternal and umbilical cord serum after vitamin d2 supplementation in human pregnancy. Am. J. Clin. Nutr. 1984, 40, 1057-1063.

29. Heaney, R.P.; Recker, R.R.; Grote, J.; Horst, R.L.; Armas, L.A. Vitamin d3 is more potent than vitamin d2 in humans. J. Clin. Endocrinol. Metab. 2011, 96, E447-E452.

30. Pramyothin, P.; Biancuzzo, R.M.; Lu, Z.; Hess, D.T.; Apovian, C.M.; Holick, M.F. Vitamin d in adipose tissue and serum 25-hydroxyvitamin d after roux-en-y gastric bypass. Obesity (Silver Spring) 2011, 19, 2228-2234.

31. Brouwer, D.A.; van Beek, J.; Ferwerda, H.; Brugman, A.M.; van der Klis, F.R.; van der Heiden, H.J.; Muskiet, F.A. Rat adipose tissue rapidly accumulates and slowly releases an orally-administered high vitamin d dose. Br. J. Nutr. 1998, 79, 527-532.

32. Mason, C.; Xiao, L.R.; Imayama, I.; Duggan, C.R.; Bain, C.; Foster-Schubert, K.E.; Kong, A.; Campbell, K.L.; Wang, C.Y.; Neuhouser, M.L.; et al. Effects of weight loss on serum vitamin d in postmenopausal women. Am. J. Clin. Nutr. 2011, 94, 95-103. 

Chapter 7

Mental Disorders 



\title{
Vitamin D and Depression: A Systematic Review and Meta-Analysis Comparing Studies with and without Biological Flaws
}

\section{Simon Spedding}

\begin{abstract}
Efficacy of Vitamin D supplements in depression is controversial, awaiting further literature analysis. Biological flaws in primary studies is a possible reason meta-analyses of Vitamin D have failed to demonstrate efficacy. This systematic review and meta-analysis of Vitamin D and depression compared studies with and without biological flaws. The systematic review followed the Preferred Reporting Items for Systematic Reviews and Meta-Analyses (PRISMA) guidelines. The literature search was undertaken through four databases for randomized controlled trials (RCTs). Studies were critically appraised for methodological quality and biological flaws, in relation to the hypothesis and study design. Meta-analyses were performed for studies according to the presence of biological flaws. The $15 \mathrm{RCT}$ identified provide a more comprehensive evidence-base than previous systematic reviews; methodological quality of studies was generally good and methodology was diverse. A meta-analysis of all studies without flaws demonstrated a statistically significant improvement in depression with Vitamin D supplements $(+0.78 \mathrm{CI}+0.24,+1.27)$. Studies with biological flaws were mainly inconclusive, with the meta-analysis demonstrating a statistically significant worsening in depression by taking Vitamin D supplements $(-1.1$ CI $-0.7,-1.5)$. Vitamin D supplementation ( $\geq 800$ I.U. daily) was somewhat favorable in the management of depression in studies that demonstrate a change in vitamin levels, and the effect size was comparable to that of anti-depressant medication.
\end{abstract}

Reprinted from Nutrients. Cite as: Spedding, S. Vitamin D and Depression: A Systematic Review and Meta-Analysis Comparing Studies with and without Biological Flaws. Nutrients 2014, 6, 1501-1518.

\section{Introduction}

Depression affects 350 million people worldwide, is the leading cause of disability and the fourth-leading cause of the global disease burden [1]. However, the effectiveness of conventional treatments for depression is questioned: meta-analyses of drug treatments demonstrate minimal difference from placebo, comparisons of real and sham electroconvulsive therapy show little difference after a month, and the evidence for the use of specific cognitive interventions is weak [2]. Therefore we examined the evidence for other approaches to the management of depression.

The association between depressive disorders and Vitamin D deficiency from a lack of sun exposure is well established and was first noted two thousand years ago [3], therefore we considered the evidence for the effectiveness of Vitamin D supplementation.

Vitamin D is a unique secosteroid hormone formed mainly by photosynthesis, so an indoor lifestyle and sun-avoidance leads to deficiency $(25 \mathrm{OHD}<50 \mathrm{nmol} / \mathrm{L})$ [4]. Vitamin D deficiency is now a global public health problem affecting a billion people worldwide [5]. Even in sunny Australia, deficiency affects one third of the population [6], with much higher rates observed in migrant populations $[7,8]$. 
There has been an increase in the prevalence of Vitamin D deficiency [9] and a ten-fold increase in spending on supplements in the US over the last decade [10].

Knowledge of Vitamin D has grown exponentially [11] and 95\% of our current knowledge was published in the last 15 years [12]. This demonstrates new mechanisms and diseases associated with deficiency including cancer, cardiovascular disease, diabetes, and premature mortality [4]. Whilst Vitamin D was believed to follow Funk's model of vitamins, having a single mechanism and function limited to calcium and bone metabolism [13], the mechanisms of action of Vitamin D are now recognized to be endocrine, paracrine and autocrine via Vitamin D receptors (VDRs) [14] affecting most physiological systems, including the brain [15]. The enzymes necessary for the hydroxylation of 25hydroxyvitamin D (25OHD) to the active form 1,25dihydroxyvitamin D are present in the hypothalamus, cerebellum, and substantia nigra [16]. Vitamin D modulates the hypothalamic-pituitary-adrenal axis, regulating adrenalin, noradrenaline and dopamine production through VDRs in the adrenal cortex [17]; and protects against the depletion of dopamine and serotonin centrally [18]. Therefore, biological plausibility for the action of Vitamin D in depression has been established.

Epidemiological evidence shows that Vitamin D deficiency is associated with an $8 \%-14 \%$ increase in depression [19-22] and a 50\% increase in suicide [23]; however, causality and efficacy of supplementation remain controversial $[10,24]$ awaiting confirmation by systematic review and meta-analysis.

Four systematic reviews of Vitamin D efficacy in depression, but no meta-analysis, have been published [25-28]. These reviews provide conflicting results due to the limited number of studies found and the inclusion of inappropriate studies. Based on six RCTs deemed relevant, the Institute of Medicine (IOM) [25] concluded there was "inconclusive evidence of an effect" although four of these RCTs showed a beneficial effect of Vitamin D supplementation in depression. The inclusion of the other two studies [29,30] described by the IOM as "RCTs of Vitamin D" was inappropriate as; one used calcium and not Vitamin D as the intervention, and the other was not an RCT in the opinion of the study authors as the intervention decreased 25OHD levels. Similarly, consistent conclusions could not be drawn from the other systematic reviews [26-28], as these found so few of the primary studies.

These reviews mirror the inconsistent results found across Vitamin D research as demonstrated by the twenty four conflicting meta-analyses for falls, fractures, and all-cause mortality [31]. The reason Vitamin D meta-analyses fail to produce useful results is thought to be biological flaws in primary studies. These flaws lead to null results [32] as the intervention does not change the Vitamin D status however these flaws may be overlooked when evaluating the research for Vitamin D and other nutrients [33,34].

The concept of "biological flaws" arises from the work of Heaney and others [33,34], and refers to limitations in the design of primary studies which preclude them from testing the research hypothesis. The hypothesis being addressed in this review is that rectifying Vitamin D deficiency decreases depressive symptoms. However some trials have limitations in their study design that prevent this evaluation. This hypothesis can only be tested if participants are Vitamin D deficient at baseline and then receive a large enough dose of Vitamin D supplements to achieve Vitamin D sufficiency during the trial. Vitamin D deficiency cannot be demonstrated if the level of 25OHD is sufficient or higher or not tested at baseline. An ineffective dose of Vitamin D is one that would not be expected to increase the level of 25OHD from deficient to sufficient.

Trials with these biological flaws may demonstrate the limitations of the study design rather than the effectiveness of Vitamin D supplements for changing health outcomes. The parallel in pharmaceutical 
research to these nutrient studies with biological flaws would be trialling a drug known to be ineffective or on patients already taking a full dose of the drug. Thus biological flaws are a critical element that differentiates nutrient research from pharmaceutical research.

This review was designed to estimate the effect of Vitamin D supplementation in depression and examine the influence of biological flaws in primary studies on the meta-analyses.

\section{Methods}

This review followed the PRISMA (Preferred Reporting Items for Systematic Reviews and Meta-Analyses) guidelines, systematically identifying and appraising peer-reviewed RCTs reporting on the effect of Vitamin D supplementation for individuals with symptoms of depression with the objectives of investigating:

- the primary evidence for Vitamin D supplementation and depression from RCTs;

- the types of subjects, the dose of Vitamin D supplementation, the control interventions and the measures of outcome used;

- methodological quality of the studies;

- biological flaws in the study design, and

- estimates of the size of the effect.

\subsection{Search Approach}

A systematic search for relevant RCTs was performed evaluating oral Vitamin D supplementation that included data on depression using four library databases of PsychINFO, MedLine, PubMed and Cochrane online library. Search approaches for the different databases can be obtained from the researchers. All databases were searched from inception to October 2012, with eligible papers limited to English language and human subjects.

\subsection{Independence}

Two independent researchers investigated the library databases to reduce errors/bias in accessing evidence. The reference lists of four systematic reviews [25-28] were hand-searched to identify other RCTs.

\subsection{Eligible Studies}

RCTs were included where the intervention was Vitamin D supplementation and excluded where trials were not RCTs or used surrogate interventions. Studies were not excluded on their methodological quality as the entire evidence base was required to address the aims of this research. 


\subsection{Decision-Making}

Relevant publications were identified from title, abstract and study descriptors by one researcher; the decision to include was independently validated by a second and disagreements were referred to third for an independent ruling.

\subsection{Critical Appraisal}

Methodological quality of articles was critically appraised with PEDro [35]. Trials were rated with a checklist, the PEDro scale. This considers two aspects of trial quality; internal validity of the trial and whether the trial contains sufficient statistical information to make it interpretable. It does not rate external validity or the effect size.

\subsection{Data Extraction}

Data was extracted for participants, 25OHD levels, study timeframes, interventions, outcome measures, measures of effect, methodological quality scores, and biological flaws.

\subsection{Biological Flaws}

Biological flaws in primary studies were identified. These studies included:

- inappropriate interventions (interventions that did not include Vitamin D), or

- interventions producing the opposite effect of that intended (interventions that included Vitamin D, but reduced the 25OHD level in the intervention group), or

- ineffective interventions that did not improving Vitamin D status (did not significantly change the 25OHD level), or

- where the baseline 25OHD level was not measured in the majority of participants, or

- where the baseline 25OHD level indicated sufficiency (not deficiency) at baseline.

Studies were grouped according to the presence of biological flaws, and compared by date of publication, methodological quality, outcome measure, and study outcome.

\subsection{Meta-Analysis}

Meta-analyses were performed using MedCalc where data was available on diagnosis, dose, outcome measure, and biological flaws. Estimates of the size of effect using the standardised mean difference (SMD) were compared according to the presence of biological flaws in primary studies.

For meta-analysis of studies with a continuous measure, MedCalc uses the "Hedges g" statistic as a formulation for the SMD under the fixed effects model. The SMD is the difference between the two means divided by the pooled standard deviation, with a correction for small sample bias. Next the heterogeneity statistic is incorporated to calculate the summary SMD under the random effects model. The total SMD with 95\% CI is given both for the Fixed effects model and the Random effects model.

The SMD has no units or dimensions, however using Cohen's rule of thumb for interpretation of the SMD statistic: a value of 0.2 indicates a small effect, a value of 0.5 indicates a medium effect, and a value of 0.8 or larger indicates a large effect. 


\section{Results}

\subsection{Systematic Review}

From all databases 465 relevant articles were identified with 390 articles remaining after removal of duplicates. After applying inclusion criteria, 375 were removed and 15 articles remained. These included 15 RCTs [30,36-49], nine new RCTs and six identified by previous reviews. Seven of the 15 were published in 2011 and 2012 (Table 1).

There was wide variation in study methodology. The study populations were diverse (Table 1). Smaller studies were performed in patients with specific disorders (depression, seasonal affective disorder, obesity, post-menstrual tension and hospitalized patients) [30,37-39,41-44,47-49], and studies in University students $[45,46]$.

Table 1. Study populations, sample sizes (numbers entering intervention and control groups respectively) and methodological quality score (PEDro Scale).

\begin{tabular}{|c|c|c|c|c|c|}
\hline Author & Year & $\begin{array}{l}\text { Reference } \\
\text { Citation \# }\end{array}$ & Population & Sample Size & $\begin{array}{c}\text { Quality } \\
\text { Score }\end{array}$ \\
\hline Arvold et al. & 2009 & {$[36]$} & $\begin{array}{l}\text { Individuals with Vit } D \text { deficiency } \\
(10-25 \mathrm{ng} / \mathrm{mL}) \text { seen for medical } \\
\text { care at a primary healthcare clinic }\end{array}$ & 100 (I 50, C 50) & 10 \\
\hline $\begin{array}{l}\text { Belcaro } \\
\text { et al. }\end{array}$ & 2010 & {$[42]$} & $\begin{array}{l}\text { Menopausal women with signs of } \\
\text { depression and mood disorder }\end{array}$ & 65 (I 33, C 32) & 8 \\
\hline $\begin{array}{l}\text { Bertone-Johnson } \\
\quad \text { et al. }\end{array}$ & 2012 & {$[38]$} & $\begin{array}{l}\text { Postmenopausal } \\
\text { Women with depressive symptoms }\end{array}$ & 36,282 (I 18176, C 18106) & 11 \\
\hline Dean et al. & 2011 & {$[45]$} & $\begin{array}{l}\text { Young healthy adults (University } \\
\text { students) }\end{array}$ & 128 (I 63, C 65) & 11 \\
\hline $\begin{array}{l}\text { Dumville } \\
\text { et al. }\end{array}$ & 2006 & [43] & $\begin{array}{l}\text { Older women with seasonal } \\
\text { affective disorder }\end{array}$ & 2117 (I 912,C 1205) & 11 \\
\hline Gloth et al. & 1999 & [44] & Adults with Season Affective Disorder & 15 (I 8,C 7) & 6.5 \\
\hline $\begin{array}{c}\text { Harris \& } \\
\text { Dawson-Hughes }\end{array}$ & 1993 & [30] & $\begin{array}{l}\text { Women with seasonal affective } \\
\text { disorder }\end{array}$ & 250 (I 125, C 125) & 5 \\
\hline Jorde et al. & 2008 & [37] & Overweight and obese adults & 441 (IH 150, ILl 142, C 149) & 8 \\
\hline $\begin{array}{l}\text { Khajehei } \\
\text { et al. }\end{array}$ & 2009 & {$[46]$} & $\begin{array}{l}\text { University female students with } \\
\text { premenstrual syndrome }\end{array}$ & 180 (IOes 60, I 60, C 60) & 9 \\
\hline $\begin{array}{l}\text { Khoraminya } \\
\text { et al. }\end{array}$ & 2013 & [49] & $\begin{array}{l}\text { Adults with major depressive } \\
\text { disorder based on DSM-IV criteria, } \\
\text { without psychosis }\end{array}$ & 40 (I 20, C 20) & 10 \\
\hline $\begin{array}{c}\text { Landsdowne \& } \\
\text { Provost }\end{array}$ & 1998 & [39] & $\begin{array}{l}\text { Adults with seasonal affective } \\
\text { disorder }\end{array}$ & 44 (I 22, C 22) & 8 \\
\hline $\begin{array}{l}\text { Sanders } \\
\text { et al. }\end{array}$ & 2011 & [47] & $\begin{array}{l}\text { Community dwelling older women } \\
\text { with seasonal mood disorders } \\
\text { Adults with serum } 25(\mathrm{OH}) \mathrm{D}\end{array}$ & 2012 (I 1001, C 1011) & 11 \\
\hline Veith et al. & 2004 & [40] & $\begin{array}{l}<61 \mathrm{nmol} / \mathrm{L} \text { in summer, expected to } \\
\text { develop } 25(\mathrm{OH}) \mathrm{D} \text { concentrations } \\
<40 \mathrm{nmol} / \mathrm{L} \text { by winter }\end{array}$ & 64 ( I 32, C 32) & 10 \\
\hline $\begin{array}{l}\text { Yalamanchilli \& } \\
\text { Gallagher }\end{array}$ & 2012 & [48] & $\begin{array}{l}\text { Older post-menopausal women with } \\
\text { depression }\end{array}$ & $\begin{array}{l}488 \text { (Ioes+Calcitrol 122, Ioes } 122, \\
\text { Calcitrol 123, placebo } 123 \text { ) }\end{array}$ & 11 \\
\hline Zhang et al. & 2011 & [41] & Hospitalized patients & 32 (I 17, C 15) & 9 \\
\hline
\end{tabular}

$\mathrm{C}=$ control group and $\mathrm{I}=$ intervention group. Where there are two intervention groups; IH is used to indicate where a high dose and IL for where a low dose of Vitamin D supplements were given. Where one intervention group took a hormone, this was designated IOes. 
Baseline 25OHD levels were not reported in six papers [36-41] but were performed in eight studies [42-49] (Table 2). For one study [30], Vitamin D data was sought from an earlier paper [50] showing 25OHD levels were not measured at baseline. However 25OHD levels were measured twice during the study. This demonstrated that the 25OHD levels decreased $5 \%$ in the intervention group during this part of the study due to the decreased availability of sunlight with the change in season, overwhelming the effect of the low dose of Vitamin D supplements provided.

Daily doses varied from 400 I.U. to 18,400 I.U. across the 15 trials (Figure 1). Three studies [30,38,43] used doses lower that 800 I.U./day. In the Women's Health Initiative [38], the Vitamin D dose would be inadequate to change vitamin levels; the actual dose ingested was $\approx 200$ I.U., as the stipulated dose was 400 I.U. but compliance was $46 \%$. The doses shown in two papers were misprints; reported as $200 \mathrm{mg}$ Vitamin D [42] and $0.25 \mathrm{~g}$ of calcitriol [48], equating to millions of international units. However, attempts to clarify this with authors and editors were unsuccessful. The intervention in another study [47] was high dose Vitamin D (500,000 I.U.) probably inducing side effects; a 15\% increase in falls and 26\% increase in fractures.

Figure 1. Daily dose of Vitamin D per study. This shows the range of equivalent daily doses. (These were calculated after estimating the actual dose rather than using the dose shown in their published papers).

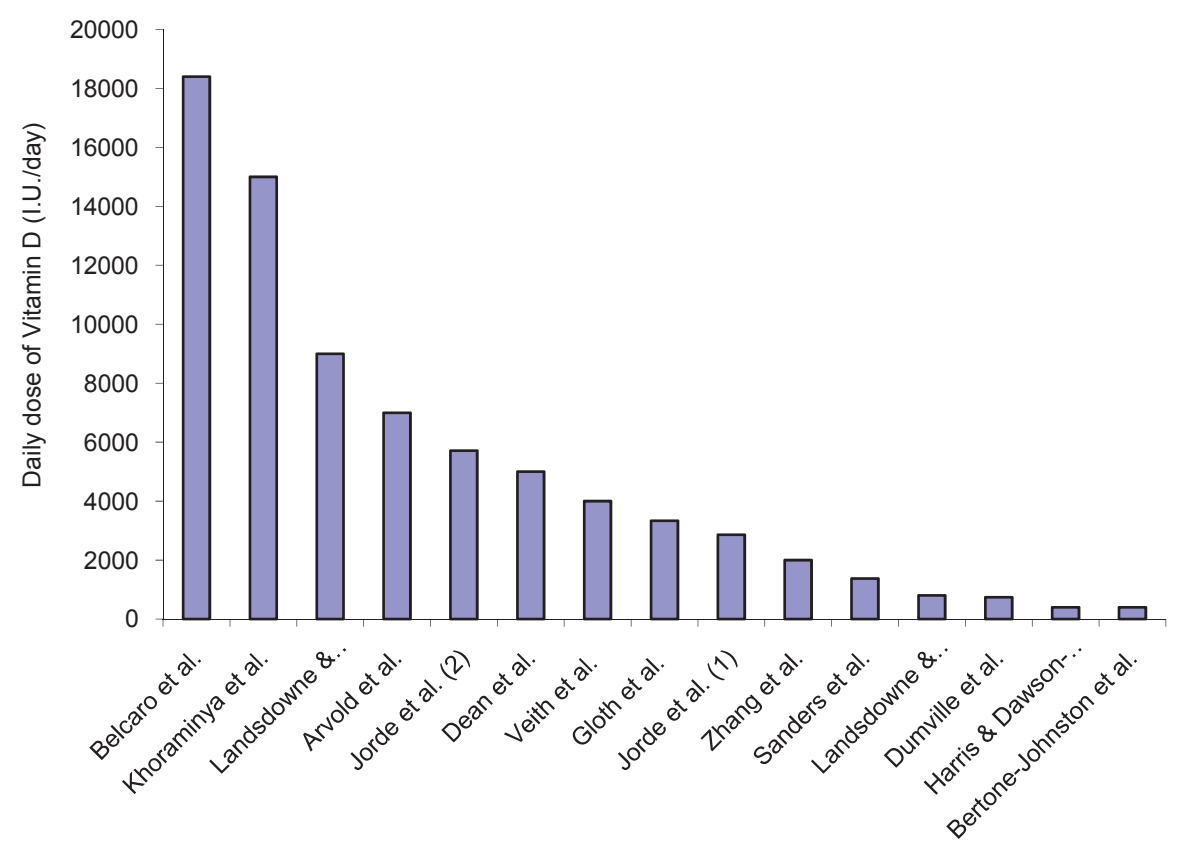

Low doses of 400 I.U. in Harris \& Dawson-Hughes [30] and Bertone-Johnson et al. [38]; High doses were over 15,000 I.U. per day in Belcaro et al. [42] and Khoraminya et al. [49]; Jorde et al. [37] and Landsdowne \& Provost [39] both tested three groups; two differing dosages and one placebo.

Validated outcome measures of depression (Table 2) included Beck Depression Index in three studies $[37,45,49]$ the Profile of Mood States in two studies [30,41] and the mental component score of the SF12 in two studies [43,47]. Questionnaires about pre-menstrual syndrome [46], fibromyalgia [36], and menopause [42] included depression as a domain. One early study used an unvalidated questionnaire [39]. There was no significant differences at baseline measures and methodological quality of studies was generally high (9 out of 11) (Table 1). 


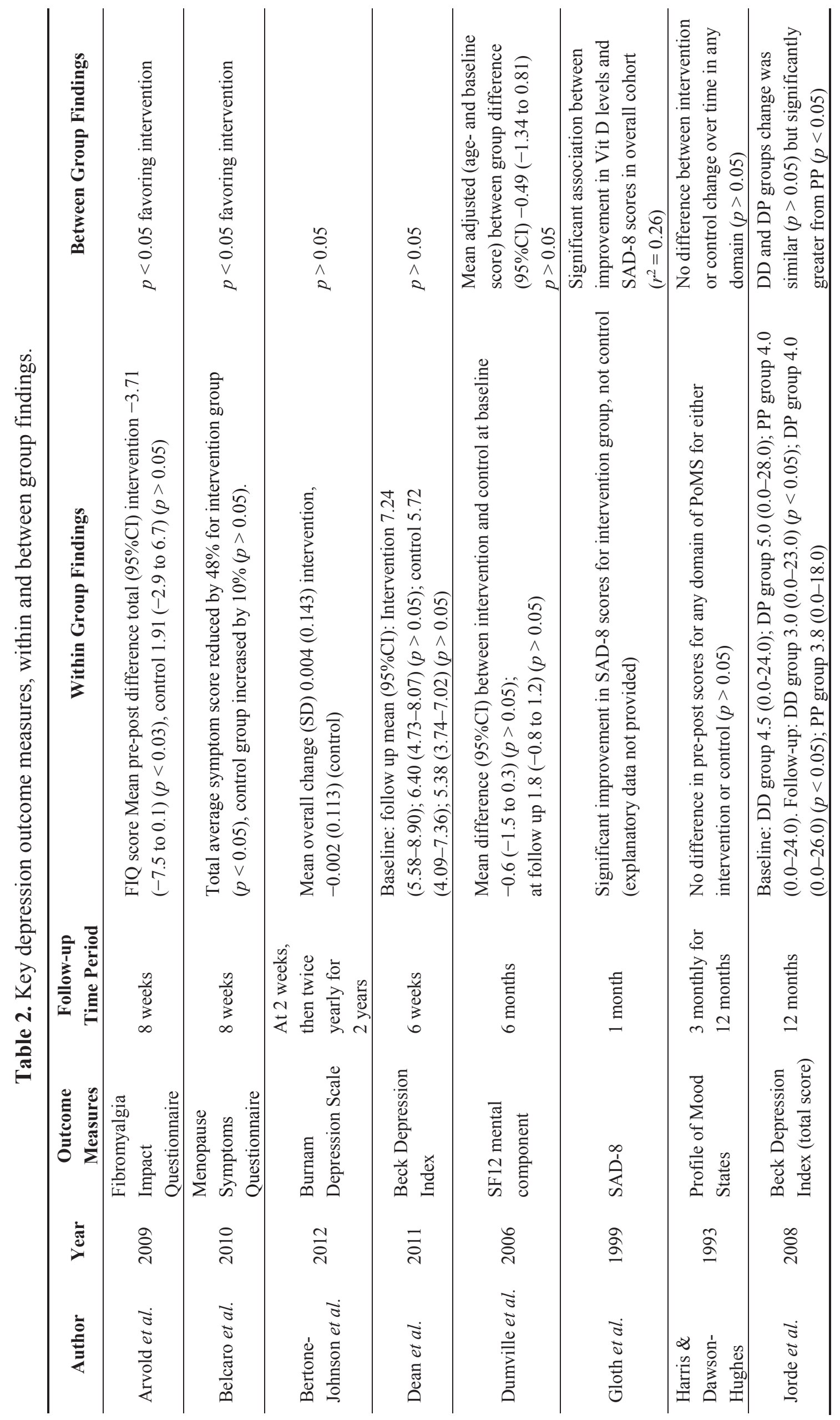




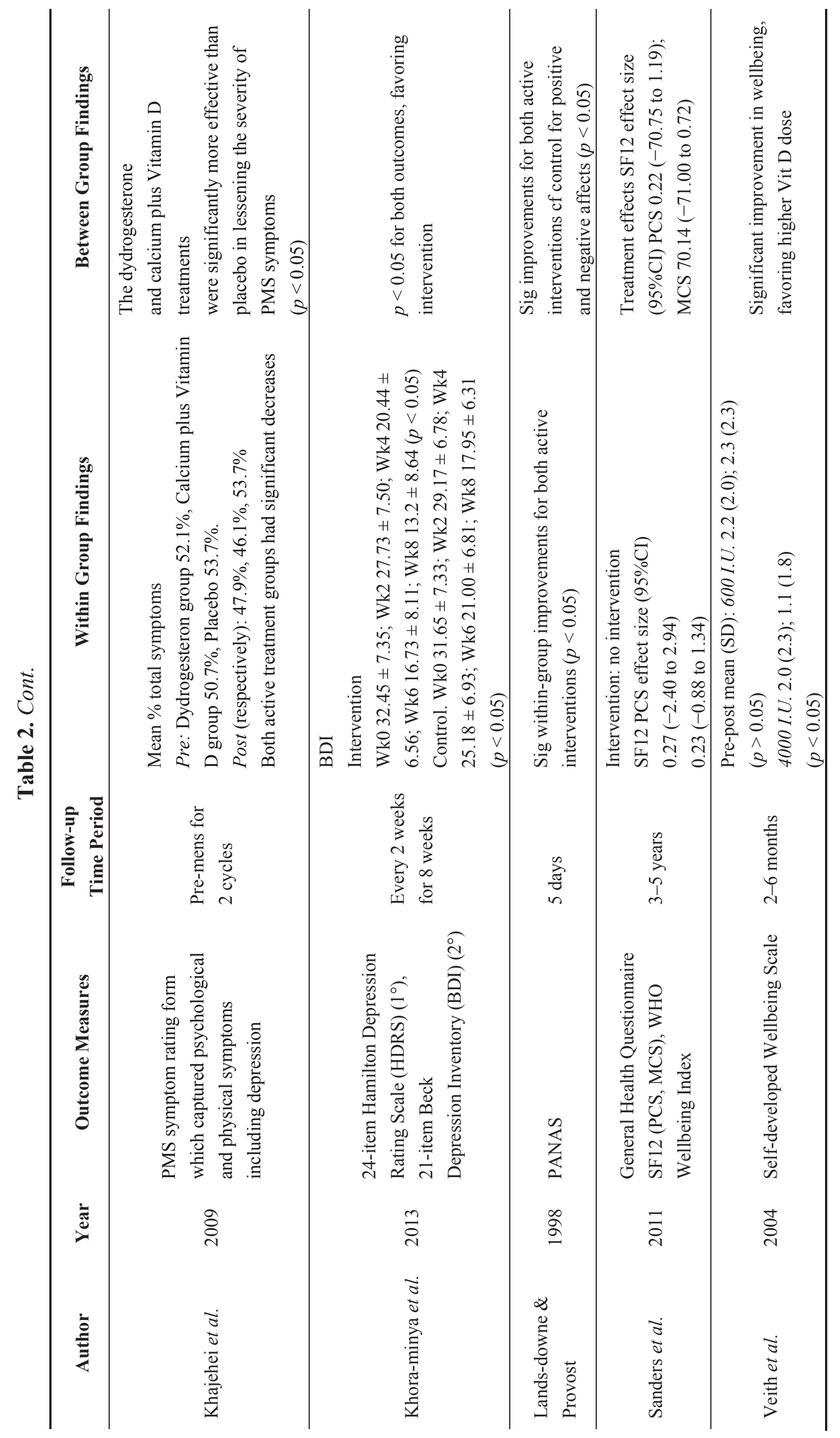




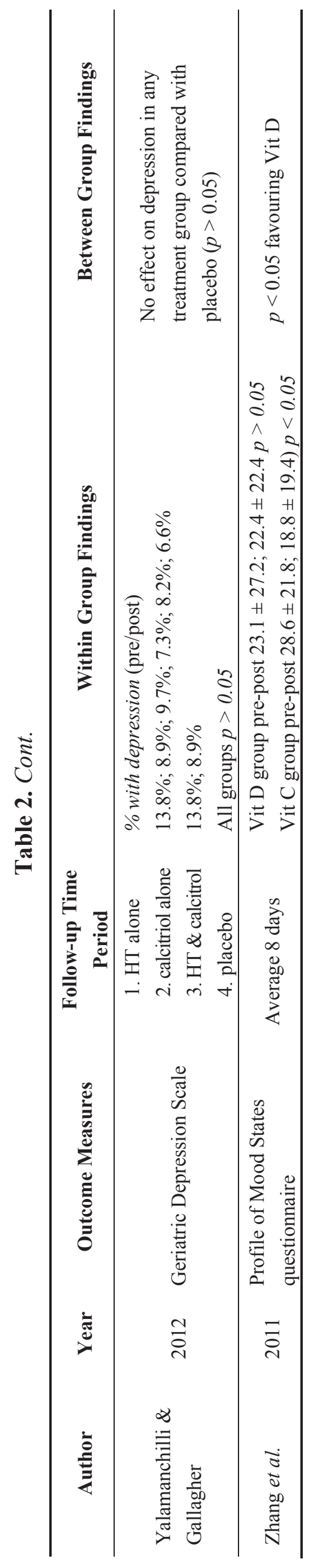




\subsection{Biological Flaws}

Biological flaws were found in eight of the 15 studies (Table 3). These flaws limit the ability of these studies to demonstrate a change in vitamin status in the intervention group. The most common flaw, occurring in five studies, was not measuring 25OHD. Two studies [30,38] utilized doses below the minimum effective dose of 600-800 I.U. [51] and one study [45] had such high baseline 25OHD levels that supplements could not improve the Vitamin D status of participants. One intervention was associated with a decrease in 25OHD level [30], and another caused falls and fractures minimising the potential to see any health benefits [47]. Biological flaws were more prevalent $(70 \%)$ in recent studies (since 2010) than in earlier studies (50\%), and in larger studies than in smaller studies (Table 3 ).

Table 3. Comparison of studies by presence of biological flaws to the study findings and methodological quality.

\begin{tabular}{|c|c|c|c|c|c|c|c|}
\hline \multirow[b]{2}{*}{ Study } & \multirow{2}{*}{$\begin{array}{c}\text { Biological } \\
\text { Flaws NOT } \\
\text { Present }\end{array}$} & \multirow{2}{*}{$\begin{array}{c}\text { Biological } \\
\text { Flaw(s) } \\
\text { Present } \\
\end{array}$} & \multicolumn{2}{|c|}{ Type of Flaw } & \multirow{2}{*}{$\begin{array}{l}\text { Quality Score } \\
\text { (Max 11) }\end{array}$} & \multirow{2}{*}{\multicolumn{2}{|c|}{$\begin{array}{c}\text { Date of } \\
\text { Publication }\end{array}$}} \\
\hline & & & $\begin{array}{c}25 O H D \text { not } \\
\text { Assessed }\end{array}$ & $\begin{array}{c}\text { Dose not } \\
\text { Appropriate }\end{array}$ & & & \\
\hline Belcaro et al. & & $\mathrm{X}$ & $\mathrm{X}$ & & 8 & & 2010 \\
\hline Bertone-Johnson et al. & & $\mathrm{X}$ & $\mathrm{X}$ & $X(L)$ & 11 & & 2012 \\
\hline Dumville et al. & & $X$ & $\mathrm{X}$ & & 11 & 2006 & \\
\hline Harris \& Dawson-Hughes & & $X$ & $\mathrm{X}$ & $X(L)$ & 5 & 1993 & \\
\hline Dean et al. & & $\mathrm{X}$ & $\mathrm{X}$ & $\mathrm{X}(\mathrm{H})$ & 11 & & 2011 \\
\hline Khajehei et al. & & $\mathrm{X}$ & & $\mathrm{X}(\mathrm{I})$ & 9 & 2009 & \\
\hline Sanders et al. & & $\mathrm{X}$ & & $\mathrm{X}(\mathrm{SE})$ & 11 & & 2011 \\
\hline Yalamanchilli \& Gallagher & & $\mathrm{X}$ & & $\mathrm{X}(\mathrm{I})$ & 11 & & 2012 \\
\hline $\begin{array}{c}\text { Total-8 Studies with } \\
\text { Biological Flaws }\end{array}$ & $\mathbf{0}$ & 8 & 5 & 6 & & 3 & 5 \\
\hline Arvold et al. & $\mathbf{X}$ & & & & 10 & 2009 & \\
\hline Gloth et al. & $\mathbf{X}$ & & & & 6.5 & 1999 & \\
\hline Jorde et al. & $\mathbf{X}$ & & & & 8 & 2008 & \\
\hline Khoraminya et al. & $\mathbf{X}$ & & & & 10 & & 2013 \\
\hline Landsdowne \& Provost & $\mathbf{X}$ & & & & 8 & 1998 & \\
\hline Veith et al. & $\mathbf{X}$ & & & & 10 & 2004 & \\
\hline Zhang et al. & $\mathbf{X}$ & & & & 9 & & 2011 \\
\hline $\begin{array}{c}\text { Total-7 studies } \\
\text { without flaws }\end{array}$ & 7 & $\mathbf{0}$ & $\mathbf{0}$ & $\mathbf{0}$ & & 5 & 2 \\
\hline
\end{tabular}

Of the seven studies without flaws, six [36,37,39,40,44,49] showed improvement in depression with supplementation, whereas six of the nine flawed studies [30,38,42,45-48] had a null result (Table 3). The positive results in two flawed studies maybe due to the unknown contents [46] or the effects of the herbs [42] used in these studies. 


\subsection{Meta-Analysis}

\subsubsection{Meta-Analysis of Studies without Biological Flaws (Right Panel of Figure 2)}

Two studies (Jorde et al. [37] and Khoraminya et al. [49]) were included as they used the same outcome measure; the Beck Depression Inventory.

The standardized mean difference for these studies without flaws is shown in the Right Panel of Figure 2. It shows a statistically significant positive effect of Vitamin D in depression of 0.78 (CI $0.24,1.27)$. The random effects model was used due to the diverse populations studied.

Figure 2. The figures show the meta-analysis of studies from the systematic review.

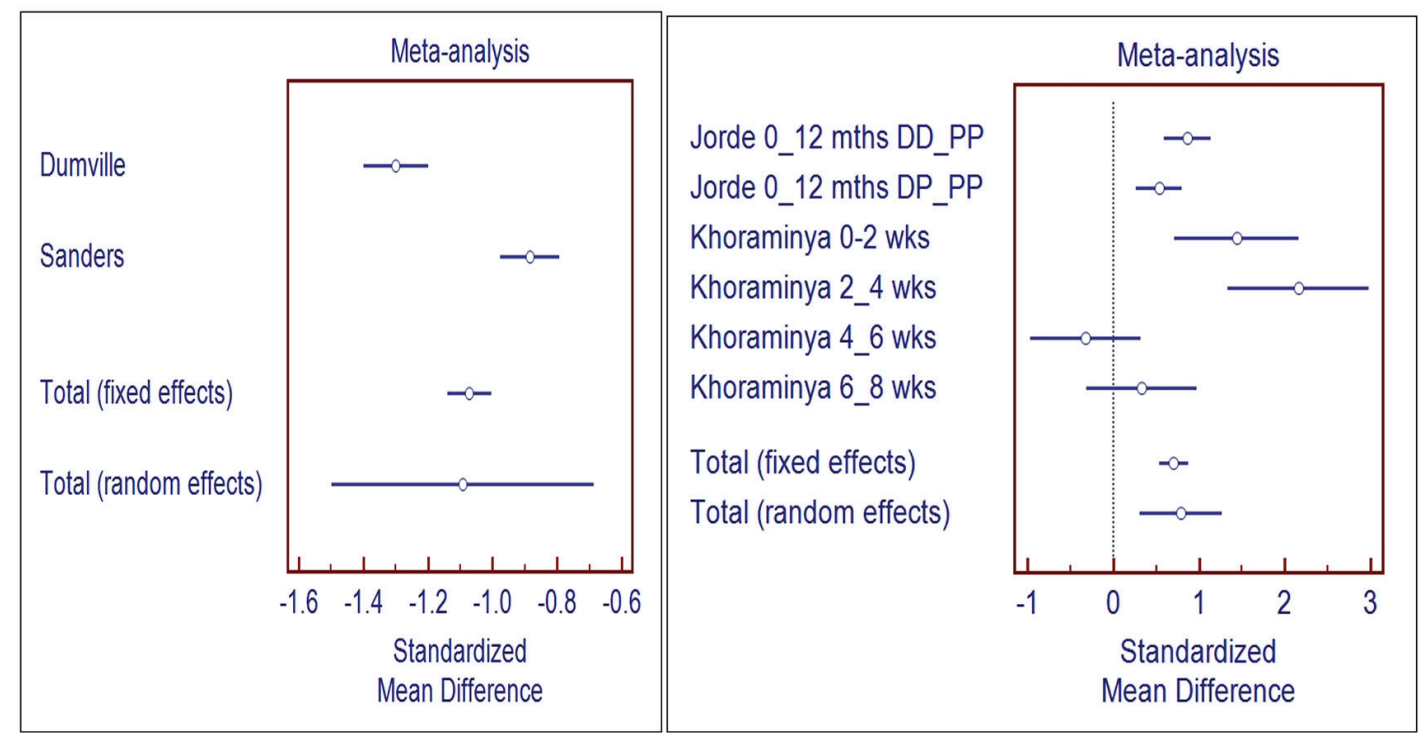

Left Panel-Two studies with biological flaws were combined, Dumville et al. [43] and Sanders et al. [47];

Right Panel—Two studies without biological flaws were combined, Jorde et al. [37] and Khoraminya et al. [49], showing two intervention groups for Jorde et al. [37] (high and low dose Vitamin D) and the data from the Khoraminya et al. [49] at 2, 4, 6, and 8 weeks.

The Jorde et al. [37] trial $(n=387)$ had three study groups; two interventions with different doses of Vitamin D and a control. The Khoraminya et al. [49] trial $(n=40)$ compared Vitamin D plus fluoxetine to fluoxetine alone. The studies had similar baseline level of 25OHD (Jorde et al. [37] $55 \mathrm{nmol} / \mathrm{L}$ ) (Khoraminya et al. [49] $57 \mathrm{nmol} / \mathrm{L}$ ), and the doses of Vitamin D over $800 \mathrm{nmol} / \mathrm{L}$ in both studies. The participants in both studies were patients; Khoraminya et al. [49] depressed patients and Jorde et al. [37] obese patients. Depression and obesity overlap, as there is a reciprocal relationship between obesity and depression indicated by the $50 \%$ increase in one condition when the other is present [52].

\subsubsection{Meta-Analysis of Studies with Biological Flaws (Left Panel of Figure 2)}

Options for meta-analysis were examined and performed combining the Dumville et al. [43] and Sanders et al. [47] studies, due to the diverse outcome variables used in other studies. There was a 
statistically significant negative effect of Vitamin D administration evident from the forest plot in the standardized mean differences as shown in the Left Panel of Figure 2. The effect size was -1.1 (CI $-0.7,-1.5)$ (random effects). These studies were of high methodological quality, had similar subjects (community dwelling women aged $>70$ years) and baseline $25 \mathrm{OHD}$, and used the same outcome measure. The studies differed in the dosing schedule, daily and annually.

\section{Discussion}

This is the most comprehensive systematic review of randomized controlled trials investigating the effectiveness of Vitamin D in the management of depression. Fifteen RCTs were found, whilst previous reviews captured few of the available RCTs. Although the methodological quality was good, biological flaws were common and more prevalent in recent studies.

For the meta-analysis of studies without biological flaws, the size of the effect was statistically significant being +0.78 (CI $0.24,1.27$ ). As the measure of effect size was the standardized mean difference (SMD), this was 0.78, using Cohen's Rule-of-Thumb, a SMD of 0.8 is considered to indicate a large effect.

As less than half the study population were deficient the effect of the intervention was diluted such that if all subjects had been deficient the size of the effect would have been higher, perhaps double, 1.5 points on the BDI scale. This is similar to the size of effect seen in a large RCT of antidepressant medication, which was 0.8 point on the BDI scale for the blinded parts of the study and 1.7 points overall [53]. A review of antidepressant efficacy published in the NEJM [54] shows that the effect size of antidepressant medication was increased by selective publication of trials and altering the effect size. However the overall mean weighted effect size value for antidepressants was only 0.15 (CI $0.08,0.22$ ) for unpublished studies and 0.37 (CI $0.33,0.41)$ for published studies. Thus, the effect size of Vitamin D demonstrated in our meta-analysis may be comparable with that of anti-depressant medication. For the meta-analysis of studies with biological flaws, the size of the effect was statistically significant and negative being -1.1 (CI $-0.7,-1.5)$, indicating that Vitamin D supplementation in flawed studies may lead to deterioration in depression.

The main finding is that all studies without flaws and the meta-analysis of studies without biological flaws support the efficacy of Vitamin D supplementation for depression, as compared with the negative results of meta-analysis for studies with biological flaws. The Womens Health Initiative [38] (WHI), with more participants that all the other studies combined, had the highest methodological quality and the most biological flaws leading to non-significant outcomes for both bone strength and mood. Due to its sheer size, the WHI has dominated previous meta-analysis leading to null results.

The main limitation of this review was the diversity of study methodology precluding more extensive meta-analyses, and leaving only two studies in each meta-analysis. The variability in outcome measures and reporting suggest agreement should be sought within the research community to underpin standard conduct and reporting of future studies to support meta-analysis. 


\section{Conclusions}

Traditional evidence, biological plausibility and epidemiological studies indicate Vitamin D has therapeutic effects in depression. There are no previous meta-analyses of Vitamin D and depression as the evidence was deemed to be insubstantial [25]. This may be due to previous systematic reviews identifying few of the available studies and including RCTs with inappropriate methodology and biological flaws.

Meta-analysis of studies without biological flaws demonstrates that improving Vitamin D levels improves depression, whereas the meta-analysis of flawed studies had a negative result. Heaney [34] identified the most common flaw "baseline status" and the most pernicious flaw "(in)effective dosing". However we found other flaws: not measuring 25OHD levels throughout the study limits the ability to know if the 25OHD level actually changed. In this case, there would be no reason to believe that the intervention caused a biological difference in Vitamin D levels between intervention and control groups. We also found more fundamental biological flaws where the intervention was not Vitamin D but calcium, and caused a decreased in the 25OHD level. These two studies were included in previous systematic reviews but rejected by this review.

The finding that meta-analyses for studies with biological flaws had the statistically significant effect of increasing depression, may lead to a conclusion that some of these trials led to levels for Vitamin D above the therapeutic range. This would be supported by a recent paper indicating that the therapeutic range for 25OHD in depression is 50 and $85 \mathrm{nmol} / \mathrm{L}$ [55].

It may be argued that meta-analysis including flawed RCTs reflect the trial methodology more than the efficacy of the intervention, leaving reviewers unable to make valid conclusions about efficacy [34], resulting in uncertainty amongst researchers and clinicians. This has led to calls for more RCTs and less "torturing of the data" by meta-analysis [56]. However, as this review demonstrates, it is excluding biological flaws that will lead to greater understanding of Vitamin D, not simply increasing the quantity of studies.

We note that biological flaws are more frequent in recent studies; this may be due to the belief that vitamins exert a function beyond deficiency. Hence RCTs should test whether using supplementation to correct deficiency is beneficial, rather than testing whether additional supplementation on top of the recommended doses is beneficial in reducing disease [57]. Thus, it is unremarkable that Vitamin D supplementation would not benefit a population that are not deficient or where the dose was ineffective. To test the hypothesis that correcting Vitamin D deficiency leads to an improvement in depression, it is critical to exclude biological flaws from future studies.

The effect size for Vitamin D in depression demonstrated in this meta-analysis is comparable with the effect of anti-depressant medication, an accepted treatment for depression. Should these results be verified by future research, these findings may have important clinical and public health implications.

\section{Acknowledgments}

The authors would like to acknowledge Karen Grimmer, Kate Beaton, Khushnum Pastakia and Ellie King for their invaluable technical support, Howard Morris for his advice, and Jan Drewery-Clark, the journal editors and staff for their assistance preparing the manuscript for publication. 


\section{References}

1. Hyman, S.; Chisholm, D.; Kessler, R.; Patel, V.; Whiteford, H. Mental disorders. In Disease Control Priorities in Developing Countries, 2nd ed.; Jamison, D.T., Breman, J.G., Measham, A.R., Alleyne, G., Claeson, M., Evans, D.B., Jha, P., Mills, A., Musgrove, P., Eds.; Oxford University Press: New York, NY, USA, 2006; pp. 605-626.

2. Bracken, P.; Thomas, P.; Timimi, S. Psychiatry beyond the current paradigm. Br. J. Psychiat. 2012, 201, 430-434.

3. Jordanes. The Origin and Deeds of the Goths; Mierow, C.C.; Princeton University Press: Princeton, NJ, USA; 2012; pp. 19-21. Available Online: http://people.ucalgary.ca/ /vandersp/ Courses/texts/jordgeti.html (accessed on 8 April 2014).

4. Holick, M.F. The Vitamin D deficiency pandemic: A forgotten hormone important for health. Public Health Rev. 2010, 32, 267-283.

5. Hollick, M.F. Vitamin D deficiency. N. Engl. J. Med. 2007, 357, 266-281.

6. Daly, R.M.; Gagnon, C.; Lu, Z.X.; Magliano, D.J.; Dunstan, D.W.; Sikaris, K.A.; Zimmet, P.Z.; Ebeling, P.R.; Shaw, J.E. Prevalence of Vitamin D deficiency and its determinants in Australian adults aged 25 years and older: A national, population-based study. Clin. Endocrinol. 2011, 77, 26-35.

7. Munns, C.F.; Simm, P.J.; Rodda, C.P. Incidence of Vitamin D deficiency rickets among Australian children: An Australian Paediatric Surveillance Unit study. Med. J. Aust. 2012, 196, 466-468.

8. Thacher, T.D.; Fischer, P.R.; Strand, M.A.; Pettifor, J.M. Nutritional rickets around the world: Causes and future directions. Ann. Trop. Paediatr. 2006, 26, 1-16.

9. Ginde, A.A.; Liu, M.C.; Camargo, C.A. Vitamin D deficiency seems to be increasing in the US population. Arch. Intern. Med. 2009, 169, 616-32.

10. Maxmen, A. Nutrition advice: The Vitamin D-lemma. A vociferous debate about vitamin-D supplementation reveals the difficulty of distilling strong advice from weak evidence. Nature 2011, 475, 23-25.

11. Scragg, R. Vitamin D and public health: An overview of recent research on common diseases and mortality in adulthood. Public Health Nutr. 2011, 14, 1515-1532.

12. Heaney, R.P. Does inconclusive evidence for Vitamin D supplementation to reduce risk for cardiovascular disease warrant pessimism? [Letter to the editor]. Ann. Intern. Med. 2010, 153, 208-209.

13. Spedding, S. Vitamins are more adaptable than Casimir Funk thought. Australasian J. Med. 2013, in press.

14. Hendrix, I.; Anderson, P.; May, B.; Morris, H. Regulation of gene expression by the CYP27B1 promoter-study of a transgenic model. J. Steroid Biochem. Mol. Biol. 2004, 89-90, 139-142.

15. Ramagopalan, S.V.; Heger, A.; Berlanga, A.J.; Maugeri, N.J.; Lincoln, M.R.; Burrell, A. A ChIP-seq defined genome-wide map of Vitamin D receptor binding: Associations with disease and evolution. Genome Res. 2010, 20, 1352-1360. 
16. Obradovic, D.; Gronemeyer, H.; Lutz, B.; Rein, T. Cross-talk of Vitamin D and glucocorticoids in hippocampal cells. J. Neurochem. 2006, 96, 500-509.

17. Puchacz, E.; Stumpf, W.; Stachowiak, E.K.; Stachowiak, M.K. Vitamin D increases expression of the tyrosine hydroxylase gene in adrenal medullary cells. Mol. Brain Res. 1996, 36, 193-196.

18. Cass, W.A.; Smith, M.P.; Peters, L.E. Calcitriol protects against the dopamine- and serotonin-depleting effects of neurotoxic doses of methamphetamine. Ann. N. Y. Acad. Sci. 2006, 1074, 261-271.

19. Ganji, V.; Milone, C.; Cody, M.; McCarty, F.; Wang, Y.T. Serum Vitamin D concentrations are related to depression in young adult US population: The Third National Health and Nutrition Examination Survey. Int. Arch. Med. 2010, 3, 29.

20. May, H.T.; Bair, T.L.; Lappé, D.L.; Anderson, J.L.; Horne, B.D.; Carlquist, J.F.; Muhlestein, J.B. Association of Vitamin D levels with incident depression among a general cardiovascular population. Am. Heart J. 2010, 159, 1037-1043.

21. Hoang, M.T.; DeFina, L.F.; Willis, B.L. Association between low serum 25-hydroxyVitamin D and depression in a large sample of healthy adults: The Cooper Center Longitudinal Study. Mayo Clin. Proc. 2011, 86, 1050-1055.

22. Kjærgaard, M.; Joakimsen, R.; Jorde, R. Low serum 25-hydroxyVitamin D levels are associated with depression in an adult Norwegian population. Psychiatry Res. 2011, 190, 221-225.

23. Umhau, J.C.; George, D.T.; Heaney, R.P.; Lewis, M.D.; Ursano, R.J. Low Vitamin D status and suicide: A case-control study of active duty military service members. PLoS One 2013, 8, e51543.

24. Li, G.; Mbuagbaw, L.; Samaan, Z.; Zhang, S.; Adachi, J.D.; Papaioannou, A. ThabaneL Efficacy of vitamin D supplementation in depression in adults: a systematic review protocol. Syst. Rev. 2013, 2, 64. doi:10.1186/2046-4053-2-64.

25. Institute of Medicine. Dietary Reference Intakes for Calcium and Vitamin D; The National Academics Press: Washington DC, WA, USA, 2011.

26. Morgan, A.; Jorm, A. Self-help interventions for depressive disorders and depressive symptoms: A systematic review. Ann. Gen. Psychiatry 2008, 7, 13.

27. Murphy, P.K.; Wagner, C.L. Vitamin D and mood disorders among women: An integrative review. J. Midwifery Womens Health 2008, 53, 440-446.

28. Barnard, K.; Colon-Emeric, C. Extraskeletal effects of Vitamin D in older adults: Cardiovascular disease, mortality, mood, and cognition. Am. J. Geriatr. Pharmacother. 2010, 8, 4-33.

29. Thys-Jacobs, S.; Starkey, P.; Bernstein, D.; Tian, J. Calcium carbonate and the premenstrual syndrome: Effects on premenstrual and menstrual symptoms. Premenstrual Syndrome Study Group. Am. J. Obstet. Gynecol. 1998, 179, 444-452.

30. Harris, S.; Dawson-Hughes, B. Seasonal mood changes in 250 normal women. Psychiatry Res. 1993, 49, 77-87.

31. Scragg, R. Do we need to take calcium with Vitamin D supplements to prevent falls, fractures, and death? Curr. Opin. Clin. Nutr. Metab. Care 2012, 15, 614-624. 
32. Biesalski, H.K.; Aggett, P.J.; Anton, R.; Bernstein, P.S.; Blumberg, J.; Heaney, R.P. Scientific substantiation of health claims: Evidence-based nutrition. 26th Hohenheim Consensus Conference. Nutrition 2011, 27, S1-S20.

33. Lappe, J.M.; Heaney, R.P. Why randomized controlled trials of calcium and Vitamin D sometimes fail. Dermato Endocrinol. 2012, 4, 95-100.

34. Heaney, R.P. Vitamin D-Baseline status and effective dose. [Letters to the editor]. N. Engl. J. Med. 2012, 367, 1.

35. Sherrington, C.; Herbert, R.D.; Maher, C.G.; Moseley, A.M. PEDro. A database of randomized trials and systematic reviews in physiotherapy. Man. Ther. 2000, 5, 223-226.

36. Arvold, D.S.; Odean, M.J.; Dornfeld, M.P.; Regal, R.R.; Arvold, J.G.; Karwoski, G.C.; Mast, D.J.; Sanford, P.B.; Sjoberg, R.J. Correlation of symptoms with Vitamin D deficiency and symptom response to cholecalciferol treatment: A randomized controlled trial. Endocr. Pract. 2009, 15, 203-212.

37. Jorde, R.; Sneve, M.; Figenschau, Y.; Svartberg, J.; Waterloo, K. Effects of Vitamin D supplementation on symptoms of depression in overweight and obese subjects: Randomized double blind trial. J. Intern. Med. 2008, 264, 599-609.

38. Bertone-Johnson, E.R.; Powers, S.I.; Spangler, L.; Larson, J.; Michael, Y.L.; Millen, A.E.; Bueche, M.N.; Salmoirago-Blotcher, E.; Wassertheil-Smoller, S.; Brunner, R.L. Vitamin D supplementation and depression in the women's health initiative calcium and Vitamin D trial. Am. J. Epidemiol. 2012, 176, 1-13.

39. Lansdowne, A.T.; Provost, S.C. Vitamin D3 enhances mood in healthy subjects during winter. Psychopharmacol. 1998, 135, 319-323.

40. Veith, R.; Kimball, S.; Hu, A.; Walfish, P.G. Randomized comparison of the effects of the Vitamin D3 adequate intake versus $100 \mathrm{mcg}$ (4000 IU) per day on biochemical responses and the wellbeing of patients. Nutr. J. 2004, 3, 8.

41. Zhang, M.; Robitaille, L.; Eintracht, S.; Hoffer, L.J. Vitamin C provision improves mood in acutely hospitalized patients. Nutrition 2011, 27, 530-533.

42. Belcaro, G.; Cesarone, M.R.; Cornelli, U.; Dugall, M. MF Afragil ${ }^{\circledR}$ in the treatment of 34 menopause symptoms: A pilot study. Panminerva Med. 2010, 52, 49-54.

43. Dumville, J.C.; Miles, J.N.; Porthouse, J.; Cockayne, S.; Saxon, L.; King, C. Can Vitamin D supplementation prevent winter-time blues? A randomised trial among older women. J. Nutr. Health Aging 2006, 10, 151-153.

44. Gloth, F.M., III; Alam, W.; Hollis, B. Vitamin D vs. broad spectrum phototherapy in the treatment of seasonal affective disorder. J. Nutr. Health Aging 1999, 3, 5-7.

45. Dean, A.J.; Bellgrove, M.A.; Hall, T.; Phan, W.M.; Eyles, D.W.; Kvaskoff, D.; McGrath, J.J. Effects of Vitamin D supplementation on cognitive and emotional functioning in young adults-A randomised controlled trial. PLoS One 2011, 6, e25966.

46. Khajehei, M.; Abdali, K.; Parsanezhad, M.E.; Tabatabaee, H.R. Effect of treatment with dydrogesterone or calcium plus Vitamin D on the severity of premenstrual syndrome. Int. J. Gynecol. Obstet. 2009, 105, 158-161. 
47. Sanders, K.M.; Stuart, A.L.; Williamson, E.J.; Jacka, F.N.; Dodd, S.; Nicholson, G.; Berk, M. Annual high-dose Vitamin D3 and mental well-being: randomised controlled trial. $\mathrm{Br} . \mathrm{J}$. Psychiatry 2011, 198, 357-364.

48. Yalamanchili, V.V.; Gallagher, J.C. Treatment with hormone therapy and calcitriol did not affect depression in older postmenopausal women: No interaction with estrogen and Vitamin D receptor genotype polymorphisms. Menopause 2012, 19, 697-703.

49. Khoraminya, N.; Tehrani-Doost, M.; Jazayeri, S.; Hosseini, A.; Djazayery, A. Therapeutic effects of Vitamin D as adjunctive therapy to fluoxetine in patients with major depressive disorder. Aust. N.Z. J. Psychiatry 2013, 47, 271-275.

50. Dawson-Hughes, B.; Dallal, G.E.; Krall, E.A.; Harris, S.; Sokoll, L.J.; Falconer, G. Effect of Vitamin D supplementation on wintertime and overall bone loss in healthy postmenopausal women. Ann. Intern. Med. 1991, 115, 505-512.

51. Gallagher, J.C.; Sai, A.; Templin, T., II; Smith, L. Dose response to Vitamin D supplementation in postmenopausal women: A randomized trial. Ann. Intern. Med. 2012, 156, 425-437.

52. Luppino, F.S.; Wit, L.M.; Bouvy, P.F. Overweight, obesity and depression. A systematic review and meta-analysis of longitudinal studies. Arch. Gen. Psychiatry 2010, 67, 220-229.

53. Kendrick, T.; Chatwin, J.; Dowrick, C.; Tylee, A.; Morriss, R.; Peveler, R. Randomised controlled trial to determine the clinical effectiveness and cost-effectiveness of selective serotonin reuptake inhibitors plus supportive care, versus supportive care alone, for mild to moderate depression with somatic symptoms in primary care: The THREAD (THREshold for AntiDepressant response) study. Health Technol. Assess. 2009, 13, 1-159.

54. Turner, E.H.; Matthews, A.M.; Linardatos, E.; Tell, R.; Rosenthal, R. Selective publication of antidepressant trials and its influence on apparent efficacy. N. Engl. J. Med. 2008, 358, 252-260.

55. Maddock, J.; Berry, D.J.; Geoffroy, M.-C.; Power, C.; Hyppönen, E. Vitamin D and common mental disorders in mid-life: Cross-sectional and prospective findings. Clin. Nutr. 2013, 32, 758-764.

56. Bolland, M.J.; Grey, A.; Reid, I.R. Time for a moratorium on meta-analyses of Vitamin D? Letter to the editor. Br. Med. J. 2009, 339, 987.

57. Moser, U. Vitamins -Wrong approaches. Int. J. Vitamin Nutr. Res. 2012, 82, 327-332. 


\title{
Serum 25-Hydroxyvitamin D Concentrations and Depressive Symptoms among Young Adult Men and Women
}

\author{
Maria A. Polak, Lisa A. Houghton, Anthony I. Reeder, Michelle J. Harper and Tamlin S. Conner
}

Abstract: There has been an increased interest in the role of vitamin D in depression; however, there have been few studies conducted in younger population groups. Our aim was to investigate the association between vitamin D status and depressive symptoms in a non-clinical young adult sample living in Dunedin, New Zealand. A cross-sectional sample of 615 young adults completed a questionnaire including demographics and the Centre for Epidemiological Studies Depression Scale (CES-D). Height, weight and a blood sample for 25-hydroxyvitamin D [25(OH)D] was obtained. Serum 25(OH)D was used to predict depression scores, adjusting for potential confounders including time spent outdoors for 13 consecutive days, BMI, age, sex and ethnicity. Prevalence of low vitamin D was high even in this age group, and serum $25(\mathrm{OH}) \mathrm{D}$ was negatively associated with depression symptoms before and after adjustment. When investigating the relationship between the presence versus absence of depressive symptoms and quartiles of $25(\mathrm{OH}) \mathrm{D}$, participants in the lowest quartile were more likely to report depressive symptoms compared with those in the highest quartile. Although our findings suggest that vitamin D is a predictor of depression symptomatology, even when controlling for time spent outdoors, a randomised controlled trial in this young adult target group is needed to confirm the association.

Reprinted from Nutrients. Cite as: Polak, M.A.; Houghton, L.A.; Reeder, A.I.; Harper, M.J.; Conner, T.S. Serum 25-Hydroxyvitamin D Concentrations and Depressive Symptoms among Young Adult Men and Women. Nutrients 2014, 6, 4720-4730.

\section{Introduction}

Recently, there has been an increased interest in the potential role of vitamin D in mental health and wellbeing. Vitamin D receptors are present in many different types of cells, including neurons and glial cells [1-3]. Most importantly, vitamin D is now accepted for its neurosteroid activity and impact on brain serotonin [2,4,5], suggesting a possible role in mood regulation. There is some evidence that low circulating concentration of serum 25-hydroxyvitamin D [25(OH)D], the marker for vitamin D status, is associated with mood disorders, including major depressive disorder, seasonal affective disorder, and premenstrual dysphoric disorder [6,7].

Sun exposure is the major source of vitamin D for humans [8,9], and thus, in the absence of adequate supplementation or suitable fortification, the concentration of circulating serum $25(\mathrm{OH}) \mathrm{D}$ decreases significantly during winter in non-equatorial locations [10]. At the same time, modern lifestyles often reduce the time in sunlight, decreasing opportunities to endogenously produce vitamin D in sufficient quantities [11]. Primary vitamin D deficiency is highly prevalent worldwide [12-14]. In European countries, $2 \%$ to $30 \%$ of all adults are estimated to be deficient in vitamin D, with up to $75 \%$ when considering older adults only [15]. A similarly high prevalence of vitamin D deficiency is present in most other areas, including those close to the equator [13]. 
While there is ample evidence of an association between serum 25(OH)D and mood disorders such as depression in older and vulnerable populations $[6,16]$, there has been very little research on younger and non-clinical populations. Some studies in depressed populations did show evidence of less time spent outdoors in depressed individuals [17], leading to suggestion that depressive symptoms might keep people inside and thus limit their opportunities for the manufacture of endogenous vitamin D. Therefore, the aim of the present study was to investigate the association between vitamin D status and depression symptoms in a non-clinical sample of young men and women, taking into account time spent outdoors during daylight hours. We hypothesised that higher vitamin D status would be associated with lower depression scores from the CES-D controlling for gender, age, ethnicity, BMI, and the time spent outdoors during daylight hours.

\section{Experimental Section}

\subsection{Participants}

Participants were healthy university student volunteers recruited through the University of Otago, which is located in Dunedin, New Zealand ( $\left.45^{\circ} 52^{\prime} 0^{\prime \prime} \mathrm{S}\right)$. Participants were recruited for the Daily Life Study, a cross-sectional study of experiences and biological markers of wellbeing carried out between March and May (southern hemisphere autumn) during the academic years 2011 and 2012. Inclusion criteria were as follows: aged between 17 and 25 years, enrolled at the University of Otago, and access to the Internet. Of the total 666 participants who completed the initial survey, 615 participants completed at least 7 of 13 daily diaries and provided a venous blood sample. The study was approved by the University of Otago Human Ethics Committee (\#10/177). All participants provided written informed consent.

\subsection{Procedure}

Participants who fulfilled the inclusion criteria were invited to an onsite session where they completed an initial computerised survey including the demographics variables and depression scale and obtained instructions for completing the 13-day online diaries (day 1 of study). Starting on day 2 of the study, participants completed an online daily diary for 13 consecutive days including a question about time spent outdoors that day. The diary took about 5-10 min to complete and also included questions about other daily experiences of participants (not part of the current study). The diary was available from any on- or off-campus computer through a password-protected website between 3 p.m. and 8 p.m. After completing the 13-day daily diary protocol, participants attended a session in the Human Nutrition Clinic of the University of Otago between 8.30 a.m. and 11.00 a.m. (day 15 of the study). During the clinic visit, their height and body composition were measured using standardised techniques, and they provided a non-fasting venous blood sample for 25(OH)D analysis.

\subsection{Measures}

Participants' depressive symptoms were assessed using the Center for Epidemiologic Studies Depression Scale (CES-D). The CES-D has been developed specifically for research in the general 
population (i.e., not clinical evaluation). The scale consists of 20 items that encompass four areas: depressed affect, positive affect, somatic vegetative signs, and interpersonal distress, with an emphasis on the affective component [18]. Participants rated how often they experienced symptoms associated with depression, including problems sleeping, poor appetite, and feeling sad over the past week. Response options range from 0 to 3 for each item $(0=$ rarely or none of the time, $1=$ some or little of the time, 2 = moderately or much of the time, 3 = most or almost all the time). Four items $(4,8,12$ and 16) are worded positively and were reverse coded for analyses. Six participants did not answer one CES-D item each, and individual means were imputed. The final score was obtained by calculating the total sum across all items, which could range from 0 to 60 , with higher scores indicating greater presence of depressive symptoms. A total score of 16 or higher is equivalent to experiencing five or more symptoms all or most of the time and is indicative of possible clinical depression [18].

To measure time spent outdoors, we modified the sun habits questionnaire developed by Glanz and colleagues [19] to create one question suitable for close-to-real-time reporting of actual daily sun exposure, asking participants to report on the time they spent outdoors during daylight hours on that day. This question was presented in the daily diary. Participants selected one of 12 categories ranging from 0 to $15 \mathrm{~min}$ to $10 \mathrm{~h}$ or more, and the answers were scored from 0 to 11 .

During the clinic visit (day 15), participants' height and weight were measured using standardised techniques [20]. All measurements were taken in duplicate by the same trained anthropometrist using calibrated equipment (Seca) with the participants wearing light clothing and no shoes. The mean of the two closest measurements was calculated. A third measure was taken if the first two measures were outside the acceptable range of variance for the given measurement. These measures were used to calculate body mass index [BMI $\left.\left(\mathrm{kg} / \mathrm{m}^{2}\right)\right]$. Participants also provided a venous non-fasting blood sample for serum total $25(\mathrm{OH}) \mathrm{D}$ analyses.

\subsection{Blood Sample Processing and 25(OH)D Analysis}

Following blood collection, the samples were centrifuged at $3000 \mathrm{rpm}$ for 15 min at $4{ }^{\circ} \mathrm{C}$, the serum was aliquoted then stored at $-80{ }^{\circ} \mathrm{C}$ until analysis. Serum $25(\mathrm{OH}) \mathrm{D}$ was analysed using isotope-dilution liquid chromatography tandem mass spectrometry based on the method of Maunsell et al. [21], using an API 3200 instrument (Applied Biosystems) connected to a Dionex Ultimate 3000 HPLC system. The limit of quantification for the assay was $<5 \mathrm{nmol} / \mathrm{L}$. To assess accuracy and interassay variability, external quality control serum material (UTAK Laboratories) containing low and medium concentration of $25(\mathrm{OH}) \mathrm{D}_{3}$ and $25(\mathrm{OH}) \mathrm{D}_{2}$ were analysed with every run.

A different batch of controls was used for each year of the study. In 2011, the 25(OH)D 3 low control mean was $26.0 \mathrm{nmol} / \mathrm{L}$ (verified value $27.5 \mathrm{nmol} / \mathrm{L}$ ); CV $3.8 \%$, and the medium control mean $75.7 \mathrm{nmol} / \mathrm{L}$ (verified value $77.5 \mathrm{nmol} / \mathrm{L}$ ); CV $2.9 \%$. For $25(\mathrm{OH}) \mathrm{D}_{2}$, the low control mean was $22.5 \mathrm{nmol} / \mathrm{L}$ (verified value $24.2 \mathrm{nmol} / \mathrm{L}$ ); $\mathrm{CV} 7.0 \%$ and the medium control mean $71.2 \mathrm{nmol} / \mathrm{L}$ (verified value $72.7 \mathrm{nmol} / \mathrm{L}$ ); CV $4.5 \%$.

In 2012, the 25(OH)D 3 low control mean was $29.6 \mathrm{nmol} / \mathrm{L}$ (verified value $29.9 \mathrm{nmol} / \mathrm{L}$ ); CV $3.1 \%$, and the medium control mean $86.1 \mathrm{nmol} / \mathrm{L}$ (verified value $79.9 \mathrm{nmol} / \mathrm{L})$; CV 3.7\%. For $25(\mathrm{OH}) \mathrm{D}_{2}$ the low control mean was $24.8 \mathrm{nmol} / \mathrm{L}$ (verified value $26.6 \mathrm{nmol} / \mathrm{L}$ ); $\mathrm{CV} 6.2 \%$ and the medium control mean $79.5 \mathrm{nmol} / \mathrm{L}$ (verified value $77.5 \mathrm{nmol} / \mathrm{L}$ ); CV $4.9 \%$. 
Internal quality control pooled serum samples were also analysed; the inter-assay $\mathrm{CV}$ for $25(\mathrm{OH}) \mathrm{D}_{3}$ was $5.8 \%$ at $20.3 \mathrm{nmol} / \mathrm{L}(2011)$ and $3.9 \%$ at $60.7 \mathrm{nmol} / \mathrm{L}(2012)$. The concentration of $25(\mathrm{OH}) \mathrm{D}_{2}$ in the internal controls was below the limit of quantification for both years.

\subsection{Statistical Analyses}

Descriptive statistics were used to summarise the demographics and anthropometric characteristics of our sample including age, gender, and BMI. Descriptive variables were reported as means (standard deviation) by gender for all participants. We calculated a mean score for time spent outdoors during daylight hours for each participant by averaging each person's scores across the maximum 13 daily diaries. Mean values were used instead of the total score since we did not have the same number of daily reports for each participant. We performed a linear regression analysis to examine the relationship between vitamin $\mathrm{D}$ status as a continuous predictor variable and depression as a continuous outcome variable taking into account gender, age, ethnic background, and BMI. We controlled for gender (coded 0 for men and 1 for women) because women showed both higher depressive symptoms and higher $25(\mathrm{OH}) \mathrm{D}$ concentrations than men in our sample. We controlled for age, even though we had a narrow age range, for two reasons: the prevalence of depression changes with age [22], and the efficiency of manufacturing vitamin D in response to sunlight can also vary with age [3]. Ethnicity categories were NZ European, Māori/Pacific Islander, Asian and Other in line with the top level ethnicity classification used by Statistics New Zealand. Dummy variables were created for the Māori/Pacific Islander, Asian, and Other categories with NZ European as the reference category. Finally, to test whether time outdoors explains the association between 25(OH)D and depression, we added average time spent outdoors score as a control variable. Statistical analyses were carried out using the IBM SPSS Statistics package version 19 (IBM Corp. Released 2010. IBM SPSS Statistics for Windows, Armonk, NY, USA.).

\section{Results}

The mean age of participants was 19.5 years ( $S D=1.5$; range 17 to 25 years), $75 \%$ were in their first or second year of university, and more than half were female $(62 \% ; 381 / 615)$. The majority of participants were of European origin (79.8\%), 9.9\% were Asian, 2.9\% Māori or Pacific Islander, and $7.4 \%$ of other ethnicities. Of our sample, $2.3 \%(14 / 615)$ were classified as underweight $(\mathrm{BMI}<18.5)$, $68.5 \%$ as normal weight (BMI 18.5-24.9), while $23.3 \%$ and $6.0 \%$ of our sample were classified as overweight (BMI 25-29.9) and obese (BMI $\geq 30$ ), respectively. Sample characteristics did not differ statistically across the two years of data collection. There were also no statistically significant differences in total serum 25(OH)D and total CES-D scores between 2011 and 2012.

CES-D, serum 25(OH)D concentrations and confounding variables are reported in Table 1. The mean total serum 25(OH)D concentration was $64.1 \mathrm{nmol} / \mathrm{L}(\mathrm{SD}=26.6 \mathrm{nmol} / \mathrm{L})$ ranging from $8.2 \mathrm{nmol} / \mathrm{L}$ to $177.0 \mathrm{nmol} / \mathrm{L}$. When participants were divided into groups by gender, differences were found in CES-D scores, serum 25-hydroxyvitamin D, and BMI (Table 1). Total CES-D score in our sample ranged from 0 to 46 with 219 participants (35.4\%) scoring 16 or above, representing the presence of depressive symptoms indicative of clinical depression. The mean score for time spent 
outdoors was 2.0 ( $\mathrm{SD}=1.2)$, meaning that, on average, participants spent between half an hour to one hour outside per day. Average time spent outdoors during daylight hours was not a significant predictor of depressive symptoms or 25(OH)D. Participants with a CES-D score of 16 or higher had lower 25(OH)D even though they did not differ on mean time spent outdoors (Table 2).

Table 1. Average CES-D score, serum 25(OH) vitamin D, time spent outdoors, and BMI for all participants, and men and women separately.

\begin{tabular}{llllllll}
\hline \multirow{2}{*}{ Variable } & \multicolumn{2}{l}{ ALL $(\boldsymbol{n}=\mathbf{6 1 5})$} & \multicolumn{3}{l}{ Men $(\boldsymbol{n}=\mathbf{2 3 4})$} & \multicolumn{3}{l}{ Women $(\boldsymbol{n}=\mathbf{3 8 1})$} & \\
\cline { 2 - 7 } & Mean & $(\mathrm{SD})$ & Mean & $(\mathrm{SD})$ & Mean & $(\mathbf{S D})$ & \\
\hline CES-D score & 14.1 & $(8.4)$ & 12.9 & $(8.2)$ & 14.8 & $(8.5)$ & $p=0.005$ \\
Serum 25(OH) D (nmol/L) & 64.1 & $(26.6)$ & 56.5 & $(21.9)$ & 68.7 & $(28.2)$ & $p<0.001$ \\
Time spent outdoors score & 2.0 & $(1.2)$ & 2.2 & $(1.4)$ & 1.8 & $(1.1)$ & $p=0.001$ \\
BMI & 23.6 & $(3.7)$ & 24.1 & $(3.7)$ & 23.4 & $(3.7)$ & $p=0.014$ \\
\hline
\end{tabular}

Abbreviations: CES-D, Center for Epidemiologic Studies Depression Scale; BMI, body mass index; $p$ value is based on the results of a $t$-test comparing men and women.

Multiple regression analyses showed that serum 25(OH)D status was significantly negatively associated with the total CES-D scores (controlling for gender, age, BMI, and ethnicity) ( $\mathrm{B}=-0.048$, $t=-3.828, p<0.001)$ Moreover, the relationship between 25(OH)D and depression scores remained significant even after adjusting for mean time outdoors $(\mathrm{B}=-0.053, t=-3.567, p<0.001$. (Figure 1). Additionally, we examined the relationship between the presence versus absence of depressive symptoms and quartiles of 25(OH)D adjusted for age, gender, ethnic origin, BMI and time spent outdoors (Table 3). Participants in quartiles I to III and compared with those in the highest quartile were more likely to report depressive symptoms; however, this relationship was present for men but not women.

Table 2. Participant characteristics by presence of depressive symptomatology (CES-D score below 16 vs. 16 and over).

\begin{tabular}{llllllll}
\hline \multirow{2}{*}{ Variable } & \multicolumn{2}{c}{ ALL $(\boldsymbol{n}=\mathbf{6 1 5})$} & \multicolumn{2}{l}{$\begin{array}{l}\text { CES-D score }<\mathbf{1 6} \\
(\boldsymbol{n}=\mathbf{3 9 6})\end{array}$} & \multicolumn{2}{l}{$\begin{array}{l}\text { CES-D score } \geq 16 \\
(\boldsymbol{n}=\mathbf{2 1 9})\end{array}$} & \\
\cline { 2 - 7 } & Mean & $(\mathbf{S D})$ & Mean & $(\mathbf{S D})$ & Mean & (SD) & \\
\hline CES-D score & 14.1 & $(8.4)$ & 8.9 & $(3.7)$ & 23.4 & $(6.3)$ & $p<0.001$ \\
Serum 25(OH) D (nmol/L) & 64.1 & $(26.6)$ & 67.4 & $(26.3)$ & 58.0 & $(26.2)$ & $p<0.001$ \\
Time spent outdoors score & 2.0 & $(1.2)$ & 2.0 & $(1.1)$ & 2.0 & $(1.4)$ & $p=0.783$ \\
BMI & 23.6 & $(3.7)$ & 23.8 & $(3.9)$ & 23.4 & $(3.4)$ & $p=0.220$ \\
\hline
\end{tabular}

Abbreviations: CES-D, Center for Epidemiologic Studies Depression Scale; BMI, body mass index; $p$ value is based on the results of a $t$-test comparing participants with CES-D scores $<16$ and $\geq 16$. 
Figure 1. CES-D scores by serum 25(OH)D concentration in 615 young adults aged $17-25$ years: LOWESS plot.

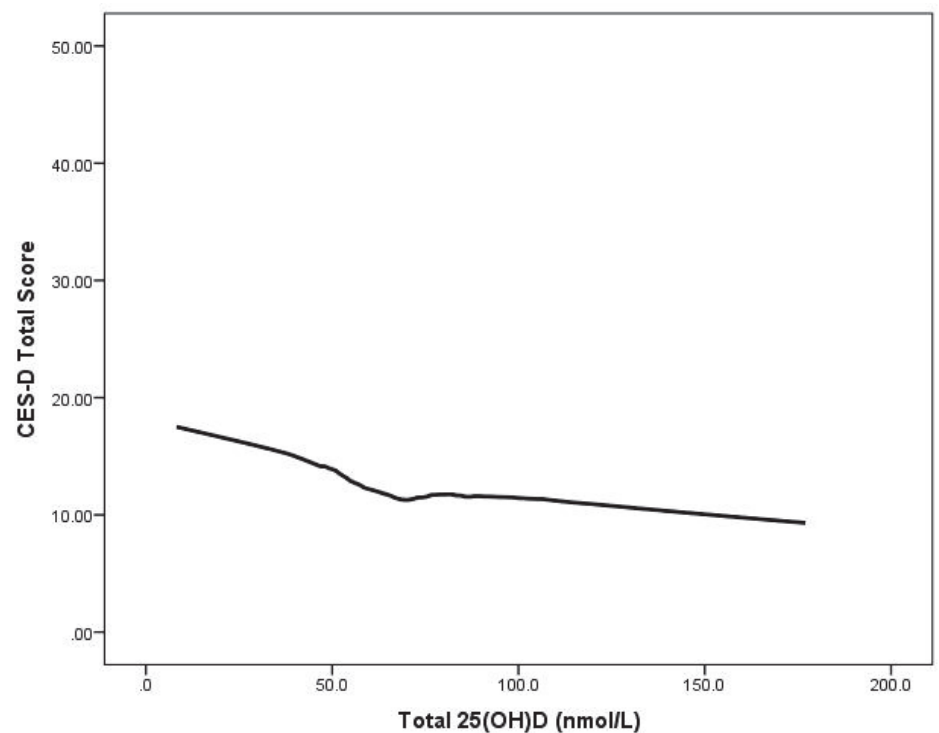

Table 3. Adjusted odds ratios (95\% confidence intervals) for the presence of depressive symptoms (CES-D $\geq 16)$ and 25-hydroxyvitamin D quartile.

\begin{tabular}{lllllll}
\hline $\begin{array}{l}\text { 25(OH)D Quartile } \\
\text { (Range in nmol/L) }\end{array}$ & $\boldsymbol{n}$ & All & $\boldsymbol{n}$ & Men & $\boldsymbol{n}$ & Women \\
\hline IV ( $\geq 80.0)$ & 155 & reference & 27 & reference & 128 & reference \\
III (64.0-79.9) & 153 & $1.34(0.81,2.23)$ & 62 & $2.51(1.14,5.50)$ & 91 & $0.64(0.31,1.32)$ \\
II (44.0-63.9) & 156 & $2.22(1.27,3.86)$ & 75 & $3.21(1.34,7.63)$ & 81 & $1.51(0.72,3.18)$ \\
I ( $<5.0-43.9)$ & 151 & $2.24(1.27,3.97)$ & 70 & $7.14(1.83,27.78)$ & 81 & $1.96(0.62,2.59)$ \\
\hline
\end{tabular}

Adjusted for age, gender, age, ethnic origin, BMI, and time spent outdoors.

\section{Discussion}

In this population-based study of over 600 young adults, lower vitamin D status was associated with higher depression scores even after taking potential confounders including time spent outdoors into consideration. While several studies have evaluated the relationship between vitamin D and depression among older adults [23,24], the relationship in younger adults has not yet been investigated. On average, in practical terms, our analysis showed that for every one standard deviation increase in serum $25(\mathrm{OH}) \mathrm{D}(27 \mathrm{nmol} / \mathrm{L})$, there was a 4.5 point decrease in the CES-D score among our participants aged $17-25$ years.

To date, few large epidemiological studies have been performed in a population-based setting; however, our results are in accordance with survey-based findings in the USA and Europe. Specifically, Ghanji and colleagues utilizing data reported in the U.S. National Health and Nutrition Examination Survey (NHANES) found an independent association between lower 25(OH)D concentration and higher depression status and depression severity among a group of participants aged 15-39 years $(n=7970)$ surveyed between 1988 and 1994 [25]. In their study, depression status was assessed using the Diagnostic Interview Schedule (DIS) developed by the National Institute of 
Mental Health. In a large sample of older adults aged 65-95 years randomly selected from 11 municipalities in the Netherlands, Hoogendijk and colleagues also demonstrated an association of depression status and depression severity assessed using CES-D with decreased serum 25(OH)D concentration [24]. Lee and colleagues also reported an association between 25(OH)D concentration and depression assessed using BDI-II among a large sample of middle-aged and older European men [26]. Another case control study found low concentration of $25(\mathrm{OH}) \mathrm{D}$ associated with depressive disorder in 18-65-year-old participants in the Netherlands [27].

In contrast to our findings, Zhao and colleagues found no significant association between serum $25(\mathrm{OH}) \mathrm{D}$ concentrations and the presence of depression among 3916 U.S adults aged 20 years and over from the 2006-06 NHANES [28]. Participants' depressive symptoms were assessed using the Patient Health Questionnaire (PHQ-9) which is a standard instrument for use in depression diagnosis in primary care [29]. Pan and colleagues also reported no significant association between vitamin D status and depressive symptoms in 3262 Chinese older adults aged 50-70 years [30].

There are a number of possible reasons for the conflicting results among studies, including differences in population groups (i.e., ethnic and other cultural differences), age of the participants surveyed, measures of depression obtained, type of assay used for the analysis of serum vitamin D, and statistical adjustment of known confounding factors including sun exposure. Importantly, in a recent study by Nanri and colleagues, the authors found an association between $25(\mathrm{OH}) \mathrm{D}$ and depression symptoms during winter, but not in summer or overall [31], thereby suggesting the season of study has a major influence on results.

One of the strengths of our study is that vitamin D status was measured using the nominal gold standard method. Other strengths include the inclusion of several variables that may influence depression outcome in the data analysis - in particular, taking into account time spent outside as a proxy for recent sun exposure. This is in contrast to the recent findings that higher levels of reported sun exposure, rather than $25(\mathrm{OH}) \mathrm{D}$ concentration, were associated with less depressive symptoms in sample of multiple sclerosis patients [32]. However, the authors used a different instrument - a recall questionnaire rather than a close-to-real time diary of the current study and they appropriately highlight the need to consider reverse causality as an explanation for the findings between sun exposure and depression. Interestingly, we found no association between average daily time spent outdoors during a recent period relevant for subcutaneous vitamin D manufacture and $25(\mathrm{OH}) \mathrm{D}$ concentration in our study participants. Even though we obtained daily reports of time spent outdoors for each day on 13 consecutive days, this measure only serves as a proxy of potential opportunities to produce endogenous vitamin $\mathrm{D}$, and vitamin $\mathrm{D}$ status is ultimately affected by exposure longer than two weeks. Overall sun exposure and thus the production of vitamin D varies in individuals not only as a function of time spent outdoors, but also time of day, clothing, and current weather conditions [33]. In addition, we did not have information about the exact time of day participants spent the time outside, actual weather, and sunscreen use by participants during the time they were outside during the two-week study period. Moreover, a plateau of vitamin D production is reached after about 15-30 min of sun exposure [9], suggesting that additional time outdoors may not provide additional benefits as far as vitamin D production is concerned. Future studies could consider using real-time reporting of time outdoors including current weather conditions and actual use of sun 
protection. However, such close monitoring of sun exposure and sun protective behaviours could lead to behavioural changes during the study due to social expectations of sun protection use.

Limitations of the study included the cross-sectional design, which limits the ability to evaluate the cause and effect relation between depression and vitamin D deficiency; however, from a public health perspective, the coexistence of vitamin D deficiency and depression in this sample of young adults is a concern. Depression and chronic low mood is a major global health problem with concomitant consequences on morbidity, mortality, and quality of life. Although the prevalence of major depression is relatively low among community-dwelling adults, the occurrence of subsyndromal depression or depressive symptomatology is high and estimated to affect around $12 \%$ of adults [34,35]. Our sample mean for the CES-D score of 14.1 was very similar to that found in a previous study of self-reported mood scales in the general population [22], suggesting that we have obtained a representative sample of young adults on the dimension of depression. Interestingly, over one-third of our participants had a total CES-D score of 16 or above, indicating the possibility of the presence of clinical depression. Most importantly, a recent study elaborated on the possible mechanism of vitamin D effects on the serotonin system implicated in depression [36]. The presence of vitamin D is likely to upregulate transcription of serotonin-synthesising gene tryptophan hydroxylase 2 (TPH2) in the brain and concurrently to downregulate the transcription of THP1 outside the brain [5].

\section{Conclusions}

The present study found evidence of an association between $25(\mathrm{OH}) \mathrm{D}$, a marker of vitamin $\mathrm{D}$ status, and depression scores in a young adult sample. The association of vitamin $\mathrm{D}$ and depression remained significant even after adjusting for age, gender, ethnicity, BMI, and time spent outdoors. Our results support the previous findings, suggesting a direct effect of vitamin D on the serotonin system that should be followed up with an appropriately designed, randomised controlled trial of supplementation with vitamin D among young adults in the general population.

\section{Acknowledgments}

The authors gratefully acknowledge the Daily Experiences Lab members for assistance with data collection, the staff at the Human Nutrition Clinic and laboratories, and all study participants. The Daily Life Study was funded by a University of Otago Research Grant to Tamlin Conner. Reeder and the Cancer Society Social and Behavioural Research Unit receive support from the Cancer Society of New Zealand Inc. and the University of Otago.

\section{Author Contributions}

Maria Polak was the lead researcher, collected the data, and took a lead in statistical analysis of the results. Lisa Houghton contributed to the design of the study and interpretation of the results. Anthony Reeder provided expertise in the area of sun exposure and measurement of time spent outdoors. Michelle Harper undertook the laboratory analyses of serum 25-hydroxyvitamin D in the Department of Human Nutrition at the University of Otago. Tamlin Conner contributed substantially 
to the design of the study, was the lead investigator of the Daily Life Study, and provided statistical analysis expertise. All authors contributed to the production of the manuscript.

\section{Conflicts of Interest}

The authors declare no conflict of interest.

\section{References}

1. Eyles, D.W.; Smith, S.; Kinobe, R.; Hewison, M.; McGrath, J.J. Distribution of the vitamin D receptor and $1 \alpha$-hydroxylase in human brain. J. Chem. Neuroanat. 2005, 29, 21-30.

2. Garcion, E.; Wion-Barbot, N.; Montero-Menei, C.N.; Berger, F.; Wion, D. New clues about vitamin D functions in the nervous system. Trends Endocrinol. Metab. 2002, 13, 100-105.

3. Zittermann A. Vitamin D in preventive medicine: Are we ignoring the evidence? Br. J. Nutr. 2003, 89, 552-572.

4. Stumpf, W.E.; Sar, M.; Clark, S.A.; DeLuca, S.A. Brain target sites for 1,25-dihydroxyvitamin D. Science 1982, 215, 1403-1405.

5. Patrick, R.P.; Ames, B.N. Vitamin D hormone regulates serotonin synthesis. part 1: Relevance for autism. FASEB 2014, doi:10.1096/fj.13-246546.

6. Bertone-Johnson, E.R.; Powers, S.I.; Spangler, L.; Brunner, R.L.; Michael, Y.L.; Larson, J.C.; Millen, A.E.; Bueche, M.N.; Salmoirago-Blotcher, E.; Liu, S.; Wassertheil-Smoller, S.; et al. Vitamin D intake from foods and supplements and depressive symptoms in a diverse population of older women. Am. J. Clin. Nutr. 2011, 94, 1104-1112.

7. Kjærgaard, M.; Joakimsen, R.; Jorde, R. Low serum 25-hydroxyvitamin D levels are associated with depression in an adult Norwegian population. Psychiatr. Res. 2011, 190, 221-225.

8. Holick, M.F. Vitamin D deficiency. NEJM 2007, 357, 266-281.

9. Holick, M.F. Environmental factors that influence the cutaneous production of vitamin D. Am. J. Clin. Nutr. 1995, 61, 638S-645S.

10. McKenna, M.J. Differences in vitamin D status between countries in young adults and the elderly. Am. J. Med. 1992, 93, 69-77.

11. Vieth, R. Why the optimal requirement for vitamin $\mathrm{D}_{3}$ is probably much higher than what is officially recommended for adults. J. Steroid Biochem. Mol. Biol. 2004, 89/90, 575-579.

12. Looker, A.C.; Pfeiffer, C.M.; Lacher, D.A. Serum 25-hydroxyvitamin D status of the US population: 1988-1994 compared with 2000-2004. Am. J. Clin. Nutr. 2008, 88, 1519-1527.

13. Prentice, A. Vitamin D deficiency: A global perspective. Nutr. Rev. 2008, 66, S153-S164.

14. Rockell, J.E.; Skeaff, C.M.; Williams, S.M.; Green, T.J. Serum 25-hydroxyvitamin D concentrations of New Zealander aged 15 years and older. Osteoporos. Int. 2006, 17, 1382-1389.

15. Mithal, A.; Wahl, D.A.; Bonjour, J.-P.; Burckhardt, P.; Dawson-Hughes, B.; Eisman, J.A.; El-Haji Fuleihan, G.; Josse, R.G.; Lips, P.; Morales-Torres, J.; et al. Global vitamin D status and determinants of hypovitaminosis D. Osteoporos. Int. 2009, 20, 1807-1820. 
16. Jorde, R.; Sneve, M.; Figenschau, Y.; Svartberg, J.; Waterloo, K. Effects of vitamin D supplementation on symptoms of depression in overweight and obese subjects: Randomized double blind trial. J. Int. Med. 2008, 264, 599-609.

17. Kripke, D.F.; Girardin, J.-L.; Elliott, J.A.; Klauber, M.R.; Rex, K.M.; Tuunainen, A.; Langer, R.D. Ethnicity, sleep, mood, and illumination in postmenopausal women. $B M C$ Psychiatry 2004, 4, 8.

18. Radloff, L.S. The CES-D scale: A self-report depression scale for research in the general population. Appl. Psych. Meas. 1977, 1, 385-401.

19. Glanz, K.; Yaroch, A.L.; Dancel, M.; Saraiya, M.; Crane, L.A.; Buller, D.B.; Manne, S.; O'Riordan, D.L.; Heckman, C.J.; Hay, J.; et al. Measures of sun exposure and sun protection practices for behavioral and epidemiologic research. Arch. Dermatol. 2008, 144, 217-222.

20. Lohman, T.G.; Roche, A.F.; Matorell, R. Anthropometric Standardization Reference Manual; Human Kinetics Books: Champaign, IL, USA, 1988.

21. Maunsell, Z.; Wright, D.J.; Rainbow, S.J. Routine isotope-dilution liquid chromatography-tandem mass spectrometry assay for simultaneous measurement of the 25-hydroxy metabolites of vitamins $\mathrm{D}_{2}$ and $\mathrm{D}_{3}$. Clin. Chem. 2005, 51, 1683-1690.

22. Crawford, J.; Cayley, C.; Lovibond, P.F.; Wilson, P.H.; Hartley, C. Percentile norms and accompanying interval estimates from an Australian general adult population sample for self-report mood scales (BAI, BDI, CRSD, CES-D, DASS, DASS-21, STAI-X, STAI-Y, SRDS, and SRAS). Aus. Psychol. 2011, 26, 3-14.

23. Wilkins, C.H.; Sheline, Y.I.; Roe, C.M.; Birge, S.J.; Morris, J.C. Vitamin D deficiency is associated with low mood and worse cognitive performance in older adults. Am. J. Ger. Psychiatry 2006, 14, 1032-1040.

24. Hoogendijk, W.J.; Lips, P.; Dik, M.G.; Deeg, D.J.; Beekman, A.T.F.; Penninx, B.W.J.H. Depression is associated with decreased 25-hydroxyvitamin D and increased parathyroid hormone levels in older adults. Arch. Gen. Psychiatry 2008, 65, 508-512.

25. Ganji, V.; Milone, C.; Cody, M.M.; McCarty, F.; Wang, Y.T. Serum vitamin D concentrations are related to depression in young adult US population: The Third National Health and Nutrition Examination Survey. Int. Arch. Med. 2010, 3, 29.

26. Lee, D.M.; Tajar, A.; O’Neill, T.W.; O’Connor, D.B.; Bartfai, G.; Boonen, S.; Bouillon, R.; Casanueva, F.F.; Finn, J.D.; Forti, G.; et al. Lower vitamin D levels are associated with depression among community-dwelling European men. J. Psychopharmacol. 2011, 25, 1320-1328.

27. Milaneschi, Y.; Hoogendijk, W.; Lips, P.; Heijboer, A.C.; Schoevers, R.; van Hemert, A.M.; Beekman, A.T.; Smit, J.H.; Penninx, B.W. The association between low vitamin D and depressive disorders. Mol. Psychiatry 2013, 19, 444-451.

28. Zhao, G.; Ford, E.S.; Li, C.; Balluz, L.S. No associations between serum concentrations of 25-hydroxyvitamin D and parathyroid hormone and depression among US adults. Br. J. Nutr. 2010, 104, 1696-1702.

29. Spitzer, R.L.; Kroenke, K.; Williams, J.B.W. Validation and utility of a self-report verion of PRIME-MD: The PHQ primary care study. JAMA 1999, 282, 1737-1744. 
30. Pan, A.; Lu, L.; Franco, O.H.; Yu, Z.; Li, H.; Lin, X. Association between depressive symptoms and 25-hydroxyvitamin D in middle-aged and elderly Chinese. J. Affect. Disord. 2009, 118, 240-243.

31. Nanri, A.; Mizoue, T.; Matsushita, Y.; Poudel-Tandukar, K.; SatoM Ohta, M.; Mishima, N. Association between serum 25-hydroxyvitamin D and depressive symptoms in Japanese: Analysis by survey season. Eur. J. Clin. Nutr. 2009, 63, 1444-1447.

32. Knippenberg, S.; Damoiseaux, J.; Bol, Y.; Hupperts, R.; Taylor, B.V.; Ponsonby, A.-L.; Dwyer, T.; Simpson, S.; van der Mei, I.A. Higher levels of reported sun exposure, and not vitamin D status, are associated with less depressive symptoms and fatigue in multiple sclerosis. Acta Neurol. Scand. 2013, 129, 123-131.

33. Lucas, R.M.; McMichael, A.J.; Armstrong, B.K.; Smith, W.T. Estimating the global disease burden due to ultraviolet radiation exposure. Int. J. Epidemiol. 2008, 37, 654-667.

34. Goldney, R.D.; Fisher, J.; Dal Grande, E.; Taylor, A.W. Subsyndromal depression: Prevalence, use of health services and quality of life in an Australian population. Soc. Psychiat. Psychiatr. Epidemiol. 2004, 39, 293-298.

35. Pietrzak, R.H.; Kinley, J.; Afifi, T.O.; Enns, M.W.; Fawcett, J.; Sareen, J. Subsyndromal depression in the United States: Prevalence, course, and risk for incident psychiatric outcomes. Psych. Med. 2012, 43, 1401-1414.

36. Baldwin, R.; Rudge, S. The role of serotonin in depression and anxiety. Int. Clin. Psychopharmacol. 1995, 9, 41-45. 
Chapter 8

Sports Medicine 



\title{
Vitamin D and the Athlete: Risks, Recommendations, and Benefits
}

\section{Dana Ogan and Kelly Pritchett}

\begin{abstract}
Vitamin D is well known for its role in calcium regulation and bone health, but emerging literature tells of vitamin D's central role in other vital body processes, such as: signaling gene response, protein synthesis, hormone synthesis, immune response, plus, cell turnover and regeneration. The discovery of the vitamin $\mathrm{D}$ receptor within the muscle suggested a significant role for vitamin $\mathrm{D}$ in muscle tissue function. This discovery led researchers to question the impact that vitamin $\mathrm{D}$ deficiency could have on athletic performance and injury. With over $77 \%$ of the general population considered vitamin D insufficient, it's likely that many athletes fall into the same category. Research has suggested vitamin D to have a significant effect on muscle weakness, pain, balance, and fractures in the aging population; still, the athletic population is yet to be fully examined. There are few studies to date that have examined the relationship between vitamin D status and performance, therefore, this review will focus on the bodily roles of vitamin $\mathrm{D}$, recommended $25(\mathrm{OH}) \mathrm{D}$ levels, vitamin $\mathrm{D}$ intake guidelines and risk factors for vitamin $\mathrm{D}$ insufficiency in athletes. In addition, the preliminary findings regarding vitamin D's impact on athletic performance will be examined.
\end{abstract}

Reprinted from Nutrients. Cite as: Ogan, D.; Pritchett, K. Vitamin D and the Athlete: Risks, Recommendations, and Benefits. Nutrients 2013, 5, 1856-1868.

\section{Introduction}

As research has progressed, the importance and versatility of vitamin D in the body has become quite evident, therefore the prevalence of vitamin D insufficiency has been heavily examined in recent years. Research suggests vitamin D's active role in immune function, protein synthesis, muscle function, inflammatory response, cellular growth and regulation of skeletal muscle [1-4]. In addition, a common symptom of clinical vitamin D deficiency is muscle weakness. Due to the many essential roles of vitamin D within the body, it has been suggested that physical performance may be influenced by serum vitamin D status, especially in those who are clinically deficient.

Vitamin D insufficiencies are estimated to affect over one billion people worldwide [5]. The Third National Health and Nutrition Examination Survey (NHANES III) data showed a significant increase in vitamin D insufficiency in the USA over the last 30 years, with over $77 \%$ of Americans considered vitamin D insufficient [6]. The alarming rates of insufficiency and the vast metabolic properties of vitamin D have led researchers to examine the influence of vitamin D, not only on disease prevention, but also on physical performance and injury. Vitamin D has been identified in most tissues within the body, including skeletal muscle, which has led to further examination of vitamin D's influence on athletes and physical performance.

Because athletes and sports medicine physicians are primarily concerned with performance, the risk of vitamin D insufficiency among athletes has received growing interest and is under current examination by many researchers. In the last decade, researchers have examined $25(\mathrm{OH}) \mathrm{D}$ levels 
among various groups of athletes, ranging from gymnasts to runners to jockeys. Some findings have suggested that vitamin D levels in athletes are comparable to those of the general population; however, results depended largely on geographical location and type of sport (indoor vs. outdoor). It is apparent that the athlete is at an equal risk for vitamin D insufficiency, therefore the potential impact of vitamin D status on performance is now under examination. There are few studies to date that have examined the relationship between vitamin $\mathrm{D}$ status and performance, therefore, this review will focus on the bodily roles of vitamin D, recommended serum 25(OH)D level, vitamin D intake guidelines and risk factors for vitamin $\mathrm{D}$ insufficiency in athletes. In addition, the preliminary findings regarding vitamin D's impact on athletic performance will be examined.

\section{Physiology \& Bone Health}

Vitamin D functions in two distinct ways within the body, through endocrine and autocrine mechanisms. The first, and most well-known, mechanism is the endocrine function, which enhances intestinal calcium absorption and osteoclast activity [1]. Vitamin D is essential for bone growth, density and remodeling, and without adequate amounts, bone loss or injury will occur [7]. When vitamin $\mathrm{D}$ is low, parathyroid hormone $(\mathrm{PTH})$ increases to activate bone resorption in order to satisfy the body's demand for calcium [8]. Low vitamin D increases bone turnover, which increases the risk for a bone injury, like a stress fracture.

A study examining male Finnish military recruits found vitamin D status to be a significant determinant of maximal peak bone mass and also discovered that 25(OH)D levels below $30 \mathrm{ng} / \mathrm{mL}$ significantly increased the risk of stress fractures in this subject group [9]. In a large $(n=3700)$ vitamin D supplementation trial using female navy recruits, subjects receiving $800 \mathrm{IU} /$ day of vitamin D for eight weeks, had a $20 \%$ lower incidence in stress fractures than the placebo group [8]. These studies in active populations, such as military recruits, display the critical role that vitamin D plays in optimal bone health. These findings also suggest that sufficient vitamin D status may prevent injuries, such as stress fractures. Stress fractures are quite common among athletes; most commonly seen among track and field sports, in up to $10 \%-31 \%$ of athletes [8]. Stress fractures can significantly influence performance due to debilitating pain and even cause permanent disability [8].

Vitamin D's other pathway is the autocrine pathway. It is less recognized, but many essential metabolic processes take place in this pathway. On a daily basis, over $80 \%$ of the vitamin $\mathrm{D}$ within the body is utilized through the autocrine pathway [10]. The autocrine pathway is involved in essential body processes like signaling gene response/expression, synthesizing proteins, hormone synthesis, immune/inflammatory response, plus, cell turnover and synthesis [10]. "Without vitamin D, the ability of the cell to respond adequately to pathologic and physiologic signals is impaired" [10]. 


\section{Vitamin D and Muscle Tissue}

The autocrine pathway appears to be of utmost importance and has recently received a great deal of attention in regards to vitamin D's influence on skeletal muscle function [11]. Vitamin D receptor (VDR) sites have been identified in virtually every tissue within the body [12]. VDR regulates expression in hundreds of genes that perform essential bodily functions. The discovery of VDR within the muscle suggested a significant role for vitamin D in muscle tissue and has since been identified as a regulator of skeletal muscle [3,11,13-16]. There are two proposed mechanisms by which vitamin D status may influence muscular strength. One possible explanation involves the direct role of 1,25-dihydroxyvitamin $\mathrm{D}\left[1,25(\mathrm{OH})_{2} \mathrm{D}\right]$ on VDRs within the muscle cells $[11,17,18]$. A second explanation suggests that vitamin D modifies the transportation of calcium in the sarcoplasmic reticulum by increasing the efficiency or number of calcium binding sites involved in muscle contraction. This indirect mechanism however, has only been examined in rat models [11]. On the contrary, one study disputes the evidence for the presence of VDRs within the skeletal muscle cells and suggests that the immunocytochemical staining to detect VDR may be responsible for the false positives results in previous studies $[18,19]$.

Furthermore, it has been suggested that vitamin D supplementation in individuals with low vitamin D status may improve muscle strength. This is believed to be due to an increase in the size and amount of type II (fast twitch) muscle fibers associated with vitamin D supplementation [11,20]. It should be noted that type II fibers are predominant in power and anaerobic activities, and are recruited first to prevent falls, associated with muscle strength in the aging population [11].

Various researchers have found vitamin D to have a significant effect on muscle weakness, pain, balance and fractures in aging individuals $[3,4]$. It is difficult, however to compare the results given the variety of outcome measures and differences in populations used in the studies [14]. Several observational studies have suggested that vitamin D status influences muscular strength and function in the elderly [11,21]. Contrary to these findings, Chan et al., (2012) found no association between baseline vitamin $\mathrm{D}$ status and changes in performance measures over a four year period $[14,22]$. Replacing vitamin D stores in the elderly population may be protective against fall risk and declining physical function $[11,14]$.

Few studies to date have examined this relationship in the adolescent population. Foo et al. (2009) examined the relationship between $25(\mathrm{OH}) \mathrm{D}$ status and bone mass, bone turnover, and muscle strength in Chinese adolescent females $(n=301)$ and found that poor vitamin D status $(<20 \mathrm{ng} / \mathrm{mL})$ was associated with reduced forearm strength, (using a handgrip dynanmometer) when compared to individuals with adequate vitamin D levels $(>20 \mathrm{ng} / \mathrm{mL})$ [17]. Ward et al. (2004) suggested that $25(\mathrm{OH}) \mathrm{D}$ levels were positively associated with muscle power, and jump height in postmenarchal females $(n=91)$, however physical activity levels were not taken into consideration $[11,23]$.

These findings in regard to muscle tissue and function suggest that vitamin D status may have a significant effect on muscle performance and injury prevention, therefore possibly influencing athletic performance. However, further research is warranted to determine the magnitude of effect of vitamin $\mathrm{D}$ on muscle strength and performance. 


\section{Vitamin D Recommendations (Intake and Desirable Levels)}

Although the sun is the most plentiful source of vitamin D, there are also some dietary sources. Some common foods contain significant levels of vitamin D, naturally, including salmon, fatty fish, egg yolks, plus, fortified products also exist, such as, milk, cereal and orange juice [24]. While these dietary sources may appear significant, the process of absorbing dietary vitamin D is only about $50 \%$ efficient; therefore, much of the nutrient value is lost in digestion [25]. The lack of dietary vitamin $\mathrm{D}$ is yet another factor that increases the risk of vitamin D insufficiency. Most experts agree that a higher intake of vitamin D, through dietary sources, ultraviolet B (UVB) exposure, and supplementation, is necessary to obtain optimal serum vitamin D levels [10,26-28].

In November of 2010, the Institute of Medicine (IOM) released new recommendations for dietary intake of vitamin D, 400-600 IU/day for children \& adults (0-70 years), $800 \mathrm{IU} /$ day for older adults (>70 years) [29]. These values are only slightly higher than past recommendations [29]. Many experts argue that while IOM intake recommendations may adequately prevent clinical vitamin D deficiency, they are significantly lower than the level necessary to achieve optimal vitamin D status $[5,6,10,26]$. The Recommended Dietary Allowance (RDA) for Vitamin D, according to the National Institute of Medicine (IOM) [29] is compared to the Endocrine Society's [30] recommended intake in Table 1. Many believe that the RDA is grossly underestimated [5,6,10,26], including the Endocrine Society, who released vitamin intake guidelines that are significantly higher [30]. The Endocrine Society recommends 400-1000 IU/day for infants, 600-1000 IU/day in children (1-18 years) and 1500-2000 IU/day in adults, in addition to sensible sun exposure [30].

Another area of debate among vitamin D researchers is the terminology and reference values used to define optimal vitamin D status, deficiency, and insufficiency. Optimal serum 25(OH)D concentrations have yet to be defined; however, most researchers have similar reference values [31]. Vitamin D deficiency is often defined as $<20 \mathrm{ng} / \mathrm{mL}(50 \mathrm{nmol} / \mathrm{L})$, and insufficiency defined as $20-32 \mathrm{ng} / \mathrm{mL}$ (50-80 nmol/L) and optimal levels are $>40 \mathrm{ng} / \mathrm{mL}(100 \mathrm{nmol} / \mathrm{L})[5,10,12,32]$. The term insufficiency "appears to be the currently favored term for the range of marginal deficiency and is the theoretical serum concentration that is not high enough to protect against certain chronic diseases" [32].

Table 1. Recommended vitamin D intake levels of the Institute of Medicine vs. Endocrine Society [29,30].

\begin{tabular}{|c|c|c|}
\hline Age & Recommended Intake (IU/day) & Upper Limit (IU/day) \\
\hline National Institute of Medicine \\
\hline Children (0-18 years) & $400-600$ & $\begin{array}{c}2500(1-3 \text { years) } \\
3000(4-8 \text { years }) \\
4000(13-18 \text { years })\end{array}$ \\
\hline Adults (19-70 years) & 600 & 4000 \\
\hline Older Adults ( 70 years) & 800 & 4000 \\
\hline Pregnancy/Lactation & 600 & 4000 \\
\hline The Endocrine Society & & $2000-4000$ \\
\hline Children (0-18 years) & $400-1000$ & 10,000 \\
\hline Adults (19-70 years) & $1500-2000$ & 10,000 \\
\hline Older Adults ( $>70$ years) & $1500-2000$ & 10,000 \\
\hline Pregnancy/Lactation & $600-1000(14-18$ years $)$ & \\
\hline
\end{tabular}


Optimal levels of serum 25(OH)D are no exception to the controversy. When serum levels reach $>32 \mathrm{ng} / \mathrm{mL}$, parathyroid hormone (PTH) levels become stable and reduce the risk of secondary hypoparathyroidism, which is commonly associated with low vitamin D status. In addition, intestinal calcium absorption is enhanced, reducing the risk of secondary bone disease [5,28]. These basic vitamin D functions are efficiently demonstrated at $25(\mathrm{OH}) \mathrm{D}$ levels $>32 \mathrm{ng} / \mathrm{mL}$; however, superior benefits are observed at even greater levels. For example, only at 25(OH)D levels $>40 \mathrm{ng} / \mathrm{mL}$, does vitamin $\mathrm{D}$ begin to be stored in the muscle and fat for future use [20,28]. Therefore, at levels $<40 \mathrm{ng} / \mathrm{mL}$, the body relies on a daily replenishment of vitamin D to directly satisfy its daily requirements, which is not likely to be present in the common diet. At levels $<40 \mathrm{ng} / \mathrm{mL}$, there appears to be just enough circulating $25(\mathrm{OH}) \mathrm{D}$ available for all of the immediate metabolic needs; however, stored vitamin D is not likely available for the advanced processes involved in the critical autocrine pathways [20].

It is estimated that the body requires 3000-5000 IU of vitamin D per day to meet the needs of "essentially every tissue and cell in the body" [12]. The IOM recommends 600 IU of vitamin D for most adults (18-70 years of age) to prevent clinical vitamin D deficiency, defined as $25(\mathrm{OH}) \mathrm{D} \leq 20 \mathrm{ng} / \mathrm{mL}$ [29]. In contrast, most expert's recommendations are much higher than $600 \mathrm{IU}$ per day, because their recommendations are designed to help reach optimal 25(OH)D levels of at least $40 \mathrm{ng} / \mathrm{mL}$. Intake levels recommended by most experts not only allow support for daily metabolic requirements, but also allow for vitamin D storage and increased availability, which appears to reduce the risk of many diseases and possibly enhance performance. The recommended daily vitamin D intake, according to most experts, is at least 1000 IU per day to maintain optimal $25(\mathrm{OH}) \mathrm{D}$ status; however, more is required if levels begin suboptimal $[5,10,28]$. With over $77 \%$ of Americans considered insufficient in vitamin $\mathrm{D}$, it is apparent that the current recommendations are suboptimal $[5,6,10,26]$.

Intake recommendations increase with age, pregnancy, and lactation. In addition, experts recommend much higher initial dosages if 25(OH)D levels begin deficient, ranging from 2000 to 200,000 IU, until optimal 25(OH)D levels are met, then 1000-2000 IU/day for maintenance $[5,28,32]$. A commonly prescribed treatment to quickly correct vitamin D deficiency is a weekly dose of 50,000 IU of vitamin D for eight weeks [12].

The tolerable upper limit for vitamin D has been set by the IOM at $4000 \mathrm{IU}$ for adults, compared to $10,000 \mathrm{IU} /$ day by the Endocrine Society [29,30] (Table 1). Leading experts have claimed that a daily intake of 10,000 IU would take months, or even years to manifest symptoms of toxicity [28]. A recent publication found no cases of toxicity with daily intakes of 30,000 IU per day for an extended period of time [10]. Regardless of the current dietary intake value, the amount of vitamin D produced from 15 min of unprotected sun exposure is 10,000 to $20,000 \mathrm{IU}$, in a light-skinned individual, making most experts believe toxicity to be a rare and unlikely event $[10,12]$. During the months that UVB rays are available from the sun, five to $15 \mathrm{~min}$ of unprotected sun exposure between the hours of 10 a.m. and 3 p.m. appear to provide adequate amounts of vitamin D [12].

There have never been any reported cases of vitamin D toxicity from over exposure to the sun; however, symptoms of intoxication, such as hypercalcemia, have been observed when $25(\mathrm{OH}) \mathrm{D}$ levels are greater than $150 \mathrm{ng} / \mathrm{mL}$ [12]. Serum 25(OH)D levels in individuals living close to the 
equator and working outdoors are often around $50 \mathrm{ng} / \mathrm{mL}$, supporting the theory that vitamin $\mathrm{D}$ toxicity from the sun is extremely unlikely, and suggesting that any toxicities would result only from over supplementation [28]. Regardless, many experts agree than $1000 \mathrm{IU} /$ day in the absence of proper sun exposure can maintain $25(\mathrm{OH}) \mathrm{D}$ levels of at least $32 \mathrm{ng} / \mathrm{mL}$ [12].

\section{Vitamin D Status of Athletes}

The distance from the equator, season, and time of day dictate whether vitamin $\mathrm{D}$ is available from the sun. Production of vitamin D from the sun is also dictated by cloud cover, pollution, sunblock, skin pigment and age. During the summer months, UVB radiation from the sun can be absorbed in adequate amounts to synthesize vitamin D [5]. However, during winter months, the angle of the sun prevents UVB radiation from reaching latitudes greater than 35-37 degrees, therefore, vitamin $\mathrm{D}$ cannot be synthesized from in these areas [5,20].

Research has suggested that low levels of vitamin D are widespread in populations living south of the 35th parallel [26]. Even if one spends ample time in the sun, sunscreen with a sun protection factor (SPF) of 15 results in a 99\% decrease in vitamin D absorption [5]. Individuals who spend ample time outdoors may still need vitamin D supplementation to maintain adequate levels during the winter [33,34]. Many outdoor athletes avoid peak sunlight hours, opting to practice early in the morning or late at night, which greatly reduces UVB exposure, putting them at considerable risk of vitamin D insufficiency. Various studies have found many athletes to be at high risk for vitamin D insufficiencies. Table 2 displays prevalence of vitamin D insufficiencies among diverse athletic groups.

Hamilton et al. (2009) revealed that $90 \%$ of Middle Eastern sportsmen were vitamin D deficient between April and October [33]. Although these sportsmen were training at favorable latitudes, Qatar $\left(25.4^{\circ} \mathrm{N}\right)$, they averaged less than $30 \mathrm{~min}$ of sun exposure per day. Another study conducted at favorable latitude (Israel $31.8^{\circ} \mathrm{N}$ ), suggested that $73 \%$ of athletes were vitamin D insufficient [35]. The majority (83\%) of female, Australian indoor athletes were also found to be vitamin D insufficient [36]. In contrast, a study conducted at less favorable latitude (Laramie, WY $41.3^{\circ} \mathrm{N}$ ), revealed vitamin D insufficiency in 63\% of indoor/outdoor athletes during winter, compared to the fall (12\%) and spring (20\%) in indoor and outdoor athletes [37]. Finally, a study conducted even further from the equator (Ellensburg, WA $46.9^{\circ} \mathrm{N}$ ), using exclusively outdoor athletes, found $25 \%-30 \%$ with vitamin D insufficiency from fall to winter [38]. Storlie et al. suggested that $1000 \mathrm{IU} /$ day of vitamin D was not enough to prevent seasonal decline of vitamin D status in this cohort [38]. Although the results are variable, geographical location (latitude) and gender do not appear to be the major risk factors for vitamin D insufficiency in athletes. Lack of sun exposure appears to be the main risk factor, putting indoor athletes and those who avoid peak daylight hours, regardless of latitudinal location, at the greatest risk for vitamin D insufficiency [2,9,33,35-38]. 
Table 2. Prevalence of Vitamin D deficiency $(<20 \mathrm{ng} / \mathrm{mL})$ and insufficiency $(<32$ $\mathrm{ng} / \mathrm{mL}$ ) in various athletic populations.

\begin{tabular}{|c|c|c|c|c|}
\hline Type of Athlete & Indoor/Outdoor & Gender & Vitamin D Status & Reference \\
\hline Finnish military recruits & Combination & Male & $39 \%$ deficient & Valimaki et al. [8] \\
\hline $\begin{array}{l}\text { UK professional athletes } \\
\text { (jockeys, rugby, soccer) }\end{array}$ & Combination & Male & $62 \%$ deficient & Close et al. [39] \\
\hline UK athletes (football, rugby) & Combination & Male & $57 \%$ deficient & Close et al. [40] \\
\hline Middle Eastern sportsman & Combination & Male & $\begin{array}{l}32 \% \text { insufficient } \\
58 \% \text { deficient }\end{array}$ & Hamilton et al. [33] \\
\hline Australian gymnasts & Indoor & Female & $33 \%$ insufficient & Lovell [36] \\
\hline Israeli athletes \& dancers & Indoor & Male \& Female & $73 \%$ insufficient & Constantini et al. [35] \\
\hline USA indoor/outdoor athletes & Combination & Male \& Female & $12 \%$ insufficient & Halliday et al. [37] \\
\hline USA endurance athletes (runners) & Outdoor & Male \& Female & $\begin{array}{c}42 \% \text { insufficient } \\
11 \% \text { deficient }\end{array}$ & Willis et al. [2] \\
\hline $\begin{array}{l}\text { USA outdoor athletes (rugby, } \\
\text { football, track, cross country) }\end{array}$ & Outdoor & Male & $25 \%$ insufficient & Storlie et al. [38] \\
\hline
\end{tabular}

\section{Vitamin D and Athletic Performance}

Original research concerning vitamin D and athletic performance dates back to the early twentieth century, but current performance trials are quite limited and inconclusive. Russian and German researchers were the first to report the convincing effects of ultraviolet light irradiation for improving athletic performance and decreasing chronic sports related pain [20]. These early European researchers suggested significant improvements in time trials, cardiovascular fitness, and strength with treatment of UVB irradiation prior to performance [20]. German Olympic officials considered these effects significant enough for UVB radiation (vitamin D) to be considered an ergogenic aid. In support of this concept, many athletes claim to peak in physical fitness during the time of year that vitamin D (UVB) levels are at their highest, summer and fall [20].

Unfortunately, there are limited experimental studies available and even fewer that demonstrate a performance enhancement from vitamin D supplementation. However, research examining the aging population ( $>65$ years of age) suggests benefits from vitamin D supplementation. Multiple performance studies in older adults have related low vitamin D levels to decreased reaction time, poor balance, and an increased risk of falling [3]. Furthermore, vitamin D supplementation (800 IU/ day) in older adults showed improvements in strength, and walking distance, and a decrease in general discomfort [3]. These favorable results in older adults support the need for further research on athletic performance and vitamin $\mathrm{D}$.

The current research available to support vitamin D's influence on performance is quite limited. An $(n=39)$, unpublished thesis examined $25(\mathrm{OH}) \mathrm{D}$ and maximal oxygen uptake ( $\left.\mathrm{VO}_{2} \mathrm{max}\right)$ to determine vitamin D's effect on aerobic fitness in physically active college males [41]. Higher 25(OH)D levels were associated with an increased VO2max, compared to those with lower vitamin $\mathrm{D}$ levels $(p<0.01)$ [41]. These findings suggest that a favorable vitamin D status may improve aerobic performance. 
Close et al. (2013) examined, young, United Kingdom $\left(\mathrm{UK}, 53^{\circ} \mathrm{N}\right)$ based athletes $(n=30)$, and examined the effects that vitamin D supplementation (20-40,000 IU/week for 12 weeks) had on muscle performance (1-RM bench press, leg press and vertical jump height) [39]. Subjects were assigned to a placebo, 20,000 IU/week or 40,000 IU/week of vitamin D for 12 weeks. Muscle performance and 25(OH)D was measured at six and 12 weeks, revealing that six weeks of supplementation was enough to correct vitamin D deficiency, however, it was not enough to obtain optimal vitamin D levels $>40 \mathrm{ng} / \mathrm{mL}$ [39]. Contrary to the findings in the elderly population, no significant improvements in muscle performance were observed after 6 or 12 weeks of vitamin D supplementation, although serum 25(OH)D levels significantly increased over this time, from an average of $20.43 \mathrm{ng} / \mathrm{mL}$ to $31.65-39.26 \mathrm{ng} / \mathrm{mL}$ [39]. In this study, lower baseline concentrations appeared to respond greater to supplementation, therefore, future studies may find more substantial results by dividing subjects into groups based on their baseline levels.

Although final 25(OH)D concentrations obtained by the athletes were no longer considered deficient $(>20 \mathrm{ng} / \mathrm{mL})$, researchers hypothesized that higher total serum levels may be necessary to document enhanced muscle performance in young athletes [8,39]. According to Close et al. (2013), higher 25(OH)D levels may be necessary to induce a physiological response within skeletal muscle [39]. To explain the lack of response, the author suggested that skeletal muscle may require higher serum concentrations for a response, compared to other tissues [39]. The significant response shown in elderly subjects, however, may be explained by sarcopenia. If the elderly were actively losing muscle mass, they may have a more sensitive response to vitamin D supplementation in the skeletal muscle [39]. The authors suggested that more convincing results may be observed by giving supplemental doses of vitamin D to increase serum 25(OH)D above $40 \mathrm{ng} / \mathrm{mL}$.

A larger ( $n=61$ athletes, $n=31$ healthy control subjects) UK-based vitamin D supplementation trial resulted in higher mean $25(\mathrm{OH}) \mathrm{D}$ levels, as a result of $5000 \mathrm{IU} /$ day of vitamin D3 for eight weeks and found promising muscle performance results [40]. This supplementation regime significantly increased mean 25(OH)D levels from (mean $\pm \mathrm{SD}$ ) $11.62 \pm 10.02 \mathrm{ng} / \mathrm{mL}$ to $41.27 \pm 10.02 \mathrm{ng} / \mathrm{mL}$, whereas a placebo group showed no significant changes. The supplementation group also displayed significant improvements $(p=0.008)$ in 10-meter sprint times and vertical jump (with no improvements in 1-RM bench and squat tests) compared to the placebo group [40]. One athlete's 25(OH)D levels increased from $22.40 \mathrm{ng} / \mathrm{mL}$ to $55.69 \mathrm{ng} / \mathrm{mL}$ and showed improvements in all performance areas, this is only one athlete however. These findings support the aforementioned hypothesis that higher serum 25(OH)D levels $(>40 \mathrm{ng} / \mathrm{mL})$ may generate more convincing performance improvements [40]. Findings also suggest that a daily dose of vitamin D (5000 IU/day) may be superior in raising 25(OH)D levels when compared to a weekly dose $(40,000 \mathrm{IU} /$ week) $[39,40]$. Based off of these two preliminary studies and guidelines from leading experts, $25(\mathrm{OH}) \mathrm{D}$ levels above $40 \mathrm{ng} / \mathrm{mL}$ are likely necessary to significantly improve anaerobic athletic performance. There are no studies available that have examined the effect of vitamin D on aerobic or endurance athletic performance.

To maintain 25(OH)D levels of $40 \mathrm{ng} / \mathrm{mL}$, vitamin D supplementation, especially during the winter months, is warranted $[20,28,39,40]$. The $25(\mathrm{OH}) \mathrm{D}$ goal of $40 \mathrm{ng} / \mathrm{mL}$ is recommended for athletes because at this level, vitamin $\mathrm{D}$ begins to be stored in the muscle and fat for future use. 
Furthermore, at levels below $32 \mathrm{ng} / \mathrm{mL}$, vitamin $\mathrm{D}$ is not likely to be readily available for the advanced processes involved in the autocrine pathways, which is the pathway that is most likely to influence performance [20,25]. This level is also supported by the two comparable Close et al. studies, where the study achieving $25(\mathrm{OH}) \mathrm{D}$ levels greater than $40 \mathrm{ng} / \mathrm{mL}$ showed significant effects on performance $[39,40]$.

Besides the two UK based performance trials $[39,40]$, recent research on vitamin D and athletes has focused on the prevalence of vitamin D insufficiency among athletes, not the effects on performance. Although performance trials are limited, various other studies have resulted in alternative findings to support vitamin D's positive impact on performance. Willis et al. (2012) revealed that decreased vitamin $\mathrm{D}$ was associated with an increased marker for inflammation in endurance athletes [2]. These results call for future investigation to determine whether decreased vitamin D may increase the risk for inflammatory-related injuries [2]. Razavi et al. (2011) found that vitamin D and aerobic exercise improved exercise tolerance in asthmatic patients (compared to a control, only aerobic exercise or only vitamin D supplementation groups), suggesting that vitamin D and aerobic exercise together, may provide anti-inflammatory effects within the lungs [42].

As previously mentioned, the body requires an estimated 3000-5000 IU/day of vitamin D and the high levels of physical activity in athletes may result in increased physiological demands for vitamin D [12]. Since vitamin D is actively used in many metabolic pathways, it is possible that the athlete may require increased intake of vitamin $\mathrm{D}$ to assure adequate availability and storage for optimal performance [32]. This hypothesis may explain the lack of response observed from Close et al., when $25(\mathrm{OH}) \mathrm{D}$ levels above $40 \mathrm{ng} / \mathrm{mL}$ were not achieved and may also support increased vitamin D intake recommendations for athletes [40]. At this point, the appropriate vitamin D supplementation regime for athletes appears to depend on current $25(\mathrm{OH}) \mathrm{D}$ levels, season and sun exposure, with the goal of $>40 \mathrm{ng} / \mathrm{mL}$ in mind. Considering these factors, many athletes, especially indoor athletes and those who are insufficient, will require up to $5000 \mathrm{IU}$ of vitamin D/day for eight weeks, to reach $40 \mathrm{ng} / \mathrm{mL}$, then 1000-2000 IU/day for maintenance.

Although the results of performance trials are not yet convincing enough to support vitamin D as a direct performance enhancer, obtaining optimal 25(OH)D levels can reduce the risk of debilitating stress fracture among athletes, which may indirectly influence performance through prevention of injury $[8,9]$. In addition, because of its active role in muscle, resolution of vitamin D insufficiency has the potential to impact performance $[11,14]$.

\section{Conclusions}

Vitamin D is established as a major factor in preventing stress factors and optimizing bone health, both of which are of great importance to the athlete [8,9]. Rates of vitamin D insufficiency in athletes vary among studies, but most researchers agree that athletes should be evaluated regarding vitamin D status and given intake recommendations to maintain optimal $25(\mathrm{OH}) \mathrm{D}$ levels $>40 \mathrm{ng} / \mathrm{mL}$. Not only does vitamin D assist in growth and maintenance of the bone, but it also aids in regulation of electrolyte metabolism, protein synthesis, gene expression, and immune function [10,28]. These vital functions are essential for all individuals, especially the elite and recreational athlete. 
Therefore, regardless of the limited literature available in support of a positive effect from vitamin D on performance, obtaining optimal $25(\mathrm{OH}) \mathrm{D}$ levels should be a goal for all athletes.

The data are not conclusive to support vitamin D supplementation as a direct performance enhancer, however, research supports the role of vitamin D in the prevention of chronic and acute diseases, such as: cancer, cardiovascular disease, type 2 diabetes, autoimmune diseases and infectious diseases [18]. Athlete or not, optimal vitamin D status is essential to countless fundamental body functions, making it important for all individuals to obtain appropriate levels. Further research is warranted to appropriately define supplementation regimes for specific populations (elderly, athletes, those who are deficient, altering levels for the seasons), establish definite serum 25(OH)D goals, and investigate the effect of vitamin D on physical performance, especially endurance training.

While there is still limited evidence to support vitamin D as a performance enhancer, sports physicians should consider the importance of optimal vitamin D status to prevent stress fractures and muscle injury. Further research is warranted to determine the magnitude of effect from vitamin D on muscle strength and performance. Based off of the prevalence data, high-risk athletes, such as indoor athletes and those who avoid peak daylight hours, should have $25(\mathrm{OH}) \mathrm{D}$ levels assessed annually.

\section{Conflicts of Interest}

The authors declare no conflict of interest.

\section{References}

1. Larsen-Meyer, D.E.; Willis, K.S. Vitamin D and athletes. Curr. Sports Med. Rep. 2010, 9, 220-226.

2. Willis, K.S.; Smith, D.T.; Broughton, K.S.; Larson-Meyer, D.E. Vitamin D status and biomarkers of inflammation in runners. Open Access J. Sports Med. 2012, 3, 35-42.

3. Campbell, P.M.F.; Allain, T.J. Muscle strength and vitamin D in older people. Gerontology 2006, 52, 335-338.

4. Ceglia, L. Vitamin D and skeletal muscle tissue and function. Mole Aspects Med. 2008, 29, 407-414.

5. Holick, M.F. Vitamin D: A D-lightful health perspective. Nutr. Rev. 2008, 66, 182-194.

6. Ginde, A.A.; Liu, M.C.; Camargo, C.A. Demographic differences and trends of vitamin D insufficiency in the U.S. population, 1988-2004. Arch. Intern. Med. 2009, 169, 626-632.

7. DeLuca, H.F. Overview of general physiologic features and functions of vitamin D. Am. J. Clin. Nutr. 2004, 80, 1689-1696.

8. Lappe, J.; Cullen, D.; Haynatzki, G.; Recker, R.; Ahlf, R.; Thompson, K. Calcium and vitamin D supplementation decreased incidence of stress fractures in female navy recruits. $J$. Bone Miner. Res. 2008, 23, 741-749.

9. Valimaki, V.V.; Alfthan, H.; Lehmuskallio, E.; Loyttyniemi, E.; Sahi, T.; Stenman, U.H.; Suominen, H.; Valimaki, M.J. Vitamin D status as a determinant of peak bone mass in young Finnish men. J. Clin. Endocr. Metab. 2004, 89, 76-80.

10. Heaney, R.P. Vitamin D in health and disease. Clin. J. Am. Soc. Nephrol. 2008, 3, 1535-1541. 
11. Ceglia, L.; Harris, S.S. Vitamin D and its role in skeletal muscle. Calcif. Tissue Int. 2013, 92, $151-162$.

12. Holick, M.F. The vitamin D epidemic and its health consequences. J. Nutr. 2005, 135, 2739S-2748S.

13. Bischoff-Ferrari, H.A. Relevance of vitamin D in muscle health. Rev. Endocr. Metab. Disord. 2012, 13, 71-77.

14. Girgis, C.M.; Clifton-Bligh, R.J.; Hamrick, M.W.; Holick, M.F.; Gunton, J.E. The roles of vitamin D in skeletal muscle: Form, function, and metabolism. Endocr. Rev. 2013, 34, 33-83.

15. Hamilton, B. Vitamin D and athletic performance: The potential role of muscle. Asian J. Sports Med. 2011, 2, 211-219.

16. Marantes, I.; Achenbach, S.J.; Atkinson, E.J.; Khosla, S.; Melton, L.J., III; Amin, S. Is vitamin D a determinant of muscle mass and strength? J. Bone Miner. Res. 2011, 26, 2860-2871.

17. Foo, L.H.; Zhang, Q.; Zhu, K.; Ma, G.; Hu, X.; Greenfield, H.; Fraser, D.R. Low vitamin D status has an adverse influence on bone mass, turnover, and muscle strength in adolescent female girls. J. Nutr. 2009, 139, 1002-1007.

18. Wacker, M.; Holick, M.F. Vitamin D-Effects on skeletal and extraskeletal health and the need for supplementation. Nutrients 2013, 5, 111-148.

19. Wang, Y.; DeLuca, H.F. Is the vitamin D receptor found in muscle? Endocrinology 2011, 152, 354-363.

20. Cannell, J.J.; Hollis, B.W.; Sorenson, M.B.; Taft, T.N.; Anderson, J.J.B. Athletic performance and vitamin D. Med. Sci. Sport Exerc. 2009, 41, 1102-1110.

21. Bischoff-Ferrari, H.A.; Dietrich, T.; Orav, E.J.; Hu, F.B.; Zhang, Y.; Karison, E.W.; Dawson-Hughes, B. Higher 25-hydroxyvitamin D concentrations are associated with better lower-extremity function in both active and inactive persons aged $>$ or $=60 \mathrm{y}$. Am. J. Clin. Nutr. 2004, 80, 752-758.

22. Chan, R.; Chan, D.; Woo, J.; Ohlsson, C.; Mellstrom, D.; Kwok, T.; Leung, P.C. Not all elderly people benefit from vitamin D supplementation with respect to physical function: Results from the osteoporotic fractures in men study, Hong Kong. J. Ame. Geriatr. Soc. 2012, 60, 290-295.

23. Ward, K.A.; Das, G.; Berry, J.L.; Roberts, S.A.; Rawer, R.; Adams, J.E.; Mughal, Z. Vitamin D status and muscle function in postmenarchal adolescent girls. J. Clin. Endocrinol. Metab. 2004, 94, 559-563.

24. Chen, T.C.; Chimeh, F.; Zhiren, L.; Mathieu, J.; Person, K.S.; Zhang, A.; Holick, M.F. Factors that influence the cutaneous synthesis and dietary sources of vitamin D. Arch. Biochem. Biophys. 2007, 460, 213-217.

25. Mahan, L.K.; Escott-Stump, S. In Krause's Food, Nutrition and Diet Therapy, 11st ed.; Gallagher, M.G., Ed.; Elsevier: Philadelphia, PA, USA, 2004; pp. 83-88.

26. Moyad, M.A. Vitamin D: A rapid review. Dermatol. Nurs. 2009, 21, 25-30, 55.

27. Holick, M.F. Vitamin D and health: Evolution, biologic functions, and recommended dietary intakes for vitamin D. Clin. Rev. Bone Min. Metab. 2009, 7, 2-19.

28. Cannell, J.J.; Hollis, B.W. Use of vitamin D in clinical practice. Altern. Med. Rev. 2008, 13, 6-20. 
29. Institute of Medicine of the National Academies. Dietary Reference Intakes for Calcium and Vitamin D; Catharine Ross, A., Taylor, C.L., Yaktine, A.L., Eds.; The National Academy of Sciences: Washington, DC, USA, 2011.

30. Holick, M.F.; Binkley, N.C.; Bischoff-Ferrari, H.A.; Gordon, C.M.; Hanley, D.A.; Heaney, R.P.; Murad, M.H.; Weaver, C.M. Evaluation, treatment, and prevention of vitamin D deficiency: An endocrine society clinical practice guideline. J. Clin. Endocrinol. Metab. 2011, 96, 1911-1930.

31. Bischoff-Ferrari, H.A.; Giovannucci, E.; Willett, W.C.; Dietrich, T.; Dawson-Hughes, B. Estimation of optimal serum concentrations of 25-hydroxyvitamin D for multiple health outcomes. Am. J. Clin. Nutr. 2006, 84, 18-28.

32. Willis, K.S.; Peterson, N.J.; Larson-Meyer, D.E. Should we be concerned about the vitamin D status of athletes? Int. J. Sport Nutr. Exerc. Metab. 2008, 18, 204-224.

33. Hamilton, B.; Grantham, J.; Racinais, S.; Hakim, C. Vitamin D deficiency is endemic in Middle Eastern sportsman. Public Health Nutr. 2009, 10, 1528-1534.

34. Tseng, M.; Giri, V.; Bruner, D.W.; Giovannucci, E. Prevalence and correlates of vitamin D status in African American men. BMC Public Health 2009, 9, 191-198.

35. Contantini, N.W.; Arieli, R.; Chodick, G.; Dubnov-Raz, G. High prevalence of vitamin D insufficiency in athletes and dancers. Clin. J. Sport Med. 2010, 20, 368-371.

36. Lovell, G. Vitamin D status of females in an elite gymnastics program. Clin. J. Sport Med. 2008, $18,159-161$.

37. Halliday, T.M.; Peterson, N.J.; Thomas, J.J.; Kleppinger, K.; Hollis, B.W.; Larson-Meyer, D.E. Vitamin D status relative to diet, lifestyle, injury and illness in college athletes. Med. Sci. Sport Exerc. 2010, 42, 335-343.

38. Storlie, D.M.; Pritchett, K.; Pritchett, R.; Cashman, L. 12-Week vitamin D supplementation trial does not significantly influence seasonal $25(\mathrm{OH}) \mathrm{D}$ status in male collegiate athletes. Int. J. Health Nutr. 2011, 2, 8-13.

39. Close, G.L.; Leckey, J.; Patterson, M.; Bradley, W.; Owens, D.J.; Fraser, W.D.; Morton, J.P. The effects of vitamin D3 supplementation on serum total $25(\mathrm{OH}) \mathrm{D}$ concentration and physical performance: A randomised dose-response study. Br. J. Sports Med. 2013, in press.

40. Close, G.L.; Russel, J.; Cobley, J.N.; Owens, D.J.; Wilson, G.; Gregson, W.; Fraser, W.D.; Morton, J.P. Assessment of vitamin D concentration in non-supplemented professional athlettes and healthy adults during the winter months in the UK: Implications for skeletal muscle function. J. Sports Sci. 2013, 31, 344-353.

41. Forney, L. Vitamin D status, adiposity and athletic performance measures in college-aged students. M.S. Thesis, Louisiana State University, Baton Rouge, LA, USA, June 2012.

42. Razavi, M.Z.; Nazarali, P.; Hanachi, P. The effect of an exercise programme and consumption of vitamin D on performance and respiratory indicators in patients with asthma. Sport Sci. Health 2011, 6, 89-92. 


\title{
Higher Serum 25-Hydroxyvitamin D Concentrations Associate with a Faster Recovery of Skeletal Muscle Strength after Muscular Injury
}

\author{
Tyler Barker, Vanessa T. Henriksen, Thomas B. Martins, Harry R. Hill, Carl R. Kjeldsberg, \\ Erik D. Schneider, Brian M. Dixon and Lindell K. Weaver
}

\begin{abstract}
The primary purpose of this study was to identify if serum 25-hydroxyvitamin D $(25(\mathrm{OH}) \mathrm{D})$ concentrations predict muscular weakness after intense exercise. We hypothesized that pre-exercise serum $25(\mathrm{OH}) \mathrm{D}$ concentrations inversely predict exercise-induced muscular weakness. Fourteen recreationally active adults participated in this study. Each subject had one leg randomly assigned as a control. The other leg performed an intense exercise protocol. Single-leg peak isometric force and blood $25(\mathrm{OH}) \mathrm{D}$, aspartate and alanine aminotransferases, albumin, interferon (IFN)- $\gamma$, and interleukin-4 were measured prior to and following intense exercise. Following exercise, serum 25(OH)D concentrations increased $(p<0.05)$ immediately, but within minutes, subsequently decreased $(p<0.05)$. Circulating albumin increases predicted $(p<0.005)$ serum 25(OH)D increases, while IFN- $\gamma$ increases predicted $(p<0.001)$ serum 25(OH)D decreases. Muscular weakness persisted within the exercise leg $(p<0.05)$ and compared to the control leg $(p<0.05)$ after the exercise protocol. Serum 25(OH)D concentrations inversely predicted $(p<0.05)$ muscular weakness (i.e., control leg vs. exercise leg peak isometric force) immediately and days (i.e., 48-h and 72-h) after exercise, suggesting the attenuation of exercise-induced muscular weakness with increasing serum 25(OH)D prior to exercise. Based on these data, we conclude that pre-exercise serum $25(\mathrm{OH}) \mathrm{D}$ concentrations could influence the recovery of skeletal muscle strength after an acute bout of intense exercise.
\end{abstract}

Reprinted from Nutrients. Cite as: Barker, T.; Henriksen, V.T.; Martins, T.B.; Hill, H.R.; Kjeldsberg, C.R.; Schneider, E.D.; Dixon, B.M.; Weaver, L.K. Higher Serum 25-Hydroxyvitamin D Concentrations Associate with a Faster Recovery of Skeletal Muscle Strength after Muscular Injury. Nutrients 2013, 5, 1253-1275.

\section{Introduction}

Vitamin D is a pleiotropic micronutrient that influences health across a range of physiological and pathophysiological conditions in humans. In the body, 25-hydroxyvitamin D (25(OH)D) is the most abundant circulating metabolite and most reliable indicator of vitamin D intake and storage [1]. Maintaining an adequate circulating concentration of $25(\mathrm{OH}) \mathrm{D}$ is, therefore, important to the physiological events moderated by vitamin $\mathrm{D}$.

In addition to its well-known regulation of calcium and mineral homeostasis, vitamin $\mathrm{D}$ also regulates genomic and non-genomic events that govern skeletal muscle function, for review see, for review [2-6]. These functions include the regulation of calcium handling and transport, the expression of cytoskeletal proteins, phosphate metabolism, cell proliferation and differentiation, and the activation of mitogen activated protein kinase signaling pathways in skeletal muscle [7-22]. 
These functions are established in experimental animal and cell culture studies, but less clear is the regulatory influence of vitamin D on skeletal muscle function in humans.

In physiological and pathophysiological conditions, muscular strength associates with serum 25(OH)D concentrations [23-35]. This association is prominent at deficient $(<20 \mathrm{ng} / \mathrm{mL})$ and insufficient (20 to $32 \mathrm{ng} / \mathrm{mL}$ ) serum 25(OH)D concentrations [25,36,37]. Despite the efficacy of supplemental vitamin D to increase serum $25(\mathrm{OH}) \mathrm{D}$ concentrations, however, ambiguity persists regarding the influence of supplemental vitamin D on skeletal muscle strength [30,38-43]. Nevertheless, it is inferred that increasing serum 25(OH)D from deficient to insufficient or sufficient (>32 ng/mL) concentrations attenuates muscular weakness.

Immediate (i.e., minutes to hours) and persistent (i.e., days) muscular weakness can follow intense exercise or a muscle-damaging event $[44,45]$. Muscular weakness after intense exercise or a muscle-damaging event could cause a predisposition to additional trauma. For example, muscular weakness due to injury could contribute to the development of stress fractures in military recruits, which interestingly, could be prevented by increasing serum $25(\mathrm{OH}) \mathrm{D}$ concentrations $[46,47]$. Likewise, low serum 25(OH)D concentrations contribute to muscular weakness and the increase in falls and bone fractures in elderly [36,48-54]. Increasing or maintaining an adequate serum $25(\mathrm{OH}) \mathrm{D}$ concentration, therefore, could preserve muscular strength after injury and protect against further injury.

Following intense exercise, serum 25(OH)D concentrations were not associated with muscular strength [55]. In that previous study, however, the serum 25(OH)D concentration and muscular strength relationship was examined 2-days after eccentric contractions [55]. In experimental rats, vitamin D (activated 7-dehyrocholesterol at 332,000 IU/kg of body mass) promoted muscle regeneration and accelerated the recovery of skeletal muscle strength after crush injury [19], which could possibly relate to the ability of vitamin D to regulate satellite cells in the repair of skeletal muscle [21].

Eccentric (i.e., active-lengthening) contractions of skeletal muscle induce muscle injury and a systemic inflammatory response [56], which in theory, could contribute to serum $25(\mathrm{OH}) \mathrm{D}$ concentration decreases [57-59]. Although the causative mechanism responsible for the serum $25(\mathrm{OH}) \mathrm{D}$ decrease during inflammation awaits future elucidation in humans, persuasive results reveal the regulatory influence of inflammatory cytokines on vitamin D metabolism in immune cells.

Interferon (IFN)- $\gamma$ is an inflammatory cytokine that influences vitamin D metabolism in immune cells $[60,61]$. In isolated peripheral immune cells, IFN- $\gamma$ increases $1 \alpha$-hydroxylase activity and mediates the conversion of $25(\mathrm{OH}) \mathrm{D}$ to 1,25-dihydroxyvitamin $\mathrm{D}(1,25(\mathrm{OH}) \mathrm{D})$ [60-65]. In contrast to IFN- $\gamma$, interleukin (IL)-4 is a cytokine that initiates the catabolism of $25(\mathrm{OH}) \mathrm{D}$ to 24,25 -dihydroxyvitamin D [65]. Following intense exercise, IFN- $\gamma$ decreases or remains unchanged [66-68] while IL-4 remains unchanged or below detection limits in the circulation $[69,70]$. Thus, given its regulation of vitamin D metabolism in immune cells, IFN- $\gamma$ changes could be inversely related to serum 25(OH)D concentration alterations after exercise.

Data suggest that serum 25(OH)D concentrations and muscular strength are not associated after exercise [55]. However, in that earlier study [55], it is possible that the imposed exercise protocol induced a systemic inflammatory response [56]. This is an important consideration because inflammation could modulate serum 25(OH)D concentrations, and consequentially, confound the interpretation between vitamin $\mathrm{D}$ and muscle strength [71]. To address this conundrum, the primary aim of this 
study was to identify if pre-exercise serum $25(\mathrm{OH}) \mathrm{D}$ concentrations predict immediate and persistent (i.e., several days) muscular weakness after intense exercise. We hypothesized that pre-exercise serum 25(OH)D concentrations inversely predict immediate and persistent muscular weakness after intense exercise. As the secondary aim of this study, we sought to identify if circulating cytokines predict serum $25(\mathrm{OH}) \mathrm{D}$ concentrations after intense exercise. We hypothesized that IFN- $\gamma$ changes inversely predict serum $25(\mathrm{OH}) \mathrm{D}$ concentration alterations.

\section{Experimental Section}

The Institutional Review Board at Intermountain Healthcare (Murray, UT, USA) approved this study. Recreationally active (i.e., at least $30 \mathrm{~min}$ of continuous exercise 3 times per week for 1 year prior to enrollment) subjects were informed of the experimental protocol and procedures and provided both written and verbal consent prior to participation. Subjects were excluded if: they were taking any dietary supplements or anti-inflammatory medications, suffered a lower leg injury during the previous year that required the use of crutches, were taking digoxin and antiarrhythmic medication(s), diagnosed with diabetes mellitus, impaired liver or kidney function, pregnant, morbidly obese (body mass index $(\mathrm{BMI})>40 \mathrm{~kg} / \mathrm{m}^{2}$ ), using corticosteroid medication, planning on increasing or decreasing the amount of time spent in the sun or tanning bed, or traveling south of $37^{\circ} \mathrm{N}$ in latitude during study participation. Subjects were also excluded if they had a history of: metabolic bone disease, skeletal muscle pathology, cardiac or peripheral cardiovascular system abnormalities, clotting disorders, coronary artery disease, peripheral vascular disease, stroke, cancer, hypercalcemia or parathyroid dysfunction, iron deficiency within the past year or a potential concern of iron deficiency, high cholesterol or triglycerides, high blood pressure, or the presence of strength or power output asymmetry (i.e., $>5 \%$ difference in peak isometric between legs). Potential subjects were excluded from study participation if they reported or showed any medical condition, under physician treatment, or taking any prescribed medication. During study participation, subjects were asked to keep their diet consistent with their regular eating habits during the previous year.

Data was collected during the winter (December to March) in Salt Lake City, UT, USA ( $40^{\circ} \mathrm{N}$ latitude). Subjects were asked to refrain from physical activity, and using steroids, aspirin, ibuprofen, naproxen sodium, acetaminophen, or other anti-inflammatory agents 72 -h prior to the blood draw and strength testing procedures to remove the potential confounding influences of activity and anti-inflammatory agents on blood and strength testing results.

\subsection{Exercise Protocol}

In an attempt to minimize the bias of leg dominance, subjects that displayed greater than a $5 \%$ difference in peak isometric force between legs were excluded from study participation. Following study inclusion, each subject had one leg randomly assigned as the control (CON) leg. The other leg performed an intense-stretch shortening contraction (SSC) protocol (Figure 1). The rationale for performing this protocol unilaterally was to assess isometric force within and between legs across time, and to allow each subject to serve as their own control. 
Figure 1. Examples of the single-leg strength testing and the intense-stretch shortening contraction (SSC) protocol on the horizontal Plyo-press. (A) The weight stack resistance was overloaded to prevent sled movement in order to achieve isometric testing. (A-C) Subjects performed repetitive single-leg jumps during the SSC protocol. Illustrated below is an example of a subject in the push-off position (A), at peak height of the jump (B), and the subsequent landing (C) during the SSC protocol. Subjects performed 10 sets of 10 jumps with a 20 -s rest between each set at $75 \%$ of body mass on one leg only (i.e., SSC leg). Note: This figure is adapted with permission from [68]. Copyright (C) Barker et al.; licensee BioMed Central Ltd.

A

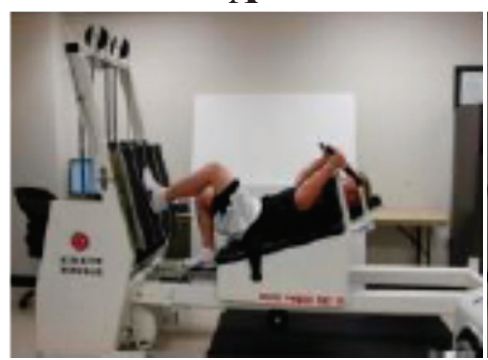

B

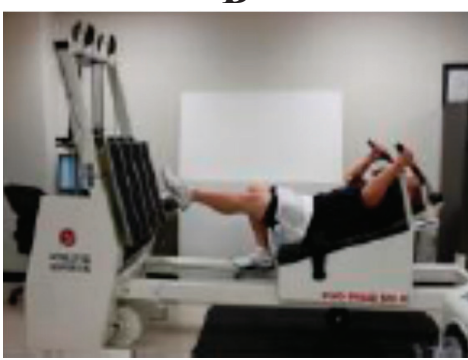

C

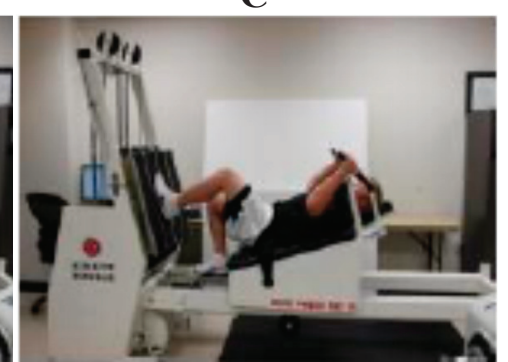

The purpose of the SSC protocol was to induce peak isometric force deficits that would persist for several days [68]. The SSC protocol consisted of 10 sets of 10 repetitive jumps at $75 \%$ of body mass with a $20 \mathrm{~s}$ rest between each set. Subjects were instructed and verbally encouraged to perform each set with maximal effort and to jump as high as possible through a full range of motion $\left(90^{\circ}\right.$ of knee flexion-to-full extension). Subjects performed presses through a full range of motion if they were unable to complete the jumps. If they were unable to complete presses, the exercise protocol was terminated. The mean number of jumps and presses completed during the SSC protocol were 62 \pm 9 and $23 \pm 5$, respectively, while 7 ( 3 males and 4 females; age, $32 \pm 3$ years; height, $167 \pm 5 \mathrm{~cm}$; body mass, $76.8 \pm 8.7 \mathrm{~kg}$; body mass index, $27.1 \pm 1.7 \mathrm{~kg} / \mathrm{m}^{2}$ ) of the 14 subjects were unable to complete the SSC protocol due to fatigue or exhaustion. Of the subjects who completed the exercise protocol, 3 subjects (all males; age, $29 \pm 2$ years; height, $182 \pm 4 \mathrm{~cm}$; body mass, $85.3 \pm 5.4 \mathrm{~kg}$; body mass index, $25.7 \pm 1.6 \mathrm{~kg} / \mathrm{m}^{2}$ ) completed all the jumps and four subjects ( 3 males and 1 female; age, $33 \pm 2$ years; height, $176 \pm 7 \mathrm{~cm}$; body mass, $78.0 \pm 7.5 \mathrm{~kg}$; body mass index, $24.8 \pm 0.8 \mathrm{~kg} / \mathrm{m}^{2}$ ) completed the exercise protocol by performing a combination of jumps and presses. Circulating chemistries and leg strength were not significantly different between subjects who completed the exercise protocol compared to those who were unable to complete the exercise protocol (data not shown). Although 7 subjects were unable to complete the acute exercise protocol, all subjects ( $n=$ 14), completed the remainder of study protocol following the exercise protocol.

Subjects were allowed to consume water ad libitum during and following exercise protocol. The study protocol is illustrated in Figure 2. 
Figure 2. Study protocol. Each subject provided eight fasting blood draws. The first blood draw was performed at baseline (Bsl) and 28-day before the SSC protocol. The rationale for collecting this sample 28-day before the SSC protocol was to allow for the seasonal decrease in serum $25(\mathrm{OH}) \mathrm{D}$ concentrations. The second blood draw was obtained immediately before (Pre) the SSC protocol. The six remaining blood draws were performed immediately (i.e., Post), 1-h, 24-h, 48-h, 72-h and 168-h (i.e., 7 days) after the SSC protocol. Subjects were familiarized with the single-leg peak isometric force testing procedure at Bsl. Thereafter, single-leg peak isometric force measurements were performed on six different occasions: (1) immediately before (Pre) the SSC protocol; and (2) immediately (Post); (3) 24-h; (4) 48-h; (5) 72-h and (6) 168-h after the SSC protocol.

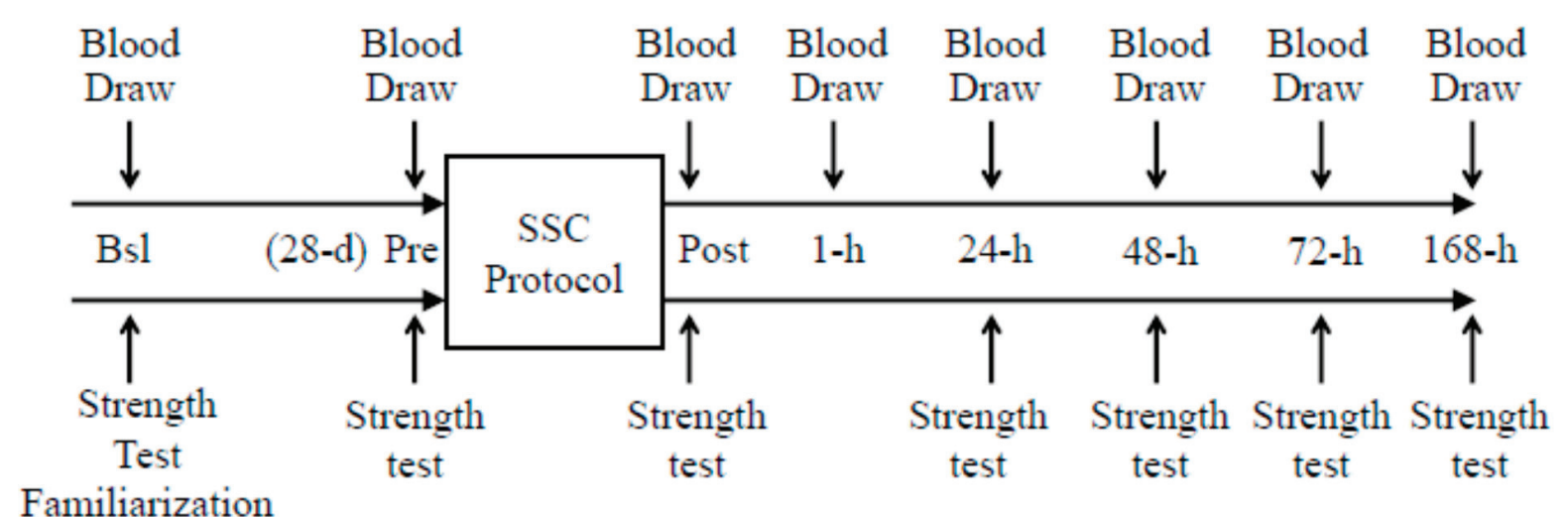

\subsection{Analytical Procedures}

Blood draws were performed from the antecubital vein and collected in one $6.0 \mathrm{~mL}$ red-top serum Becton Dickinson (BD; Franklin Lake, NJ, USA) vacutainer tube, one $4.0 \mathrm{~mL}$ purple-top BD Vacutainer tube (K2 EDTA $7.2 \mathrm{mg}$ plasma), and one $4.5 \mathrm{~mL}$ light green-top BD Vacutainer tube (PST Gel and Lithium Heparin, 83 units). After coagulation, serum was separated by centrifugation (VWR International, Clinical 50 Centrifuge, Radnor, PA, USA) at $1100 \times \mathrm{g}$ for $20 \mathrm{~min}$ at room temperature. Serum samples were immediately aliquoted to several $500 \mu \mathrm{L}$ microcentrifuge tubes and stored at $-80^{\circ} \mathrm{C}$ (Revco Freezer, GC Laboratory Equipment, Asheville, NC, USA) until the day of $25(\mathrm{OH}) \mathrm{D}$ and cytokine concentration analyses (see below). Plasma was separated by centrifugation (Heraeus Labofuge 400 series, Buckinghamshire, England) at 2400× $\mathrm{g}$ for 6 min at room temperature within 20 min of sample collection. Following separation, plasma samples were sent to ARUP Laboratories (Salt Lake City, UT, USA) for parathyroid hormone (PTH), calcium, albumin, aspartate aminotransferase (AST), and alanine aminotransferase (ALT) chemistries (see below).

\subsubsection{Serum 25(OH)D Concentration}

Serum 25(OH)D concentrations $(\mathrm{ng} / \mathrm{mL}$ ) were measured in duplicate (coefficient of variation = $3.19 \%$ ) at USANA Health Sciences, Inc. (Salt Lake City, UT, USA) [39]. Specifically, $100 \mu \mathrm{L}$ of serum was added to $400 \mu \mathrm{L}$ of a 2:1 methanol:chloroform solution containing deuterated $25(\mathrm{OH}) \mathrm{D}_{3}$ as an internal standard $(10 \mathrm{ng} / \mathrm{mL}$ in the stock solution; $40 \mathrm{ng} / \mathrm{mL}$ final concentration) in a $2 \mathrm{~mL}$ 
centrifuge tube. Samples were immediately vortexed and allowed to sit on ice for $10 \mathrm{~min}$. Samples were then spun at $15,000 \times g$ for $5 \mathrm{~min}$ and the supernatant was transferred to a new $2 \mathrm{~mL}$ centrifuge tube containing $500 \mu \mathrm{L}$ of chloroform. Samples were vortexed and allowed to stand for 5 min. To achieve phase separation, $750 \mu \mathrm{L}$ of $\mathrm{ddH}_{2} \mathrm{O}$ was added, vortexed, and centrifuged for 2 min at $15,000 \times g$. The aqueous phase (top), and any debris between the two phases, was removed and discarded. The remaining organic phase was dried down to completeness in a centrifugal vacuum concentrator for $18 \mathrm{~min}$ at $45{ }^{\circ} \mathrm{C}$ under negative pressure. The pellet was then resuspended in $100 \mu \mathrm{L}$ of methanol and added to high performance-liquid chromatography (HPLC) vials.

Analytes were separated by injecting $10 \mu \mathrm{L}$ into an Agilent HPLC (series 6410, Model G6410B, Santa Clara, CA, USA) and a Phenomenex Inertsil 3 micron, $150 \times 4.60 \mathrm{~mm}$ column. Method conditions were: $0-8 \mathrm{~min}, 90 \% \mathrm{MeOH} / 10 \%$ (0.03\% formic acid in water); $8-15 \mathrm{~min}, 100 \%$ 2-propanol; $15-20 \mathrm{~min}, 90 \% \mathrm{MeOH} / 10 \%$ (0.03\% formic acid in water). $25(\mathrm{OH}) \mathrm{D}_{2}$ and $25(\mathrm{OH}) \mathrm{D}_{3}$ were detected on an Agilent tandem mass spectrometer (Series 6410, Model G6410B, Santa Clara, CA, USA) using atmospheric pressure chemical ionization (APCI) detection $\left(350{ }^{\circ} \mathrm{C}\right.$ gas temperature, $400{ }^{\circ} \mathrm{C}$ vaporizer). The $25(\mathrm{OH}) \mathrm{D}_{3}$, deuterated $25(\mathrm{OH}) \mathrm{D}_{3}$, and $25(\mathrm{OH}) \mathrm{D}_{2}$ precursor ions were 383.3 , 386.3 , and 395.4, respectively. The $25(\mathrm{OH}) \mathrm{D}_{3}$, deuterated $25(\mathrm{OH}) \mathrm{D}_{3}$, and $25(\mathrm{OH}) \mathrm{D}_{2}$ product ions were 365.3 , 368.3, and 208.9, respectively. Serum $25(\mathrm{OH}) \mathrm{D}_{2}$ and $25(\mathrm{OH}) \mathrm{D}_{3}$ concentrations were determined relative to authentic standards and corrected for recovery of the $25(\mathrm{OH}) \mathrm{D}_{3}$ internal standard. The detection limit was $<1 \mathrm{ng} / \mathrm{mL}$ for all analytes. The sum of $25(\mathrm{OH}) \mathrm{D}_{2}$ and $25(\mathrm{OH}) \mathrm{D}_{3}$ concentrations was used as the $25(\mathrm{OH}) \mathrm{D}$ total concentration. Serum $25(\mathrm{OH}) \mathrm{D}_{2}$ was not detected in any of the subjects, and therefore, serum $25(\mathrm{OH}) \mathrm{D}$ total concentrations are referred to as serum $25(\mathrm{OH}) \mathrm{D}$ concentrations hereafter.

\subsubsection{Serum Cytokine Concentrations}

Serum IFN- $\gamma$ and IL-4 were quantitated using a multiplexed sandwich capture assay developed in the ARUP Institute for Clinical and Experimental Pathology (University of Utah, Salt Lake City, UT, USA) using the Luminex Multi-Analyte Profiling system (Luminex, Austin, TX, USA) [72,73]. Monoclonal capture antibodies for IFN- $\gamma$ and IL-4 were coupled to microspheres (Luminex). The monoclonal antibody for IL-4 was purchased from Pharmingen/BD Biosciences (San Diego, CA, USA) and the monoclonal antibody for IFN- $\gamma$ was purchased from Biosciences (San Diego, CA, USA).

Standard curves for each cytokine were made using known concentrations of recombinant human cytokine of interest and performed during the same run as the subjects' serum analyses. All incubations were conducted at room temperature on an orbital plate shaker while protected from light for $10 \mathrm{~min}$. Following incubation, beads $(25 \mu \mathrm{L})$ were added to each well after 2 repeated bouts of vortexing (10 s) and sonication (20 s). The plate was then incubated for $1 \mathrm{~h}$, washed $3 \times$ by vacuum filtration with phosphate-buffered saline containing Tween 20 (PBST). Then, $100 \mu \mathrm{L}$ of a mixture of 2 different biotinylated secondary antibodies were added and incubated for $30 \mathrm{~min}$. Following washes $(3 \times)$ with PBST, $100 \mu \mathrm{L} 5 \mu \mathrm{g}$ streptavidin-conjugated $R$-phycoerythrin/mL (Moss Substrates, Pasadena, MD, USA) were added to each well. Plates were incubated for $20 \mathrm{~min}$, washed $3 \times$ with PBST, then beads were re-suspended in $150 \mu \mathrm{L}$ PBST and mixed for $5 \mathrm{~min}$. 
Microtiter plates were then placed in a Luminex 100 instrument for analysis. Microspheres pass through a flow cell where dual lasers identify the microsphere and quantitate the amount of analyte bound to the microsphere by measuring the median fluorescent intensity of the reporter molecule (phycyoerythrin). The median fluorescence intensity is then converted in $\mathrm{pg} / \mathrm{mL}$ based on the known concentrations of the standard curve.

\subsubsection{Clinical Chemistries}

Plasma AST (U/L), ALT (U/L), PTH (pg/mL), and calcium $(\mathrm{mg} / \mathrm{dL})$ concentrations were measured using a quantitative electrochemiluminescent immunoassay, and plasma albumin $(\mathrm{g} / \mathrm{dL})$ concentrations were determined using spectrophotometry (ARUP Laboratories, Salt Lake City, UT, USA).

\subsubsection{Single-Leg Strength Testing}

Single-leg strength testing was performed on a horizontal Plyo-Press (Athletic Republic, Park City, UT, USA). The reliability (intraclass reliability coefficients $=0.98$ ) for the peak isometric force measurement on the Plyo-Press has been reported previously [68] along with the testing procedures $[39,74]$. In brief, the Plyo-Press sled was adjusted for each subject to align the knee and hip joint flexion angles to $90^{\circ}$ with the abdominal, low back region secured and stabilized to the Plyo-Press sled with a harness. Hip and knee extension-isometric contractions were accomplished by overloading the weight stack resistance $(>2260 \mathrm{~N})$. Leg selection (i.e., CON or SSC leg) at the start of each testing session was randomized and followed by an alternating sequence of leg contractions. Peak isometric contractions on each leg were performed in triplicate (CON coefficient of variation $=3.62 \%$; SSC leg coefficient of variation $=4.70 \%$ ), and each isometric contraction was $3 \mathrm{~s}$ in duration and separated by $1 \mathrm{~min}$ of rest. Subjects were verbally instructed and strongly encouraged to exert maximal force against the mounted force platform during each isometric contraction. Force output was measured from signals obtained from a mounted force plate (Advanced Mechanical Technology, Watertown, MA, USA) on the Plyo-press. Data were sampled at $200 \mathrm{~Hz}$ with a low-pass filter at $10 \mathrm{~Hz}$ using DartPower software (Athletic Republic, Park City, UT, USA, version 2.0). Peak isometric force was defined as the highest resultant force produced from the three isometric tests and was expressed relative to body mass $(\mathrm{N} / \mathrm{kg})$.

\subsection{Statistical Analyses}

Data were checked for normality prior to all statistical analyses with a Kolmogorov-Smirnov test. Statistical significance of serum 25(OH)D, cytokine, PTH, AST, and ALT concentration data were assessed with a Friedman repeated measures analysis of variance (ANOVA) on ranks followed by a Tukey's Honestly Significant Difference (HSD) to test multiple pairwise comparisons. Statistical significance of calcium and albumin concentration data were assessed with a one-way ANOVA with repeated measures followed by a Tukey's HSD to test multiple pairwise comparisons. Statistical significance of the muscle strength data was assessed with a two-way (time, leg) ANOVA with repeated measures and followed by a Holm-Sidak method to test for multiple pairwise comparisons. 
Separate multiple linear regression analyses were performed to determine if Pre serum 25(OH)D concentrations or the change in serum $25(\mathrm{OH}) \mathrm{D}$ concentrations from Bsl to Pre predict the immediate (i.e., Post) and persistent (i.e., 24-h, 48-h, and 72-h) deficits in muscle strength after a muscle-damaging event. Along with Pre serum 25(OH)D concentrations and the change in serum 25(OH)D concentrations from Bsl to Pre, body mass index and age were included as independent variables in the multiple linear regression model. Another multiple linear regression was performed to determine if circulating deviations in IFN- $\gamma$, calcium, PTH, or albumin predict serum 25(OH)D alterations. Prior to all multiple linear regressions, serum 25(OH)D, IFN- $\gamma$, and PTH concentrations were rank transformed in order to achieve normality. Selection of independent variables for the multiple linear regression models were based on their biological significance on the dependent variable.

Secondary analyses consisted of a one (gender)-way ANOVA with repeated measures were performed to determine if a statistical difference existed between gender data (i.e., serum 25(OH)D, peak isometric force, peak isometric force difference between the CON and SSC legs at Post, 24-h, 48-h, and 72-h, AST, ALT, albumin, calcium, iPTH, IFN- $\gamma$ and IL-4). Statistical analyses were performed with SysStat software (SigmaPlot 10.0, SigmaStat 3.5, Chicago, IL, USA) and significance was set at a $p<0.05$. Data presented as mean \pm SEM, unless otherwise noted.

\section{Results}

\subsection{Subject Characteristics}

Fourteen non-smoking subjects (male, $n=9$; female, $n=5$; age, $32 \pm 1$ year; height, $173 \pm 5 \mathrm{~cm}$; body mass, $79.0 \pm 4.8 \mathrm{~kg}$; body mass index, $26.1 \pm 0.9 \mathrm{~kg} / \mathrm{m}^{2}$ ) participated in this study. No subjects reported being highly trained, actively competing at a professional or amateur level, or performing recreational activities on a daily basis or for extended durations (i.e., >60 min per session) consistently one year prior to study enrollment.

\subsection{Serum 25(OH)D Concentrations}

The average serum 25(OH)D concentration was $28.0 \pm 2.5 \mathrm{ng} / \mathrm{mL}$ upon study enrollment (Figure 3). Upon enrollment, five subjects $(36 \%)$ had a serum $25(\mathrm{OH}) \mathrm{D}$ concentration greater than $32 \mathrm{ng} / \mathrm{mL}$. Of the nine participants $(64 \%)$ with serum $25(\mathrm{OH}) \mathrm{D}$ concentrations less than $32 \mathrm{ng} / \mathrm{mL}$, three $(21 \%)$ subjects had a concentration less than $20 \mathrm{ng} / \mathrm{mL}$, and one $(7 \%)$ of which had a concentration less than $10 \mathrm{ng} / \mathrm{mL}$. 
Figure 3. Serum $25(\mathrm{OH}) \mathrm{D}$ concentrations $(\mathrm{ng} / \mathrm{mL})$. Serum $25(\mathrm{OH}) \mathrm{D}$ concentrations were significantly increased at Bsl ( ${ }^{1} p<0.05 v s$. Pre, 48-h, and 72-h) and Post $\left({ }^{2} p<0.05\right.$ vs. Pre, 24-h, 48-h, 72-h, and 168-h). Data presented as mean \pm SEM.

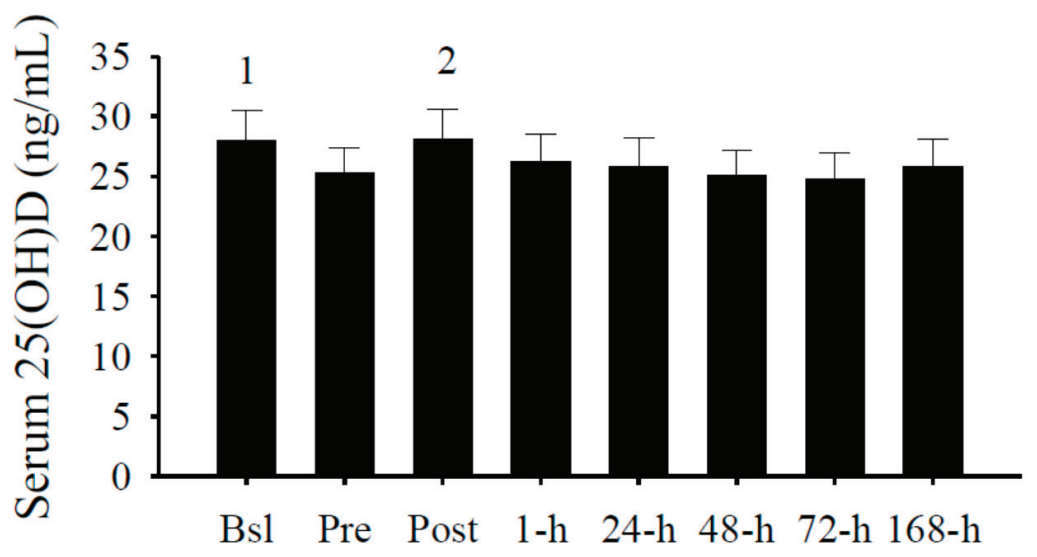

Consistent with the seasonal regulation [75], serum 25(OH)D concentrations significantly $(p<0.05)$ decreased by approximately $10 \%$ from Bsl to Pre (Figure 2). Surprisingly, serum 25(OH)D concentrations significantly $(p<0.05)$ increased immediately after the intense exercise protocol. Within minutes, however, serum 25(OH)D concentrations subsequently decreased $(p<0.05)$ and leveled during the following days.

\subsection{Peak Isometric Force}

Peak isometric force significantly $(p<0.05)$ decreased immediately and remained significantly $(p<0.05)$ impaired several days after the exercise protocol (Figure 4$)$. The immediate and persistent deficits in peak isometric force were apparent within the SSC leg across time $(p<0.05)$ and compared the corresponding CON leg $(p<0.05)$. At 168-h, peak isometric force returned to Pre and CON leg values. 
Figure 4. Peak isometric force $(\mathrm{N} / \mathrm{kg})$. Single leg peak isometric forces were significantly (leg, time interaction, $p<0.05$ ) different following the SSC protocol. Single-leg peak isometric forces were not significantly different within the control (CON) leg across time, but within the SSC leg, decreased immediately $\left({ }^{1} p<0.05 v s\right.$. Bsl, Pre, 24-h, 72-h, and 168-h) and remained impaired several days $\left({ }^{2} p<0.05\right.$ vs. Bsl, Pre, and 168-h) after the intense exercise protocol. Peak isometric forces were also significantly $(* p<0.05)$ decreased in the SSC leg compared to those in the CON leg at Post, 24-h, 48-h, and 72-h. Data presented as mean \pm SEM.

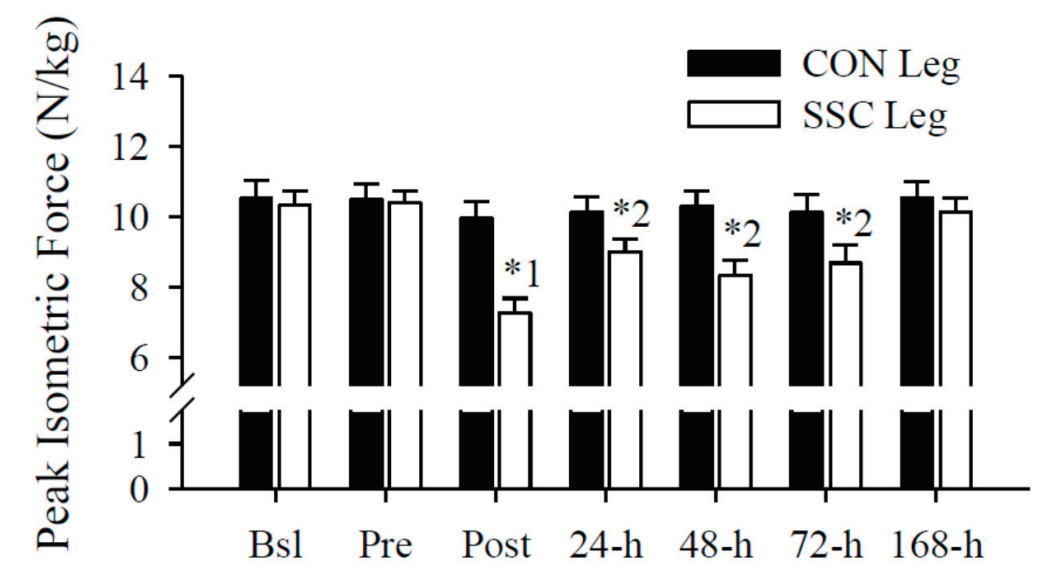

\subsection{Serum 25(OH)D Predicts Immediate and Persistent Muscular Weakness after a Muscle-Damaging Event}

Pre serum 25(OH)D concentrations significantly $(p<0.05)$ predicted muscular weakness $(\mathrm{CON}$ vs. SSC Leg) immediately (i.e., Post) but not 24 -h after the intense exercise protocol (Table 1). Serum $25(\mathrm{OH}) \mathrm{D}$ concentrations at Pre significantly $(p<0.05)$ predicted muscular weakness (CON vs. SSC Leg) at 48-h and 72-h after the intense exercise protocol (Table 1).

In a sensitivity analysis, we repeated the multiple linear regression analyses (Table 1) regarding the prediction of muscular weakness following the intense exercise protocol. Excluding the $\Delta$ in Serum 25(OH)D from the multiple linear regression analyses did not change the significance of the models reported in Table 1. However, excluding the $\Delta$ in serum 25(OH)D increased the $p$-value from 0.04 to 0.08 for Pre serum 25(OH)D and decreased the model-statistical power from $86 \%$ to $59 \%$. It is likely that the shift in significance and decrease in statistical power at 72-h after excluding the $\Delta$ in serum $25(\mathrm{OH}) \mathrm{D}$ from the multiple linear regression model is due to the size of our sample. 
Table 1. Higher serum 25(OH)D concentrations predict a lower deficit ${ }^{1}$ in muscle strength immediately (i.e., Post) and days (i.e., 48-h and 72-h) after intense exercise.

\begin{tabular}{|c|c|c|c|}
\hline & \multicolumn{2}{|c|}{ Coefficient } & \multirow[b]{2}{*}{$p$} \\
\hline & Unstandardized $( \pm \mathrm{SE})$ & Standardized & \\
\hline \multicolumn{4}{|c|}{ Post } \\
\hline Constant & $6.23( \pm 4.12)$ & & \\
\hline Pre serum $25(\mathrm{OH}) \mathrm{D}$ & $-0.28( \pm 0.11)$ & -0.68 & 0.03 \\
\hline$\Delta$ serum $25(\mathrm{OH}) \mathrm{D}$ & $-0.11( \pm 0.10)$ & -0.27 & 0.29 \\
\hline Age & $-0.06( \pm 0.08)$ & -0.17 & 0.50 \\
\hline BMI & $-0.28( \pm 0.13)$ & -0.57 & 0.06 \\
\hline \multicolumn{4}{|c|}{$r^{2}=0.51$, adjusted $r^{2}=0.30$} \\
\hline \multicolumn{4}{|c|}{$24-h$} \\
\hline Constant & $2.41( \pm 4.00)$ & & \\
\hline Pre serum $25(\mathrm{OH}) \mathrm{D}$ & $-0.17( \pm 0.10)$ & -0.52 & 0.14 \\
\hline$\Delta$ Serum $25(\mathrm{OH}) \mathrm{D}$ & $-0.04( \pm 0.10)$ & -0.12 & 0.70 \\
\hline Age & $-0.03( \pm 0.07)$ & -0.13 & 0.67 \\
\hline BMI & $-0.04( \pm 0.13)$ & -0.10 & 0.77 \\
\hline \multicolumn{4}{|c|}{$r^{2}=0.24$, adjusted $r^{2}=0.00$} \\
\hline \multicolumn{4}{|c|}{$48-h$} \\
\hline Constant & $7.24( \pm 4.43)$ & & \\
\hline Pre serum $25(\mathrm{OH}) \mathrm{D}$ & $-0.28( \pm 0.12)$ & -0.68 & 0.04 \\
\hline$\Delta$ Serum $25(\mathrm{OH}) \mathrm{D}$ & $-0.14( \pm 0.11)$ & -0.33 & 0.23 \\
\hline Age & $-0.07( \pm 0.08)$ & -0.23 & 0.40 \\
\hline BMI & $-0.10( \pm 0.11)$ & -0.29 & 0.33 \\
\hline \multicolumn{4}{|c|}{$r^{2}=0.45$, adjusted $r^{2}=0.21$} \\
\hline \multicolumn{4}{|c|}{$72-h$} \\
\hline Constant & $6.40( \pm 3.34)$ & & \\
\hline Pre serum $25(\mathrm{OH}) \mathrm{D}$ & $-0.21( \pm 0.09)$ & -0.63 & 0.04 \\
\hline$\Delta$ Serum $25(\mathrm{OH}) \mathrm{D}$ & $-0.15( \pm 0.08)$ & -0.45 & 0.09 \\
\hline Age & $-0.08( \pm 0.06)$ & -0.29 & 0.26 \\
\hline BMI & $-0.10( \pm 0.11)$ & -0.26 & 0.36 \\
\hline \multicolumn{4}{|c|}{$r^{2}=0.52$, adjusted $r^{2}=0.31$} \\
\hline
\end{tabular}

\subsection{Plasma AST and ALT}

Increases in circulating AST and ALT are indices of skeletal muscle damage [76,77]. Days after the exercise protocol, plasma AST (Figure 5A) and ALT (Figure 5B) concentrations were significantly (both $p<0.05$ ) increased, thereby imply the presence of skeletal muscle damage. 
Figure 5. Plasma aspartate aminotransferase (AST) (U/L) and ALT (U/L) concentrations. (A) Plasma AST was significantly increased at $72-\mathrm{h}\left({ }^{1} p<0.05 v s\right.$. Bsl). (B) Plasma ALT was significantly $\left({ }^{2} p<0.05\right.$ vs. Post) increased at 24-h, 72-h, and $168-$ h. Data presented as mean \pm SEM.

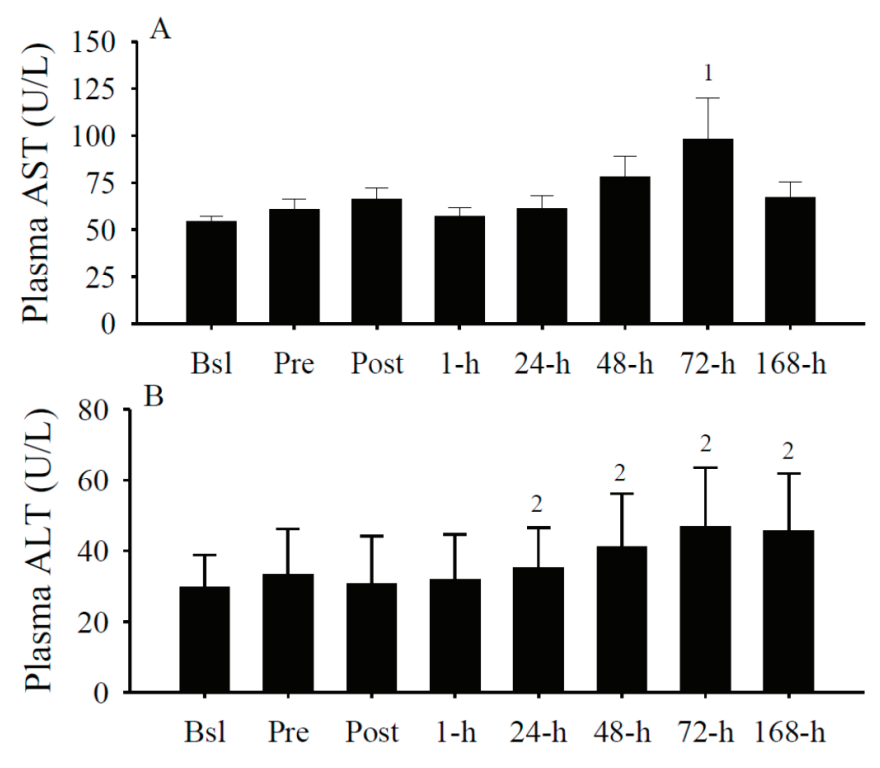

\subsection{Serum Cytokine Concentrations}

Serum IFN- $\gamma$ concentrations (Figure 6A) were significantly $(p<0.05)$ increased immediately after the exercise protocol. Although serum IL-4 concentrations were also elevated (Figure 6B), concentrations were not significantly different. Thus, IFN- $\gamma$ was elevated while IL-4 was not significantly changed immediately after intense exercise.

Figure 6. Serum IFN- $\gamma$ and IL-4 concentrations (pg/mL). Serum IFN- $\gamma$ concentrations (A) were significantly ( ${ }^{1} p<0.05$ vs. 1 -h, $48-\mathrm{h}$, and $\left.72-\mathrm{h}\right)$ increased at Post. Serum IL-4 concentrations $(\mathbf{B})$ were not significantly different. Data presented as mean $\pm \mathrm{SEM}$.

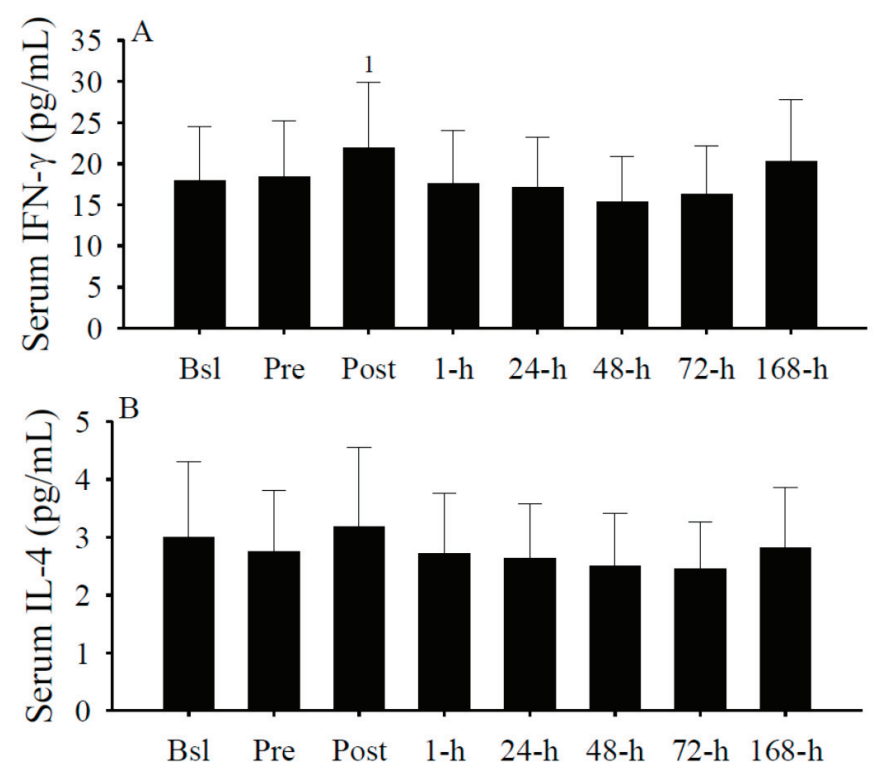




\subsection{Plasma Calcium, PTH, and Albumin Concentrations}

Immediately following the exercise protocol (i.e., Post), plasma calcium (Figure 7A) and PTH (Figure 7B) concentrations were significantly (both $p<0.05$ ) increased. At 1-h, calcium concentrations remained significantly $(p<0.05)$ increased and plasma PTH concentrations significantly $(p<0.05)$ decreased. Immediately following intense exercise, albumin concentrations significantly $(p<0.05)$ increased (Figure 7C).

Figure 7. Plasma calcium $(\mathrm{mg} / \mathrm{dL})$, parathyroid hormone $(\mathrm{PTH} ; \mathrm{pg} / \mathrm{mL})$, and albumin (g/dL) concentrations. (A) Total plasma calcium concentrations were significantly increased at Post ( ${ }^{1} p<0.05$ vs. Bsl, Pre, 24-h, 48-h, 72-h, and 168-h) and 1-h ( ${ }^{2} p<0.05$ vs. 24-h, 48-h, 72-h, and 168-h). (B) Plasma PTH concentrations were significantly increased at Post ( ${ }^{3} p<0.05$ vs. Bsl, Pre, 1-h, and 72-h) and significantly decreased at 1-h ( ${ }^{4} p<0.05$ vs. 24-h, 48-h, and 168-h). (C) Plasma albumin concentrations were significantly increased at Post $\left({ }^{5} p<0.05\right.$ vs. Bsl, Pre, 1-h, 24-h, 48-h, 72-h, and 168-h). Data presented as mean \pm SEM.

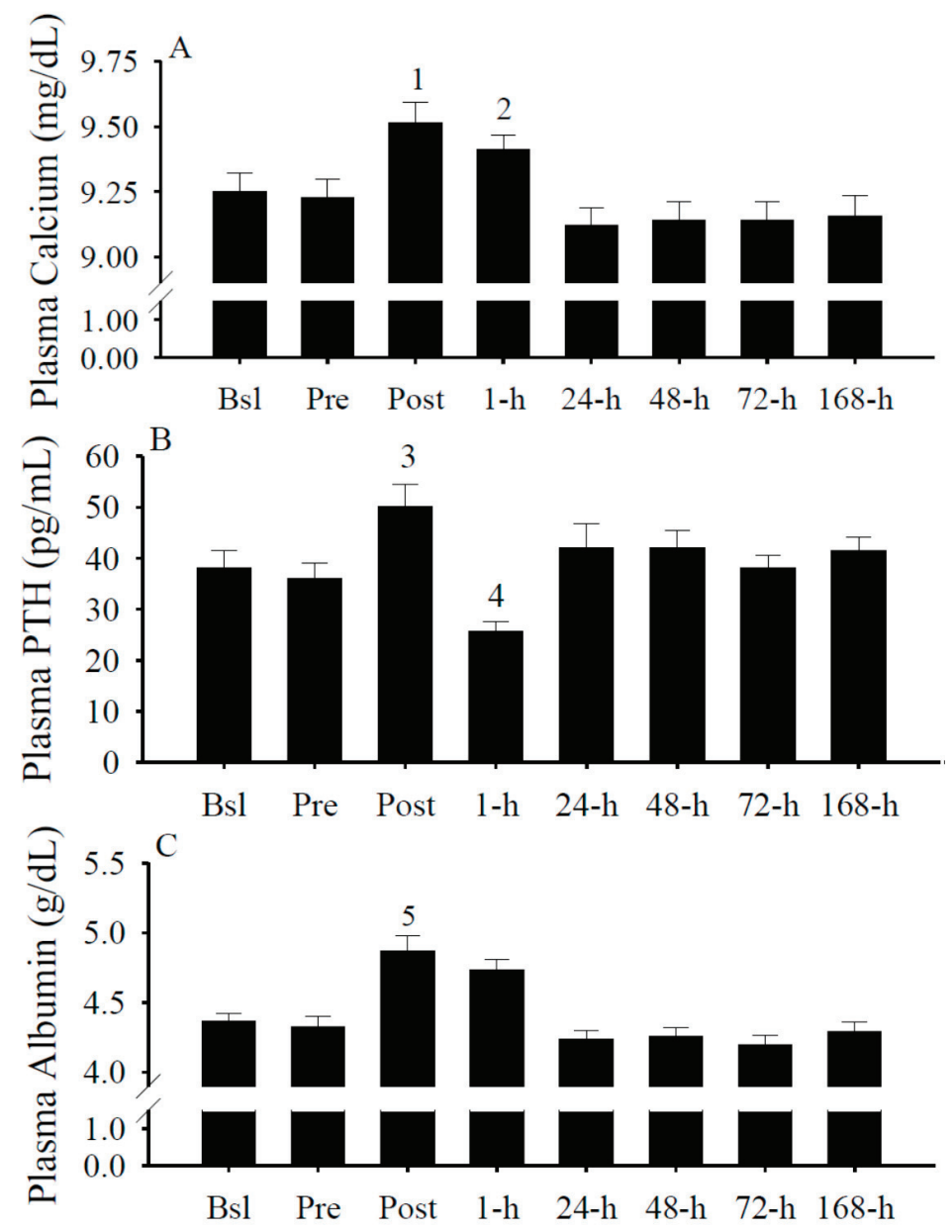




\subsection{IFN- $\gamma$ and Albumin Predict Serum 25(OH)D Changes}

Circulating IFN- $\gamma$ increases predicted $(p<0.001)$ serum $25(\mathrm{OH}) \mathrm{D}$ decreases, while albumin increases predicted $(p<0.005)$ serum 25(OH)D increases (Table 2$)$.

Table 2. Circulating albumin increases predict serum 25(OH)D increases, while IFN- $\gamma$ increases predict serum $25(\mathrm{OH}) \mathrm{D}$ decreases.

\begin{tabular}{cccc}
\hline & \multicolumn{2}{c}{ Coefficient } & \\
\cline { 2 - 3 } & Unstandardized $( \pm$ SE) & Standardized & $p$ \\
\hline Constant & $75.99( \pm 6.32)$ & & \\
$\Delta$ IFN- $\gamma$ & $-0.60( \pm 0.09)$ & -0.60 & $<0.001$ \\
$\Delta$ iPTH & $0.06( \pm 0.09)$ & 0.06 & 0.48 \\
$\Delta$ Calcium & $19.7( \pm 10.6)$ & 0.19 & 0.07 \\
$\Delta$ Albumin & $28.1( \pm 9.3)$ & 0.32 & $<0.005$ \\
& $r^{2}=0.36$, adjusted $r^{2}=0.33$ & & \\
\hline
\end{tabular}

$\Delta$, change from Bsl.

\subsection{Gender Differences}

In the secondary analysis investigating the difference between genders, serum $25(\mathrm{OH}) \mathrm{D}$, IFN- $\gamma$, IL-4, PTH, AST, ALT, peak isometric force, and the difference in peak isometric force between the CON and SSC legs were not significantly different between males and females (data not shown). However, circulating calcium (males, $9.33 \pm 0.06 v s$. females, $9.09 \pm 0.08 \mathrm{mg} / \mathrm{dL}$ ) and albumin (males, $4.48 \pm 0.05 v s$. females, $4.16 \pm 0.07 \mathrm{~g} / \mathrm{dL}$ ) concentrations were significantly (both $p<0.05$ ) different between genders.

\section{Discussion}

In the present investigation, we provide new data identifying that muscular weakness immediately (i.e., Post) and several days (i.e., 48-h and 72-h) after intense exercise were inversely predicted by pre-exercise serum $25(\mathrm{OH}) \mathrm{D}$ concentrations. Additionally, IFN- $\gamma$ increases predicted serum $25(\mathrm{OH}) \mathrm{D}$ decreases, while albumin increases predicted serum 25(OH)D increases. These unique findings establish the importance of IFN- $\gamma$ and albumin on the regulation of serum $25(\mathrm{OH}) \mathrm{D}$, and that the serum 25(OH)D concentration prior to exercise could be a determinant of muscular strength after intense exercise.

The novel finding of the present investigation was the inverse association between pre-exercise serum 25(OH)D and both immediate and persistent muscular weakness after intense exercise. Pre-exercise serum 25(OH)D concentrations, however, did not predict muscular weakness 24-h after the intense exercise protocol, collectively suggesting a temporal sensitivity of skeletal muscle strength to vitamin D levels following muscular insult.

Muscular weakness is a unique measure of muscle recovery because it spans the degenerative and regenerative events occurring at the molecular and cellular levels [44]. Furthermore, muscular weakness persists until repair is complete, suggesting that a faster recovery in skeletal muscle 
strength reflects a faster progression through the degenerative and regenerative events following muscular insult. In experimental rats, vitamin D treatment accelerated the recovery in muscular strength after injury [19]. Vitamin D treatment also increased serum 25(OH)D concentrations [19], which presumably, maintained substrate availability for the conversion of vitamin $\mathrm{D}$ to $1,25(\mathrm{OH}) \mathrm{D}$ and increased the expression (message and protein) of the vitamin D-receptor (VDR) [21]. Despite cell and experimental animal data supporting the therapeutic influence of vitamin $\mathrm{D}$ on the muscle recovery after muscular insult, results in human suggest otherwise.

Contrasting with the results here, Ring et al. [55] reported that vitamin D status was not influential on muscular weakness after exercise. The reason for the conflicting reports could relate to different experimental protocols, including the muscle(s) or muscle group(s) exercised, damaged and/or tested, disparate contraction modalities (i.e., eccentric vs. eccentric-concentric contraction cycles), or the volume, duration and intensity of the exercise protocol. Additionally, the non-significant linear regression reported previously consisted of 2-d post isometric force and serum $25(\mathrm{OH}) \mathrm{D}$ concentration data [55]. Although we did not observe a significant difference 48-h after the intense exercise protocol, it is probable that serum $25(\mathrm{OH}) \mathrm{D}$ concentrations were modulated by intense-eccentric contractions [55], and consequentially, confounded the interpretation between vitamin D and muscular strength. To avoid the potential confounding influence of exercise in the present study, pre-exercise serum 25(OH)D concentrations were related to post-exercise muscle weakness.

Consistent with the postulate that exercise modulates circulating vitamin $\mathrm{D}$, we identify a transient $25(\mathrm{OH}) \mathrm{D}$ increase in the circulation immediately after intense exercise. The serum $25(\mathrm{OH}) \mathrm{D}$ concentration increases immediately after the intense exercise protocol was preceded by an initial (i.e., Bsl to Pre) and followed by a second (i.e., Post to 1-h) decrease. The initial decrease is consistent with data demonstrating the serum $25(\mathrm{OH}) \mathrm{D}$ concentration response during the winter [75]. The second decrease, however, suggests that serum $25(\mathrm{OH}) \mathrm{D}$ is temporally sensitive to intense exercise. Interestingly, cytokine and protein shifts in the circulation could contribute, in part, to the observed 25(OH)D fluctuations (see Table 2).

Interferon $-\gamma$ is an inflammatory cytokine that regulates vitamin D metabolism $[60,61,78,79]$. In immune and endothelial cells, IFN- $\gamma$ impairs CYP27A1 message and protein expression [80], and thereby implying its potential role of impeding $25(\mathrm{OH}) \mathrm{D}$ production. Furthermore, IFN- $\gamma$ accelerates the conversion of $25(\mathrm{OH}) \mathrm{D}$ to $1,25(\mathrm{OH}) \mathrm{D}[60,61,78,79]$ by increasing $1 \alpha \mathrm{OHase}$ expression $[62,64,65,81,82]$. Supporting the influence of IFN- $\gamma$ on vitamin D metabolism in vivo, we illustrated previously that IFN- $\gamma$ tends to inversely correlate with $25(\mathrm{OH}) \mathrm{D}$ and significantly correlates (positive) with 1,25(OH)D [59], suggesting 25(OH)D decreases and 1,25(OH)D increases with increasing IFN- $\gamma$ in the circulation. Based on the results here and those reported elsewhere, we propose that IFN- $\gamma$ contributes to the circulating $25(\mathrm{OH}) \mathrm{D}$ decrease by impairing the production and accelerating the catabolism of $25(\mathrm{OH}) \mathrm{D}$. However, confirmation of the proposed hypothesis requires further investigation.

Interleukin-4 is another inflammatory cytokine that has been implicated in the regulation of vitamin D metabolism. In human monocytes, IL-4 upregulated the message expression of CYP27B1 and the VDR [65]. Although message expression increased, IL-4 did not increase the activity of CYP27B1 [65]. Strikingly, IL-4 increased the activity of CYP24A1 and the conversion of 25(OH)D 
to $24,25(\mathrm{OH}) \mathrm{D}$ in monocytes [65]. These unique findings suggest that IL-4 could decrease $25(\mathrm{OH}) \mathrm{D}$ concentrations by accelerating catabolism. However, we were unable to detect a significant difference in IL-4 concentrations prior to or following the intense exercise protocol. Thus, based on our findings, it is unclear if IL-4 contributes to the fluctuating serum 25(OH)D concentrations observed during the winter and following intense exercise.

Another unique finding of the present investigation was the prediction of serum $25(\mathrm{OH}) \mathrm{D}$ concentrations by albumin. Albumin is a plasma protein synthesized in the liver that maintains colloid osmotic pressure [83]. Following short-term, high intensity exercise, albumin increases in the circulation and expands blood volume [84-86]. In the circulation, albumin binds to vitamin D metabolites [87]. The predication of increasing 25(OH)D by albumin is plausibly the product of increased hepatic secretion of albumin and its binding to vitamin D in the blood. However, additional research investigating the influence of albumin on vitamin D concentration in the circulation is required.

Calcium and PTH regulate circulating 25(OH)D concentrations. Low calcium concentrations in the blood stimulate the parathyroid gland to secrete PTH. The increase in PTH stimulates the conversion of $25(\mathrm{OH}) \mathrm{D}$ to $1,25(\mathrm{OH}) \mathrm{D}$ [88-90] and increases the circulating calcium. Increases in dietary and serum calcium increase the catabolism of $25(\mathrm{OH}) \mathrm{D}$ to $24,25(\mathrm{OH}) \mathrm{D}$ [91]. In this investigation, we found an increase in plasma calcium concentrations immediately following the intense exercise protocol that was resolved by the following day (Figure 7A). PTH concentrations displayed a transient fluctuation after (i.e., Post and 1-h) the intense exercise protocol (Figure 7B). Although vitamin D metabolism is regulated by calcium and $\mathrm{PTH}$, neither the calcium or PTH predicted the deviation in serum $25(\mathrm{OH}) \mathrm{D}$ concentrations after intense exercise (Table 2).

In addition to those discussed above, there are limitations to this study that should be considered with future investigations. First, we did not obtain skeletal muscle biopsies to assess local damage, vitamin D metabolites, or the enzymes involved with vitamin D metabolism. Second, we did not control fluid or dietary intake prior to, during, or following intense exercise. Third, it is unknown if the results here can be extrapolated to other inflammatory modalities. Fourth, calcium and albumin concentrations were significantly different between genders. Calcium and albumin concentrations, however, were within the recommended clinical reference ranges (calcium, 8.4-10.4 mg/dL; albumin, 3.3-4.8 g/dL) for both genders, and therefore, it is unclear if this statistical difference is of physiological impact. Along these lines, serum 25(OH)D concentrations and peak isometric forces were not significantly different between male and female subjects as well. Next, this study consisted of 14 subject's total. Future investigations are encouraged to include larger sample sizes when investigating the potential influence of vitamin D on skeletal muscle strength after injury. Finally, it is unknown skeletal muscle damage or fatigue-related factors, or both, were contributing to muscular weakness after the imposed exercise protocol. However, it is noteworthy that circulating biomarkers of skeletal muscle damage, and specifically AST and ALT, were elevated concurrently with deficits in skeletal muscle strength during the days following the intense exercise protocol. Thus, these results tend to support the possibility of the presence of skeletal muscle damage. 


\section{Conclusions and Implications}

Muscle weakness hinders millions of people worldwide every year and is mediated by a variety of conditions, including aging, disease, inactivity, limb immobilization, repetitive use, and intense or unaccustomed exercise. In the present investigation, we reveal that muscular weakness after an intense exercise bout is abrogated with increasing serum $25(\mathrm{OH}) \mathrm{D}$ concentration prior to exercise. However, this was apparent immediately and several days (2-day and 3-day), but not 1-day, after intense exercise. Additionally, IFN- $\gamma$ and albumin predicted serum 25(OH)D concentration fluctuations, and due on these fluctuations, we recommend caution when interpreting serum $25(\mathrm{OH}) \mathrm{D}$ concentrations immediately after intense exercise and/or during an acute inflammatory response. We conclude that maintaining an adequate serum $25(\mathrm{OH}) \mathrm{D}$ concentration could attenuate muscular weakness after intense exercise. Given the feasibility of increasing $25(\mathrm{OH}) \mathrm{D}$ concentrations in the blood, future research investigating the influence of diverse vitamin $\mathrm{D}$ interventions on the alleviation of muscular weakness after muscular insult are encouraged in humans.

\section{Acknowledgments}

We would like to thank the subjects who participated in this study; Penny Snow, Kristi Thunell and Michelle (Shelly) Oliver (The Orthopedic Specialty Hospital) for the phlebotomy; and the late Adam S. Dern (USANA Health Sciences, Inc.) for serum sample preparations. This study was funded in part by the Intermountain Research and Medical Foundation (Intermountain Healthcare, Salt Lake City, UT, USA) and the ARUP Institute for Clinical and Experimental Pathology (Salt Lake City, UT, USA).

\section{Conflicts of Interest}

The authors declare no conflict of interest.

\section{References}

1. Jones, G. Pharmacokinetics of vitamin D toxicity. Am. J. Clin. Nutr. 2008, 88, 582S-586S.

2. Girgis, C.M.; Clifton-Bligh, R.J.; Hamrick, M.W.; Holick, M.F.; Gunton, J.E. The roles of vitamin D in skeletal muscle: form, function, and metabolism. Endocr. Rev. 2013, 34, 33-83.

3. Ceglia, L.; Harris, S.S. Vitamin D and its role in skeletal muscle. Calcif. Tissue Int. 2013, 92, 151-162.

4. Bischoff-Ferrari, H.; Stahelin, H.B.; Walter, P. Vitamin D effects on bone and muscle. Int. J. Vitam. Nutr. Res. 2011, 81, 264-272.

5. Ceglia, L. Vitamin D and skeletal muscle tissue and function. Mol. Aspects Med. 2008, 29, 407-414.

6. Boland, R.L. VDR activation of intracellular signaling pathways in skeletal muscle. Mol. Cell. Endocrinol. 2011, 347, 11-16.

7. Boland, R. Role of vitamin D in skeletal muscle function. Endocr. Rev. 1986, 7, 434-448. 
8. Boland, R.; de Boland, A.R.; Marinissen, M.J.; Santillan, G.; Vazquez, G.; Zanello, S. Avian muscle cells as targets for the secosteroid hormone 1,25-dihydroxy-vitamin D3. Mol. Cell. Endocrinol. 1995, 114, 1-8.

9. Birge, S.J.; Haddad, J.G. 25-Hydroxycholecalciferol stimulation of muscle metabolism. J. Clin. Invest. 1975, 56, 1100-1107.

10. De Boland, A.R.; Boland, R. In vitro cellular muscle calcium metabolism. Characterization of effects of 1,25-dihydroxy-vitamin D3 and 25-hydroxy-vitamin D3. Z. Naturforsch. C 1985, 40, 102-108.

11. De Boland, A.R.; Boland, R.L. Rapid changes in skeletal muscle calcium uptake induced in vitro by 1,25-dihydroxyvitamin D3 are suppressed by calcium channel blockers. Endocrinology 1987, 120, 1858-1864.

12. Walters, M.R.; Ilenchuk, T.T.; Claycomb, W.C. 1,25-Dihydroxyvitamin D3 stimulates 45Ca2+ uptake by cultured adult rat ventricular cardiac muscle cells. J. Biol. Chem. 1987, 262, 2536-2541.

13. Giuliani, D.L.; Boland, R.L. Effects of vitamin D3 metabolites on calcium fluxes in intact chicken skeletal muscle and myoblasts cultured in vitro. Calcif. Tissue Int. 1984, 36, 200-205.

14. Matthews, C.; Heimberg, K.W.; Ritz, E.; Agostini, B.; Fritzsche, J.; Hasselbach, W. Effect of 1,25-dihydroxycholecalciferol on impaired calcium transport by the sarcoplasmic reticulum in experimental uremia. Kidney Int. 1977, 11, 227-235.

15. Morelli, S.; Buitrago, C.; Vazquez, G.; de Boland, A.R.; Boland, R. Involvement of tyrosine kinase activity in 1alpha,25(OH)2-vitamin D3 signal transduction in skeletal muscle cells. J. Biol. Chem. 2000, 275, 36021-36028.

16. Selles, J.; Boland, R. Rapid stimulation of calcium uptake and protein phosphorylation in isolated cardiac muscle by 1,25-dihydroxyvitamin D3. Mol. Cell. Endocrinol. 1991, 77, 67-73.

17. Buitrago, C.; Boland, R.; de Boland, A.R. The tyrosine kinase c-Src is required for 1,25(OH)2-vitamin D3 signalling to the nucleus in muscle cells. Biochim. Biophys. Acta 2001, 1541, 179-187.

18. Buitrago, C.G.; Arango, N.S.; Boland, R.L. 1alpha,25(OH)2D3-dependent modulation of Akt in proliferating and differentiating $\mathrm{C} 2 \mathrm{C} 12$ skeletal muscle cells. J. Cell. Biochem. 2012, 113, 1170-1181.

19. Stratos, I.; Li, Z.; Herlyn, P.; Rotter, R.; Behrendt, A.K.; Mittlmeier, T.; Vollmar, B. Vitamin D increases cellular turnover and functionally restores the skeletal muscle after crush injury in rats. Am. J. Pathol. 2012, 182, 895-904.

20. Alami-Durante, H.; Cluzeaud, M.; Bazin, D.; Mazurais, D.; Zambonino-Infante, J.L. Dietary cholecalciferol regulates the recruitment and growth of skeletal muscle fibers and the expressions of myogenic regulatory factors and myosin heavy chain in European sea bass larvae. J. Nutr. 2011, 141, 2146-2151.

21. Srikuea, R.; Zhang, X.; Park-Sarge, O.K.; Esser, K.A. VDR and CYP27B1 are expressed in $\mathrm{C} 2 \mathrm{C} 12$ cells and regenerating skeletal muscle: Potential role in suppression of myoblast proliferation. Am. J. Physiol. Cell. Physiol. 2012, 303, C396-C405. 
22. Garcia, L.A.; Ferrini, M.G.; Norris, K.C.; Artaza, J.N. 1,25(OH)(2)vitamin D(3) enhances myogenic differentiation by modulating the expression of key angiogenic growth factors and angiogenic inhibitors in $\mathrm{C}(2) \mathrm{C}(12)$ skeletal muscle cells. J. Steroid. Biochem. Mol. Biol. 2013, $133,1-11$.

23. Ward, K.A.; Das, G.; Berry, J.L.; Roberts, S.A.; Rawer, R.; Adams, J.E.; Mughal, Z. Vitamin D status and muscle function in post-menarchal adolescent girls. J. Clin. Endocrinol. Metab. 2009, 94, 559-563.

24. Ward, K.A.; Das, G.; Roberts, S.A.; Berry, J.L.; Adams, J.E.; Rawer, R.; Mughal, M.Z. A randomized, controlled trial of vitamin D supplementation upon musculosketal health in postmenarchal females. J. Clin. Endocrinol. Metab. 2010, 95, 4643-4651.

25. Foo, L.H.; Zhang, Q.; Zhu, K.; Ma, G.; Hu, X.; Greenfield, H.; Fraser, D.R. Low vitamin D status has an adverse influence on bone mass, bone turnover, and muscle strength in Chinese adolescent girls. J. Nutr. 2009, 139, 1002-1007.

26. Ceglia, L.; Chiu, G.R.; Harris, S.S.; Araujo, A.B. Serum 25-hydroxyvitamin D concentration and physical function in adult men. Clin. Endocrinol. (Oxf.) 2011, 74, 370-376.

27. Ziambaras, K.; Dagogo-Jack, S. Reversible muscle weakness in patients with vitamin D deficiency. West. J. Med. 1997, 167, 435-439.

28. Russell, J.A. Osteomalacic myopathy. Muscle Nerve 1994, 17, 578-580.

29. Stockton, K.A.; Kandiah, D.A.; Paratz, J.D.; Bennell, K.L. Fatigue, muscle strength and vitamin D status in women with systemic lupus erythematosus compared to healthy controls. Lupus 2011, 21, 271-278.

30. Glerup, H.; Mikkelsen, K.; Poulsen, L.; Hass, E.; Overbeck, S.; Andersen, H.; Charles, P.; Eriksen, E.F. Hypovitaminosis D myopathy without biochemical signs of osteomalacic bone involvement. Calcif. Tissue Int. 2000, 66, 419-424.

31. Barker, T.; Martins, T.B.; Hill, H.R.; Kjeldsberg, C.R.; Trawick, R.H.; Weaver, L.K.; Traber, M.G. Low vitamin D impairs strength recovery after anterior cruciate ligament surgery. J. Evid. Based Complement. Altern. Med. 2011, 16, 201-209.

32. Prineas, J.W.; Mason, A.S.; Henson, R.A. Myopathy in metabolic bone disease. Br. Med. J. 1965, 1, 1034-1036.

33. Gordon, P.L.; Doyle, J.W.; Johansen, K.L. Association of 1,25-dihydroxyvitamin D levels with physical performance and thigh muscle cross-sectional area in chronic kidney disease stage 3 and 4. J. Ren. Nutr. 2012, 22, 423-433.

34. Grimaldi, A.S.; Parker, B.A.; Capizzi, J.A.; Clarkson, P.M.; Pescatello, L.S.; White, C.M.; Thompson, P.D. 25(OH) vitamin D is associated with greater muscle strength in healthy men and women. Med. Sci. Sports Exerc. 2013, 45, 157-162.

35. Von Hurst, P.R.; Conlon, C.; Foskett, A. Vitamin D status predicts hand-grip strength in young adult women living in Auckland, New Zealand. J. Steroid. Biochem. Mol. Biol. 2012, doi:10.1016/j.jsbmb.2012.11.015.

36. Wicherts, I.S.; van Schoor, N.M.; Boeke, A.J.; Visser, M.; Deeg, D.J.; Smit, J.; Knol, D.L.; Lips, P. Vitamin D status predicts physical performance and its decline in older persons. J. Clin. Endocrinol. Metab. 2007, 92, 2058-2065. 
37. Barker, T.; Martins, T.B.; Hill, H.R.; Kjeldsberg, C.R.; Dixon, B.M.; Schneider, E.D.; Henridsen, V.T.; Weaver, L.K. Circulating pro-inflammatory cytokines are elevated and peak power output correlates with 25 -hydroxyvitamin D in vitamin D insufficient adults. Eur. J. Appl. Physiol. 2013, doi:10.1007/s00421-012-2582-7.

38. Carrillo, A.E.; Flynn, M.G.; Pinkston, C.; Markofski, M.M.; Jiang, Y.; Donkin, S.S.; Teegarden, D. Impact of vitamin D supplementation during a resistance training intervention on body composition, muscle function, and glucose tolerance in overweight and obese adults. Clin. Nutr. 2012, doi:10.1016/j.clnu.2012.08.014.

39. Barker, T.; Martins, T.B.; Hill, H.R.; Kjeldsberg, C.R.; Henriksen, V.T.; Dixon, B.M.; Schneider, E.D.; Dern, A.; Weaver, L.K. Different doses of supplemental vitamin D maintain interleukin-5 without altering skeletal muscle strength: A randomized, double-blind, placebo-controlled study in vitamin D sufficient adults. Nutr. Metab. (Lond.) 2012, 9, 16.

40. Sato, Y.; Iwamoto, J.; Kanoko, T.; Satoh, K. Low-dose vitamin D prevents muscular atrophy and reduces falls and hip fractures in women after stroke: A randomized controlled trial. Cerebrovasc. Dis. 2005, 20, 187-192.

41. Janssen, H.C.; Samson, M.M.; Verhaar, H.J. Muscle strength and mobility in vitamin D-insufficient female geriatric patients: A randomized controlled trial on vitamin D and calcium supplementation. Aging Clin. Exp. Res. 2010, 22, 78-84.

42. Verhaar, H.J.; Samson, M.M.; Jansen, P.A.; de Vreede, P.L.; Manten, J.W.; Duursma, S.A. Muscle strength, functional mobility and vitamin D in older women. Aging (Milano) 2000, 12, $455-460$.

43. Floyd, M.; Ayyar, D.R.; Barwick, D.D.; Hudgson, P.; Weightman, D. Myopathy in chronic renal failure. Q. J. Med. 1974, 43, 509-524.

44. Warren, G.L.; Lowe, D.A.; Armstrong, R.B. Measurement tools used in the study of eccentric contraction-induced injury. Sports Med. 1999, 27, 43-59.

45. Komi, P.V. Stretch-shortening cycle: A powerful model to study normal and fatigued muscle. J. Biomech. 2000, 33, 1197-1206.

46. Burgi, A.A.; Gorham, E.D.; Garland, C.F.; Mohr, S.B.; Garland, F.C.; Zeng, K.; Thompson, K.; Lappe, J.M. High serum 25-hydroxyvitamin D is associated with a low incidence of stress fractures. J. Bone Miner. Res. 2011, 26, 2371-2377.

47. Lappe, J.; Cullen, D.; Haynatzki, G.; Recker, R.; Ahlf, R.; Thompson, K. Calcium and vitamin $\mathrm{D}$ supplementation decreases incidence of stress fractures in female navy recruits. $J$. Bone Miner. Res. 2008, 23, 741-749.

48. Snijder, M.B.; van Schoor, N.M.; Pluijm, S.M.; van Dam, R.M.; Visser, M.; Lips, P. Vitamin D status in relation to one-year risk of recurrent falling in older men and women. J. Clin. Endocrinol. Metab. 2006, 91, 2980-2985.

49. Broe, K.E.; Chen, T.C.; Weinberg, J.; Bischoff-Ferrari, H.A.; Holick, M.F.; Kiel, D.P. A higher dose of vitamin $\mathrm{D}$ reduces the risk of falls in nursing home residents: A randomized, multiple-dose study. J. Am. Geriatr. Soc. 2007, 55, 234-239. 
50. Bischoff, H.A.; Stahelin, H.B.; Dick, W.; Akos, R.; Knecht, M.; Salis, C.; Nebiker, M.; Theiler, R.; Pfeifer, M.; Begerow, B.; et al. Effects of vitamin D and calcium supplementation on falls: A randomized controlled trial. J. Bone Miner. Res. 2003, 18, 343-351.

51. Visser, M.; Deeg, D.J.; Lips, P. Low vitamin D and high parathyroid hormone levels as determinants of loss of muscle strength and muscle mass (sarcopenia): The Longitudinal Aging Study Amsterdam. J. Clin. Endocrinol. Metab. 2003, 88, 5766-5772.

52. Chapuy, M.C.; Arlot, M.E.; Delmas, P.D.; Meunier, P.J. Effect of calcium and cholecalciferol treatment for three years on hip fractures in elderly women. BMJ 1994, 308, 1081-1082.

53. Dawson-Hughes, B.; Harris, S.S.; Krall, E.A.; Dallal, G.E. Effect of calcium and vitamin D supplementation on bone density in men and women 65 years of age or older. N. Engl. J. Med. 1997, 337, 670-676.

54. Flicker, L.; Mead, K.; MacInnis, R.J.; Nowson, C.; Scherer, S.; Stein, M.S.; Thomasx, J.; Hopper, J.L.; Wark, J.D. Serum vitamin D and falls in older women in residential care in Australia. J. Am. Geriatr. Soc. 2003, 51, 1533-1538.

55. Ring, S.M.; Dannecker, E.A.; Peterson, C.A. Vitamin D status is not associated with outcomes of experimentally-induced muscle weakness and pain in young, healthy volunteers. $J$. Nutr. Metab. 2010, 2010, 674240.

56. Peake, J.M.; Nosaka, K.; Muthalib, M.; Suzuki, K. Systemic inflammatory responses to maximal versus submaximal lengthening contractions of the elbow flexors. Exerc. Immunol. Rev. 2006, 12, 72-85.

57. Louw, J.A.; Werbeck, A.; Louw, M.E.; Kotze, T.J.; Cooper, R.; Labadarios, D. Blood vitamin concentrations during the acute-phase response. Crit. Care Med. 1992, 20, 934-941.

58. Reid, D.; Toole, B.J.; Knox, S.; Talwar, D.; Harten, J.; O’Reilly, D.S.; Blackwell, S.; Kinsella, J.; McMillan, D.C.; Wallace, A.M. The relation between acute changes in the systemic inflammatory response and plasma 25-hydroxyvitamin D concentrations after elective knee arthroplasty. Am. J. Clin. Nutr. 2011, 93, 1006-1011.

59. Barker, T.; Martins, T.B.; Kjeldsberg, C.R.; Trawick, R.H.; Hill, H.R. Circulating interferon-gamma correlates with $1,25(\mathrm{OH}) \mathrm{D}$ and the $1,25(\mathrm{OH}) \mathrm{D}$-to-25(OH)D ratio. Cytokine 2012, 60, 23-26.

60. Koeffler, H.P.; Reichel, H.; Bishop, J.E.; Norman, A.W. Gamma-interferon stimulates production of 1,25-dihydroxyvitamin D3 by normal human macrophages. Biochem. Biophys. Res. Commun. 1985, 127, 596-603.

61. Reichel, H.; Koeffler, H.P.; Barbers, R.; Norman, A.W. Regulation of 1,25-dihydroxyvitamin D3 production by cultured alveolar macrophages from normal human donors and from patients with pulmonary sarcoidosis. J. Clin. Endocrinol. Metab. 1987, 65, 1201-1209.

62. Stoffels, K.; Overbergh, L.; Giulietti, A.; Verlinden, L.; Bouillon, R.; Mathieu, C. Immune regulation of 25-hydroxyvitamin-D3-1alpha-hydroxylase in human monocytes. J. Bone Miner. Res. 2006, 21, 37-47.

63. Stoffels, K.; Overbergh, L.; Bouillon, R.; Mathieu, C. Immune regulation of 1alpha-hydroxylase in murine peritoneal macrophages: unravelling the IFNgamma pathway. J. Steroid. Biochem. Mol. Biol. 2007, 103, 567-571. 
64. Overbergh, L.; Stoffels, K.; Valckx, D.; Giulietti, A.; Bouillon, R.; Mathieu, C. Regulation of 25-hydroxyvitamin D-1alpha-hydroxylase by IFNgamma in human monocytic THP1 cells. J. Steroid. Biochem. Mol. Biol. 2004, 89-90, 453-455.

65. Edfeldt, K.; Liu, P.T.; Chun, R.; Fabri, M.; Schenk, M.; Wheelwright, M.; Keegan, C.; Krutzik, S.R.; Adams, J.S.; Hewison, M.; Modlin, R.L. T-cell cytokines differentially control human monocyte antimicrobial responses by regulating vitamin D metabolism. Proc. Natl. Acad. Sci. USA 2010, 107, 22593-22598.

66. Weinstock, C.; Konig, D.; Harnischmacher, R.; Keul, J.; Berg, A.; Northoff, H. Effect of exhaustive exercise stress on the cytokine response. Med. Sci. Sports Exerc. 1997, 29, 345-354.

67. Malm, C.; Sjodin, B.; Sjoberg, B.; Lenkei, R.; Renstrom, P.; Lundberg, I.E.; Ekblom, B. Leukocytes, cytokines, growth factors and hormones in human skeletal muscle and blood after uphill or downhill running. J. Physiol. 2004, 556, 983-1000.

68. Barker, T.; Henriksen, V.T.; Martins, T.B.; Kjeldsberg, C.R.; Hill, H.R. Fluctuations in the skeletal muscle power-velocity relationship and interferon-gamma after a muscle-damaging event in humans. Extrem. Physiol. Med. 2012, 1, 1-6.

69. Peake, J.M.; Suzuki, K.; Hordern, M.; Wilson, G.; Nosaka, K.; Coombes, J.S. Plasma cytokine changes in relation to exercise intensity and muscle damage. Eur. J. Appl. Physiol. 2005, 95, $514-521$.

70. Hirose, L.; Nosaka, K.; Newton, M.; Laveder, A.; Kano, M.; Peake, J.; Suzuki, K. Changes in inflammatory mediators following eccentric exercise of the elbow flexors. Exerc. Immunol. Rev. 2004, 10, 75-90.

71. Duncan, A.; Talwar, D.; McMillan, D.C.; Stefanowicz, F.; O’Reilly, D.S. Quantitative data on the magnitude of the systemic inflammatory response and its effect on micronutrient status based on plasma measurements. Am. J. Clin. Nutr. 2012, 95, 64-71.

72. Martins, T.B.; Pasi, B.M.; Pickering, J.W.; Jaskowski, T.D.; Litwin, C.M.; Hill, H.R. Determination of cytokine responses using a multiplexed fluorescent microsphere immunoassay. Am. J. Clin. Pathol. 2002, 118, 346-353.

73. Martins, T.B.; Anderson, J.L.; Muhlestein, J.B.; Horne, B.D.; Carlquist, J.F.; Roberts, W.L.; Carlquist, J.F. Risk factor analysis of plasma cytokines in patients with coronary artery disease by a multiplexed fluorescent immunoassay. Am. J. Clin. Pathol. 2006, 125, 906-913.

74. Barker, T.; Leonard, S.W.; Hansen, J.; Trawick, R.H.; Ingram, R.; Burdett, G.; Lebold, K.M.; Walker, J.A.; Traber, M.G. Vitamin E and C supplementation does not ameliorate muscle dysfunction following anterior cruciate ligament surgery. Free Radic. Biol. Med. 2009, 47, $1611-1618$.

75. Webb, A.R.; Kline, L.; Holick, M.F. Influence of season and latitude on the cutaneous synthesis of vitamin D3: Exposure to winter sunlight in Boston and Edmonton will not promote vitamin D3 synthesis in human skin. J. Clin. Endocrinol. Metab. 1988, 67, 373-378.

76. Brancaccio, P.; Limongelli, F.M.; Maffulli, N. Monitoring of serum enzymes in sport. Br. J. Sports Med. 2006, 40, 96-97. 
77. Nie, J.; Tong, T.K.; George, K.; Fu, F.H.; Lin, H.; Shi, Q. Resting and post-exercise serum biomarkers of cardiac and skeletal muscle damage in adolescent runners. Scand. J. Med. Sci. Sports 2011, 21, 625-629.

78. Dusso, A.S.; Kamimura, S.; Gallieni, M.; Zhong, M.; Negrea, L.; Shapiro, S.; Slatopolsky, E. Gamma-interferon-induced resistance to 1,25-(OH)2 D3 in human monocytes and macrophages: A mechanism for the hypercalcemia of various granulomatoses. J. Clin. Endocrinol. Metab. 1997, 82, 2222-2232.

79. Adams, J.S.; Gacad, M.A. Characterization of 1 alpha-hydroxylation of vitamin D3 sterols by cultured alveolar macrophages from patients with sarcoidosis. J. Exp. Med. 1985, 161, 755-765.

80. Reiss, A.B.; Awadallah, N.W.; Malhotra, S.; Montesinos, M.C.; Chan, E.S.; Javitt, N.B.; Cronstein, B.N. Immune complexes and IFN-gamma decrease cholesterol 27-hydroxylase in human arterial endothelium and macrophages. J. Lipid Res. 2001, 42, 1913-1922.

81. Overbergh, L.; Decallonne, B.; Valckx, D.; Verstuyf, A.; Depovere, J.; Laureys, J.; Rutgeerts, O.; Saint-Arnaud, R.; Bouillon, R.; Mathieu, C. Identification and immune regulation of 25-hydroxyvitamin D-1-alpha-hydroxylase in murine macrophages. Clin. Exp. Immunol. 2000, $120,139-146$.

82. Monkawa, T.; Yoshida, T.; Hayashi, M.; Saruta, T. Identification of 25-hydroxyvitamin D3 1alpha-hydroxylase gene expression in macrophages. Kidney Int. 2000, 58, 559-568.

83. Quinlan, G.J.; Martin, G.S.; Evans, T.W. Albumin: Biochemical properties and therapeutic potential. Hepatology 2005, 41, 1211-1219.

84. Green, H.J.; Thomson, J.A.; Ball, M.E.; Hughson, R.L.; Houston, M.E.; Sharratt, M.T. Alterations in blood volume following short-term supramaximal exercise. J. Appl. Physiol. 1984, 56, 145-149.

85. Gillen, C.M.; Lee, R.; Mack, G.W.; Tomaselli, C.M.; Nishiyasu, T.; Nadel, E.R. Plasma volume expansion in humans after a single intense exercise protocol. J. Appl. Physiol. 1991, 71, 1914-1920.

86. Yang, R.C.; Mack, G.W.; Wolfe, R.R.; Nadel, E.R. Albumin synthesis after intense intermittent exercise in human subjects. J. Appl. Physiol. 1998, 84, 584-592.

87. Bikle, D.D.; Gee, E.; Halloran, B.; Kowalski, M.A.; Ryzen, E.; Haddad, J.G. Assessment of the free fraction of 25-hydroxyvitamin $\mathrm{D}$ in serum and its regulation by albumin and the vitamin D-binding protein. J. Clin. Endocrinol. Metab. 1986, 63, 954-959.

88. Garabedian, M.; Holick, M.F.; DeLuca, H.F.; Boyle, I.T. Control of 25-hydroxycholecalciferol metabolism by parathyroid glands. Proc. Natl. Acad. Sci. USA 1972, 69, 1673-1676.

89. Rasmussen, H.; Wong, M.; Bikle, D.; Goodman, D.B. Hormonal control of the renal conversion of 25-hydroxycholecalciferol to 1,25-dihydroxycholecalciferol. J. Clin. Invest. 1972, 51, 2502-2504.

90. Fraser, D.R.; Kodicek, E. Regulation of 25-hydroxycholecalciferol-1-hydroxylase activity in kidney by parathyroid hormone. Nat. New Biol. 1973, 241, 163-166.

91. Boyle, I.T.; Gray, R.W.; DeLuca, H.F. Regulation by calcium of in vivo synthesis of 1,25-dihydroxycholecalciferol and 21,25-dihydroxycholecalciferol. Proc. Natl. Acad. Sci. USA 1971, 68, 2131-2134. 

Chapter 9

Risk Factors 



\title{
Vitamin D Insufficiency and Bone Mineral Status in a Population of Newcomer Children in Canada
}

\section{Hassanali Vatanparast, Christine Nisbet and Brian Gushulak}

\begin{abstract}
Background: Low levels of circulating vitamin D are more likely to be found in those with darker skin pigmentation, who live in areas of high latitude, and who wear more clothing. We examined the prevalence of vitamin D deficiency and inadequacy in newcomer immigrant and refugee children. Methods: We evaluated circulating vitamin D status of immigrant children at the national level. Subsequently, we investigated vitamin D intake, circulating vitamin D status, and total body bone mineral content (TBBMC) in newcomer children living in Saskatchewan. Results: In the sample of newcomer children in Saskatchewan, the prevalence of inadequacy in calcium and vitamin D intakes was $76 \%$ and $89.4 \%$, respectively. Vitamin D intake from food/supplement was significantly higher in immigrants compared to refugees, which accords with the significant difference in serum status. Circulating vitamin D status indicated that $29 \%$ of participants were deficient and another $44 \%$ had inadequate levels of serum $25(\mathrm{OH}) \mathrm{D}$ for bone health. Dietary vitamin D intake, sex, region of origin, and length of stay in Canada were significant predictors of serum vitamin D status. Results for TBBMC revealed that $38.6 \%$ were found to have low TBBMC compared to estimated values for age, sex, and ethnicity. In the regression model, after controlling for possible confounders, children who were taller and had greater circulating vitamin D also had greater TBBMC. Nationally, immigrant children, particularly girls, have significantly lower plasma $25(\mathrm{OH}) \mathrm{D}$ than non-immigrant children. Interpretation: Newcomer immigrant and refugee children are at a high risk of vitamin D deficiency and inadequacy, which may have serious negative consequences for their health.
\end{abstract}

Reprinted from Nutrients. Cite as: Vatanparast, H.; Nisbet, C.; Gushulak, B. Vitamin D Insufficiency and Bone Mineral Status in a Population of Newcomer Children in Canada. Nutrients 2013, 5, $1561-1572$.

\section{Introduction}

Recent studies report various health benefits for vitamin D along with its important role in bone health [1]. Limited food sources of vitamin D and long winters resulting in reduced sun exposure in Canada have raised concerns, particularly in growing children where vitamin D is needed for bone mineral accrual. National data report a low prevalence of vitamin D deficiency in children aged 6-11 years compared to other age groups [2]. However, the growing proportion of immigrants in Canada, particularly children, may represent communities at greater risk for vitamin D deficiency. Some new arrivals have high skin pigmentation, and social and cultural factors that can result in insufficient vitamin D intake from food [3-6]. From 2002 to 2004, 150 cases of rickets were reported in Canadian children [7]. Darker-skinned individuals represented $89 \%$ of cases, while $24 \%$ were immigrants [7]. In a cross-sectional study in Edmonton, vitamin D concentrations decreased in children as age increased [8]. The authors speculated that this is possibly due to less consumption of milk fortified with vitamin $D$ and less exposure to the sun [8]. In response to the paucity of 
information in this area, we evaluated circulating vitamin D status of immigrant and refugee children new to Canada. Additionally, we examined the association between circulating vitamin D with bone mineral status.

\section{Methods}

Two studies were conducted to address the following research objectives: (i) to obtain a national perspective on vitamin D status of immigrant children in comparison to non-immigrant children; (ii) to evaluate determinants of vitamin $\mathrm{D}$ and its association with bone mineral status in children new to Canada.

\subsection{Study 1. Vitamin D Status of Canadian Immigrant and Non-Immigrant Children}

We used data from the Canadian Health Measures Survey (CHMS), cycle 1, 2007-2009, conducted by Statistics Canada [9]. The CHMS is a nationally representative survey among approximately 5500 Canadians aged 6-79 years, representative of $96.3 \%$ of Canadians. Circulating 25-hydroxyvitamin D [25(OH)D] levels were obtained for Canadian non-immigrant and first-generation immigrant children aged 6-11 years. In CHMS data, immigrants are not differentiated from refugees. The information on vitamin D measurement in the CHMS is found elsewhere [2]. Descriptive data are presented as mean \pm SEM. A statistically significant difference in vitamin D status among immigrant versus non-immigrant children is indicated by no overlap in 95\% Confidence Intervals [10]. As per Statistics Canada's recommendation, analyses were weighted and bootstrapped to obtain estimates representative of the Canadian population. Degree of freedom was considered 11 due to sampling structure, and alpha was set at 0.05. Data manipulation, cleaning, and creating new variables were done using SPSS 19. All analyses were conducted by STATA SE 11.

\subsection{Study 2. Healthy Immigrant Children (HIC)}

\subsubsection{Study Design and Participants}

In the absence of official data sources that identifies immigration status in Saskatchewan, a convenience sample of study participants $(n=72)$ were recruited with the assistance of several organizations that have regular contact with immigrant and refugees. These organizations include Saskatoon Open Door Society, Saskatchewan Intercultural Association, and ethno-cultural associations. Participant recruitment posters were also placed in accessible public locations including the universities, public libraries, health care facilities and commercial locations. Interpreters were used for 50 (69\%) participants while 22 (31\%) were able to speak English and, therefore, did not require an interpreter.

In a cross-sectional design (2010-2011), health and nutrition measures were collected from immigrant $(n=33)$ and refugee $(n=39)$ children aged $7-11$ years who had been living in Saskatoon, Saskatchewan, Canada for no more than five years. An immigrant is someone who "comes as a permanent resident to a country other than one's native land" [11]. A refugee is someone who "owing to well-founded fear of being persecuted for reasons of race, religion, nationality, membership of a 
particular social group or political opinion", is unable or unwilling to return to his/her country of birth due to fear for their safety [12]. This age range is an important time for bone acquisition and for establishing healthy dietary habits. Ethics approval was obtained from the University of Saskatchewan Biomedical Research Ethics Board (Bio \# 09-197).

\subsubsection{Data Collection}

Data collection for the study included multiple variables. Demographics and socioeconomic status were evaluated using questionnaires from the Canadian Community Health Survey [13]. Food security status was evaluated using the version of the United States Department of Agriculture questionnaire that was modified by Canada [14].

Anthropometric measurements included height, weight and waist circumference. Values were recorded in centimeters to the nearest millimeter. All measurements were taken twice for consistency to help eliminate human error. Percentile Body Mass Index (BMI) was categorized as normal, overweight, or obese according to World Health Organization (WHO) child classifications [15].

To assess physical activity, we used the CHMS children's physical activity questionnaire (CPA). Another variable was derived to examine sedentary activity in hours per day. Based on the CHMS, two questions on sun exposure were also included [9].

Three 24-h dietary recalls were administered to each child to obtain a usual intake. All 24-h recalls were administered at least three weeks apart and conducted in person. The child's caregiver assisted in the dietary assessments. Diet analyses were performed in "The Food Processor Nutrition and Fitness Software version SQL 10.5”, Esha Research, Salem, USA. Foods were categorized into food groups consistent with Eating Well with Canada's Food Guide [16]. To examine the overall nutrition status, we used the Canadian version of the Healthy Eating Index (HEIC) [17]. The HEIC has been validated and incorporates recommendations from Eating Well with Canada's Food Guide [17]. This index categorizes diets into one of three categories, "poor", "needs improvement", and "good" [17].

\subsubsection{Outcome Measures}

Blood samples were taken from 1 May to 30 September 2010 using a finger prick on eight 6-mm spots on a filter card. Circulating $25(\mathrm{OH}) \mathrm{D}$ was analyzed from dried blood spots, using a standard LC-MS/MS assay by ZRT Laboratory which is widely used for measuring 25-Hydroxy Vitamin D2/D3 $[18,19]$. The ZRT Laboratory participates in DEQAS, the Vitamin D Quality Assessment Scheme, which provides control samples to ensure assay accuracy [18,19]. Plasma and blood spot determinations were deemed to be equivalent because of the high stability of $25(\mathrm{OH}) \mathrm{D}$ in serum or plasma [20]. The blood spot card measurements of vitamin D levels are in agreement with serum and whole blood specimen [21]. Recent recommendations by the Institute of Medicine's dietary reference intake (DRI) panel on vitamin D were used to define vitamin D status including less than $30 \mathrm{nmol} / \mathrm{L}$ as deficient, $30-50 \mathrm{nmol} / \mathrm{L}$ as inadequate, and more than $50 \mathrm{nmol} / \mathrm{L}$ as sufficient [22].

Bone mineral status was assessed using Dual-energy X-ray Absorptiometry (DXA), Hologic Inc., Discovery-Wi, Bedford, USA, Serial \#80964. This study focused on Total Body Bone Mineral Content (TBBMC) as the most accurate measure in children [23]. The coefficient of variation for 
TBBMC in our laboratory is $0.5 \%$. Values obtained for TBBMC were compared to estimated normal values for each child's age, sex, and ethnicity based on data from four longitudinal studies [24].

\subsubsection{Statistical Analyses}

Descriptive statistics were analyzed by calculating means and standard deviations of variables of interest as well as the distribution of participants in various categories. We used a two-sided independent Student's $t$-test or non-parametric equivalent (Mann Whitney $U$-test) to investigate differences between refugees and immigrants and between males and females. Pearson's Chi square was used to analyze categorical variables. Finally, multivariate analyses (linear and logistic regression) were conducted to examine the association between variables of interest and health outcomes (vitamin D status, TBBMC), controlling for possible confounders. Analyses were conducted using PASW Statistics 18 by Polar Engineering and Consulting, Chicago, USA. In all analyses, alpha was set at 0.05. All data, when possible, was compared to Canadian published data.

\section{Results}

Descriptive data from CHMS revealed that mean plasma $25(\mathrm{OH}) \mathrm{D}$ concentrations in non-immigrant children was significantly higher than in immigrant children. This difference is over $20 \mathrm{nmol} / \mathrm{L}$ in immigrant girls (Table 1).

Table 1. Plasma 25-hydroxyvitamin D [25(OH)D] (nmol/L) concentrations in immigrant and non-immigrant children aged 6-11 years in Canada ${ }^{1}$.

\begin{tabular}{cc}
\hline Children aged 6-11 years & $\begin{array}{c}\text { Plasma 25-hydroxyvitamin D [25(OH)D] } \\
\text { (Mean } \pm \text { SEM) }\end{array}$ \\
\hline All children $(n=1,817,260)$ & $75.1 \pm 2.4$ \\
Boys & $79.9 \pm 2.0$ \\
Girls & $73.1 \pm 3.0$ \\
Immigrant children $(n=134,833)$ & $61.1 \pm 5.9 *$ \\
Immigrant boys & $69.0 \pm 7.9$ \\
Immigrant girls & $54.1 \pm 4.5 * *$ \\
Non-immigrant children $(n=1,682,427)$ & $76.2 \pm 2.2$ \\
Non-immigrant boys & $77.5 \pm 1.9$ \\
Non-immigrant girls & $74.7 \pm 2.8$ \\
\hline
\end{tabular}

${ }_{1}$ Data from the Canadian Health Measures Survey Cycle 1, 2007-2009. * Significantly lower than non-immigrant children. ** Significantly lower than non-immigrant boys and girls.

In the HIC study, the mean \pm SD age of participants was $8.9 \pm 1.4$ years, with no significant difference between immigrants $(n=33)$ and refugees $(n=39)$. There was also no significant difference in distribution of males $(n=48)$ and females $(n=24)$ according to immigration status. Most immigrant children in the study were from the Middle East (73\%), while refugee children were from various regions including South East Asia, Africa, the Middle East and Latin America in descending order. In 2009, approximately $78 \%$ of newcomers in Saskatchewan were from Asia and the Pacific (59.9\%), Africa and the Middle East (15.4\%) and South and Central America (2.5\%) [25]. 
These regions contribute to around $80 \%$ of immigrants in Canada [26]. The mean length of stay in Canada was $2.6 \pm 1.5$ years. Over $55 \%$ of families were from the lowest income bracket according to Statistics Canada classification.

Table 2 presents general characteristics of participants. Percentile height (mean $\pm \mathrm{SD}$ ) was lower than the 50th percentile with no significant difference between immigrants and refugees. The majority of children were found to have a normal BMI while $22.2 \%$ were overweight and $6.9 \%$ were obese. Results showed $87.5 \%$ of immigrant and refugee newcomer children were obtaining the recommended number of hours per day of physical activity ( $\geq 60 \mathrm{~min}$ ) according to the Canadian Society for Exercise Physiology [27]. However, many participants were spending too much time $(>2 \mathrm{~h} /$ day) in sedentary activities such as time spent watching television or movies, playing video games, or using the computer.

Table 2. General characteristics of healthy immigrant children study participants.

\begin{tabular}{cccc}
\hline Characteristics & $\begin{array}{c}\text { Immigrants } \\
\boldsymbol{n = 3 3 ( 4 5 . 8 \% )}\end{array}$ & $\begin{array}{c}\text { Refugees } \\
\boldsymbol{n}=\mathbf{3 9}(\mathbf{5 4 . 2 \% )})\end{array}$ & $\begin{array}{c}\text { All participants } \\
\boldsymbol{n}=\mathbf{7 2}(\mathbf{1 0 0} \%)\end{array}$ \\
\hline Age (Mean \pm SD) & $8.9 \pm 1.6$ & $8.9 \pm 1.3$ & $8.9 \pm 1.4$ \\
Sex & & & \\
Male & $22(66.7 \%)$ & $26(66.7 \%)$ & $48(66.7 \%)$ \\
Female & $11(33.3 \%)$ & $13(33.3 \%)$ & $24(33.3 \%)$ \\
Length of stay in Canada in years (Mean \pm SD) & $2.6 \pm 1.5$ & $2.5 \pm 1.1$ & $2.5 \pm 1.3$ \\
Height in cm (Mean \pm SD) & $132.6 \pm 13.2$ & $130.3 \pm 10.9$ & $131.4 \pm 12.0$ \\
Percentile Height (Mean \pm SD) & $49.7 \pm 31.1$ & $42.6 \pm 30.8$ & $45.8 \pm 31.0$ \\
Weight in kg (Mean \pm SD) & $32.1 \pm 10.6$ & $29.9 \pm 7.8$ & $30.9 \pm 9.2$ \\
Percentile Weight (Mean \pm SD) & $68.8 \pm 27.4$ & $64.9 \pm 28.8$ & $66.7 \pm 28.0$ \\
Physical activity in h/week (Mean \pm SD) & $13.1 \pm 5.4$ & $12.0 \pm 3.7$ & $12.5 \pm 4.6$ \\
Recommended level $(\geq 60$ min/day) & $29(87.9 \%)$ & $34(87.2 \%)$ & $63(87.5 \%)$ \\
Less than recommended level $(<60$ min/day) & $4(12.1 \%)$ & $5(12.8 \%)$ & $9(12.5 \%)$ \\
\hline
\end{tabular}

Dietary vitamin D and calcium intakes and HEIC are presented in Table 3. Only $24.2 \%$ of participants met recommendations for servings per day of milk and alternatives. The prevalence of calcium intake inadequacy was $76 \%$. Vitamin D intake from food and supplement was $213 \pm 195 \mathrm{IU}$ (mean $\pm \mathrm{SD}$ ) for all participants with a prevalence of inadequacy of $89.4 \%$. Only two children reported taking a vitamin D supplement regularly. Vitamin D intake from food and supplement was significantly higher in immigrants compared to refugees, which accords with the significant difference in serum status (Table 3). There was a significant difference in mean \pm SD HEIC scores between immigrants and refugees at $65.4 \pm 7.7 v s .60 .4 \pm 8.8$, respectively $(p=0.021)$. The majority of participants $(90.9 \%)$ needed to improve their diet. Only one immigrant was classified in "good" quality diet category based on HEIC, while no refugees did. There were also five refugees who had poor diets, while no immigrants did. 
Table 3. Nutrient intake related to bone health.

\begin{tabular}{cccc}
\hline Characteristics & $\begin{array}{c}\text { Immigrants } \\
\boldsymbol{n}=\mathbf{3 3 ( 4 5 . 8 \% )}\end{array}$ & $\begin{array}{c}\text { Refugees } \\
\boldsymbol{n}=\mathbf{3 9}(\mathbf{5 4 . 2} \%)\end{array}$ & $\begin{array}{c}\text { All participants } \\
\boldsymbol{n}=\mathbf{7 2}(\mathbf{1 0 0} \%)\end{array}$ \\
\hline Vitamin D intake in IU/day (Mean $\pm \mathrm{SD})$ & $249 \pm 247$ & $181 \pm 130$ & $213 \pm 195$ \\
Prevalence of vitamin D intake inadequacy $[n(\%)]$ & $25(81 \%)$ & $34(97 \%)$ & $59(89 \%)$ \\
Calcium intake in mg & $764 \pm 393$ & $676 \pm 380$ & $718 \pm 386$ \\
Prevalence of calcium intake inadequacy $[n(\%)]$ & $23(74 \%)$ & $27(77 \%)$ & $50(76 \%)$ \\
$\quad$ Milk and alternatives & & & \\
Mean intake (servings/day) & $1.9 \pm 1.2$ & $1.6 \pm 1.1$ & $1.7 \pm 1.2$ \\
Meeting Canada's Food Guide recommendations & $10(32.3 \%)$ & $6(17.1 \%)$ & $16(24.2 \%)$ \\
Healthy Eating Index Canada & & & \\
Mean Score & $65.4 \pm 7.7 *$ & $60.4 \pm 8.8$ & $62.7 \pm 8.6$ \\
$\quad$ Good Diet & $1(3.2 \%)$ & $0(0.0 \%)$ & $1(1.5 \%)$ \\
Diet Needs Improvement & $30(96.8 \%)$ & $30(85.7 \%)$ & $60(90.9 \%)$ \\
Poor Diet & $0(0.0 \%)$ & $5(14.3 \%)$ & $5(7.6 \%)$ \\
\hline
\end{tabular}

* Significantly different from refugee children, $t$-test $(P<0.05)$.

Total serum 25(OH)D (nmol/L) was significantly higher in immigrant compared to refugee children $(p=0.021$ ) (Table 4). Overall, 29\% of participants were vitamin D deficient and another $44 \%$ had inadequate levels of serum 25(OH)D for bone health [22]. Children spent $3.6 \pm 1.6 \mathrm{~h} /$ day (mean $\pm \mathrm{SD}$ ) in the sun in the summer months during peak times (11:00 am-4:00 pm), which was consistent across immigration status. Very few children regularly applied sunscreen, whereas more than half of children never did (Table 4). In linear regression analyses, after controlling for possible confounders including total caloric intake, age, sunscreen use, total hours spent in the sun in the summer months during peak times, and calcium intake; dietary vitamin D intake (Standardized Regression Coefficient: $0.46 \pm 0.11, p<0.001$ ), sex (Standardized Regression coefficient: $-0.39 \pm 0.11$, $p<0.001$ ), region of origin (Standardized Regression coefficient: $0.24 \pm 0.11, p=0.027$ ), and length of stay in Canada (Standardized Regression Coefficient: $-0.23 \pm 0.11, p=0.04$ ) were found to be significant predictors of serum vitamin D status. Females - those who had been living in Canada longer, those from regions with darker skin pigmentation, and those with lower vitamin D intake-were at greater risk of low circulating vitamin D. 
Table 4. Vitamin D status of newcomer children.

\begin{tabular}{|c|c|c|c|}
\hline Characteristics & $\begin{array}{c}\text { Immigrants } \\
n=33(45.8 \%)\end{array}$ & $\begin{array}{c}\text { Refugees } \\
n=39(54.2 \%)\end{array}$ & $\begin{array}{c}\text { All participants } \\
n=72(100 \%)\end{array}$ \\
\hline $\mathrm{H} /$ day spent in the sun during peak times $($ Mean $\pm \mathrm{SD})$ & $3.6 \pm 1.7$ & $3.5 \pm 1.5$ & $3.6 \pm 1.6$ \\
\hline \multicolumn{4}{|l|}{ Sunscreen use } \\
\hline always & $0(0.00 \%)$ & $2(5.1 \%)$ & $2(2.8 \%)$ \\
\hline often & $4(12.1 \%)$ & $0(0.00 \%)$ & $4(5.6 \%)$ \\
\hline sometimes & $5(15.2 \%)$ & $10(25.6 \%)$ & $15(20.8 \%)$ \\
\hline rarely & $3(9.1 \%)$ & $5(12.8 \%)$ & $8(11.1 \%)$ \\
\hline never & $21(63.6 \%)$ & $22(56.4 \%)$ & $43(59.7 \%)$ \\
\hline Total serum vitamin $\mathrm{D}$ in $\mathrm{nmol} / \mathrm{L}($ Mean $\pm \mathrm{SD})$ & $45.7 \pm 13.9 *$ & $37.8 \pm 15.5$ & $41.2 \pm 15.2$ \\
\hline Deficient and inadequate $<50 \mathrm{nmol} / \mathrm{L}$ & $19(63.3 \%)$ & $31(79.5 \%)$ & $50(72.5 \%)$ \\
\hline Sufficient $\geq 50 \mathrm{nmol} / \mathrm{L}$ & $11(36.7 \%)$ & $8(20.5 \%)$ & $19(27.5 \%)$ \\
\hline $\begin{array}{l}\text { Total body bone mineral content }(\mathrm{TBBMC}) \\
\text { in grams }(\text { Mean } \pm \mathrm{SD})\end{array}$ & $984.9 \pm 245.0$ & $947.8 \pm 208.8$ & $964.6 \pm 224.9$ \\
\hline Low TBBMC & $13(41.9 \%)$ & $14(35.9 \%)$ & $27(38.6 \%)$ \\
\hline
\end{tabular}

* Significant difference from refugees; for means a $t$-test was used and for categorical variables chi square was used.

TBBMC was low in $38.6 \%$ of participants compared to estimated values for age, sex, and ethnicity [24]. In the regression model, after controlling for possible confounders, including immigration status; food security; age; sex; region of origin; physical activity level; total caloric intake; and intakes of calcium, magnesium, phosphorus, sodium, and caffeine, height and serum vitamin D status were found to be determinants of TBBMC (Table 5). Children who were taller and had greater serum vitamin D also had greater TBBMC.

Table 5. Factors associated with total body bone mineral content (TBBMC) in regression analysis (using the stepwise procedure) among all subjects $(n=56)$.

\begin{tabular}{|c|c|c|c|c|c|c|}
\hline \multirow{2}{*}{$\begin{array}{l}\text { Outcome } \\
\text { variable }\end{array}$} & \multirow{2}{*}{ Constant } & \multicolumn{4}{|c|}{ Regression coefficient } & \multirow[t]{2}{*}{ Total $R^{2}$} \\
\hline & & Height (cm) & \multicolumn{2}{|c|}{ Serum vitamin D (nmol/L) } & & \\
\hline TBBMC & -1257.33 & $0.95 \pm 0.06$ & \multicolumn{2}{|c|}{$0.13 \pm 0.06$} & & \multirow[t]{3}{*}{0.82} \\
\hline Partial $R^{2}$ & & 0.90 & \multicolumn{2}{|c|}{0.27} & & \\
\hline$p$-value & & $<0.001$ & \multicolumn{2}{|c|}{0.047} & & \\
\hline \multirow[b]{2}{*}{$\begin{array}{l}\text { Outcome } \\
\text { variable }\end{array}$} & \multicolumn{6}{|c|}{ Excluded variables } \\
\hline & Sex & $\begin{array}{l}\text { Region of } \\
\text { origin }\end{array}$ & Age & Calcium (mg) & $\begin{array}{c}\text { Calories } \\
\text { (kcal) }\end{array}$ & $\begin{array}{c}\text { Immigration } \\
\text { Status }\end{array}$ \\
\hline TBBMC & 0.01 & -0.09 & -0.12 & 0.07 & 0.07 & 0.05 \\
\hline Partial $R^{2}$ & 0.03 & -0.20 & -0.17 & 0.13 & 0.14 & 0.12 \\
\hline$p$-value & 0.832 & 0.147 & 0.227 & 0.353 & 0.323 & 0.408 \\
\hline \multirow{2}{*}{$\begin{array}{l}\text { Outcome } \\
\text { variable }\end{array}$} & \multicolumn{6}{|c|}{ Excluded variables } \\
\hline & $\begin{array}{c}\text { H/week in } \\
\text { physical activities }\end{array}$ & $\begin{array}{c}\text { Food } \\
\text { security }\end{array}$ & $\begin{array}{l}\text { Magnesium } \\
\text { (mg) }\end{array}$ & $\begin{array}{l}\text { Phosphorous } \\
\text { (mg) }\end{array}$ & $\begin{array}{l}\text { Sodium } \\
\text { (mg) }\end{array}$ & $\begin{array}{c}\text { Caffeine } \\
\text { (mg) }\end{array}$ \\
\hline TBBMC & 0.02 & 0.11 & 0.06 & 0.06 & -0.04 & -0.03 \\
\hline Partial $R^{2}$ & 0.03 & 0.23 & 0.11 & 0.11 & -0.08 & -0.06 \\
\hline$p$-value & 0.805 & 0.095 & 0.419 & 0.434 & 0.574 & 0.660 \\
\hline
\end{tabular}




\section{Interpretation}

The HIC study is the first to evaluate vitamin D status of newcomer immigrant and refugee children aged 7-11 years in Canada. Data from a nationally representative sample showed alarmingly low 25(OH)D levels in immigrant children, particularly girls. HIC also showed a rate of $25(\mathrm{OH}) \mathrm{D}$ deficiency among newcomer immigrant and refugee children. Among these growing children, after controlling for biological and environmental co-factors, serum vitamin D was a significant predictor of TBBMC.

Using CHMS data, we previously reported Canadian children 6-11 years had higher 25(OH)D concentrations than adolescents and adults [2]. However, non-White Canadians were less likely to achieve recommended levels. This same study showed first generation immigrant children aged 6-11 years, particularly girls, had significantly lower 25(OH)D compared to their non-immigrant counterparts. Many newcomers have darker skin pigmentation and are, therefore, at greater risk for deficiency as melanin in the skin prevents the body from synthesizing vitamin D [28]. It is likely that females were at greater risk of deficiency than males due to cultural practices associated with greater covering of females causing insufficient skin exposure to the sun even though they may have been outdoors [28].

In the absence of comprehensive data on dietary intakes and other health measures in CHMS, specifically in newcomer children, data from HIC provides more insight into health concerns faced by this population. Many newcomer children, particularly refugees, did not have a good quality diet including dietary sources of vitamin D. However, vitamin D intake was still the main predictor of serum vitamin D. Circulating vitamin D of $75 \mathrm{nmol} / \mathrm{L}$ is considered beneficial for multiple health outcome [29]. None of the HIC participants met the 25(OH)D level of $75 \mathrm{nmol} / \mathrm{L}$ and over $29 \%$ were vitamin D deficient. This occurred even though non-Caucasian immigrant and refugee children spent almost four hours a day outside during peak times in summer months and the majority of participants rarely or never used sunscreen. It is more difficult to get the necessary vitamin D in areas of high latitude, especially during winter months [3-6]. This may explain why those who had been in Canada longer were at greater risk of deficiency; vitamin D stores are depleted each winter and not replenished enough in the summer. Girls, children with low vitamin D intake, those from regions with darker skin, and those with longer duration of stay in Canada were at higher risk of having inadequate/deficient levels of serum vitamin D.

Since bone mineral mass decreases with age, research has emphasized the importance of achieving optimal bone mineral accrual during childhood and adolescence, which will decrease skeletal-related health issues later in life [30-33]. Therefore, meeting requirements for vitamin D and calcium in these critical ages is crucial [34,35]. Most newcomer children in this study were not getting the recommended amounts of vitamin D and dietary calcium for bone health. Low intakes of calcium and vitamin D accord with low intakes of milk and alternatives, the main dietary sources of these two important nutrients [35]. Inadequate levels impede proper bone growth, which can result in stunting and increase the risk of developing osteoporosis in the future $[4,5,22]$. Serum vitamin D was a significant predictor of TBBMC in this study. TBBMC was lower than predicted values for age, sex and ethnicity in over $38 \%$ of participants. A recent Canadian study found significantly higher 
TBBMC values in Caucasian compared to Asian children [36]. There was no significant difference in TBBMC according to immigration status in HIC. Further research with a larger sample size is needed to examine TBBMC in children according to ethnicity.

The relatively small sample size in the HIC study may limit the conclusions. However, the accordance of HIC findings on serum vitamin D status of immigrant children with those from the CHMS confirms the importance of HIC results. In the absence of a large-scale comprehensive study on the nutrition and health status of newcomer children, our data could provide valuable insight into this area. HIC also distinguishes refugees from immigrants. Self-reported data on dietary intake using a 24-h recall is subject to under-reporting/over-reporting and omission of frequently forgotten items. To maximize accuracy of the data, we used three 24-h recalls where our expert research personnel used proper probing and assessment aids such as food model booklets, measuring cups and spoons, and color pictures for various food items to help recall names of food items. The premise of the study is to investigate and document disparities in and between host and mobile populations that reflect biological, social and behavioral differences between these populations. Defining these differences can lead to greater awareness of their existence in health providers who may appreciate them and assist those developing programs and policies to identify and mitigate preventable outcomes. Once a framework of existing disparities is defined, further investigation to explore and delineate specific co-founding factors can be undertaken.

\section{Conclusions}

In addition to the beneficial effects of vitamin $\mathrm{D}$ on bone, recent data on the association between vitamin D and chronic diseases including certain types of cancers, diabetes, and multiple sclerosis, demonstrate the importance of this nutrient in growing children [34,35]. Although more research is warranted, a considerably high rate of vitamin D intake inadequacy and serum deficiency/inadequacy in newcomer children is already associated with bone mineral mass during pre-adolescence. This, therefore, requires preventive interventions to minimize the risk of serious vitamin $\mathrm{D}$ related diseases.

\section{References}

1. Wacker, M.; Holick, M.F. Vitamin D-effects on skeletal and extraskeletal health and the need for supplementation. Nutrients 2013, 5, 111-148.

2. Whiting, S.J.; Langlois, K.A.; Vatanparast, H.; Greene-Finestone, L.S. The vitamin D status of Canadians relative to the 2011 Dietary Reference Intakes: An examination in children and adults with and without supplement use. Am. J. Clin. Nutr. 2011, 94, 128-135.

3. Grant, W.B.; Holick, M.F. Benefits and requirements of vitamin D for optimal health: A review. Altern. Med. Rev. 2005, 10, 94-111.

4. Hintzpeter, B.; Scheidt-Nave, C.; Muller, M.J.; Schenk, L.; Mensink, G.B. Higher prevalence of vitamin D deficiency is associated with immigrant background among children and adolescents in Germany. J. Nutr. 2008, 138, 1482-1490. 
5. Mithal, A.; Wahl, D.A.; Bonjour, J.P.; Burckhardt, P.; Dawson-Hughes, B.; Eisman, J.A.; El-Haji Fuleihan, G.; Josse, R.G.; Lips, P.; Morales-Torres, J. Global vitamin D status and determinants of hypovitaminosis D. Osteoporos. Int. 2009, 20, 1807-1820.

6. Van der Meer, I.M.; Karamali, N.S.; Boeke, A.J.; Lips, P.; Middelkoop, B.J.; Verhoeven, I.; Wuister, J.D. High prevalence of vitamin D deficiency in pregnant non-Western women in The Hague, Netherlands. Am. J. Clin. Nutr. 2006, 84, 350-353.

7. Ward, L.M.; Gaboury, I.; Ladhani, M.; Zlotkin, S. Vitamin D-decifiency rickets among children in Canada. CMAJ 2007, 177, 161-166.

8. Roth, D.E. Bones and beyond: An update on the role of vitamin D in child and adolescent health in Canada. Appl. Physiol. Nutr. Metab. 2007, 32, 770-777.

9. Statistics Canada. Canadian Health Measures Survey (Detailed information for Spring 2007 to Spring 2009 (Cycle 1)), 2010. Available online: http:/www.statcan.gc.ca/cgi-bin/imdb/ p2SV.pl?Function $=$ getSurvey $\&$ SDDS $=5071 \&$ lang $=\mathrm{en} \& \mathrm{db}=\mathrm{imdb} \& \mathrm{adm}=8 \& \mathrm{dis}=2$ (accessed on 30 August 2012).

10. Health Canada. Canadian Community Health Survey Cycle 2.2, Nutrition (2004) - A Guide to Accessing and Interpreting the Data. Available online: http://www.hc-sc.gc.ca/fn-an/alt_formats/ hpfb-dgpsa/pdf/surveill/cchs-guide-escc-eng.pdf (accessed on 25 August 2012).

11. Immigration. In Canadian Oxford Dictionary; Oxford University Press: New York, NY, USA, 2011. Available online: http://www.oxfordreference.com/views/SEARCH_RESULTS.html? $\mathrm{y}=14 \& \mathrm{q}=$ immigrate $\&$ category $=\mathrm{t} 150 \& \mathrm{x}=4 \& \mathrm{ssid}=865872107 \&$ scope $=$ book \& time $=0.83025998$ 3485593 (accessed on 15 August 2012).

12. United Nations High Commissioner for Refugees. Convention Relating to the Status of Refugees Article, 1951. Available online: http://www2.ohchr.org/English/law/refugees.htm\#wp 1037003 (accessed on 15 August 2012).

13. Statistics Canada. Master and share files derived variables documentation, 2008. Available online: http://www.statcan.gc.ca/imdb-bmdi/document/5049_D11_T9_V1-eng.pdf (accessed on 27 August 2012).

14. Health Canada. Canadian Community Health Survey, Cycle 2.2, Nutrition (2004)-Income-related household food security in Canada. Ottawa (ON): Office of Nutrition Policy and Promotion Health Products and Food Branch, 2007. Available online: http://www.hc-sc.gc.ca/fnan/alt_formats/hpfb-dgpsa/pdf/surveill/income_food_sec-sec_alim-eng.pdf (accessed on 19 July 2012).

15. World Health Organization. Growth reference 5-19 years, 2007. Available online: http://www. who.int/growthref/en/ (accessed on 19 July 2012).

16. Health Canada. Eating well with Canada's food guide, 2007. Available online: http://www. hc-sc.gc.ca/fn-an/food-guide-aliment/index-eng.php (accessed on 11 June 2012).

17. Garriguet, D. Diet quality in Canada. Health Rep. 2009, 20, 41-52.

18. Eyles, D.; Anderson, C.; Ko, P.; Jones, A.; Thomas, A.; Burne, T.; Mortensen, P.B.; Norgaard-Pedersen, B.; Hougaard, D.M.; McGrath, J. A sensitive LC/MS/MS assay of $25 \mathrm{OH}$ vitamin D3 and 25OH vitamin D2 in dried blood spots. Clin. Chim. Acta 2009, 403, 145-151. 
19. Newman, M.S.; Brandon, T.R.; Groves, M.N.; Gregory, W.L.; Kapur, S.; Zava, D.T. A liquid chromatography/tandem mass spectrometry method for determination of 25-hydroxi vitamin D2 and 25-hydroxy vitamin D3 in dried blood spots: A potential adjunct to diabetes and cardiometabolic risk screening. J. Diabetes Sci. Technol. 2009, 3, 156-162.

20. Hollis, B.W. Measuring 25-hydroxyvitamin D in a clinical environment: Challenges and needs. Am. J. Clin. Nutr. 2008, 88, 507S-510S.

21. Larkin, E.K.; Gebretsadik, T.; Koestner, N.; Newman, M.S.; Liu, Z.; Carroll, K.N.; Minton, P.; Woodward, K.; Hartert, T.V. Agreement of blood spot card measurements of vitamin D levels with serum, whole blood specimen types and a dietary recall instrument. PLoS One 2011, $6, \mathrm{e} 16602$.

22. Institute of Medicine. Dietary reference intakes for calcium and vitamin D; The National Academies Press: Washington, DC, USA, 2010. Available online: http://www.iom.edu/Reports/ 2010/Dietary-Reference-Intakes-for-Calcium-and-Vitamin-D.aspx (accessed on 27 August 2012).

23. Fewtrell, M.S. Bone densitometry in children assessed by dual X ray absorptiometry: Uses and pitfalls. Arch. Dis. Child. 2003, 88, 795-798.

24. Baxter-Jones, A.D.; Burrows, M.; Bachrach, L.K.; Lloyd, T.; Petit, M.; Macdonald, H.; Mirwald, R.L.; Bailey, D.; McKay, H. International longitudinal pediatric reference standards for bone mineral content. Bone 2009, 46, 208-216.

25. Government of Saskatchewan. Saskatchewan Statistical Immigration Report 2009, 2008. Available online http://aeel.gov.sk.ca/sk-immigration-statistical-report-2009 (accessed on 23 August 2012).

26. Citizenship and Immigration Canada facts and figures 2011. Available online: http://www. cic.gc.ca/english/pdf/research-stats/facts2011.pdf (accessed on 23 August 2012).

27. Canadian Society for Exercise Physiology, Communique, March Newsletter. Available online: http://www.csep.ca/english/view.asp? $x=804$ (accessed on 27 August 2012).

28. Nellen, J.F.; Smulders, Y.M.; Frissen, P.H.; Slaats, E.H.; Silberusch, J. Hypovitaminosis D in immigrant women: Slow to be diagnosed. BMJ 1996, 312, 570-572.

29. Wimalawansa, S.J. Vitamin D in the new millennium. Curr. Osteoporos. Rep. 2012, 10, 4-15.

30. Caradonna, P.; Rigante, D. Bone health as a primary target in the pediatric age. Eur. Rev. Med. Pharmacol. Sci. 2009, 13, 117-128.

31. Nichols, D.L.; Sanborn, C.F.; Essery, E.V.; Clark, R.A.; Letendre, J.D. Impact of curriculum-based bone loading and nutrition education program on bone accrual in children. Pediatr. Exerc. Sci. 2008, 20, 411-425.

32. Vatanparast, H.; Baxter-Jones, A.; Faulkner, R.A.; Bailey, D.A.; Whiting, S.J. Positive effects of vegetable and fruit consumption and calcium intake on bone mineral accrual in boys during growth from childhood to adolescence: The University of Saskatchewan Pediatric Bone Mineral Accrual Study. Am. J. Clin. Nutr. 2005, 82, 700-706.

33. Bailey, D.A.; Faulkner, R.A.; McKay, H.A. Growth, physical activity, and bone mineral acquisition. Exerc. Sport Sci. Rev. 1996, 24, 233-266.

34. Whiting, S.J.; Calvo, M.S. Dietary recommendations for vitamin D: A critical need for functional end points to establish an estimated average requirement. J. Nutr. 2005, 135, 304-309. 
35. Holick, M.F. Health benefits of vitamin D and sunlight: A D-bate. Nat. Rev. Endocrinol. 2011, 7, 73-75.

36. Burrows, M.; Baxter-Jones, A.; Mirwald, R.; Macdonald, H.; McKay, H. Bone mineral accrual across growth in a mixed-ethnic group of children: Are Asian children disadvantaged from an early age? Calcif. Tissue Int. 2009, 84, 366-378. 


\title{
The ABC of Vitamin D: A Qualitative Study of the Knowledge and Attitudes Regarding Vitamin D Deficiency amongst Selected Population Groups
}

\author{
Billie Bonevski, Jamie Bryant, Sylvie Lambert, Irena Brozek and Vanessa Rock
}

\begin{abstract}
Objective: In Australia, vitamin D supply in food is limited, and sun exposure is the main source of vitamin D. However skin cancer risk is high, and the need to gain some sun exposure for adequate vitamin $\mathrm{D}$ is challenging public health messages to use protection in the sun. The complex vitamin D public health message may be confusing the public and, in particular, those at highest risk for vitamin D deficiency. This study explored vitamin D and sun exposure attitudes, knowledge and practices of some groups considered at risk of vitamin D deficiency and those delivering healthy sun exposure messages to children. Method: 52 adults participated in six focus groups. Results: Results corroborated with previous research showing low levels of vitamin D knowledge. Individual and environmental barriers to receiving adequate sun exposure were also identified. Conclusions and Implications: The message advocating balanced sun exposure to produce adequate vitamin $\mathrm{D}$ needs to be made clearer and be more effectively communicated. Findings provide insights to aid development of appropriate public health messages for safe sun exposure and vitamin D, especially for vulnerable groups.
\end{abstract}

Reprinted from Nutrients. Cite as: Bonevski, B.; Bryant, J.; Lambert, S.; Brozek, I.; Rock, V. The ABC of Vitamin D: A Qualitative Study of the Knowledge and Attitudes Regarding Vitamin D Deficiency amongst Selected Population Groups. Nutrients 2013, 5, 915-927.

\section{Introduction}

While there continues to be uncertainty about recent claims of vitamin D's preventive role with some types of cancers [1,2], autoimmune disorders (such as multiple sclerosis) and possibly cardiovascular diseases [2] and diabetes [3], its essential benefits in the normal development and maintenance of bone health $[2,4]$ have been long known.

In Australia, vitamin D content in food is almost non-existent, and it is predominantly gained from exposure to ultraviolet (UV) sun exposure. Because of its relationship to UV exposure, vitamin D status is associated with geography. There is greater insufficiency of vitamin D at high latitudes, like northern European countries [5-7], but it is also found at low latitudes, like Australia, where UV levels are generally high and rates of skin cancer are amongst the highest in the world [8,9]. This co-occurrence of vitamin D insufficiency and skin cancer is puzzling and has led to the development of guidelines for a balanced approach to sun exposure for both the public [10,11] and health professionals [12]. The balance message suggests some sunlight exposure each day for adequate vitamin D production, but not so much that would lead to increased skin cancer risk.

Population groups at higher risk of vitamin D deficiency include the elderly living in residential care $(22 \%-86 \%[13,14])$, dark skinned and veiled pregnant women (80\% [15]), individuals with hip fracture (63\% deficiency [16]) and those who cover their skin for religious reasons [17]. 
Findings from Australian surveys suggest that there is a limited awareness and understanding about vitamin D in the general community [18]. For example, $80 \%$ of participants in a community survey were unable to name a health benefit of adequate vitamin D and $15 \%$ were unable to give an estimate of the amount of sun exposure needed for vitamin D maintenance [18].

Successful communication of a health message has been associated with changes in people's beliefs about and attitudes toward a risky behaviour and, in turn, changes in that behaviour [19] and the need for messages to be consistent has been highlighted [20,21]. The current vitamin D and sun "balance" message, however, is complex requiring an up-to-date understanding of factors, such as personal skin type, amount of sun exposure, time of day and UV rating, season, latitude and clothing worn [10-12]. While broad community surveys provide valuable prevalence data on the attitudes, knowledge and actions of populations, they often fail to capture more subtle influential factors. For example, is uncertainty regarding amount of sun exposure required for adequate vitamin D [18], due to the sometimes contradictory vitamin D and sun "balance" messages or other factors? Qualitative methods can address these types of issues in the development of public health messages providing a deeper understanding behind reasons for low knowledge or misperceptions. Specifically, focus groups provide an understanding of a target group's motivations, environments, belief systems and health practices [22-24]. To date, no qualitative research exploring these constructs with people with increased risk of vitamin D deficiency or individuals responsible for delivering healthy sun exposure messages to children (teachers) has been published. This study aimed to explore vitamin D and sun exposure attitudes, knowledge and practices of the selected populations using focus groups.

\section{Methods}

\subsection{Study Design}

Qualitative focus groups $(n=6)$ were conducted in November 2010, in Sydney, Australia. The Consolidated Criteria for Reporting Qualitative Research framework was used to guide the reporting of the findings [25].

Two health behaviour theories-Social Cognitive Theory [26] and The Health Belief Model [27] — informed sampling, development of the interview guide and analysis. Social Cognitive Theory suggests that behaviour is influenced by social and physical environments, along with the features of the behaviour [26]. The Health Belief Model specifies that individuals adopt a health protective behaviour (e.g., sun protection for skin cancer or sun exposure for vitamin D), to the extent that they perceive themselves to be susceptible to a health threat (i.e., skin cancer or deficiency), perceive the threat to be severe, perceive the benefits of the proposed health action for mitigating the threat and can overcome perceived barriers to the health behaviour.

\subsection{Sample \& Recruitment}

Participants were English-speaking adults aged over 18 years, living in Sydney, Australia. Purposive sampling ensured participants were recruited from the three groups of interest: (1) teachers (two groups - primary and secondary); (2) office workers (two groups); and (3) elderly (two groups - community dwelling and those in residential aged care facilities). 
Recruitment of participants was conducted by an accredited recruitment agency-Stable Research. Participants were recruited from pre-existing registers and supplemented by additional methods (e.g., contacting local aged care facilities). Equal numbers of males and females were targeted across groups. Four focus groups were held in a location with a high migrant population to increase the cultural mix of the sample. "Office workers" were defined as those working in an indoor office at least four days a week. "Community living elderly" were persons aged 65 years living independently and "Elderly living in aged care" were persons aged 65 years and over living in an aged care facility or assisted retirement village.

\subsection{Procedure}

An independent social market research organisation (IPSOS-Eureka) with experience in qualitative research was engaged to conduct the focus groups. Groups were conducted in four locations across Sydney. Following initial telephone contact by the recruitment agency to determine interest in participating, each participant was mailed an information statement and consent form prior to attending the group discussions. All focus groups were recorded. One of the authors was an observer in the focus groups (IB). All participants were offered $\$ 80$ reimbursement for time and travel expenses. Ethics approval was granted by the University of Newcastle Human Ethics Research Committee.

\subsection{Discussion Guide Content}

Each focus group was led by an experienced moderator who used a discussion guide to focus the discussion. Informed by theoretical models outlined above, the discussion guide included items within the broad domains of: knowledge of vitamin D; awareness of vitamin D message; barriers to receiving adequate sun exposure; and communicating the vitamin $\mathrm{D}$ message.

\subsection{Data Coding and Analysis}

Audio recordings were transcribed verbatim. Transcripts were coded by two independent coders (JB \& SL) using NVivo version 8 [28]. Each transcript was reviewed line-by-line, and through inductive reasoning, words, statements and paragraphs related to the broad domains of the interview guide were extracted. Through this in-depth analysis, similar excerpts were identified using the same label or code [29]. Codes were either single words (e.g., "food", "sun") or short phrases (e.g., "balance between sun exposure and protection") that captured the essence of the excerpts. Codes were grouped under broad domains of the discussion guide and theoretical constructs (e.g., personal susceptibility to health effects of vitamin D deficiency). Where appropriate, sub-categories were developed to further describe the categories. Three of the six focus groups were analysed by two coders (JB and SL), with discrepancies in coding discussed until a kappa of $>0.6$ was achieved across $75 \%$ of central nodes. The remaining transcripts $(n=3)$ were coded independently by one coder (JB or SL). 


\section{Results}

Fifty-two participants (23 males, 29 females) took part in six focus groups. Groups contained seven to nine participants and ranged from 1 to $1.5 \mathrm{~h}$ in duration. Whilst we aimed to include approximately equal numbers of males and females in each group, the group with primary school teachers included only one male participant (see Table 1).

Table 1. Focus group schedule.

\begin{tabular}{llcc}
\hline & Participants & Male N & Female N \\
\hline Group 1 & Office workers & 4 & 5 \\
Group 2 & Independent living adults (65 years + ) & 5 & 4 \\
Group 3 & Office workers & 4 & 5 \\
Group 4 & Community aged home residents (65 years + ) & 4 & 5 \\
Group 5 & Primary School teachers & 1 & 6 \\
Group 6 & Secondary School teachers & 5 & 4 \\
\hline
\end{tabular}

\subsection{Knowledge}

\subsubsection{General Vitamin D Knowledge}

Most participants felt they knew less about the benefits or role of Vitamin D in comparison to other vitamins. This was mainly attributed to comparatively limited media attention given to vitamin D compared to other vitamins, such as vitamin C or B. Many participants presumed vitamin D had to be essential and offer some health benefits, but few could name what these were.

\subsubsection{Sources of Information about Vitamin D}

Several participants could not recall a specific source of information for their knowledge of vitamin D. Sources of information on vitamin D mentioned by participants included (in descending order):

- Media: articles in newspapers, magazines and current affairs programs;

- Doctors: some participants (primarily with a deficiency in vitamin D) had learnt about vitamin D from their doctor, although often information they had received was limited;

- Family members/friends: few participants mentioned that they had heard about vitamin D through family members, friends or significant others;

- School and further education: a small number of participants said they had learnt what they know from school or further education.

\subsubsection{Vitamin D Testing and Education}

Most participants did not know whether their vitamin D level had ever been tested or assumed it had been tested as part of a blood test for a range of things.

"... I have a cholesterol test usually at least once a year, but I have got no idea whether there is a vitamin D component in that." (Independent aged) 
Most participants tested and found to be low in vitamin D did not recall being told by their doctor why it was important, the consequences of inadequate vitamin D or how much sun was needed each day to ensure adequate vitamin D. Rather, it was common for participants to report that they had simply been told they "need to get out in the sun more" or advised to take a supplement.

"No, he [doctor] didn't [explain why Vitamin D was important], he just recommended [...] I take Caltrac with vitamin D. That was it." (Office worker)

\subsection{Awareness}

\subsubsection{Knowledge of Times and Seasons for Sun Protection}

Many participants identified mid-day or the hottest time of the day as the critical period when sun protection is needed.

Early morning (e.g., before 10:00) or late afternoon (e.g., after 15:00) was perceived as the ideal time to spend time in the sun, because "you still get the sunshine, but you are not getting it as intense." (Primary school teacher).

\subsubsection{Knowledge about Amount of Sun Exposure Required for Adequate Vitamin D}

Many participants admitted they were unsure of how long was needed to be in the sun for adequate vitamin D. There was a tendency however to overestimate the time required in summer, with 15-20 min being the most common response. Participants identified that time needed in the sun to get enough vitamin D might vary according to age, skin colour/type and nutrition. Discussion about the amount of sun exposure raised many questions regarding the impact of clothing and sunscreen.

"How much exposure, too, I mean, I was out in the sun yesterday with arms exposed. I mean, is that the same as, do I need 20 min of that, whereas I can stay outside in the nude for three minutes?" (Primary school teacher)

\subsubsection{Knowledge about Groups at Higher Risk of Vitamin D Deficiency}

Participants identified groups that may be at increased risk of vitamin D deficiency in Australia, including; the elderly or individuals who might have difficulty getting outside (e.g., immobile due to disability), workers confined to an office during the day, shift workers and those who cover their skin for religious reasons.

\subsection{Personal Behaviours and Perceived Risk}

Personal UV Exposure for Adequate Vitamin D

Current guidelines for vitamin D were communicated to participants (see Table 2), and most felt they were getting adequate sunlight in the summer months on most days of the week. Most school teachers and office workers thought they would easily meet the recommendations on most days in summer, spring and autumn, largely through incidental exposure. While most adults over 65 also 
thought they would meet recommendations on most days, this was often dependent on the weather. Several participants were surprised at the length of exposure needed in winter, and most reported that they would not meet recommendations in winter.

"No. I don't think in winter I would in a day. Especially when you have a week, like days of rain and ... I don't think in winter." (Primary school teacher)

Table 2. Summary of key Australian guidelines for sun exposure for vitamin D sufficiency for the general population (moderate fair skin) and people at high risk of vitamin $\mathrm{D}$ deficiency.

\begin{tabular}{|c|c|}
\hline & General Population \\
\hline a & $\begin{array}{l}\text { Fair skinned people can achieve adequate vitamin D levels ( }>50 \mathrm{nmol} / \mathrm{L}) \text { in summer by exposing the } \\
\text { face, arms and hands or the equivalent area of skin to a few minutes of sunlight on either side of peak } \\
\text { UV periods on most days of the week. } \\
\text { In winter, in the southern regions of Australia, where UV radiation levels are less intense, maintenance } \\
\text { of vitamin D levels may require } 2-3 \mathrm{~h} \text { of sunlight exposure to the face, arms and hands or equivalent } \\
\text { area of skin over a week. }\end{array}$ \\
\hline \multirow[t]{2}{*}{$\mathrm{b}$} & $\begin{array}{l}\text { In Sydney, in December to January (Australian summer), } 6 \text { to } 8 \text { min at } 10 \text { am or } 2 \mathrm{pm} \text {. } \\
\text { In Sydney, in July to August (Australian winter), } 26 \text { to } 28 \mathrm{~min} \text { at } 10 \text { am or } 2 \mathrm{pm} \text { or } 16 \mathrm{~min} \text { at } 12 \text { noon. }\end{array}$ \\
\hline & People at high risk of vitamin D deficiency \\
\hline $\mathrm{a}$ & $\begin{array}{l}\text { Naturally dark skinned people (Fitzpatrick skin type } 5 \text { and } 6 \text { ) are relatively protected from skin cancer } \\
\text { by the pigment in their skin; they could safely increase their sun exposure. Other people at high risk of } \\
\text { vitamin D deficiency should discuss their vitamin D status with their medical practitioner, as some } \\
\text { might benefit from dietary supplementation with vitamin D. }\end{array}$ \\
\hline $\mathrm{b}$ & Vitamin D supplementation is likely to be required for this population group. \\
\hline & $\begin{array}{l}\text { a The Risks and Benefits of Sun Exposure Position Statement. Approved by the Australian and New } \\
\text { Zealand Bone and Mineral Society, Osteoporosis Australia, The Australasian College of Dermatologists }\end{array}$ \\
\hline
\end{tabular}

Communication of the guidelines prompted questions from participants seeking more detail about the type of exposure needed. Participants were unsure how sunscreen and protective clothing affected the absorption of vitamin D and whether it is possible to "store" vitamin D by having longer period of sun exposure, but on fewer days per week. Several participants also wondered whether it would be equally acceptable to spend a shorter period of time in the more 'intense' sunlight during the middle of the day to get a "boost [...] of vitamin D" (Independent aged).

\subsection{Barriers}

\subsubsection{Barriers to Receiving Sun Exposure for Adequate Vitamin D}

A number of barriers to receiving adequate sun exposure were identified: lack of information and knowledge about the effects of vitamin D deficiency, concern about skin cancer and sun burn, ability to go outside, the weather and work. Overall, participants were much more aware of the "SunSmart" message than the "vitamin D" message and almost unanimously more concerned about preventing skin cancer than about ensuring they get enough vitamin D. Two reasons seemed to underpin these 
findings. Promotion of the "SunSmart" message, as well as an awareness of the dangers of skin cancer has led to participants purposefully limiting their sun exposure. In comparison, vitamin D deficiency seemed inconsequential and not serious enough to warrant any specific action.

"The consequences I think are greater. It's [skin cancer] deadly, and you die a lot quicker from cancer than you can from vitamin D deficiency..." (Secondary school teacher)

Several participants highlighted that the "SunSmart" and "vitamin D" messages seem contradictory - on the one hand, the recommendation is to cover up and on the other hand, the recommendation is to expose skin. A few participants also mentioned that in addition to skin cancer, they were concerned about sunburn and, consequently, limited their exposure. Extremes of weather, including the summer heat and wet weather, being unable to go outside due to medical conditions and physical ability were identified as barriers to sun exposure.

\subsubsection{Ways to Address the Barriers to Increase Sun Exposure}

The majority of participants' suggestions to help them meet recommendations for sun exposure centred around increasing incidental exposure, such as by parking the car further away from their destination, a brief walk at lunchtime and eating meals outdoors. Only one office worker and a secondary school teacher identified fortifying food with vitamin D or taking a supplement as a way of receiving adequate vitamin $\mathrm{D}$.

\subsubsection{Communicating the Vitamin D Message}

Overall, participants felt the vitamin D message had not been effectively communicated. Participants made recommendations as to how to communicate the vitamin $\mathrm{D}$ message (communication medium) and what needs to be communicated (type of message).

Communication medium: Television advertising was considered the communication medium of choice, followed by newspapers, magazines and radio. New media, including the internet, Facebook and "pop-up" ads on websites, such as Google, were identified as potentially effective ways of communicating the message, particularly to adolescents and young adults.

Doctors and pharmacists were considered a good source of information about vitamin D, especially for the elderly.

Type of message: Participants suggested that messages relating to vitamin D should focus on providing education, but stressed that "It's got to be a simple message. If it's too complicated, your eyes just glaze over and you think about something else." (Independent aged).

While number of participants suggested combining recommendations for acquiring adequate vitamin D with related messages, such as the Slip! Slop! Slap! Message, an equal number of participants were concerned this could cause confusion and be counter-productive. There was particular concern that mixed messages could be used as an excuse by children and adolescents to be out in the sun without sun protection. One participant suggested that the message should only be targeted at those who are at-risk. 


\section{Discussion}

This study used qualitative methods to explore understanding and awareness of the vitamin D message and opportunities and barriers to UV exposure. Focus groups were conducted with individuals at risk for vitamin D deficiency, including people who have limited access to outdoor sunlight through the day, including indoor office workers and elderly people in aged care facilities. The study provides new and unique knowledge in this emerging area, with implications for the development of the "balance" message.

\subsection{Low Knowledge and Awareness about Balancing the Benefits and Risks of Sun Exposure}

The almost complete lack of awareness of the balance message and low levels of knowledge about vitamin D were not surprising. Previous quantitative community surveys conducted in Australia, although mostly in Queensland, have found similar results [18,30]. Furthermore, some research suggests that people who intentionally tan claim to do so for their vitamin D status. Thus, the misunderstanding about vitamin D may be placing people at risk of skin cancer. Our participants were unable to name the health benefits of vitamin D with certainty, had little awareness of UV times of the day for adequate vitamin D exposure and most were unaware of the amount of time in the sun they required. Most participants had not had previous exposure to the current guidelines for sun exposure, and when the message was communicated, it prompted several questions. These results and previous quantitative studies suggest that current communication of the "balance" message is not reaching most of the community. Unlike previous studies that have found that the vitamin D message is being misinterpreted, particularly by people who intentionally suntan putting themselves at risk of skin cancer [30], the current study found people continued to heed the sun protection message, even during winter and outside of peak UV times. These results suggest that even those at risk of deficiency are not aware of the need to increase their vitamin D intake.

One factor, which may be contributing to the low levels of knowledge and difficulty in communicating the "balance" sun exposure message, is the lack of conclusive research evidence regarding how much time the public needs in direct UV exposure in order to assist their vitamin D status. Currently, the message is complex and different according to location, season and individual characteristics. Broad recommendations for the amount of skin an individual needs to expose to the sun and the amount of time to be exposed are based on incomplete data. As further research evidence is gathered, the messages will be made clearer and communicated more confidently.

\subsection{High Levels of SunSmart Awareness and Sun Avoidance Behaviours}

Slogans, such as Slip! Slop! Slap! and SunSmart, have very high public recognition, and there has been considerable policy and practice in place in Australia since the early 1980s that reinforces sun protective behaviour [31]. Two related themes emerged in the discussions that reflect this situation. Firstly, the SunSmart message has been effectively communicated and adopted by study participants. Use of sun protection amongst this group was largely "normalised", and most participants reported frequently using sun protection measures. Secondly, the message that skin cancer is a high risk 
concern had been effectively communicated and taken up by participants. Participants stated they were more concerned about skin cancer than vitamin D deficiency.

One consequence of the success of the SunSmart messages is that some participants reported the need to use sun protection at all times, with some participants feeling that there was no safe time to be exposed, especially in summer. Encouragingly, most participants identified the middle of the day as unsuitable, and some believed that early morning or late afternoon were suitable for safe sun exposure. No participants offered "outside of peak UV times" as appropriate times to be in the sun. Instead, generic "early" and "late" in the day times were offered. This suggests that the UV Alert, which may be an appropriate tool for displaying safe and unsafe sun exposure times of the day, requires further promotion and education for people to understand and use the ratings.

\subsection{Barriers to Sun Exposure for Vitamin D}

Incidental exposure during the day was the most common type of sun exposure reported, with more time spent outside on weekends than weekdays. This type of sun exposure may not be sufficient for vitamin D, given that the recommendation of up to 10 min outside of peak UV time is based on estimates from ideal conditions of sun exposure (clear, open sky and an unshaded, horizontal surface) and may not correspond to typical outdoor behaviours [32]. This is the first study to explore barriers to sun exposure for vitamin D. Types of barriers participants in all groups reported included lack of knowledge about the need for vitamin D, concerns about sunburn, the need to use sun protection when outdoors and environmental barriers, including the weather (wet, hot and cold weather), work hours indoors (indoor workers group) and physical inabilities to go outside (aged care group). Development of vitamin D public health education and campaigns need to address these barriers.

\subsection{Strategies to Overcome the Barriers}

Increasing incidental sun exposure through routine, daily, outdoor activities was the main strategy identified by participants for increasing sun exposure for vitamin D. Examples included parking the car ten minutes away from work and walking the distance and eating lunch outside the workplace. Improving education about the need for sun exposure and vitamin D was also suggested. Channels for communicating the vitamin D message included the mass media and internet, schools, doctors and pharmacists. While the mass media has been informally used to date, it's reporting of health news is less than optimal [33] and has been accused of misrepresenting the vitamin D issue and confusing the message further [34,35]. Alternatively, doctors and pharmacists may be better placed as providers of information, where the benefits and risks can be communicated in a balanced manner and various factors considered in calculating risk and need (including latitude, skin type, season and time of day). Doctors, in particular, frequently need to manage the communication of uncertainties, risks and benefits of medical therapies [36]. However, the results of this study suggest that participants' doctors were not informing their patients about their vitamin D status. Other research suggests this may be due to low levels of knowledge about vitamin D amongst doctors [37]. Clearly, further efforts into educating both the media and health professionals about vitamin $\mathrm{D}$ is needed. 
There was a division of opinion regarding whether the vitamin D message should be linked to the Slip! Slop! Slap! Seek and Slide message. Whilst some participants felt it was a natural way to communicate the balance message, others believed this strategy would confuse the messages and cause negative consequences, including increased tanning. Others have suggested that the vitamin D message can complement SunSmart messages in Australia [31], particularly if the UV Alert is effectively incorporated. Some research has started to suggest that younger tanners are using the need for vitamin D as a reason for their sun tanning behaviours [38]. This highlights the need to carefully explore all the factors that may affect the interpretation and use of a balance message among a variety of target groups and identify methods for communicating the message. A tailored approach with messages designed for different groups may be the most effective and safe. The need for a consistent and simple message was reinforced.

\subsection{Study Strengths and Weaknesses}

This study is one of the first qualitative studies of the knowledge and attitudes of groups at risk of vitamin D deficiency. The use of the theoretical models proved instrumental to shaping aspects of the study. The focus groups found that social and physical environments, as proposed in the Social Cognitive Model, played a role in lack of UV exposure, both for office workers and elderly persons. As predicted by the Health Belief Model, perceived susceptibility to skin cancer and sunburn was greater than risk of vitamin D deficiency, and perceptions of the severity of the skin cancer and sunburn threats were greater than the threat of vitamin D deficiency. An examination of the barriers to the desired health behaviour revealed suggestions for overcoming the barriers. As a result, the study provides valuable insight into peoples' understanding of the vitamin D and sun exposure message. Other strengths of this formative research are its inclusion of groups at higher risk for vitamin D deficiency and a high number of participants. The use of multiple researchers to collect and analyse the qualitative data also reduced the potential for investigator bias in interpreting the findings. The generalizability of study results is limited, due to two main reasons. First, only select groups were included in the study. Secondly, detailed demographic or skin cancer history information about participants was not collected. Further research is required to generalise these findings to other types of community groups or individuals, including those with darker skin types or those who wear veiled clothing.

\section{Author Contributions}

BB and VR conceived of the study and developed the study protocol. BB and JB developed study materials. IB was an observer during focus groups. JB and SL conducted coding and analysis. All authors contributed to paper writing.

\section{Conflicts of Interest}

The authors declare no conflict of interest. 


\section{References}

1. Garland, C.F.; Garland, F.C.; Gorham, E.D.; Lipkin, M.; Newmark, H.; Mohr, S.B.; Holick, M. The role of vitamin D in cancer prevention. Am. J. Public Health 2005, 96, 252-261.

2. Holick, M.F. Sunlight and vitamin D for bone health and the prevention of autoimmune diseases, cancers and cardiovasular disease. Am. J. Clin. Nutr. 2004, 80, 1678s-1688s.

3. Hypponen, E. Vitamin D and the Risk of Type 1 Diabetes. In Vitamin D: Physiology, Molecular Biology, and Clinical Applications (Nutrition and Health); Hollick, M.F., Ed.; Humana Press: New York, NY, USA, 2010; pp. 867-879.

4. Holick, M.F. vitamin D and bone health. J. Nutr. 1996, 126, 11595-11645.

5. Moan, J.; Porojnicu, A.C.; Dahlback, A.; Setlow, R.B. Addressing the health benefits and risks, involving vitamin D or skin cancer, of increased sun exposure. Proc. Natl. Acad. Sci. USA 2008, $105,668-673$.

6. Vik, T.; Try, K.; Stromme, J.H. The vitamin D status of man at 70 degrees north. Scand. J. Clin. Lab. Invest. 1980, 40, 227-232.

7. Brustad, M.; Alaser, E.; Engelsen, O.; Aksnes, L.; Lund, E. Vitamin D status of middle aged women at 65-71 degrees north in relation to dietary intake and exposure to ultraviolet radiation. Public Health Nutr. 2004, 7, 327-335.

8. McGrath, J.J.; Kimlin, M.G.; Saha, S.; Eyles, D.; Parisi, A. Vitamin D insufficiency in southeast queensland. Med. J. Aust. 2001, 174, 150-151.

9. Pasco, J.A.; Henry, M.J.; Nicholson, G.C.; Sanders, K.M.; Kotowicz, M.A. Vitamin D status of women in the geelong osteoporosis study: Association with diet and casual exposure to sunlight. Med. J. Aust. 2001, 175, 401-405.

10. Australian New Zealand Bone and Mineral Society; Osteoporosis Australia; The Australasian College of Dermatologists; and the Cancer Council Australia. Risks and benefits of sun exposure position statement, updated 2007. Available online: http://www.Dermcoll.Asn.Au/ downloads/ccrisksandbenefitsmarch8.Pdf (accessed on 20 January 2011).

11. American Academy of Dermatology Updated position statement on vitamin D. Available online: http://www.aad.org/media/background/news/Releases/American_Academy_of_Dermatology_Iss ues_Updated_Pos/ (accessed on 20 January 2011).

12. Osteoporosis Australia. Calcium, vitamin D and osteoporosis. A guide for gps, 2nd ed.; 2008. Available online: http:/www.Osteoporosis.Org.Au/files/factsheets/oth-7665-eng.Pdf (accessed on 20 January 2011).

13. Flicker, L.; Mead, K.; Macinnis, R.J.; Nowson, C.; CScherer, S.; Stein, M.S.; Thomasx, J.; Hopper, J.L.; Wark, J.D. Serum vitamin D and falls in older women in residential care in australia. J. Am. Geriatr. Soc. 2003, 51, 1533-1538.

14. Sambrook, P.N.; Cameron, I.D.; Cumming, R.G.; Lord, S.R.; Schwarz, J.M.; Trube, A.; March, L.M. Vitamin D deficiency is common in frail institutionalised older people in northern sydney. Med. J. Aust. 2002, 176, 560.

15. Grover, S.R.; Morley, R. Vitamin D deficiency in veiled or dark skinned pregnant women. Med. J. Aust. 2001, 175, 251-252. 
16. Diamond, T.; Smerdely, P.; Kormas, N.; Sekel, R.; Vu, T.; Day, P. Hip fracture in elderly men: The importance of subclinical vitamin D deficiency and hypogonadism. Med. J. Aust. 1998, $169,138-141$.

17. Hatun, S.; Islam, O.; Cizmecioglu, F.; Kara, B.; Babaoglu, K.; Berk, F.; Gökalp, A.S. Subclinical vitamin D deficiency is increased in adolescent girls who wear concealing clothing. J. Nutr. 2005, 135, 218-222.

18. Janda, M.; Youl, P.; Bolz, K.; Niland, C.; Kimlin, M. Knowledge about the health benefits of vitamin D in queensland australia. Prev. Med. 2010, 50, 215-216.

19. Rosenstock, I.M.; Strecher, V.J.; Becker, M.H. Social learning theory and the health belief model. Health Educ. Q. 1988, 15, 175-183.

20. McGuire, W.J. Public communication as a strategy for inducing health-promoting behavioural change. Prev. Med. 1984, 13, 299-319.

21. Rogers, E.M. Diffusion of Innovations; The Free Press: New York, NY, USA, 1993.

22. Kirby, S.; Baranowski, T.; Reynolds, K.D.; Taylor, G.; Binkley, D. Children's fruit and vegetable intake: Socioeconomic, adult-child, regional, and urban-rural influences. J. Nutr. Educ. 1995, 27, 261-271.

23. Green, L.W.; Kreuter, M. Health Promotion Planning: An Educational and Environmental Approach, 2nd ed.; Mayfield: Mountain View, CA, USA, 1991.

24. Glanz, K.; Lewis, F.M.; Rimer, B.K. Health Behavior and Health Education: Theory, Research, and Practice, 2nd ed.; Jossey-Bass: San Francisco, CA, USA, 1997.

25. Tong, A.; Sainsbury, P.; Craig, J. Consolidated criteria for reporting qualitative research (coreq): A 32-item checklist for interviews and focus groups. Int. J. Qual. Health Care 2007, 19, 349-357.

26. Bandura, A. Social Foundations of Thought and Action: A Social Cognitive Theory; Prentice-Hall: Englewood Cliffs, NJ, USA, 1986.

27. Strecher, V.J.; Rosenstock, I.M. The Health Belief Model, 2nd ed.; Jossey-Bass: San Francisco, CA, USA, 1997.

28. NVivo Qualitative Data Analysis Software, version 8; QSR International Pty Ltd.: Doncaster, Australia, 2008.

29. Holloway, I.; Wheeler, S. Qualitative Research in Nursing, 2nd ed.; Blackwell Publishing: Melbourne, Australia, 2002.

30. Youl, P.; Janda, M.; Kimlin, M.G. vitamin D and sun protection: The impact of mixed messages in australia. Int. J. Cancer 2008, 124, 1963-1970.

31. Sinclair, C. Risks and benefits of sun exposure: Implications for public health practice based on the australian experience. Prog. Biophys. Mol. Biol. 2006, 92, 173-178.

32. Diffey, B.L. Is casual exposure to summer sunlight effective at maintaining adequate vitamin D status? Photodermatol. Photoimmunol. Photomed. 2010, 26, 172-176.

33. Wilson, A.; Bonevski, B.; Jones, A.; Henry, D. Deconstructing cancer: What makes a good quality news story. Med. J. Aust. 2010, 193, 702-706.

34. Kemp, G.A.; Eagle, L.; Verne, J. Mass media barriers to social marketing interventions: The example of sun protection in the uk. Health Promot. Int. 2010, 26, 37-45. 
35. Scully, M.; Wakefield, M.; Dixon, H. Trends in news coverage about skin cancer prevention, 1993-2006: Increasingly mixed messages for the public. Aust. N. Z. J. Public Health 2008, 32, 461-466.

36. Politi, M.C.; Han, P.K.J.; Col, N.F. Communicating the uncertainty of harms and benefits of medical interventions. Med. Decis. Mak. 2007, 27, 681.

37. Bonevski, B.; Girgis, A.; Magin, P.; Horton, G.; Brozek, I.; Armstrong, B. Prescribing sunshine: A survey of general practitioners' knowledge, attitudes and practices relating to the sun and vitamin D. Int. J. Cancer 2011, 130, 2138-2145.

38. Woo, D.K.; Eide, M.J. Tanning beds, skin cancer, and vitamin D: An examination of the scientific evidence and public health implications. Dermatol. Ther. 2010, 23, 61-71. 


\title{
Calcidiol Deficiency in End-Stage Organ Failure and after Solid Organ Transplantation: Status quo
}

\section{Ursula Thiem, Bartosz Olbramski and Kyra Borchhardt}

\begin{abstract}
Among patients with organ failure, vitamin D deficiency is extremely common and frequently does not resolve after transplantation. This review crystallizes and summarizes existing data on the status quo of vitamin D deficiency in patients with organ failure and in solid organ transplant recipients. Interventional studies evaluating different treatment strategies, as well as current clinical practice guidelines and recommendations on the management of low vitamin D status in these patients are also discussed.
\end{abstract}

Reprinted from Nutrients. Cite as: Thiem, U.; Olbramski, B.; Borchhardt, K. Calcidiol Deficiency in EndStage Organ Failure and after Solid Organ Transplantation: Status quo. Nutrients 2013, 5, 2352-2371.

\section{Introduction}

Vitamin D deficiency is a commonly observed phenomenon in patients with organ failure and solid organ transplant recipients. It occurs in patients with different types of solid organ transplant and frequently persists even in the long-term, post-transplant period (reviewed in [1]). The causes of vitamin D deficiency in these patients are diverse. Vitamin D deficiency may be primarily ascribed to lifestyle and environmental factors that result in reduced exposure to sunlight, as the main source of vitamin D is the skin, where it is synthesized from 7-dehydrochoesterol under the influence of ultraviolet light (reviewed in [2]). On the other hand, in patients with end-stage organ disease, there may be additional disease-specific factors that contribute to vitamin D deficiency, such as liver dysfunction [3] or uremia, which reduces the capacity of the skin to synthesize vitamin D [4,5]. After transplantation, the avoidance of sunlight, due to the increased risk of skin cancer in immunosuppressed patients [6], may be the main factor causing vitamin D deficiency [7]. Additional factors might involve the use of glucocorticoids, which were shown to enhance the catabolism of calcidiol [8].

Vitamin D deficiency is also commonly observed in the general population [9]. The National Health and Nutrition Examination Survey (NHANES) between 2002 and 2004, for example, assessed calcidiol levels in a representative sample of more than 20,000 persons in the USA and revealed that calcidiol levels below $20 \mathrm{ng} / \mathrm{mL}$ occur in approximately one third of the studied population [10]. In non-institutionalized elderly people across 11 European countries, 36\% of men and 47\% of women showed calcidiol levels below $12 \mathrm{ng} / \mathrm{mL}$ during winter [11]. For the general population, the Institute of Medicine in 2011 released their report on dietary reference intakes for calcium and vitamin D. For optimal bone health, the recommended dietary allowances of 600 International Units of vitamin D for ages up to 70 years and 800 International Units for ages above 70 years are suggested, corresponding to calcidiol levels above $20 \mathrm{ng} / \mathrm{mL}$ [12].

Herein, we review the prevalence of vitamin D deficiency in patients with end-stage organ failure and organ transplant recipients, as well as clinical trials on supplementation strategies and current guidelines on the recommendation of vitamin $\mathrm{D}$ intake in these patients. In this review, we consider 
25-hydroxyvitamin D (calcidiol) levels below $30 \mathrm{ng} / \mathrm{mL}$ as insufficiency or hypovitaminosis, below $20 \mathrm{ng} / \mathrm{mL}$ as deficiency and below $10 \mathrm{ng} / \mathrm{mL}$ as severe deficiency $(\times 2.5$ for conversion to $\mathrm{nmol} / \mathrm{L})$.

\section{Congestive Heart Failure and Cardiac Transplantation}

\subsection{Vitamin D Status in Patients with End-Stage Heart Failure}

Hypovitaminosis D is highly prevalent among patients with congestive heart failure, with $17 \%$ to $57 \%$ of the patients displaying severe vitamin D deficiency $[13,14]$. The vitamin D status was reported to be related to the severity of the disease. In particular, patients evaluated for cardiac transplantation and classified United Network of Organ Sharing (UNOS) status 1 (i.e., hospitalization and dependence on intravenous inotropic agents or left ventricular assist devices) had significantly lower calcidiol levels as compared with patients classified UNOS status 2, who were well enough to be managed as outpatients, (19 ng/mL vs. $24 \mathrm{ng} / \mathrm{mL}$ ). While $23 \%$ of status 1 patients displayed severe vitamin D deficiency, only $8 \%$ of status 2 patients did so [13]. Similarly, mean serum calcidiol levels were reported to be lower in end-stage congestive heart failure patients awaiting cardiac transplantation who were classified as urgent or high urgent candidates according to the Eurotransplant listing criteria as compared with elective candidates $(9.3 \mathrm{ng} / \mathrm{mL} v s .14 \mathrm{ng} / \mathrm{mL})$. None of the urgent or high urgent and only about $5 \%$ of the elective transplant candidates had sufficient vitamin D levels. Severe vitamin D deficiency was present in 57\% of urgent or high urgent and in 50\% of elective transplant candidates [14].

\subsection{Vitamin D Status in Cardiac Transplant Recipients}

Only one study reported the vitamin D status at the time of cardiac transplantation. Almost $90 \%$ of the patients presented with vitamin D insufficiency and 10\% were found to be severely deficient [15]. In short-term heart transplant recipients, mean serum calcidiol levels increased from $18.7 \mathrm{ng} / \mathrm{mL}$, analyzed within 12 months pre-transplant, to $24.5 \mathrm{ng} / \mathrm{mL}$ at one year post-transplant. Even though intake of 400 to 800 International Units of vitamin D was recommended to all patients, approximately three quarters of the patients displayed vitamin D insufficiency at one year post-transplant [16]. Similar results were obtained from an Iranian cohort of short-term heart transplant recipients, where two thirds were reported to be vitamin D deficient [17]. Even in the long-term post-transplant period, vitamin D deficiency frequently persists. In cardiac transplant recipients with a mean allograft age of approximately four years, more than $90 \%$ of the patients were reported to have hypovitaminosis D, with more than one third of patients displaying severe deficiency [18]. A summary of the vitamin D status in patients with end-stage heart failure and cardiac transplant recipients is presented in Table 1.

\subsection{Interventional Studies and Guidelines}

Despite the high prevalence of vitamin D insufficiency among patients with advanced heart failure and cardiac transplant recipients, interventional studies are sparse or lacking. In a randomized controlled trial in patients with heart failure (New York Heart Association class II and higher), the effect of daily $500 \mathrm{mg}$ calcium and 2000 International Units vitamin $\mathrm{D}_{3}$ on survival, cytokine profiles and echocardiographic parameters was studied and compared with calcium treatment alone. After nine months, treatment with vitamin $\mathrm{D}_{3}$ increased the mean serum calcidiol level by $26.8 \mathrm{ng} / \mathrm{mL}$, while an 
increase of only $3.6 \mathrm{ng} / \mathrm{mL}$ was observed in placebo treated patients. Treatment with vitamin $\mathrm{D}_{3}$ prevented an increase in tumor necrosis factor alpha, as observed in placebo-treated patients, and increased interleukin 10 levels [19]. Moreover, a recent study investigated the effect of vitamin $\mathrm{D}_{3}$ on biochemical and functional parameters of congestive heart failure in vitamin D insufficient patients with New York Heart Association class I to III heart failure. The treatment consisted of 50,000 International Units per week for eight weeks. Thereafter, the patients received 50,000 International Units every month for two months. Mean serum calcidiol level increased by $17 \mathrm{ng} / \mathrm{mL}$ after the four month treatment period. Interestingly, a decrease in pro-brain natriuretic peptide and high-sensitivity C-reactive protein, as well as an improvement in six minute walk distance and New York Heart Association class was observed [20].

Current guidelines for vitamin D intake in heart transplant candidates and cardiac transplant recipients are based on the beneficial effects of vitamin D therapy on corticosteroid-induced bone loss. In particular, based on expert consensus (Level of Evidence C), the International Society of Heart and Lung Transplantation recommends a daily intake of 1000 to $1500 \mathrm{mg}$ of calcium and 400 to 1000 International Units of vitamin D to all heart transplant candidates and recipients. Serum calcidiol levels should be maintained above $30 \mathrm{ng} / \mathrm{mL}$ [21]. 


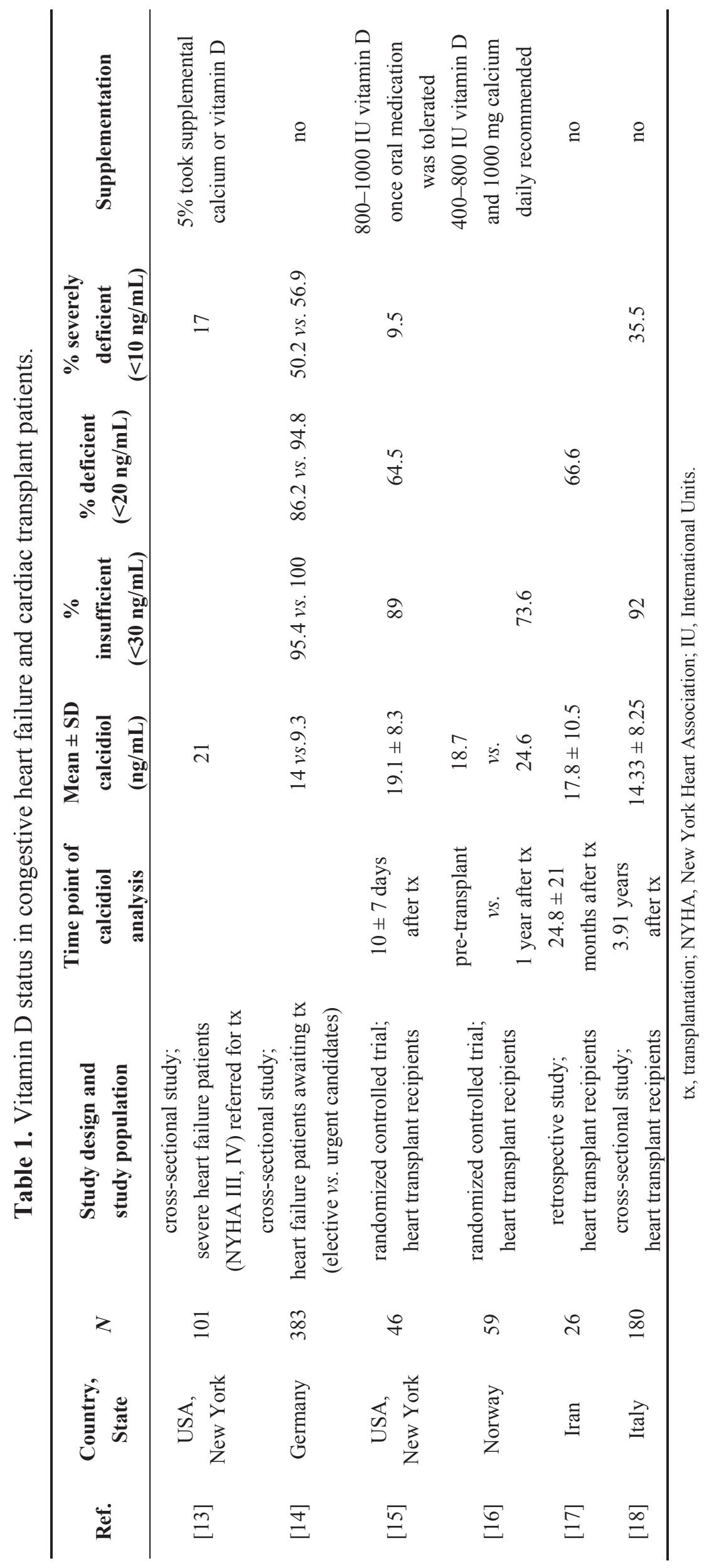




\section{End-Stage Pulmonary Disease and Lung Transplantation}

\subsection{Vitamin D Status in Patients with End-Stage Pulmonary Disease}

In end-stage pulmonary disease patients, varying prevalence of vitamin $\mathrm{D}$ deficiency was reported depending on the underlying disease. Severe vitamin D deficiency was seen in $14 \%$ to $40 \%$ of patients with cystic fibrosis [22-24], 20\% to $42 \%$ of patients with chronic obstructive pulmonary disease [22,23], 14\% of patients with pulmonary fibrosis [23] and $18 \%$ of patients with pulmonary hypertension [23]. Even though in one study, the majority of the patients received multivitamin supplements containing 400 to 800 International Units of vitamin D, $40 \%$ had severe vitamin D deficiency, and only $25 \%$ of the patients showed serum calcidiol levels above $20 \mathrm{ng} / \mathrm{mL}$ [24].

\subsection{Vitamin D Status in Lung Transplant Recipients}

In short-term lung transplant recipients in the United States, the proportion of patients with vitamin D insufficiency decreased from $79 \%$ at the time of transplantation to $26 \%$ at one year post-transplant. Approximately half of the patients received vitamin D supplements at the time of transplantation, while all of them did so after lung transplantation. Vitamin D deficiency at the time of transplantation was associated with an increased risk of experiencing acute rejection episodes or infections [25]. Similarly, a European study reported an improvement in vitamin D status after one year of lung transplantation. Mean serum calcidiol levels increased from $25.1 \mathrm{ng} / \mathrm{mL}$ at the time of transplantation to $29.4 \mathrm{ng} / \mathrm{mL}$ at one year post-transplant. Intake of 400 to 800 International Units of vitamin D was recommended to all patients. Still, approximately half of the patients displayed vitamin D insufficiency at one year post-transplant [16].

Data on the vitamin D status in long-term lung transplant recipients is sparse. In a Belgian cohort of 131 prevalent lung transplant recipients with an allograft age ranging from one to four years, approximately half of the patients were reported to have vitamin D insufficiency, despite daily treatment with 880 to 1000 International Units of cholecalciferol for the prevention of osteoporosis. A subgroup-analysis revealed that the proportion of vitamin D insufficient patients was similar between patients with one, two, three or four years of follow-up (ranging from $42 \%$ at one and two years post-transplant to $53 \%$ at three and four years post-transplant). Interestingly, after multivariate adjustment, vitamin D deficiency was associated with lower $\mathrm{FEV}_{1}$ (forced expiratory volume in one second), and patients deficient in vitamin D experienced more episodes of moderate to severe B-grade rejection (lymphocytic bronchiolitis) [26]. Details on the vitamin D status in patients with end-stage pulmonary disease and lung transplant recipients are summarized in Table 2. 


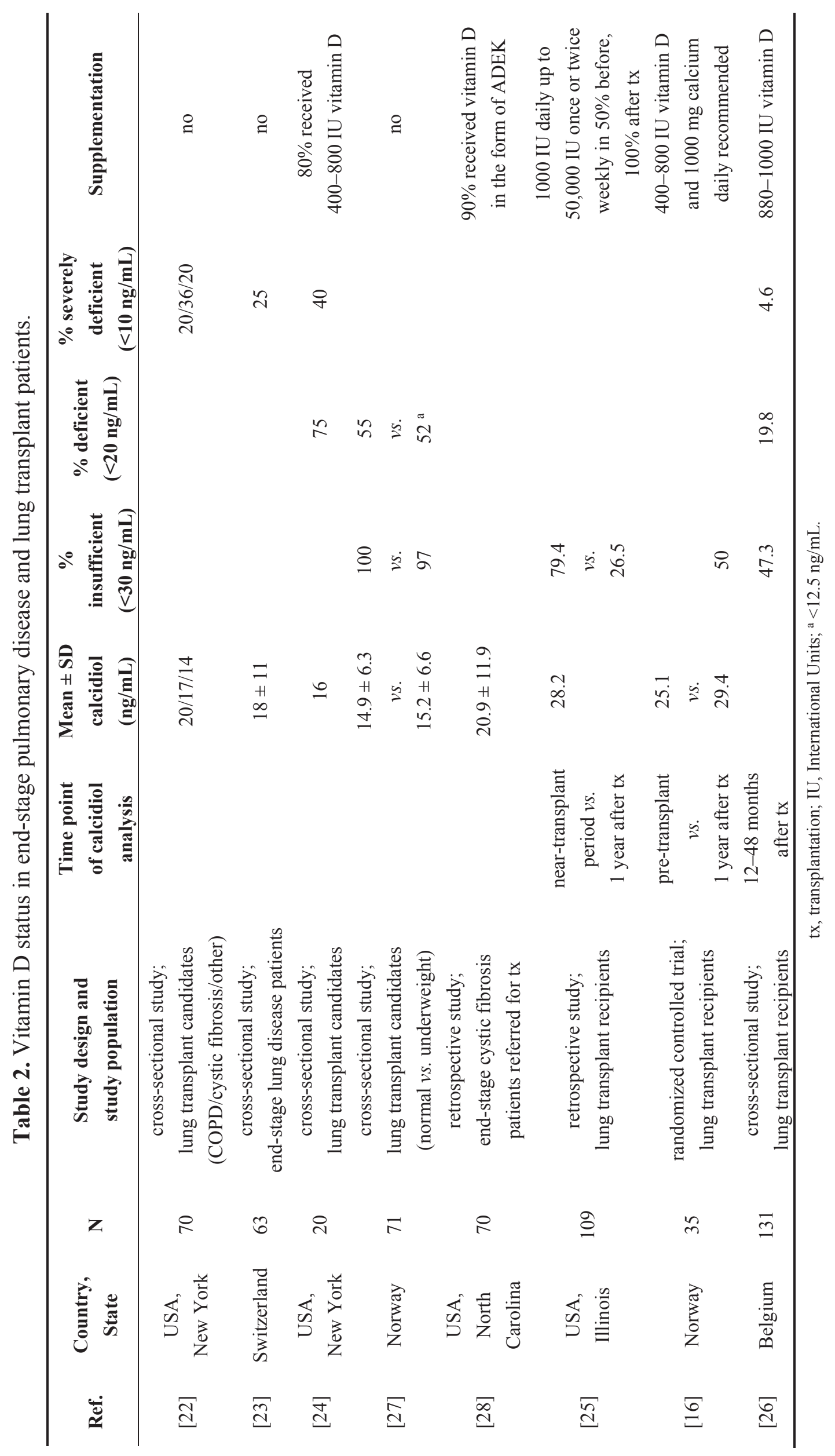

393 


\subsection{Interventional Studies and Guidelines}

Based on the finding that vitamin D deficiency in lung transplant recipients is associated with an increased risk of developing rejection episodes, a randomized controlled trial is currently investigating the effect of a two year therapy of monthly 100,000 International Units of vitamin D in incident lung transplant recipients on the development of bronchiolitis obliterans syndrome, a frequent manifestation of chronic rejection (clinicaltrials.gov NCT01212406).

To our knowledge, there are no guidelines on the management of vitamin D deficiency in patients with end-stage pulmonary disease or lung transplant patients.

\section{Liver Failure and Liver Transplantation}

\subsection{Vitamin D Status in Patients with End-Stage Liver Disease}

The liver is the main site where hydroxylation of vitamin D at position C-25 takes place. Thus, it is not surprising that the degree of liver dysfunction correlates with calcidiol levels [3] and that the prevalence of vitamin D insufficiency is particularly high in patients with chronic liver disease [29-32]. At the time of transplantation, between $80 \%$ and $95 \%$ of the patients with end-stage liver failure were reported to have hypovitaminosis $\mathrm{D}$, with varying prevalence of severe vitamin $\mathrm{D}$ deficiency (ranging from 3\% up to 50\%) [15,33-35]. Notably, in one study, more than one fifth of the patients had serum calcidiol below the detection limit of $6.8 \mathrm{ng} / \mathrm{mL}$ [15].

\subsection{Vitamin D Status in Liver Transplant Recipients}

At three months after liver transplantation, Reese and colleagues observed a marked increase in serum calcidiol levels with a median change of $17.8 \mathrm{ng} / \mathrm{mL}$ (interquartile range 8.6 to $25.9 \mathrm{ng} / \mathrm{mL}$ ). The prevalence of vitamin D deficiency dropped from $84 \%$ at the time of transplantation to $24 \%$ after three months post-transplant. Moreover, serum vitamin D binding protein and albumin substantially increased, which according to the authors, might have contributed to the marked improvement in vitamin D status by facilitating a shift of calcidiol from the adipose tissue to the circulation. Even though the prevalence of vitamin D deficiency was similar in black and non-black patients at the time of transplantation, median calcidiol levels were significantly lower in black patients (4.9 vs. 9.6 ng/mL). At three months after transplantation, vitamin D deficiency was more prevalent among black liver transplant recipients as compared with non-black patients (38\% vs. 20\%) [36]. In contrast, in an Iranian cohort of liver transplant recipients, a high prevalence of vitamin D deficiency persisted in the early post-transplant period [17]. In a Spanish study, 45 liver transplant recipients were followed up to three years after transplantation. Before transplantation, severe vitamin D deficiency was present in $62 \%$ of the patients, with a mean serum calcidiol of $9.4 \mathrm{ng} / \mathrm{mL}$. In comparison, 40 healthy age-matched controls displayed a mean serum calcidiol of $23.1 \mathrm{ng} / \mathrm{mL}$. Serum calcidiol levels continuously increased over time $(9.5 \mathrm{ng} / \mathrm{mL}$ at one month, $16.5 \mathrm{ng}$ at three months, $15.9 \mathrm{ng}$ at six months, $19 \mathrm{ng} / \mathrm{mL}$ at 12 months, $19.9 \mathrm{ng} / \mathrm{mL}$ at $18 \mathrm{months}, 18 \mathrm{ng} / \mathrm{mL}$ at 24 months and $19.5 \mathrm{ng} / \mathrm{mL}$ at 36 months post-transplant). At one year and three years after transplantation, severe vitamin D deficiency was observed in only $14 \%$ and $10 \%$ of the patients, respectively [37]. In contrast, in an Israelian cohort of long-term liver transplant 
recipients with a mean allograft age of 7.5 years, more than one third was reported to have severe vitamin D deficiency [38]. Table 3 summarizes the prevalence of vitamin D insufficiency, deficiency and severe deficiency in patients with end-stage liver disease and liver transplant recipients.

\subsection{Interventional Studies and Guidelines}

Recently, a clinical practice guideline on the evaluation, treatment and prevention of vitamin D deficiency has been published by the Endocrine Society. According to this guideline, patients with hepatic failure are considered at high risk for vitamin D deficiency. Therefore, calcidiol measurements are reasonable, and supplementation in case of calcidiol levels below $20 \mathrm{ng} / \mathrm{mL}$ is recommended. For bone health, adults are considered to require at least 600 to 800 International Units daily. For reaching calcidiol levels above $30 \mathrm{ng} / \mathrm{mL}$, however, higher doses may be required (1500 to 2000 International Units). This recommendation is based on lower quality evidence [39]. For the management of liver transplant patients, the American Association for the Study of Liver Disease and the American Society of Transplantation released a practice guideline, which recommends calcidiol levels to be maintained above $30 \mathrm{ng} / \mathrm{mL}$ for optimal bone health. This daily use of 400 to 1000 International Units of vitamin D is suggested; however, calcidiol levels should be measured at least annually to check if the treatment regimen is appropriate. Treatment with vitamin D supplements is recommended in osteopenic liver transplant recipients with a Level of Evidence A [40].

\section{Chronic Kidney Disease and Kidney Transplantation}

\subsection{Vitamin D Status in Patients with Chronic Kidney Disease}

Vitamin D deficiency is very common in patients with chronic kidney disease (CKD) across all stages. In North America, a prevalence of vitamin D insufficiency of approximately $85 \%$ was reported among patients with advanced kidney disease [41,42]. Severe vitamin D deficiency $(<15 \mathrm{ng} / \mathrm{mL})$ was more pronounced in patients with CKD stage 5 (56\%), as compared with CKD stage 4 (37\%) [42], and mean serum calcidiol was reported to be significantly lower in diabetic as compared with non-diabetic patients [43]. Similarly, in a cohort of chronic kidney disease stage 3 and 4 in the UK, hypovitaminosis D was very common, with $80 \%$ of the patients showing vitamin D insufficiency and $37 \%$ severe vitamin D deficiency $(<15 \mathrm{ng} / \mathrm{mL})$. After exclusion of patients who received vitamin D supplements or other drugs known to interfere with calcidiol levels, mean serum calcidiol remained unchanged. Likewise, the proportional distribution of patients with vitamin D insufficiency and severe deficiency was similar [44]. 


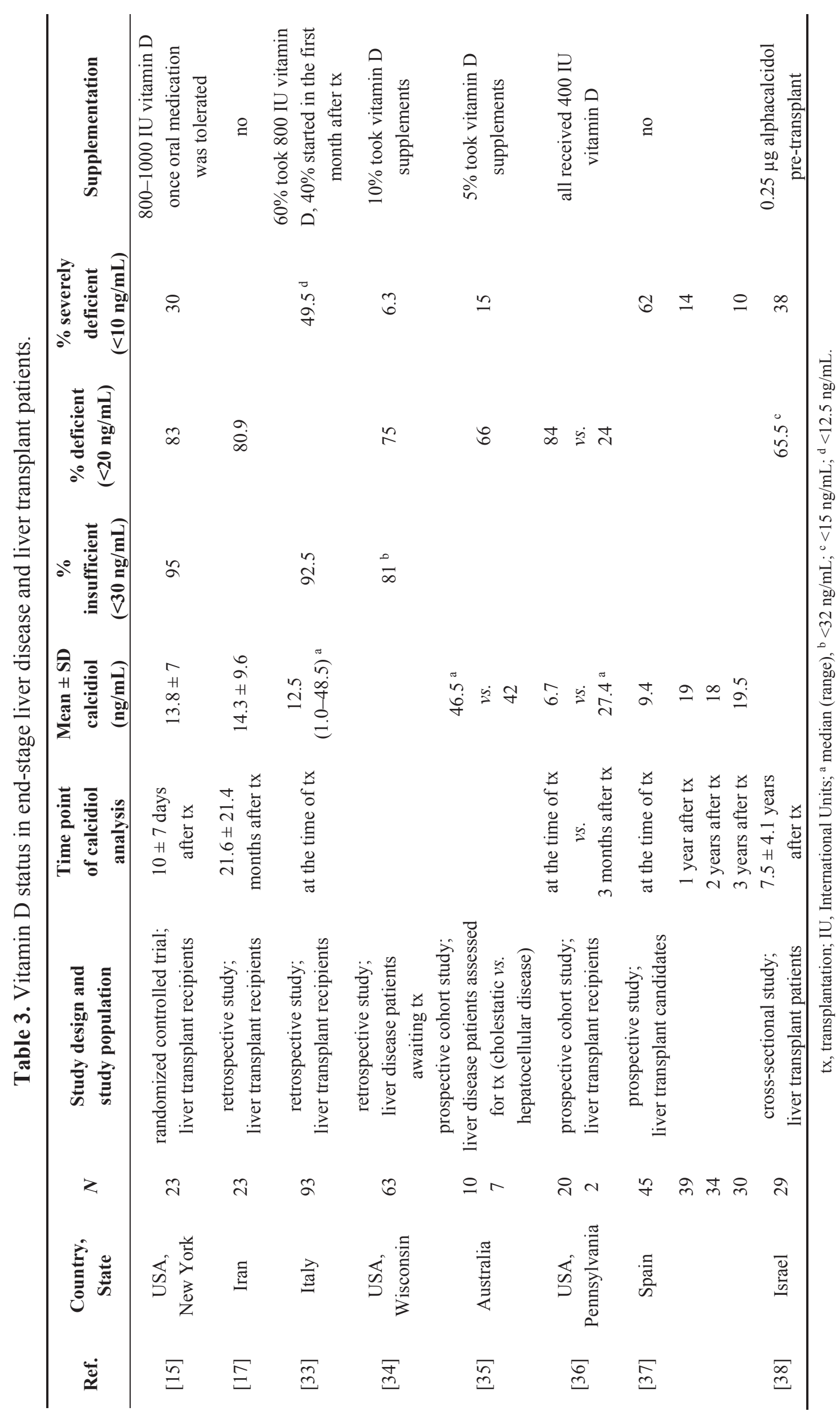




\subsection{Vitamin D Status in Kidney Transplant Recipients}

Only a few studies investigated the vitamin D status at the time of transplantation. Almost $90 \%$ of incident kidney transplant recipients were reported to have insufficient calcidiol levels, with a mean serum calcidiol of $16.6 \mathrm{ng} / \mathrm{mL}$ and a trend towards higher calcidiol levels in summer as compared with winter. Notably, vitamin D levels of black kidney transplant recipients were significantly lower compared with non-black patients $(13.6 \mathrm{ng} / \mathrm{mL} v s .17 .5 \mathrm{ng} / \mathrm{mL})$ [45]. Similar findings were obtained from another cohort of African American renal transplant recipients in the early post-transplant period, where $95 \%$ of the patients presented with calcidiol levels below $30 \mathrm{ng} / \mathrm{mL}$ and $58 \%$ showed severe vitamin deficiency $(<16 \mathrm{ng} / \mathrm{mL})$, even at the end of summer [46].

Even though vitamin D status was reported to improve in the early post-transplant period [47], low vitamin D levels are frequently observed in long-term kidney transplant recipients $[7,48,49]$. Querings et al. investigated the vitamin D status of 31 long-term kidney transplant recipients with a mean allograft age of seven years at the end of winter and found a mean serum calcidiol of $10.9 \mathrm{ng} / \mathrm{mL}$. Notably, vitamin D sufficiency was observed in one patient only, and almost one third of the patients had serum calcidiol levels below the detection limit $(<4 \mathrm{ng} / \mathrm{mL})$ [7]. In contrast, in a Danish cohort of long-term kidney transplant recipients, approximately one fifth of the patients were found to have sufficient vitamin D during the winter months, with a median calcidiol level of $19.8 \mathrm{ng} / \mathrm{mL}$. A subgroup analysis revealed that $60 \%$ of these patients received vitamin D supplements at a median dose of $7.8 \mu \mathrm{g}$ per day (in the form of ergocalciferol or cholecalciferol or alphacalcidol), which might explain the lower prevalence of vitamin D insufficiency in this cohort. In addition, a median alimentary intake of approximately 3.2 $\mu \mathrm{g}$ vitamin D per day was reported [49]. Moreover, clear seasonal variations in serum calcidiol levels were found in long-term kidney transplant recipients, with 3.5-times more patients reaching calcidiol above $30 \mathrm{ng} / \mathrm{mL}$ during summer months compared with winter months. Still, the majority of the patients exhibited vitamin D insufficiency, even during summer [50,51]. In Table 4, details on the vitamin D status in CKD patients and renal transplant patients are presented. 


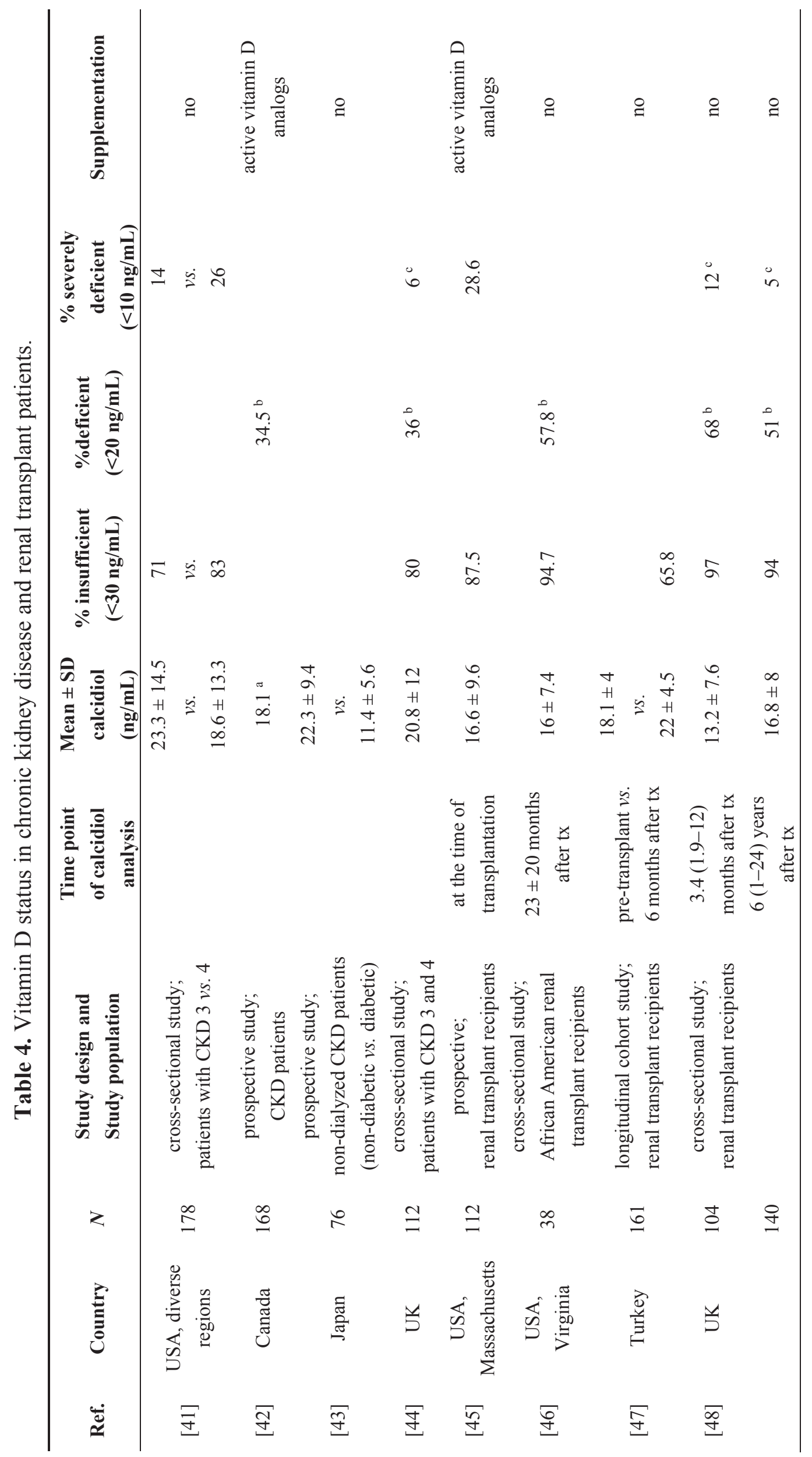




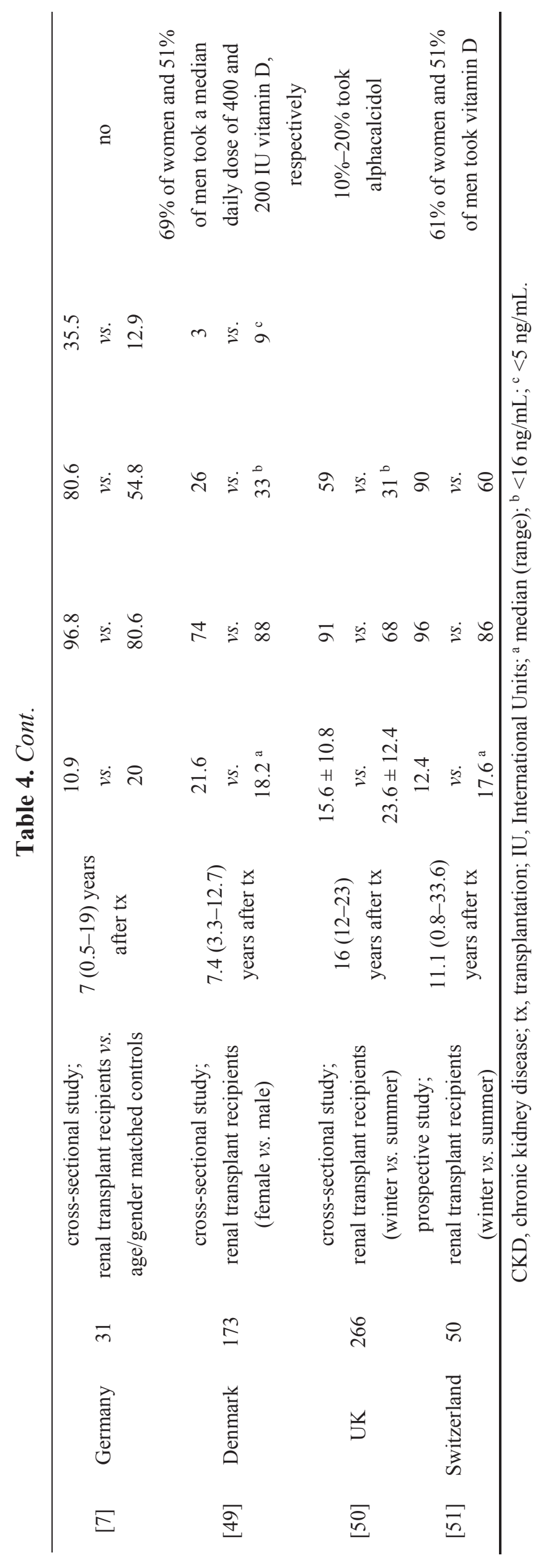




\subsection{Interventional Studies and Guidelines}

Even though vitamin D deficiency is extremely common among patients with chronic kidney disease, including renal transplant recipients, there is no consensus on how to treat vitamin D deficiency in these patients. Current guidelines of the National Kidney Foundation for patients with chronic kidney disease recommend the measurement of calcidiol in patients with CKD stage 3 or 4 only in case of elevated parathyroid hormone levels. Oral supplementation with ergocalciferol for six months, with 50,000 International Units per week for four weeks in the case of mild (5 to $15 \mathrm{ng} / \mathrm{mL}$ ) and 12 weeks in the case of severe (below $5 \mathrm{ng} / \mathrm{mL}$ ) vitamin D deficiency is proposed. Thereafter, a monthly dose of 50,000 International Units is recommended. For vitamin D insufficiency, ergocalciferol at a monthly dose of 50,000 International Units orally for six months is suggested. All of these recommendations are opinion-based [52]. For patients after renal transplantation, Clinical Practice Guidelines for the Care of Kidney transplant recipients (KDIGO) suggest correction of vitamin D deficiency or insufficiency as done for the general population (Level of Evidence C) [53].

A recent meta-analysis of seventeen observational and five randomized controlled trials in patients with various forms of CKD evaluated the effect of vitamin D supplementation and found a significant improvement of the vitamin D status, with a mean difference of $24.1 \mathrm{ng} / \mathrm{mL}$ in observational and $14 \mathrm{ng} / \mathrm{mL}$ in randomized controlled trials. Treatment regimens ranged from 4000 International Units daily up to 50,000 International Units daily, with an average treatment period of half a year [41].

The few studies available indicate that renal transplant recipients have a higher need for vitamin $\mathrm{D}$ to correct insufficiency than what is known from the general population. In particular, in a randomized controlled trial in kidney transplant recipients, Wissing and colleagues evaluated the effect of $400 \mathrm{mg} /$ day calcium and 25,000 International Units of cholecalciferol per month on bone mineral density one year after transplantation. Surprisingly, this dose was not sufficient to correct vitamin D deficiency in these patients [54]. In contrast, Courbebaisse and colleagues used 100,000 International Units of cholecalciferol once every two weeks for two months and, thereafter, treated the patients with 100,000 International Units of cholecalciferol every two months for another six months. After the initial intensive treatment period, more than $90 \%$ of the patients showed calcidiol levels above $30 \mathrm{ng} / \mathrm{mL}$, while only 50\% were vitamin D sufficient after the maintenance treatment period, indicating that 100,000 International Units of cholecalciferol every two months is still not sufficient to maintain optimal vitamin D levels [55]. Moreover, these studies demonstrated that spontaneous recovery of vitamin D deficiency after kidney transplantation does not occur. In the untreated control groups, calcidiol either remained stable [55], or even decreased further [54], over time. Based on their previous study, the group of Courbebaisse established a pharmacokinetic model that describes calcidiol levels after treatment with cholecalciferol in kidney transplant recipients within the first year after transplantation. According to this model, a treatment regimen with six doses of 100,000 International Units cholecalciferol every two weeks, followed by 100,000 International Units once per month is proposed to maintain calcidiol levels between 30 and $80 \mathrm{ng} / \mathrm{mL}$ [56]. Several clinical trials are currently ongoing with different treatment regimens ([57], NCT00752401, NCT00748618, NCT01431430), which will help to identify strategies to maintain optimal vitamin D status. 


\section{Conclusions}

In summary, studies in patients with organ failure consistently show that vitamin D insufficiency or deficiency is widespread among these patients, even in the healthier sub-population of patients awaiting transplantation. After transplantation, calcidiol levels frequently remain low and, in many cases, do not recover in the long-term post-transplant period. Minor discrepancies in the reported prevalence of vitamin D insufficiency or deficiency might result from the different treatment regimens used (if patients received vitamin D supplements or not), different habits and customs of the studied populations (sun protection and nutrition), but also from the different assays used to measure calcidiol.

From the current evidence, it is not clear whether vitamin D deficiency is one of the causative factors or a consequence in the development and progression of organ failure. For example, the active vitamin $\mathrm{D}$ metabolite, 1,25-dihydroxyvitamin $\mathrm{D}$, might affect the development and progression of cardiovascular disease by different mechanisms of action, such as regulation of the mineral metabolism, interaction with the renin-angiotensin-aldosterone system or modulation of immune responses (reviewed in [58,59]). Similarly, it might exert renoprotective effects and, thus, delay the progression of $\mathrm{CKD}$, e.g., by inhibition of the renin-angiotensin-aldosterone system, regulation of the immune system or increase of insulin sensitivity (reviewed in [60]). On the other hand, low calcidiol levels might simply reflect poorer health status in patients with advanced stages of organ failure.

At present, there are only a few recommendations on how to manage vitamin D deficiency in these patients; most of them are based on expert consensus and derived from the beneficial effects of vitamin D on the skeleton. However, especially in the setting of organ transplantation, the effects of vitamin D might go beyond bone health. In particular, the active metabolite, 1,25-dihydroxyvitamin D, has immunomodulatory activity, which is supported by extensive experimental research (reviewed in $[61,62])$. Recent clinical trials indicate that modulation of the immune system can be achieved by administration of nutritional vitamin D [63-69]. These immunomodulatory effects may be exploited in various diseases, including organ tolerance after transplantation. The increase in regulatory T-cells by nutritional vitamin $\mathrm{D}$, as shown in a randomized controlled trial, could be of particular relevance in organ transplant recipients [63]. To date, however, we do not have sufficient evidence to recommend treatment with vitamin D based on its immunomodulatory actions, and further clinical trials are clearly warranted.

\section{Acknowledgments}

U.T. is a recipient of a DOC-fFORTE fellowship of the Austrian Academy of Sciences (\#23478) at the Division of Nephrology and Dialysis in cooperation with the Institute of Pathophysiology and Allergy Research, Medical University of Vienna, and was funded by the Marie Curie Initial Training Network, "Multifaceted CaSR", FP7-264663.

\section{Conflicts of Interest}

The authors declare no conflict of interest. 


\section{References}

1. Stein, E.M.; Shane, E. Vitamin D in organ transplantation. Osteoporos. Int. 2011, doi:10.1007/s00198-010-1523-8.

2. Holick, M.F. Vitamin D deficiency. N. Engl. J. Med. 2007, 357, 266-281.

3. Putz-Bankuti, C.; Pilz, S.; Stojakovic, T.; Scharnagl, H.; Pieber, T.R.; Trauner, M.; Obermayer-Pietsch, B.; Stauber, R.E. Association of 25-hydroxyvitamin D levels with liver dysfunction and mortality in chronic liver disease. Liver Int. 2012, 32, 845-851.

4. Nessim, S.J.; Jassal, S.V.; Fung, S.V.; Chan, C.T. Conversion from conventional to nocturnal hemodialysis improves vitamin D levels. Kidney Int. 2007, 71, 1172-1176.

5. Jacob, A.I.; Sallman, A.; Santiz, Z.; Hollis, B.W. Defective photoproduction of cholecalciferol in normal and uremic humans. J. Nutr. 1984, 114, 1313-1319.

6. Euvrard, S.; Kanitakis, J.; Claudy, A. Skin cancers after organ transplantation. N. Engl. J. Med. 2003, 348, 1681-1691.

7. Querings, K.; Girndt, M.; Geisel, J.; Georg, T.; Tilgen, W.; Reichrath, J. 25-hydroxyvitamin D deficiency in renal transplant recipients. J. Clin. Endocrinol. Metab. 2006, 91, 526-529.

8. Pascussi, J.M.; Robert, A.; Nguyen, M.; Walrant-Debray, O.; Garabedian, M.; Martin, P.; Pineau, T.; Saric, J.; Navarro, F.; Maurel, P.; et al. Possible involvement of pregnane X receptorenhanced CYP24 expression in drug-induced osteomalacia. J. Clin. Investig. 2005, 115, 177-186.

9. Mithal, A.; Wahl, D.A.; Bonjour, J.P.; Burckhardt, P.; Dawson-Hughes, B.; Eisman, J.A.; El-Hajj Fuleihan, G.; Josse, R.G.; Lips, P.; Morales-Torres, J. Global vitamin D status and determinants of hypovitaminosis D. Osteoporos. Int. 2009, 20, 1807-1820.

10. Yetley, E.A. Assessing the vitamin D status of the US population. Am. J. Clin. Nutr. 2008, 88, 558S-564S.

11. Van der Wielen, R.P.; Lowik, M.R.; van den Berg, H.; de Groot, L.C.; Haller, J.; Moreiras, O.; van Staveren, W.A. Serum vitamin D concentrations among elderly people in Europe. Lancet 1995, 346, 207-210.

12. Ross, A.C.; Manson, J.E.; Abrams, S.A.; Aloia, J.F.; Brannon, P.M.; Clinton, S.K.; Durazo-Arvizu, R.A.; Gallagher, J.C.; Gallo, R.L.; Jones, G.; et al. The 2011 report on dietary reference intakes for calcium and vitamin D from the Institute of Medicine: What clinicians need to know. J. Clin. Endocrinol. Metab. 2011, 96, 53-58.

13. Shane, E.; Mancini, D.; Aaronson, K.; Silverberg, S.J.; Seibel, M.J.; Addesso, V.; McMahon, D.J. Bone mass, vitamin D deficiency, and hyperparathyroidism in congestive heart failure. Am. $J$. Med. 1997, 103, 197-207.

14. Zittermann, A.; Schleithoff, S.S.; Gotting, C.; Dronow, O.; Fuchs, U.; Kuhn, J.; Kleesiek, K.; Tenderich, G.; Koerfer, R. Poor outcome in end-stage heart failure patients with low circulating calcitriol levels. Eur. J. Heart Fail. 2008, 10, 321-327.

15. Stein, E.M.; Cohen, A.; Freeby, M.; Rogers, H.; Kokolus, S.; Scott, V.; Mancini, D.; Restaino, S.; Brown, R.; McMahon, D.J.; et al. Severe vitamin D deficiency among heart and liver transplant recipients. Clin. Transplant. 2009, 23, 861-865. 
16. Forli, L.; Bollerslev, J.; Simonsen, S.; Isaksen, G.A.; Kvamsdal, K.E.; Godang, K.; Gadeholt, G.; Pripp, A.H.; Bjortuft, O. Dietary vitamin K2 supplement improves bone status after lung and heart transplantation. Transplantation 2010, 89, 458-464.

17. Movassaghi, S.; Nasiri Toosi, M.; Bakhshandeh, A.; Niksolat, F.; Khazaeipour, Z.; Tajik, A. Frequency of musculoskeletal complications among the patients receiving solid organ transplantation in a tertiary health-care center. Rheumatol. Int. 2012, 32, 2363-2366.

18. Dalle Carbonare, L.; Zanatta, M.; Braga, V.; Sella, S.; Vilei, M.T.; Feltrin, G.; Gambino, A.; Pepe, I.; Rossini, M.; Adami, S.; et al. Densitometric threshold and vertebral fractures in heart transplant patients. Transplantation 2011, 92, 106-111.

19. Schleithoff, S.S.; Zittermann, A.; Tenderich, G.; Berthold, H.K.; Stehle, P.; Koerfer, R. Vitamin D supplementation improves cytokine profiles in patients with congestive heart failure: A double-blind, randomized, placebo-controlled trial. Am. J. Clin. Nutr. 2006, 83, 754-759.

20. Amin, A.; Minaee, S.; Chitsazan, M.; Naderi, N.; Taghavi, S.; Ardeshiri, M. Can Vitamin D supplementation improve the severity of congestive heart failure? Congest. Heart Fail. 2013, doi:10.1111/chf.12026.

21. Costanzo, M.R.; Dipchand, A.; Starling, R.; Anderson, A.; Chan, M.; Desai, S.; Fedson, S.; Fisher, P.; Gonzales-Stawinski, G.; Martinelli, L.; et al. The International Society of Heart and Lung Transplantation Guidelines for the care of heart transplant recipients. J. Heart Lung Transplant. 2010, 29, 914-956.

22. Shane, E.; Silverberg, S.J.; Donovan, D.; Papadopoulos, A.; Staron, R.B.; Addesso, V.; Jorgesen, B.; McGregor, C.; Schulman, L. Osteoporosis in lung transplantation candidates with end-stage pulmonary disease. Am. J. Med. 1996, 101, 262-269.

23. Tschopp, O.; Boehler, A.; Speich, R.; Weder, W.; Seifert, B.; Russi, E.W.; Schmid, C. Osteoporosis before lung transplantation: Association with low body mass index, but not with underlying disease. Am. J. Transplant. 2002, 2, 167-172.

24. Donovan, D.S., Jr.; Papadopoulos, A.; Staron, R.B.; Addesso, V.; Schulman, L.; McGregor, C.; Cosman, F.; Lindsay, R.L.; Shane, E. Bone mass and vitamin D deficiency in adults with advanced cystic fibrosis lung disease. Am. J. Respir. Crit. Care Med. 1998, 157, 1892-1899.

25. Lowery, E.M.; Bemiss, B.; Cascino, T.; Durazo-Arvizu, R.A.; Forsythe, S.M.; Alex, C.; Laghi, F.; Love, R.B.; Camacho, P. Low vitamin D levels are associated with increased rejection and infections after lung transplantation. J. Heart Lung Transplant. 2012, 31, 700-707.

26. Verleden, S.E.; Vos, R.; Geenens, R.; Ruttens, D.; Vaneylen, A.; Dupont, L.J.; Verleden, G.M.; van Raemdonck, D.E.; Vanaudenaerde, B.M. Vitamin D deficiency in lung transplant patients: Is it important? Transplantation 2012, 93, 224-229.

27. Forli, L.; Bjortuft, O.; Boe, J. Vitamin D status in relation to nutritional depletion and muscle function in patients with advanced pulmonary disease. Exp. Lung Res. 2009, 35, 524-538.

28. Aris, R.M.; Renner, J.B.; Winders, A.D.; Buell, H.E.; Riggs, D.B.; Lester, G.E.; Ontjes, D.A. Increased rate of fractures and severe kyphosis: Sequelae of living into adulthood with cystic fibrosis. Ann. Intern. Med. 1998, 128, 186-193.

29. Arteh, J.; Narra, S.; Nair, S. Prevalence of vitamin D deficiency in chronic liver disease. Dig. Dis. Sci. 2010, 55, 2624-2628. 
30. Malham, M.; Jorgensen, S.P.; Ott, P.; Agnholt, J.; Vilstrup, H.; Borre, M.; Dahlerup, J.F. Vitamin D deficiency in cirrhosis relates to liver dysfunction rather than aetiology. World $J$. Gastroenterol. 2011, 17, 922-925.

31. Miroliaee, A.; Nasiri-Toosi, M.; Khalilzadeh, O.; Esteghamati, A.; Abdollahi, A.; Mazloumi, M. Disturbances of parathyroid hormone-vitamin D axis in non-cholestatic chronic liver disease: A cross-sectional study. Hepatol. Int. 2010, 4, 634-640.

32. Fisher, L.; Fisher, A. Vitamin D and parathyroid hormone in outpatients with noncholestatic chronic liver disease. Clin. Gastroenterol. Hepatol. 2007, 5, 513-520.

33. Bitetto, D.; Fabris, C.; Falleti, E.; Fornasiere, E.; Fumolo, E.; Fontanini, E.; Cussigh, A.; Occhino, G.; Baccarani, U.; Pirisi, M.; et al. Vitamin D and the risk of acute allograft rejection following human liver transplantation. Liver Int. 2010, 30, 417-444.

34. Venu, M.; Martin, E.; Saeian, K.; Gawrieh, S. High prevalence of vitamin A deficiency and vitamin D deficiency in patients evaluated for liver transplantation. Liver Transpl. 2013, doi:10.1002/lt.23646.

35. Abbott-Johnson, W.J.; Kerlin, P.; Abiad, G.; Clague, A.E.; Cuneo, R.C. Dark adaptation in vitamin A-deficient adults awaiting liver transplantation: Improvement with intramuscular vitamin A treatment. Br. J. Ophthalmol. 2011, 95, 544-548.

36. Reese, P.P.; Bloom, R.D.; Feldman, H.I.; Huverserian, A.; Thomasson, A.; Shults, J.; Hamano, T.; Goral, S.; Shaked, A.; Olthoff, K.; et al. Changes in vitamin D binding protein and vitamin D concentrations associated with liver transplantation. Liver Int. 2012, 32, 287-296.

37. Monegal, A.; Navasa, M.; Guanabens, N.; Peris, P.; Pons, F.; Martinez de Osaba, M.J.; Ordi, J.; Rimola, A.; Rodes, J.; Munoz-Gomez, J. Bone disease after liver transplantation: A long-term prospective study of bone mass changes, hormonal status and histomorphometric characteristics. Osteoporos. Int. 2001, 12, 484-492.

38. Segal, E.; Baruch, Y.; Kramsky, R.; Raz, B.; Tamir, A.; Ish-Shalom, S. Predominant factors associated with bone loss in liver transplant patients-After prolonged post-transplantation period. Clin. Transplant. 2003, 17, 13-19.

39. Holick, M.F.; Binkley, N.C.; Bischoff-Ferrari, H.A.; Gordon, C.M.; Hanley, D.A.; Heaney, R.P.; Murad, M.H.; Weaver, C.M. Evaluation, treatment, and prevention of vitamin D deficiency: An Endocrine Society clinical practice guideline. J. Clin. Endocrinol. Metab. 2011, 96, 1911-1930.

40. Lucey, M.R.; Terrault, N.; Ojo, L.; Hay, J.E.; Neuberger, J.; Blumberg, E.; Teperman, L.W. Long-term management of the successful adult liver transplant: 2012 practice guideline by the American Association for the Study of Liver Diseases and the American Society of Transplantation. Liver Transpl. 2013, 19, 3-26.

41. LaClair, R.E.; Hellman, R.N.; Karp, S.L.; Kraus, M.; Ofner, S.; Li, Q.; Graves, K.L.; Moe, S.M. Prevalence of calcidiol deficiency in CKD: A cross-sectional study across latitudes in the United States. Am. J. Kidney Dis. 2005, 45, 1026-1033.

42. Ravani, P.; Malberti, F.; Tripepi, G.; Pecchini, P.; Cutrupi, S.; Pizzini, P.; Mallamaci, F.; Zoccali, C. Vitamin D levels and patient outcome in chronic kidney disease. Kidney Int. 2009, $75,88-95$. 
43. Ishimura, E.; Nishizawa, Y.; Inaba, M.; Matsumoto, N.; Emoto, M.; Kawagishi, T.; Shoji, S.; Okuno, S.; Kim, M.; Miki, T.; et al. Serum levels of 1,25-dihydroxyvitamin D, 24,25-dihydroxyvitamin D, and 25-hydroxyvitamin D in nondialyzed patients with chronic renal failure. Kidney Int. 1999, 55, 1019-1027.

44. Stavroulopoulos, A.; Porter, C.J.; Roe, S.D.; Hosking, D.J.; Cassidy, M.J. Relationship between vitamin D status, parathyroid hormone levels and bone mineral density in patients with chronic kidney disease stages 3 and 4. Nephrology (Carlton) 2008, 13, 63-67.

45. Sadlier, D.M.; Magee, C.C. Prevalence of 25(OH) vitamin D (calcidiol) deficiency at time of renal transplantation: A prospective study. Clin. Transplant. 2007, 21, 683-688.

46. Tripathi, S.S.; Gibney, E.M.; Gehr, T.W.; King, A.L.; Beckman, M.J. High prevalence of vitamin D deficiency in African American kidney transplant recipients. Transplantation 2008, $85,767-770$.

47. Yilmaz, M.I.; Sonmez, A.; Saglam, M.; Yaman, H.; Kilic, S.; Turker, T.; Unal, H.U.; Gok, M.; Cetinkaya, H.; Eyileten, T.; et al. Longitudinal analysis of vascular function and biomarkers of metabolic bone disorders before and after renal transplantation. Am. J. Nephrol. 2013, 37, 126-134.

48. Stavroulopoulos, A.; Cassidy, M.J.; Porter, C.J.; Hosking, D.J.; Roe, S.D. Vitamin D status in renal transplant recipients. Am. J. Transplant. 2007, 7, 2546-2552.

49. Ewers, B.; Gasbjerg, A.; Moelgaard, C.; Frederiksen, A.M.; Marckmann, P. Vitamin D status in kidney transplant patients: Need for intensified routine supplementation. Am. J. Clin. Nutr. 2008, 87, 431-437.

50. Penny, H.; Frame, S.; Dickinson, F.; Garrett, G.; Young, A.R.; Sarkany, R.; Chitalia, N.; Hampson, G.; Goldsmith, D. Determinants of vitamin D status in long-term renal transplant patients. Clin. Transplant. 2012, 26, E617-E623.

51. Burkhalter, F.; Schaub, S.; Dickenmann, M. Preserved circannual rhythm of vitamin D in kidney transplant patients. Swiss Med. Wkly. 2012, 142, w13672.

52. National Kidney Foundation. K/DOQI clinical practice guidelines for bone metabolism and disease in chronic kidney disease. Am. J. Kidney Dis. 2003, 42, S1-S201.

53. KDIGO. KDIGO clinical practice guideline for the care of kidney transplant recipients. Am. J. Transplant. 2009, 9 (Suppl. 3), 1-155.

54. Wissing, K.M.; Broeders, N.; Moreno-Reyes, R.; Gervy, C.; Stallenberg, B.; Abramowicz, D. A controlled study of vitamin D3 to prevent bone loss in renal-transplant patients receiving low doses of steroids. Transplantation 2005, 79, 108-115.

55. Courbebaisse, M.; Thervet, E.; Souberbielle, J.C.; Zuber, J.; Eladari, D.; Martinez, F.; Mamzer-Bruneel, M.F.; Urena, P.; Legendre, C.; Friedlander, G.; et al. Effects of vitamin D supplementation on the calcium-phosphate balance in renal transplant patients. Kidney Int. 2009, 75, 646-651.

56. Benaboud, S.; Urien, S.; Thervet, E.; Prie, D.; Legendre, C.; Souberbielle, J.C.; Hirt, D.; Friedlander, G.; Treluyer, J.M.; Courbebaisse, M. Determination of optimal cholecalciferol treatment in renal transplant recipients using a population pharmacokinetic approach. Eur J. Clin. Pharmacol. 2013, 69, 499-506. 
57. Thiem, U.; Heinze, G.; Segel, R.; Perkmann, T.; Kainberger, F.; Muhlbacher, F.; Horl, W.; Borchhardt, K. VITA-D: Cholecalciferol substitution in vitamin D deficient kidney transplant recipients: A randomized, placebo-controlled study to evaluate the post-transplant outcome. Trials 2009, 10, 36.

58. Meems, L.M.; van der Harst, P.; van Gilst, W.H.; de Boer, R.A. Vitamin D biology in heart failure: Molecular mechanisms and systematic review. Curr. Drug Targets 2011, 12, 29-41.

59. Pilz, S.; Tomaschitz, A.; Marz, W.; Drechsler, C.; Ritz, E.; Zittermann, A.; Cavalier, E.; Pieber, T.R.; Lappe, J.M.; Grant, W.B.; et al. Vitamin D, cardiovascular disease and mortality. Clin. Endocrinol. (Oxf.) 2011, 75, 575-584.

60. Shroff, R.; Wan, M.; Rees, L. Can vitamin D slow down the progression of chronic kidney disease? Pediatr. Nephrol. 2012, 27, 2167-2173.

61. Di Rosa, M.; Malaguarnera, M.; Nicoletti, F.; Malaguarnera, L. Vitamin D3: A helpful immuno-modulator. Immunology 2011, 134, 123-139.

62. Thiem, U.; Borchhardt, K. Vitamin D in solid organ transplantation with special emphasis on kidney transplantation. Vitam. Horm. 2011, 86, 429-468.

63. Bock, G.; Prietl, B.; Mader, J.K.; Holler, E.; Wolf, M.; Pilz, S.; Graninger, W.B.; Obermayer-Pietsch, B.M.; Pieber, T.R. The effect of vitamin D supplementation on peripheral regulatory $\mathrm{T}$ cells and beta cell function in healthy humans: A randomized controlled trial. Diabetes Metab. Res. Rev. 2011, 27, 942-945.

64. Hopkins, M.H.; Owen, J.; Ahearn, T.; Fedirko, V.; Flanders, W.D.; Jones, D.P.; Bostick, R.M. Effects of supplemental vitamin D and calcium on biomarkers of inflammation in colorectal adenoma patients: A randomized, controlled clinical trial. Cancer Prev Res. (Phila.) 2011, 4, $1645-1654$.

65. Bucharles, S.; Barberato, S.H.; Stinghen, A.E.; Gruber, B.; Piekala, L.; Dambiski, A.C.; Custodio, M.R.; Pecoits-Filho, R. Impact of cholecalciferol treatment on biomarkers of inflammation and myocardial structure in hemodialysis patients without hyperparathyroidism. J. Ren. Nutr. 2012, 22, 284-291.

66. Bischoff-Ferrari, H.A.; Dawson-Hughes, B.; Stocklin, E.; Sidelnikov, E.; Willett, W.C.; Edel, J.O.; Stahelin, H.B.; Wolfram, S.; Jetter, A.; Schwager, J.; et al. Oral supplementation with 25(OH)D3 versus vitamin D3: Effects on 25(OH)D levels, lower extremity function, blood pressure, and markers of innate immunity. J. Bone Miner. Res. 2012, 27, 160-169.

67. Barker, T.; Martins, T.B.; Hill, H.R.; Kjeldsberg, C.R.; Henriksen, V.T.; Dixon, B.M.; Schneider, E.D.; Dern, A.; Weaver, L.K. Different doses of supplemental vitamin D maintain interleukin-5 without altering skeletal muscle strength: A randomized, double-blind, placebo-controlled study in vitamin D sufficient adults. Nutr. Metab (Lond.) 2012, 9, 16.

68. Alvarez, J.A.; Zughaier, S.M.; Law, J.; Hao, L.; Wasse, H.; Ziegler, T.R.; Tangpricha, V. Effects of high-dose cholecalciferol on serum markers of inflammation and immunity in patients with early chronic kidney disease. Eur J. Clin. Nutr. 2013, 67, 264-269.

69. Hossein-Nezhad, A.; Spira, A.; Holick, M.F. Influence of vitamin d status and vitamin d3 supplementation on genome wide expression of white blood cells: A randomized double-blind clinical trial. PLoS One 2013, 8, e58725. 


\title{
Vitamin D Status Is Associated with Disease Activity among Rheumatology Outpatients
}

\section{Zohreh Sabbagh, Janet Markland and Hassanali Vatanparast}

\begin{abstract}
The co-existence of high prevalence of vitamin D inadequacy among Canadians and high prevalence of systematic autoimmune rheumatic diseases (SARDs) raise the question on relationship between the two situations. Objective: To determine vitamin D status in known cases of common SARDs and compare to those with non-autoimmune diseases; further, to evaluate the impact of vitamin $\mathrm{D}$ on disease activity in rheumatoid arthritis (RA) cases. Methods: In a retrospective case-control study design, we evaluated 116 patients in a community clinic classified in two groups, Control group: patients with non-rheumatic disease $(n=56)$, and Case group: those with rheumatic diseases $(n=60)$. We compared plasma vitamin D status $(25(\mathrm{OH}) \mathrm{D})$, indicators of disease activity and other potential confounders. Further, we determined factors associated with disease activity in RA cases. Results: The plasma 25(OH)D was significantly lower in Case group (64.8 \pm 29.8$)$ compared to Control group ( $86.8 \pm 37.7)$. High number of SARDs outpatients $56 \%)$ had considerably low plasma $25(\mathrm{OH}) \mathrm{D}$ concentration. RA cases with low plasma $25(\mathrm{OH}) \mathrm{D}$ had over five times higher risk of disease activity $(\mathrm{OR}=5.1595 \% \mathrm{CI} 1.16,22.9 ; p=0.031)$. Conclusion: Inadequate vitamin D status in SARDs cases, along with considerably strong association with disease activity in RA cases, indicate the need for proper evaluation of vitamin D status in this clinical population. Moreover, appropriate training should be given to the patients to ensure the intake of the recommended amount of vitamin D per day through diet or supplement.
\end{abstract}

Reprinted from Nutrients. Cite as: Sabbagh, Z.; Markland, J.; Vatanparast, H. Vitamin D Status Is Associated with Disease Activity among Rheumatology Outpatients. Nutrients 2013, 5, 2268-2275.

\section{Introduction}

Systematic autoimmune rheumatic disorders (SARDs) are a cluster of chronic autoimmune disorders associated with significant mortality and morbidity in developed countries, including Canada [1]. Rheumatoid arthritis (RA) as the most common disease in this group, affects around $0.9 \%$ of Canadian population; which will increase to $1.3 \%$ over the next 30 years [2]. The chronic progressive nature of the disease disables over $50 \%$ of cases within 10 years if not treated properly [2]. Early diagnosis and proper intervention strategies are key factors in managing the SARDs and decreasing the burden of disease. Since the prevalence of SARDs is affected by genetic and environmental factors [3], nutrition intervention might have impact in disease prevention and risk reduction.

The co-existence of a high prevalence of vitamin D inadequacy among Canadians, particularly in long winters [4], and the high prevalence of SARDs may raise the question on the relationship between these two. Vitamin D is traditionally known for its role in bone mineral homeostasis [5]. However, recent research reveals the existence of vitamin D receptors (VDR) in a variety of cells including anti-presenting cells (APC) $[3,5,6]$. This indicates the influence of vitamin D in various 
physiologic processes; one of them is the immune system. Active metabolite of vitamin D $(1,25(\mathrm{OH}) 2 \mathrm{D})$ inhibits the synthesis of IL-1, IL-6, IL-12 and TNF- $\alpha$ by macrophages [7]. It also decreases MHC-II expression on cell surface and molecules such as CD86, CD80 and CD 40 [3,7]. Finally, it increases apoptosis induced by DC and T lymphocytes. Animal studies report the role of vitamin D in preventing autoimmune encephalomyelitis, systemic lupus erythematosus (SLE), collagen-induced arthritis, and inflammatory bowel disease [7]. Along with these findings, evidence suggests an increase in the emergence of self-reactive $\mathrm{T}$ cells where the development of immune system co-exists with low vitamin D status [7]. All this evidence suggests vitamin D deficiency might trigger an autoimmune response and appropriate vitamin D status presents immunosuppressive effect [7].

A systematic review of studies on vitamin D and SARDs reported, comparing to healthy control groups, many case-control studies found lower vitamin D status in SARDs cases including SLE, RA, ankylosing spondylitis, scleroderma, Type 1 diabetes, Multiple Sclerosis and Crohn's disease [8]. Further, current evidence from mainly small scale heterogeneous studies indicate possible role of vitamin D in improving disease activity in SARD cases such as RA, Multiple Sclerosis, type 1 diabetes, SLE and Crohn's disease [8]. The association between vitamin D status and diseases activity in RA was evaluated in a recent meta-analyses of current studies including data from three cohort, six cross-sectional and two case-control studies $(n=215,757)$ [9]. Song et al. report inverse association between RA disease activity and serum vitamin D levels [9]. Recent advances in the role of vitamin $\mathrm{D}$ in various diseases and very limited dietary sources has promoted the intake of vitamin D, particularly in Canada's high latitude areas with long winters such as Saskatoon [4,10], where vitamin D synthesis through sun is limited for at least seven months of the year. However, whether adult patients diagnosed with common autoimmune diseases in Canada have adequate levels of serum vitamin $\mathrm{D}$ is not known. The purpose of this study is to determine serum vitamin $\mathrm{D}$ status of known cases of common autoimmune diseases and compare to those with non-autoimmune diseases; also to evaluate the impact of vitamin D on disease activity.

\section{Materials and Methods}

In a retrospective case-control study design, we evaluated the charts of over 4000 patients and selected 116 patients with measured plasma 25-hydoxy vitamin D (25(OH)D). Using information on final diagnosis we categorized patients in case and control groups; 60 patients with autoimmune rheumatologic disease, as well as 56 patients with non-autoimmune conditions who were visited in one of the private rheumatology clinics in Saskatoon from January 2010 to the end of December 2010. Information on age, sex, BMI, plasma 25(OH)D, vitamin D and calcium supplement use, serum calcium, serum phosphate, erythrocyte sedimentation rate (ESR), C-reactive protein (CRP), glomerular filtration rate (GFR) and season were collected and recorded. We also collected data on disease activity score (DAS 28-ESR) only in cases with rheumatoid arthritis. DAS28-ESR is a quantitative measure of disease activity in rheumatoid arthritis, calculated by using a formula that considers the number of tender joints and swollen joints within 28 joints, as well as ESR [11]. The disease activity is considered high with the score of $>5.1$, low with $<3.2$ score, and the score of $<2.6$ for diseases in remission [11]. 
Independent $t$-test was used to evaluate the differences between patients in case and control group. Pearson correlation was used to determine the relationship between disease activity and serum vitamin D status in RA cases. We used the threshold of $50 \mathrm{nmol} / \mathrm{L}$ to define vitamin $\mathrm{D}$ deficiency based on recent literature $[6,12]$. To evaluate distribution of cases and controls across vitamin D status groups (deficient and optimal), Chi-square test was applied. Logistic regression was used to identify the association between vitamin D status and disease (autoimmune vs. non-auto immune). Data manipulation, cleaning, and creation of new variables and statistical analyses were done using SPSS IBM (Version 19, Armonk, NY, USA). In all analyses, alpha was set at the level of 0.05. Ethics approval was obtained from the Royal University Hospital Ethics Board, at the University of Saskatchewan.

\section{Results}

The characteristics of participants in case and control groups are presented in Table 1. In the control group, most patients were suffering from osteoarthritis, osteoporosis, fibromyalgia and gout, in descending order. In case group, rheumatoid arthritis $(n=39)$ was the most common autoimmune disease. Other autoimmune diseases included SLE, Sjogren, mixed connective tissue disease (MCTD), Wegner, psoriasis arthritis, and scleroderma.

Table 1. Characteristics of participants in Case and Control groups.

\begin{tabular}{ccccc}
\hline & \multicolumn{2}{c}{ Case group $(\boldsymbol{n}=\mathbf{6 0})$} & \multicolumn{2}{c}{ Control group $(\boldsymbol{n}=\mathbf{5 6})$} \\
\cline { 2 - 5 } & Mean & SD & Mean & SD \\
\hline Age * & 54.5 & 13.0 & 65.0 & 11.7 \\
BMI & 29.0 & 8.1 & 29.7 & 5.4 \\
Total 25 vitamin D value (nmol/L) * & 64.8 & 29.8 & 86.8 & 37.7 \\
Vitamin D supplement use (IU) & 1043.6 & 649.4 & 1008.0 & 664.7 \\
Serum Calcium * & 2.3 & 0.1 & 2.4 & 0.1 \\
Calcium supplement use (mg/day) * & 459.3 & 439 & 715.1 & 591 \\
Pho4 & 1.0 & 0.2 & 1.1 & 0.2 \\
ESR & 23.68 & 20.5 & 19.18 & 14.6 \\
CRP $*$ & 12.9 & 19.3 & 6.07 & 9.6 \\
Creatinine & 72.1 & 44.4 & 78.8 & 33.9 \\
GFR & 69.9 & 28.7 & 71.6 & 20.5 \\
\hline
\end{tabular}

* Significant difference $(p<0.05)$, independent student $t$-test.

Patients in case group were significantly younger. Only 13 participants were males ( 3 in control group and 10 in case group). Although most patients in both case $(91.7 \%)$ and control (83.9\%) groups reported taking approximately 1000 IU vitamin D supplement intake (median of 1000 IU in both case and control group), the mean plasma $25(\mathrm{OH}) \mathrm{D}$ was significantly lower in patients with autoimmune rheumatic diseases compared to control patients $(p<0.05)$ (Figure 1). Also, serum calcium was lower in case group ( $p=0.01$ ). In case group, $91.7 \%$ of participants reported vitamin $\mathrm{D}$ supplement use, among them $47.5 \%$ had vitamin D supplement prescription. The significantly higher CRP in patients with autoimmune rheumatic disease may indicate the higher disease activity in those 
patients ( $p=0.035$ ). A negative correlation was observed between plasma 25(OH)D and CRP with a borderline significance, probably due to a small sample size $(r=-0.2, p=0.087)$.

Figure 1. Plasma concentration of $25(\mathrm{OH})$ D across case and control groups.

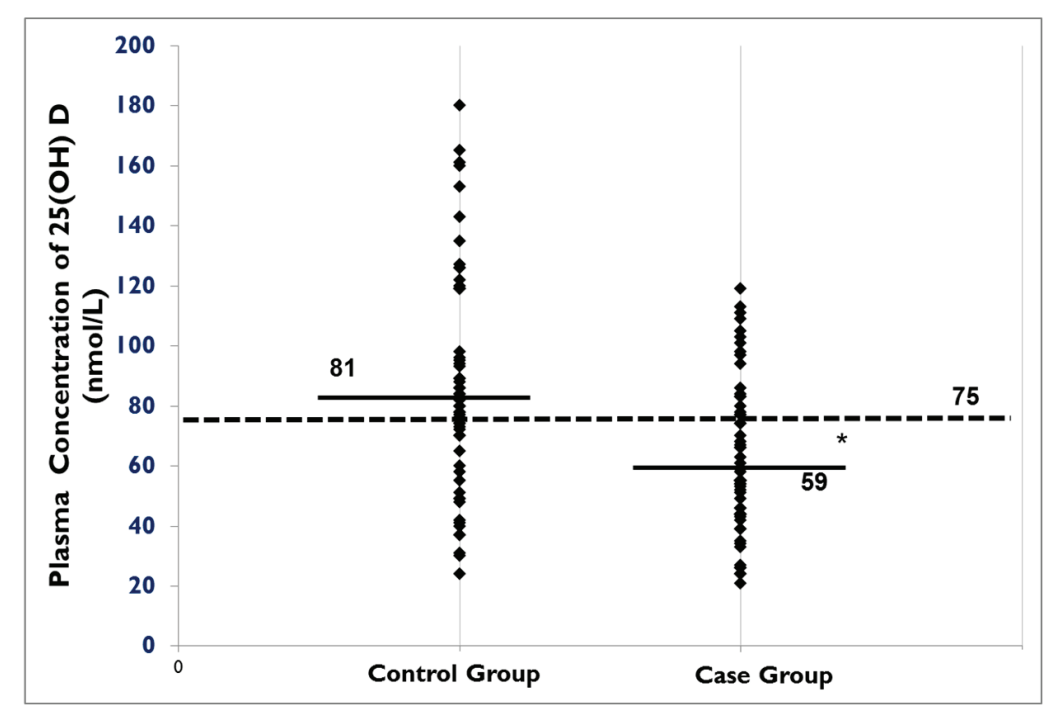

In the control group 11 patients $19.6 \%$ ) with no autoimmune diseases were vitamin D deficient (plasma $25(\mathrm{OH}) \mathrm{D}<50 \mathrm{nmol} / \mathrm{L})$. Whereas in the case group, 20 patients $(34.5 \%)$ were vitamin $\mathrm{D}$ deficient. The distribution of patients across plasma 25(OH)D groups (deficient, optimal) presented a borderline significant difference $(p=0.05)$ between case and control groups with higher distribution of patients with autoimmune diseases in deficient vitamin $\mathrm{D}$ group.

In all subjects, serum vitamin D was significantly higher in those who were measured in summer compared to the winter months $(85.1 \pm 33.4$ vs. $70.8 \pm 35.9, p<0.05)$. While no significant difference in plasma $25(\mathrm{OH}) \mathrm{D}$ was observed by season in control group, plasma $25(\mathrm{OH}) \mathrm{D}$ was significantly higher in summer compared to the winter months in case group (82.2 \pm 30.3 vs. $61.9 \pm 32.7$, $p=0.019$ ). Evaluating the seasonal difference between case and control groups showed no significant difference in plasma $25(\mathrm{OH}) \mathrm{D}$ between case and control groups in summer. However, in winter, plasma $25(\mathrm{OH}) \mathrm{D}$ was significantly lower in case group compared to the control group $(52.3 \pm 22.4 v s .86 .0 \pm 36.3, p=0.02$, in case and control groups respectively).

A considerable proportion of RA cases (59.6\%) had a disease activity score of above 2.6 (cut off for disease remission). Further, the significant negative correlation between plasma $25(\mathrm{OH}) \mathrm{D}$ and disease activity $(r=-0.43, p=0.01)$ may indicate lower disease activity with increase in plasma $25(\mathrm{OH}) \mathrm{D}$. In logistic regression analyses to evaluate the association between vitamin $\mathrm{D}$ status and disease activity in RA cases, adjusted for age, sex and season, the odds of having active disease was 5.15 times higher in patients with low plasma $25(\mathrm{OH}) \mathrm{D}$ compared to those with adequate vitamin $\mathrm{D}$ $(\mathrm{O} R=5.1595 \%$ CI 1.16, 22.9; $p=0.031)$. 


\section{Discussion}

Our study is the first Canadian study to evaluate the vitamin D status of rheumatology outpatients in two distinct groups: those who are suffering from SARD in comparison to non-SARD patients. We found significantly low plasma $25(\mathrm{OH}) \mathrm{D}$ in SARD group compared to non-SARD group despite similar self-reported vitamin D supplement use, as well as seasonal difference in plasma $25(\mathrm{OH}) \mathrm{D}$ concentration in SARD cases. Further, among RA cases, considerably higher risk of disease activity in individuals with low $25(\mathrm{OH}) \mathrm{D}$ concentration is in agreement with other studies suggesting disturbance in vitamin D metabolism in RA and association between vitamin D and disease activity in rheumatic patients $[8,9,13-17]$.

The low plasma 25(OH)D concentration $(<75 \mathrm{nmol} / \mathrm{L})$ in a considerable number of patients with SARD (56\%) and its relationship with indicators of disease activity justifies the need for thorough evaluation of vitamin D status in this at-risk population in clinical settings. In our study, only $2.9 \%$ of patients with SARD and non-SARD diseases, who were visited in a community clinic, were evaluated for vitamin D status. Osteoporotic patients, as one of the common non-SARD cases visited in rheumatology clinics, receive clear instructions on vitamin D intake as adjunct therapy as indicated by Osteoporosis Canada [18]. This might explain why the influence of season on serum vitamin D likely has been minimized by supplement use during winter in control group, whereas in case group due to lack of clear guidelines for this group and consequently low supplement use during long winters. Meanwhile, similar year-around levels of supplement intake between the case and control group (1000 IU), but different mean and median 25(OH)D may indicate different requirements for this specific clinical population who are suffering from SARD. Sainaghi et al. [19] evaluating $25(\mathrm{OH}) \mathrm{D}$ plasma concentration of 245 patients SARD and non-SARD groups, report the 750-1000 IU/day vitamin D might not be enough to provide minimum optimal $25(\mathrm{OH}) \mathrm{D}$ concentration of $\geq 75 \mathrm{nmol}$ in this clinical population. Based on recent evidence, Holick [6] suggests daily vitamin D intake of 1500-2000 IU for healthy adults in general population. Most Canadian adults are not meeting this recommendation [20]. A recent study examined the effect of high-dose loading treatment in RA cases [21]. They reported a single oral dose of 300,000 IU followed by oral vitamin D3 of 800-1000 IU is effective in correcting hypovitaminosis D and PTH level [21]. The potential risks of high loading dose of vitamin D require further evaluations. In a randomized controlled trial, Sanders et al. [22] reported increased risk of falling in older women with annual dose of 500,000 IU vitamin D. Whether suppressing disease activity in SARDs requires more vitamin D, and the risks and benefits of high dose vitamin D requires more investigation in this clinical population.

Although many studies suggest the "overall" immuno-suppressive effect of vitamin D is SARDs, particularly RA, the Dietary Reference Intake Panel at the Institute of Medicine, responsible for setting recommendations for dietary intake, reported no conclusive evidence on the impact of vitamin D on SARDs [5]. The panel indicated that well-designed randomized controlled trials and large scale prospective cohort studies are needed to support the inverse relationship between vitamin D and autoimmune rheumatic diseases [5]. Since their report, more research was done. A meta-analyses reported vitamin D receptor polymorphism in RA and SLE, although only a few existing studies were analysed [21]. Others emphasised the impact of vitamin D on disease activity and elucidated 
the mechanisms [7-9,19,22]. Our evaluation of current evidence identified the following gaps in research:

1. Vitamin D receptor polymorphism and SARDs, and role of ethnicity in association between vitamin D and SARDs;

2. Differences in vitamin D requirements between SARD cases and the general population as well as the requirements for individuals who are at risk of SARD and individuals with established SARD;

3. Mechanisms of the impact of vitamin $\mathrm{D}$ in different pathways in immune system;

4. The potential interaction between vitamin D and drugs in SARD cases and optimal supplementation approaches.

Our study adds a Canadian perspective to existing literature on the impact of vitamin D on SARDs, mainly RD, however some limitations should be noted. The retrospective nature of our casecontrol study limited us to access information on other factors that might have impact on disease status such as smoking, physical activity, dietary intake, and sunlight exposure. Further, data on PTH plasma concentration and glucocorticoid use are not available. Sainaghi et al. reported high PTH level in RA patients irrespective of plasma vitamin D level [19]. Some medications such as glucocorticoids increase the destruction of vitamin D [23]. Finally, a single center study might under/over-estimate the hypovitaminosis D in outpatient rheumatology clinics.

\section{Conclusions}

Although more research is needed, the considerably low plasma $25(\mathrm{OH}) \mathrm{D}$ concentration in a considerably high number of SARDs outpatients (56\%), as well as over five times higher risk of disease activity in RA cases with low plasma 25(OH)D concentration indicates the need for proper evaluation of vitamin D status in this clinical population. Further, appropriate training should be given to the patients to ensure the intake of the recommended amount of vitamin D per day through diet or supplement.

\section{Acknowledgments}

Z.S. and H.V. designed the study. Z.S. conducted research, analyzed data and wrote the paper, and H.V. contributed in data analyses and its revisions. J.M. was involved in study design and operation in her clinic and contributed in revising the manuscript. H.V. has primary responsibility for final content. All authors read and approved the final manuscript.

\section{Conflicts of Interest}

The author declares no conflict of interest.

Support for this manuscript obtained from University of Saskatchewan. 


\section{References}

1. Bernatsky, S.; Lix, L.; Hanly, G.J.; Hudson, M.; Badley, E.; Peschken, C.; Pineau, C.A.; Clarke, A.E.; Fortin, R.; Smith, M.; et al. Surveillance of systemic autoimmune rheumatic diseases using administrative data. Rheumatol. Int. 2011, 31, 549-554.

2. The Impact of Arthritis in Canada: Today and over the Next 30 Years. Available online: http://www.arthritisnetwork.ca/downloads/20111022_Impact_of_arthritis.pdf (accessed on 12 November 2012).

3. Cutolo, M.; Straub, R.H. Insights into endocrine-immunological disturbances in autoimmunity and their impact on treatment. Arthritis Res. Ther. 2009, 11, 218.

4. Whiting, S.J.; Langlois, K.A.; Vatanparast, H.; Greene-Finestone, L.S. The vitamin D status of Canadians relative to the 2011 Dietary Reference Intakes: An examination in children and adults with and without supplement use. Am. J. Clin. Nutr. 2011, 94, 128-135.

5. Institute of Medicine, Food and Nutrition Board. Dietary Reference Intakes for Calcium and Vitamin D. Available online: http://www.iom.edu/ /media/Files/Report\%20Files/2010/DietaryReference-Intakes-for-Calcium-and-VitaminD/Vitamin\%20D\%20and\%20Calcium\%202010\%20 Report\%20Brief.pdf (accessed on 26 September 2012).

6. Holick, M.F. Evidence-based D-bate on health benefits of vitamin D revisited. Dermatoendocrinology 2012, 4, 183-190.

7. Cutolo, M.; Pizzorni, C.; Sulli, A. Vitamin D endocrine system involvement in autoimmune rheumatic diseases. Autoimmun. Rev. 2011, 11, 84-87.

8. Kriegel, M.A.; Manson, J.E.; Costenbader, K.H. Does vitamin D affect risk of developing autoimmune disease? A systematic review. Semin. Arthritis Rheum. 2011, 40, 512-531.

9. Song, G.G.; Bae, S.C.; Lee, Y.H. Association between vitamin D intake and the risk of rheumatoid arthritis: A meta-analysis. Clin. Rheumatol. 2012, 31, 1733-1739.

10. Webb, A.R.; Kline, L.; Holick, M.F. Influence of season and latitude on the cutaneous synthesis of vitamin D3: Exposure to winter sunlight in Boston and Edmonton will not promote vitamin D3 synthesis in human skin. J. Clin. Endocrinol. Metab. 1988, 67, 373-378.

11. The DAS28 Score. National Rheumatoid Arthritis Society. Available online: http://www.nras. org.uk/about_rheumatoid_arthritis/established_disease/managing_well/the_das 28 _score.aspx (accessed on 11 September 2011).

12. Holick, M.F.; Binkley, N.C.; Bischoff-Ferrari, H.A.; Gordon, C.M.; Hanley, D.A.; Heaney, R.P.; Murad, M.H.; Weaver, C.M.; Endocrine Society. Evaluation, treatment, and prevention of vitamin D deficiency: An Endocrine Society clinical practice guideline. J. Clin. Endocrinol. Metab. 2011, 96, 1911-1930.

13. Cutolo, M.; Otsa, K.; Laas, K.; Yprus, M.; Lehtme, R.; Secchi, M.E.; Sulli, A.; Paolino, S.; Seriolo, B. Circannual vitamin D serum levels and disease activity in rheumatoid arthritis: Northern vs. Southern Europe. Clin. Exp. Rheumatol. 2006, 24, 702-704.

14. Merlino, L.A.; Curtis, J.; Mikuls, T.R.; Cerhan, J.R.; Criswell, L.A.; Saag, K.G. Iowa Women's Health Study. Vitamin D intake is inversely associated with rheumatoid arthritis: Results from the Iowa Women's Health Study. Arthritis Rheum. 2004, 50, 72-77. 
15. Garcia-Lozano, J.R.; Gonzalez-Escribano, M.; Valenzuela, A.; Garcia, A.; Núñez-Roldán, A. Association of vitamin D receptor genotypes with early onset rheumatoid arthritis. Eur. J. Immunogenet. 2001, 28, 89-93.

16. Kröger, H.; Penttilä, I.M.; Alhava, E.M. Low serum vitamin D metabolites in women with rheumatoid arthritis. Scand. J. Rheumatol. 1993, 22, 172-177.

17. Oelzner, P.; Müller, A.; Deschner, F.; Hüller, M.; Abendroth, K.; Hein, G.; Stein, G. Relationship between disease activity and serum levels of vitamin D metabolites and PTH in rheumatoid arthritis. Calcif. Tissue Int. 1998, 62, 193-198.

18. Osteoporosis Canada. Available online: http://www.osteoporosis.ca/index.php/ci_id/5536/ la_id/1.htm (accessed on 15 September 2012).

19. Sainaghi, P.P.; Bellan, M.; Carda, S.; Cerutti, C.; Sola, D.; Nerviani, A.; Molinari, R.; Cisari, C.; Avanzi, G.C. Hypovitaminosis D and response to cholecalciferol supplementation in patients with autoimmune and non-autoimmune rheumatic diseases. Rheumatol. Int. 2012, 32, 3365-3372.

20. Vatanparast, H.; Calvo, M.S.; Green, T.J.; Whiting, S.J. Despite mandatory fortification of staple foods, vitamin D intakes of Canadian children and adults are inadequate. J. Steroid Biochem. Mol. Biol. 2010, 121, 301-303.

21. Sainaghi, P.P.; Bellan, M.; Nerviani, A.; Sola, D.; Molinari, R.; Cerutti, C.; Pirisi, M. Superiority of a high loading dose of cholecalciferol to correct hypovitaminosis D in patients with inflammatory/autoimmune rheumatic diseases. J. Rheumatol. 2013, 40, 166-172.

22. Sanders, K.M.; Stuart, A.L.; Williamson, E.J.; Simpson, J.A.; Kotowicz, M.A.; Young, D.; Nicholson, G.C. Annual high-dose oral vitamin D and falls and fractures in older women: A randomized controlled trial. JAMA 2010, 303, 1815-1822.

23. Lee, Y.H.; Bae, S.C.; Choi, S.J.; Ji, J.D.; Song, G.G. Associations between vitamin D receptor polymorphisms and susceptibility to rheumatoid arthritis and systemic lupus erythematosus: A meta-analysis. Mol. Biol. Rep. 2011, 38, 3643-3651. 


\title{
Vitamin D Level and Risk of Community-Acquired Pneumonia and Sepsis
}

\author{
Anna J. Jovanovich, Adit A. Ginde, John Holmen, Kristen Jablonski, Rebecca L. Allyn, \\ Jessica Kendrick and Michel Chonchol
}

\begin{abstract}
Previous research has reported reduced serum 25-hydroxyvitamin D (25(OH)D) levels is associated with acute infectious illness. The relationship between vitamin D status, measured prior to acute infectious illness, with risk of community-acquired pneumonia (CAP) and sepsis has not been examined. Community-living individuals hospitalized with CAP or sepsis were age-, sex-, race-, and season-matched with controls. ICD-9 codes identified CAP and sepsis; chest radiograph confirmed CAP. Serum 25(OH)D levels were measured up to 15 months prior to hospitalization. Regression models adjusted for diabetes, renal disease, and peripheral vascular disease evaluated the association of 25(OH)D levels with CAP or sepsis risk. A total of 132 CAP patients and controls were $60 \pm 17$ years, $71 \%$ female, and 86\% Caucasian. The 25(OH)D levels $<37 \mathrm{nmol} / \mathrm{L}$ (adjusted odds ratio (OR) $2.57,95 \%$ CI 1.08-6.08) were strongly associated with increased odds of CAP hospitalization. A total of 422 sepsis patients and controls were $65 \pm 14$ years, 59\% female, and 91\% Caucasian. The 25(OH)D levels $<37 \mathrm{nmol} / \mathrm{L}$ (adjusted OR 1.75, 95\% CI 1.11-2.77) were associated with increased odds of sepsis hospitalization. Vitamin D status was inversely associated with risk of CAP and sepsis hospitalization in a community-living adult population. Further clinical trials are needed to evaluate whether vitamin D supplementation can reduce risk of infections, including CAP and sepsis.
\end{abstract}

Reprinted from Nutrients. Cite as: Jovanovich, A.J.; Ginde, A.A.; Holmen, J.; Jablonski, K.; Allyn, R.L.; Kendrick, J.; Chonchol, M. Vitamin D Level and Risk of Community-Acquired Pneumonia and Sepsis. Nutrients 2014, 6, 2196-2205.

\section{Introduction}

Relatively little progress has been made in improving mortality associated with community-acquired pneumonia (CAP) [1] which is a leading cause of death in the United States [2]. Likewise, the incidence of sepsis continues to rise, the population-adjusted incidence of sepsis increased $8.7 \%$ per year between 1979 and 2000 [3]. While mortality associated with sepsis has improved [3], it remains a substantial cause of death in the United States [2].

The major circulating form of vitamin D, 25-hydroxyvitamin $\mathrm{D}(25(\mathrm{OH}) \mathrm{D})$, and its active form, 1,25-dihydroxyvitamin $\mathrm{D}\left(1,25(\mathrm{OH})_{2} \mathrm{D}\right)$, were originally recognized as important endocrine hormones in calcium homeostasis and bone health. However, studies over the past twenty years suggest a broader role of $25(\mathrm{OH}) \mathrm{D}$ in endothelial function, cell proliferation, and immunity. The vitamin D receptor (VDR) is essentially ubiquitous, including immune cells. It responds to $1,25(\mathrm{OH})_{2} \mathrm{D}[4,5]$ and regulates antimicrobial peptides cathelicidin and beta-defensing [5]. Furthermore, $25(\mathrm{OH}) \mathrm{D}$ deficiency is common; $32 \%$ of the U.S. population have $25(\mathrm{OH}) \mathrm{D}$ levels $<50 \mathrm{nmol} / \mathrm{L}$ and $77 \%$ have levels $<75 \mathrm{nmol} / \mathrm{L}$ [6]. Large epidemiological studies have shown an association between 
25(OH)D deficiency and chronic diseases [7] including diabetes [8], renal disease [9], and peripheral vascular disease [10].

Lower serum 25(OH)D levels are associated with increased risk of upper respiratory tract infection [11-13]. When measured during hospital admission for acute illness, 25(OH)D deficiency is associated with increased risk of mortality in patients with CAP [14], more severe sepsis [15], and mortality in septic patients [16]. Most studies linking infection risk with 25(OH)D levels measure $25(\mathrm{OH}) \mathrm{D}$ during acute illness, which may not reflect pre-illness $25(\mathrm{OH}) \mathrm{D}$ status. There are no data assessing existing 25(OH)D deficiency with risk of hospital admission for CAP or sepsis. In this case-control study of community-living adults, serum $25(\mathrm{OH}) \mathrm{D}$ levels and the risk of hospital admission for CAP and sepsis was evaluated.

\section{Experimental Section}

\subsection{Data Source}

A retrospective matched cohort study was performed using the Intermountain Healthcare Enterprise Data Warehouse, which incorporates comprehensive electronic health and administrative data for over 10 years [17]. Intermountain Healthcare is a non-profit organization with 22 hospitals and over 150 outpatient clinics that serves the states of Utah and southeastern Idaho. The latitude of the hospitals and outpatient clinics is approximately $40^{\circ}$ N. Facilities range from major adult tertiary-level care centers to small clinics and hospitals that are the only source of care in rural communities. There were 160,979 admissions from 2008 to 2010 [17]. The institutional review board at Intermountain Healthcare System and University of Colorado Denver approved the project.

\subsection{Cohort Definition}

Case and control selection occurred between 1 January 2008 and 31 December 2010. CAP cases were identified through ICD-9 codes (480-488) and confirmed with chest radiograph. There were 187,132 CAP admissions of which 43,460 had discharge ICD-9 codes indicating pneumonia. Of these, 11,455 had chest radiography confirming pneumonia. 4352 Sepsis cases were identified through ICD-9 codes $(995.91,995.92)$. Controls were randomly selected from a pool of 62,757 adult patients without a CAP or sepsis diagnosis admitted within the same time period and matched 1:1 with cases by age, sex, race, and season of $25(\mathrm{OH}) \mathrm{D}$ measurement. Cases and control had to have a serum $25(\mathrm{OH})$ level in the electronic medical record 3-15 months prior to admission; therefore, only 132 and 422 patients were included in the final CAP and sepsis analyses, respectively. These time points were chosen arbitrarily to assess the relationship between pre-infection serum $25(\mathrm{OH}) \mathrm{D}$ levels and infectious episodes.

\subsection{5(OH) D Measurements}

Serum 25(OH)D level were measured in all participants using an INCSTAR 25(OH)D two step assay procedure with a coefficient of variation of less than $10 \%$. The first step in the procedure involves the rapid extraction of $25(\mathrm{OH}) \mathrm{D}$ from the serum using acetonitrile. Following extraction, 
the treated sample is assayed by using an equilibrium radioimmunoassay procedure. This method is based on an antibody with specificity to $25(\mathrm{OH}) \mathrm{D}$. The sample, antibody, and tracer are incubated at 20-25 ${ }^{\circ} \mathrm{C}$ for ninety minutes. A second antibody-precipitating complex is used to achieve phase separation. The radioimmunoassay method tends to overestimate the level of $25(\mathrm{OH}) \mathrm{D}$ because the antibody recognizes all forms of dihydroxy-vitamin D and D steroids.

\subsection{Statistical Analysis}

The associations of serum 25(OH)D levels with CAP or sepsis admission were evaluated separately. The $\chi^{2}$ Test of Independence tested the distribution of categorical variables and the Wilcoxon Rank Sum tested for differences in 25(OH)D levels among cases and controls. Cox logistic regression was performed using log-transformed $25(\mathrm{OH}) \mathrm{D}$ as a continuous variable and non-transformed $25(\mathrm{OH}) \mathrm{D}$ as a categorical variable $(<75 \mathrm{nmol} / \mathrm{L} v s . \geq 75 \mathrm{nmol} / \mathrm{L},<50 \mathrm{nmol} / \mathrm{L} v s . \geq 50 \mathrm{nmol} / \mathrm{L}$, and $<37 \mathrm{nmol} / \mathrm{L} v s . \geq 37 \mathrm{nmol} / \mathrm{L})$. These thresholds were chosen using established definitions of $25(\mathrm{OH}) \mathrm{D}$ deficiency/insufficiency $[18,19]$. Models adjusted for diabetes, renal disease, and peripheral vascular disease, which were chosen as confounding variables on the basis of previous studies $[20,21]$ and obtained from the Charlson Comorbidity Index score. Two-tailed values of $p<0.05$ were considered statistically significant.

\section{Results}

\subsection{Community-Acquired Pneumonia}

The demographic and clinical characteristics of the 66 cases and 66 controls for the CAP cohort are described in Table 1 . The mean (SD) age of the participants was $60 \pm 17$ years, $71 \%$ were female, and $86 \%$ were Caucasian. There was no statistically significant difference in median [IQR] 25(OH)D levels in controls $v s$. cases $(79.3[71.1-88.1]$ vs. $70.1[62.2-79.6] \mathrm{nmol} / \mathrm{L}, p=0.33)$. Renal disease was more prevalent in cases than controls $(31.8 \%$ vs. 10.6\%, $p=0.003)$. Serum 25(OH)D levels were recorded in 28 cases 3-5 months prior, in 18 cases 6-11 months prior, in 10 cases each 9-11 and 12-15 months prior to admission.

Table 1. Baseline characteristics for community-acquired pneumonia and sepsis cases and matched controls.

\begin{tabular}{ccc}
\hline & Case & Control \\
\hline Number & & \\
\hline CAP & 66 & 66 \\
Sepsis & 211 & 211 \\
\hline Age in years & \\
\hline CAP & $60 \pm 17$ & $60 \pm 17$ \\
Sepsis & $65 \pm 14$ & $65 \pm 14$ \\
\hline Females & & \\
\hline CAP & 47 & 47 \\
Sepsis & 125 & 125 \\
\hline
\end{tabular}


Table 1. Cont.

\begin{tabular}{|c|c|c|}
\hline & Case & Control \\
\hline \multicolumn{3}{|c|}{ Race_-White } \\
\hline CAP & 57 & 57 \\
\hline Sepsis & 189 & 189 \\
\hline \multicolumn{3}{|c|}{ Race-Hispanic } \\
\hline CAP & 6 & 6 \\
\hline Sepsis & 13 & 13 \\
\hline \multicolumn{3}{|c|}{ Race-Other } \\
\hline CAP & 3 & 3 \\
\hline Sepsis & 5 & 5 \\
\hline \multicolumn{3}{|l|}{ Diabetes } \\
\hline CAP & $24(36.4 \%)$ & $19(28.8 \%)$ \\
\hline Sepsis * & $97(46.0 \%)$ & $64(30.3 \%)$ \\
\hline \multicolumn{3}{|c|}{ Renal Disease } \\
\hline $\mathrm{CAP}^{*}$ & $21(31.8 \%)$ & $7(10.6 \%)$ \\
\hline Sepsis * & $76(36.0 \%)$ & $46(21.8 \%)$ \\
\hline \multicolumn{3}{|c|}{ Peripheral Vascular } \\
\hline CAP & $13(19.7 \%)$ & $9(13.6 \%)$ \\
\hline Sepsis & $61(28.9 \%)$ & $45(21.3 \%)$ \\
\hline \multicolumn{3}{|c|}{$25(\mathrm{OH}) \mathrm{D}(\mathrm{nmol} / \mathrm{L}) * *$} \\
\hline CAP & $70.1[62.2-79.6]$ & $79.3[71.1-88.1]$ \\
\hline Sepsis & $61.2[55.9-66.4]$ & $69.1[64.2-74.1]$ \\
\hline \multicolumn{3}{|c|}{$25(\mathrm{OH}) \mathrm{D}>75 \mathrm{nmol} / \mathrm{L}$} \\
\hline CAP & $34(52.3 \%)$ & $35(53.0 \%)$ \\
\hline Sepsis & $84(39.8 \%)$ & $99(46.9 \%)$ \\
\hline \multicolumn{3}{|c|}{ 25(OH)D 51-75 nmol/L } \\
\hline CAP & $19(28.8 \%)$ & $19(28.8 \%)$ \\
\hline Sepsis & $56(26.5 \%)$ & $60(28.4 \%)$ \\
\hline \multicolumn{3}{|c|}{$25(\mathrm{OH}) \mathrm{D} 37-50 \mathrm{nmol} / \mathrm{L}$} \\
\hline CAP & $7(10.6 \%)$ & $10(15.2 \%)$ \\
\hline Sepsis & $31(14.7 \%)$ & $29(13.7 \%)$ \\
\hline \multicolumn{3}{|c|}{$25(\mathrm{OH}) \mathrm{D}<37 \mathrm{nmol} / \mathrm{L}$} \\
\hline CAP & $9(9.1 \%)$ & $2(3.0 \%)$ \\
\hline Sepsis * & $40(19.0 \%)$ & $23(10.9 \%)$ \\
\hline
\end{tabular}

In unadjusted logistic regression, log-transformed $25(\mathrm{OH}) \mathrm{D}$ as a continuous variable was not associated with CAP (0.99 [0.90-1.09], $p=0.84)$. A lack of association remained after adjustment for diabetes, renal disease, and peripheral vascular disease (OR 0.94 [0.59-1.48], $p=0.78$ ). Likewise, $25(\mathrm{OH}) \mathrm{D}<75 \mathrm{nmol} / \mathrm{L} v s . \geq 75 \mathrm{nmol} / \mathrm{L}$ and $<50 \mathrm{nmol} / \mathrm{L} v s . \geq 50 \mathrm{nmol} / \mathrm{L}$ were not associated with CAP in adjusted analyses (Table 2). However, when $25(\mathrm{OH}) \mathrm{D}$ was categorized as $<37 \mathrm{nmol} / \mathrm{L} v s$. $\geq 37 \mathrm{nmol} / \mathrm{L}$, there was an association with increased odds of CAP (OR 2.57 [1.08-6.08], $p=0.03$; Table 2) in the adjusted model. 
Table 2. Adjusted odds ratios for Community-Acquired Pneumonia (CAP) and Sepsis Cases relative to controls by serum $25(\mathrm{OH}) \mathrm{D}$ levels.

\begin{tabular}{|c|c|c|}
\hline Model § & OR $(95 \% \mathrm{CI})$ & $p$-Value \\
\hline \multicolumn{3}{|c|}{$25(\mathrm{OH}) \mathrm{D}<75 \mathrm{nmol} / \mathrm{L} v \mathrm{~s}_{.} \geq 75 \mathrm{nmol} / \mathrm{L}$} \\
\hline CAP & $1.03(0.51-2.09)$ & 0.93 \\
\hline Sepsis & $1.24(0.84-1.83)$ & 0.28 \\
\hline \multicolumn{3}{|c|}{$25(\mathrm{OH}) \mathrm{D}<50 \mathrm{nmol} / \mathrm{L} v s . \geq 50 \mathrm{nmol} / \mathrm{L}$} \\
\hline CAP & $0.96(0.35-2.61)$ & 0.94 \\
\hline Sepsis & $1.75(1.11-2.77)$ & 0.02 \\
\hline \multicolumn{3}{|c|}{$25(\mathrm{OH}) \mathrm{D}<37 \mathrm{nmol} / \mathrm{L} v s . \geq 37 \mathrm{nmol} / \mathrm{L}$} \\
\hline CAP & $2.57(1.08-6.08)$ & 0.03 \\
\hline Sepsis & $1.89(1.09-3.31)$ & 0.02 \\
\hline
\end{tabular}

$\S$ Adjustments made for diabetes, renal disease, peripheral vascular disease; CAP, community-acquired pneumonia; 25(OH)D, 25 hydroxyvitamin D.

\subsection{Sepsis}

The demographic and clinical characteristics of the 211 cases and 211 controls for the sepsis cohort are described in Table 1 . The mean (SD) age of the participants was $65 \pm 14$ years, 59\% were female and $91 \%$ were Caucasian. There was no statistically significant difference in median [IQR] $25(\mathrm{OH}) \mathrm{D}$ levels in controls $v s$. cases $(69.1$ [64.2-74.1] nmol/L vs. 61.2 [55.9-66.4] nmol/L, $p=0.05)$. Comorbid conditions were more prevalent in sepsis cases than in controls: diabetes $(46.0 \%$ $v s .30 .3 \%, p=0.0009)$ and renal disease $(36.0 \% v s .21 .2 \%, p=0.001)$. Serum 25(OH)D levels were recorded in 93 cases 3-5 months prior, in 50 cases 6-11 months prior, in 47 cases 9-11 months prior, and 21 cases $12-15$ months prior to admission.

In unadjusted logistic regression, log-transformed $25(\mathrm{OH}) \mathrm{D}$ as a continuous variable was not associated with sepsis (OR 0.99 [0.93-1.05], $p=0.70$ ). A lack of association remained after adjustment for diabetes, renal disease, and peripheral vascular disease (OR 0.82 [0.64-1.05], $p=0.12$ ). $25(\mathrm{OH}) \mathrm{D}<75 \mathrm{nmol} / \mathrm{L} v s . \geq 75 \mathrm{nmol} / \mathrm{L}$ was not associated with sepsis in adjusted analyses (Table 2). However, when $25(\mathrm{OH}) \mathrm{D}$ was categorized as $<50 \mathrm{nmol} / \mathrm{L} v s . \geq 50 \mathrm{nmol} / \mathrm{L}$ and $<37 \mathrm{nmol} / \mathrm{L}$ $v s . \geq 37 \mathrm{nmol} / \mathrm{L}$, there was an association with increased odds of sepsis (Table 2, OR 1.75 [1.11-2.77], $p=0.02$ and 1.89 [1.09-3.31], $p=0.02$, respectively) in adjusted analyses.

\section{Discussion}

In a cohort of community-living adults, increased risk of hospitalization for CAP was associated with serum 25(OH)D levels $<37 \mathrm{nmol} / \mathrm{L}$ and for sepsis with serum 25(OH)D levels $<50 \mathrm{nmol} / \mathrm{L}$. This association was not observed for $25(\mathrm{OH}) \mathrm{D}<75 \mathrm{nmol} / \mathrm{L}$, suggesting that $25(\mathrm{OH}) \mathrm{D}<37 \mathrm{nmol} / \mathrm{L}$ confers a greater risk of infection than vitamin D insufficiency.

These findings are consistent with other epidemiologic studies linking vitamin D deficiency with increased risk of infection and infection-associated complications. A large observational study using National Health and Nutrition Examination Survey data showed that in a diverse cohort of 18,883 individuals greater than 12 years of age, those with a serum $25(\mathrm{OH}) \mathrm{D}$ level $<25 \mathrm{nmol} / \mathrm{L}$ and 
a serum $25(\mathrm{OH}) \mathrm{D} 25-75 \mathrm{nmol} / \mathrm{L}$ had a $36 \%$ and $24 \%$ increased risk of upper respiratory tract infection, respectively, compared to those with a serum $25(\mathrm{OH}) \mathrm{D} \geq 75 \mathrm{nmol} / \mathrm{L}$ [11]. Likewise, in a prospective observational study evaluating 25(OH)D levels in Finnish military recruits, 25(OH)D levels $<40 \mathrm{nmol} / \mathrm{L}$ were associated with a higher likelihood of physician diagnosed respiratory tract infections and lost days of work in the subsequent six months [13]. Ginde and colleagues reported that upon presentation to an urban emergency department, 81 patients with sepsis and $25(\mathrm{OH}) \mathrm{D}$ levels $<75 \mathrm{nmol} / \mathrm{L}$ were more likely to have severe sepsis compared to those with 25(OH)D levels $\geq 75 \mathrm{nmol} / \mathrm{L}(61 \%$ vs. $24 \%, p=0.006)$ at initial evaluation and at $24 \mathrm{~h}(67 \%$ vs. $29 \%, p=0.005)$ [15]. While other studies have measured $25(\mathrm{OH}) \mathrm{D}$ levels at acute illness onset, our study evaluates $25(\mathrm{OH}) \mathrm{D}$ levels at least 3 months prior to hospital admission, thus suggesting that there is an increased risk of CAP with a $25(\mathrm{OH}) \mathrm{D}$ level $<37 \mathrm{nmol} / \mathrm{L}$ and sepsis with a $25(\mathrm{OH}) \mathrm{D}$ level $<50 \mathrm{nmol} / \mathrm{L}$. It also eliminates the potential for confounding by acute illness altering serum $25(\mathrm{OH}) \mathrm{D}$ levels.

Both the VDR and CYP27B1, the gene encoding 1- $\alpha$-hydroxylase, which converts $25(\mathrm{OH}) \mathrm{D}$ to its active form $1,25(\mathrm{OH})_{2} \mathrm{D}$, are expressed in immune cells, suggesting that $25(\mathrm{OH}) \mathrm{D}$ has paracrine or autocrine function. Furthermore, $1-\alpha$-hydroxylase in macrophages is not regulated by parathyroid hormone $(\mathrm{PTH})[22]$ but depends on circulating $25(\mathrm{OH}) \mathrm{D}$ concentrations or may be induced by cytokines [23]. When toll-like receptors on macrophages bind bacterial wall lipopolysaccharides (LPS), 1- $\alpha$-hydroxylase and VDR expression is increased ${ }^{4}$ resulting in local conversion of $25(\mathrm{OH}) \mathrm{D}$ to $1,25(\mathrm{OH})_{2} \mathrm{D}$, which in turn increases the expression of cathelicidin and beta-defensing, bactericidal proteins. There is evidence that cathelicidin transcription is particularly dependent on sufficient levels of 25(OH)D [4]. Indeed, in a study of critically ill patients with and without sepsis, Jeng and colleagues report a positive relationship between $25(\mathrm{OH}) \mathrm{D}$ levels and LL-37 (cathelicidin) levels [24].

In sepsis, $25(\mathrm{OH}) \mathrm{D}$ and $1,25(\mathrm{OH})_{2} \mathrm{D}$ may have other effects beyond those associated with immunity such as endothelial function, coagulation, and hemodynamic stability. In rats with induced sepsis, pretreatment with $1,25(\mathrm{OH})_{2} \mathrm{D}$ resulted in a more normal coagulation profile compared to placebo [25]. The VDR is also found in arterioles and the myocardium [26] and $1,25(\mathrm{OH})_{2} \mathrm{D}$ has been shown to enhance the effect of inotropes [27] suggesting a possible positive hemodynamic effect of $1,25(\mathrm{OH})_{2} \mathrm{D}$ in sepsis [25]. Taken together, sufficient circulating 25(OH)D levels, independent of the classic vitamin D-PTH axis, play a pivotal role in immunity. Moreover, 25(OH)D may also be protective against the adverse physiologic changes that occur in sepsis.

The findings of this study are consistent with other epidemiologic reports linking serum $25(\mathrm{OH}) \mathrm{D}<75 \mathrm{nmol} / \mathrm{L}$ with increased risk of infection and associated complications [11-16]. While other studies measured $25(\mathrm{OH}) \mathrm{D}$ levels during acute illness, this is the first to evaluate $25(\mathrm{OH}) \mathrm{D}$ levels $\geq 3$ months prior to hospital admission for CAP or sepsis, eliminating potential confounding by acute illness altering serum $25(\mathrm{OH}) \mathrm{D}$ levels. This study has important strengths. Chest radiograph confirmed CAP ICD-9 codes. Serum 25(OH)D level was measured prior to the onset of illness, which is different from other studies that measured $25(\mathrm{OH}) \mathrm{D}$ at the time of presentation. This is important because vitamin $\mathrm{D}$ binding protein may be decreased resulting in potential urinary wasting of $25(\mathrm{OH}) \mathrm{D}$ in sepsis [24]. Finally, this study used a community-living cohort that included a relatively large number of patients with sepsis. 
This study has several limitations. First, it is retrospective and observational so causation cannot be assumed. Second, the use of ICD-9 codes to identify admissions for sepsis limits details of cause and severity. Third, while cases and controls were well-matched and regression models adjusted for comorbidities, there may be other unmeasured confounders that could potentially affect the results. Fourth, ascertainment bias may exist because only cases and controls with a serum 25(OH)D level available prior to admission were considered. Additionally, no information is available on outcomes during the hospitalization.

\section{Conclusions}

In conclusion, serum $25(\mathrm{OH}) \mathrm{D}$ level $<37 \mathrm{nmol} / \mathrm{L}$ in a community-living cohort was associated with increased risk of hospital admission for CAP and sepsis. Large randomized controlled trials are needed to establish whether or not $25(\mathrm{OH}) \mathrm{D}$ repletion will decrease CAP and sepsis incidence in community-living adult populations.

\section{Acknowledgments}

This work was supported by the National Institute of Health and the American Geriatrics Society Beeson Career Development Award [grant number K23AG040708] and the American Geriatrics Society Jahnigen Career Development Scholars Award to AG; the National Institute of Diabetes and Digestive and Kidney Diseases [grant numbers 1R01DK081473-01A, 1R01DK078112-01A2] to $\mathrm{MC}$; the National Institute of Diabetes and Digestive and Kidney Diseases [grant number K23DK087859-01A1] to JK.

\section{Author Contributions}

Anna Jovanovich, John Holmen, Jessica Kendrick and Michel Chonchol had full access to all of the data in the study and take responsibility for the integrity of the data and the accuracy of the data analysis. Study concept and design: Jovanovich, Ginde, Holmen, Jablonski, ALlyn, Kendrick and Chonchol. Collection, management, analysis and interpretation of data: Jovanovich, Ginde, Holmen, Jablonski, Allyn, Kendrick and Chonchol. Preparation, review, or approval of manuscript: Jovanovich, Ginde, Holmen, Jablonski, Allyn, Kendrick and Chonchol.

\section{Conflicts of Interest}

The authors declare no conflict of interest.

\section{References}

1. Mandell, L.A.; Wunderink, R.G.; Anzueto, A.; Bartlett, J.G.; Campbell, G.D.; Dean, N.C.; Dowell, S.F.; File, T.M., Jr.; Musher, D.M.; Niederman, M.S.; et al. Infectious Diseases Society of America/American Thoracic Society consensus guidelines on the management of community-acquired pneumonia in adults. Clin. Infect. Dis. 2007, 44, S27-S72. 
2. Deaths: Preliminary Data for 2009. National Vital Statistics Reports. CDC. 2011. Available online: http://www.cdc.gov/nchs/data/nvsr/nvsr59/nvsr59_04.pdf (accessed on 16 March 2011).

3. Martin, G.S.; Mannino, D.M.; Eaton, S.; Moss, M. The epidemiology of sepsis in the United States from 1979 through 2000. N. Engl. J. Med. 2003, 348, 1546-1554.

4. Liu, P.T.; Stenger, S.; Li, H.; Wenzel, L.; Tan, B.H.; Krutzik, S.R.; Ochoa, M.T.; Schauber, J.; $\mathrm{Wu}, \mathrm{K}$.; Meinken, C.; et al. Toll-like receptor triggering of a vitamin D-mediated human antimicrobial response. Science 2006, 311, 1770-1773.

5. White, J.H. Vitamin D metabolism and signaling in the human immune system. Rev. Endocr. Metab. Disord. 2012, 13, 21-29.

6. Ginde, A.A.; Liu, M.C.; Camargo, C.A., Jr. Demographic differences and trends of vitamin D insufficiency in the US population, 1988-2004. Arch. Intern. Med. 2009, 169, 626-632.

7. Holick, M.F. Vitamin D for health and in chronic kidney disease. Semin. Dial. 2005, 18, 266-275.

8. Scragg, R.; Sowers, M.; Bell, C.; Third National Health and Nutrition Examination Survey. Serum 25-hydroxyvitamin D, diabetes, and ethnicity in the Third National Health and Nutrition Examination Survey. Diabetes Care 2004, 27, 2813-2818.

9. Chonchol, M.; Scragg, R. 25-Hydroxyvitamin D, insulin resistance, and kidney function in the Third National Health and Nutrition Examination Survey. Kidney Int. 2007, 71, 134-139.

10. Melamed, M.L.; Muntner, P.; Michos, E.D.; Uribarri, J.; Weber, C.; Sharma, J.; Raggi, P. Serum 25-hydroxyvitamin D levels and the prevalence of peripheral arterial disease: Results from NHANES 2001 to 2004. Arterioscler. Thromb. Vasc. Biol. 2008, 28, 1179-1185.

11. Ginde, A.A.; Mansbach, H.M.; Camargo, C.A., Jr. Association between serum 25-hydroxyvitamin D level and upper respiratory tract infection in the Third National Health and Nutrition Examination Survey. Arch. Intern. Med. 2009, 169, 384-390.

12. Sabetta, J.R.; DePetrillo, P.; Cipriani, R.J.; Smardin, J.; Burns, L.A.; Landry, M.L. Serum 25-hydroxyvitamin D and the incidence of acute viral respiratory tract infection in healthy adults. PLoS One 2010, 5, e11088.

13. Laaksi, I.; Ruohola, J.P.; Tuohimaa, P.; Auvinen, A.; Haataja, R.; Pihajamaki, H.; Ylikomi, T. An association of serum vitamin $\mathrm{D}$ concentrations $<40 \mathrm{nmol} / \mathrm{L}$ with acute respiratory tract infection in young Finnish men. Am. J. Clin. Nutr. 2007, 86, 714-717.

14. Leow, L.; Simpson, T.; Cursons, R.; Karalus, N.; Hancox, R.J. Vitamin D, innate immunity and outcomes in community acquired pneumonia. Respirology 2011, 16, 611-616.

15. Ginde, A.A.; Camargo, C.A., Jr.; Shapiro, N.I. Vitamin D insufficiency and sepsis severity in emergency department patients with suspected infection. Acad. Emerg. Med. 2011, 18, 551-554.

16. Braun, A.B.; Gibbons, F.K.; Litonjua, A.A.; Giovannucci, E.; Christopher, K.B. Low serum 25-hydroxyvitamin D at critical care initiation is associated with increased mortality. Crit. Care Med. 2012, 40, 63-72.

17. Intermoutain Healthcare Annual Report. Intermountain Healthcare 2010. Available online: http://intermountainhealthcare.org/about/overview/Documents/annualreport2010.pdf (accessed on 12 December 2012). 
18. Mehrotra, R.; Kermah, D.A.; Salusky, I.B.; Wolf, M.S.; Thadhani, R.I.; Chiu, Y.W.; Martins, D.; Adler, S.G.; Norris, K.C. Chronic kidney disease, hypovitaminosis D, and mortality in the United States. Kidney Int. 2009, 76, 977-983.

19. Institute of Medicine. Dietary References Intakes for Calcium and Vitamin D. Available online: http://www.iom.edu/Activities/Nutrition/DRIVitDCalcium/2010-Nov-30.aspx (accessed on 12 December 2012).

20. Anderson, J.L.; May, H.T.; Horne, B.D.; Bair, T.L.; Hall, N.L.; Carlquist, J.F.; Lappé, D.L.; Muhlestein, J.B.; Intermountain Heart Collaborative (IHC) Study Group. Relation of vitamin d deficiency to cardiovascular risk factors, disease status, and incident events in a general healthcare population. Am. J. Cardiol. 2010, 106, 953-958.

21. Sterling, K.A.; Eftekhari, P.; Girndt, M.; Kimmel, P.L.; Raj, D.S. The immunoregulatory function of vitamin D: Implications in chronic kidney disease. Nat. Rev. Nephrol. 2012, 8, 403-412.

22. Wu, S.; Ren, S.; Nguyen, L.; Adams, J.S.; Hewison, M. Splice variants of the CYP27b1 gene and the regulation of 1,25-dihydroxyvitamin D3 production. Endocrinology 2007, 148, 3410-4318.

23. Van Etten, E.; Stoffels, K.; Gysemans, C.; Mathieu, C.; Overbergh, L. Regulation of vitamin D homeostasis: Implications for the immune system. Nutr. Rev. 2008, 66, S125-S134.

24. Jeng, L.; Yamshchikov, A.V.; Judd, S.E.; Blumberg, H.M.; Martin, G.S.; Ziegler, T.R.; Tangpricha, V. Alterations in vitamin D status and anti-microbial peptide levels in patients in the intensive care unit with sepsis. J. Transl. Med. 2009, 7, 28.

25. Moller, S.; Laigaard, F.; Olgaard, K.; Hemmingsen, C. Effect of 1,25-dihydroxy-vitamin $\mathrm{D}_{3}$ in experimental sepsis. Int. J. Med. Sci. 2007, 4, 190-195.

26. Bukoski, R.D.; Xue, H. On the vascular inotropic action of 1,25-(OH)2 vitamin D3. Am. J. Hypertens. 1993, 6, 388-396.

27. Walters, M.R.; Wicker, D.C.; Riggle, P.C. 1,25-Dihydroxyvitamin D3 receptors identified in the rat heart. J. Mol. Cell. Cardiol. 1986, 18, 67-72. 


\title{
Increased Plasma Concentrations of Vitamin D Metabolites and Vitamin D Binding Protein in Women Using Hormonal Contraceptives: A Cross-Sectional Study
}

\author{
Ulla K. Møller, Susanna við Streym, Lars T. Jensen, Leif Mosekilde, Inez Schoenmakers, \\ Shailja Nigdikar and Lars Rejnmark
}

\begin{abstract}
Use of hormonal contraceptives (HC) may influence total plasma concentrations of vitamin D metabolites. A likely cause is an increased synthesis of vitamin D binding protein (VDBP). Discrepant results are reported on whether the use of $\mathrm{HC}$ affects free concentrations of vitamin D metabolites. Aim: In a cross-sectional study, plasma concentrations of vitamin D metabolites, VDBP, and the calculated free vitamin D index in users and non-users of HC were compared and markers of calcium and bone metabolism investigated. Results: 75 Caucasian women aged 25-35 years were included during winter season. Compared with non-users $(n=23)$, users of HC $(n=52)$ had significantly higher plasma concentrations of 25-hydroxyvitamin D (25OHD) (median 84 interquartile range: [67-111] vs. 70 [47-83] nmol/L, $p=0.01), 1,25$-dihydroxyvitamin $\mathrm{D}\left(1,25(\mathrm{OH})_{2} \mathrm{D}\right)$ (198 [163-241] vs. 158 [123-183] pmol/L, $p=0.01)$ and VDBP (358 [260-432] vs. 271 [179-302] $\mu \mathrm{g} / \mathrm{mL}, p<0.001$ ). However, the calculated free indices (FI-25OHD and FI-1,25(OH) $2 \mathrm{D}$ ) were not significantly different between groups $(p>0.10)$. There were no significant differences in indices of calcium homeostasis (plasma concentrations of calcium, parathyroid hormone, and calcitonin, $p>0.21$ ) or bone metabolism (plasma bone specific alkaline phosphatase, osteocalcin, and urinary $\mathrm{NTX} /$ creatinine ratio) between groups. In conclusion: Use of HC is associated with $13 \%-25 \%$ higher concentrations of total vitamin D metabolites and VDBP. This however is not reflected in indices of calcium or bone metabolism. Use of $\mathrm{HC}$ should be considered in the interpretation of plasma concentrations vitamin D metabolites.
\end{abstract}

Reprinted from Nutrients. Cite as: Møller, U.K.; við Streym, S.; Jensen, L.T.; Mosekilde, L.; Schoenmakers, I.; Nigdikar, S.; Rejnmark, L. Increased Plasma Concentrations of Vitamin D Metabolites and Vitamin D Binding Protein in Women Using Hormonal Contraceptives: A Cross-Sectional Study. Nutrients 2013, 5, 3470-3480.

\section{Introduction}

Vitamin D (calciferol) is obtained from endogenous synthesis in the skin in response to solar UV-B radiation and intake from the diet and supplements [1,2]. Once in the circulation, calciferol is converted to 25-hydroxyvitamin D (25OHD) in the liver and, subsequently, to its circulating biologically active form 1,25-dihydroxyvitamin $\mathrm{D}\left(1,25(\mathrm{OH})_{2} \mathrm{D}\right)$ in the kidney [3]. This conversion may also occur in other tissues for auto- or paracrine actions [4]. It has been estimated that $85 \%$ to $90 \%$ of $25 \mathrm{OHD}$ and $1,25(\mathrm{OH})_{2} \mathrm{D}$ is bound to vitamin $\mathrm{D}$ binding protein (VDBP) [5], $10 \%$ to $15 \%$ to albumin, whereas only a very small fraction $(<0.1 \%)$ circulates in its free form $[5,6]$. VDBP binding protects vitamin D metabolites from hydroxylase-mediated catabolism, affects their cellular uptake, and modulates their biological activity $[5,6]$. 
Total plasma concentrations of $25 \mathrm{OHD}$ are considered an indicator of vitamin D status due to its long plasma half-life (approximately 15-35 days) and lack of hormonal control of the hepatic 25-hydroxylase [3].

Vitamin D is known to affect several health outcomes. Classically, low vitamin D concentrations are known to be associated with an increased risk of myopathy, rickets or osteomalacia, and low bone mineral density and fracture. In a number of recent studies, an impaired vitamin D status has also been associated with various adverse non-skeletal health outcomes such as an increased risk of malignancies or cardiovascular diseases [1].

Plasma 25OHD concentrations are influenced by many factors. In addition to variations in UVB-exposure and dietary intake, 25OHD concentrations are influenced by several host factors such as age, adiposity $[2,7,8]$, ethnicity, and skin tone as well as certain genotypes [8,9], and plasma VDBP concentrations [5].

Pregnancy is known to be associated with an increase in VDBP through its oestrogen mediated increase in synthesis $[10,11]$. Plasma concentrations of 25OHD are reported to be unaltered and $1,25(\mathrm{OH})_{2} \mathrm{D}$ to be elevated compared to non-pregnant women [12-14]. The use of hormonal contraceptives (HC) may also affect 25OHD concentrations and metabolism due to their oestrogenic components. The limited data on the effects of $\mathrm{HC}$ on $25 \mathrm{OHD}$ concentrations report no change or an increase in total 25OHD [9,12,15-17], whereas most studies consistently report an increase in levels of $1,25(\mathrm{OH})_{2} \mathrm{D}$ and VDBP $[10,15,18-20]$.

These data suggest that $\mathrm{HC}$ may cause differential effects on $25 \mathrm{OHD}$ and $1,25(\mathrm{OH})_{2} \mathrm{D}$; the free $25 \mathrm{OHD}$ index (the molar ratio of 25OHD- to VDBP-concentrations) may be decreased due to an absence of a parallel increase in VDBP and 25OHD, whereas the free index of $1,25(\mathrm{OH})_{2} \mathrm{D}$ may remain unchanged.

In order to study the possible effects of $\mathrm{HC}$, we compared plasma concentrations of $25 \mathrm{OHD}$, $1,25(\mathrm{OH})_{2} \mathrm{D}$, VDBP, and the calculated free vitamin D index in users and non-users of HC. In addition, we assessed possible impacts of $\mathrm{HC}$ on calcium homeostasis and bone metabolism.

\section{Subjects and Methods}

This paper reports a secondary analysis of the effects of $\mathrm{HC}$ on vitamin D metabolism in a subset of women participating in a population based controlled cohort study, using cross-sectional data obtained at baseline. The design of the study has previously been reported in detail [12,21]. In brief, we included 153 healthy Caucasian women, aged 25-35 years, trying to conceive, and 75 age-matched women not planning a pregnancy for the next 21 months. All women were recruited by direct mailing of 11,175 randomly selected women from a population of 21,317 women aged 25-35 years living in the community of Aarhus, Denmark. We obtained names and addresses from the Danish Civil Registration System. A total of 561 wished to participate, from which 333 were excluded as based on predefined exclusion criteria (Pregnant or breastfeeding at the start of the study $(n=85)$, known infertility $(n=46)$, miscarriage within last 6 months $(n=3)$, withdrawal or moved residence $(n=84)$, age, illness, foreign origin $(n=25)$, or responded after closure of recruitment $(n=90))$. Analyses reported in this paper only include data obtained in the group of women $(n=75)$ not planning a pregnancy, of which 52 were using hormonal contraception (including oral, subdermal contraceptive 
implant, or hormonal spiral methods). They were all included between October 2006 and April 2007. The study was performed according to The Helsinki Declaration II. The study was notified to the Danish Data Protection Agency (\#2004-41-4737) and approved by the Regional Scientific Ethical Committee of Aarhus County (\#20040186).

\subsection{Measurements}

Standing height and body weight were measured (Seca, Sa-med, Kvistgaard, Denmark) wearing indoor clothing. Incident diseases and the use of drugs were recorded. Participants were asked to fill in a questionnaire on medical conditions, smoking habits, and dietary intake of calcium as well as use of calcium and vitamin D containing supplements. Dietary intake of calcium was assessed as previously described [22] and total calcium intake was calculated as dietary intake plus intake from supplements.

\subsection{Biochemistry}

A non-fasting blood sample was drawn between 8 a.m. and 2 p.m. according to standardized procedures and centrifuged at $4{ }^{\circ} \mathrm{C}$ with a relative centrifugal force of $2500 \mathrm{~g}$ for $10 \mathrm{~min}$. Plasma was separated and stored at $-80{ }^{\circ} \mathrm{C}$ until analyzed. Urine and plasma samples were assessed in batches, i.e., all samples from each participant were analyzed in the same run, except for analysis of calcium, creatinine, and phosphate, which were analyzed within two hours after collection. A second void morning urine sample was collected at home. Urine samples were collected under fasting conditions or before any consumption of calcium rich foods. Plasma 25-hydroxyvitamin D (25OHD) concentrations were measured by isotope dilution liquid chromatography-tandem mass spectrometry (LC-MS/MS) by a method adapted from Maunsell et al. [23,24]. The method separately quantifies $25 \mathrm{OHD}_{2}$ and total $25 \mathrm{OHD}_{3}$ (including the 3 -epimer). The total $25 \mathrm{OHD}$ concentration was calculated and used for further analyses. Calibrators traceable to NIST SRM 972 (Chromsystems, DE) were used. The inter-assay $\mathrm{CV}$ was $<10 \%$, at plasma concentrations of $23.4 \mathrm{nmol} / \mathrm{L}\left(25 \mathrm{OHD}_{2}\right)$ and $24.8 \mathrm{nmol} / \mathrm{L}$ $\left(25 \mathrm{OHD}_{3}\right)$. We determined plasma 1,25-dihydroxyvitamin $\mathrm{D}\left(1,25(\mathrm{OH})_{2} \mathrm{D}\right)$ concentrations by a radioimmunoassay (Gamma-B 1,25-Dihydroxy Vitamin D, Immunodiagnostic Systems (IDS) Ltd., Boldon, England). The inter- and intra-assay CV was $9.0 \%$ and $8.0 \%$, respectively, at $220 \mathrm{pmol} / \mathrm{L}$.

Vitamin D binding protein concentration was determined by ELISA (R \& D Systems, Abingdon, $\mathrm{UK})$ with both an inter- and intra-assay $\mathrm{CV}<6 \%$. Assay performance was monitored using kit and in-house controls and under strict standardization according to ISO 9001:2000.

The free fraction of $25 \mathrm{OHD}$ and $1,25(\mathrm{OH})_{2} \mathrm{D}$ were calculated as the free 25OHD index (FI-25OHD) and the free $1,25(\mathrm{OH})_{2} \mathrm{D}$ index $\left(\mathrm{FI}-1,25(\mathrm{OH})_{2} \mathrm{D}\right)$ using the molar ratio of $25 \mathrm{OHD}$ and $1,25(\mathrm{OH})_{2} \mathrm{D}$ to VDBP [11].

We determined plasma and urinary concentrations of calcium and creatinine $(\mathrm{Cr})$ by standard laboratory methods and calculated the albumin adjusted calcium concentration according to the formula: plasma calcium, adjusted $[\mathrm{mmol} / \mathrm{L}]=$ plasma calcium, total $[\mathrm{mmol} / \mathrm{L}]+0.00086 \times(650$-plasma albumin $\mu \mathrm{mol} / \mathrm{L})[22]$. 
Calcitonin was measured by a radioimmunoassay as described by Schifter [25]. The plasma concentrations of intact parathyroid hormone (PTH) and osteocalcin were measured with electro-chemiluminescence immunoassays using an automated instrument (Cobas 601e, Roche Diagnostics, GmbH, Mannheim, Germany). We measured plasma bone specific alkaline phosphatase concentrations by an immunoassay (METRA BAP EIA kit, Quidel Corporation, San Diego, CA, USA). The renal excretion of cross-linked $N$-terminal telopeptide of type 1 collagen (NTx) was quantified by ELISA using an automated instrument (Vitros ECI, Ortho Clinical Diagnostics, Amersham, UK). Results were expressed relative to creatinine ( $\mathrm{Cr}$ ) excretion (NTx/Cr), as nmol of bone collagen equivalents (nMmol BCE) per mmol of creatinine. The CV was $9.6 \%$ at $41.5 \mathrm{nmol}$ $\mathrm{BCE} / \mathrm{mmol} \mathrm{Cr}$.

We measured bone mineral density (BMD) of the whole body, the lumbar spine, and total hip. Total body fat and lean mass were measured. All DXA scans were performed using a Hologic Discovery scanner (Hologic, Waltham, MA, USA). We assessed long-term stability through daily scans of an anthropometrical phantom. Precision error for BMD was $1 \%$ at the lumbar spine and $2 \%$ at the total hip.

\subsection{Statistics}

The majority of the data were non-normally distributed, therefore descriptive statistics are reported as medians with the 25 and 75 -percentile (p25; p75), unless stated otherwise. We explored the differences between groups using chi-square tests for categorical variables and a Mann-Whitney $U$-test for continuous variables. Spearman's rho correlation was used to calculate the magnitude and direction of the correlations between measured variables.

Vitamin D status was described according to plasma 250HD concentrations categorized into three groups: $25 \mathrm{OHD}<50 \mathrm{nmol} / \mathrm{L} ; 25 \mathrm{OHD}$ between 50.1 and $75 \mathrm{nmol} / \mathrm{L}, 25 \mathrm{OHD}>75.1 \mathrm{nmol} / \mathrm{L}$ [26].

All statistical analyses were performed using the Statistical Package for Social Sciences (SPSS 17, Chicago, IL, USA) for Windows. $P$-values below 0.05 were considered statistically significant.

\section{Results}

Table 1 shows characteristics of the 75 included women. Anthropometric-, diet-, and lifestyle-characteristics did not differ between women using hormonal contraceptives (HC) $(n=52)$ and non-users $(n=23)$, except that daily calcium intake was slightly higher in users- compared with non-users of HC $(p=0.02)$. 
Table 1. Characteristic of the 75 studied women stratified by use of hormonal contraceptives. Median with interquartile (p25; p75) ranges unless otherwise indicated.

\begin{tabular}{lllll}
\hline & All, $\boldsymbol{n}=\mathbf{7 5}$ & $\begin{array}{l}\text { Users of } \\
\text { hormonal } \\
\text { contraceptive } \\
(\boldsymbol{n}=\mathbf{5 2})\end{array}$ & $\begin{array}{l}\text { Non-users of } \\
\text { hormonal } \\
\text { contraceptives } \\
(\boldsymbol{n}=\mathbf{2 3})\end{array}$ & $\boldsymbol{p ~ v a l u e ~}^{\mathbf{1}}$ \\
\hline Age, mean & $29(27 ; 32)$ & $29(27 ; 32)$ & $29(26 ; 33)$ & 0.81 \\
Weight, kg & $67(60 ; 77)$ & $69(63 ; 77)$ & $63(57 ; 80)$ & 0.12 \\
Height, cm & $168(163 ; 172)$ & $167(162 ; 172)$ & $169(163 ; 172)$ & 0.61 \\
BMI & $24(22 ; 27)$ & $25(23 ; 27)$ & $23(20 ; 27)$ & 0.12 \\
Total calcium intake, mg/day & $800(660 ; 975)$ & $850(700 ; 1000)$ & $700(500 ; 853)$ & 0.02 \\
Use of vitamin D & & & & \\
supplements, $n(\%)$ & $24(32)$ & $17(33)$ & $7(30)$ & 1.00 \\
Vitamin D intake from & $5(5 ; 9)$ & $5(5 ; 10)$ & $5(5 ; 5)$ & \\
supplements $(\mu \mathrm{g} /$ day) & & & $4(17)$ & 0.71 \\
Smoking, $n(\%)$ & $11(15)$ & $7(14)$ & & \\
\hline
\end{tabular}

${ }^{1}$ Independent-Samples Mann-Whitney U Test.

Of the $52 \mathrm{HC}$ users, 44 used oral $\mathrm{HC}$, six used intrauterine hormonal device, one used sub-dermal contraceptive implant, and one used a vaginal ring. No differences in biochemical markers were seen between the 44 using oral $\mathrm{HC}$ and the six using intrauterine hormonal device (data not shown).

Physical activity, time spend outdoor, and the time of the day for blood sampling did not differ between the $\mathrm{HC}$ users and no-users of $\mathrm{HC}$ (data not shown). The number of women with a BMI above 25 was not different between users- and non-HC users. BMD at whole body, lumbar spine, and total hip, as well as fat and lean mass did not differ between the HC users and no-users of HC (data not shown). However, given the sample size of 23 non-HC users and 52 users our statistical power to detect a $5 \%$ difference between groups in lumbar spine BMD $(2 \alpha=0.05$ and $\beta=0.20)$ was only approximately $60 \%$.

When all data were pooled, the plasma concentrations of 25OHD was significantly and positively correlated with $\operatorname{VDBP}\left(r_{\mathrm{s}}=0.26, p=0.03\right)$ (Figure $\left.1 \mathrm{~A}\right)$ and further with $1,25(\mathrm{OH})_{2} \mathrm{D}\left(r_{\mathrm{s}}=0.43\right.$, $p<0.01$ ). 
Figure 1. (A) Scatter plot of linear relations between plasma 25OHD and VDBP in 75 healthy Caucasian women stratified by use of hormonal contraceptives; (B) Scatter plot of linear relations between plasma $1,25 \mathrm{OH}_{2} \mathrm{D}$ and VDBP in 75 healthy Caucasian women stratified by use of hormonal contraceptives.

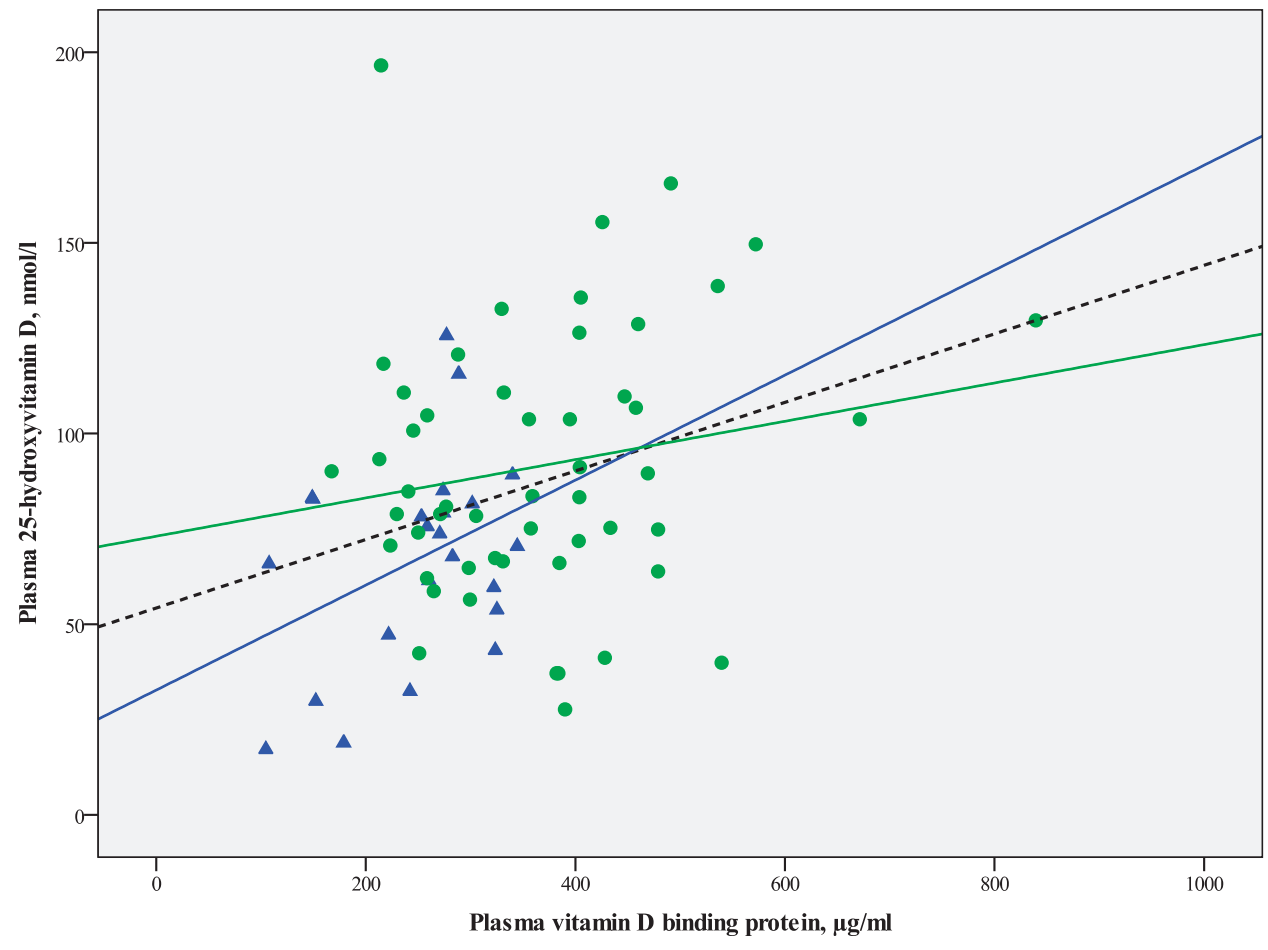

Hormonal contraceptive use

Non hormonal contraceptive users Hormonal contraceptive user

Non hormonal contraceptive users

Hormonal contraceptive users

The group as one

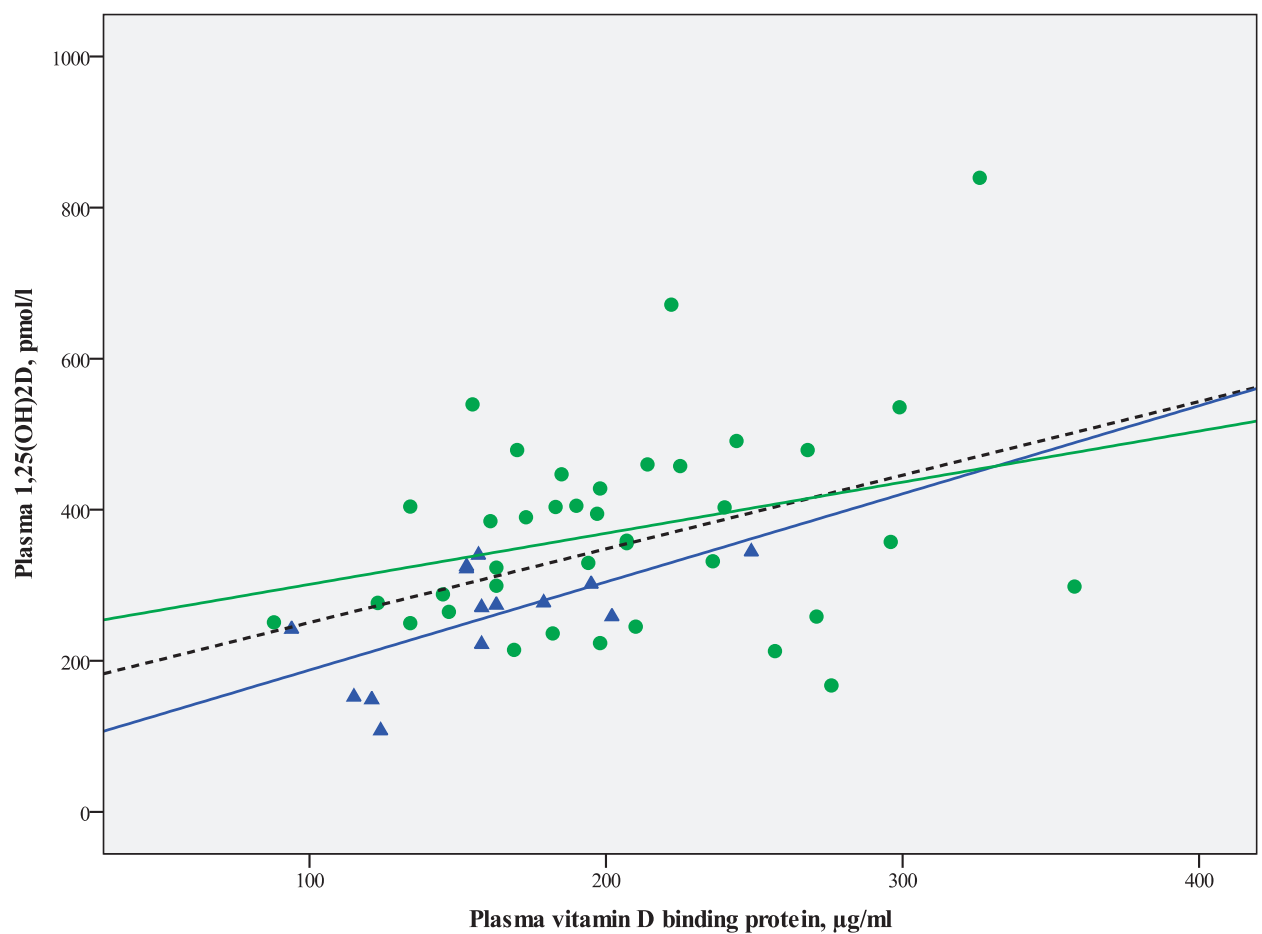

Hormonal contraceptive use

Non hormonal contraceptive users Hormonal contraceptive users

Non hormonal contraceptive users Hormonal contraceptive users

"The group as one

Plasma $1,25(\mathrm{OH})_{2} \mathrm{D}$ was significantly and positively correlated with VDBP concentrations $\left(r_{\mathrm{s}}=0.39, p<0.01\right)$ when data for all women (HC users and non users) were pooled (Figure 1B). 


\subsection{The Effect of Use of Hormonal Contraceptives on $\mathrm{P}-25 \mathrm{OHD}, \mathrm{P}-1,25 \mathrm{OH}_{2} \mathrm{D}$, and $P-V D B P$ Concentrations}

Table 2 details the biochemical indices measured as stratified by whether studied women used HC.

Table 2. Biochemical characteristics as stratified by use of hormonal contraceptives. Data are presented as Median with interquartile (p25; p75) ranges unless otherwise indicated.

\begin{tabular}{|c|c|c|c|c|}
\hline & All, $n=75$ & $\begin{array}{l}\text { Users of } \\
\text { hormonal } \\
\text { contraceptive } \\
\text { users } \\
(n=52)\end{array}$ & $\begin{array}{l}\text { Non-users of } \\
\text { hormonal } \\
\text { contraceptives } \\
(n=23)\end{array}$ & $p$ value \\
\hline Plasma vitamin D binding protein, $\mu \mathrm{g} / \mathrm{mL}$ & $305(251 ; 404)$ & $358(260 ; 432)$ & $271(179 ; 302)$ & $<0.001$ \\
\hline Plasma 25-hydroxyvitamin $\mathrm{D}, \mathrm{nmol} / \mathrm{L}$ & $79(64 ; 104)$ & $84(67 ; 111)$ & $70(47 ; 83)$ & 0.01 \\
\hline Free index 25 -hydroxyvitamin $\mathrm{D}\left(\times 10^{-3}\right)$ & $14(10 ; 19)$ & $14(10 ; 19)$ & $15(10 ; 17)$ & 0.84 \\
\hline Plasma 1,25-dihydroxyvitamin $\mathrm{D}, \mathrm{pmol} / \mathrm{L}$ & $185(156 ; 224)$ & $198(163 ; 241)$ & $158(123 ; 183)$ & 0.01 \\
\hline Free index 1,25 -dihydroxyvitamin $\mathrm{D}\left(\times 10^{-6}\right)$ & $31(26 ; 41)$ & $29(25 ; 41)$ & $36(27 ; 43)$ & 0.10 \\
\hline \multicolumn{5}{|l|}{ Vitamin D status, $N(\%)$} \\
\hline$<50 \mathrm{nmol} / \mathrm{L}$ & $12(16)$ & $6(12)$ & $6(50)$ & \\
\hline $50-75 \mathrm{nmol} / \mathrm{L}$ & $19(25)$ & $12(23)$ & $7(37)$ & $0.15^{2}$ \\
\hline$>75 \mathrm{nmol} / \mathrm{L}$ & $44(59)$ & $34(65)$ & $10(23)$ & \\
\hline Plasma PTH, pmol/L & $3.6(2.9 ; 4.6)$ & $3.3(2.5 ; 4.3)$ & $3.8(3.4 ; 4.6)$ & 0.36 \\
\hline $\begin{array}{l}\text { Plasma calcium total, } \\
\text { albumin adjusted, mmol/L }\end{array}$ & $2.45(2.42 ; 2.52)$ & $2.46(2.43 ; 2.51)$ & $2.45(2.40 ; 2.53)$ & 0.94 \\
\hline Plasma phosphate, $\mathrm{mmol} / \mathrm{L}$ & $1.00(0.93 ; 1.12)$ & $0.97(0.89 ; 1.09)$ & $1.03(0.95 ; 1.21)$ & 0.05 \\
\hline Plasma creatinine, $\mu \mathrm{mol} / \mathrm{L}$ & $64(57 ; 72)$ & $65(57 ; 73)$ & $61(58 ; 69)$ & 0.22 \\
\hline Plasma calcitonin, $\mathrm{pmol} / \mathrm{L}$ & $10(9 ; 12)$ & $10(9 ; 12)$ & $9(8 ; 11)$ & 0.21 \\
\hline $\begin{array}{l}\text { Plasma bone specific } \\
\text { alkaline phosphatase, U/L }\end{array}$ & $17.9(14.8 ; 23.0)$ & $16.5(14.6 ; 21.0)$ & $21.1(14.8 ; 23.8)$ & 0.22 \\
\hline Plasma osteocalcin, $\mu \mathrm{g} / \mathrm{L}$ & $26.9(19.3 ; 30.9)$ & $25.9(19.0 ; 29.6)$ & $29.6(20.6 ; 39.2)$ & 0.07 \\
\hline Urine $\mathrm{NTx} /$ creatinine ratio $(\mathrm{mmol} / \mathrm{mmol})$ & $42.6(30.9 ; 53.0)$ & $39.3(29.5 ; 50.8)$ & $48.7(38.8 ; 57.8)$ & 0.11 \\
\hline Urine calcium/creatinine-ratio (mmol/mmol) & $0.2(0.1 ; 0.4)$ & $0.2(0.1 ; 0.4)$ & $0.3(0.1 ; 0.4)$ & 0.52 \\
\hline
\end{tabular}

${ }^{1}$ Independent-Samples Mann-Whitney U Test; ${ }^{2}$ Chi-Square Tests.

Compared with the non-users, $\mathrm{HC}$ users has a significantly higher plasma concentrations of 25OHD, $1,25(\mathrm{OH})_{2} \mathrm{D}$, and VDBP $(p<0.01)$. The median plasma concentrations of 25OHD, $1,25(\mathrm{OH})_{2} \mathrm{D}$, and VDBP were respectively $16 \%, 13 \%$, and $25 \%$, higher in users compared to non-users of HC. However, FI-25OHD and FI-1,25(OH $)_{2} \mathrm{D}$ did not differ between groups.

Adjustment for between group differences in body weight did not change results.

The prevalence of a $25 \mathrm{OHD}$ concentration below $50 \mathrm{nmol} / \mathrm{L}$ was equal between groups, whereas there were three times as many users with a $25 \mathrm{OHD}$ concentration above $75.1 \mathrm{nmol} / \mathrm{L}$ as non-users of HC (Table 2).

The correlation between the plasma concentration of $25 \mathrm{OHD}$ and $1,25(\mathrm{OH})_{2} \mathrm{D}$ was near significant in both users of $\mathrm{HC}\left(r_{\mathrm{s}}=0.31, p=0.06\right)$ and in non-users of $\mathrm{HC}(r=0.49, p=0.07)$. 
However, 25OHD and VDBP concentrations were not significantly correlated when groups were analyzed separately (in HC users: $r_{\mathrm{s}}=0.15, p=0.29$ and in non-users: $r_{\mathrm{s}}=0.29, p=0.18$ ). Plasma $1,25(\mathrm{OH})_{2} \mathrm{D}$ tended to be positively correlated with VDBP in non-users of $\mathrm{HC}\left(r_{\mathrm{s}}=0.52, p=0.06\right)$, but not in HC users $\left(r_{\mathrm{s}}=0.21, p=0.21\right)$ (Figure 1B).

\subsection{The Effect of Use of Hormonal Contraceptives on Calcium Homeostasis and Bone Turnover}

As shown in Table 2, plasma concentrations of phosphate and osteocalcin were borderline significant lower in users-compared with non-users of HC; whereas no other measured indices differed between groups.

\section{Discussion}

We have studied a group of healthy young Danish women among whom 52 used HC and 23 did not. Our analyses showed significantly higher plasma concentrations of $25 \mathrm{OHD}, 1,25(\mathrm{OH})_{2} \mathrm{D}$ and VDBP in users compared with non-users of HC, FI-25OHD and FI-1,25OH $\mathrm{OH}_{2} \mathrm{D}$ were however not different between groups.

Our findings of increased VDBP concentrations in users of HC agrees with the findings in postmenopausal women receiving postmenopausal hormone substitution. In an earlier study from our group, initiation of postmenopausal oestrogen therapy caused a significant $8 \%$ increase in VDBP concentrations [19]. Similar results have been reported in pregnancy, during which an increase in VDBP concentration is observed [10,18].

Plasma $1,25(\mathrm{OH})_{2} \mathrm{D}$ is known to suppress the secretion of $\mathrm{PTH}$, stimulate the synthesis of osteocalcin and enhance intestinal absorption of calcium and phosphate [27,28]. The latter may be reflected in an increase renal excretion of these minerals [27]. Despite a significant increase in plasma $1,25(\mathrm{OH})_{2} \mathrm{D}$ our data did not show any significant effect of $\mathrm{HC}$ on indices of calcium and phosphate homeostasis or bone metabolism. These findings may support the notion of the free hormone hypothesis, i.e., that only the free fraction of the hormone has biological effects [29].

We assume based on our results and previous reports $[5,10,15,18,19]$ that the estrogen component of HC may increase VDBP synthesis or decrease its catabolism. The concomitant increase in the total plasma $1,25(\mathrm{OH})_{2} \mathrm{D}$ concentration may mirror a compensatory adjustment to maintain an unaltered concentration of the free fraction $[10,16,18]$.

VDBP binding protects vitamin D metabolites from hydroxylase-mediated catabolism; an increase in VDBP may therefore reduce further metabolism of vitamin D metabolites, increasing their half-life. An alternative explanation is that, in parallel with the up regulation of the $1,25(\mathrm{OH})_{2} \mathrm{D}$ concentration, the total $25 \mathrm{OHD}$ concentration is unregulated via unknown mechanisms, to maintain the free concentration of $25 \mathrm{OHD}$, available to tissues. This may potentially explain the higher plasma concentration of the largely unregulated plasma concentration of 25OHD in HC users, however, this needs further investigation.

An important limitation of our study is the relative small sample size and the fact that women were healthy and all had plasma $25 \mathrm{OHD}$ concentrations over $25 \mathrm{nmol} / \mathrm{L}$. This may have limited our ability to detect further potential effects of $\mathrm{HC}$ on calcium homeostasis and bone metabolism through 
variations in VDBP, 25OHD, and 1,25(OH) $2 \mathrm{D}$ in vitamin $\mathrm{D}$ deficiency $(25 \mathrm{OHD}<25 \mathrm{nmol} / \mathrm{L})$. Further studies should therefore aim to investigate effects of $\mathrm{HC}$ in women with vitamin D deficiency. Moreover, investigations in larger groups are needed to assess the effects of $\mathrm{HC}$ on vitamin D metabolites and its effect on muscle and bone, as well as other health outcomes.

\section{Conclusions}

In conclusion, use of $\mathrm{HC}$ is associated with an elevated plasma concentration of VDBP and concomitant higher plasma $25 \mathrm{OHD}$ and $1,25(\mathrm{OH})_{2} \mathrm{D}$. The free-indices of these vitamin $\mathrm{D}$ metabolites are however similar to non-users of $\mathrm{HC}$. The point of emphasis: the use of $\mathrm{HC}$ should be considered in the interpretation of $25 \mathrm{OHD}$ and $1,25(\mathrm{OH})_{2} \mathrm{D}$ vitamin $\mathrm{D}$ concentrations in women. Further studies should aim to clarify whether also in women with a low vitamin D supply, an HC induced increase in VDBP is accompanied by an increase in plasma 25OHD to maintain the free 25OHD level. Further research is also required to assess whether the free 25OHD index is a better marker of 25OHD tissue availability and has a higher correlation with indices of calcium homeostasis and bone metabolism than total 25OHD levels.

\section{Acknowledgments}

We are grateful for the financial support provided to the project from: The Danish Agency for Science, Technology and Innovation, The Aarhus University Research Foundation, AP Moeller Foundation for the Advancement of Medical Science, Svend Fældings Humanitære Fond, The Lundbeck Foundation, Aarhus University Fellowship, and Helga and Peter Kornings Foundation. Inez Schoenmakers and Shailja Nigdikar are supported through the core programme of the Nutrition and Bone Research group at MRC Human Nutrition Research funded by UK MRC (grant code U105960371).

\section{Conflicts of Interest}

The authors are not aware of any affiliations, memberships, funding, or financial holdings that might be perceived as affecting the objectivity of this study.

\section{References}

1. Holick, M.F. Vitamin D status: Measurement, interpretation, and clinical application. Ann. Epidemiol. 2009, 19, 73-78.

2. Mosekilde, L. Vitamin D and the elderly. Clin. Endocrinol. 2005, 62, 265-281.

3. Holick, M.F. Resurrection of vitamin D deficiency and rickets. J. Clin. Investig. 2006, 116, 2062-2072.

4. Hewison, M.; Burke, F.; Evans, K.N.; Lammas, D.A.; Sansom, D.M.; Liu, P.; Modlin, R.L.; Adams, J.S. Extra-renal 25-hydroxyvitamin D3-1alpha-hydroxylase in human health and disease. J. Steroid Biochem. Mol. Biol. 2007, 103, 316-321. 
5. Bikle, D.D.; Gee, E.; Halloran, B.; Kowalski, M.A.; Ryzen, E.; Haddad, J.G. Assessment of the free fraction of 25-hydroxyvitamin $\mathrm{D}$ in serum and its regulation by albumin and the vitamin D-binding protein. J. Clin. Endocrinol. Metab. 1986, 63, 954-959.

6. Bikle, D.D.; Siiteri, P.K.; Ryzen, E.; Haddad, J.G. Serum protein binding of 1,25-dihydroxyvitamin D: A reevaluation by direct measurement of free metabolite levels. J. Clin. Endocrinol. Metab. 1985, 61, 969-975.

7. Holick, M.F.; Chen, T.C. Vitamin D deficiency: A worldwide problem with health consequences. Am. J. Clin. Nutr. 2008, 87, 1080S-1086S.

8. Chan, J.; Jaceldo-Siegl, K.; Fraser, G.E. Determinants of serum 25 hydroxyvitamin D levels in a nationwide cohort of blacks and non-Hispanic whites. Cancer Causes Control 2010, 21, 501-511.

9. Nesby-O’Dell, S.; Scanlon, K.S.; Cogswell, M.E.; Gillespie, C.; Hollis, B.W.; Looker, A.C.; Allen, C.; Doughertly, C.; Gunter, E.W.; Bowman, B.A. Hypovitaminosis D prevalence and determinants among African American and white women of reproductive age: Third National Health and Nutrition Examination Survey, 1988-1994. Am. J. Clin. Nutr. 2002, 76, 187-192.

10. Bouillon, R.; van Assche, F.A.; van Baelen, H.; Heyns, W.; de Moor, P. Influence of the vitamin D-binding protein on the serum concentration of 1,25-dihydroxyvitamin D3. Significance of the free 1,25-dihydroxyvitamin D3 concentration. J. Clin. Investig. 1981, 67, 589-596.

11. Haddad, J.G., Jr.; Walgate, J. Radioimmunoassay of the binding protein for vitamin D and its metabolites in human serum: Concentrations in normal subjects and patients with disorders of mineral homeostasis. J. Clin. Investig. 1976, 58, 1217-1222.

12. Møller, U.; Streym, S.; Heickendorff, L.; Mosekilde, L.; Rejnmark, L. Effects of 25OHD concentrations on chances of pregnancy and pregnancy outcomes. A cohort study in healthy Danish women. Eur. J. Clin. Nutr. 2012, 66, 862-868.

13. Cross, N.A.; Hillman, L.S.; Allen, S.H.; Krause, G.F. Changes in bone mineral density and markers of bone remodeling during lactation and postweaning in women consuming high amounts of calcium. J. Bone Miner. Res. 1995, 10, 1312-1320.

14. Ritchie, L.D.; Fung, E.B.; Halloran, B.P.; Turnlund, J.R.; van Loan, M.D.; Cann, C.E.; King, J.C. A longitudinal study of calcium homeostasis during human pregnancy and lactation and after resumption of menses. Am. J. Clin. Nutr. 1998, 67, 693-701.

15. Aarskog, D.; Aksnes, L.; Markestad, T.; Rodland, O. Effect of estrogen on vitamin D metabolism in tall girls. J. Clin. Endocrinol. Metab. 1983, 57, 1155-1158.

16. Harris, S.S.; Dawson-Hughes, B. The association of oral contraceptive use with plasma 25-hydroxyvitamin D levels. J. Am. Coll. Nutr. 1998, 17, 282-284.

17. Gagnon, C.; Baillargeon, J.P.; Desmarais, G.; Fink, G.D. Prevalence and predictors of vitamin $\mathrm{D}$ insufficiency in women of reproductive age living in northern latitude. Eur. J. Endocrinol. 2010, 163, 819-824.

18. Van Hoof, H.J.; de Sevaux, R.G.; van Baelen, H.; Swinkels, L.M.; Klipping, C.; Ross, H.A.; Sweep, C.G. Relationship between free and total 1,25-dihydroxyvitamin D in conditions of modified binding. Eur. J. Endocrinol. 2001, 144, 391-396. 
19. Rejnmark, L.; Lauridsen, A.L.; Brot, C.; Vestergaard, P.; Heickendorff, L.; Nexo, E.; Mosekilde, L. Vitamin D and its binding protein Gc: Long-term variability in peri- and postmenopausal women with and without hormone replacement therapy. Scand. J. Clin. Lab. Investig. 2006, 66, 227-238.

20. Gravholt, C.H.; Leth-Larsen, R.; Lauridsen, A.L.; Thiel, S.; Hansen, T.K.; Holmskov, U.; Naeraa, R.W.; Christiansen, J.S. The effects of GH and hormone replacement therapy on serum concentrations of mannan-binding lectin, surfactant protein D and vitamin D binding protein in Turner syndrome. Eur. J. Endocrinol. 2004, 150, 355-362.

21. Moller, U.K.; við Streym, S.; Mosekilde, L.; Rejnmark, L. Changes in bone mineral density and body composition during pregnancy and postpartum. A controlled cohort study. Osteoporos. Int. 2012, 23, 1213-1223.

22. Hermann, A.P.; Thomsen, J.; Vestergaard, P.; Mosekilde, L.; Charles, P. Assessment of kalcium intake. A quick method comparerd to a 7 days food diary. Calcif. Tissue Int. 1999, 64, S82.

23. Maunsell, Z.; Wright, D.J.; Rainbow, S.J. Routine isotope-dilution liquid chromatography-tandem mass spectrometry assay for simultaneous measurement of the 25-hydroxy metabolites of vitamins D2 and D3. Clin. Chem. 2005, 51, 1683-1690.

24. Hojskov, C.S.; Heickendorff, L.; Moller, H.J. High-throughput liquid-liquid extraction and LCMSMS assay for determination of circulating $25(\mathrm{OH})$ vitamin D3 and D2 in the routine clinical laboratory. Clin. Chim. Acta 2010, 411, 114-116.

25. Schifter, S. A new highly sensitive radioimmunoassay for human calcitonin useful for physiological studies. Clin. Chim. Acta 1993, 215, 99-109.

26. Mosekilde, L. Vitamin D requirement and setting recommendation levels: Long-term perspectives. Nutr. Rev. 2008, 66, S170-S177.

27. Favus, M. Primer on the Metabolic Bone Diseases and Disorders of Mineral Metabolism, 6th ed.; Bikle, D.D., Christakos, S., Goldring, S.R., Guise, T.H., Holick, M.F., Jan de Beur, S., Kaplan, F.S., Kleerekoper, M., Langman, C.B., Lian, J.B., et al., Eds.; American Society for Bone and Mineral Research: Washington, DC, USA, 2006; pp. 50-132.

28. Henry, H.L.; Norman, A.W. Vitamin D: Metabolism and biological actions. Annu. Rev. Nutr. 1984, 4, 493-520.

29. Mendel, C.M. The free hormone hypothesis: A physiologically based mathematical model. Endocr. Rev. 1989, 10, 232-274. 


\title{
Total Vitamin D Assay Comparison of the Roche Diagnostics "Vitamin D Total" Electrochemiluminescence Protein Binding Assay with the Chromsystems HPLC Method in a Population with both D2 and D3 forms of Vitamin D
}

\author{
Laila Abdel-Wareth, Afrozul Haq, Andrew Turner, Shoukat Khan, Arwa Salem, \\ Faten Mustafa, Nafiz Hussein, Fasila Pallinalakam, Louisa Grundy, Gemma Patras \\ and Jaishen Rajah
}

\begin{abstract}
This study compared two methods of assaying the 25-hydroxylated metabolites of cholecalciferol (vitamin D3) and ergocalciferol (vitamin D2). A fully automated electrochemiluminescence assay from Roche Diagnostics and an HPLC based method from Chromsystems were used to measure vitamin D levels in surplus sera from 96 individuals, where the majority has the D2 form of the vitamin. Deming regression, concordance rate, correlation and Altman Bland agreement were performed. Seventy two subjects $(75 \%)$ had a D2 concentration $>10 \mathrm{nmol} / \mathrm{L}$ while the remaining twenty four subjects had vitamin D2 concentration of less than $10 \mathrm{nmol} / \mathrm{L}$ by HPLC. Overall, the Roche Diagnostics method showed a negative bias of $-2.59 \pm 4.11 \mathrm{nmol} / \mathrm{L}$ on the e602 as compared to the HPLC with a concordance rate of $84 \%$. The concordance rate was $91 \%$ in samples with D2 of less than $10 \mathrm{nmol} / \mathrm{L}$ and $82 \%$ in those with $\mathrm{D} 2$ concentration $>10 \mathrm{nmol} / \mathrm{L}$. The overall correlation had an $r$ value of 0.77 . The $r$ value was higher in samples with D2 levels of less than $10 \mathrm{nmol} / \mathrm{L}, r=0.96$, as compared to those with $\mathrm{D} 2$ values of greater than $10 \mathrm{nmol} / \mathrm{L}$, $r=0.74$. The observed bias had little impact on clinical decision and therefore is clinically acceptable.
\end{abstract}

Reprinted from Nutrients. Cite as: Abdel-Wareth, L.; Haq, A.; Turner, A.; Khan, S.; Salem, A.; Mustafa, F.; Hussein, N.; Pallinalakam, F.; Grundy, L.; Patras, G.; Rajah, J. Total Vitamin D Assay Comparison of the Roche Diagnostics "Vitamin D Total" Electrochemiluminescence Protein Binding Assay with the Chromsystems HPLC Method in a Population with both D2 and D3 forms of Vitamin D. Nutrients 2013, 5, 971-980.

\section{Introduction}

The last decade has witnessed a dramatic increase in both clinical and public awareness of the health implications associated with vitamin D status [1]. Consequently, clinical laboratories are receiving an increasing number of requests to measure vitamin D levels, which has led to the need for highly automated assays. A recent publication evaluated the performance of six routine 25-Hydroxyvitamin D assays in relation to variation in vitamin D binding protein concentration [2]. The majority of the study population had the D3 form of the vitamin (cholecalciferol). In contrast, our study evaluates the performance of one of those assays, the Roche Diagnostics "Vitamin D total" electrochemiluminescence protein binding assay, in a population where the majority have the D2 form of the vitamin (ergocalciferol). The technical performance of the Roche assay was evaluated by comparison to the Chromsystems HPLC-UV assay and the clinical performance was assessed in terms of classification of subjects as sufficient or deficient for the vitamin. 


\section{Material and Methods}

\subsection{Samples}

Left over sera obtained from specimens analyzed at the SKMC Pathology \& Laboratory Medicine Institute $(n=96)$ were used in this study. The use of this material was approved by the institutional ethics review board and is in accordance with the general consent signed by all patients prior to treatment at SKMC. Serum aliquots were given unique sample numbers, to conceal the identity of the patient from the staff performing the study. These aliquots were stored at $2-8{ }^{\circ} \mathrm{C}$ and analyzed within two days.

\subsection{Analytical Platforms}

\subsubsection{Chromsystems HPLC Assay}

The Chromsystems reagent kit used on the Waters HPLC 2695 allows the main metabolites of vitamin D3 and D2 to be determined in a simultaneous chromatographic manner by using a fully validated, modified high-performance liquid chromatography (HPLC) method [3]. The Waters HPLC 2695 analyzer uses a simple isocratic HPLC system, with a HPLC pump, injector and a UV detector. In summary, protein is precipitated, and through selective solid phase extraction, interfering components are removed and the analytes are concentrated. A stable vitamin D derivative is used as an internal standard in order to allow for accurate quantification. The chromatographic separation takes approximately 12 min (Chromsystems Instruments \& Chemicals GmbH, Heimburgstrasse, Munich, Germany) [3]. The assay has within run imprecision of $3.0 \%$ and total (between days) imprecision of $4.6 \%$.

\subsubsection{Roche Diagnostics Vitamin D Total Assay}

The Roche Diagnostics Vitamin D total assay is a competitive electrochemiluminescence protein binding assay intended for the quantitative determination of total $25-\mathrm{OH}$ vitamin $\mathrm{D}$ in human serum and plasma. The assay employs a vitamin D binding protein (VDBP) as capture protein, which binds to both 25-OH D3 and 25-OH D2 (Roche Diagnostics, Mannheim, Germany) [4].

The assay utilizes a 3-step incubation process, which has a duration of 27 minutes. In step 1, the sample is incubated with pretreatment reagent, which releases bound $25-\mathrm{OH}$ vitamin $\mathrm{D}$ from the VDBP. In step 2, the pretreated sample is incubated with ruthenium labeled VDBP creating a complex between the 25-OH vitamin D and the ruthenylated VDBP. The third incubation step sees the addition of streptavidin-coated microparticles and 25-OH vitamin D labeled with biotin. The free sites of the ruthenium labeled VDBP become occupied, forming a complex consisting of the ruthenium labeled vitamin $\mathrm{D}$ binding protein and the biotinylated $25-\mathrm{OH}$ vitamin $\mathrm{D}$. The entire complex becomes bound to the solid phase via interaction of biotin and streptavidin.

Between day precision was $\mathrm{CV}=4.9 \%$ and $1.9 \%$ at mean concentrations of 43.3 and $105 \mathrm{nmol} / \mathrm{L}$ respectively using quality control material provides by Roche Diagnostics. 
Both assays were validated in our laboratory following "Clinical Laboratory Standards Institute" (CLSI) protocols for validation of precision, linearity and accuracy.

Reference ranges used in this study were based upon the recommendations of the American Society for Bone and Mineral Research, 28th Annual Meeting 2006 and the Canadian consensus conference on osteoporosis, 2006 [5,6] and were defined as follows: Deficiency: $<25 \mathrm{nmol} / \mathrm{L}$, Optimal/Sufficiency: 75-200 nmol/L, Insufficiency: 25-75 nmol/L and Toxicity: $>250 \mathrm{nmol} / \mathrm{L}$. This study also considered the latest recommendations published by the Institute of Medicine (IOM) for dietary reference intake for calcium and vitamin D. According to the latest IOM recommendations, $25(\mathrm{OH}) \mathrm{D}$ levels corresponding to a serum $25(\mathrm{OH}) \mathrm{D}$ status of at least $50 \mathrm{nmol} / \mathrm{L}$ indicates sufficiency [1].

\subsubsection{Statistical Analysis}

All data points were included in the study. Results were classified into three groups; the entire population, those with vitamin D2 concentration of less than $10 \mathrm{nmol} / \mathrm{L}$ and those with vitamin D2 concentration greater than $10 \mathrm{nmol} / \mathrm{L}$. Method comparison was performed by using Deming regression. Method agreement was analyzed by the mean difference method of Bland and Altman. Pearson correlation was also calculated for the three groups. In addition, linear regression for the difference between the Roche method and the HPLC method in relation to concentration of vitamin D2 and D3 was performed to determine the influence of increasing concentrations of each of the forms respectively. Analysis was performed using "Analyse-it" (The Tannery, 91 Kirkstall Road, Leeds, LS3 1HS, UK) and Microsoft Excel softwares (Thames Valley Park Reading, Berkshire, RG6 $1 \mathrm{WG}, \mathrm{UK})$.

The concordance rate was calculated by classifying the results obtained on each platform as sufficient, insufficient or deficient using the criteria already established in our laboratory as stated earlier as well as those recently suggested by the Institute of Medicine (IOM) [1].

Secondary analysis was performed to determine the predicted bias as the data demonstrated a non-constant scatter. The data was classified into three groups (low, middle and high) in order of increasing values based on the HPLC results. Predicted bias was then calculated using partitioned residuals as described in CLSI guideline EP09-A2-IR Section 6.3. Polynomial regression analysis was performed to determine the polynomial fit equation for each group.

\section{Results}

\section{Samples}

A total of 96 samples were analyzed. Seventy two samples had D2 concentrations greater than $10 \mathrm{nmol} / \mathrm{L}$ as detected by the HPLC, while in the remaining twenty four the concentration of D2 was less than $10 \mathrm{nmol} / \mathrm{L}$. None of the samples analyzed were hemolyzed or lipemic. The mean, standard deviation, median and the range were; mean 65.22 and $62.12 \mathrm{nmol} / \mathrm{L} \pm 30.38$ and 29.54 , median 60.90 and $57.8 \mathrm{nmol} / \mathrm{L}$, range $16.3-180.9$ and $7.5-175.0 \mathrm{nmol} / \mathrm{L}$, for the Chomsystem HPLC and Roche Diagnostics Cobas e602 respectively. 
The Roche Diagnostics method demonstrated a negative bias of $-2.59 \mathrm{nmol} / \mathrm{L}(95 \% \mathrm{CI}=-6.7-1.52)$ when compared to the HPLC method using the Bland-Altman analysis (Figure 1).

Figure 1. Deming regression of total 25(OH) D comparison: Chromsystems HPLC as the reference method vs. Roche Diagnostics Vitamin D total method on the Cobas e602.

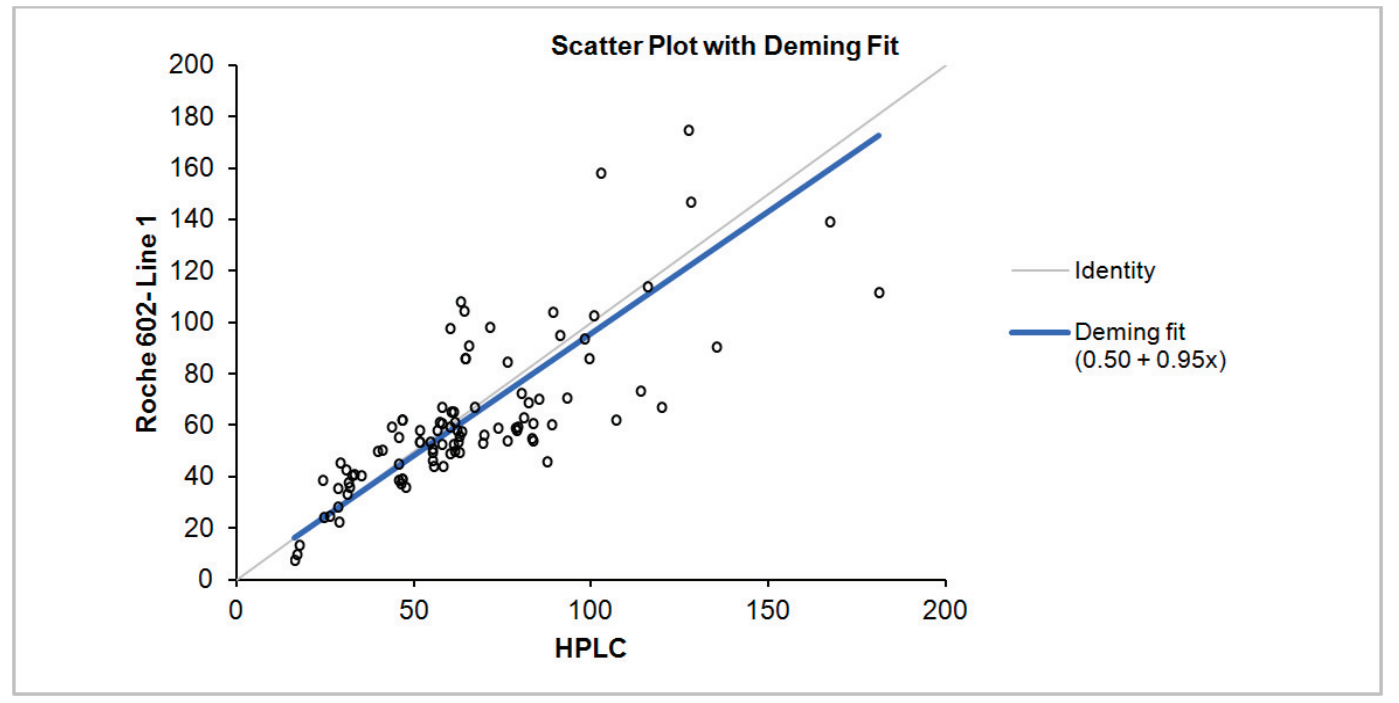

Deming regression result, correlation, slope and intercept for all samples, samples with D2 concentrations less than and greater than $10 \mathrm{nmol} / \mathrm{L}$ is summarized in Table 1. The difference between the two methods is dependent on the concentration of D2 and D3 with negative bias observed more with increasing D2 concentrations and positive bias with increasing D3 concentration (Figure 2). Linear regression analysis scatter plot of the difference in concentration between Roche Diagnostics total $25(\mathrm{OH})$ and HPLC as a function of D2 and D3 concentrations is shown in Figure 3.

Table 1. Person correlation, bias as calculated by Bland-Altman comparison, slope and intercept according to Deming regression for all samples, samples with D2 concentration less and more than $10 \mathrm{nmol} / \mathrm{L}$ as determined by HPLC.

\begin{tabular}{ccccccc}
\hline Sample Group & $\boldsymbol{n}$ & Concentration Range in nmol/L & $\boldsymbol{r}$ & Bias & Slope & Intercept \\
\hline All samples & 96 & $16.30-180.9$ & 0.77 & -2.59 & 0.95 & 0.5 \\
D2 concentration $<10 \mathrm{nmol} / \mathrm{L}$ & 24 & $16.30-127.80$ & 0.96 & 10.14 & 1.43 & -11.81 \\
D2 concentration $>10 \mathrm{nmol} / \mathrm{L}$ & 72 & $16.90-180.90$ & 0.74 & -6.63 & 0.79 & 8.07 \\
\hline
\end{tabular}


Figure 2. Bland-Altman plot showing means of paired difference between the HPLC method and the Roche Diagnostics Cobas e602 in samples with D2 greater than and less than $10 \mathrm{nmol} / \mathrm{L}$ respectively.
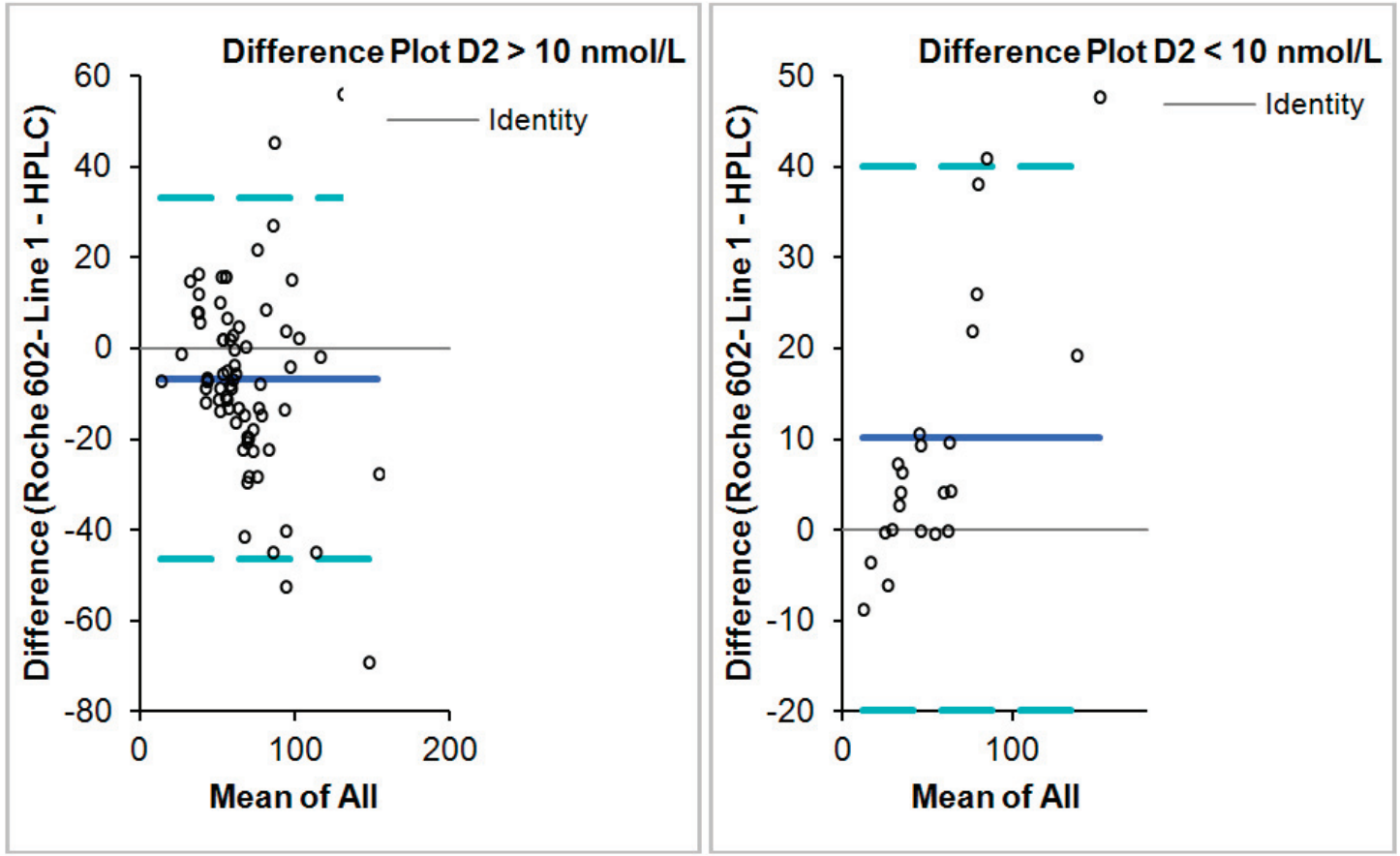

Figure 3. Linear regression analysis scatter plot of the difference in concentration between Roche Diagnostics total $25(\mathrm{OH})$ and HPLC as a function of D2 and D3 concentrations.
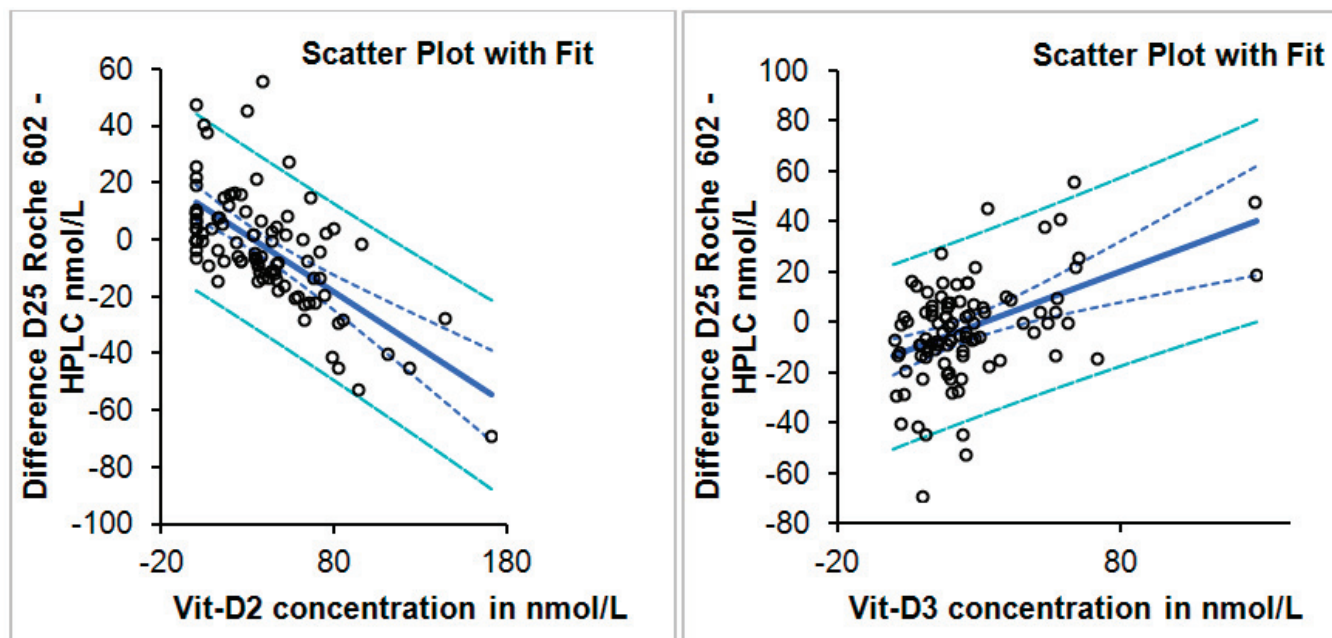

The concordance rate between the Cobas e602 and HPLC was $74 \%$ using the $75 \mathrm{nmol} / \mathrm{L}$ cutoff value as indication of vitamin D sufficiency. When applying the cutoff proposed by the IOM [1] the concordance rate increased to $84 \%$. The concordance rate was $91 \%$ in samples with D2 of less than $10 \mathrm{nmol} / \mathrm{L}$ and $82 \%$ in those with D2 concentration $>10 \mathrm{nmol} / \mathrm{L}$. The majority of the disagreement was in the sufficient to insufficient range as the Roche method slightly underestimated at the higher 
concentrations ( $>50 \mathrm{nmol} / \mathrm{L}$ ). There was no major discrepancy as to sufficiency versus deficiency noted. The detail of the sub classification is summarized in Table 2 for the two cutoffs respectively.

Table 2. Concordance of HPLC and Roche Diagnostics assays to $25(\mathrm{OH}) \mathrm{D}$ based on 2 decision criteria.

\begin{tabular}{|c|c|c|c|c|c|c|c|c|c|}
\hline \multicolumn{5}{|c|}{ Concordance Based on the $75 \mathrm{nmol} / \mathrm{L}$ Cutoff } & \multicolumn{5}{|c|}{ Concordance Based on the $50 \mathrm{nmol} / \mathrm{L}$ Cutoff } \\
\hline \multirow{6}{*}{$\begin{array}{l}\frac{0}{0} \\
\stackrel{0}{0}\end{array}$} & $70-25$ & & & & \multirow{6}{*}{$\begin{array}{l}\frac{0}{8} \\
0 \\
0\end{array}$} & $50-30$ & \multirow{2}{*}{\multicolumn{3}{|c|}{ HPLC }} \\
\hline & Cutoff & & HPLC & & & Cutoff & & & \\
\hline & \multirow[b]{2}{*}{ Sufficient } & Sufficient & Insufficient & Dificient & & \multirow[b]{2}{*}{ Sufficient } & Sufficient & \multirow{2}{*}{$\begin{array}{c}\text { Insufficient } \\
6\end{array}$} & \multirow{2}{*}{$\begin{array}{c}\text { Dificient } \\
0\end{array}$} \\
\hline & & 13 & 7 & 0 & & & 60 & & \\
\hline & Insufficient & 17 & 53 & 1 & & Insufficient & 7 & 15 & 2 \\
\hline & Dificient & 0 & 0 & 5 & & Dificient & 0 & 0 & 6 \\
\hline
\end{tabular}

The calculated predicted biases as absolute values and percentages at four medical decision limits ( 25 and $75 \mathrm{nmol} / \mathrm{L})$ and (30 and $50 \mathrm{nmol} / \mathrm{L})$ are summarized in Table 3.

Table 3. Calculated predicted biases at four medical decision cutoffs for low, middle and high groups.

\begin{tabular}{|c|c|c|c|}
\hline $\begin{array}{l}\text { Medical } \\
\text { Decision Cutoff }\end{array}$ & $\begin{array}{l}\text { Group } 1 \\
(16.3-51.5 \mathrm{nmol} / \mathrm{L}) \\
\text { Predicted Bias (\%) }\end{array}$ & $\begin{array}{l}\text { Group } 2 \\
(54.2-69.6 \mathrm{nmol} / \mathrm{L}) \\
\text { Predicted Bias (\%) }\end{array}$ & $\begin{array}{l}\text { Group } 3 \\
(71.1-180.9 \mathrm{nmol} / \mathrm{L}) \\
\text { Predicted Bias (\%) }\end{array}$ \\
\hline $25 \mathrm{nmol} / \mathrm{L}$ & $2.6(10.4 \%)$ & Not applicable for this group & Not applicable for this group \\
\hline $30 \mathrm{nmol} / \mathrm{L}$ & $2.9(9.6 \%)$ & Not applicable for this group & Not applicable for this group \\
\hline $50 \mathrm{nmol} / \mathrm{L}$ & Not applicable for this group & $-4.0(8 \%)$ & $-1.57(3 \%)$ \\
\hline $75 \mathrm{nmol} / \mathrm{L}$ & Not applicable for this group & $11.27(15 \%)$ & $-7.7(10.3 \%)$ \\
\hline
\end{tabular}

\section{Discussion}

Testing for vitamin D is required not only to screen for its deficiency, but also increasingly to adjust "therapeutic targets" and monitor efficacy of treatment. In addition to the skeletal effects, vitamin D may have a role in relation to diabetes, cancer and cardiovascular diseases [7-13]. Growing awareness of the clinical importance of vitamin D has resulted in clinical laboratories receiving a surge in requests for the assay. This has led to a need to migrate vitamin $\mathrm{D}$ testing from labor intensive methods, such as HPLC, to more highly automated testing platforms. Until recently the major challenge with vitamin D analysis has been the lack of standardization and the wide analytical variation between methods due to absence of reference standards [14]. The situation is further complicated by the discovery of C-3 epimers of vitamin D2 and D3 particularly in infants that might interfere with the accurate measurement and interpretation of vitamin D status in infants [15]. Recently, the National Institute of Standards and Technology (NIST) developed standard reference materials (SRMs) for 25(OH) D3/D2 in both human serum (SRM 972) and in solution (SRM 2972) [16]. This was supported by the recent introduction of reference measurement procedures using isotope - dilution liquid chromatography-tandem mass spectrometry [17]. 
The Roche Diagnostics Total Vitamin D kit has $80 \%$ cross reactivity to D2 and $100 \%$ cross reactivity to $\mathrm{D} 3$. This method was recently evaluated and its performance was deemed satisfactory in a population where D3 constituted the main form of total vitamin D [2]. In the United Arab Emirates, vitamin D deficiency is prevalent and the majority of the deficient population is receiving D2 supplementation [18]. In this study $75 \%$ of the participants had D2 levels $>10 \mathrm{nmol} / \mathrm{L}$. In this population, the Roche Diagnostics method had an overall negative bias, which was directly dependent on the increasing concentration of D2 as compared to the HPLC method. However, it was also observed that the method has positive bias, which was dependent on the concentration of D3 and therefore the overall negative bias was attenuated when both forms are present. More subjects were classified as "insufficient" by the Roche Diagnostics method. However, the clinical impact of the slight underestimation, one can argue, is of little clinical significance giving the wide range for the optimum level of vitamin D $(50-150 \mathrm{nmol} / \mathrm{L})$. The analytical quality goals for 25 -vitamin $\mathrm{D}$ based on biological variation were a subject of a recent publication [19]. According to this publication, the desirable analytical bias goal is around $10 \%$ and $6 \%$ for the imprecision [20]. The calculated predicted bias using the was found to be within 10\% when the IOM decision criteria of 30 and 50 $\mathrm{nmol} / \mathrm{L}$ were applied and within $15 \%$ when the criteria of American Society for Bone and Mineral Research were applied. The method has an imprecision of less than $5 \%$. We subscribe to the UK-based DEQAS vitamin D external quality assessment scheme. According to DEKAS 2012 review report, the Roche total vitamin D method had a mean \% bias of less than $10 \%$ from all laboratory trimmed mean (ALTM) while the IDS RIA and IDS iSYS assays had a high positive bias which reached up to $21 \%$. LC-MS method had positive bias according to this review of less than $10 \%$ while HPLC method had a positive bias slightly higher than $10 \%$ in one of the cycles [21]. Since both methods in this study used different standards, a clear cut $100 \%$ concordance on all samples might not be achievable. Adding to the lack of standardization are the inherent technical limitations of the protein binding assays relative to the direct chemical methods, the effect of vitamin $\mathrm{D}$ binding protein concentration of the results and the possible interferences from the C-3 epimers of D2 or D3 forms on the HPLC method [15].

\section{Conclusions}

In conclusion, Roche Diagnostics "Vitamin D total" assay performance in a population where the D2 component is negatively biased when compared to the HPLC Chromsystems method, and this is directly related the concentration of D2. The negative bias is less often observed when D3 is also present. The negative bias may impact classification of individuals receiving the test in terms of sufficiency or insufficiency for vitamin D and it should be taken in consideration when interpreting results of patients on D2 supplementation. The observed bias had little impact on clinical decision when the IOM criteria were applied and therefore is clinically acceptable.

\section{Acknowledgments}

We thank Bindu Madhavi and Aurora Mabini for their help in formatting this manuscript. We also thank Roche Diagnostics for donating the total Vitamin D kits used in this study. 


\section{Conflicts of Interest}

Competing interests: The authors have no competing interests to disclose.

Financial interests: The authors have none to declare.

Funding: This research was funded by Sheikh Khalifa Medical City. Roche diagnostics provided free kits for evaluation, and purchased from the College of American Pathologist vitamin D accuracy based survey material that was used in this study.

Ethical approval: This study was approved by the institution review board of Sheikh Khalifa Medical City (reference number: REC-04.08.2011 approval number RS:177).

Guarantor: LA-W.

Contribution: LA-W conceived the study, analyzed the data, submitted the research protocol to the institutional review board and wrote the manuscript. AH \& SK, researched the literature and contributed to the introduction and discussion sections of the manuscript. AS, FM, NH \& GP performed the actual analysis on both platforms. LG \& FP wrote the methods section and provided technical supervision for the analytical part of study. AT assisted in the statistical analysis. JR was involved in the protocol development, review of the data, review of the results \& conclusion and assisted in gaining ethical approval by providing guidelines.

\section{References}

1. Ross, C.; Manson, J.A.E.; Abrams, S.A.; Aloia, J.F.; Brannon, P.M.; Clinton, S.K.; Durazo-Arvizu, R.A.; Gallagher, J.C.; Gallo, R.L.; Jones, G.; et al. The 2011 report on dietary reference intakes for calcium and Vitamin D from the Institute of Medicine: What clinicians need to know. J. Clin. Endocrinol. Metab. 2011, 96, 53-58.

2. Heijboer, A.; Blankenstein, M.; Kema, I.P.; Buijs, M.M. Accuracy of 6 Routine 25-Hydroxyvitamin D assays: Influence of vitamin D binding protein concentration. Clin. Chem. 2012, 58, 543-548.

3. Haq, A.; Rajah, J.; Abdel-Wareth, L.O. Routine HPLC analysis of vitamin D3 and D2. DIALOG (Ger.) 2007, 2, 1-2.

4. Cobas E411 Vitamin D Total Reagent Insert (06268668001V1). Roche Diagnostics Web site. Available online: http:/www.captodayonline.com/productguides/instruments/automatedimmunoassay-analyzers-july-2012/roche-diagnostics-cobas-e411-immunoassay-analyzers-june2011.html (accessed on 19 March 2013).

5. Brown, J.P.; Fortier, M.; Frame, H.; Lalonde, A.; Papaioannou, A.; Senikas, V.; Yuen, C.K. Canadian Consensus Conference on Osteoporosis, Update. J. Gynecol. Canad. 2006, 172, S95-S112.

6. Hollick, M.F. Vitamin D deficiency. N. Engl. J. Med. 2007, 357, 266-281.

7. Joergensen, C.; Hovind, P.; Schmedes, A.; Parving, H.H.; Rossing, P. Vitamin D Levels, microvascular cmplications and mortality in Type 1 Diabetes. Diabetes Care 2011, 34, $1081-1085$. 
8. Wang, T.J.; Pencina, M.J.; Booth, S.L.; Jacques, P.F.; Ingelsson, E.; Lanier, K.; Benjamin, E.J.; D’Agostino, R.B.; Wolf, M.; Vasan, R.S. Vitamin D deficiency and risk of cardiovascular disease. Circulation 2008, 117, 503-511.

9. Lappe, J.M.; Travers-Gustafson, D.; Davies, K.M.; Recker, R.R.; Heaney, R.P. Vitamin D and calcium supplementation reduces cancer risk: Results of a randomized trial. Am. J. Clin. Nutr. 2007, 85, 1586-1591.

10. Rosen, C.J. Vitamin D insufficiency. N. Engl. J. Med. 2011, 364, 248-254.

11. Hanley, D.A.; Cranney, A.; Jones, G.; Whiting, S.J.; Leslie, W.D.; Cole, D.E.C.; Atkinson, S.A.; Josse, R.G.; Feldman, S.; Kline, G.A.; et al. Vitamin D in adult health and disease: A review and guideline statement from osteoporosis Canada. Can. Med. Assoc. J. 2010, 182, E610-E618.

12. Norman, A.W.; Bouillon, R.; Whiting, S.J.; Veith, R.; Lips, P. 13th Workshop consensus for vitamin D nutritional guidelines. J. Steroid Biochem. Mol. Biol. 2007, 103, 204-205.

13. Manson, J.E.; Mayne, S.T.; Clinton, S.K. Vitamin D and prevention of cancer-Ready for prime time? N. Engl. J. Med. 2011, 364, 1385-1387.

14. Phinney, K.W. Development of a standard reference material for vitamin D in serum. Am. J. Clin. Nutr. 2008, 88, 511S-512S.

15. Singh, R.J.; Taylor, R.L.; Reddy, G.S.; Grebe, S.K.G. C-3 Epimers can account for a significant proportion of total circulating 25-Hydroxyvitamin D in infants, complicating accurate measurement and interpretation of Vitamin D status. J. Clin. Endocrinol. Metab. 2006, 91, 3055-3061.

16. May, W.; Parris, R.; Beck, C.; Fassett, J.; Greenberg, R.; Guenther, F.; Kramer, G.; Wise, S.; Gills, T.; Colbert, J.; et al. Definitions of Terms and Modes Used at NIST for Value-Assignment of Reference Materials for Chemical Measurements; National Institute of Standards and Technology: Gaithersburg, MD, USA, 2000; Available online: http://www.cstl.nist.gov/nist839/ NIST_special_publications.htm (accessed on 19 March 2013).

17. Gaithersburg. Certificate of Analysis, Standard Reference Material 2972: 25-Hydroxivitamin D2 and D3 Calibration Solutions. Standard Reference Materials Program; National Institute of Standards and Technology: Gaithersburg, MD, USA, 2009; Available online: http://www.nist. gov/srm/upload/March-2010-Spotlight-3.pdf (accessed on 19 March 2013).

18. Al Anouti, F.; Thomas, J.; Abdel-Wareth, L.; Rajah, J.; Grant, W.; Haq, A. Vitamin D deficiency and sun avoidance among university students at Abu Dhabi, United Arab Emirates. Dermato-Endocrinology 2011, 3, 235-239.

19. Stepman, H.C.M.; Vanderroost, A.; Uytfanghe, K.V.; Thienpont, L.M. Candidate reference measurement procedures for serum 25-Hydroxyvitamin D3 and 25-Hydroxyvitamin D2 by using isotope-dilution liquid chromatography-tandem mass spectrometry. Clin. Chem. 2011, 57, 441-448.

20. Viljoen, A.; Singh, D.; Farrington, K.; Twomey, P. Analytical quality goals for 25-vitamin D based on biological variation. J. Clin. Lab. Anal. 2011, 25, 130-133.

21. Knudsen, C.S.; Nexo, E.; Højskov, C.S.; Heickendorff, L. Analytical validation of the Roche 25-OH Vitamin D Total assay. Clin. Chem. Lab. Med. 2012, 50, 1965-1968. 
MDPI AG

Klybeckstrasse 64

4057 Basel, Switzerland

Tel. +41 616837734

Fax +41613028918

http://www.mdpi.com/

Nutrients Editorial Office

E-mail: nutrients@mdpi.com

http://www.mdpi.com/journal/nutrients 


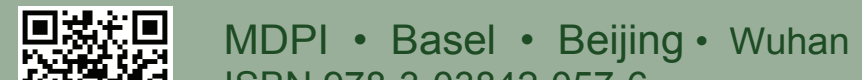

34 ISBN 978-3-03842-057-6

回梠! www.mdpi.com

MPPI

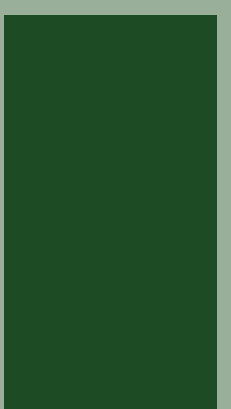

